\title{
WestVirginiaUniversity
}

THE RESEARCH REPOSITORY @ WVU

Graduate Theses, Dissertations, and Problem Reports

2012

\section{The Effects of Index Storage on Ranked Information Retrieval}

James E. Mantheiy Jr.

West Virginia University

Follow this and additional works at: https://researchrepository.wvu.edu/etd

\section{Recommended Citation}

Mantheiy, James E. Jr., "The Effects of Index Storage on Ranked Information Retrieval" (2012). Graduate Theses, Dissertations, and Problem Reports. 636.

https://researchrepository.wvu.edu/etd/636

This Thesis is protected by copyright and/or related rights. It has been brought to you by the The Research Repository @ WVU with permission from the rights-holder(s). You are free to use this Thesis in any way that is permitted by the copyright and related rights legislation that applies to your use. For other uses you must obtain permission from the rights-holder(s) directly, unless additional rights are indicated by a Creative Commons license in the record and/ or on the work itself. This Thesis has been accepted for inclusion in WVU Graduate Theses, Dissertations, and Problem Reports collection by an authorized administrator of The Research Repository @ WVU. For more information, please contact researchrepository@mail.wvu.edu. 
The Effects of Index Storage on Ranked Information Retrieval

James E. Mantheiy, Jr.

Thesis submitted to the College of Engineering and Mineral Resources at West Virginia University in partial fulfillment of the requirements for the degree of

Masters of Science

in

Electrical Engineering

Roy S. Nutter, Ph.D., Chair

Bojan Cukic, Ph.D.

Tim Menzies, Ph.D.

Lane Department of Computer Science

And Electrical Engineering

Morgantown, West Virginia

2012

Keywords: Information Retrieval, Relational Database Search, Inverted Index 


\section{ABSTRACT \\ The Effects of Index Storage on Ranked Information Retrieval}

James E. Mantheiy, Jr.

Information retrieval is the process of recalling and ordering all relevant documents based on a user's search query. Examples of information retrieval systems are Google, Bing, and Yahoo search. In order to perform an effective search, these systems utilize an inverted index for mapping content, such as words, to the original document. It is widely believed there are two options for implementing an inverted index and these options are in memory or as a file. This investigation looks at implementing an inverted index as a table in a database as compared to the other two options. In addition, this investigation will look at the optimal combination of inverted index implementation to retrieval algorithms such as TD-IDF, Best Match 25, and a unigram model with Jelinek-Mercer smoothing. This is determined by designing and developing a system which will index and search three different collections of various data, size, and complexities. By doing this, it is found that utilizing an inverted index implemented in a database is a viable option for information retrieval. It is also noteworthy that Best Match 25 or a unigram language model consistently outperforms TD-IDF. In conclusion, if the collection cannot be indexed in memory, then utilizing a database implemented index is a sufficient second option. 
"Knowledge is a process of piling up facts; wisdom lies in their simplification." -Martin H. Fischer 


\section{ACKNOWLEDGEMENTS}

I would like to take a moment to recognize those who have made my thesis, my college career, and all that I am blessed to have in my life. To my fiancée Ashley, whose faith in me and all her sacrifices that allowed to achieve all my goals over the past two years. My parents and sister whom have always been there for me even in the toughest of times and supported me in whatever endeavors I have taken on during my college career. To my grandparents who have open their home to me during the past 6.5 years as a shelter from the insanities of college.

I would like to take this time to recognize my advisor and mentor Dr. Roy S. Nutter, Jr. who convinced me to come back to West Virginia University and seek my Masters degree. His advice has been like a compass in guiding me towards excellences in and out of the classroom. To Dr. Bojan Cukic, who tried to understand my documents and my thinking when guiding me in selecting and developing my thesis. Dr. Tim Menzies, who taught to me go beyond the applicable and expand on it until you're in another world. Ms. Cindy Tanner for her answers to questions that boggled my mind for days.

Finally, I'd like to recognize the Lane Department Systems team for putting up with my endless amount of tickets and the entire Lane Department for making the last 6.5 years one of the best segments of my life. To SAIC who allowed me to keep my position and seek out the tree of knowledge and continue to learn. Lastly, I'd like to recognize WV State Police, National White Collar Crime, and NTCFA, most notably First Sergeant Chris M. Casto, Jeremiah Johnson, Michael Shoukry and, Aaron Naternicola. 


\section{Table of Contents}

ACKNOWLEDGEMENTS --:---:-

TABLE OF CONTENTS --:-

TABLE OF TABLES --:--:-

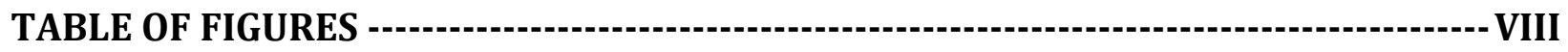

CHAPTER 1: INTRODUCTION -- 1

1.1 Introduction --1

1.2 Statement of Problem --

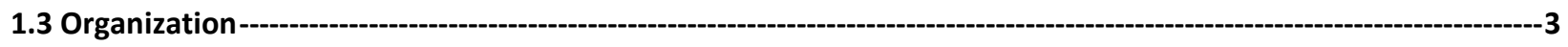

CHAPTER 2: LITERATURE REVIEW-- 4

2. 1 Current Keyword search Database Systems -

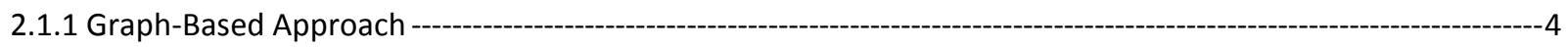

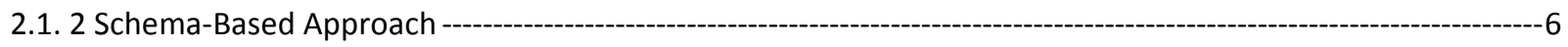

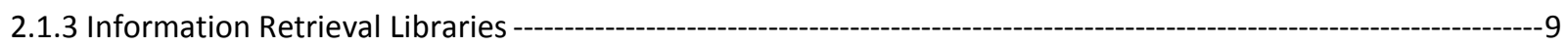

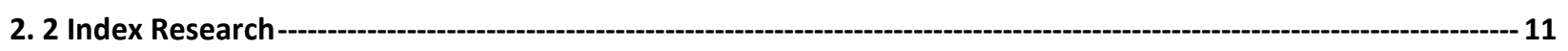

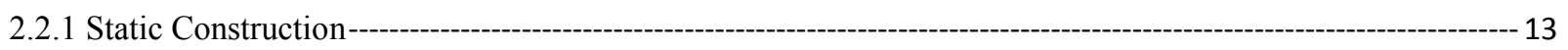

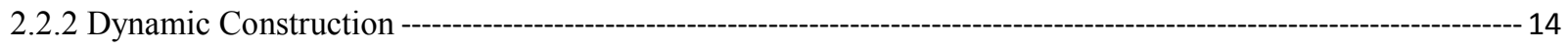

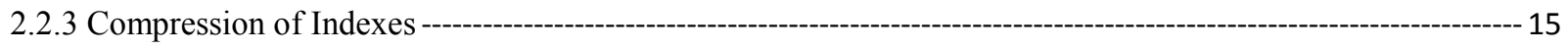

2.3 Models/Retrieval Methods -

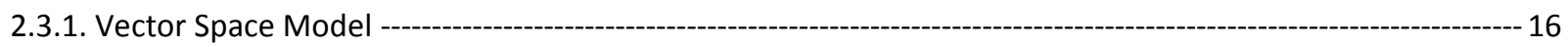

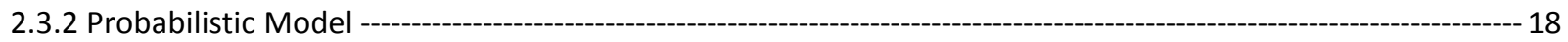

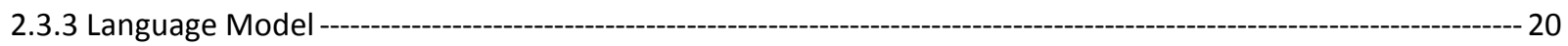

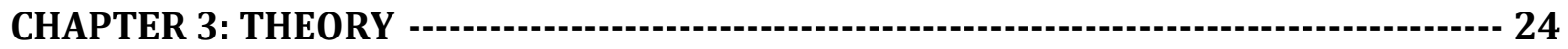

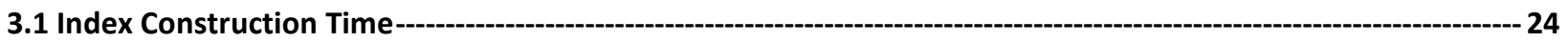

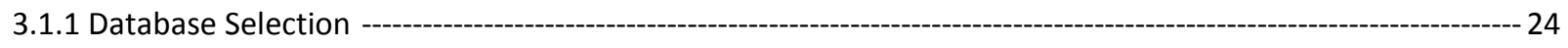

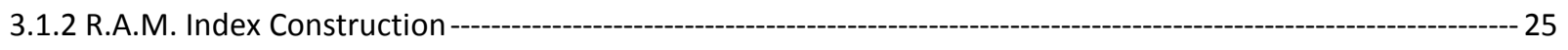

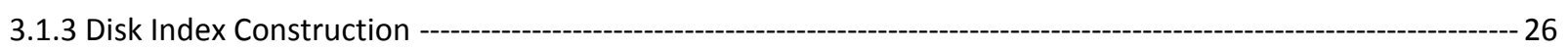

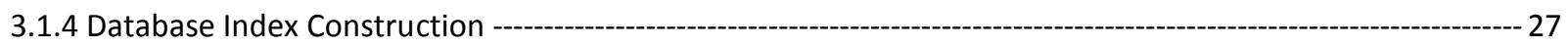




\subsection{Retrieval Time}

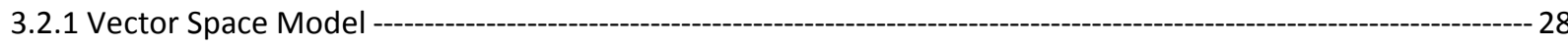

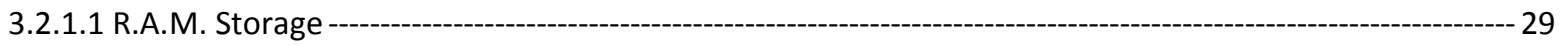

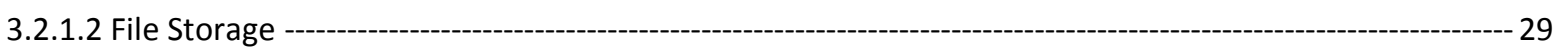

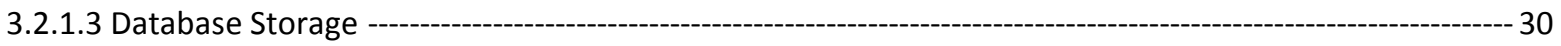

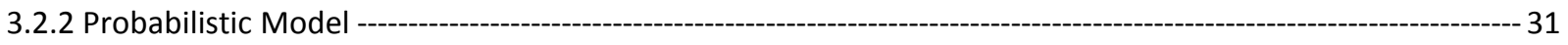

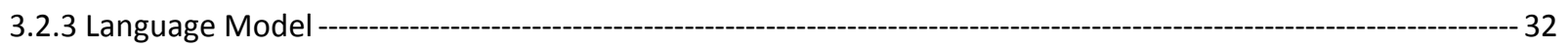

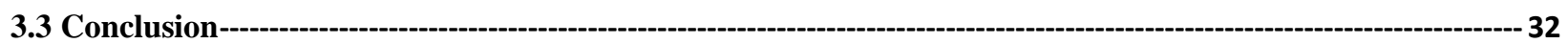

CHAPTER 4: CASE STUDIES -

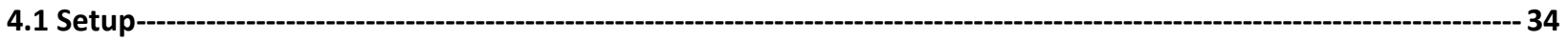

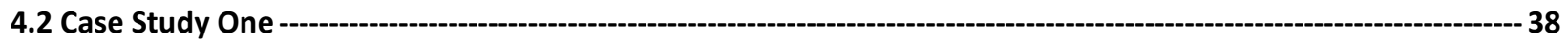

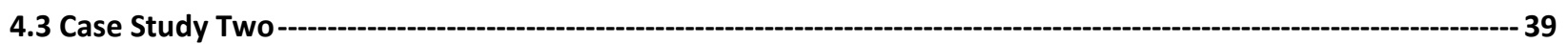

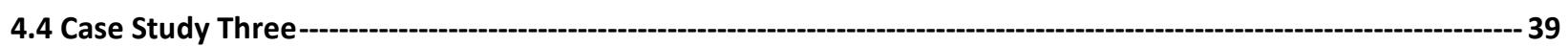

CHAPTER 5: RESULTS -

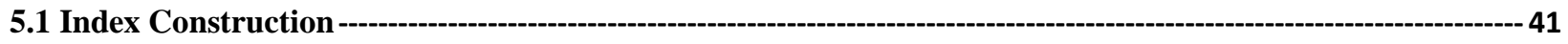

5.1.1 Case Study One Indexing ---

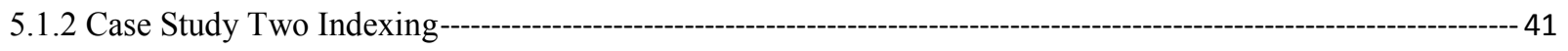

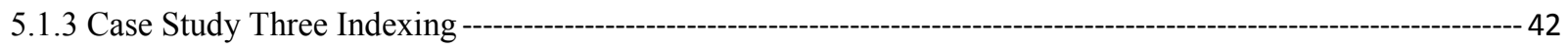

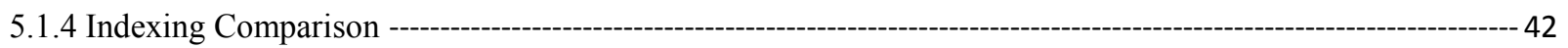

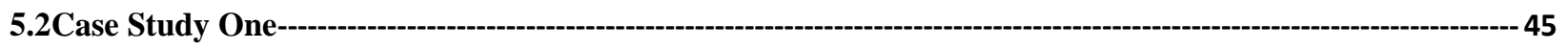

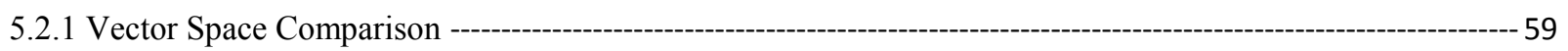

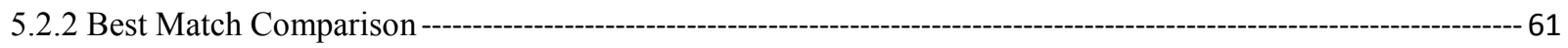

5.2.3 Language Model Comparison----------------------------------------------------------------------------------------------- 63

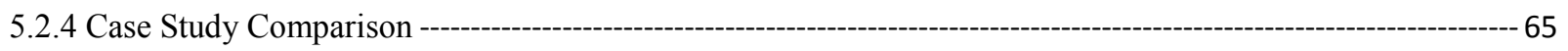

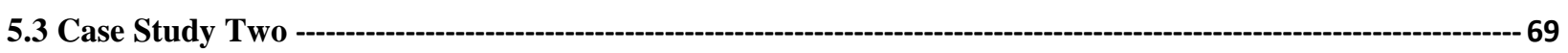

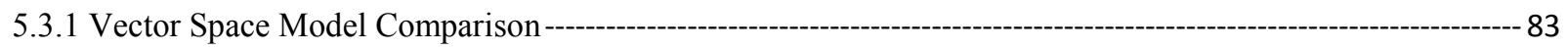

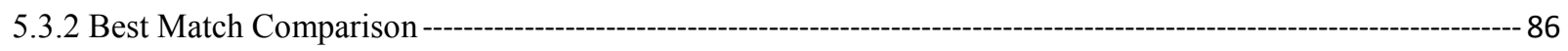

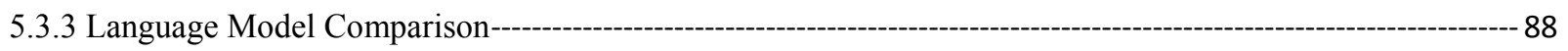

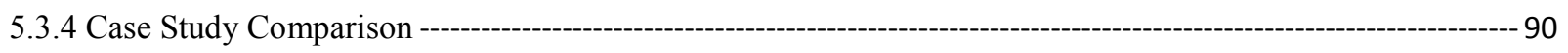

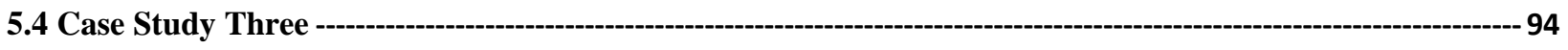

5.4.1 Vector Space Comparison ----------------------------------------------------------------------------------------------- 108

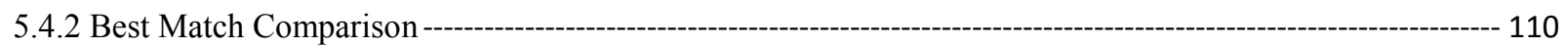

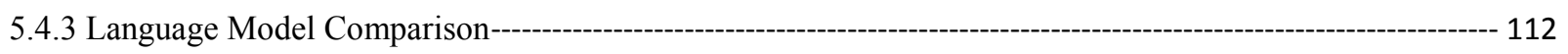

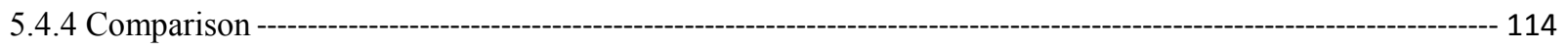




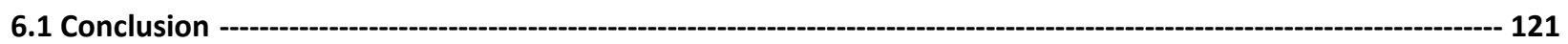

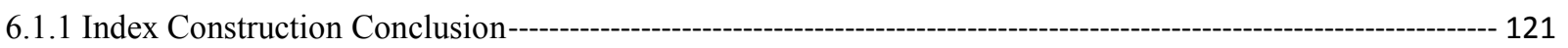

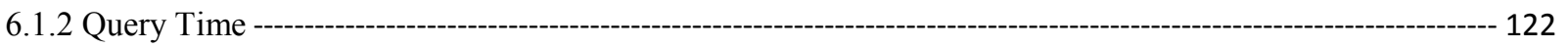

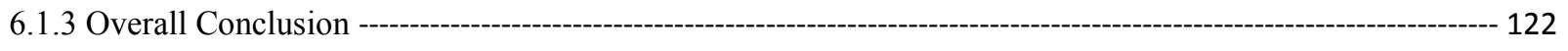

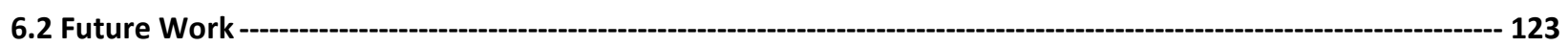

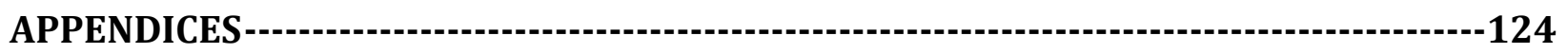

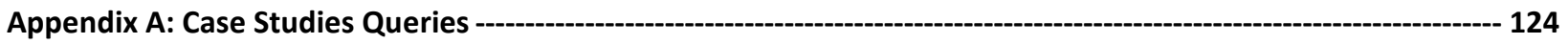

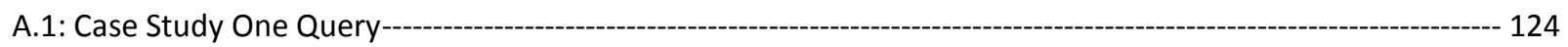

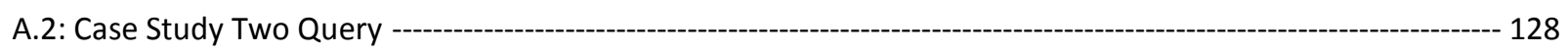

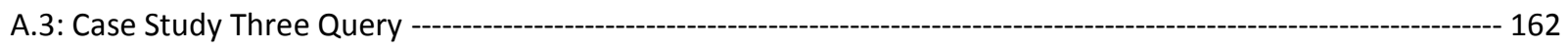

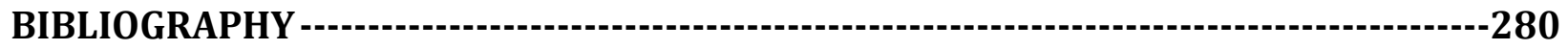

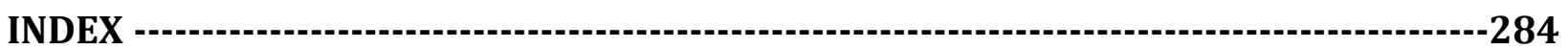




\section{Table of Tables}

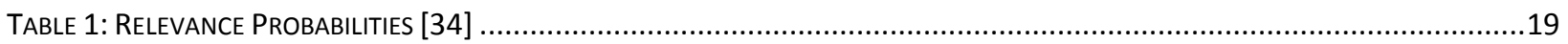

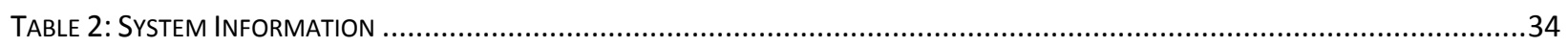

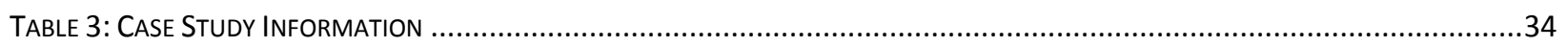

TABLE 4: DATA COLLECTION INFORMATION ..................................................................... ERROR! BOoKMARK NOT DEFINED.

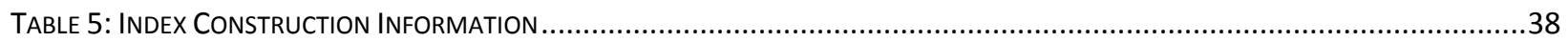

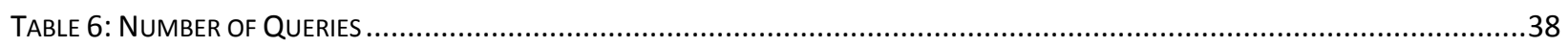

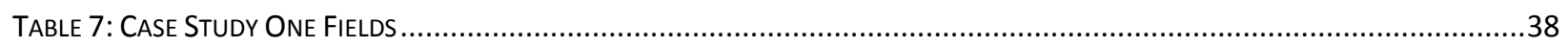

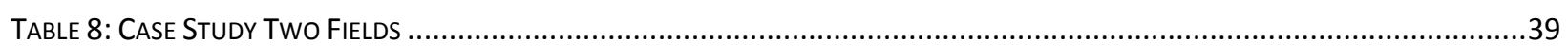

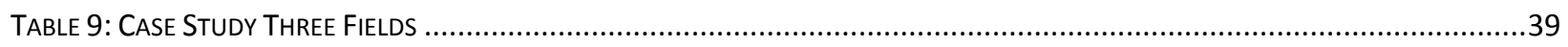

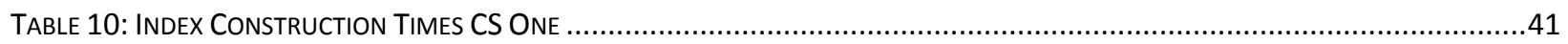

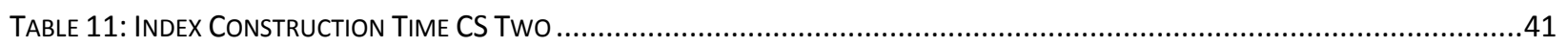

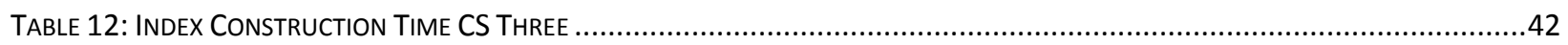

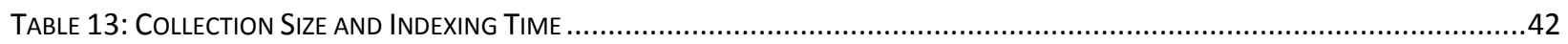

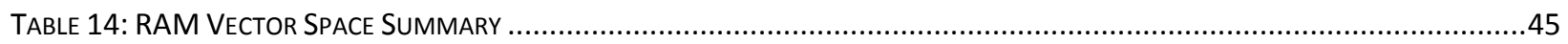

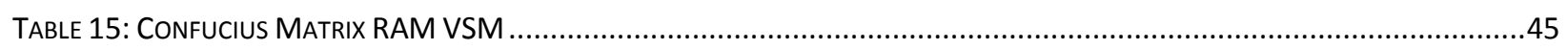

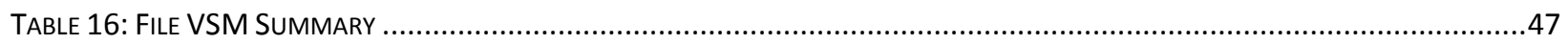

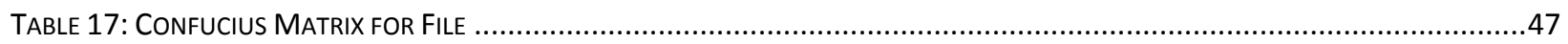

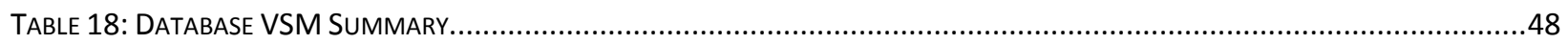

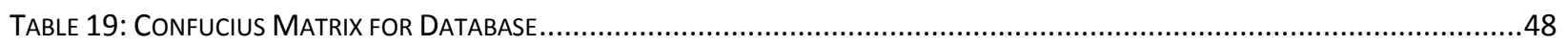

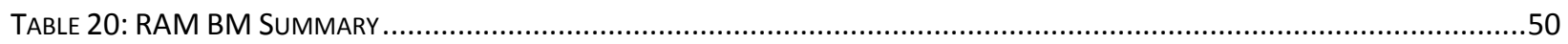

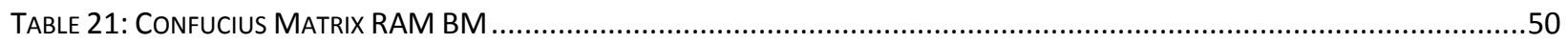

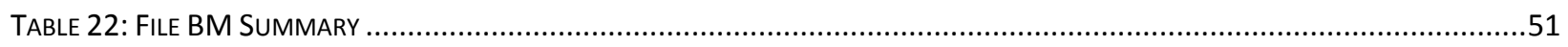

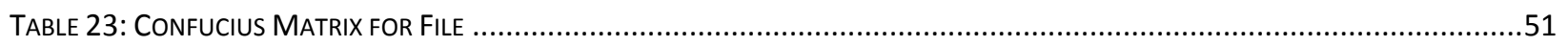

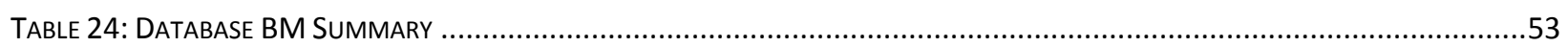

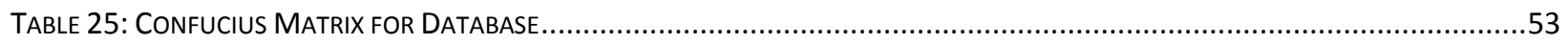

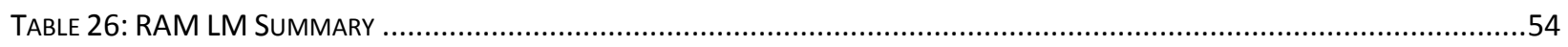

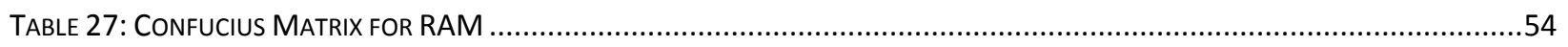

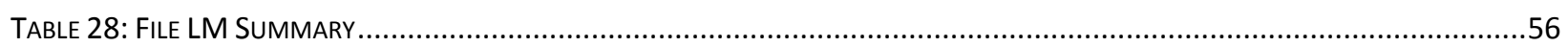

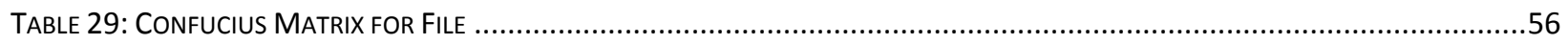

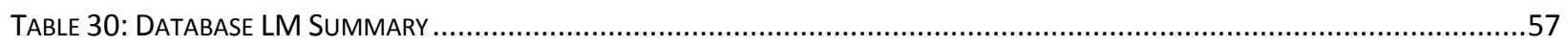

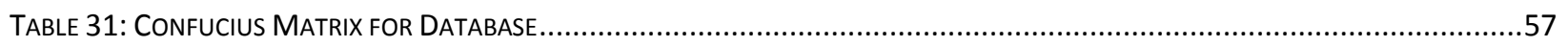

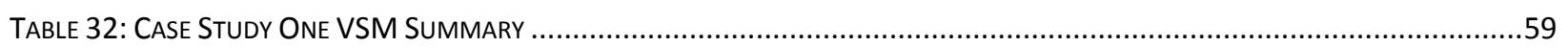

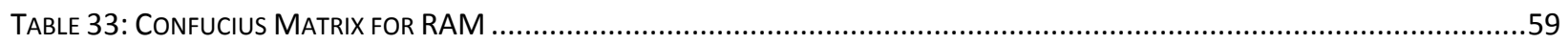

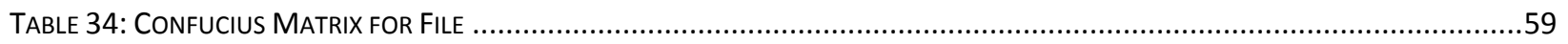

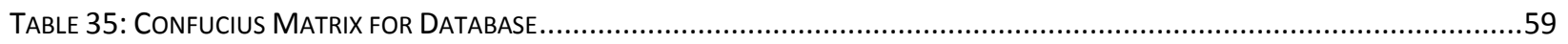

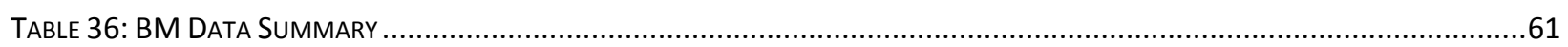

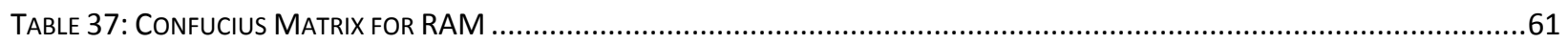

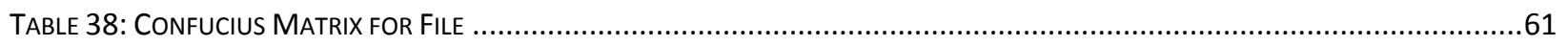

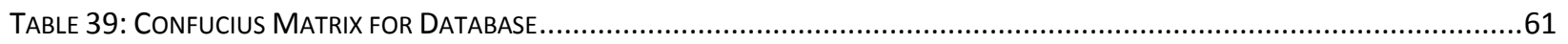

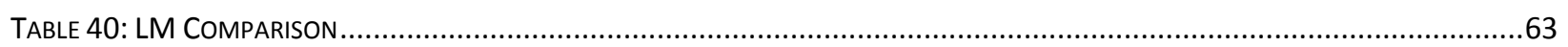

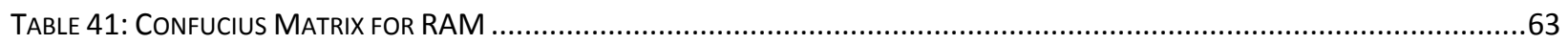

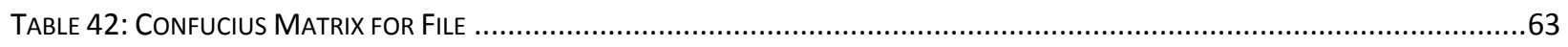

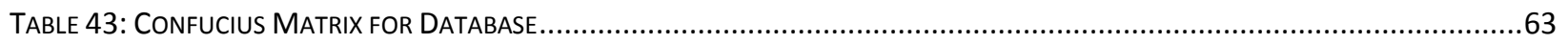


TABle 44: CASE Study ONE COMPARISON

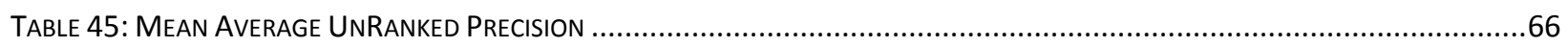

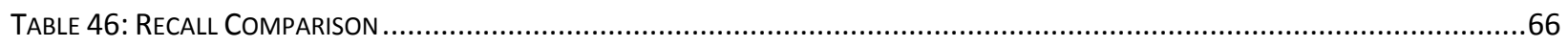

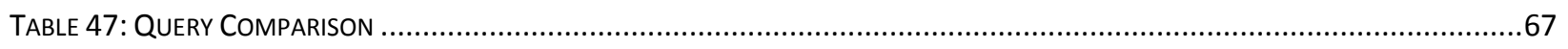

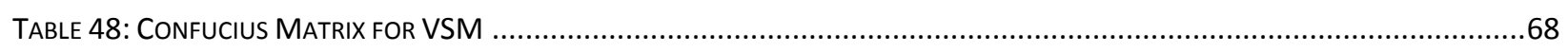

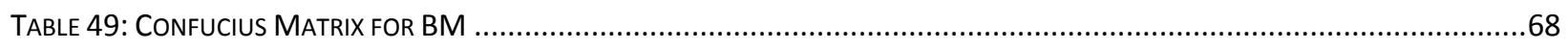

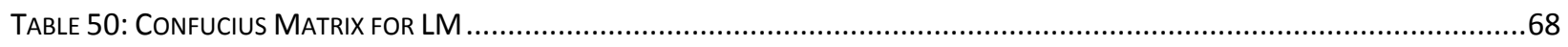

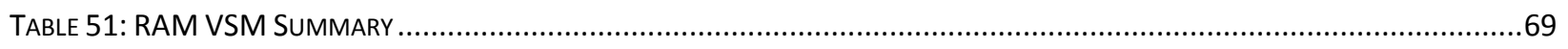

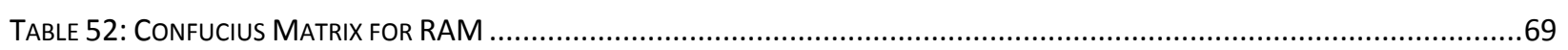

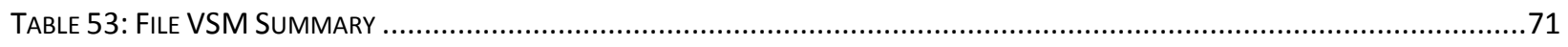

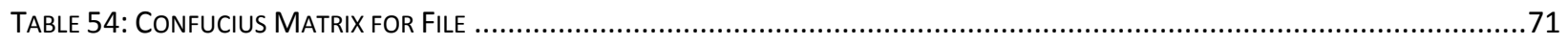

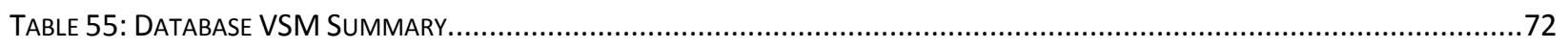

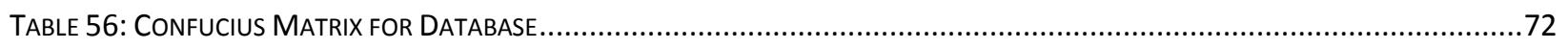

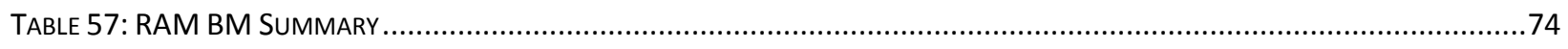

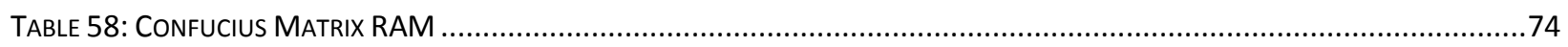

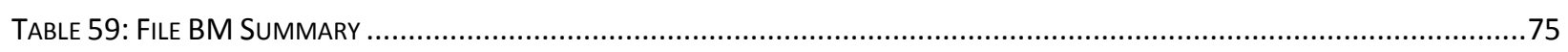

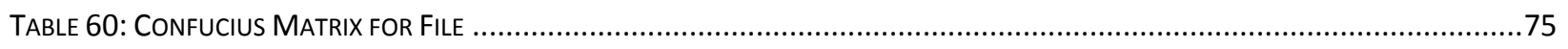

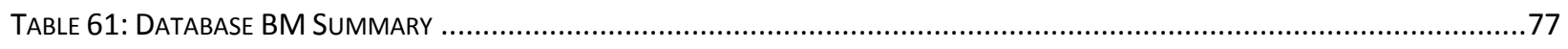

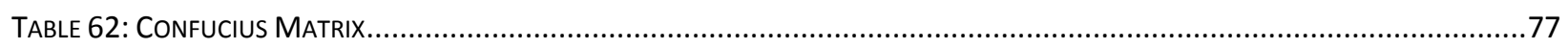

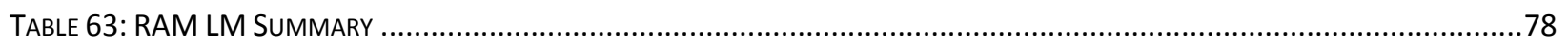

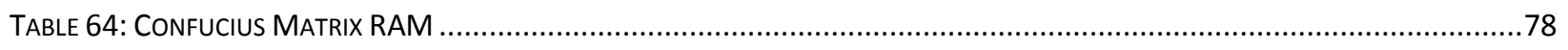

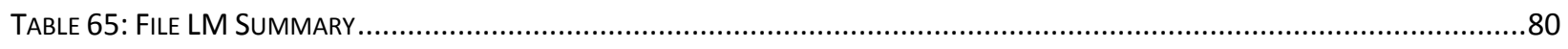

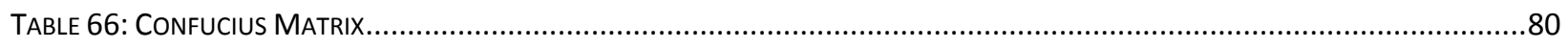

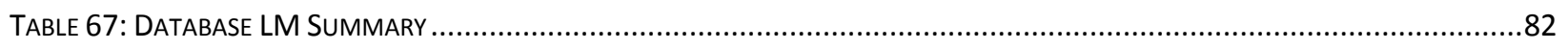

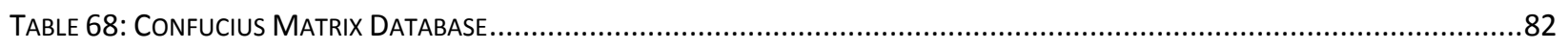

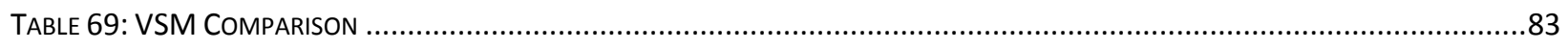

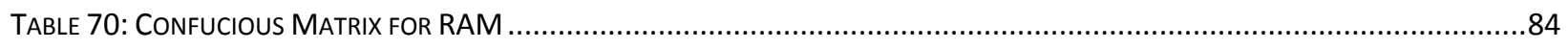

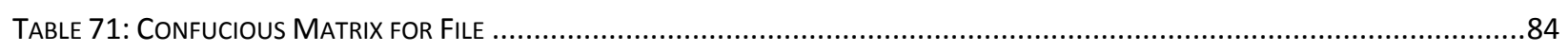

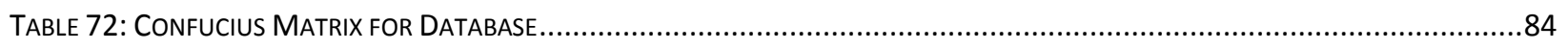

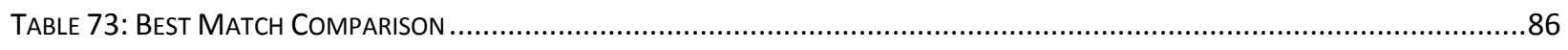

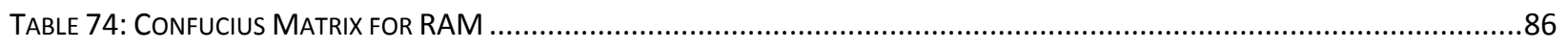

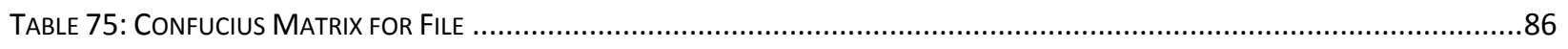

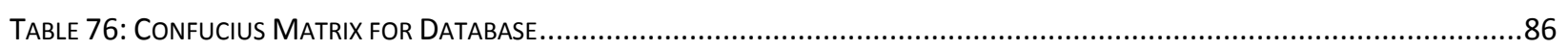

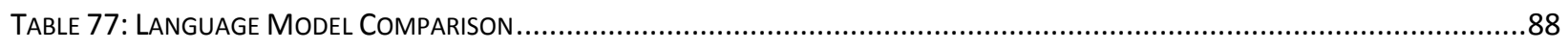

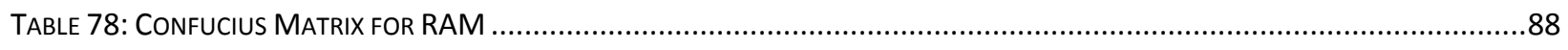

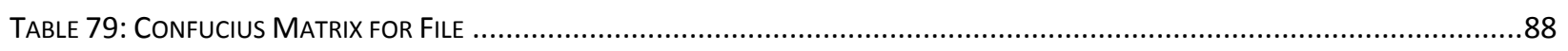

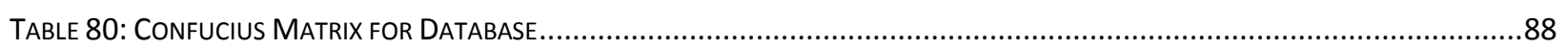

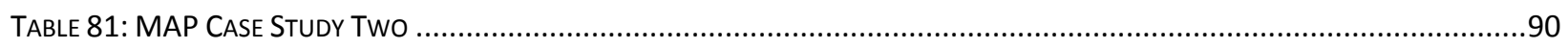

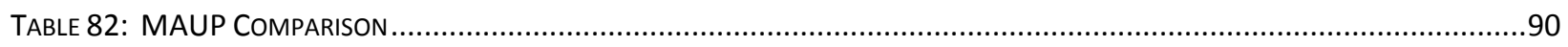

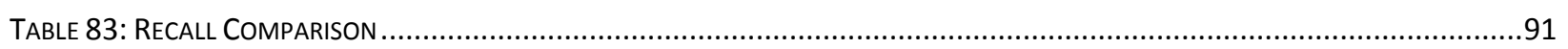

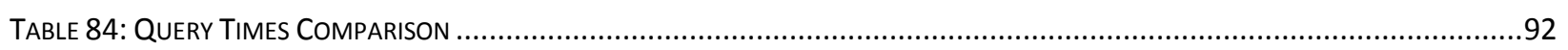

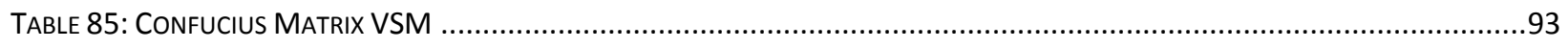

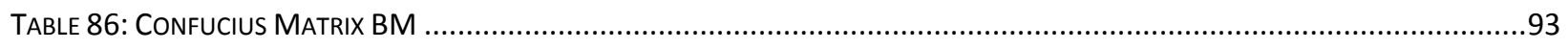

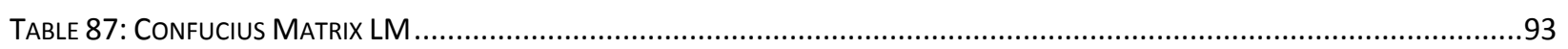

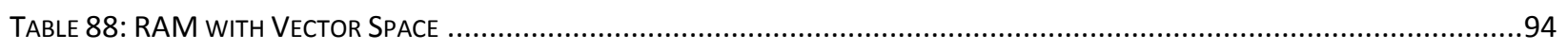

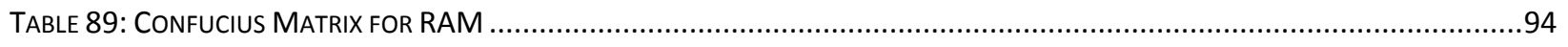




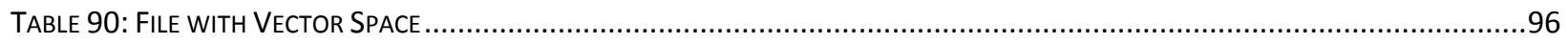

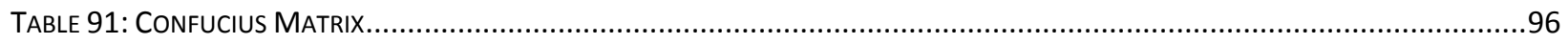

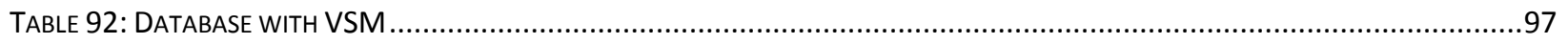

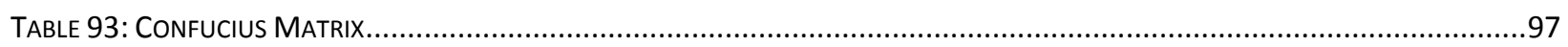

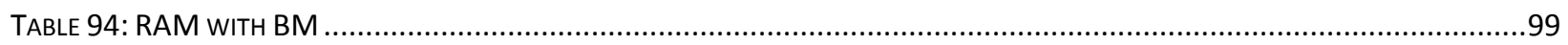

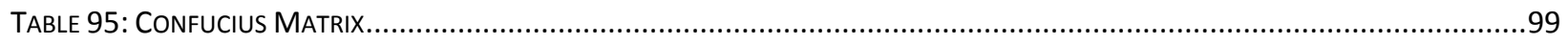

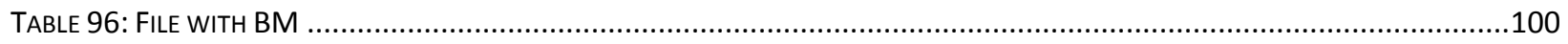

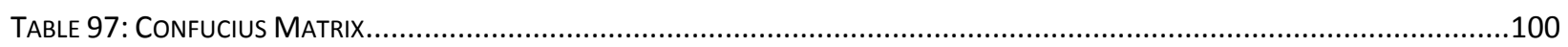

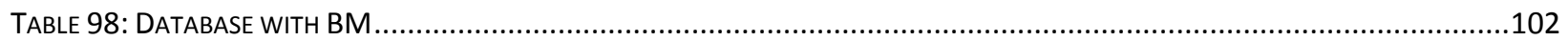

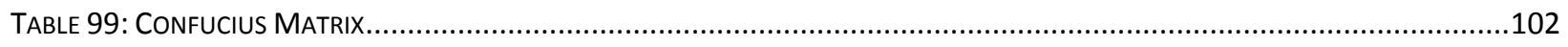

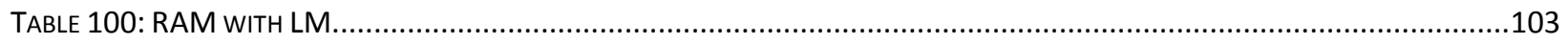

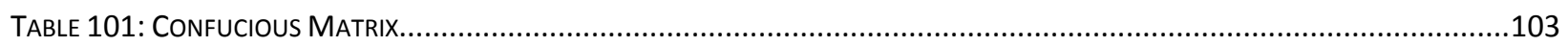

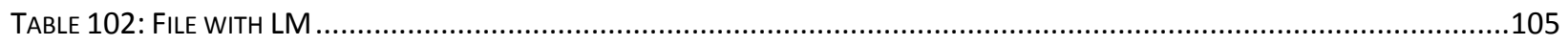

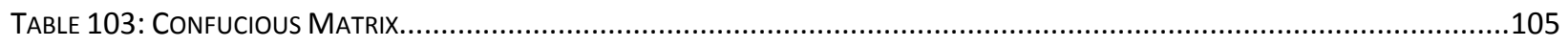

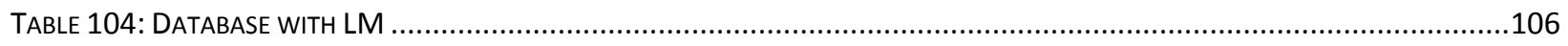

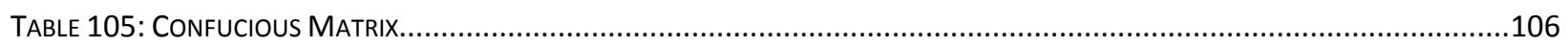

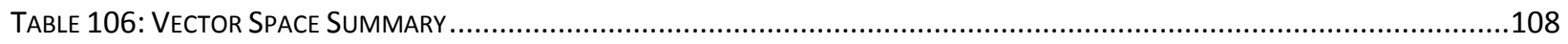

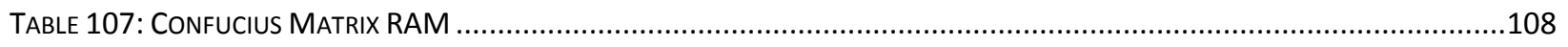

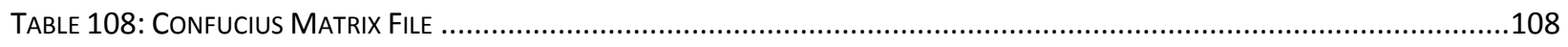

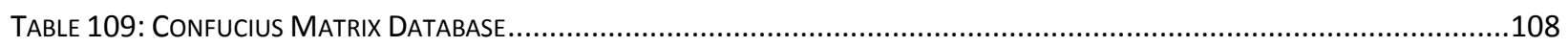

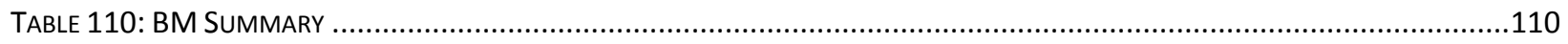

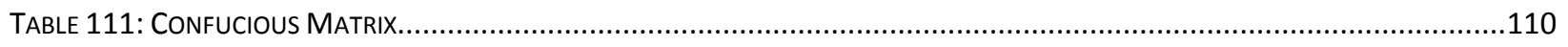

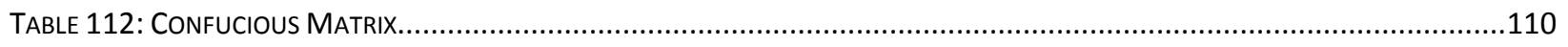

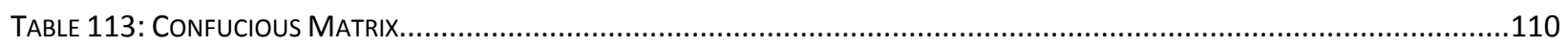

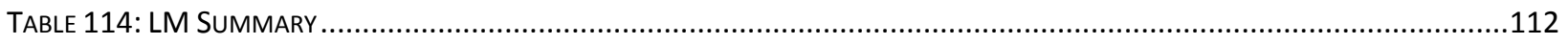

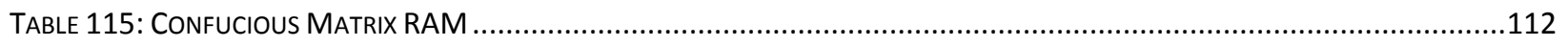

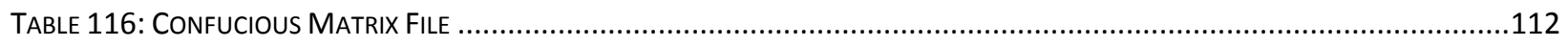

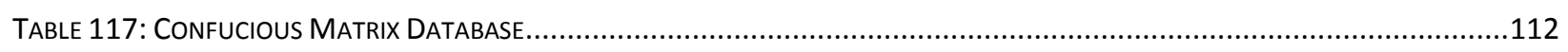

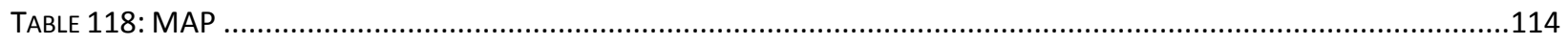

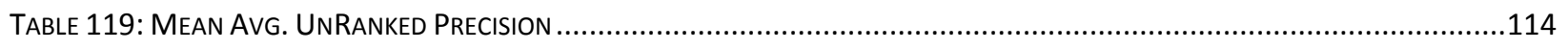

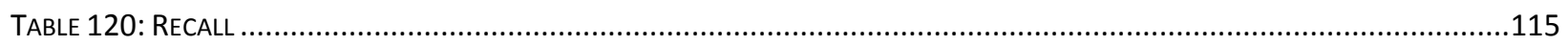

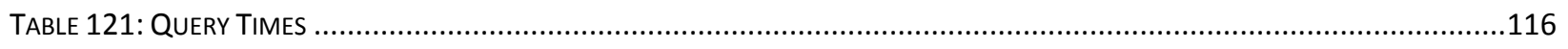

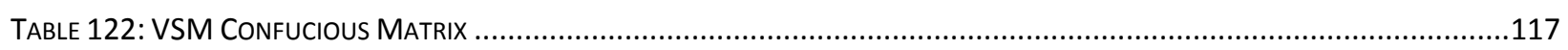

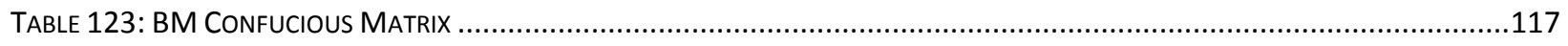

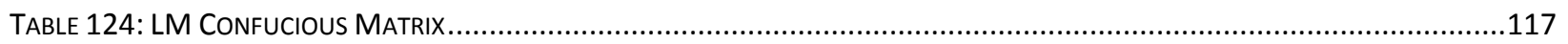

\section{Table of Figures}

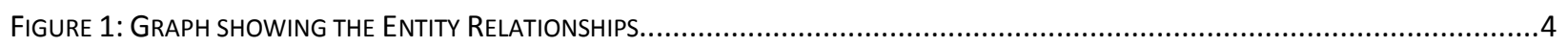

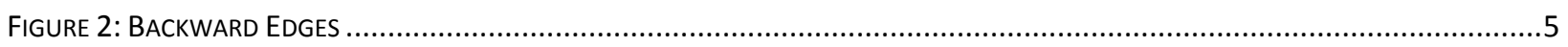

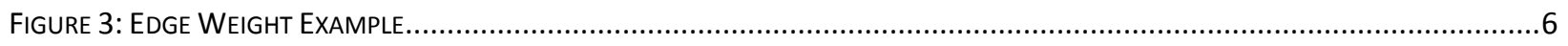

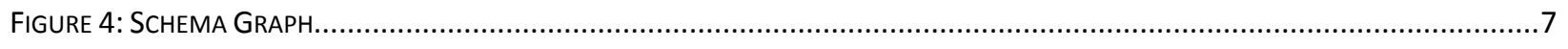

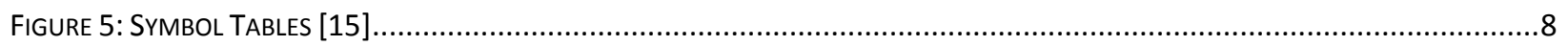




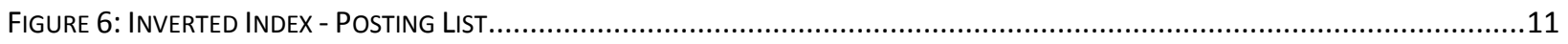

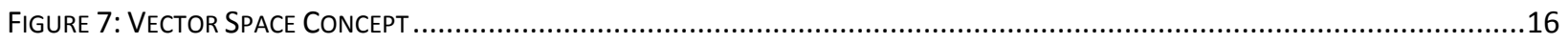

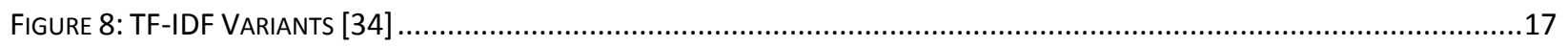

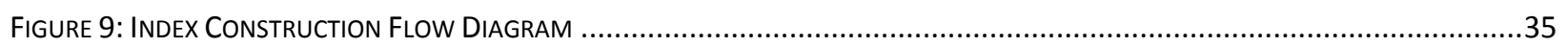

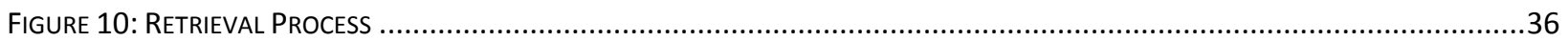

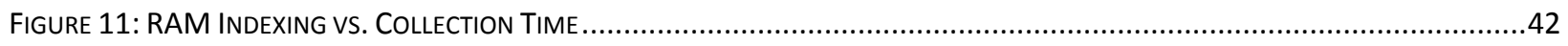

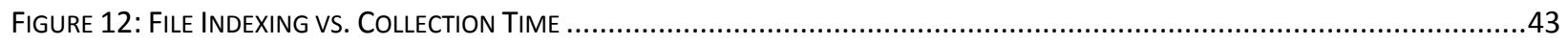

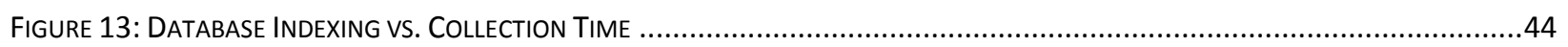

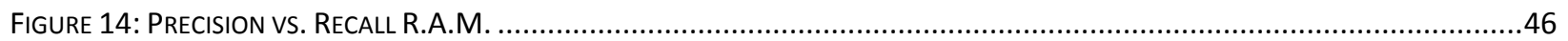

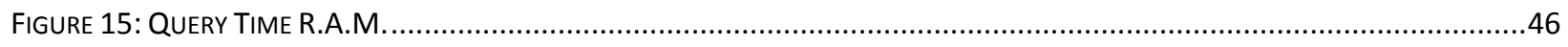

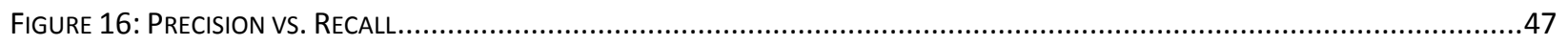

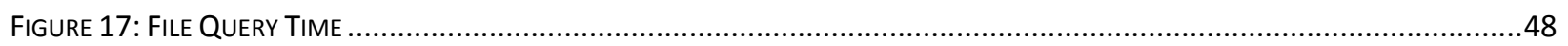

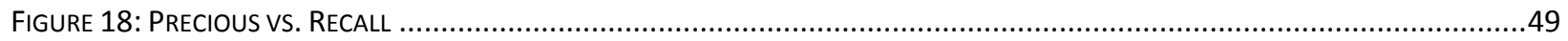

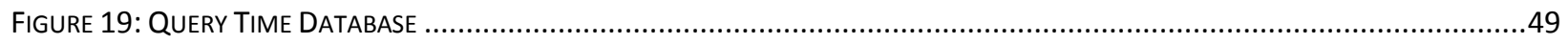

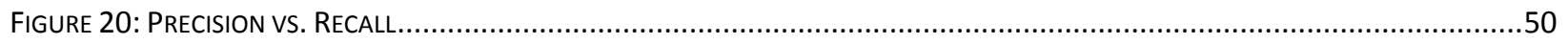

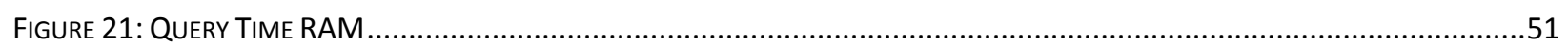

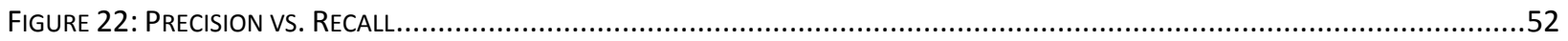

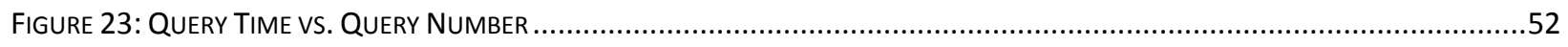

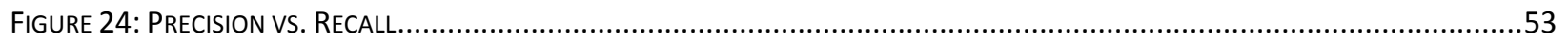

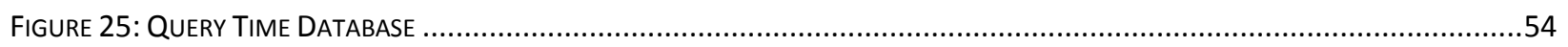

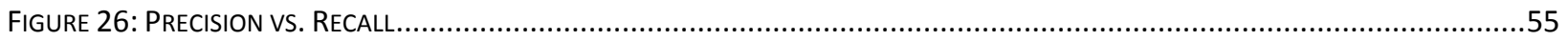

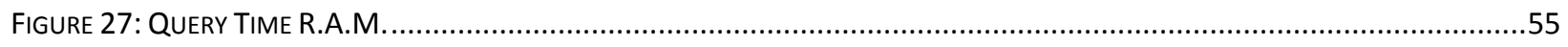

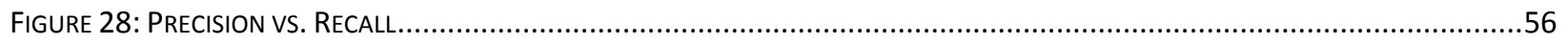

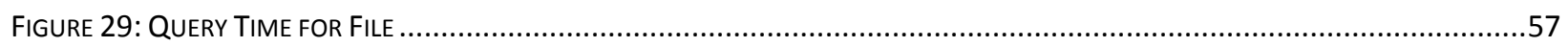

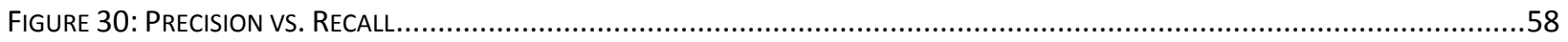

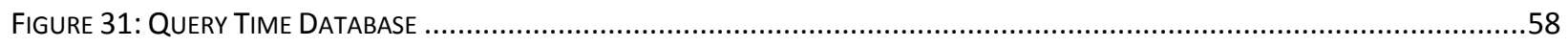

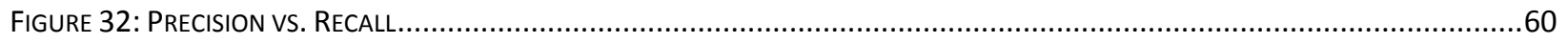

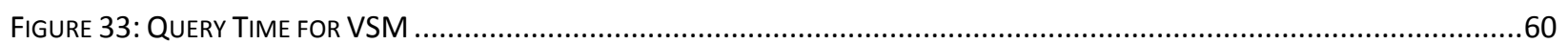

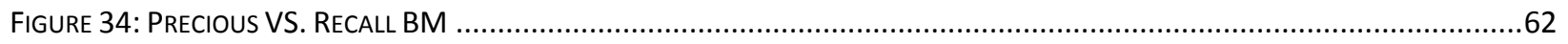

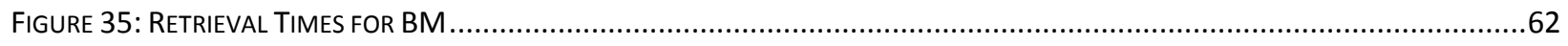

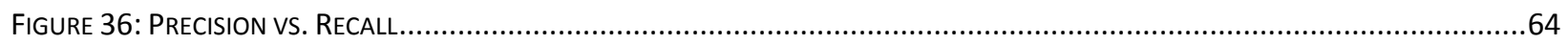

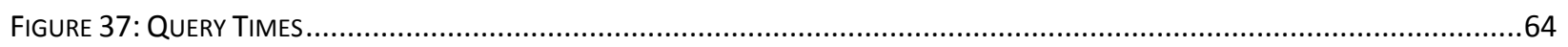

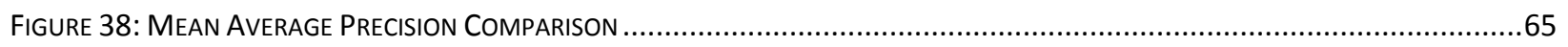

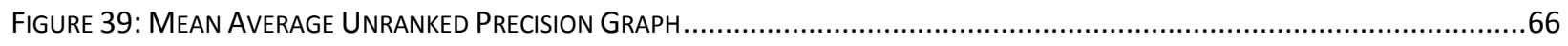

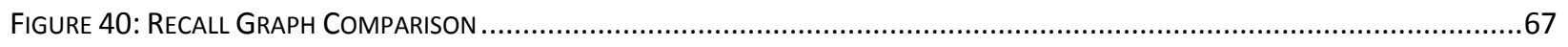

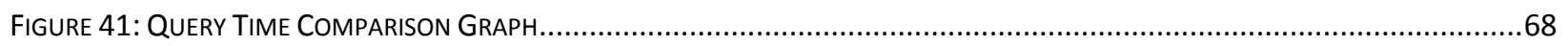

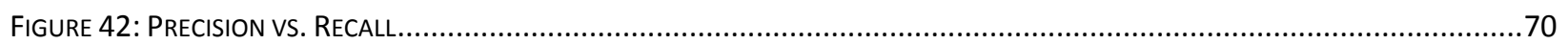

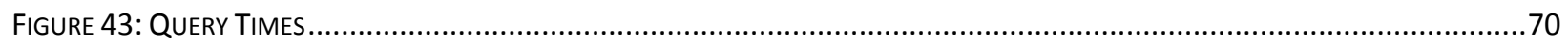

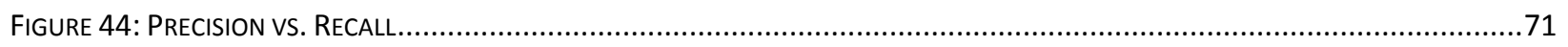

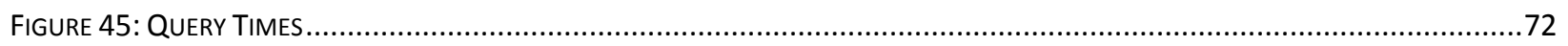

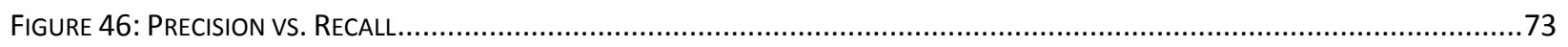

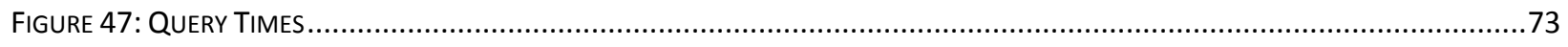

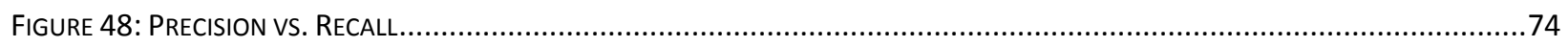

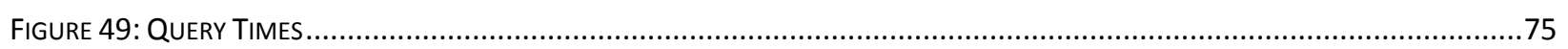

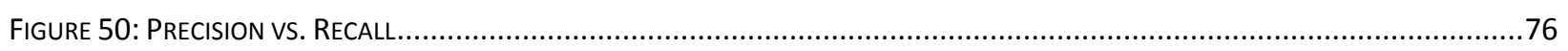

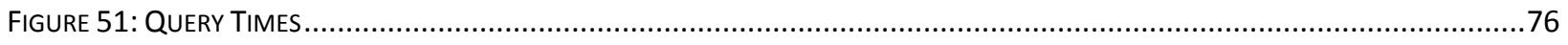




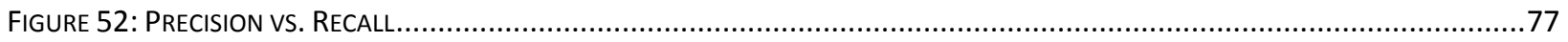

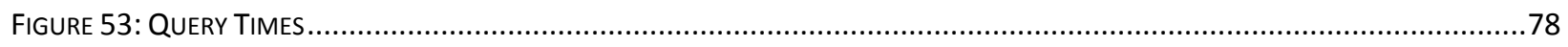

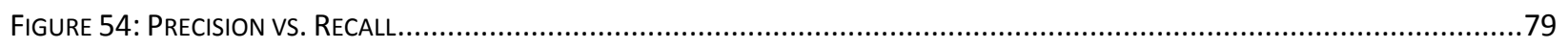

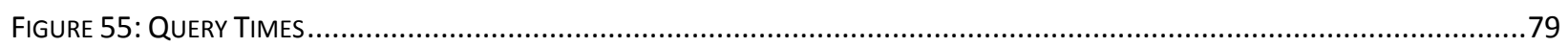

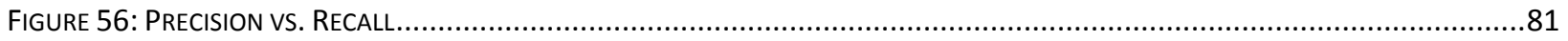

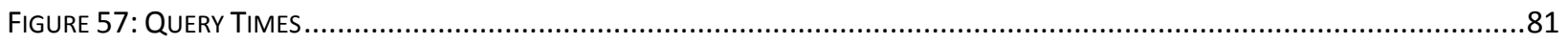

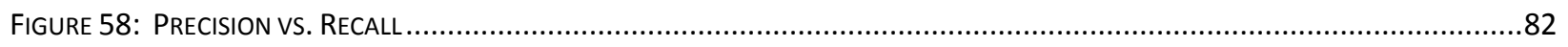

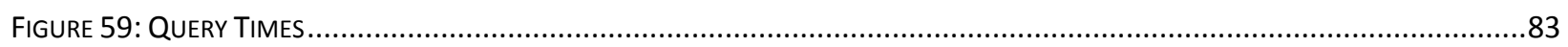

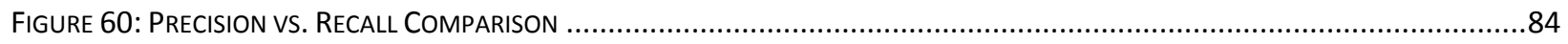

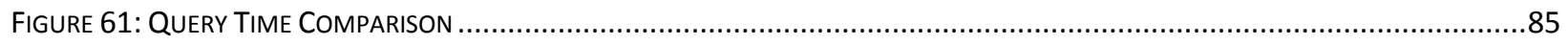

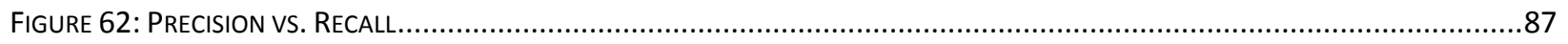

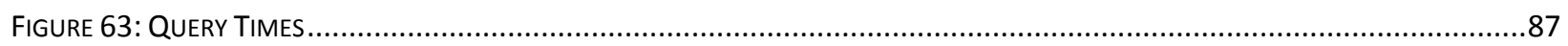

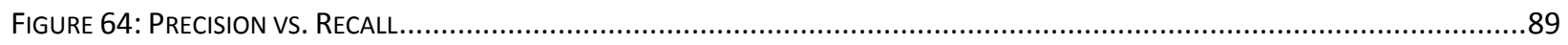

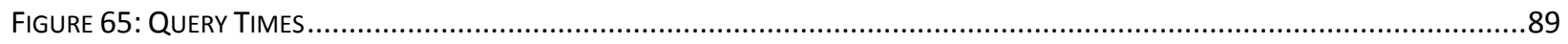

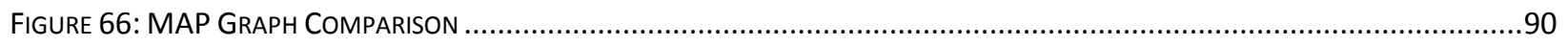

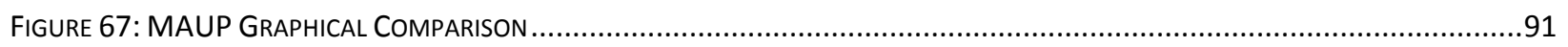

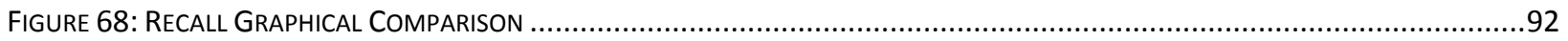

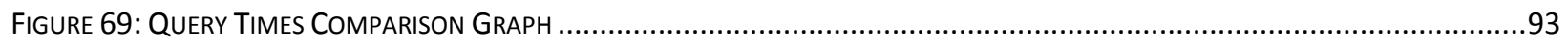

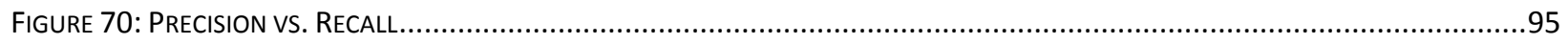

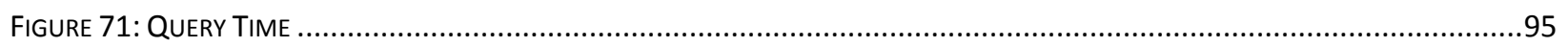

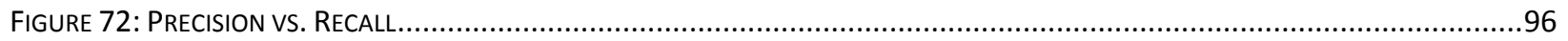

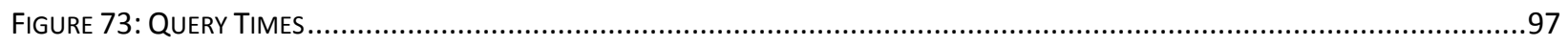

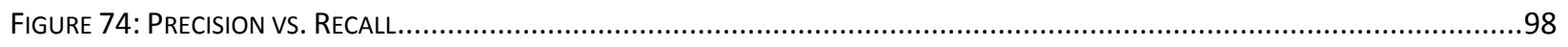

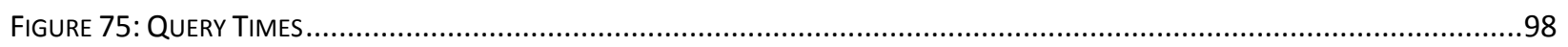

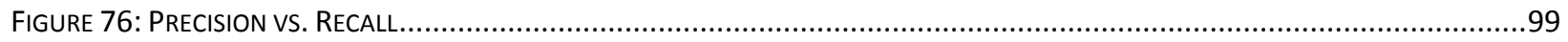

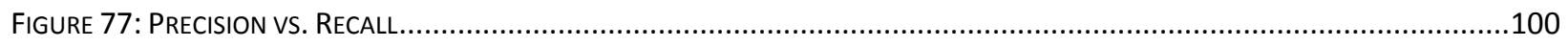

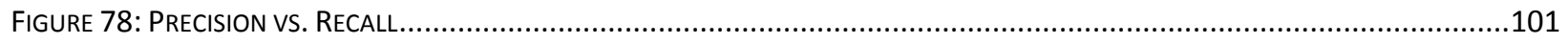

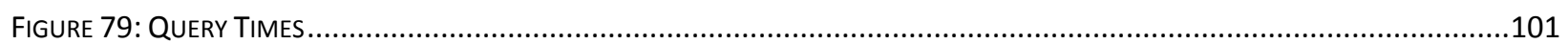

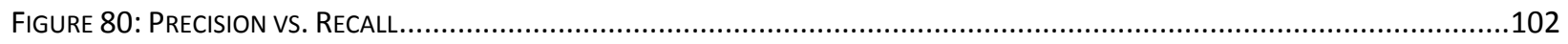

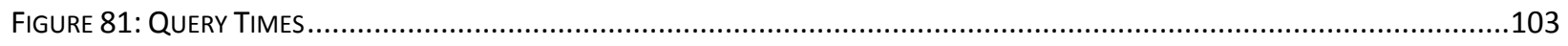

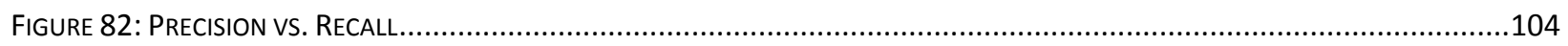

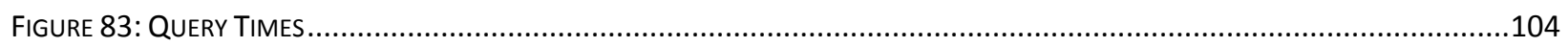

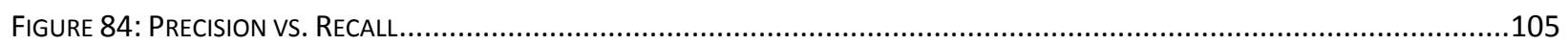

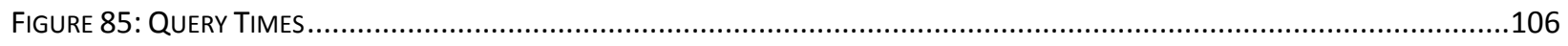

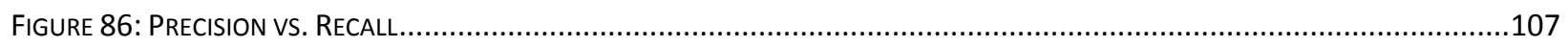

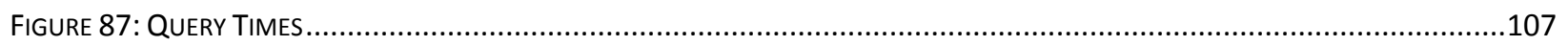

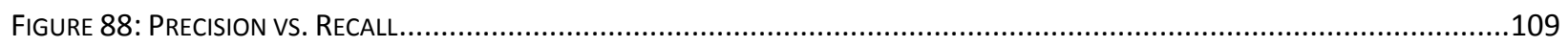

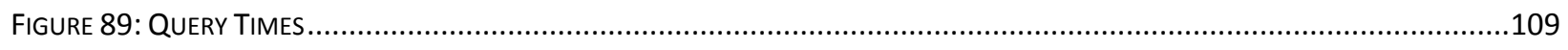

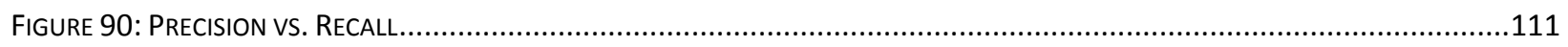

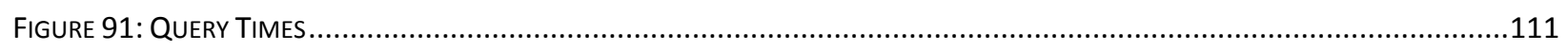

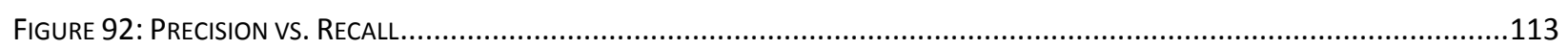

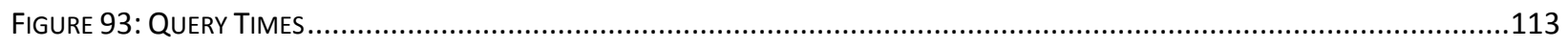

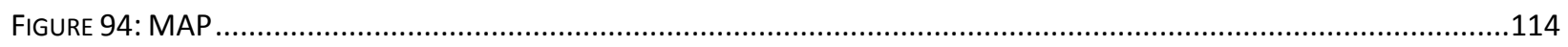

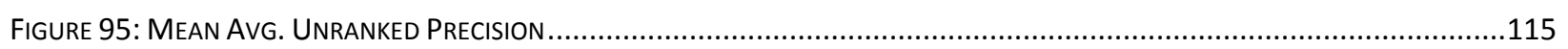

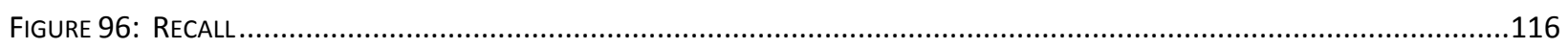

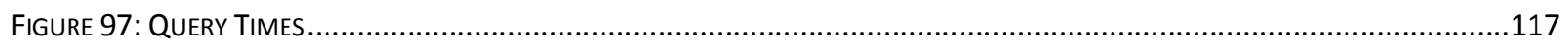




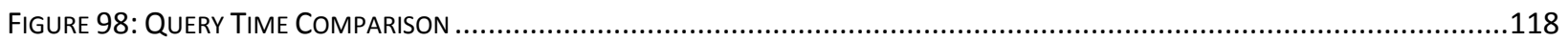

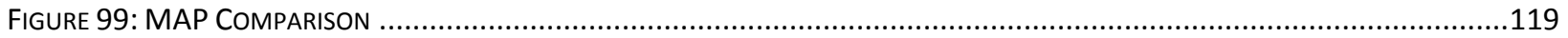

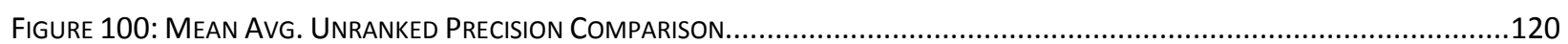

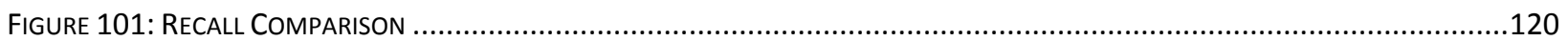

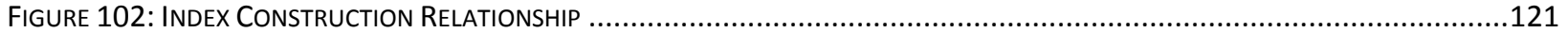

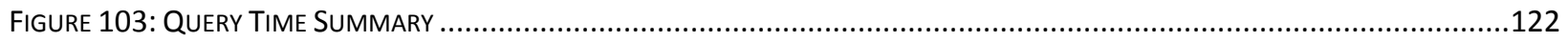




\section{Chapter 1: Introduction}

\subsection{Introduction}

In order to search a database, the user needs knowledge of the database schema and Structured Query Language (SQL). In recent years, an effort to design and build systems that allow keyword(s) searches across database data has emerged in information retrieval. Most of the solutions utilize some sort of index structure that is either stored in R.A.M. or on a disk as a file(s) and only utilize one type of ranked retrieval model.

The issue with these systems is that they are unable to store the index structure in memory due to the size of the database or security issues. One proposed solution is creating the index structure as a table within the database that is independent of the schema of the database. By doing this, the database's database management system (DBMS) can be utilized to minimize retrieval time of the keyword(s) search.

In order to validate the effectiveness of storing an index structure in a database, this investigation will compare retrieval with index storage in R.A.M., disk, and database. The comparison will be performed by using different ranked retrieval models such as vector space, probabilistic, and language models algorithms, to retrieve the most relevant document from the index structures.

The results of the comparison for each index storage medium with different rank retrieval models will determine which storage medium is the most effective for a keyword(s) search of a relational database. By doing this, database index structures can be utilize more effectively with rank retrieval algorithms to reduce data redundancy, increase data security, and optimize searches. 


\subsection{Statement of Problem}

Information Retrieval systems typically employ an inverted index structure when searching a collection of documents. Three different ranked retrieval algorithms are examined to determine if utilizing a database implemented inverted index is a better alternative than file or memory implemented inverted index. This is accomplished by comparing the search times and the effectiveness of these three algorithms and these three indexing implementations. 


\subsection{Organization}

This paper is separated into six chapters with the second chapter providing details on current research in this area of study along with the basic information needed to understand this document. The third chapter discusses the theory that the statement of problem is based on. The fourth and fifth chapter layout the simulation and the data collected to either support or refute the claim made in this document regarding database implemented inverted index structures. The final chapter will discuss the results and data collected in chapter five as well as areas where this work can be expanded on in the future. 


\section{Chapter 2: Literature Review}

This chapter provides a review of current research in the three main areas of Information Retrieval. The first section discusses three different relational search engine techniques developed. The second reviews the research of indexing data for retrieval. The last section discusses different retrieval models and algorithms.

\section{1 Current Keyword search Database Systems}

In this section an introduction to relational database search engines such as Banks[16][17], DBXplorer[15], Discover[18], Elastic Search[26], DBSight[28], and S-KWS[19]. The first class or approach is graphbased like Banks[16][17]. The second approach is schema-based like DBXplorer[15]. Finally, the third approach is index-based like RKWS[19].

\subsubsection{Graph-Based Approach}

The graph-based approach has its basis in graph theory in which the database schema or structure of the data is treated as a directed data graph [16]. Nodes in the graph are considered to be a tuples and edges are the references (foreign keys) between the tuples as represented in Figure 1.

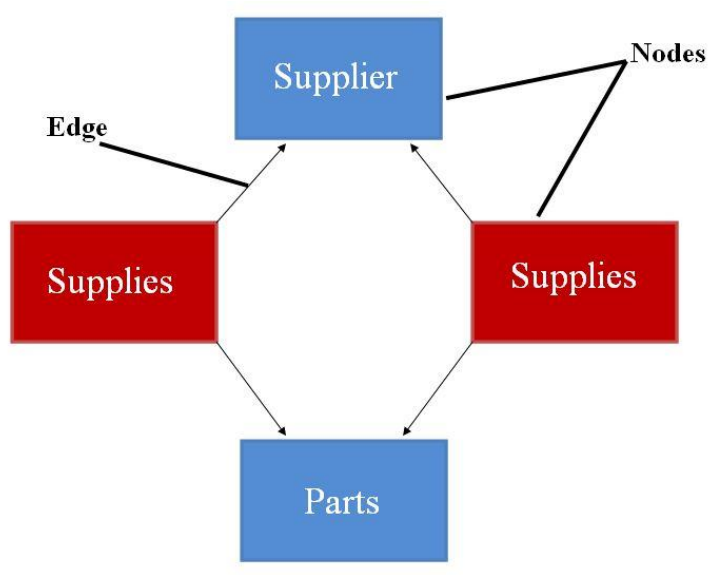


An answer to a user's search query is a sub graph of the data graph or a "rooted directed tree containing a directed path from the root to each keyword node"[16]. Banks[16] selects the ideal or prestige answer by doing a backwards expanding search. This is done by creating a backward edge from the foreign key edge. A foreign key is a key that references another value in another table. For example, an actor's name may be referenced in a movie table as the lead, which makes the actor's name a foreign key in the movie table. These backward edges have a weight value that is proportional to the number of edges of the primary node (v).

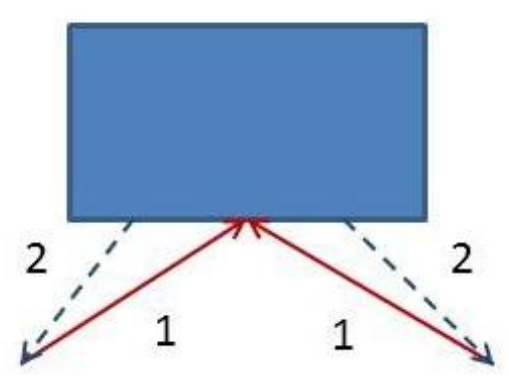

Figure 2: Backward Edges

Banks[16] then utilizes an algorithm called "Backwards Expanding Search," which is based on Dijkstra's single source shortest path algorithm [16]. The process is used to find a common vertex (root) from which a forward path exists. The rank of the answer(s) is based on the summation of the weights of each of the edges, as in the example below: 


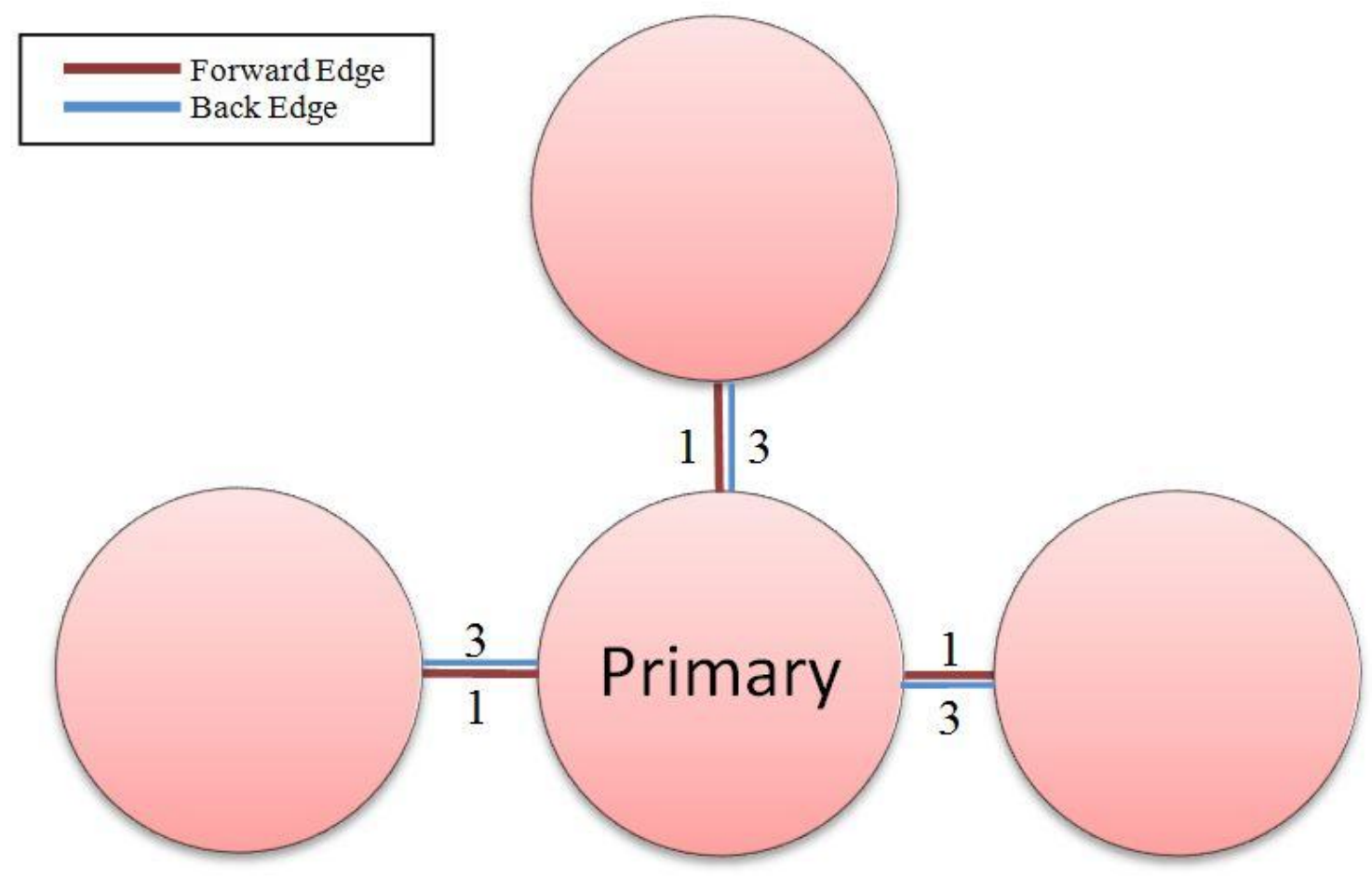

Figure 3: Edge Weight Example

Banks[16] attempts to do all processing in memory, but this uses a lot of hardware resources. Finally, the computational resources for generating the data graph and answer graphs overwhelm the CPU.

\subsection{Schema-Based Approach}

The next approach to solving the relational database keyword search dilemma is to utilize the schema of the database. From the schema a schema graph is created, but unlike the graph-based approach, the graph is generated simply upon the tables of the database as in Figure 3. 


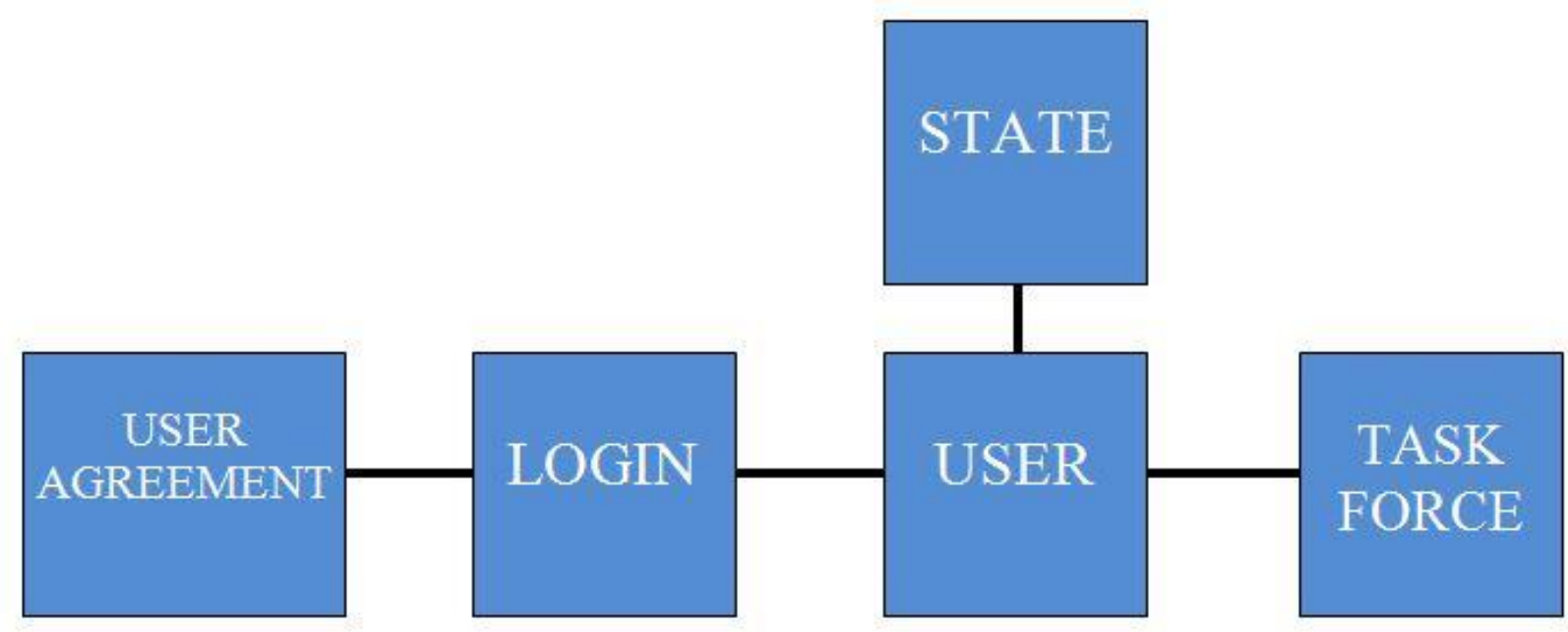

Figure 4: Schema Graph

Examples of systems that employ a schema-based approach include DBXplorer[15] and Discovery[18]. Discovery[18] uses the joining networks to find the answers to the keyword query or search. For example, a keyword query $K_{q}=$ \{smith, miller $\}$ is seen as Smith $\bowtie$ Miller in Discovery. Discovery finds the ideal solution by employing minimal joining networks trees (MJNT), which are trees of tuples in which adjacent tuples are joined via foreign key relations.

In order to find the MJNT, Discovery must first find all the candidate networks, which are tuple sets based on foreign key relationships. Due to the possibly of the candidate network growing to an enormous size, Discovery only allows candidate networks to grow to a " $\mathrm{T}$ " size, where " $\mathrm{T}$ " is the maximum size of the trees. To avoid having a candidate network achieve size "T", Discovery prunes each candidate network by finding nodes that have no keywords.

After finding the candidate networks, they are evaluated by the plan generator. The plan generator evaluates the networks based on being a tuple set or an intermediate result defined in previous assignment. For each network there is an assignment that computes joins. Basically, the less joins a network has, the more likely it will be selected as an ideal solution.

Once the candidate networks have been evaluated, the remaining ones are sent to the execution plan. Here the candidate networks, or MJNTs, that are remaining are translated into SQL and executed. The MJNTs with the least amount of joins are returned first (ranked higher) than those with 
larger number of joins. At this point, Smith $\bowtie$ Miller becomes Customers $\bowtie$ Vendors with Smith's and Miller's rows being selected and projected to the user.

A competitor to Discovery is DBXplorer[15], which comes from the research done by Microsoft of Redmond, Washington. DBXplorer like Discovery employs a schema-based searching technique, but in a slightly different fashion. DBXplorer utilizes index structures known as symbol tables to speed up the searching of keyword(s). From the symbol table, all tables, columns, and rows are identified that contain the keyword(s).

\begin{tabular}{|l|l|}
\hline HashVal & Colld \\
\hline $\mathrm{V}_{1}$ & $\mathrm{C}_{1}$ \\
\hline $\mathrm{V}_{2}$ & $\mathrm{X}$ \\
\hline $\mathrm{V}_{3}$ & $\mathrm{X}$ \\
\hline $\mathrm{V}_{4}$ & $\mathrm{X}$ \\
\hline $\mathrm{V}_{5}$ & $\mathrm{C}_{2}$ \\
\hline
\end{tabular}

Table 1: Uncompressed hash table

\begin{tabular}{|l|l|}
\hline HashVal & Colld \\
\hline $\mathrm{V}_{1}$ & $\mathrm{c}_{1}$ \\
\hline $\mathrm{V}_{5}$ & $\mathrm{c}_{2}$ \\
\hline
\end{tabular}

\begin{tabular}{|l|l|}
\hline HashVal & ColId \\
\hline $\mathrm{V}_{1}$ & $\mathrm{c}_{1}$ \\
\hline $\mathrm{V}_{2}$ & $\mathrm{c}_{1}$ \\
\hline $\mathrm{V}_{3}$ & $\mathrm{c}_{1}$ \\
\hline $\mathrm{V}_{4}$ & $\mathrm{c}_{1}$ \\
\hline $\mathrm{V}_{2}$ & $\mathrm{c}_{2}$ \\
\hline $\mathrm{V}_{3}$ & $\mathrm{c}_{2}$ \\
\hline $\mathrm{V}_{4}$ & $\mathrm{c}_{2}$ \\
\hline $\mathrm{V}_{5}$ & $\mathrm{c}_{2}$ \\
\hline
\end{tabular}

Table 2:

Compressed hash table

Table 3: ColumnsMap table

Figure 5: Symbol Tables [15]

The symbol table that is utilized in DBXplorer is analogous to the HashMap data structure. Discovery and DBXplorer's processes become very similar after the identification of all tables, columns, and rows involved. DBXplorer creates joins in which all the keywords may be contained. The join trees that are formed are ranked based on the number of joins as discussed in Discovery. Once ranked, the join trees are translated into SQL and results are provided to the user just like in Discovery.

The advantages of DBXplorer and Discovery is that they are scalable to different databases and do not require keeping graph structures in memory. However, they do have the disadvantage of requiring CPU 
resources to process their respective tree networks to find the optimal solution.

Markowetz[19] proposed a new solution based on the same theories as DBXplorer and Discover known as S-KWS[19]. S-KWS utilizes a candidate network generator based on two principles [6]:

1. By assigning a proper expansion order to the $\mathrm{CN}$, isomorphism cases can be avoided.

2. Expanding the $\mathrm{CN}$ from part of the node in a partial tree can reduce the number of partial results.

However, instead of following the same process as other schema-based systems, Markowetz[19] describes a process of transforming the candidate network into an operator tree that is integrated into a mesh structure to solve the problem of redundant trees or nodes by allowing sharing. The tree is optimized for query processing while the operator tree is in the mesh. Then the MJNTs are outputted and ranked according to their join size like the previous systems.

\subsubsection{Information Retrieval Libraries}

The third class of systems is based on Information Retrieval (IR) fundamentals that are provided by the IR libraries such as Lucene[25]. The first system we will discuss offers flexibility in the terms of dealing with traditional documents of indexing and searching, like web pages and relational database searches. Elastic Search[26] is a system that evolved out of the Compass project[27] and allows users to search multiple indexes easily with minimal setup or understanding of IR.

Its competitor, DBSight[28] is based on Lucene as well, but utilizes Apache Solr to interface with the Lucene libraries. It utilizes the speed of Lucene IR libraries, while utilizing the ease and flexibility of Apache Solr. Both of the systems utilize an inverted indexing scheme like traditional IR systems (Google) and utilize a vector space model retrieval method in which the frequency of a term in a document raises its relevancy to a user's query. 
The advantages of using a Lucene-based product are ease of implementation and execution. This appears to be an almost real-time search over a relational database. However, DBSight must be purchased after a database reaches a certain capacity.

Overall, the current research in this area ranges from developing these search engines to accepting metadata information along with search terms like "Title: "The Raven"” in which the engine would search the title field for the poem "The Raven." Research is attempting to create an efficient algorithm for solving the Steiner Tree problem, which requires extensive computational resources. The ideal solution would decrease the amount of computational resources required as well as the response time for search engines.

In addition, many of these systems utilize a Boolean Retrieval method in which the keyword is either contained or not contained in a document. While other systems utilize a vector space model based on a term's frequency in a document of a given collection. 


\section{2 Index Research}

Indexing documents, as used in Information Retrieval, is flexible and depends on the retrieval method being utilized, type of documents, and storage medium. For example: because of cost, a developer wouldn't want to store the entire document along with keywords in R.A.M., if it could be implemented in a file or a database table.

There are various index structures found in IR, such as forward (direct index), inverted index, signature files, suffix trees, and suffix arrays[22]. Forward index structures are mappings from a document ID to the list of terms that appear in the document [24]. Signature files are similar to a Bloom filter and can be used to obtain a list of documents in which a term may appear [24]. Suffix trees or suffix arrays can be used to find all occurrences of an n-gram sequence in a given text collection [24]. This structure is usually used in phrase search or regular expressions.

Most IR systems utilize an inverted index structure where the terms or tokens of the document act as the keys to a value commonly referred to as the posting list identification number. A posting list is a list of document identification numbers and each term has a posting list to keep track of what document the term occurs in. For example: there are three documents and in two of those documents the token, "Redskin," occurred. Then the inverted index and posting list would look similar to the following:

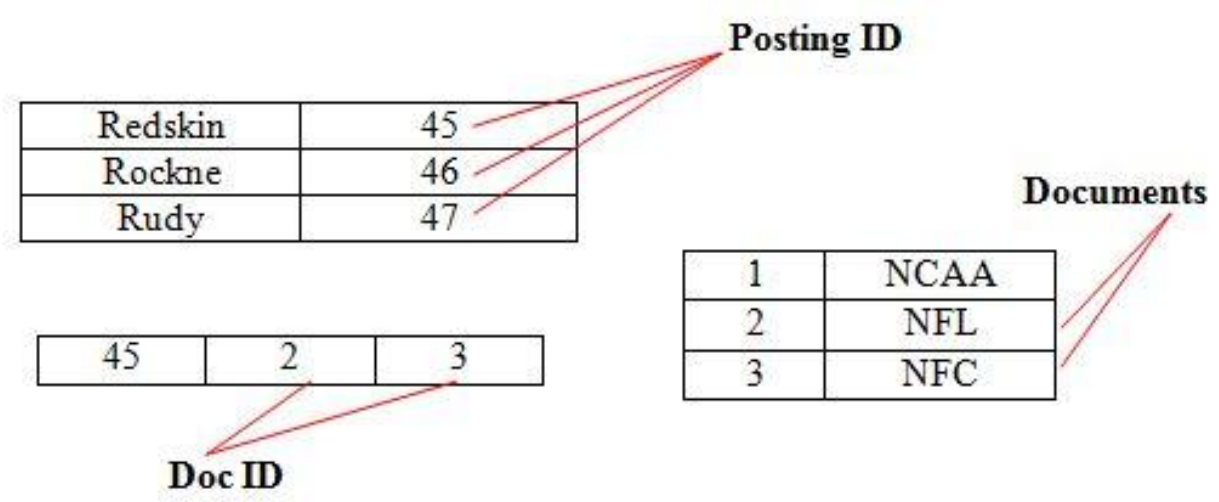

Figure 6: Inverted Index - Posting List 
There are various types of inverted indexes that are utilized in IR. The first type of inverted index is called the DocID index, which was discussed earlier. DocID indexes are employed when the retrieval model focuses on recalling all of the documents containing the keywords instead of on the relevancy of the documents.

The DocID approach usually does not satisfy many retrieval methods, so a frequency index style is implemented. In this inverted index

implementation, the index tracks the term and posting list ID. The posting list ID is a key to retrieving the correct posting list. The posting list is a linked list of all the documents containing the term and the term's document frequency. For example: a term has two documents in its posting list, and document one could have the term five times whereas document two could have the term ten times. This is a very common inverted index to see implemented in vector space models and probabilistic models, because these models incorporate term frequency into their respected scoring algorithm.

Sometimes the position of the term in a document is just as important as the occurrence of the term. For instance, if the user queried "University Maryland," without the position, then any document with "university" or "Maryland" could be considered. If the positions of the terms are kept, the retrieval method can rank documents with "University Maryland" ahead of other documents.

Inverted indexes are constructed in various ways. Some are constructed at the startup time of the IR system in R.A.M. Others maintain an active structure implemented on disk, and some combine these methods.

The construction of an inverted index can be classified as static or dynamic. Dynamic construction is when a collection either rapidly changes or is extremely large, which causes part of the index to be loaded into memory. Static construction is used on collections that don't change often or the index is small enough to fit in R.A.M. 


\subsubsection{Static Construction}

\section{In-Memory Construction}

Static approaches to index constructions include: in-memory, sort-based construction, and merge-based index construction[24]. The first inmemory concept is where the inverted index is stored in R.A.M. and the posting list is implemented in a file[24]. The other method is interleaving, where there are two structures stored together in-memory.

When adding new data to the in-memory index, one can put new information at the back of the index. This method infers that if a term occurs late in the document, then it is of little importance. The other method is to move to the front[24]. Initially a term is added to the back of the index, but if retrieved this term is relocated to the front of the index.

\section{Sort-Based Construction}

Sort-based construction is a disk-based method that sorts in-memory index structures and organizes them into a disk structure. This data can be written onto the disk as blocks in two fashions: multiday merge operation or cascaded merge operation. The difference between the operations is one writes all blocks at once and the other writes two blocks at a time. The difficulty is selecting a time when the system doesn't require availability, because the sort-based construction method is resource intensive.

\section{Merge-Base Construction}

Merge-based construction is similar to the other constructions discussed thus far, but unlike its counterparts, the merge-based construction creates index partitions on disk. Once indexing is complete, it merges all the partitions into one index. This method is handy for extremely large collections. This construction technique usually utilizes compression due to the size of the index structure. 


\subsubsection{Dynamic Construction}

Dynamic construction of an index is only necessary when the collection continuously updates like a State's DMV record collection. The primary concern with dynamic construction is updating the index with either batch or incremental updates. Updates can range from adding new items, modifying existing items, or removing items.

\section{Batch Updates}

Batch updates are updates that occur at certain time frames or increments. The two strategies that are associated with this update scheme are rebuild or remerge. Remerge is based on the merge-based construction of the static index. Whereas the rebuild method reconstructs the entire index and replaces the old index structure. According to Clarke[24], if $60 \%$ of the index has been modified, then rebuilding the index is the most efficient option.

\section{Incremental Updates}

Incremental updates are more complex, but are necessary if the collection's index needs to reflect changes immediately like

Amazon.com's inventory. Once again, the remerge option can be utilized to update the existing on disk index. However, if the collection is large enough and updates are frequent, then the overall effectiveness of the retrieval system can be harmed. This is due to "whenever the indexing process runs out of memory, it reads the entire index from disk, merges it with the in memory data and writes a new index back" [24].

The other option to remerge is in-place updates, which removes the need to read the entire index from disk upon every update. When a posting list is written to disk, there is some space left for a new entry. This allows for new postings to the same term to be transferred to disk at a later point by simply appending to the existing posting list. 


\subsubsection{Compression of Indexes}

One area of increasing popularity is index compression. Index compression can be considered from many aspects; one of the most common forms of compression is removing commonly used words known as stop words. These are words such as "a," "the," and "they" and provide little to no help in finding relevant documents regarding a query. Removing these stop words can easily reduce the size of an index. For instance, if you have a collection with 200,000 tokens (words) and of those words, there are 16,000 unique tokens. However, of the 16,000 tokens, there are 200 words that can be considered stop words. These 200 words could throw off relevancy if a word like "not" occurs $33 \%$ of the time in the collection. By removing the stop words, the index size decreases by 200 tokens and effectiveness of the search engine improves.

Another index compression method is the pruning method, which removes redundancy from the index. Researcher David Carmel suggested one method of pruning based on uniform and term-based methods to reduce the size of the index structure [31]. Carmel investigated a way of pruning the posting list for a term when the document in the list is of little relevancy to that term. By using a cutoff value to gauge relevancy of the document, he was able to achieve $35 \%$ pruning with a slight average precision loss [31].

Another proposed compression technique for inverted indexes is to compress the docID value, which takes up an extensive amount of space in the posting list. Yan[32] describes a process based on improving a compression technique known as PFD, which is a process for decoding and encoding many numbers based on a fixed size. The improvements that Yan[32] suggests are introducing a new structure to deal with possible overflow bits. Then starting with the smallest compression size possible and increasing as needed, instead of assigning a constant compression size like before. 


\subsection{Models/Retrieval Methods}

There has been an extensive amount of research done in this area over the past decade, but many of the retrieval methods are derived from the same principles. The three methods or models that will be discussed in this section are the vector space model, the probabilistic model, and the language model. There are many more models, but for the scope of this investigation, only knowledge of these three models is necessary.

\subsubsection{Vector Space Model}

One of the oldest was proposed by SMART System in the 1960s. The vector space model is sometimes called the cosine similarity method, in which two vectors, one known as document vector $\rightarrow V_{d}$ and another known as query vector $\rightarrow V_{q}$, are compared by using the cosine function. A good example is illustrated in figure 7, which shows the different vectors and how cosine is used to find the most relevant pair.

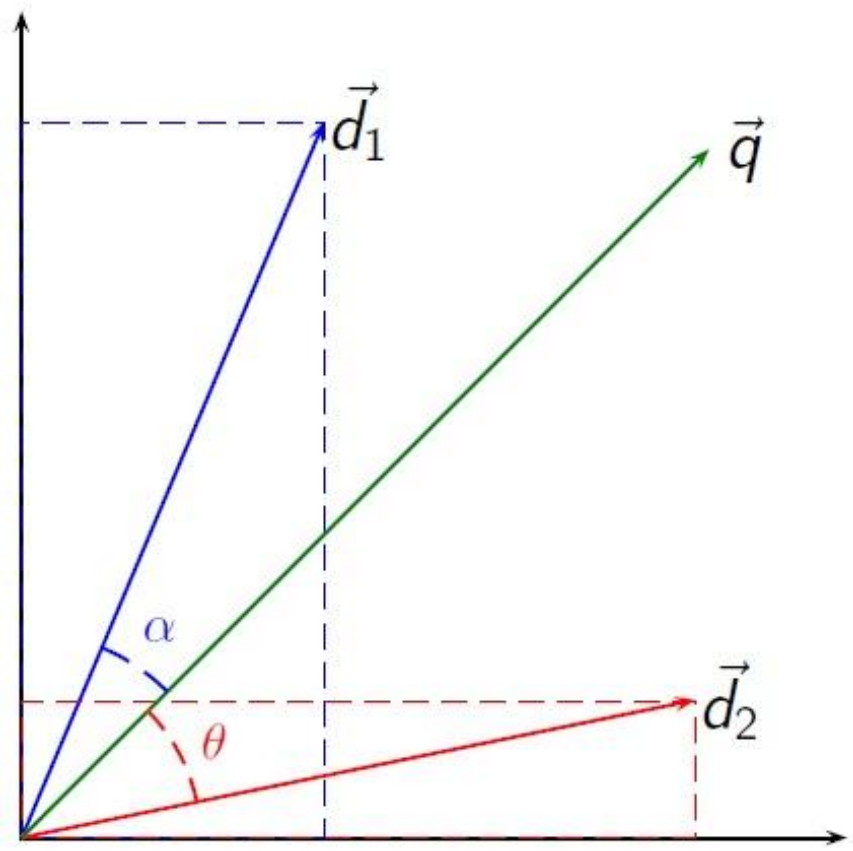

Figure 7: Vector Space Concept

In figure 7, $\alpha$ angle is smaller than angle $\theta$ and thus document 1 and the query are more similar. Whereas document two would be ranked as less 
similar than document one. The cosine similarity is calculated by the following:

$$
\cos (\theta)=\frac{\vec{d} \cdot \vec{q}}{\|\vec{d}\|\|\vec{q}\|}
$$

This model utilizes the concept of weights, where each token in the query or document is assigned a weight based on a weight scheme. Most vector space models use a variation of the TF-IDF to calculate the weights.

The TF or term frequency weight segment of the TF-IDF is the frequency of the token in a particular document. If document one has football occurring twice then the TF would be two. However, there are various formulas for term frequency because the raw term frequency can distort the scores of a document.

The IDF stands for inverted document frequency which is the log of the result when the total number of collections is divided by the number of documents in which the token occurs. This allows unique terms that rarely occur in documents to be scored higher than common occurring words.

By performing the dot product of the TF with the IDF, a balance is struck between the common occurring words and rarely occurring words in a document. Below is a table of common forms that TF-IDF formulas can take.

\begin{tabular}{|c|c|c|c|}
\hline \multicolumn{2}{|c|}{ Term Frequency } & \multicolumn{2}{|c|}{ Document Frequency } \\
\hline Natural & $t f_{t, d}$ & None & 1 \\
\hline Logarithm & $1+\log \left(t f_{t, d}\right)$ & IDF & $N\left(\frac{N}{d f_{t}}\right)$ \\
\hline Augmented & $0.5+\frac{0.5 t f_{t, d}}{\max \left(t f_{t, d}\right)}$ & Probability IDF & $\left\{\max \left(0, \log \left(\frac{n-d f_{t}}{d f_{t}}\right\}\right.\right.$ \\
\hline Boolean & $\begin{array}{l}1 \text { if } t f_{t, d}>0 \\
0 \text { otherwise }\end{array}$ & \multicolumn{2}{|}{} \\
\hline Log average & $\frac{1+\log \left(t f_{t, d}\right)}{1+\log \left(a v e\left(t f_{t, d}\right)\right)}$ & \multicolumn{2}{|}{} \\
\hline
\end{tabular}

Figure 8: TF-IDF Variants [34]

In order to incorporate this into a vector space model, the cosine equation from earlier has the TF-IDF weight scheme substituted in place of the document and query vectors $\mathrm{d}$ and $\mathrm{q}$, which results in the following: 


$$
\operatorname{sim}\left(d_{j}, q\right)=\frac{\overrightarrow{d_{j}} \cdot \vec{q}}{\left\|\overrightarrow{d_{J}}\right\|\|\vec{q}\|}=\frac{\sum_{i=1}^{N} w_{i, j} w_{i, q}}{\sqrt{\sum_{i=1}^{N} w_{i, j}^{2}} \sqrt{\sum_{i=1}^{N} w_{i, q}^{2}}}
$$

\subsubsection{Probabilistic Model}

The next important model that we will be discussing is the probabilistic model, which has its roots in statistical theory and principles.

Probabilistic model is derived from "Bayes' Rule for inverting conditional probabilities [34]", which states:

$$
P(A \mid B)=\frac{P(B \mid A) P(A)}{P(B)}=\left[\frac{P(B \mid A)}{\sum_{X \in\{A, \bar{A}\}} P(B \mid x) P(X)}\right] P(A)
$$

A retrieval algorithm must be developed from the principles stated above to utilize probabilistic theory in ranked retrieval. The ranking principle states that we can rank documents by probability of relevance, but it's only based on the data available. There are a few different algorithms that can be implemented into a retrieval system. Most algorithms are based on a traditional method known as the binary independence model; this model states that if the document is relevant to the query or token, the value of 1 is assigned and 0 if not. This model is essentially the same as the multivariate Bernoulli Naïve Bayes model [34].

In order to derive a ranking algorithm from this model to include term frequencies or other measurements of relevancy, it must be assumed that a word appearing in a document is independent of any other presence of a word appearing.

$$
O(R \mid \vec{x}, \vec{q})=\frac{P(R=1 \mid \vec{x}, \vec{q})}{P(R=0 \mid \vec{x}, \vec{q})}=\frac{\frac{P(R=1 \mid \vec{q}) P(\vec{x} \mid R=1, \vec{q})}{P(\vec{x}, \vec{q})}}{\frac{P(R=0 \mid \vec{q}) P(\vec{x} \mid R=0, \vec{q})}{P(\vec{x}, \vec{q})}}=\frac{P(R=1 \mid \vec{q})}{P(R=0 \mid \vec{q})} * \frac{P(\vec{x} \mid R=1, \vec{q})}{P(\vec{x} \mid R=0, \vec{q})}
$$

If the possibility of a term occurring in a query is the same as not occurring, then the following can be surmised: 


\begin{tabular}{|c|c|c|}
\hline Relevance & Present & Not Present \\
\hline 1 & $p_{t}$ & $1-p_{t}$ \\
\hline 0 & $u_{t}$ & $1-u_{t}$ \\
\hline
\end{tabular}

Table 1: Relevance Probabilities [34]

Then occurrence and absent terms can be treated as the same, allowing substitution in the following equation:

$O(R \mid \vec{x}, \vec{q})=O(R \mid \vec{q}) * \prod_{t: X_{t=q}} \frac{p_{t}\left(1-u_{t}\right)}{u_{t}\left(1-p_{t}\right)} * \prod_{t: q t=1} \frac{\left(1-p_{t}\right)}{\left(1-u_{t}\right)}$

The retrieval status value or RSV, which will become the basis of all retrieval algorithms developed in the model, can be derived from this equation. By taking the log of the equation above, the following equation is derived:

$$
\sum \log \left(\frac{p_{t}\left(1-u_{t}\right)}{u_{t}\left(1-p_{t}\right)}=\log \left(\frac{p_{t}}{1-p_{t}}\right)+\log \left(\frac{1-u_{t)}}{u_{t}}\right)\right.
$$

The above equation is the basis for derivation of the Best Match 25 (BM25) retrieval algorithm. BM25 has many derivations, but the one developed by Robertson and Jones will be the focus of this investigation.

$$
\operatorname{score}(D, Q)=\sum_{i=1}^{n} \operatorname{IDF}\left(q_{i}\right) \cdot \frac{f\left(q_{i}, D\right) \cdot\left(k_{1}+1\right)}{f\left(q_{i}, D\right)+k_{1} \cdot\left(1-b+b \cdot \frac{|D|}{\text { avgdl }}\right)}
$$

The IDF segment of the equation is the same as the vector space model. The variable parameters $b$ and $k$ are tuning parameters that can be changed if the queries the users will be utilizing are of a certain length. For example, if there is no normalization of a query, then $b=0$ and if a query is larger, then the $\mathrm{k}$ value is used for term frequency.

BM25 is one of the most successful retrieval algorithms that offers more precision than vector space models due to the fact that probabilistic model is a more fluid model for IR than the vector space model. 
The current research in probabilistic modeling is mainly focused on improving the BM25. One recent algorithm is the BM25F, developed by Robertson and Microsoft Research [35]. This algorithm focuses on taking the structure of the document into account for scoring. Instead of computing the scores for individual fields like "title," "author," or "year," Robertson offers a new view. He argues that the term frequencies can be calculated before "the non-linear term frequency is applied" [35]. This compensates for structure data whereas the original BM25 did not.

Another area of research is incorporating a proximity-based approach on probabilistic principles [36]. This model would incorporate BM25 traits with term weights and the distance of two queries. The position of a query term in a document is taken into account without having a special index structure. According to Microsoft Research, this improves BM25 on average $5 \%$ to $10 \%[36]$.

While there are other research motivations in this model, most of them center around improving BM25 or deriving new models like the Language model, which is the next retrieval model to be discussed.

\subsubsection{Language Model}

The language model is a unique model that attempts to capture the "language" a collection uses to better predict the most relevant document for a given query. This model improves upon pitfalls of the other two models. One example is dealing with the absence of terms in relevant documents. The language models offer a concept called smoothing, which essentially smoothes the values of a document so an absent term doesn't cause "gaps" in the scoring of the document.

Language model can be thought of as finite automata in which the query is the automata itself, while the document is evaluated to see if it matches that automata. The more a document can generate the query parts, the greater the relevance.

The first type of language model is known as a unigram language model, which estimates all terms independently. The second type of language model is called bigram language model, which is useful for speech 
recognition and other machine translation. The unigram model is more useful for the purpose of text due to the lack of training data.

In the unigram language model comes the query likelihood principle, where a language model $m_{d}$ is constructed based on each document d. As before with the probabilistic model, Bayes rule is utilized:

$$
P(q \mid d)=\frac{P(q \mid d) P(d)}{P(q)}
$$

Using naïve Bayes model from before and use an equivalent model known as the multinomial unigram model to construct a model which can determine the probability.

$$
\begin{gathered}
P(d)=\frac{L_{d} !}{t f_{t 1, d} ! t f_{t 2, d} ! \ldots t f_{t m, d !}} P\left(t_{1}\right)^{t f_{t 1, d}} P\left(t_{2}\right)^{t f_{t 2, d} \ldots P\left(t_{m}\right)^{t f_{t m, d}}} \\
P\left(q \mid M_{d}\right)=K_{q} \prod_{t \in v} P\left(t \mid M_{d}\right)^{t f_{t, d}}
\end{gathered}
$$

In order to estimate $P\left(q \mid m_{d}\right)$, a maximum likelihood estimation and a unigram model can be combined to derive equation 2.12 .

$$
\hat{P}\left(q \mid M_{d}\right)=\prod_{t \in q} \hat{P}_{m l e}\left(t \mid M_{d}\right)=\prod_{t \in q} \frac{\mathrm{t}_{t, d}}{L_{d}}
$$

In the above equation, the term frequency and length of the document is utilized in order to estimate the probability of the document's ability to generate the query. However, the issue with using the equation above is if a term doesn't occur in the document then the value is zero. The problem is a probability of zero will throw off the language model and it will not retrieve the most relevant document. For example, if a probability of zero occurs in speech recognized algorithm, then chances are the program would fail to recognize the speech correctly.

A smoothing technique must be applied to the model to eliminate the probability of zero in relevant documents. For example, suppose the query $=\{$ King Arthur $\}$ and document one only has "Arthur" in the document. Instead of applying a zero for "King" not being in the 
document, a smoothing equation is applied to that segment instead. This ensures that the document is scored as most relevant.

The first type of smoothing technique is the Jelinek-Mercer (JM) smoothing method, which is a linear combination of the document information and collection information. By combining both a document level statistic and a collection level, there will never be a zero probability with this retrieval algorithm.

$$
P(d \mid q)=\prod_{t \in q}\left((1-\lambda)\left(\frac{t f_{t, d}}{L_{d}}\right)+\lambda\left(\frac{L_{t}}{L_{c}}\right)\right)
$$

This equation takes into account the length of the document $\left(L_{d}\right)$, term frequency of the term in the document $\left(t f_{t, d}\right)$ for document level statistics and for collection level, the frequency of the term in collection $\left(L_{t}\right)$ and the length of collection $\left(L_{c}\right)$. The tuning variable $\lambda$ is used control the influence of the document to collection level statistics. Most systems utilize a value of 0.5 [24].

The other smoothing method is the Dirchlet Smoothing [24]; this method derives from the Dirichlet Distribution [37]. Like its counterpart, JelinekMercer, Dirichlet utilizes a tuning variable $(\mu)$ to distribute the collectiondocument level influence.

$$
P(d \mid q)=\prod_{t \in q}\left(\frac{t f_{t, d+\mu\left(\frac{L_{t}}{L_{c}}\right)}}{L_{d+\mu}}\right)
$$

As before, the parameters are the same, but the $\mu$ variable could be changed to create an even distribution and influence the collection to document level statistics. Usually the tuning value is adaptive based on the collection and the document combined[38] or a constant value[24]. Dirichlet vs. Jelinek-Mercer investigations have shown Dirichlet tends to outperform Jelinek-Mercer [24].

Language model offers a variety of advantages over the other "bag of words" models discussed earlier in this chapter due to its adaptive nature and smoothing the scoring of the documents. Language model research varies widely, with improvements on the current methods of application to development of new language model methods. 
One recent investigation proposes a topic-based language model as compared to a collection-based model. The reason for this is to obtain more accurate document retrieval and to remove the estimation of the document language model problem [39]. This investigation has shown that performing a two stage topic-based language model does outperform other language models. The problem with this investigation is it only utilized small test sets [39].

Another investigation by the University of California proposed a two stage Bigram model. This model was compared against a unigram model. This study found over $40 \%$ of the time the two stage Bigram outperformed the unigram model. The collection consisted of 11, 916 English queries [40].

Another problem with using a language model is that finding a mode that is not computationally exhaustive, while providing effective retrieval. In addition, language model retrieval methods can be more sensitive than and not as robust as the TD-IDF approaches. [41]

There have already been comparison studies of these three models, most notably the investigation done in 2006 by Sperious and Tashiro [42]. This investigation found BM25 and the language model were even, where BM25 outperformed until the language model parameter was modified [42]. 


\section{Chapter 3: Theory}

Chapter three will discuss the theory of different inverted index implementation. By analyzing the retrieval algorithms and index construction, an expectation can be derived.

\subsection{Index Construction Time}

The inverted index construction for each implementation for this investigation starts with the retrieval of the documents from a relational database. After the document is retrieved, each implementation's process is slightly different creating three unique scenarios.

\subsubsection{Database Selection}

Before derivation of the database retrieval can begin, a few assumptions that will assist in the derivation of the equation must be made. As stated before, it is assumed that the collection is located in a relational database management system or (RDBMS). Secondly, it is assumed that only tables exist in this database and that any views, triggers, or other components of the database can be ignored, which allows for the database schema (s) to be denoted as:

$$
S=\left\{t_{1}, t_{2}, \ldots, t_{n}\right\}
$$

Where $t$ represents the tables in the set $S$ of the schema. By deriving the schema, the primary and foreign keys (relationships) are identified. The relationships $\mathrm{R}$ are used to create the joins that binds all the tables together. Once the tables are joined, each document in the collection is a tuple that is based on the schema, thus deriving $\left(C_{r}\right)$.

$$
C_{r}=\left\{t_{1} \bowtie t_{2} \bowtie \cdots t_{n}\right\} \Rightarrow\left\{R_{1}, R_{2}, \cdots, R_{n}\right\}
$$


In order to construct an equation for the approximate time it takes to collect the data, the following steps must be outlined:

1. Selection operation on primary key (indexed attribute) A.

2. Natural Join based on the relationships R.

The first part is fairly trivial, since most RDMBS utilize a b-tree data structure for primary keys [44]. A b-tree data structure is a binary tree that can have more than two children. A b-tree operates in $O(\log (n))$ [44] for locating and retrieving the values, which is how equation 3.3 is derived.

$$
\log (n)
$$

The number of tuples per relationship is simply the cross product of $N t_{1} \times N t_{2}$ which is $\mathrm{Nr}$. However, since only the unique tuples (natural join) are of concern, the number of unique tuples must be identified. By dividing $\mathrm{N}_{\mathrm{r}}$ by the unique tuples of each table creating a low bound and an upper bound for the total number of tuples each relationship possess is derived:

$$
\frac{N_{r}}{\text { Unique tuples of } t_{1}} \leq N_{r} \leq \frac{N_{r}}{\text { Unique tuples of } t_{2}}
$$

$\mathrm{N}_{\mathrm{r}}$ will always be between the two bounds and because of that, $\mathrm{N}_{\mathrm{r}}$ can replace $n$ in equation 3.3. With the number of tuples $n$ known, an approximation of joining these relationships can be developed. Since the joins involved are natural joins, the natural joins are created by the index nested loop join algorithm. This algorithm has the time $\mathrm{O}\left(n^{2}\right)$ since it pairs the relations of table one with table two. The value of $n$ can be replace to reflect tuples in both table one and table two, which becomes $\mathrm{m}$ $+\mathrm{n}$. Thus, the following time for the join and selection of the collection:

$$
T_{\text {select join }}=(m+n)^{2}+\log (N r)
$$

Thus, the time it takes for the data of the collection to be collected from the database and analyzed can be approximated by equation 3.6.

$$
T_{D G}=(m+n)^{2}+n+\log (N r)
$$

Equation 3.6 will be used as a starting point when deriving the approximate time of constructing an index in each implementation.

\subsubsection{R.A.M. Index Construction}


R.A.M. based index implementations, typically are employed in two ways:

1. Interweaved Posting List and Inverted Index

2. Separate Posting List and Inverted Index

This investigation will focus on the separate posting list and inverted index. In addition, it will be assumed that the data structure for both is a HashMap. There are two situations that must be accounted for when constructing the overall index structure. The first one is looking up the posting list if term is already in the index. The second situation is if the term is new to the index.

In order to construct time to insert into a single structure, the following time constant is derived:

$$
T_{\text {insert }}=\frac{\text { Size of Entry }}{\text { Data Rate of } R A M} \rightarrow C_{\text {insert }}
$$

If the term is new to both structures then $T_{\text {insert }}$ would simply be multiplied by 2 . However, if a term already exsist in the inverted index and the posting list is updated, then $T_{\text {insert }}$ will change to reflect the inverted index lookup and the posting list insertion.

$$
T_{\text {insert Update }}=\frac{\text { Size of Entry }}{\text { Data Rate of RAM }}+T_{\text {lookup }} \rightarrow C_{\text {insert Post }}+C_{\text {insert }}
$$

$T_{\text {lookup }}$ is the time it takes to lookup the entry in the inverted index, this value is usually $240 \mathrm{~ns}[24]$. Since the insert and the update will not vary, it can assume they are constant, thus creating equation 3.9 to approximate the R.A.M index construction time.

$$
T_{R A M \text { Index }} \approx \sum_{i=1}^{N}\left(C_{\text {insert Post }}+C_{\text {insert }}+C_{\text {insert }}\right) \rightarrow 3 C
$$

Where $\mathrm{N}$ is the number of terms to be inserted in the index. Thus, equation 3.9 approximates the index construction time as being constant due to the consistent nature of HashMap access times.

\subsubsection{Disk Index Construction}

The disk index construction will take equation 3.9 as one of its factors for approximating the index construction time on a disk. The reason for this 
is the database implement utilizes RAM to cache the inverted index and posting list until the data is transfer to disk. Since a complete rebuild of the index is warrant when it changes more than $60 \%$ [24] and this is not a factor, the index construction will be treated as static.

Since the data is being transferred to a hard disk, the data rate of the hard disk and the size of the entry are used to determine the approximate time to write the entry to disk. Thus, to derive $T_{\text {write }}$, the transfer rate of the hard disk is utilized to formualate equation 3.10.

$$
T_{\text {write }}=\sum_{i=1}^{N} \frac{1,000,000 *(\text { Size of Entry } i)}{\frac{\text { RpM of HD }}{60} * \text { Sectors per Track } * \text { Sector Size } * 8} \rightarrow \sum_{i=1}^{N} n+C_{\text {data transfer }}
$$

Where $\mathrm{N}$ is the number of entries needing transferred to hard disk. This becomes a constant because the data rate should not change drastically. In order to determine the complete time, the time of the R.A.M. index construction and the $T_{\text {write }}$ time are combined to approximate hard disk index construction.

$$
T_{\text {Disk Time }} \approx T_{\text {Ram Time }}+T_{\text {write }}
$$

Thus, equation 3.11 is able to approximate the time of creating an index structure that is stored on the disk.

\subsubsection{Database Index Construction}

The database index construction will utilize elements of a b-tree. There will be two tables, one for the inverted index and another for the posting list. There will be some overhead time constant that is attributing to the keys and other issues. In the calculations, it is assumed that all data is committed to the database at the end of populating the inverted index and posting list.

In the derivation, the time taken to construct an R.A.M. index will be included. The R.A.M. index will act as a cache then commit the datasets to the database at once when necessary. By doing a massive commit, index construction is faster. It is also noteworthy that one benefit of utilizing DBMS, it that constraints on table prevent duplicates entries. 
In the construction of the tables, the term of the inverted index and the posting id of the posting list will be the primary key or indexed value. By keeping two tables, there will be at least two writes for a new entry, one for each table. Since the database tables use a b-tree data structure for tables with a primary key, the insertion time of a b-tree can be used like in equation 3.12 .

$$
T_{\text {write }}=\log (\mathrm{n})+\mathrm{n} * \log (\mathrm{n})=\log (\mathrm{n})+\mathrm{n} * \log (\mathrm{n})
$$

Using the write time and R.A.M. time, the following derivation of database index construction time is approximated. In equation 3.13, $\mathrm{C}$ is a constant that compensates for the delay due to commitment of data to the database.

$$
T_{\text {Database Time }} \approx T_{\text {Ram Time }}+T_{\text {write }}+\mathrm{C}
$$

Now, an equation for every index storage location has been derived and the retrieval times for the three retrieval algorithms can be estimated.

\subsection{Retrieval Time}

In this section of the theory chapter, the focus will be on the effects the inverted index implementation has on each model. By deriving the common component that will be used for each implementation to model combination an estimate of time that each model's scoring algorithm will approximate.

\subsubsection{Vector Space Model}

The algorithm that will be analyzed in vector space model is the cosine similarity algorithm. This algorithm includes fetching posting list for each query term then scoring each document in the term's posting list. In all, this algorithm is said to be $O\left(n^{2}\right)$ time, which means that for all relevant query to document terms values, there are two steps that must be performed. Based on the time complexity, equation 3.14 can be derived 
based on the two dependent steps that are included in this scoring algorithm.

$$
T_{C S}=n^{2}
$$

The next step is needed is to calculate the effects each storage location will have on the algorithm.

\subsubsection{R.A.M. Storage}

The R.A.M. storage location will have to different components besides the cosine similarity component. This includes the lookup time for both the term and posting list. In order to keep this equation simple, the preprocessing will be neglected, since the time required to process the users query will be minimal in comparison.

If assumed there will be two look ups per query term, one for the term and other for the posting list, $T_{\text {lookup }}$ becomes $2 * T_{\text {lookup }}$. As before, if assumed that the lookup time for hash map does not vary largely, then $2 * T_{\text {lookup }}$ will become a constant.

$$
T_{R A M V S M} \approx \sum_{i=1}^{N} T_{C S}+2 * T_{\text {lookup }} \stackrel{\text { yields }}{\longrightarrow} \sum_{i=1}^{N} T_{C S}+C_{\text {lookup }}
$$

Where $\mathrm{N}$ is the number of terms in the users query, which will provides an approximate time for a retrieval system using an index structure implemented in R.A.M..

\subsubsection{File Storage}

If assumed a linear search will be performed on the file that contains both the inverted index and posting list then equation 3.16 is derived.

$$
T_{\text {File Look up }}=n
$$


Where file look up refers to looking up either the term or posting list. This means that there will be a file look up for the term and the posting list containing the term information. The transfer of data will need to be taken in account, which derives the equation 3.17.

$$
T_{\text {read }}=n(3.17)
$$

Where $\mathrm{n}$ is the number of entries to be read, which then allows for the following derivation to occur:

$$
T_{\text {File VSM }} \approx \sum_{i=1}^{N} T_{C S}+2 * T_{\text {File lookup }}+T_{\text {read }}
$$

\subsubsection{Database Storage}

Due to the inverted index and posting list being implemented as a table with a primary key, b-tree seek and retrieval times can be used. The seek time for $a b$-tree is $\log (n)$ where $n$ is the number of entries in the b-tree.

$$
T_{D B \text { lookup }}=\log (n)
$$

This look up must be performed twice, one of the inverted index and another for the posting list. This means the look up will be multiplied by two to derive equation 3.20 .

$$
T_{D B} \text { lookup overall }=2 * \log (n)
$$

This in return allows for the construction of the following time approximation for an inverted index implementation with a vector space model as equation 3.21 .

$$
T_{D B V S M} \approx \sum_{i=1}^{N} T_{C S}+T_{D B \text { lookup Overall }}
$$




\subsubsection{Probabilistic Model}

The probabilistic model algorithm that will be investigated is the Best Match 25 (BM 25) algorithm, which is the more popular algorithm in information retrieval. The BM 25 is $\mathrm{O}(\mathrm{n})$ time for worst case scenario and derives equation 3.22 .

$$
T_{B M 25}=n
$$

The equations for the storage location will be the same, but instead of vector space model time values, BM 25 will be incorporated to derive equations $3.23,3.24$, and 3.25 .

$$
\begin{aligned}
& T_{R A M B M} \approx \sum_{i=1}^{N} T_{B M 25}+2 * T_{\text {lookup }} \\
& T_{\text {File BM }} \approx \sum_{i=1}^{N} T_{B M 25}+2 * T_{\text {File lookup }}+T_{\text {read }} \\
& T_{D B \text { BM }} \approx \sum_{i=1}^{N} T_{B M 25}+T_{D B \text { lookup Overall }}
\end{aligned}
$$




\subsubsection{Language Model}

The language model algorithm that will be utilized is the maximum likelihood query with a Jelinek-Mercer smoothing. The algorithm operates in $\mathrm{O}(\mathrm{n})$ time and derives equation 3.26.

$$
T_{L M}=n
$$

As before in the probabilistic mode, the index implementation time approximations will remain the same, but the model approximations will not. Equations 3.27, 3.28, and 3.29 reflect these changes and approximate the time required for retrieval.

$$
\begin{gathered}
T_{R A M ~ L M} \approx \sum_{i=1}^{N} T_{L M}+2 * T_{\text {lookup }} \\
T_{\text {File LM }} \approx \sum_{i=1}^{N} T_{L M}+2 * T_{\text {File lookup }}+T_{\text {read }} \\
T_{D B \text { LM }} \approx \sum_{i=1}^{N} T_{L M}+T_{D B \text { lookup Overall }}
\end{gathered}
$$

With all equations derived to base the case studies on, an overall conclusion can be derived.

\subsection{Conclusion}

Based on the equations derived in this section there are two conclusions. For the index construction, it can be concluded that an index structure implemented in R.A.M. will be the fastest completed. Then the file implemented inverted index construction will be second followed by the database implemented inverted index.

\section{Index Construction:}

$c<n+c<\log (n)+n \log (n)+c \rightarrow$ RAM $<$ File $<$ Database

Where $\mathrm{n}$ is the amount of terms being processed and $\mathrm{c}$ are the constants derived in the previous sections.

\section{Retrieval:}

The second conclusion is no matter what retrieval algorithm being utilized, the database implemented inverted index outperformed the file implemented inverted 
index. From the equations derived in the earlier sections, the following expectations are made.

$$
C<2 \log (n)<3 n+c \rightarrow R A M<\text { Database }<\text { File }
$$

Thus, the b-tree data structure of the database table's provides efficiency over the file implemented inverted index. 


\section{Chapter 4: Case Studies}

This chapter will discuss the setup and applications utilized. In addition, the different queries and data collected will be discussed.

\subsection{Setup}

For this investigation, a java 1.7 program will be utilized for performing the different experiments for this investigation. The program will handle the index construction of the three different implementations as well as the nine different retrieval scenarios. During the investigation, a MySQL 5 database and LOUD 12.04 Ubuntu machines will be utilized. In table 2 are the specifications of the servers being used during the investigation.

\begin{tabular}{|c|c|}
\hline Operating System & Ubuntu 12.04 LTS \\
\hline Memory & 3GB \\
\hline & AMD Athlon 64 X2 Dual Core Processor 5600+ \\
Processor & $2.8 \mathrm{GHZ}$ \\
\hline Hard Drive & $500 \mathrm{~GB}$ \\
\hline
\end{tabular}

Table 2: System Information

There will be three different case studies that will be used during this investigation. Each case study consists of a different database with a different size and contents. Each case will utilize a different parser for tokenizing the information so that it can be indexed or compared against each case studies respective index. The following is the case study information:

\begin{tabular}{|c|c|c|c|c|}
\hline Case Study & $\begin{array}{c}\text { Document } \\
\text { Count }\end{array}$ & $\begin{array}{c}\text { Size of } \\
\text { Database }\end{array}$ & $\begin{array}{c}\text { Number of } \\
\text { Tables }\end{array}$ & $\begin{array}{c}\text { Number of } \\
\text { searchable } \\
\text { Fields }\end{array}$ \\
\hline 1 & 997 & $6.63 \mathrm{MB}$ & 4 & 8 \\
\hline 2 & 9340 & $86 \mathrm{MB}$ & 4 & 4 \\
\hline 3 & 40000 & $270.5 \mathrm{MB}$ & 5 & 6 \\
\hline
\end{tabular}

Table 3: Case Study Information 
The process of index construction is the same for each case study.

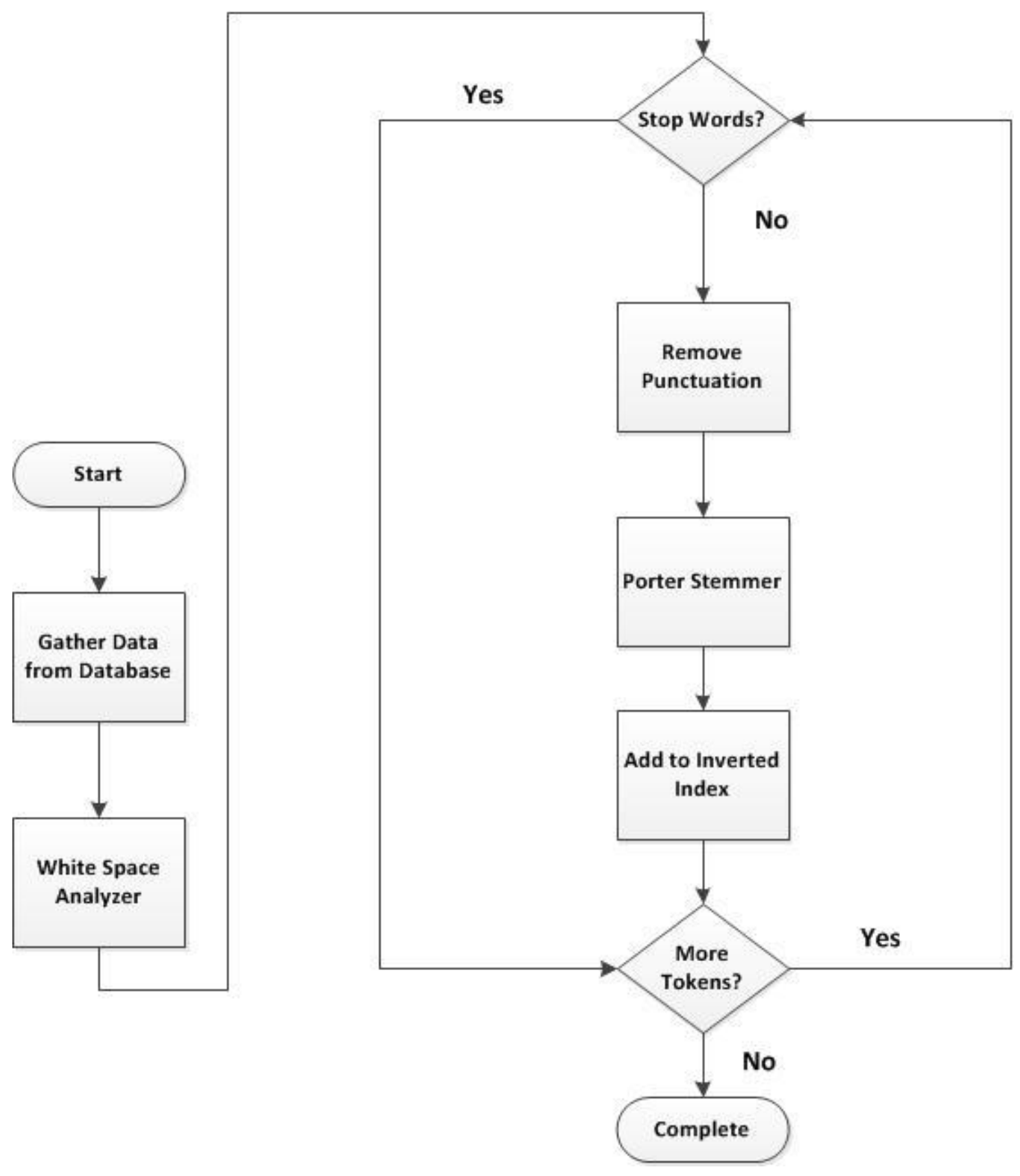

Figure 9: Index Construction Flow Diagram

The index construction process illustrated above utilizes a white space analyzer, which breaks down the documents based on white space between the words of the documents. Once this process is complete, each word is checked to see if it is on the "stop word" list, which is a list of words that are either very common in the English language such as "The" or "a" or words that serve no relevancy with respect to the content of the collection. In order for the indexing process to be 
effective when being searched, all punctuation is removed from the words and the words are stemmed. The porter stemmer is a five step stemmer that reduces words to their root or base. For example, "stories" and "story" would be reduced to "stori". Stemming was selected due to range of possible forms the user could input for one word and the demand for a high recall system. It is in this form that the word is added to the index.

Once the indexing process is complete, the collection may be searched. The process of handling a user's query is very similar to constructing an index. The only difference is that instead of inserting the words into the index, the word's posting list is retrieved. Once all the posting lists are retrieved, the ranked retrieval algorithm is applied to the documents. Afterwards, documents are sorted based on the score achieved based on the relevancy to the user's query. This process is illustrated in the diagram below:

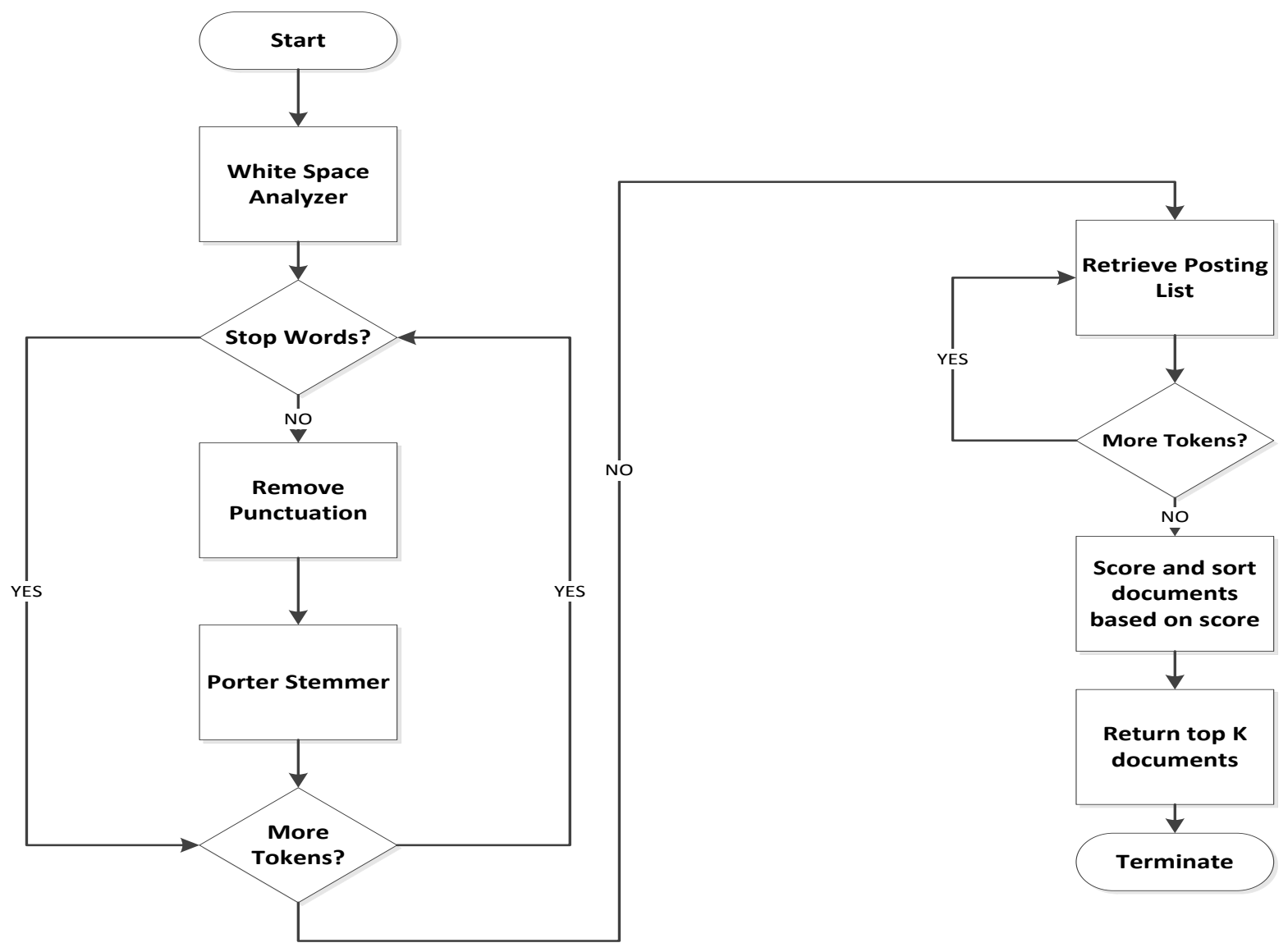

Figure 10: Retrieval Process 
During this investigation, there will be a few numerical values that we will be collecting from each situation. The precision and recall of each query will be collected, to compare the retrieval algorithms accuracy to recall ability. To normalize the precision and recall values, the irrelevant retrieved document count, relevant not retrieved documents count, and the remaining document count will be calculated. The precision and recall values will be collected for 25 trials, while the time values will be collected for 100 trials.

After the trials, the Mean Average ranked precision and unranked precision will be calculated. The reason tracking both ranked and unranked precision is to see if any drastic difference occurs between the two and to isolate any distortions that may occur in ranked precision values.

\begin{tabular}{|c|c|c|c|c|c|c|c|c|}
\hline 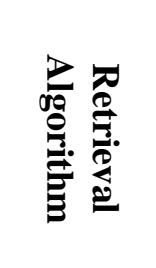 & 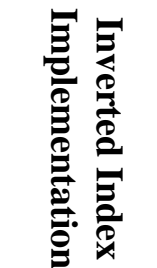 & 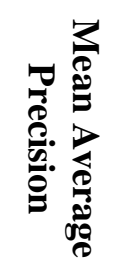 & 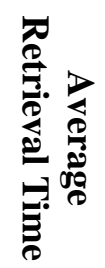 & 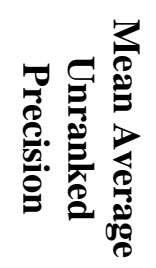 & 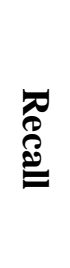 & 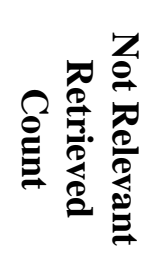 & 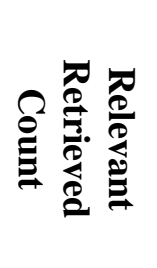 & 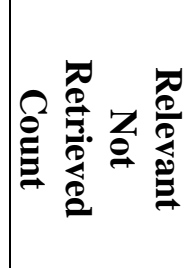 \\
\hline \multirow{3}{*}{$\begin{array}{c}\text { Cosine } \\
\text { Similarit } \\
y\end{array}$} & R.A.M. & & & & & & & \\
\hline & File & & & & & & & \\
\hline & Database & & & & & & & \\
\hline \multirow{3}{*}{$\begin{array}{c}\text { Best } \\
\text { Match } 25\end{array}$} & R.A.M. & & & & & & & \\
\hline & File & & & & & & & \\
\hline & Database & & & & & & & \\
\hline \multirow{3}{*}{$\begin{array}{c}\text { Languag } \\
\mathrm{e} \\
\text { Model }\end{array}$} & R.A.M. & & & & & & & \\
\hline & File & & & & & & & \\
\hline & Database & & & & & & & \\
\hline
\end{tabular}

The mean average ranked and unranked precisions are calculated using equation 4.1 , except the precision measurements aren't the same.

$$
M A P(Q)=\frac{1}{|Q|} \sum_{j=1}^{|Q|} \frac{1}{m_{j}} \sum_{k=1}^{m_{j}} \operatorname{Precision}\left(R_{j k}\right)
$$

Q is the set of relevant documents and $R_{j k}$ is the ranked or unranked retrieval results from the top results. Then $m_{j}$ is a subset of the set Q. 
In addition to the query data collection, the index construction times will be collected. These values will be collected for 100 trials and averaged.

\begin{tabular}{|c|l|}
\hline Storage Location & Construction Time \\
\hline RAM & \\
\hline FILE & \\
\hline Database \\
\hline \multicolumn{2}{|c|}{ Table 4: Index Construction Information }
\end{tabular}

Each case study has had a certain number of randomly generated user input queries and their SQL equivalent. The number of test queries generated is roughly $10 \%$ of the total collection size of the case study.

\begin{tabular}{|c|c|}
\hline Case Study & Number of Queries \\
\hline One & 100 \\
\hline Two & 1000 \\
\hline Three & 4285 \\
\hline
\end{tabular}

Table 5: Number of Queries

\subsection{Case Study One}

The data that resides in this database can be classified as homogenous in which most of the data does not have punctuation, foreign language vocabulary, or error/malformed text. This case study comes from the MySQL performance and tuning collection[46] and is known as the Sakila database. This database has the following fields:

\begin{tabular}{|c|c|}
\hline Table & Fields \\
\hline Film & $\begin{array}{c}\text { Title, description (desc), release year, name, } \\
\text { length, rating }\end{array}$ \\
\hline Film_Actor & $\begin{array}{c}\text { No searchable field }->\text { Join Table } \\
\text { Actor }\end{array}$ \\
\hline Language & $\begin{array}{c}\text { First_name, last_name (combined to create } \\
\text { actor field) }\end{array}$ \\
\hline \multicolumn{2}{|c|}{ Table 6: Case Study One Fields } \\
\hline
\end{tabular}

The queries used for the retrieval system and database can be seen in Appendix A.1. These queries were auto generated via a java program. 


\subsection{Case Study Two}

The data that resides in this database as compared to the previous database is more diverse with foreign language vocabulary, lots of punctuations, duplicates, and plurals of words. Case study two is known as Movielens and is part of the MySQL performance and tuning collection[46]. This database has the following fields:

\begin{tabular}{|c|c|}
\hline Tables & Fields \\
\hline Movies & Rating, title \\
\hline Users & None $->$ Join Table \\
\hline Ages_ranges & Age_ranges \\
\hline occupations & occupation \\
\hline \multicolumn{2}{|c|}{ Table 7: Case Study Two Fields }
\end{tabular}

The queries used for the retrieval system and database can be seen in Appendix A.2.

\subsection{Case Study Three}

The data that resides in this database have much longer documents, lots of punctuations, word roots, and different language vocabulary words. This database will demonstrate the importance of removing the "stop words". In addition, illustrate the weakness of a vector space model as compared to best match 25 or language model algorithm. This case study is a subset of the IMDB database collection[47]. This database has the following fields:

\begin{tabular}{|c|c|}
\hline Tables & Fields \\
\hline Movies & Name, year, rank \\
\hline Actor & $\begin{array}{c}\text { Combine first_name, last_name to } \\
\text { form actor, gender }\end{array}$ \\
\hline Role & role \\
\hline Movie_genres & genres \\
\hline
\end{tabular}

Table 8: Case Study Three Fields 
The queries used for the retrieval system and database can be seen in Appendix A.3. 


\section{Chapter 5: Results}

This chapter is divided into four sections; the first section displays the results from the index construction involving each storage location. Then the other sections discuss case study results for each combination discussed in the previous chapter.

\subsection{Index Construction}

The following are the average times collected for index construction of each case study. The averages reflect 100 trials for each case study and the different storage implementations.

\subsubsection{Case Study One Indexing}

The following table reflects the data collected for 100 trials of indexing case study one.

\begin{tabular}{|c|c|c|}
\hline Implementation & Average Time & Standard Deviation \\
\hline RAM & $0: 00: 04$ seconds & $\pm 0: 00: 00$ seconds \\
\hline File & $0: 00: 03$ minutes & $\pm 0: 00: 00$ seconds \\
\hline Database & $0: 01: 27$ minutes & $\pm 0: 00: 01$ seconds \\
\hline
\end{tabular}

\subsubsection{Case Study Two Indexing}

The following table reflects the data collected for 100 trials of indexing case study two.

\begin{tabular}{|c|c|c|}
\hline Implementation & Average Time & Standard Deviation \\
\hline RAM & $0: 00: 42$ seconds & $\pm 0: 00: 10$ seconds \\
\hline File & $0: 01: 14$ minutes & $\pm 0: 00: 09$ seconds \\
\hline Database & $0: 11: 10$ minutes & $\pm 0: 02: 51$ minutes \\
\hline
\end{tabular}




\subsubsection{Case Study Three Indexing}

The following table reflects the data collected for 100 trials of indexing case study three.

\begin{tabular}{|c|c|c|}
\hline Implementation & Average Time & Standard Deviation \\
\hline RAM & $0: 16: 26$ minutes & $\pm 0: 02: 00$ minutes \\
\hline File & $0: 08: 10$ minutes & $\pm 0: 00: 33$ seconds \\
\hline Database & $0: 59: 39$ minutes & $\pm 0: 01: 56$ minutes \\
\hline
\end{tabular}

\subsubsection{Indexing Comparison}

The following table shows the relationship of the collection size to indexing time for each index implementation.

\begin{tabular}{|c|c|c|c|}
\hline Collection Size & RAM & File & Database \\
\hline 997 & $0: 00: 04$ seconds & $0: 00: 03$ seconds & $0: 01: 27$ minutes \\
\hline 9340 & $0: 00: 42$ seconds & $0: 01: 14$ minutes & $0: 11: 10$ minutes \\
\hline 40000 & $0: 16: 26$ minutes & $0: 08: 10$ minutes & $0: 59: 39$ Minutes \\
\hline
\end{tabular}

The graph below shows the relationship that forms between the indexing time and size of the collection.

\section{Effects of Size on RAM Indexing}

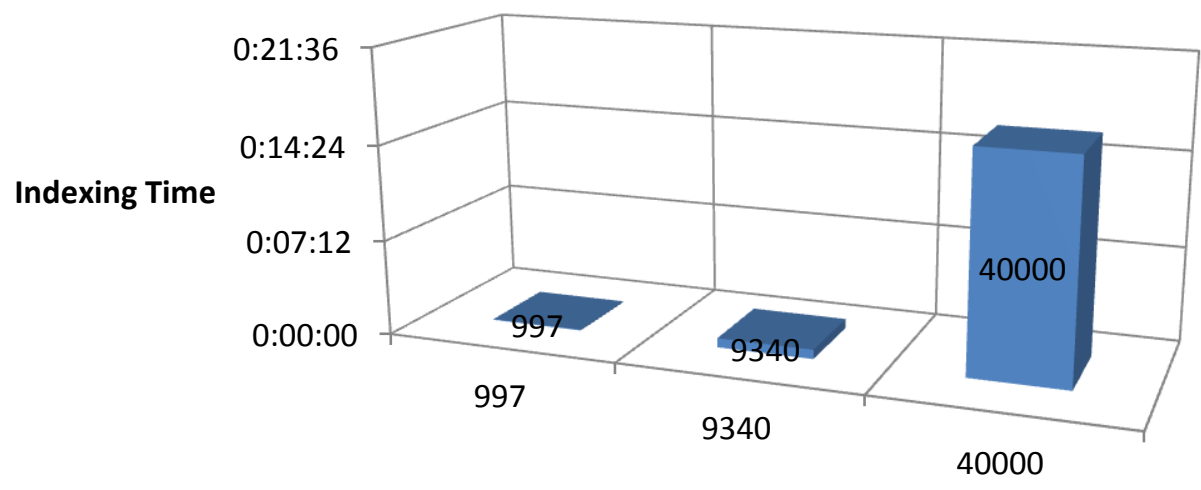

Collection Size 
The graph below shows the same increasing relationship that was displayed in the RAM graph of Figure 11.

\section{Effects of Size on File Indexing}

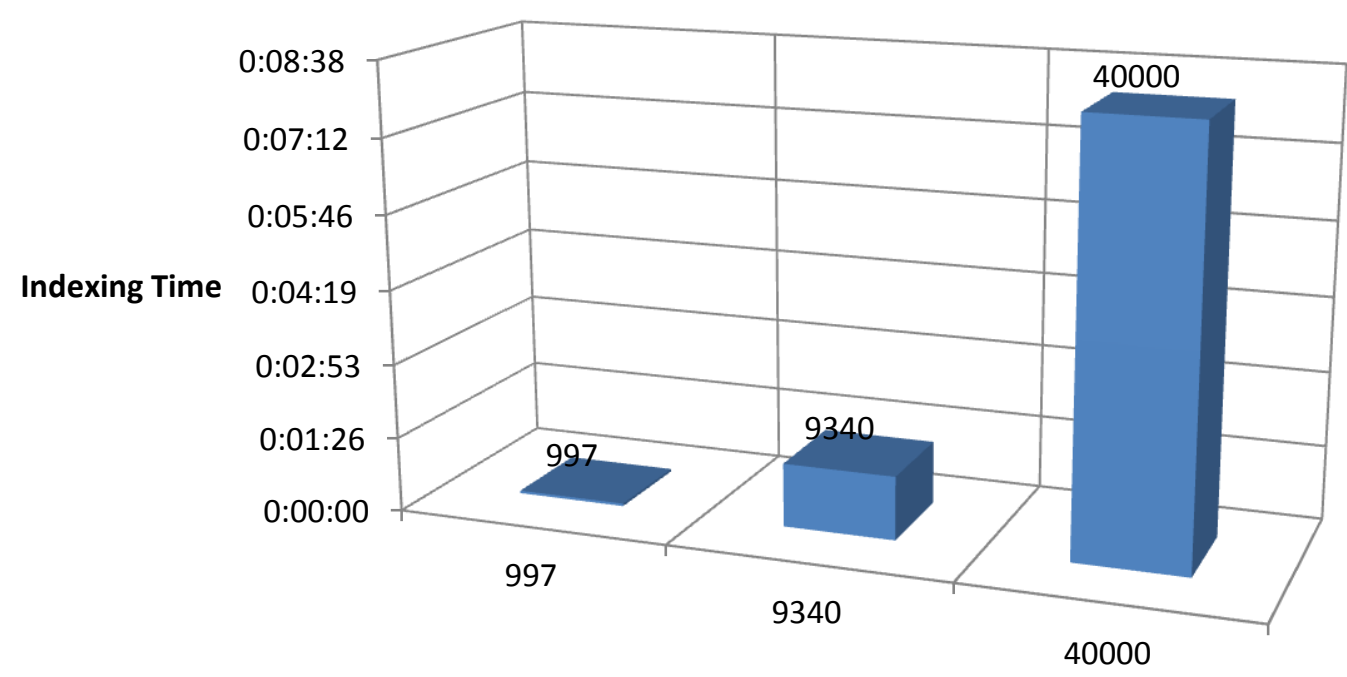

Colleciton Size

Figure 12: File Indexing vs. Collection Time 
Finally, the database indexing shows the same familiar trend that was experienced in the other indexing implementations.

\section{Effects of Size on Database Indexing}

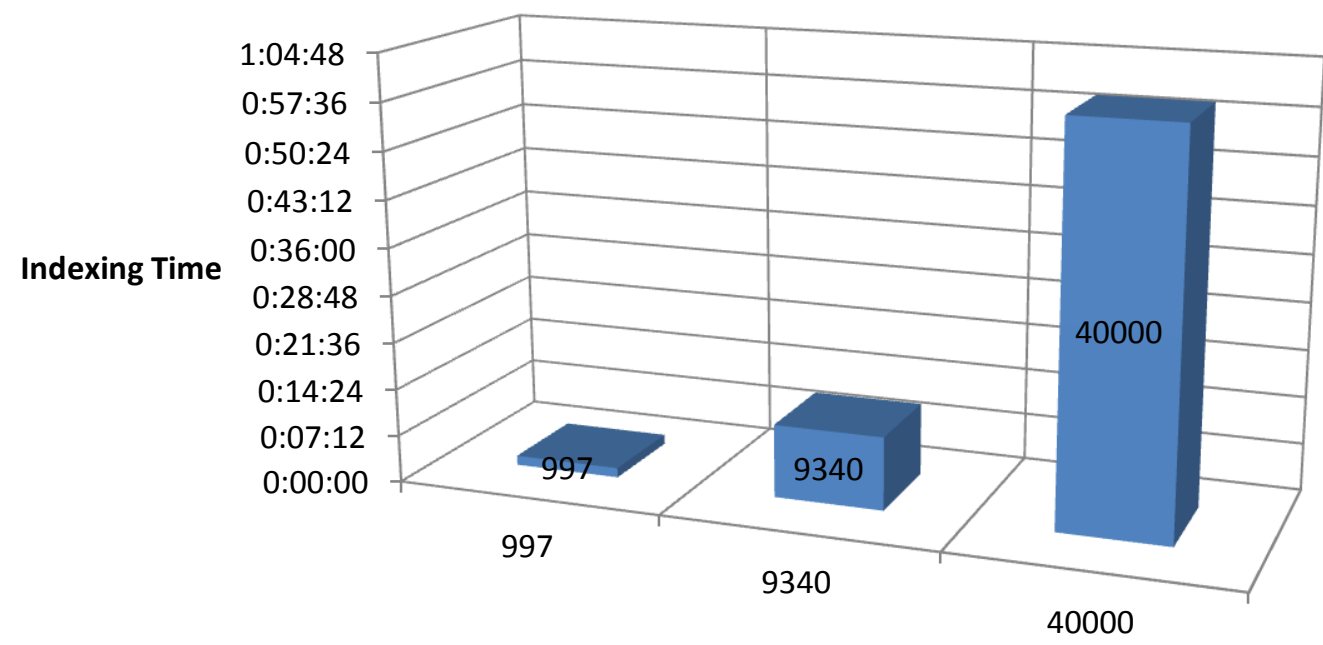

Collection Size

Figure 13: Database Indexing vs. Collection Time

The index construction time shows that as the collection size increases, the time to construction the index increases as well. 


\subsection{Case Study One}

The following section is dedicated to the results obtain from the trials of case study one database using the nine different situations.

\section{R.A.M. Implementation with Vector Space}

The R.A.M. results for the vector space model are summarized below. The confucius matrix for the data is located below the summary table.

\begin{tabular}{|c|c|c|}
\hline & Average & Standard Deviation \\
\hline Average Query Time & $00: 00: 00.106$ & $\pm 00: 00: 00.217$ \\
\hline $\begin{array}{c}\text { Mean Average Unranked } \\
\text { Precision }\end{array}$ & $12.41 \%$ & $\pm 5.45 \%$ \\
\hline Mean Average Precision & $15.90 \%$ & $\pm 6.50 \%$ \\
\hline Mean Average Recall & $96.96 \%$ & $\pm 0.00 \%$ \\
\hline
\end{tabular}

Table 13: R.A.M. Vector Space Summary

\begin{tabular}{|c|c|c|}
\hline RAM & Relevant & Not Relevant \\
\hline Retrieved & 2 & 87 \\
\hline $\begin{array}{c}\text { Not } \\
\text { Retrieved }\end{array}$ & 0 & 908 \\
\hline
\end{tabular}

Table 14: Confucius Matrix R.A.M. VSM

The graphs located below illustrate the relationship between ranked and unranked precision with recall. Then figure 15 shows the distribution of average query times for each query. The outliers in both graphs are not reflected in the above data collect. 


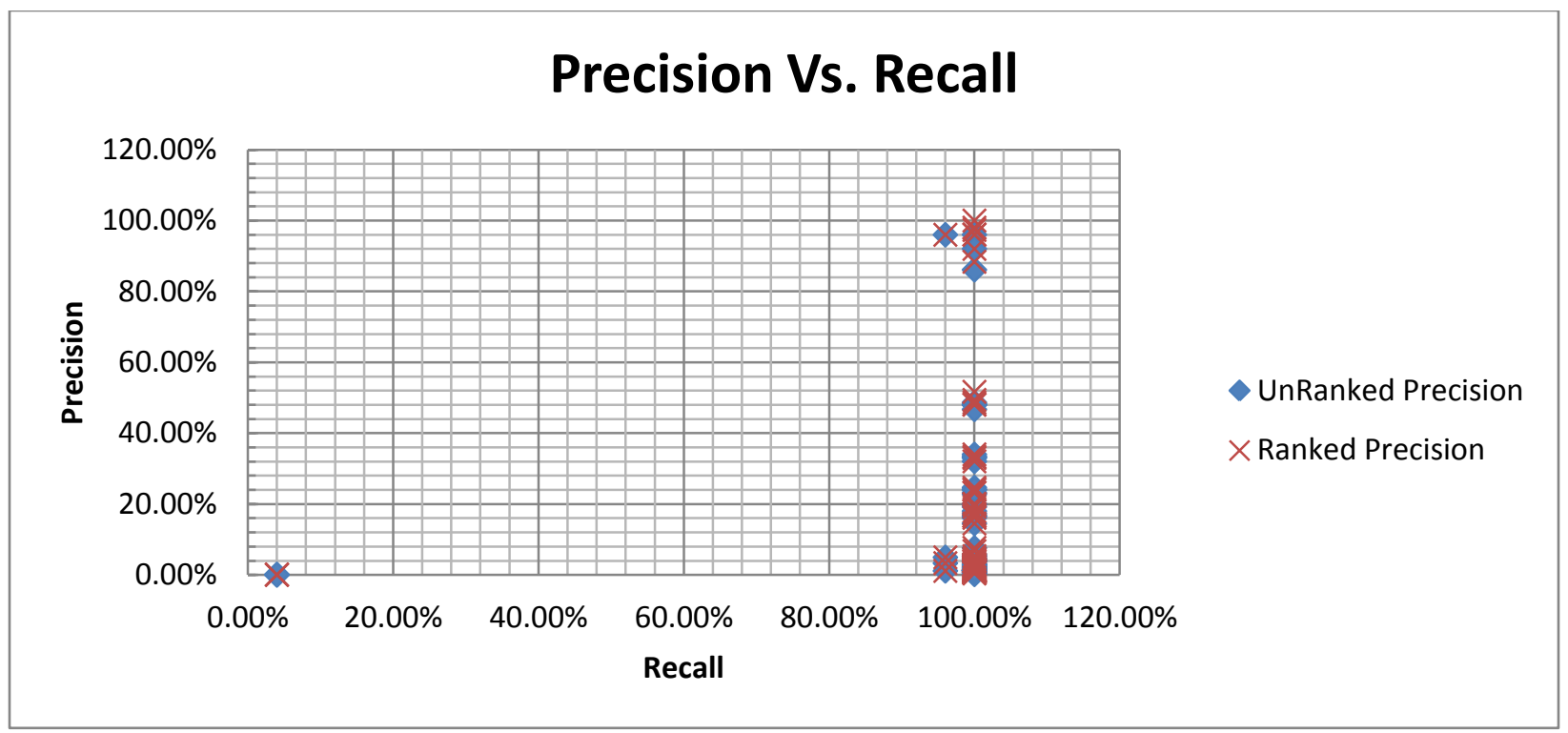

Figure 14: Precision vs. Recall R.A.M.

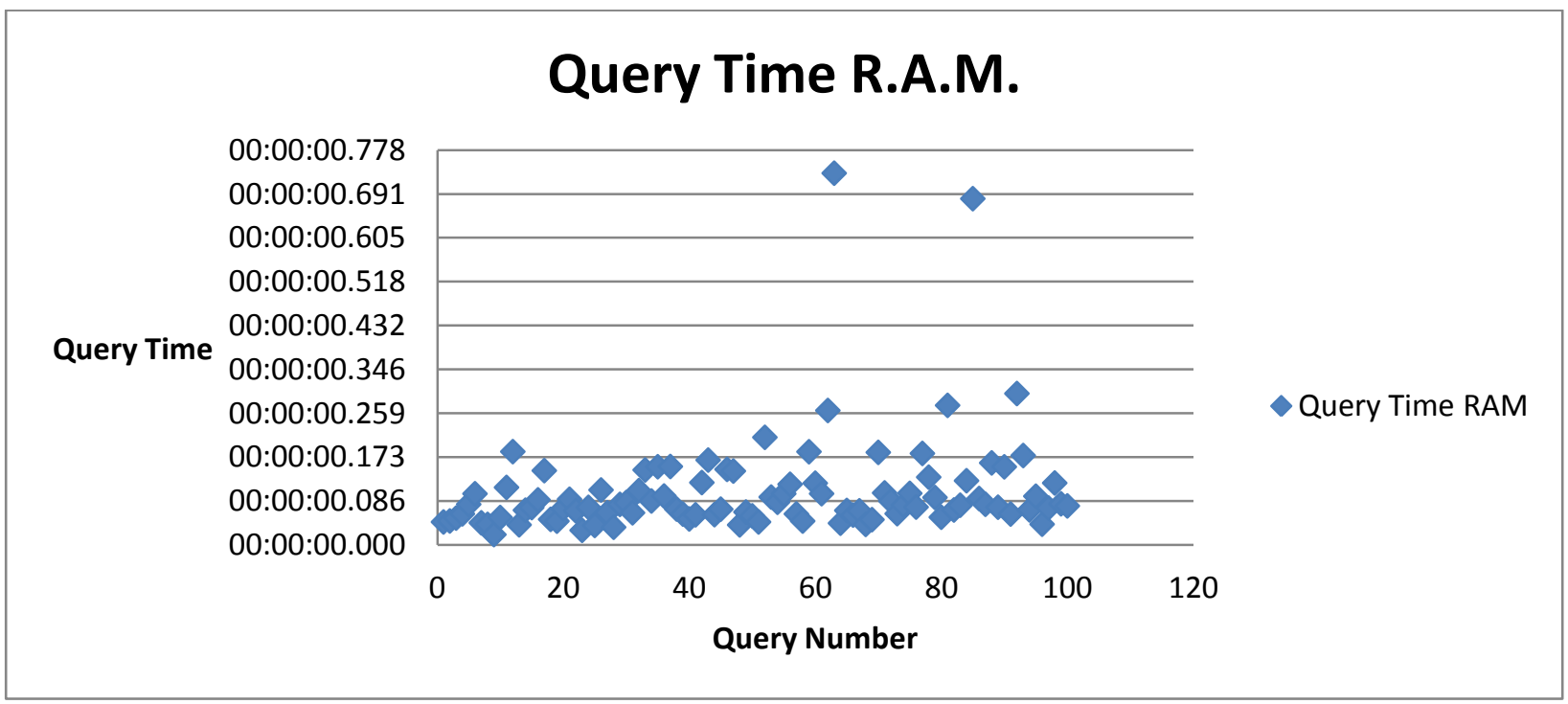

Figure 15: Query Time R.A.M. 


\section{File Implementation with Vector Space}

The data below is a summary for the file implementation using the vector space model retrieval algorithm. To normalize the data, the Confucius matrix of retrieved, not retrieved and relevant count is included below.

\begin{tabular}{|c|c|c|}
\hline & Average & Standard Deviation \\
\hline Average Query Time & $00: 00: 00.697$ & $\pm 00: 00: 00.080$ \\
\hline Mean Average Unranked Precision & $11.58 \%$ & $\pm 0.00 \%$ \\
\hline Mean Average Precision & $15.63 \%$ & $\pm 0.00 \%$ \\
\hline Mean Average Recall & $97.00 \%$ & $\pm 0.00 \%$ \\
\hline
\end{tabular}

\begin{tabular}{|c|c|c|}
\hline File & Relevant & Not Relevant \\
\hline Retrieved & 2 & 87 \\
\hline $\begin{array}{c}\text { Not } \\
\text { Retrieved }\end{array}$ & 0 & 908 \\
\hline \multicolumn{2}{|c|}{ Table 16: Confucius Matrix for File } \\
\hline
\end{tabular}

The graphs below illustrate the trends that formed during the collection of data. Any outliers that were found in the data were graphed, but not recorded in the data above.

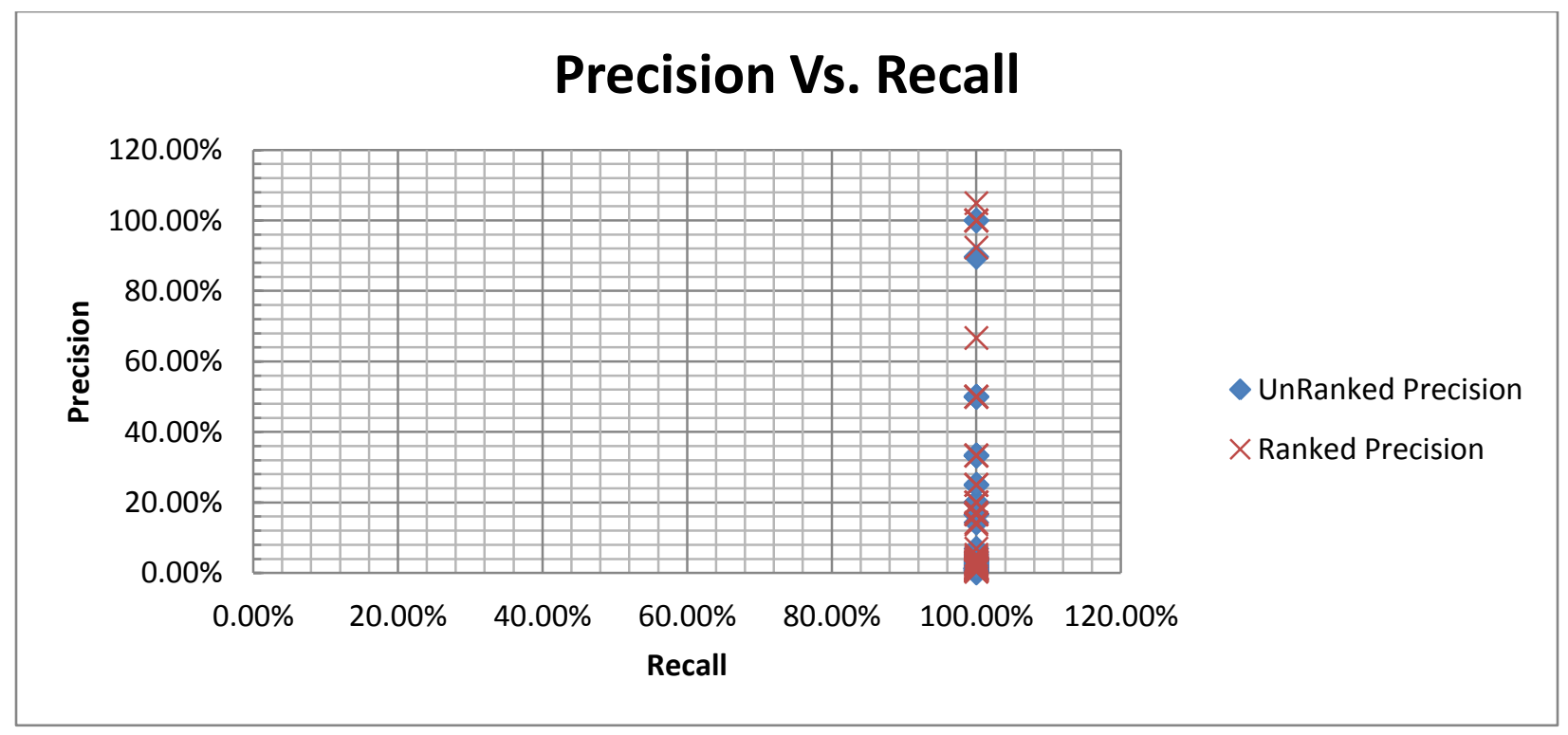

Figure 16: Precision vs. Recall 


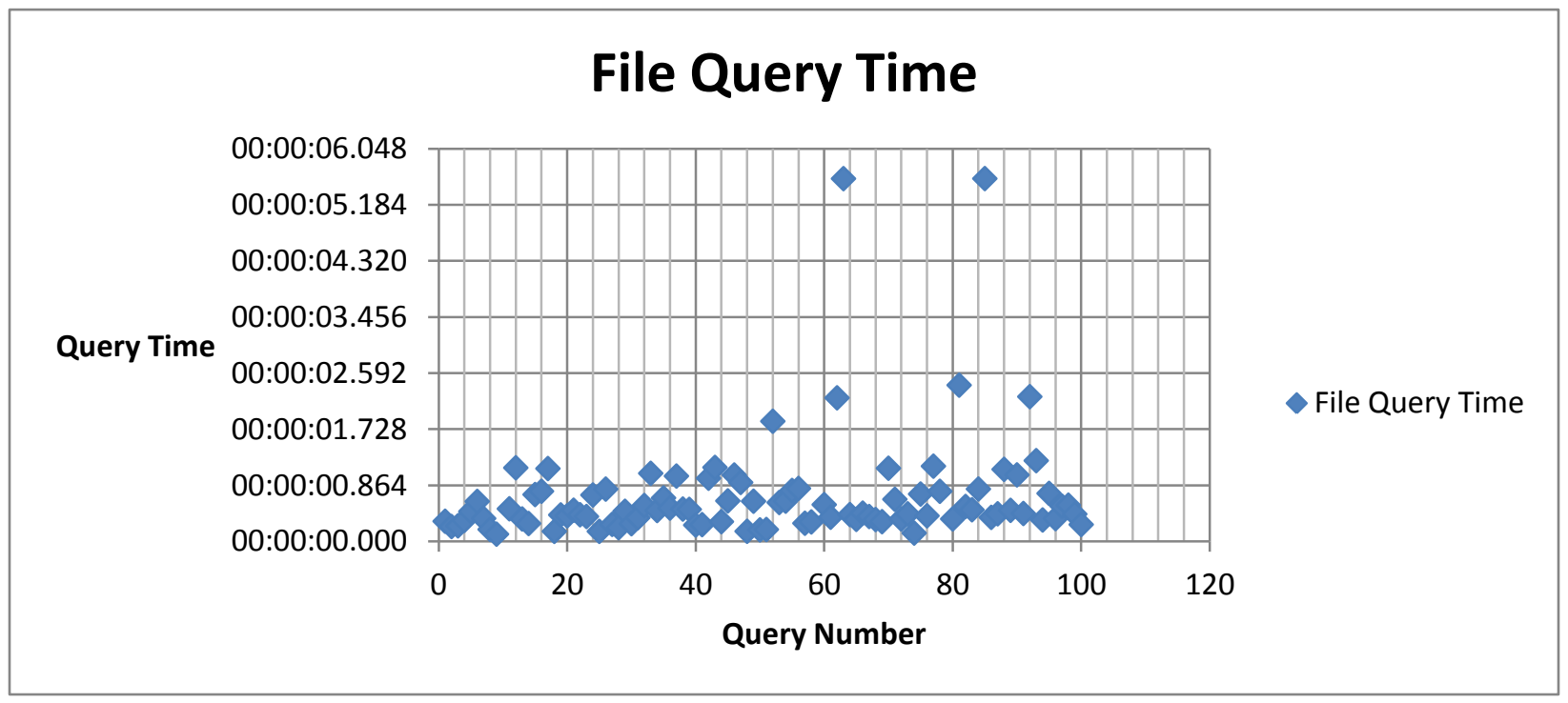

Figure 17: File Query Time

\section{Database Implementation with Vector Space}

The data below is a summary of the results of the data collected during the database implementation using a vector space model.

\begin{tabular}{|c|c|c|}
\hline & Average & Standard Deviation \\
\hline Average Query Time & $00: 00: 00.376$ & $\pm 00: 00: 00.092$ \\
\hline $\begin{array}{c}\text { Mean Average Unranked } \\
\text { Precision }\end{array}$ & $3.49 \%$ & $\pm 0.00 \%$ \\
\hline $\begin{array}{c}\text { Mean Average Precision } \\
\text { Mean Average Recall }\end{array}$ & $12.85 \%$ & $\pm 0.00 \%$ \\
\hline Table 17: Database VSM Summary & $\pm 0.00 \%$ \\
\hline
\end{tabular}

\begin{tabular}{|c|c|c|}
\hline Database & Relevant & Not Relevant \\
\hline Retrieved & 2 & 87 \\
\hline $\begin{array}{c}\text { Not } \\
\text { Retrieved }\end{array}$ & 0 & 908 \\
\hline
\end{tabular}

Table 18: Confucius Matrix for Database 


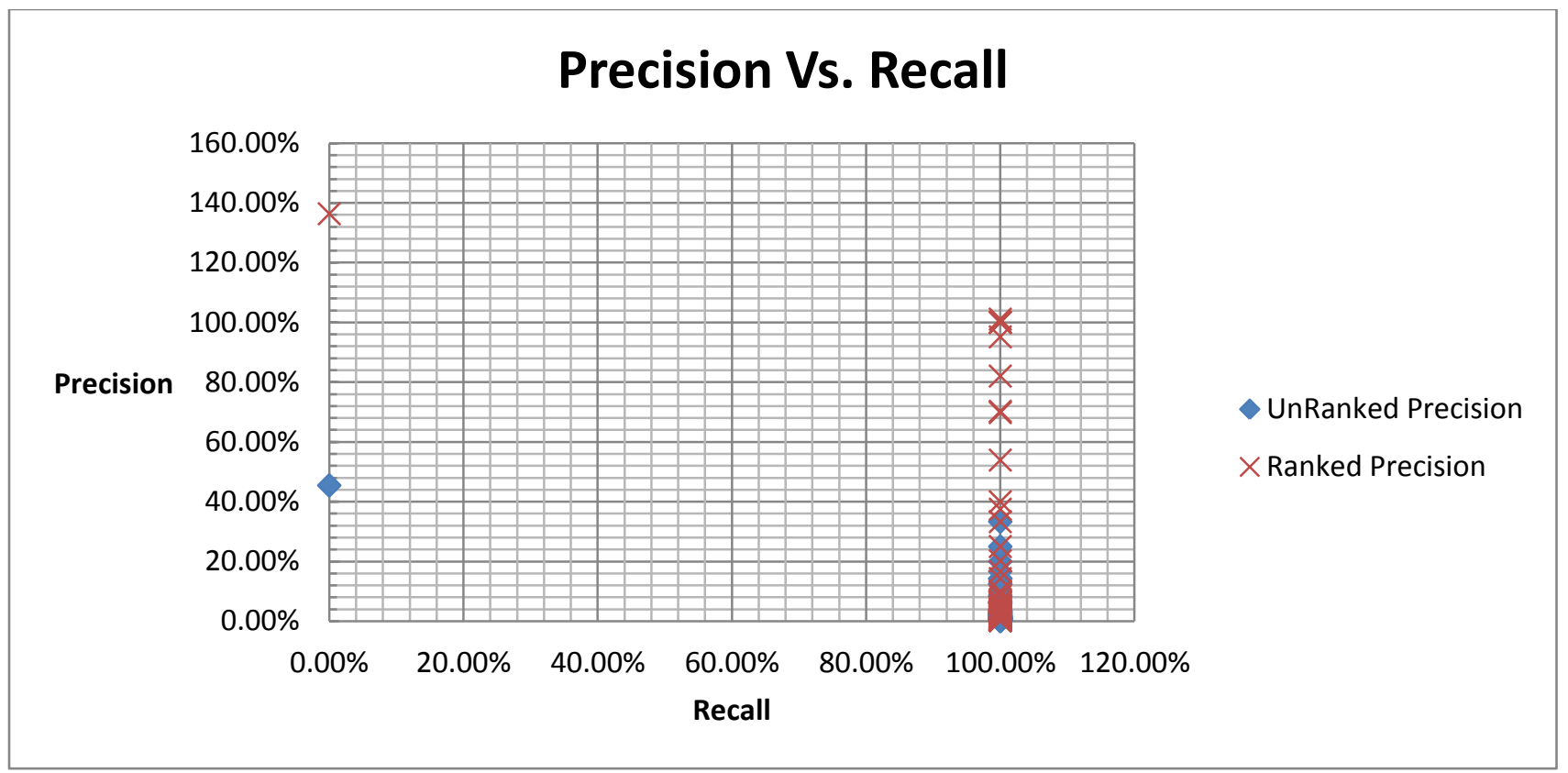

Figure 18: Precious vs. Recall

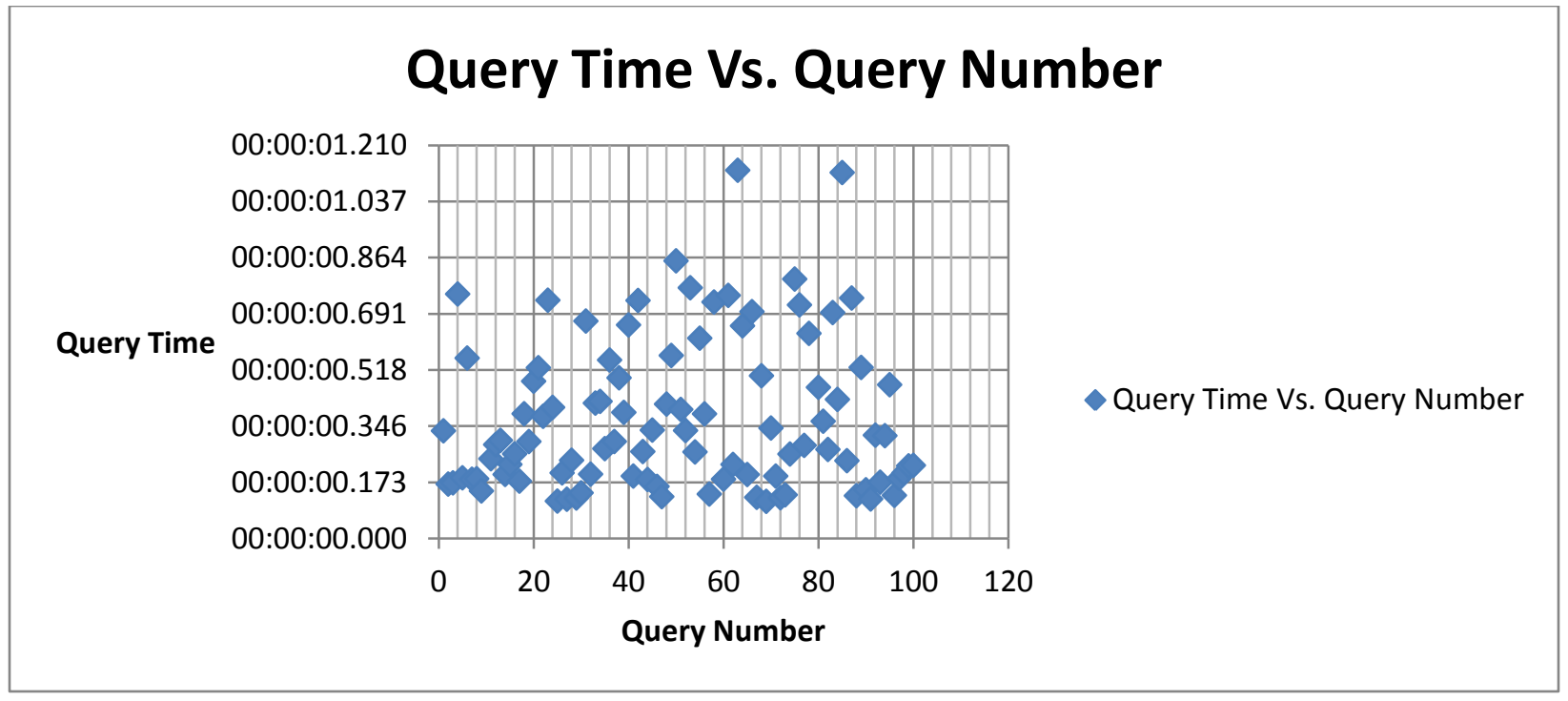

Figure 19: Query Time Database 


\section{R.A.M. Implementation with Best Match 25}

The data below is an average of all the data collected for the R.A.M. implementation using the best match 25 algorithms.

\begin{tabular}{|c|c|c|}
\hline & Average & Standard Deviation \\
\hline Average Query Time & $00: 00: 00.046$ & $\pm 00: 00: 00.011$ \\
\hline $\begin{array}{c}\text { Mean Average Unranked } \\
\text { Precision }\end{array}$ & $12.50 \%$ & $\pm 0.00 \%$ \\
\hline Mean Average Precision & $16.12 \%$ & $\pm 0.00 \%$ \\
\hline Mean Average Recall & $97.00 \%$ & $\pm 0.00 \%$ \\
\hline \multicolumn{1}{|c|}{ Table 19: R.A.M. BM Summary } \\
\hline
\end{tabular}

\begin{tabular}{|c|c|c|}
\hline RAM & Relevant & Not Relevant \\
\hline Retrieved & 2 & 87 \\
\hline $\begin{array}{c}\text { Not } \\
\text { Retrieved }\end{array}$ & 0 & 908 \\
\hline
\end{tabular}

Table 20: Confucius Matrix R.A.M. BM

The graphs below describe the trends that were seen during the investigation of RAM implementation using the best match 25 algorithm.

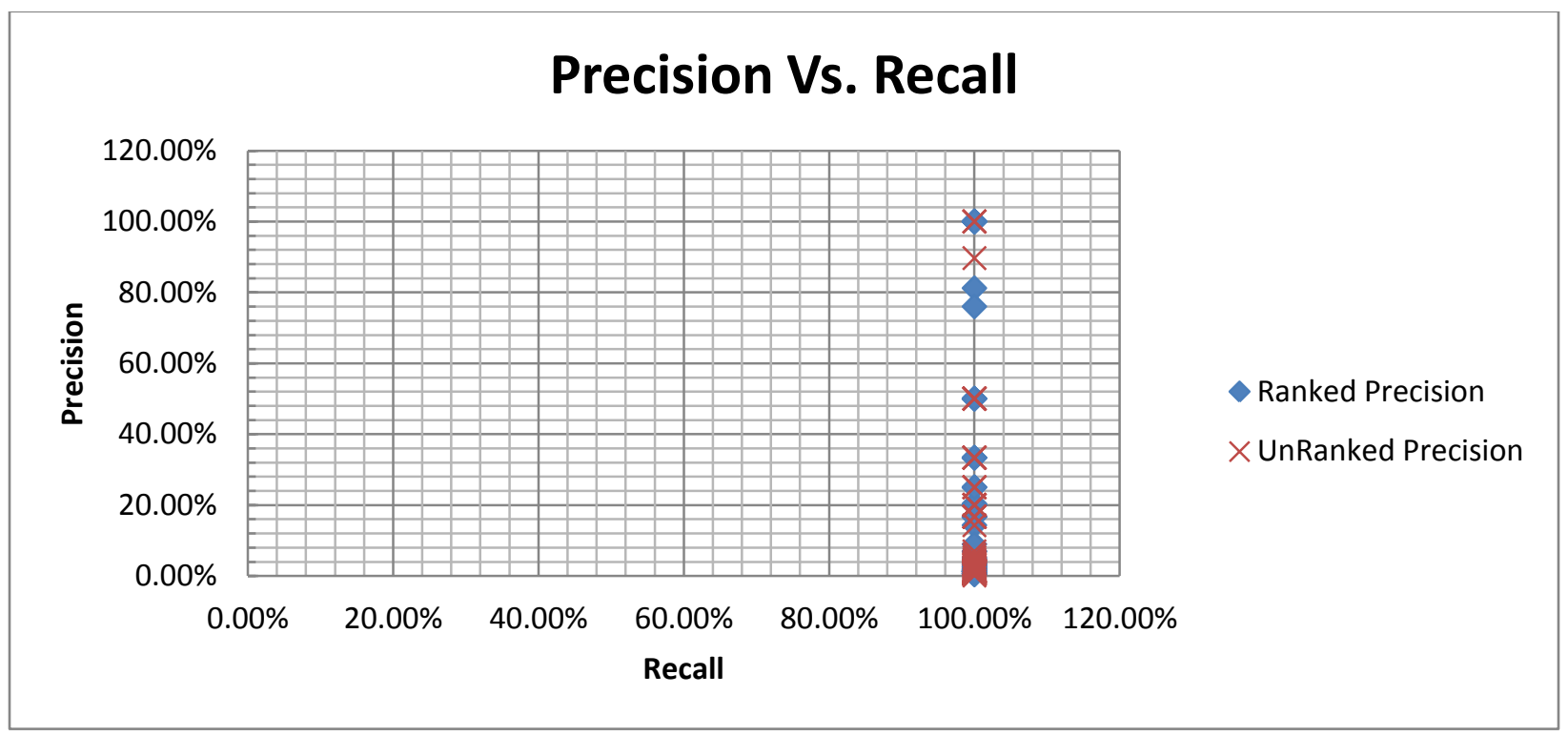

Figure 20: Precision vs. Recall 


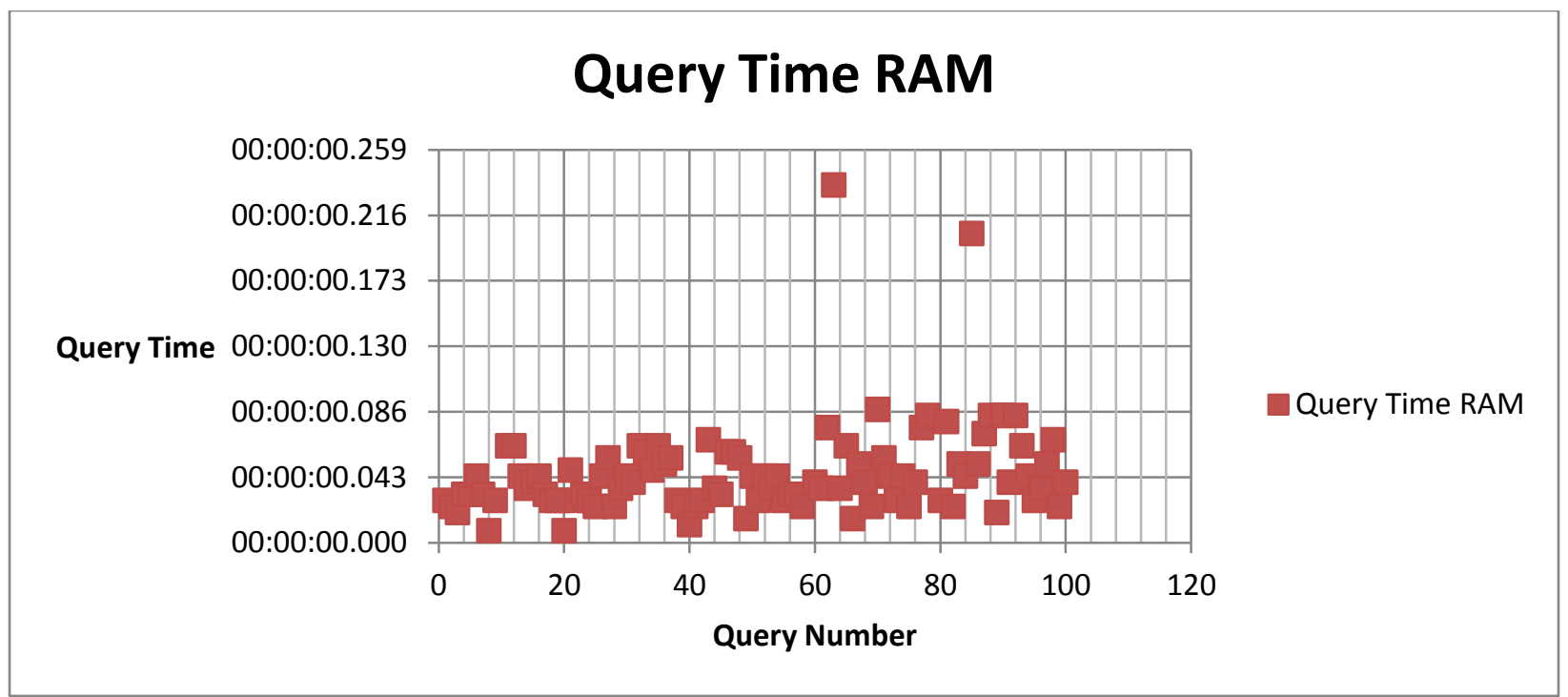

Figure 21: Query Time RAM

File Implementation with Best Match 25

The data below is the average of all the data collected during the file implementation using the best match 25 algorithm.

\begin{tabular}{|c|c|c|}
\hline & Average & Standard Deviation \\
\hline Average Query Time & $00: 00: 00.376$ & $\pm 00: 00: 00.092$ \\
\hline $\begin{array}{c}\text { Mean Average Unranked } \\
\text { Precision }\end{array}$ & $3.49 \%$ & $\pm 0.00 \%$ \\
\hline $\begin{array}{c}\text { Mean Average Precision } \\
\text { Mean Average Recall }\end{array}$ & $12.85 \%$ & $\pm 0.00 \%$ \\
\hline$\quad$ Table 21: File BM Summary & $\pm 0.00 \%$ \\
\hline
\end{tabular}

\begin{tabular}{|c|c|c|}
\hline File & Relevant & Not Relevant \\
\hline Retrieved & 2 & 152 \\
\hline $\begin{array}{c}\text { Not } \\
\text { Retrieved }\end{array}$ & 0 & 844 \\
\hline
\end{tabular}

Table 22: Confucius Matrix for File 


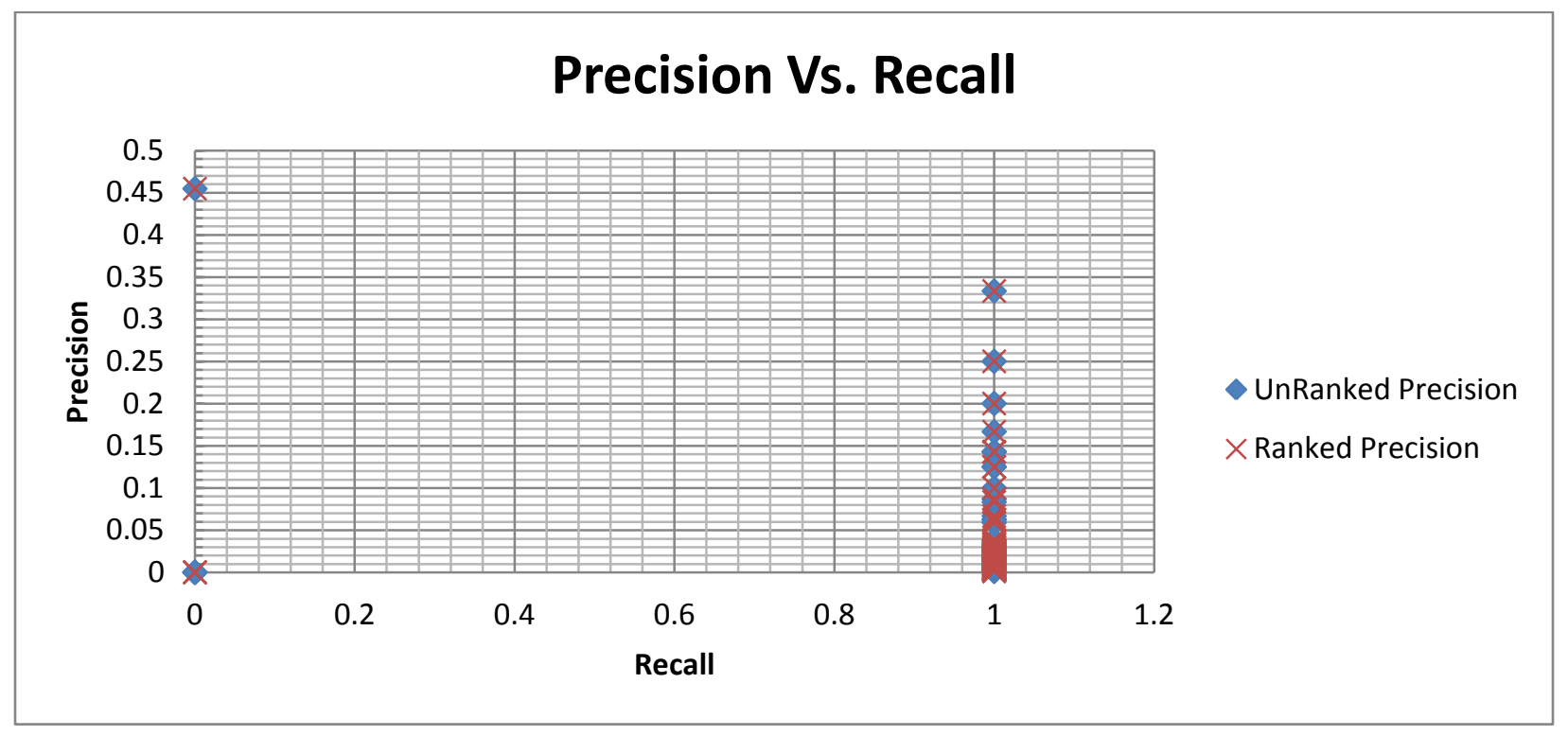

Figure 22: Precision vs. Recall

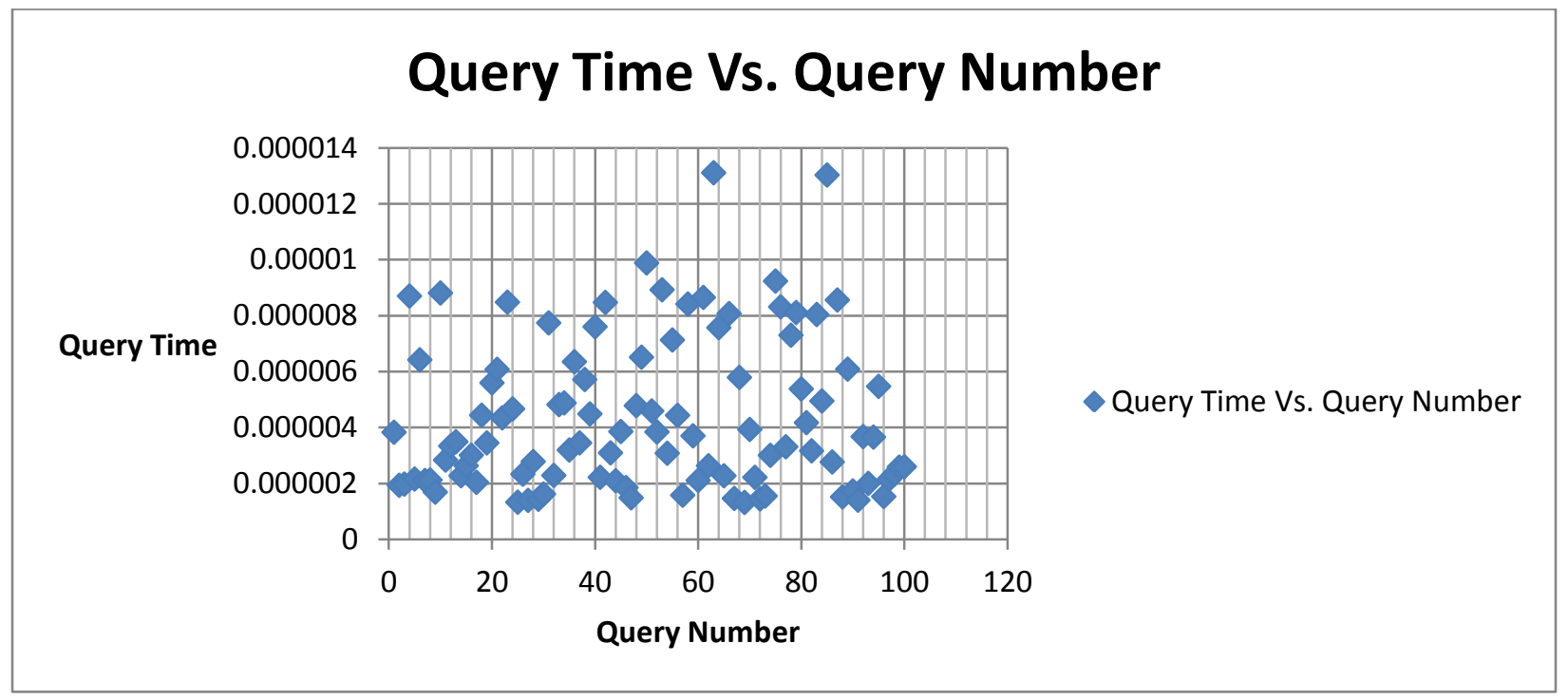

Figure 23: Query Time vs. Query Number 


\section{Database Implementation with Best Match 25}

The table below summaries the data collected during the database implementation using the best match 25 retrieval algorithm.

\begin{tabular}{|c|c|c|}
\hline & Average & Standard Deviation \\
\hline Average Query Time & $00: 00: 00.378$ & $\pm 00: 00: 00.091$ \\
\hline $\begin{array}{c}\text { Mean Average Unranked } \\
\text { Precision }\end{array}$ & $3.49 \%$ & $\pm 0.00 \%$ \\
\hline $\begin{array}{c}\text { Mean Average Precision } \\
\text { Mean Average Recall }\end{array}$ & $13.59 \%$ & $\pm 0.00 \%$ \\
\hline Table 23: Database BM Summary & $\pm 0.00 \%$ \\
\hline
\end{tabular}

\begin{tabular}{|c|c|c|}
\hline Database & Relevant & Not Relevant \\
\hline Retrieved & 2 & 152 \\
\hline $\begin{array}{c}\text { Not } \\
\text { Retrieved }\end{array}$ & 0 & 844 \\
\hline
\end{tabular}

Table 24: Confucius Matrix for Database

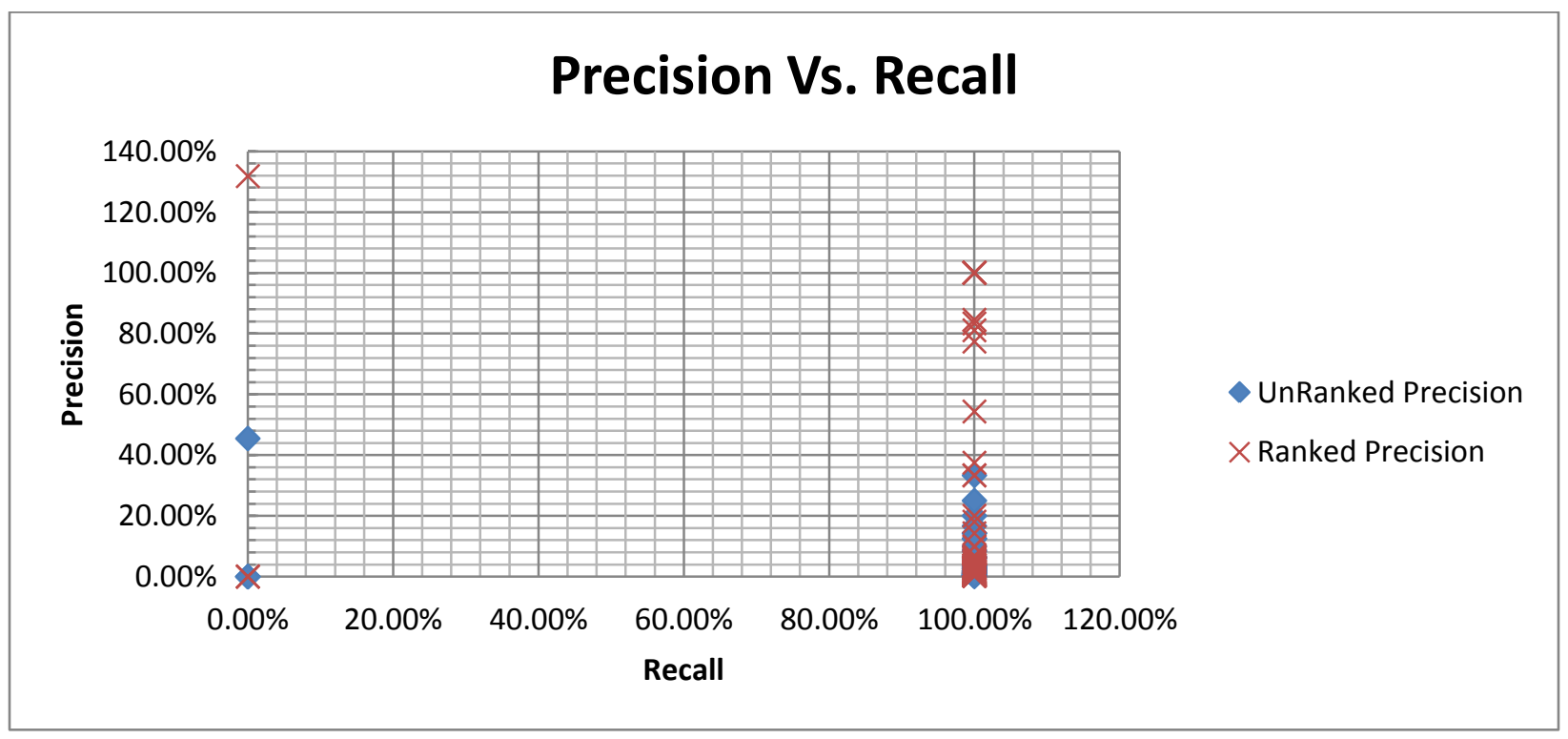

Figure 24: Precision vs. Recall 


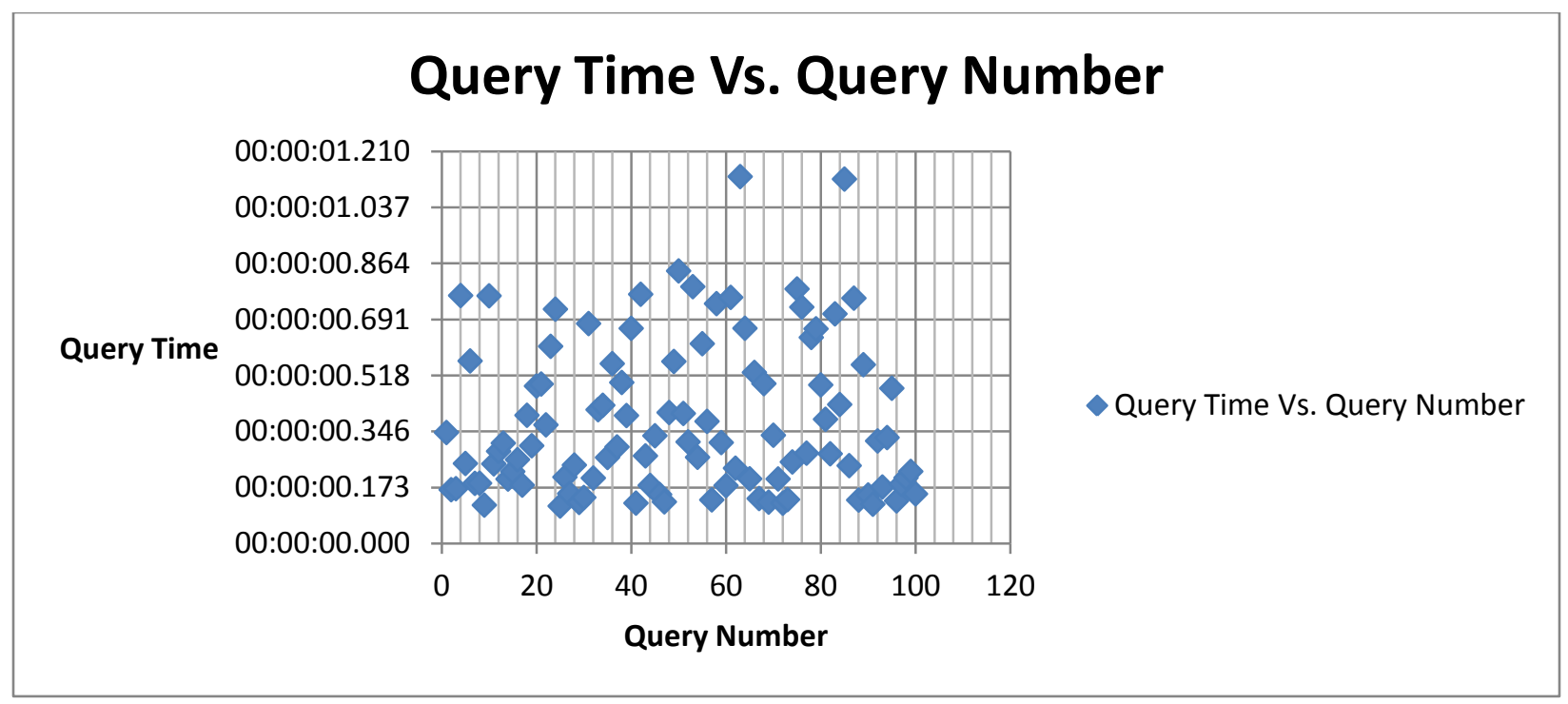

Figure 25: Query Time Database

\section{RAM Implementation with Language Model}

The tables and graphs below are a summary of the data collected during the R.A.M .implementation using a language model retrieval algorithm.

\begin{tabular}{|c|c|c|}
\hline & Average & Standard Deviation \\
\hline Average Query Time & $00: 00: 00.065$ & $\pm 00: 00: 00.015$ \\
\hline $\begin{array}{c}\text { Mean Average Unranked } \\
\text { Precision }\end{array}$ & $12.50 \%$ & $\pm 0.00 \%$ \\
\hline Mean Average Precision & $16.12 \%$ & $\pm 0.00 \%$ \\
\hline Mean Average Recall & $97.00 \%$ & $\pm 0.00 \%$ \\
\hline
\end{tabular}

\begin{tabular}{|c|c|c|}
\hline File & Relevant & Not Relevant \\
\hline Retrieved & 2 & 87 \\
\hline $\begin{array}{c}\text { Not } \\
\text { Retrieved }\end{array}$ & 0 & 908 \\
\hline
\end{tabular}

Table 26: Confucius Matrix for R.A.M. 


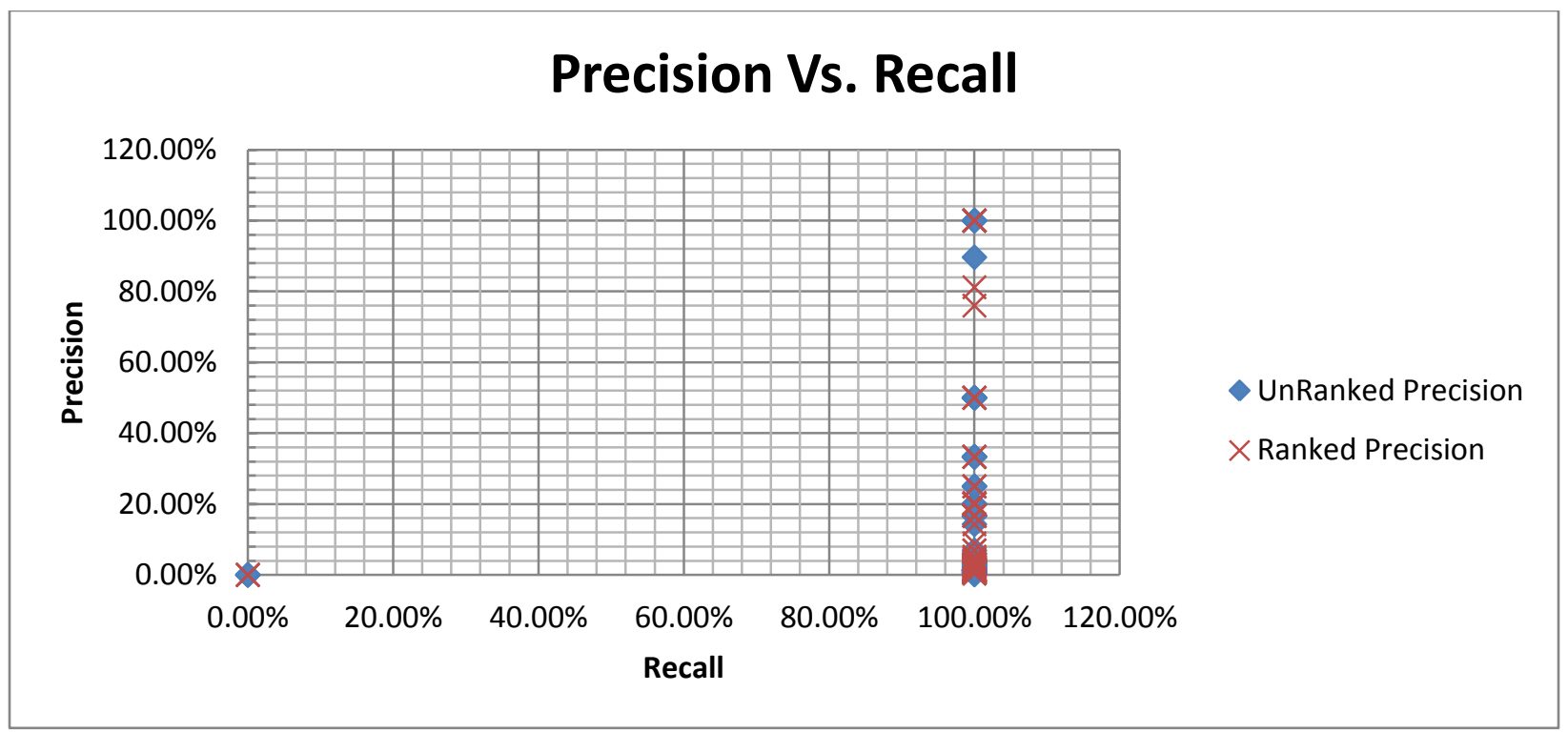

Figure 26: Precision vs. Recall

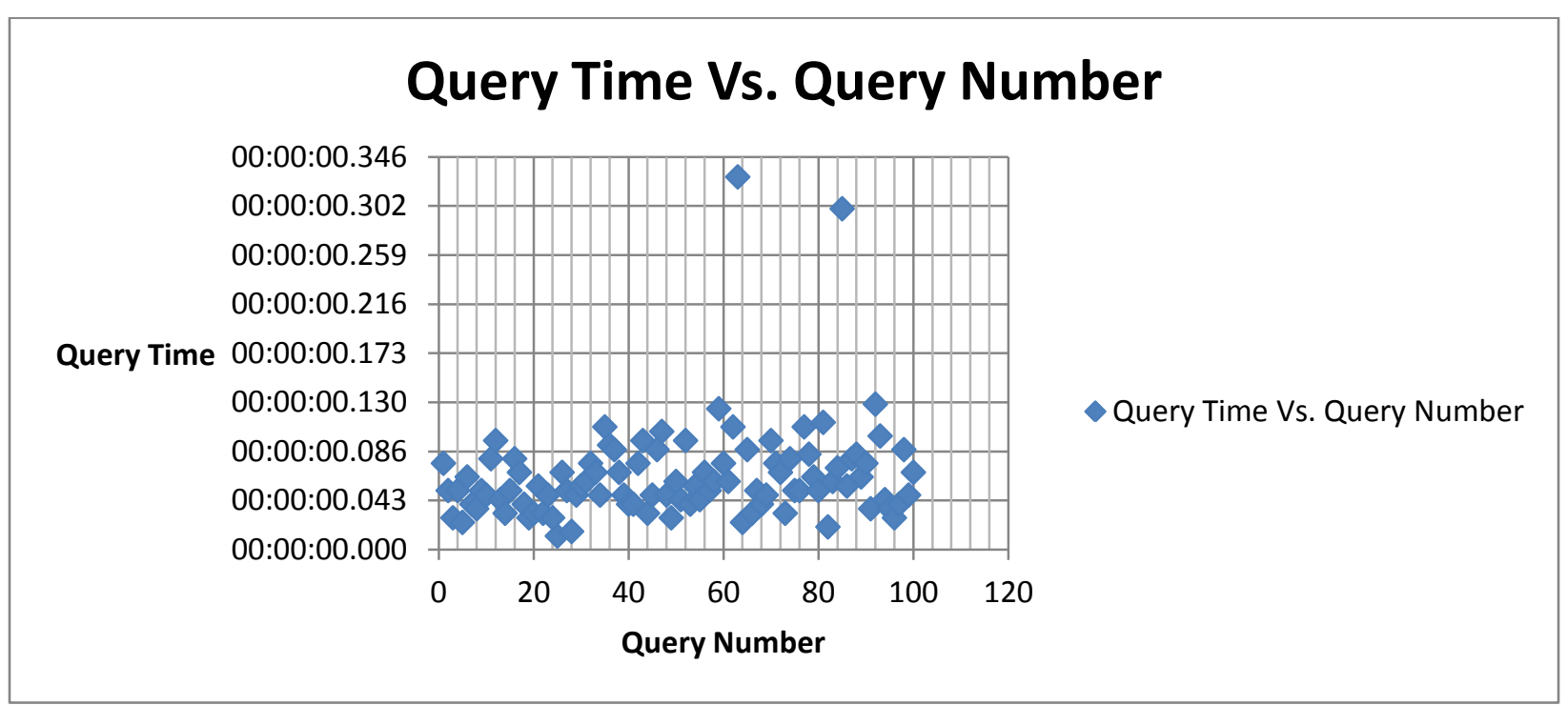

Figure 27: Query Time R.A.M. 


\section{File Implementation with Language Model}

The tables and graphs summary the data collected from the file implementation using a language model retrieval method.

\begin{tabular}{|c|c|c|}
\hline & Average & Standard Deviation \\
\hline Average Query Time & $00: 00: 01.014$ & $\pm 00: 00: 00.197$ \\
\hline $\begin{array}{c}\text { Mean Average Unranked } \\
\text { Precision }\end{array}$ & $11.66 \%$ & $\pm 1.18 \%$ \\
\hline Mean Average Precision & $15.93 \%$ & $\pm 2.96 \%$ \\
\hline Mean Average Recall & $96.96 \%$ & $\pm 0.00 \%$ \\
\hline \multicolumn{2}{|c|}{ Table 27: File LM Summary } \\
\hline
\end{tabular}

\begin{tabular}{|c|c|c|}
\hline File & Relevant & Not Relevant \\
\hline Retrieved & 2 & 86 \\
\hline $\begin{array}{c}\text { Not } \\
\text { Retrieved }\end{array}$ & 0 & 909 \\
\hline \multicolumn{2}{|c|}{ Table 28: Confucius Matrix for File } \\
\hline
\end{tabular}

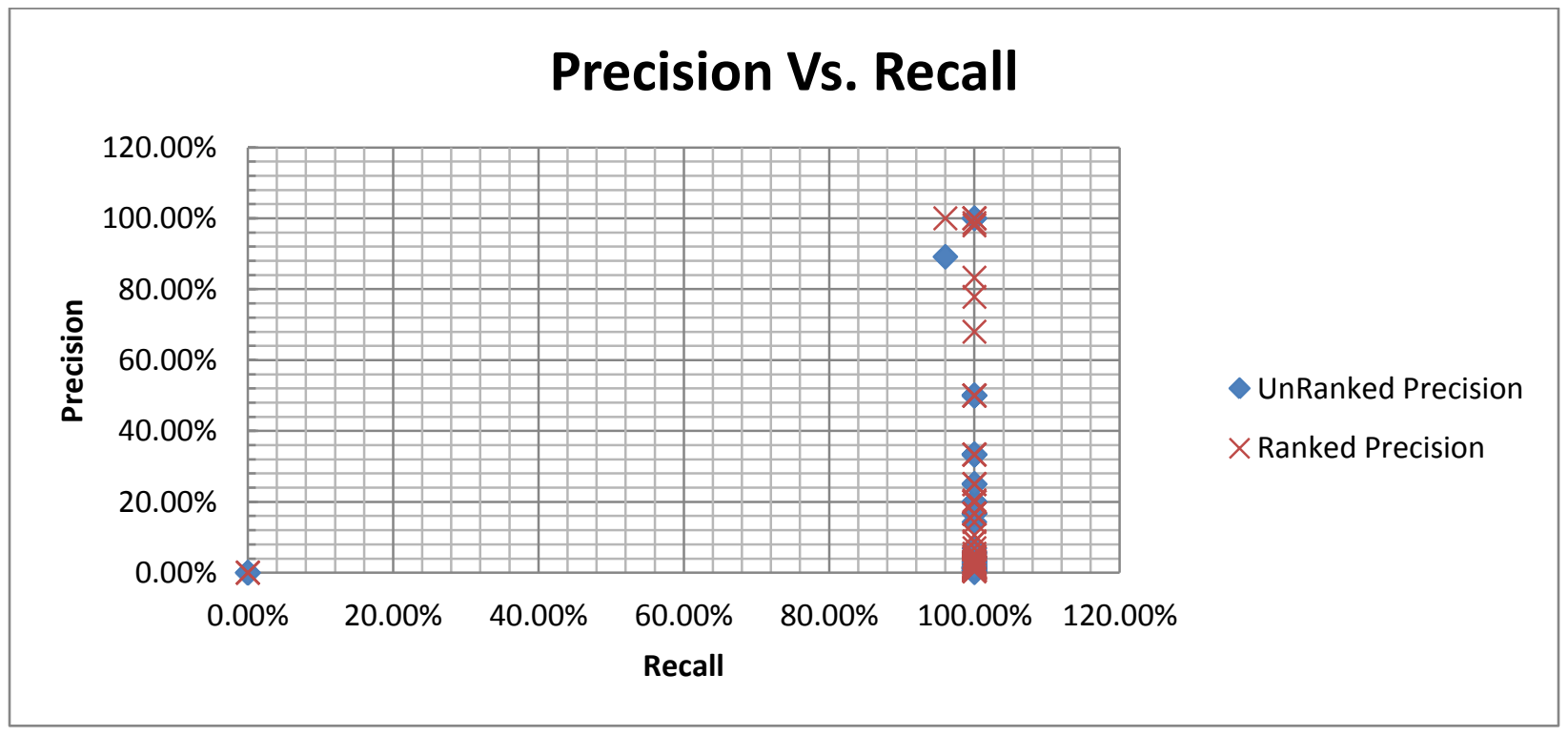

Figure 28: Precision vs. Recall 


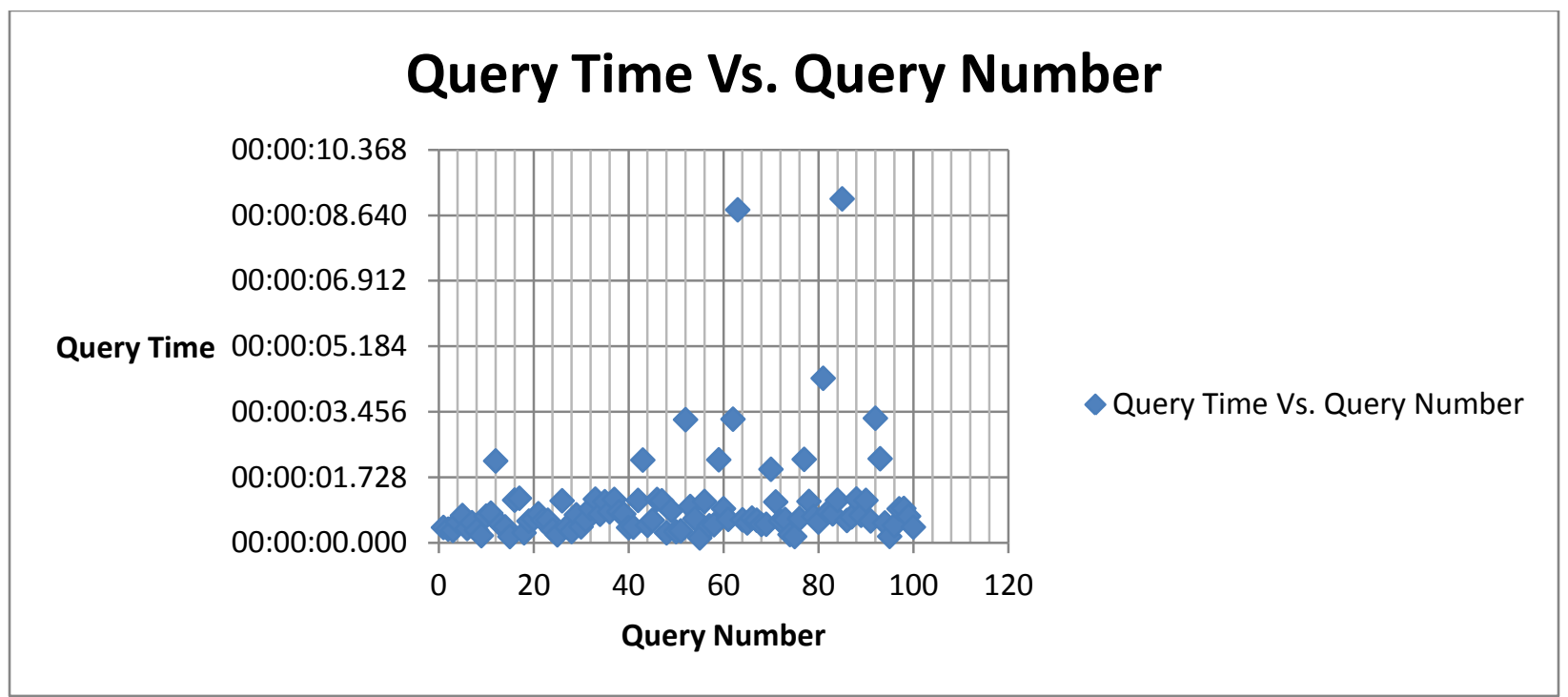

Figure 29: Query Time for File

\section{Database Implementation with Language Model}

The following tables and graphs summarize the data collected for the database implementation using a language model algorithm.

\begin{tabular}{|c|c|c|}
\hline & Average & Standard Deviation \\
\hline Average Query Time & $00: 00: 00.376$ & $\pm 00: 00: 00.077$ \\
\hline $\begin{array}{c}\text { Mean Average Unranked } \\
\text { Precision }\end{array}$ & $12.60 \%$ & $\pm 2.04 \%$ \\
\hline $\begin{array}{c}\text { Mean Average Precision } \\
\text { Mean Average Recall }\end{array}$ & $16.25 \%$ & $\pm 1.41 \%$ \\
\hline \multicolumn{2}{|c|}{ Table 29: Database LM Summary } & $\pm 0.00 \%$ \\
\hline
\end{tabular}

\begin{tabular}{|c|c|c|}
\hline File & Relevant & Not Relevant \\
\hline Retrieved & 2 & 86 \\
\hline $\begin{array}{c}\text { Not } \\
\text { Retrieved }\end{array}$ & 0 & 909 \\
\hline
\end{tabular}

Table 30: Confucius Matrix for Database 


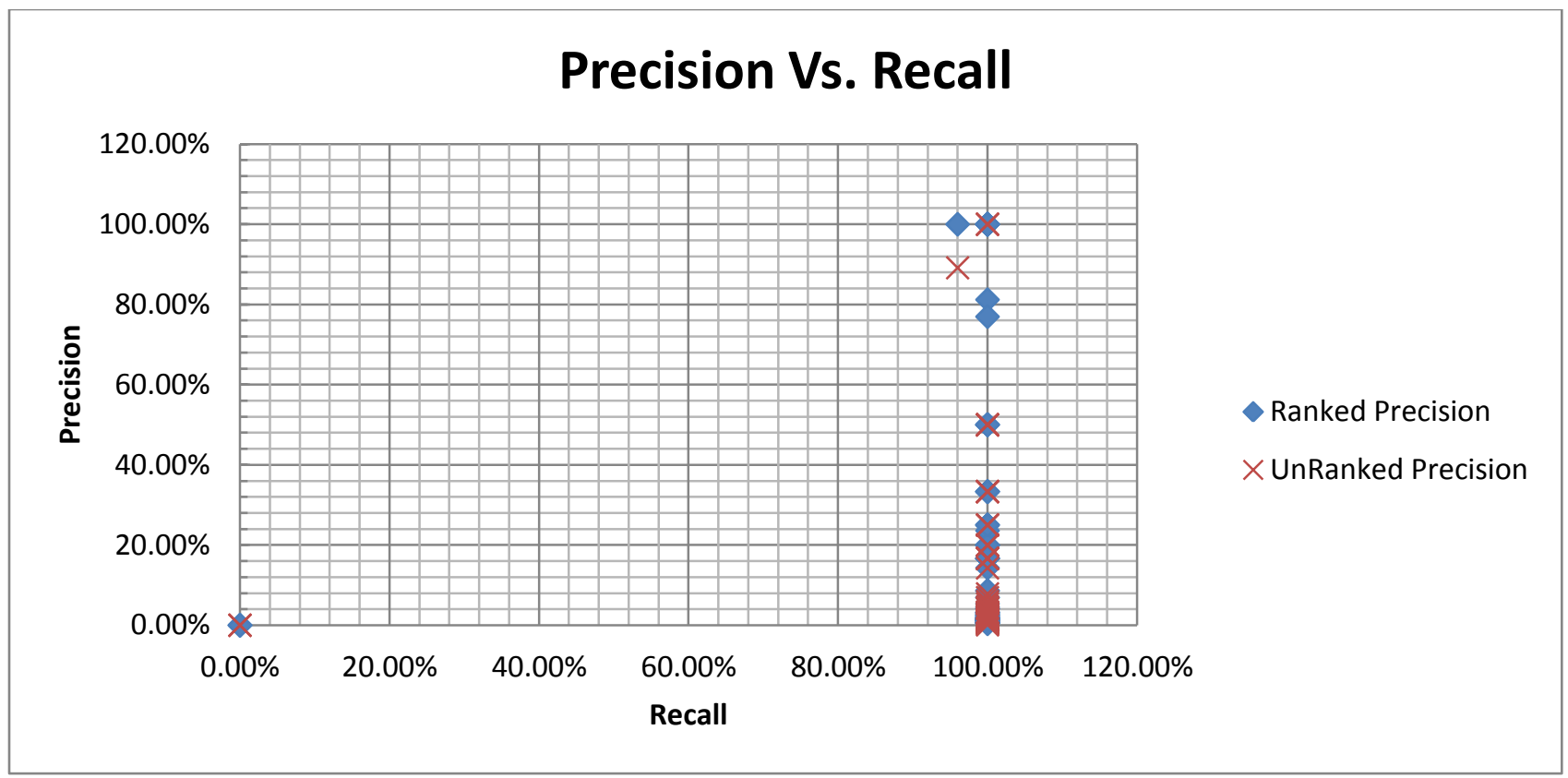

Figure 30: Precision vs. Recall

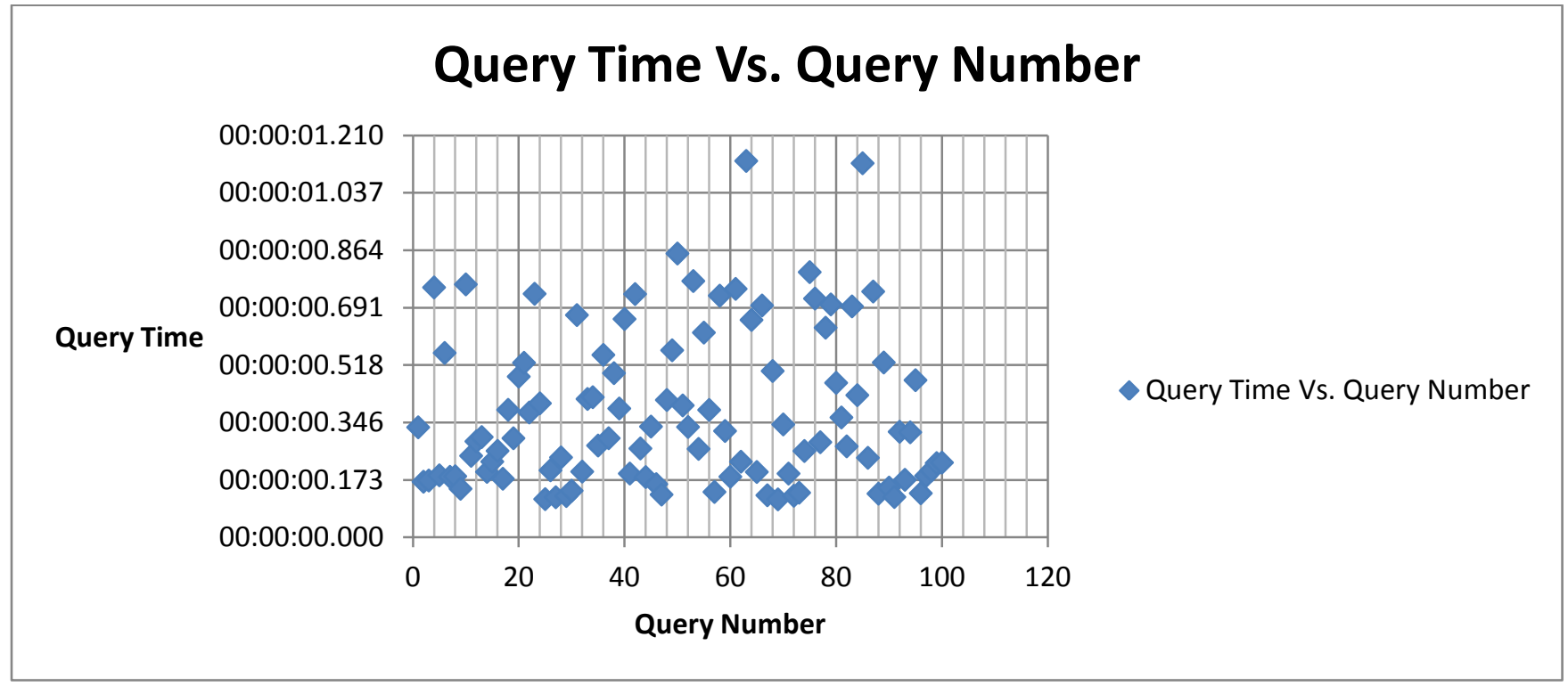

Figure 31: Query Time Database 


\subsubsection{Vector Space Comparison}

The following table summarizes the information of the vector space trials for each storage implementation.

\begin{tabular}{|c|c|c|c|c|}
\hline Storage & $\begin{array}{c}\text { Average Query } \\
\text { Time }\end{array}$ & $\begin{array}{c}\text { Mean Average } \\
\text { Unranked } \\
\text { Precision }\end{array}$ & $\begin{array}{c}\text { Mean } \\
\text { Average } \\
\text { Ranked } \\
\text { Precision }\end{array}$ & $\begin{array}{c}\text { Average } \\
\text { Recall }\end{array}$ \\
\hline RAM & $00: 00: 00.106$ & $12.41 \%$ & $15.90 \%$ & $96.96 \%$ \\
\hline File & $00: 00: 00.697$ & $11.58 \%$ & $15.63 \%$ & $97.00 \%$ \\
\hline Database & $00: 00: 00.376$ & $3.49 \%$ & $12.85 \%$ & $96.00 \%$ \\
\hline
\end{tabular}

The tables below normalize the values in the table above.

\begin{tabular}{|c|c|c|}
\hline RAM & Relevant & Not Relevant \\
\hline Retrieved & 2 & 87 \\
\hline $\begin{array}{c}\text { Not } \\
\text { Retrieved }\end{array}$ & 0 & 908 \\
\hline \multicolumn{2}{|c|}{ Table 32: Confucius Matrix for RAM } \\
\hline
\end{tabular}

\begin{tabular}{|c|c|c|}
\hline File & Relevant & Not Relevant \\
\hline Retrieved & 2 & 87 \\
\hline $\begin{array}{c}\text { Not } \\
\text { Retrieved }\end{array}$ & 0 & 908 \\
\hline \multicolumn{2}{|c|}{ Table 33: Confucius Matrix for File } \\
\hline
\end{tabular}

\begin{tabular}{|c|c|c|}
\hline Database & Relevant & Not Relevant \\
\hline Retrieved & 2 & 152 \\
\hline $\begin{array}{c}\text { Not } \\
\text { Retrieved }\end{array}$ & 0 & 844 \\
\hline
\end{tabular}

Table 34: Confucius Matrix for Database

To better illustrate the relationships that were formulated during this section of the investigation. . 


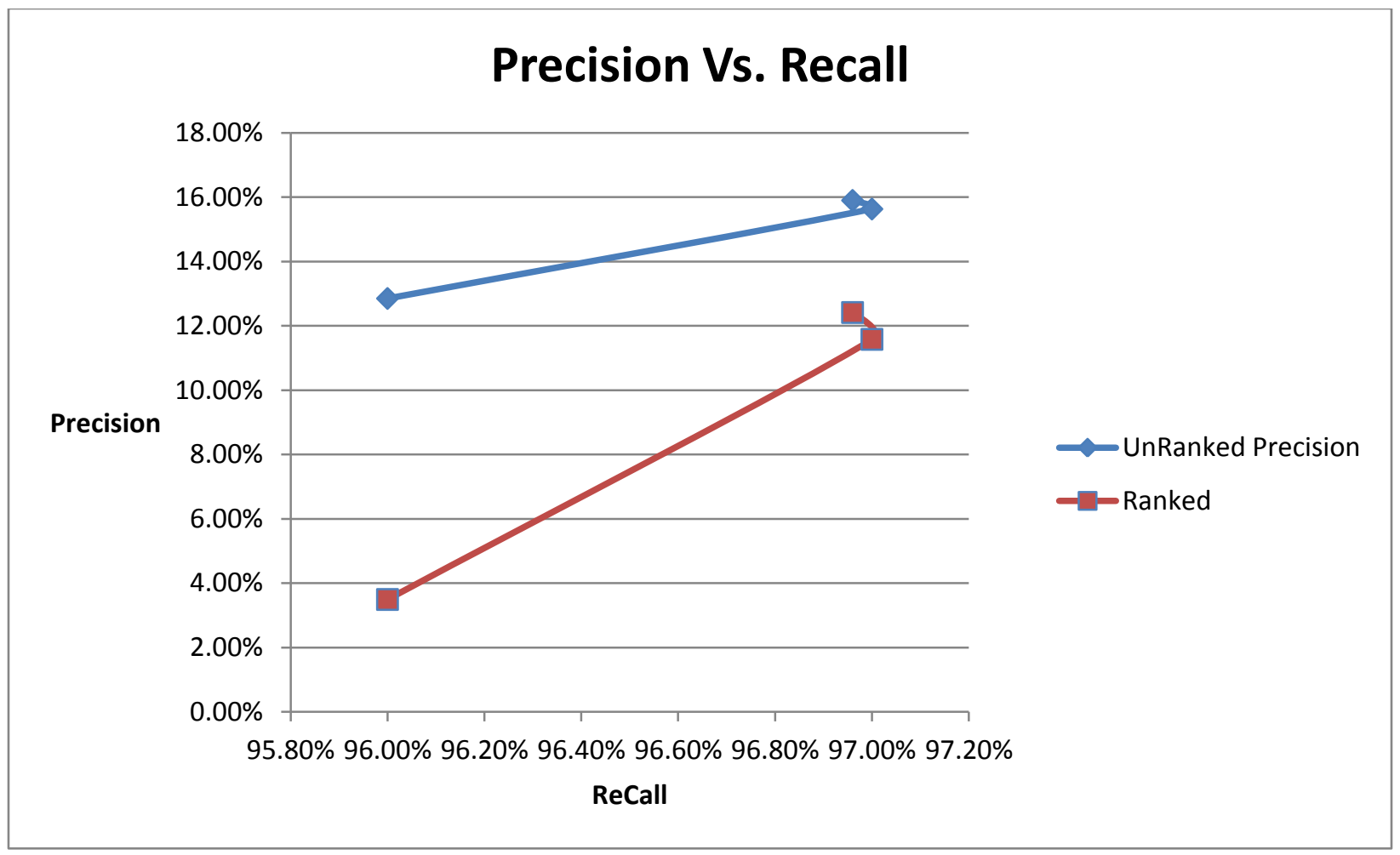

Figure 32: Precision vs. Recall

The final graph is a summary of the retrieval times obtained for each storage implementation using a vector space model.

\section{Query Times for Storage - VSM}

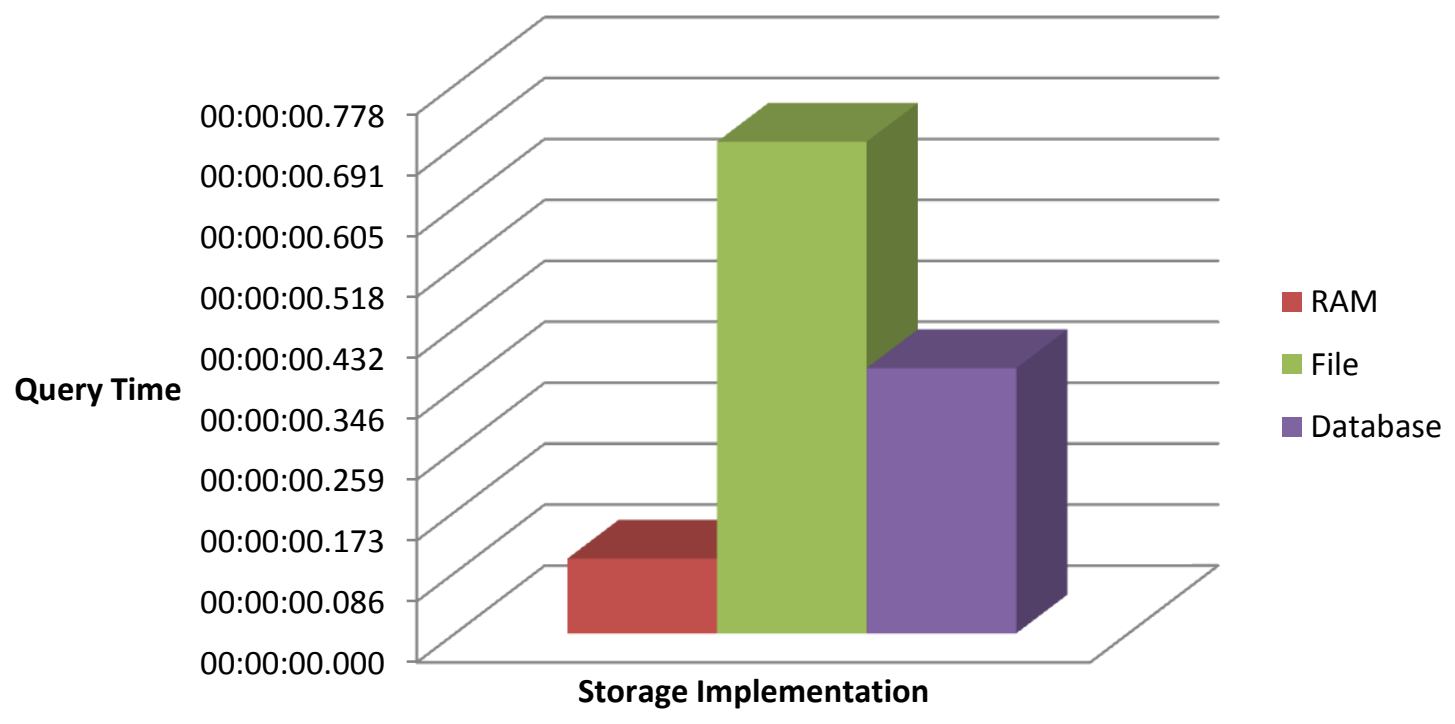

Figure 33: Query Time for VSM 


\subsubsection{Best Match Comparison}

The following data was collected during the probabilistic model algorithm known as best match 25 (BM). The table below summaries the data collected:

\begin{tabular}{|c|c|c|c|c|}
\hline Storage & $\begin{array}{c}\text { Average Query } \\
\text { Time }\end{array}$ & $\begin{array}{c}\text { Mean Average } \\
\text { Unranked Precision }\end{array}$ & $\begin{array}{c}\text { Mean } \\
\text { Average } \\
\text { Ranked } \\
\text { Precision }\end{array}$ & $\begin{array}{c}\text { Average } \\
\text { Recall }\end{array}$ \\
\hline RAM & $00: 00: 00.046$ & $12.50 \%$ & $16.12 \%$ & $97.00 \%$ \\
\hline File & $00: 00: 00.376$ & $3.49 \%$ & $12.85 \%$ & $96.00 \%$ \\
\hline Database & $00: 00: 00.378$ & $3.49 \%$ & $13.59 \%$ & $96.00 \%$ \\
\hline
\end{tabular}

The tables below normalize the summary data in table 36 .

\begin{tabular}{|c|c|c|}
\hline RAM & Relevant & Not Relevant \\
\hline Retrieved & 2 & 87 \\
\hline $\begin{array}{c}\text { Not } \\
\text { Retrieved }\end{array}$ & 0 & 908 \\
\hline
\end{tabular}

Table 36: Confucius Matrix for RAM

\begin{tabular}{|c|c|c|}
\hline File & Relevant & Not Relevant \\
\hline Retrieved & 2 & 152 \\
\hline $\begin{array}{c}\text { Not } \\
\text { Retrieved }\end{array}$ & 0 & 844 \\
\hline
\end{tabular}

Table 37: Confucius Matrix for File

\begin{tabular}{|c|c|c|}
\hline Database & Relevant & Not Relevant \\
\hline Retrieved & 2 & 152 \\
\hline $\begin{array}{c}\text { Not } \\
\text { Retrieved }\end{array}$ & 0 & 844 \\
\hline
\end{tabular}

Table 38: Confucius Matrix for Database 
The following graph summaries the relationship between precision and recall.

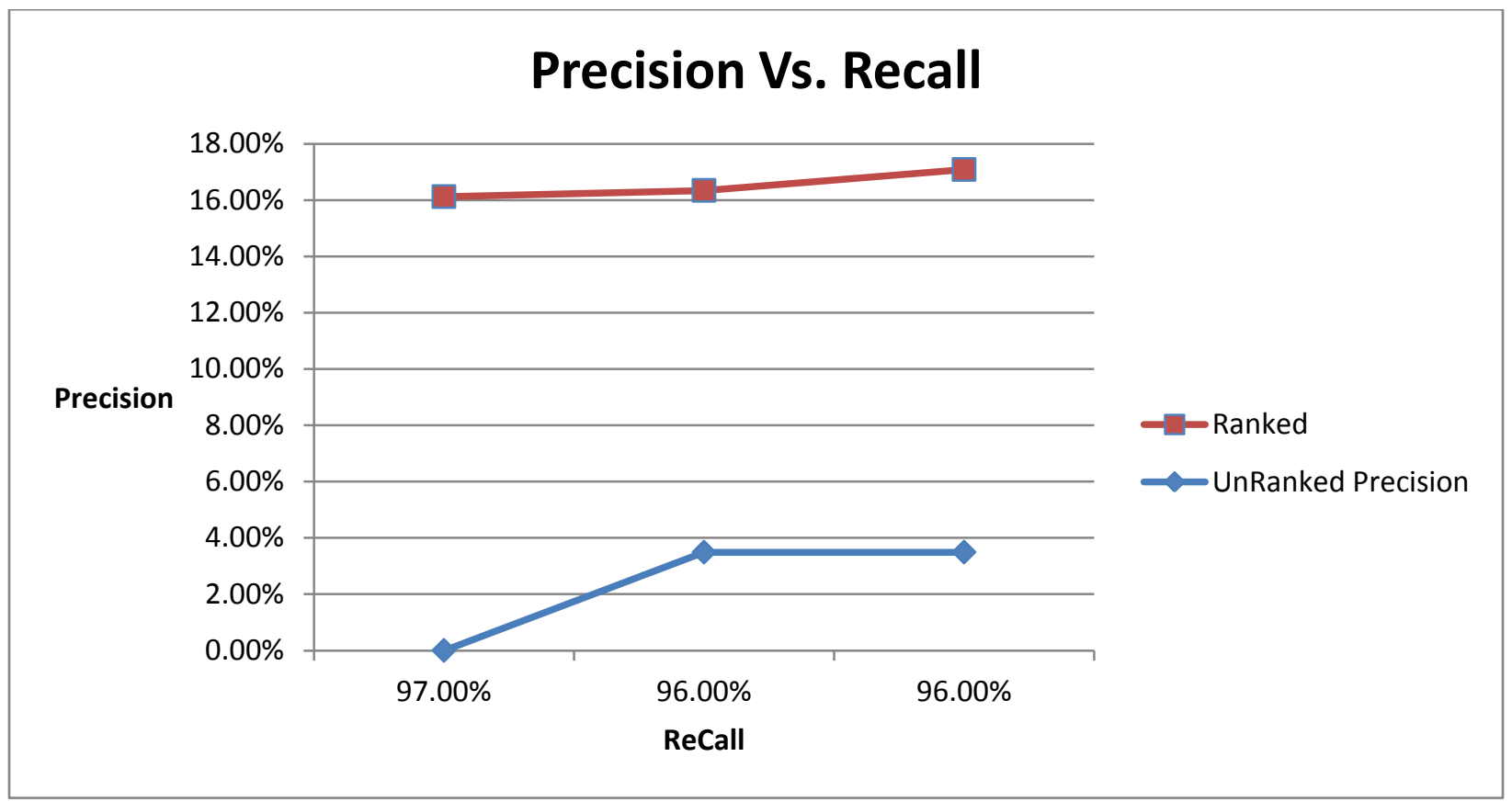

Figure 34: Precious VS. Recall BM

The final graph shows each storage implementation's effect on the retrieval process using a best match 25 algorithm.

\section{Query Times for Storage - BM}

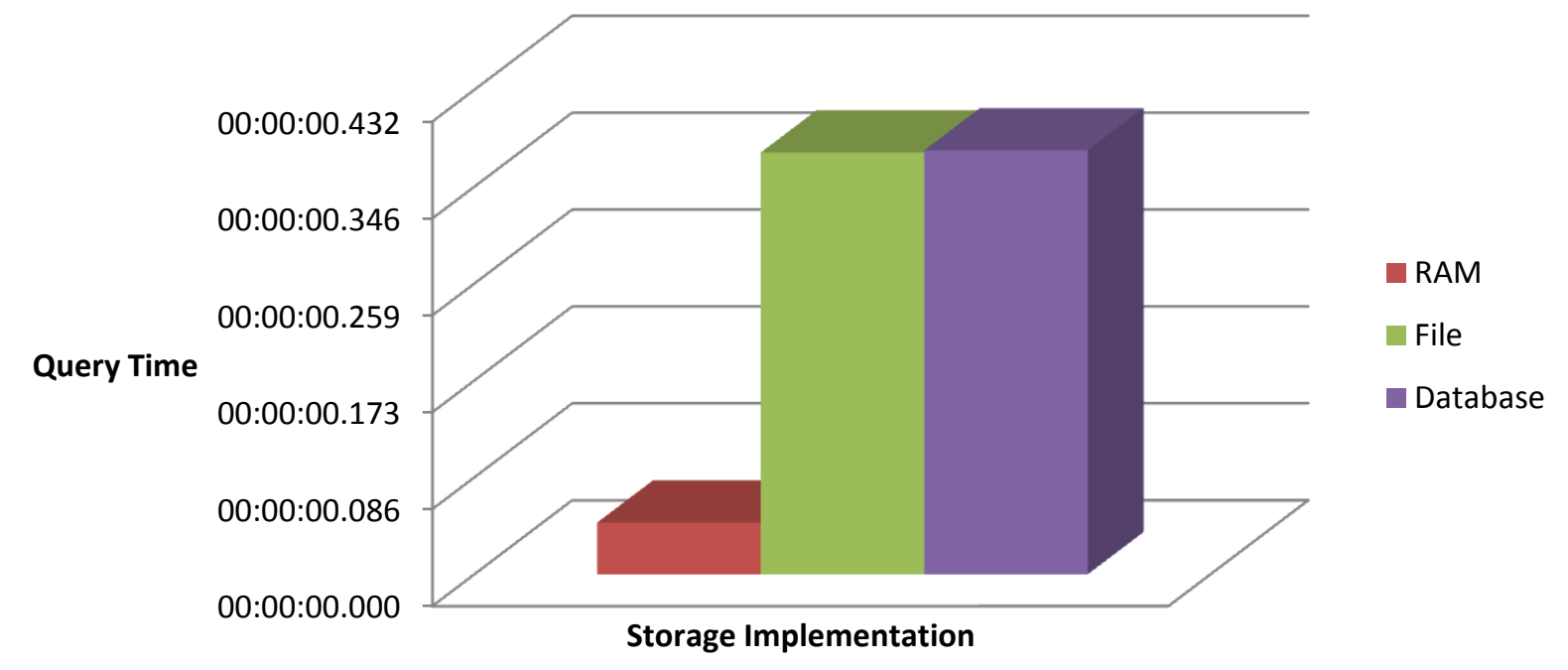

Figure 35: Retrieval Times for BM 


\subsubsection{Language Model Comparison}

The data in the tables and graphs summarizes all the index implementations compare against each other for case study one using a language model retrieval algorithm.

\begin{tabular}{|c|c|c|c|c|}
\hline Storage & $\begin{array}{c}\text { Average } \\
\text { Query Time }\end{array}$ & $\begin{array}{c}\text { Mean Average } \\
\text { Unranked } \\
\text { Precision }\end{array}$ & $\begin{array}{c}\text { Mean } \\
\text { Average } \\
\text { Ranked } \\
\text { Precision }\end{array}$ & $\begin{array}{c}\text { Average } \\
\text { Recall }\end{array}$ \\
\hline RAM & $00: 00: 00.065$ & $12.50 \%$ & $16.12 \%$ & $97.00 \%$ \\
\hline File & $00: 00: 01.014$ & $11.66 \%$ & $15.93 \%$ & $96.96 \%$ \\
\hline Database & $00: 00: 00.376$ & $12.60 \%$ & $16.25 \%$ & $96.96 \%$ \\
\hline
\end{tabular}

\begin{tabular}{|c|c|c|}
\hline RAM & Relevant & Not Relevant \\
\hline Retrieved & 2 & 87 \\
\hline Not Retrieved & 0 & 908 \\
\hline
\end{tabular}

Table 40: Confucius Matrix for RAM

\begin{tabular}{|c|c|c|}
\hline File & Relevant & Not Relevant \\
\hline Retrieved & 2 & 86 \\
\hline Not Retrieved & 0 & 909 \\
\hline
\end{tabular}

Table 41: Confucius Matrix for File

\begin{tabular}{|c|c|c|}
\hline Database & Relevant & Not Relevant \\
\hline Retrieved & 2 & 86 \\
\hline Not Retrieved & 0 & 909 \\
\hline
\end{tabular}

Table 42: Confucius Matrix for Database 


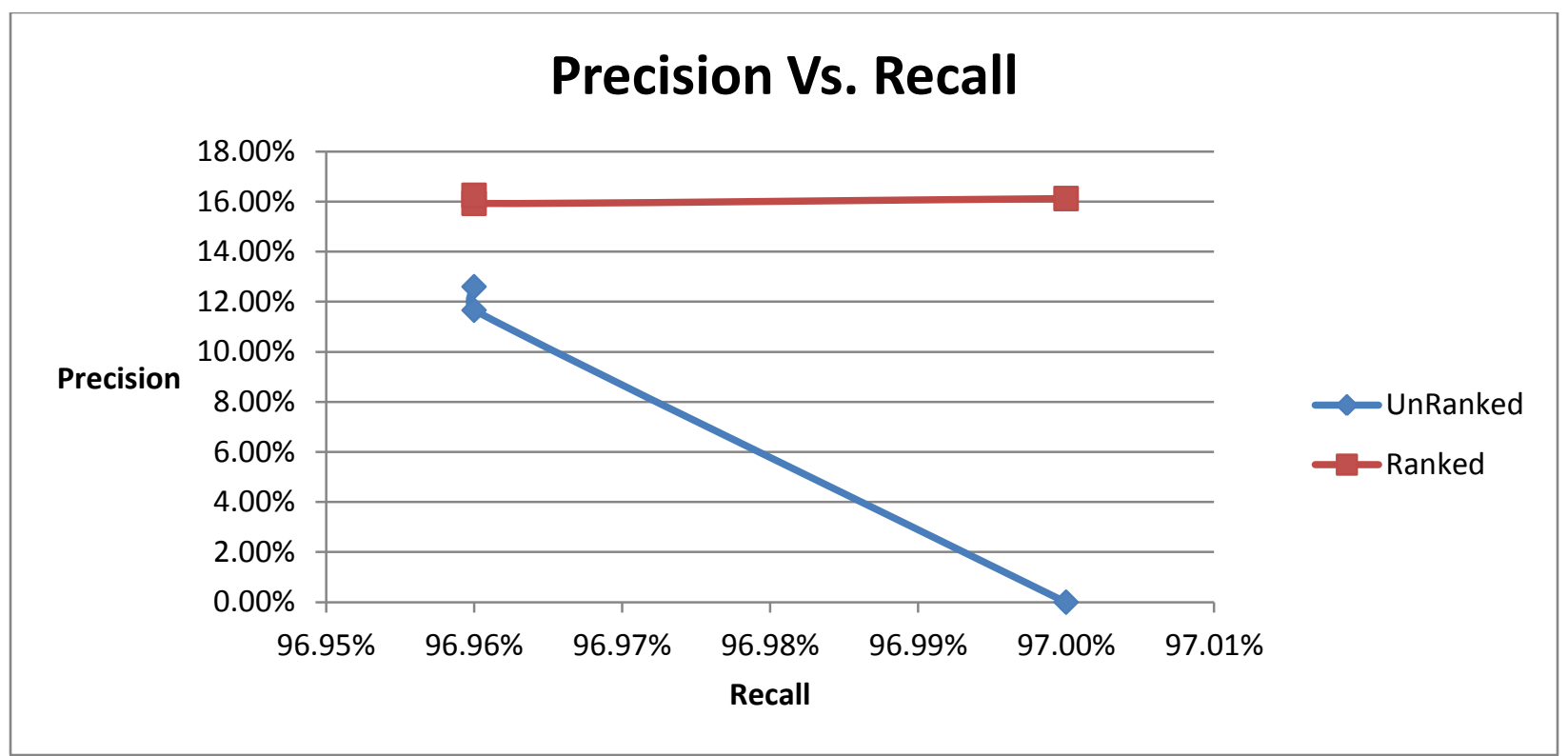

Figure 36: Precision vs. Recall

\section{Query Times for Storage-LM}

Query Times 00:00:00.518

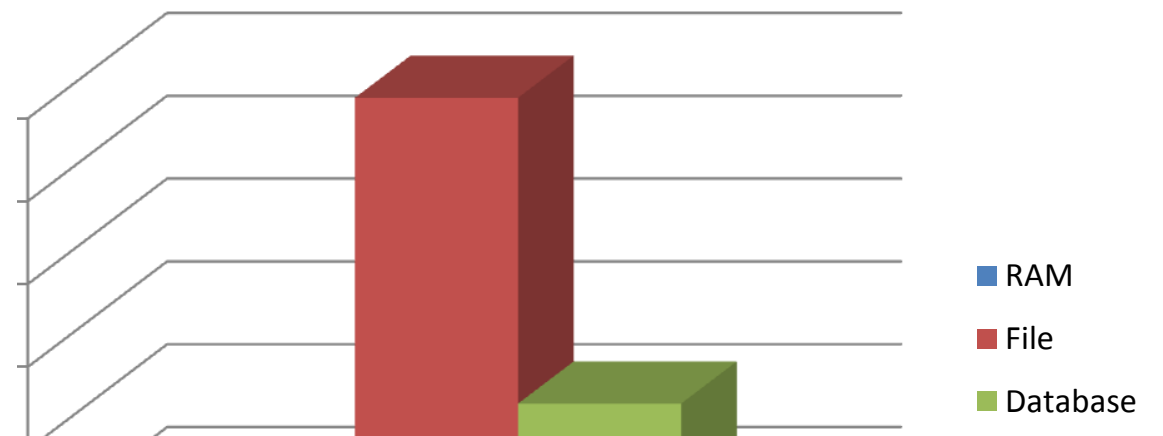

00:00:00.346

00:00:00.173

00:00:00.000

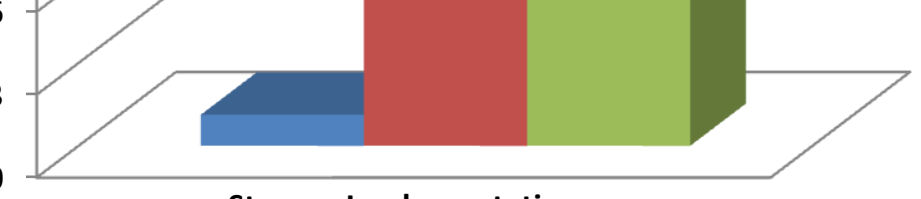

Stroage Implementation

Figure 37: Query Times 


\subsubsection{Case Study Comparison}

The following section compares all the data collected from case study one. This shows trends with precision, recall, and query times. In addition, the confucius matrix of each situation to normalize all the data gathered.

\begin{tabular}{|c|c|c|c|}
\hline Mean Avg. Precision & VSM & BM & LM \\
\hline RAM & $15.90 \%$ & $12.50 \%$ & $16.12 \%$ \\
\hline FILE & $15.63 \%$ & $3.49 \%$ & $15.93 \%$ \\
\hline Database & $12.85 \%$ & $3.49 \%$ & $16.25 \%$ \\
\hline
\end{tabular}

Table 43: Case Study One Comparison

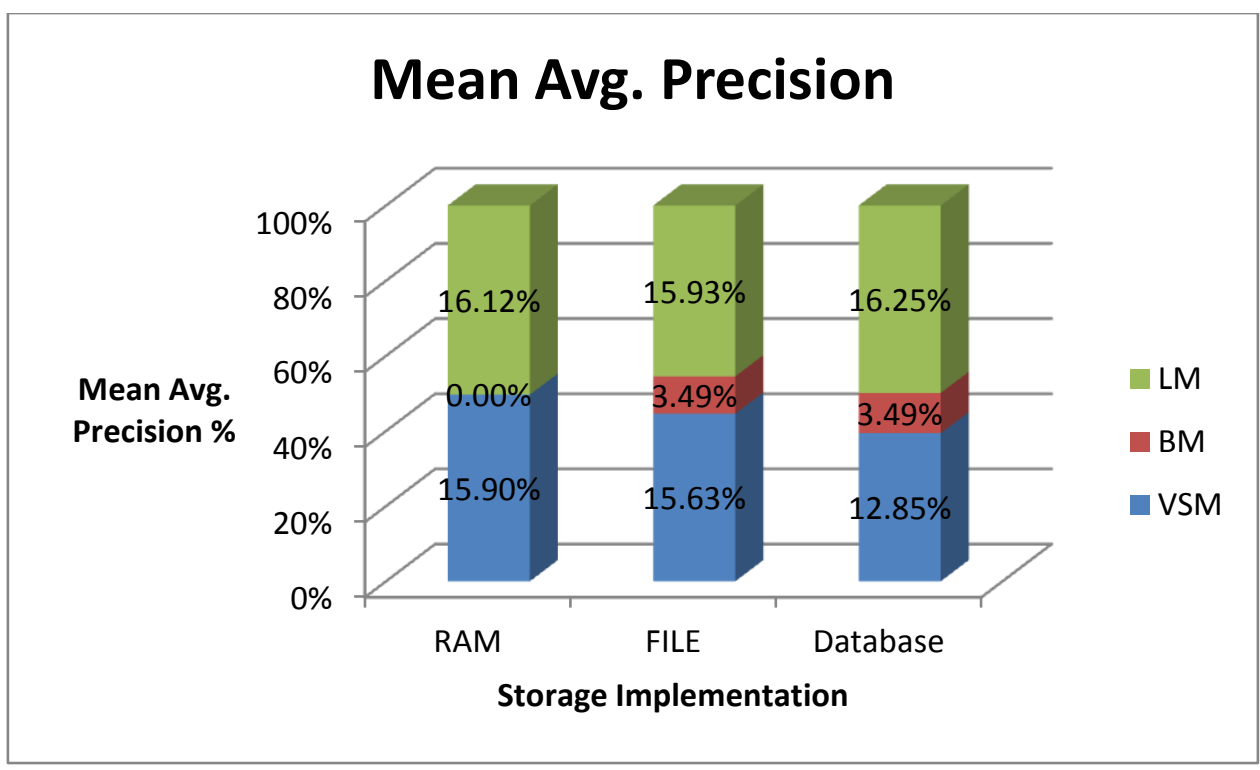

Figure 38: Mean Average Precision Comparison 


\begin{tabular}{|c|c|c|c|}
\hline Mean Avg. Unranked Precision & VSM & BM & LM \\
\hline RAM & $12.41 \%$ & $16.12 \%$ & $12.50 \%$ \\
\hline FILE & $11.58 \%$ & $12.85 \%$ & $11.66 \%$ \\
\hline Database & $3.49 \%$ & $13.59 \%$ & $12.60 \%$ \\
\hline
\end{tabular}

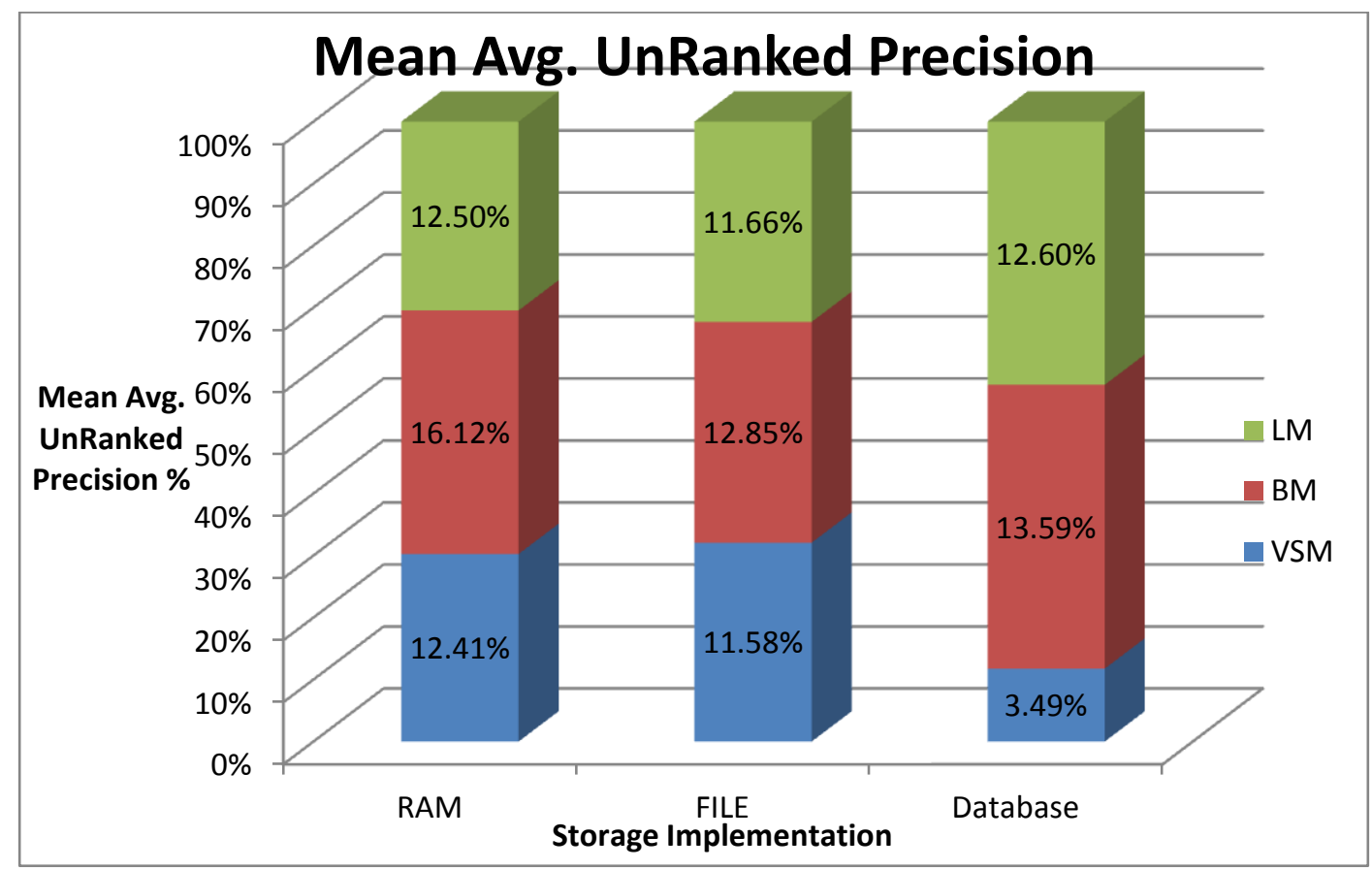

Figure 39: Mean Average Unranked Precision Graph

\begin{tabular}{|c|c|c|c|}
\hline Recall & VSM & BM & LM \\
\hline RAM & $96.96 \%$ & $97.00 \%$ & $97.00 \%$ \\
\hline FILE & $97.00 \%$ & $96.00 \%$ & $96.96 \%$ \\
\hline Database & $96.00 \%$ & $96.00 \%$ & $96.96 \%$ \\
\hline
\end{tabular}




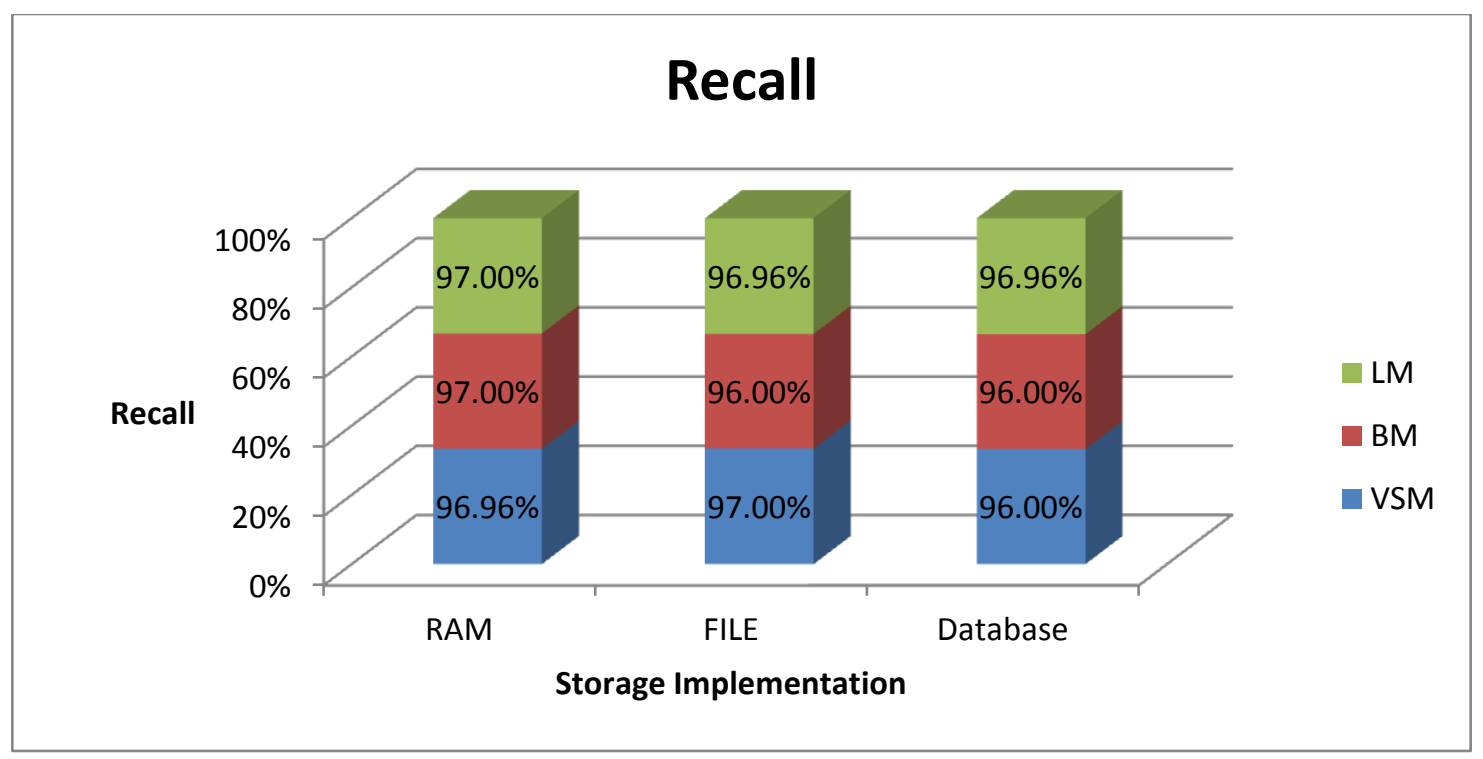

Figure 40: Recall Graph Comparison

\begin{tabular}{|c|c|c|c|}
\hline Query Time & VSM & BM & LM \\
\hline RAM & $00: 00: 00.106$ & $00: 00: 00.046$ & $00: 00: 00.065$ \\
\hline FILE & $00: 00: 00.697$ & $00: 00: 00.376$ & $00: 00: 01.014$ \\
\hline Database & $00: 00: 00.376$ & $00: 00: 00.378$ & $00: 00: 00.376$ \\
\hline
\end{tabular}




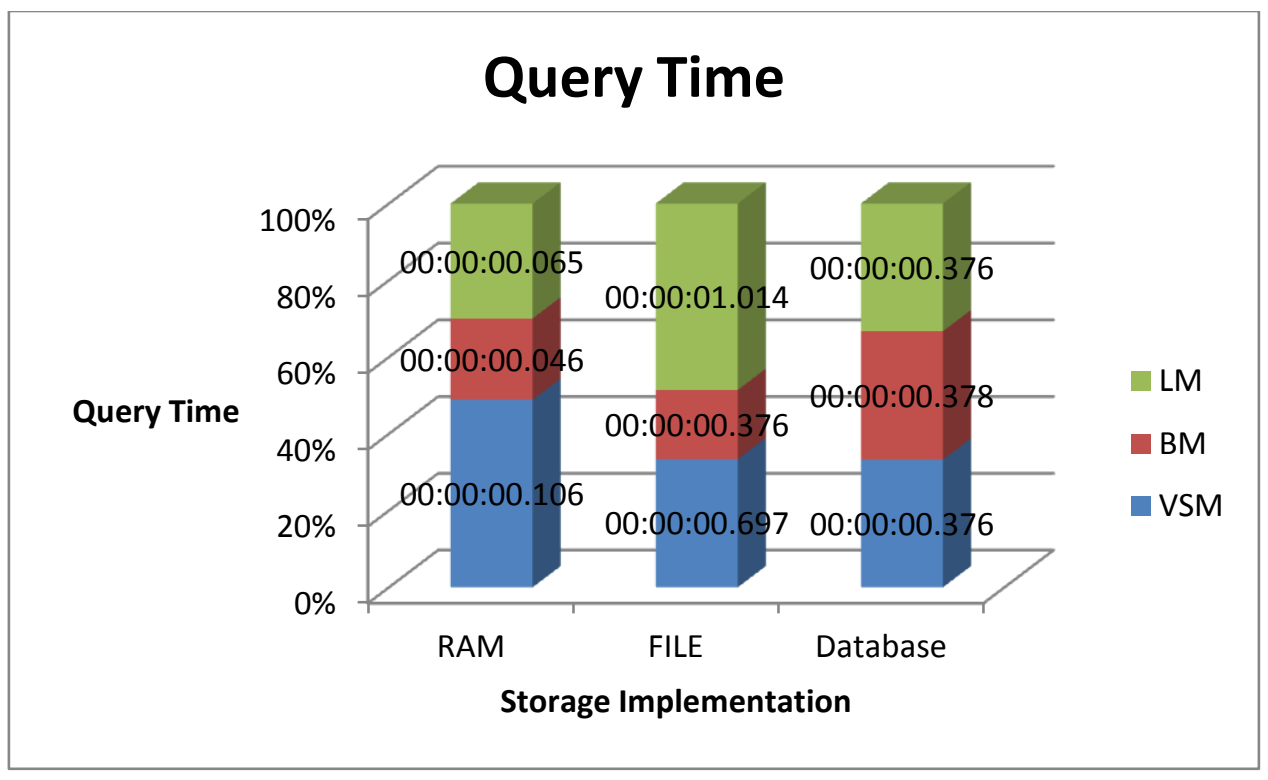

Figure 41: Query Time Comparison Graph

\begin{tabular}{|c|c|c|}
\hline VSM & Relevant & $\begin{array}{c}\text { Not } \\
\text { Relevant }\end{array}$ \\
\hline Retrieved & 2 & 108 \\
\hline $\begin{array}{c}\text { Not } \\
\text { Retrieved }\end{array}$ & 0 & 887 \\
\hline
\end{tabular}

Table 47: Confucius Matrix for VSM

\begin{tabular}{|c|c|c|}
\hline BM & Relevant & $\begin{array}{c}\text { Not } \\
\text { Relevant }\end{array}$ \\
\hline Retrieved & 2 & 130 \\
\hline $\begin{array}{c}\text { Not } \\
\text { Retrieved }\end{array}$ & 0 & 865 \\
\hline
\end{tabular}

Table 48: Confucius Matrix for BM

\begin{tabular}{|c|c|c|}
\hline LM & Relevant & $\begin{array}{c}\text { Not } \\
\text { Relevant }\end{array}$ \\
\hline Retrieved & 2 & 86 \\
\hline $\begin{array}{c}\text { Not } \\
\text { Retrieved }\end{array}$ & 0 & 909 \\
\hline
\end{tabular}

Table 49: Confucius Matrix for LM 


\subsection{Case Study Two}

\section{R.A.M. Implementation with Vector Space}

The following data was collected during the R.A.M. implementation using a vector space model for case study two.

\begin{tabular}{|c|c|c|}
\hline & Average & Standard Deviation \\
\hline Average Query Time & $00: 00: 00.081$ & $\pm 00: 00: 00.017$ \\
\hline $\begin{array}{c}\text { Mean Average Unranked } \\
\text { Precision }\end{array}$ & $11.02 \%$ & $\pm 0.00 \%$ \\
\hline Mean Average Precision & $13.68 \%$ & $\pm 0.00 \%$ \\
\hline Mean Average Recall & $97.60 \%$ & $\pm 0.00 \%$ \\
\hline
\end{tabular}

Table 50: R.A.M. VSM Summary

\begin{tabular}{|c|c|c|}
\hline RAM & Relevant & Not Relevant \\
\hline Retrieved & 2 & 180 \\
\hline Not Retrieved & 9 & 9149 \\
\hline
\end{tabular}

Table 51: Confucius Matrix for R.A.M. 


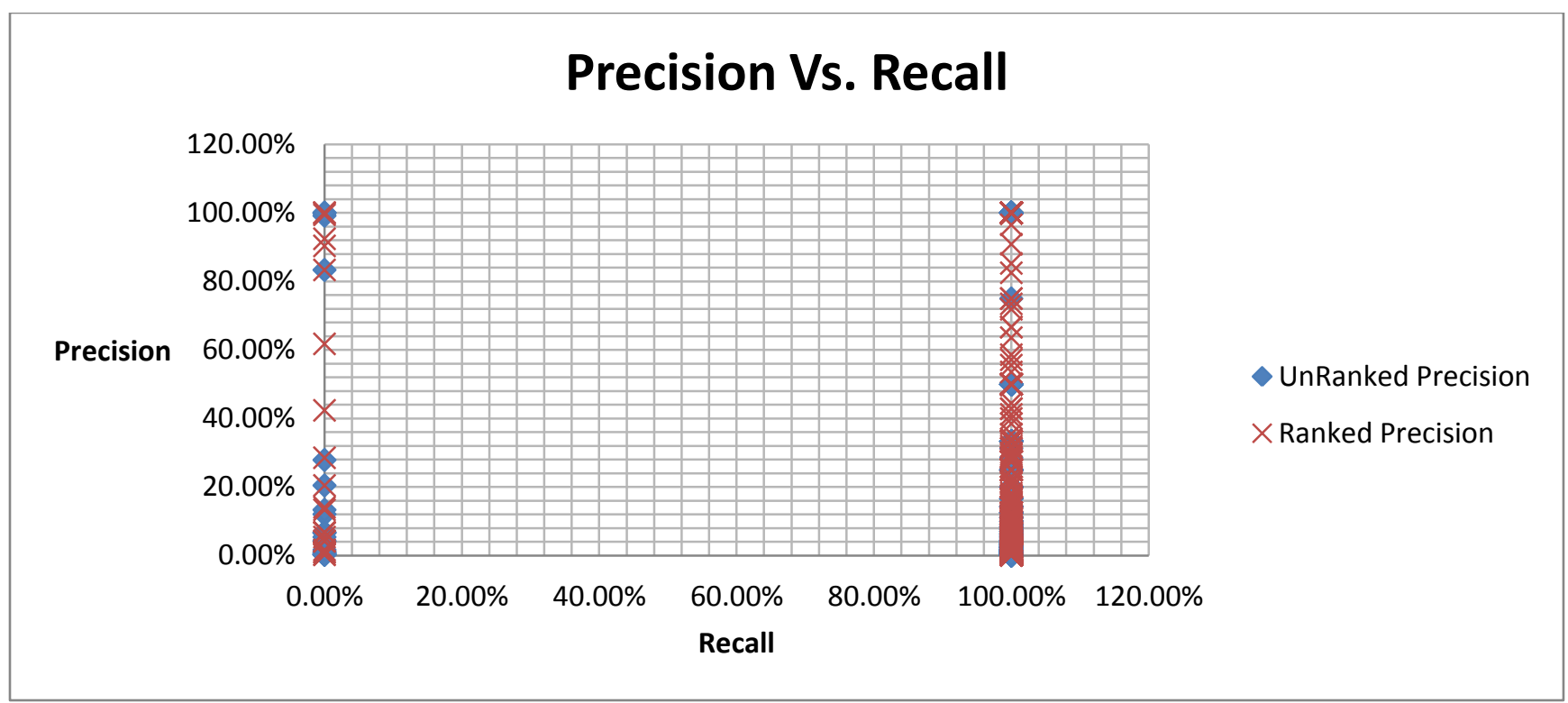

Figure 42: Precision vs. Recall

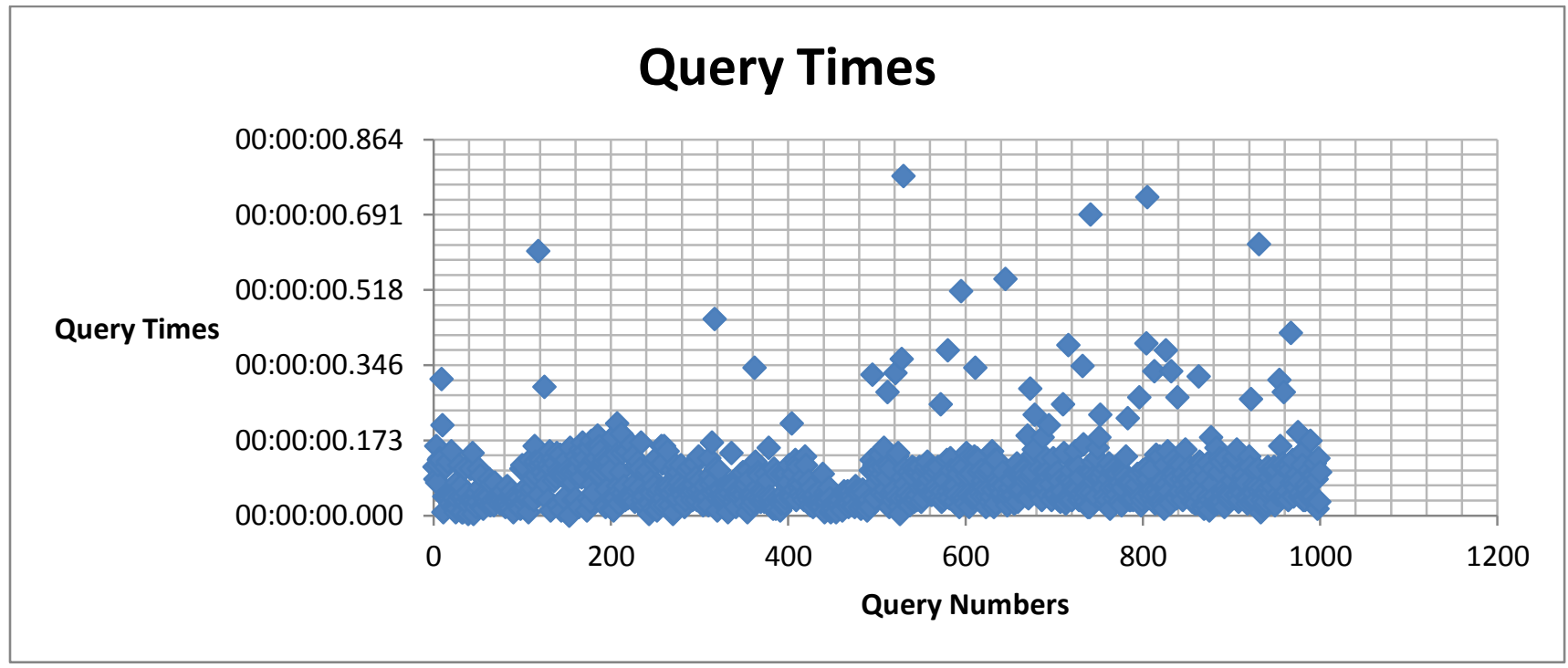

Figure 43: Query Times 


\section{File Implementation with Vector Space}

The following is a summary for the file implementation using a vector space model strategy.

\begin{tabular}{|c|c|c|}
\hline & Average & Standard Deviation \\
\hline Average Query Time & $00: 00: 04.682$ & $\pm 00: 00: 00.198$ \\
\hline $\begin{array}{c}\text { Mean Average Unranked } \\
\text { Precision }\end{array}$ & $10.51 \%$ & $\pm 0.00 \%$ \\
\hline Mean Average Precision & $13.52 \%$ & $\pm 0.00 \%$ \\
\hline Mean Average Recall & $97.60 \%$ & $\pm 0.00 \%$ \\
\hline
\end{tabular}

Table 52: File VSM Summary

\begin{tabular}{|c|c|c|}
\hline File & Relevant & Not Relevant \\
\hline Retrieved & 2 & 180 \\
\hline Not Retrieved & 9 & 9149 \\
\hline
\end{tabular}

Table 53: Confucius Matrix for File

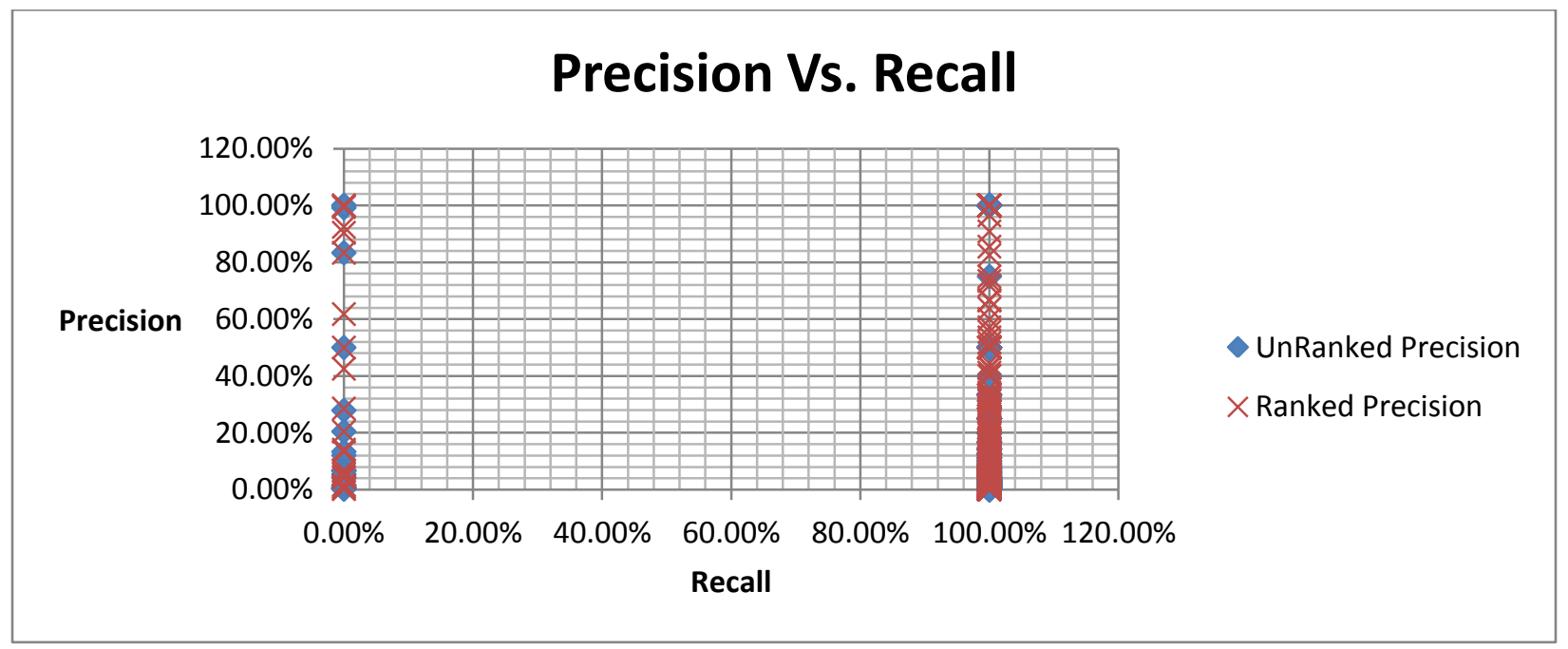

Figure 44: Precision vs. Recall 


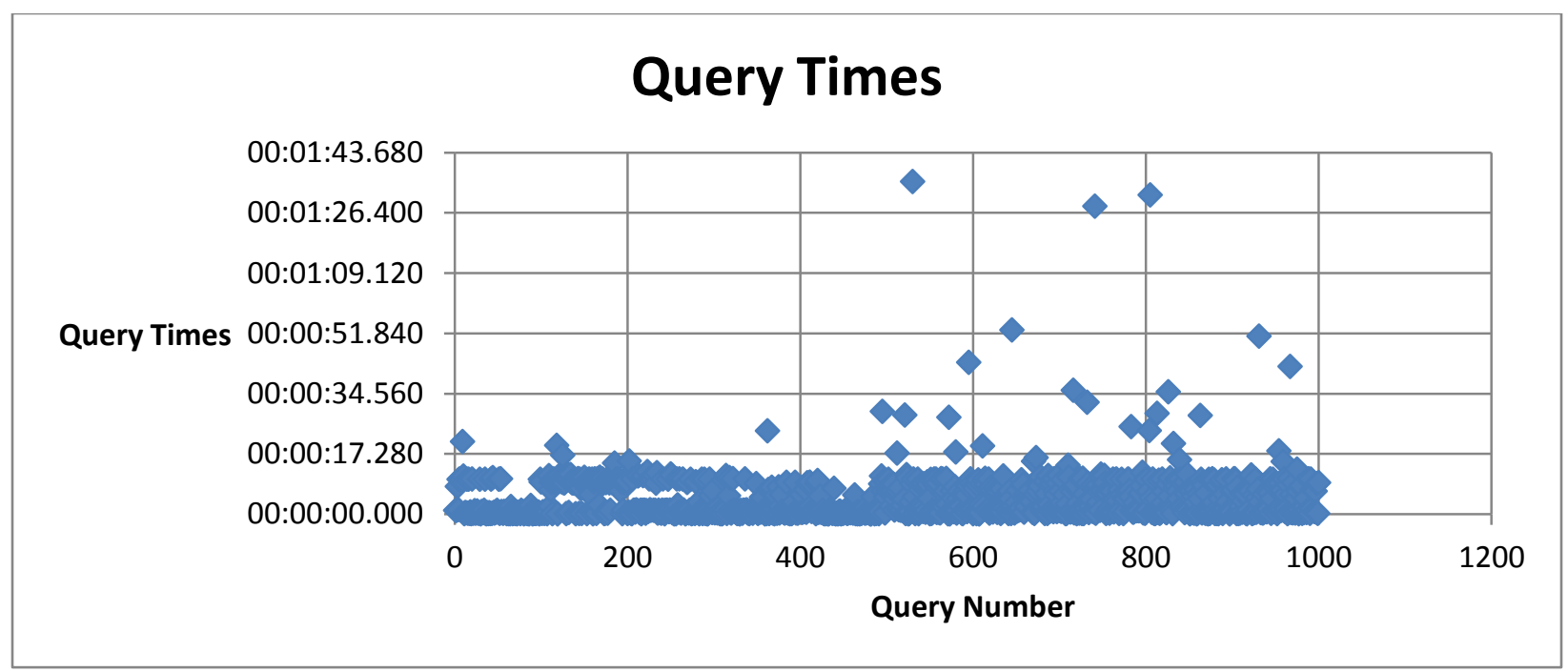

Figure 45: Query Times

\section{Database Implementation with Vector Space}

The following data summarizes the database implementation using a vector space model retrieval method.

\begin{tabular}{|c|c|c|}
\hline & Average & Standard Deviation \\
\hline Average Query Time & $00: 00: 00.341$ & $\pm 00: 00: 00.083$ \\
\hline $\begin{array}{c}\text { Mean Average Unranked } \\
\text { Precision }\end{array}$ & $6.71 \%$ & $\pm 0.00 \%$ \\
\hline $\begin{array}{c}\text { Mean Average Precision } \\
\text { Mean Average Recall }\end{array}$ & $11.89 \%$ & $\pm 0.00 \%$ \\
\hline
\end{tabular}

Table 54: Database VSM Summary

\begin{tabular}{|c|c|c|}
\hline Database & Relevant & Not Relevant \\
\hline Retrieved & 2 & 180 \\
\hline Not Retrieved & 9 & 9149 \\
\hline
\end{tabular}

Table 55: Confucius Matrix for Database 


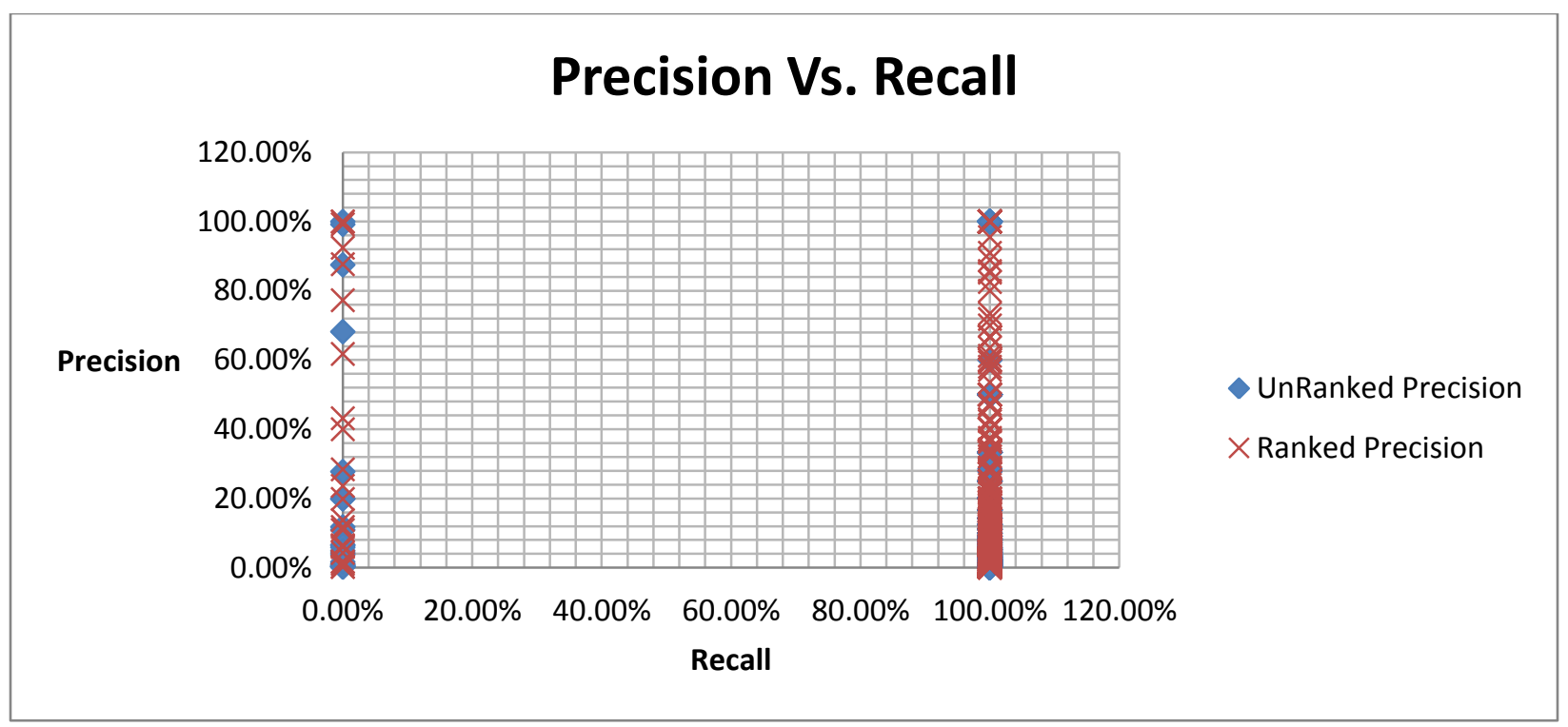

Figure 46: Precision vs. Recall

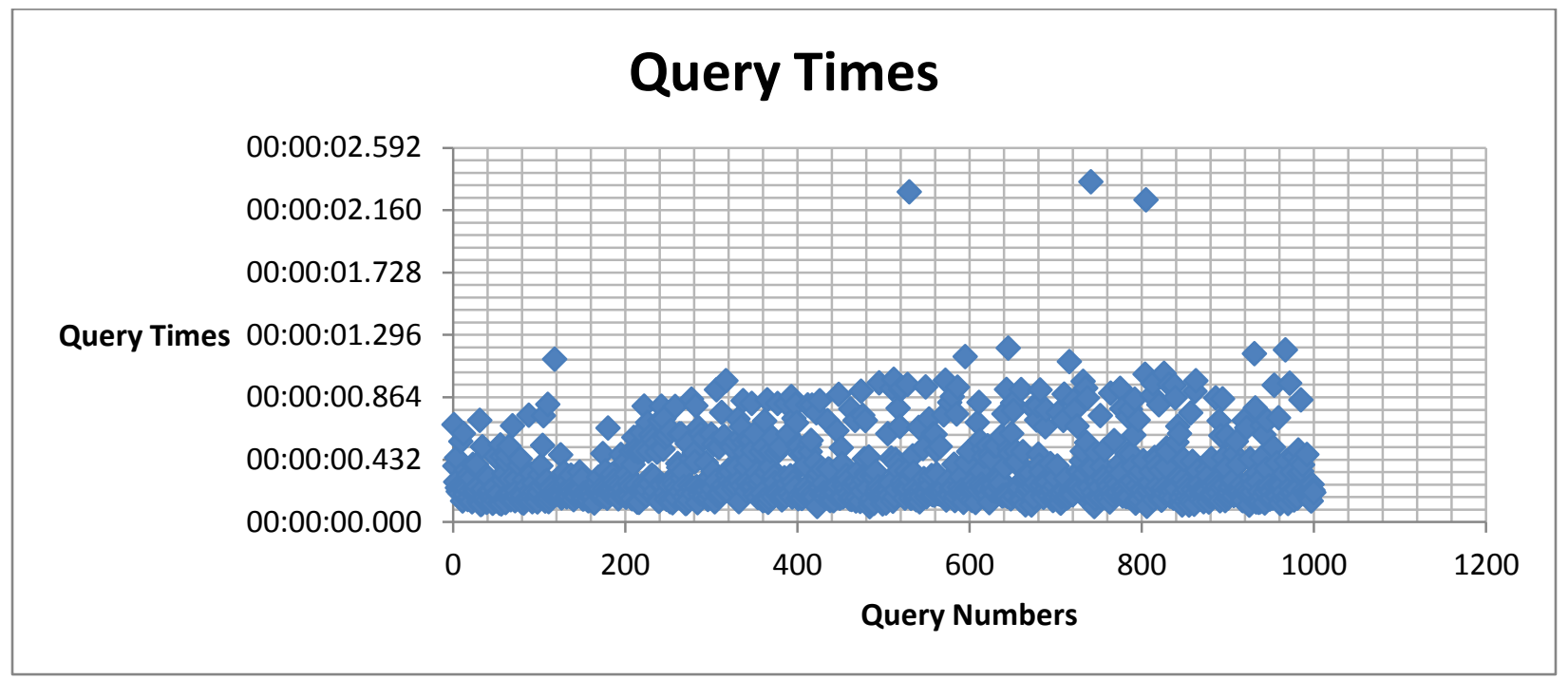

Figure 47: Query Times 


\section{R.A.M. Implementation with Best Match}

The following data is a summary of the data collected during the R.A.M implemented index using the best match 25 algorithm.

\begin{tabular}{|c|c|c|}
\hline & Average & Standard Deviation \\
\hline Average Query Time & $00: 00: 01.603$ & $\pm 00: 00: 03.636$ \\
\hline $\begin{array}{c}\text { Mean Average Unranked } \\
\text { Precision }\end{array}$ & $10.75 \%$ & $\pm 4.70 \%$ \\
\hline Mean Average Precision & $13.94 \%$ & $\pm 5.04 \%$ \\
\hline Mean Average Recall & $93.83 \%$ & $\pm 0.00 \%$ \\
\hline
\end{tabular}

Table 56: RAM BM Summary

\begin{tabular}{|c|c|c|}
\hline RAM & Relevant & Not Relevant \\
\hline Retrieved & 2 & 172 \\
\hline Not Retrieved & 9 & 9157 \\
\hline
\end{tabular}

Table 57: Confucius Matrix RAM

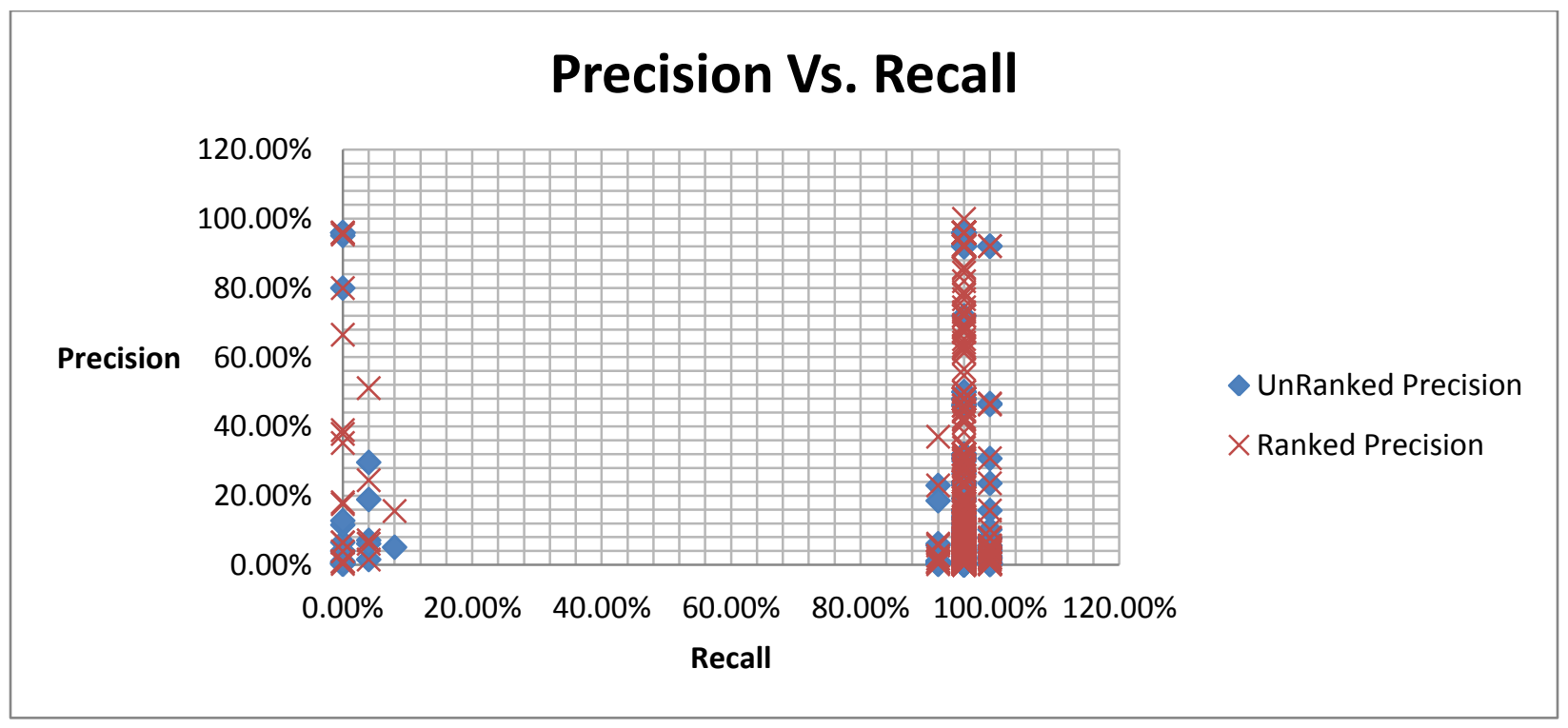

Figure 48: Precision vs. Recall 


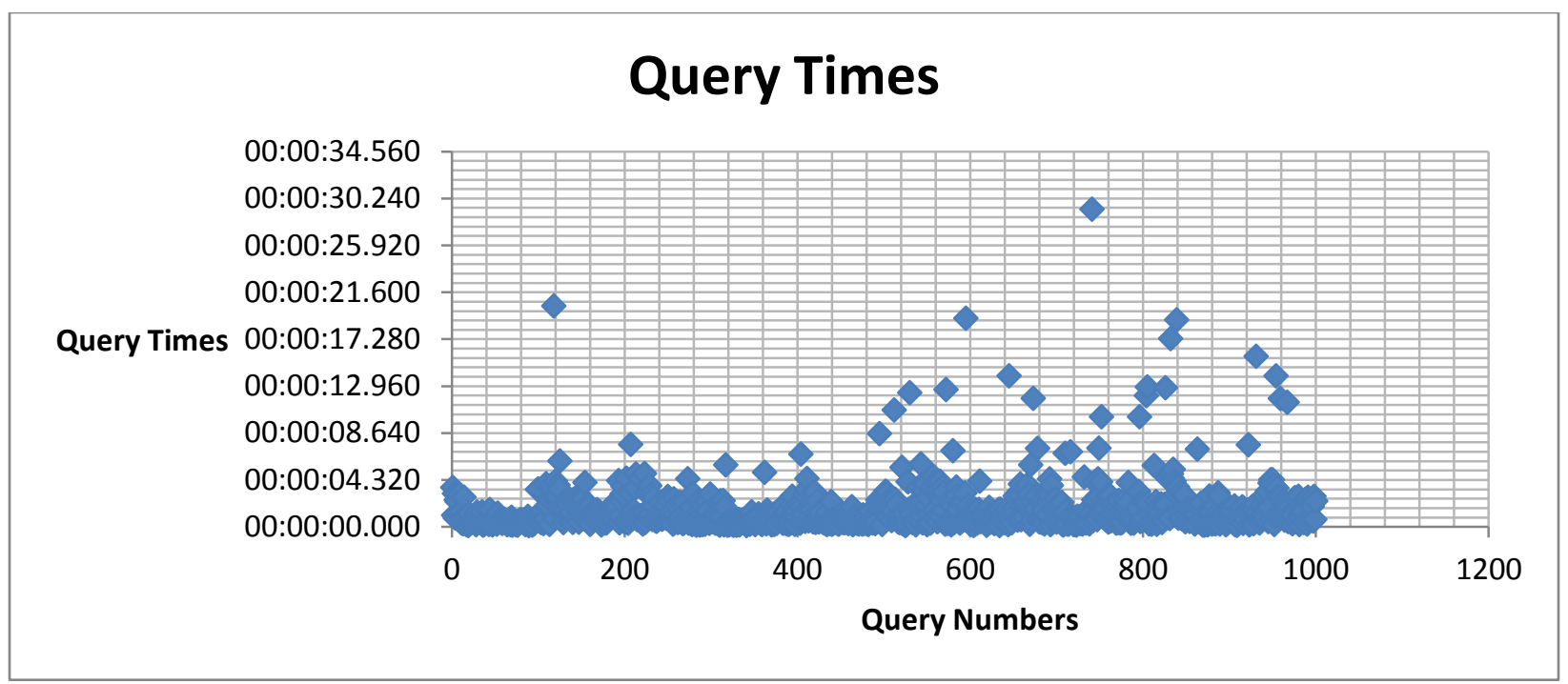

Figure 49: Query Times

\section{File Implementation with Best Match}

The following tables and graphs summarize the outcome of the file implementation using the best match 25 algorithm.

\begin{tabular}{|c|c|c|}
\hline & Average & Standard Deviation \\
\hline Average Query Time & $00: 00: 05.391$ & $\pm 00: 00: 02.792$ \\
\hline $\begin{array}{c}\text { Mean Average Unranked } \\
\text { Precision }\end{array}$ & $10.09 \%$ & $\pm 5.71 \%$ \\
\hline Mean Average Precision & $13.74 \%$ & $\pm 6.35 \%$ \\
\hline Mean Average Recall & $93.24 \%$ & $\pm 0.00 \%$ \\
\hline
\end{tabular}

Table 58: File BM Summary

\begin{tabular}{|c|c|c|}
\hline File & Relevant & Not Relevant \\
\hline Retrieved & 2 & 172 \\
\hline Not Retrieved & 9 & 9157 \\
\hline
\end{tabular}




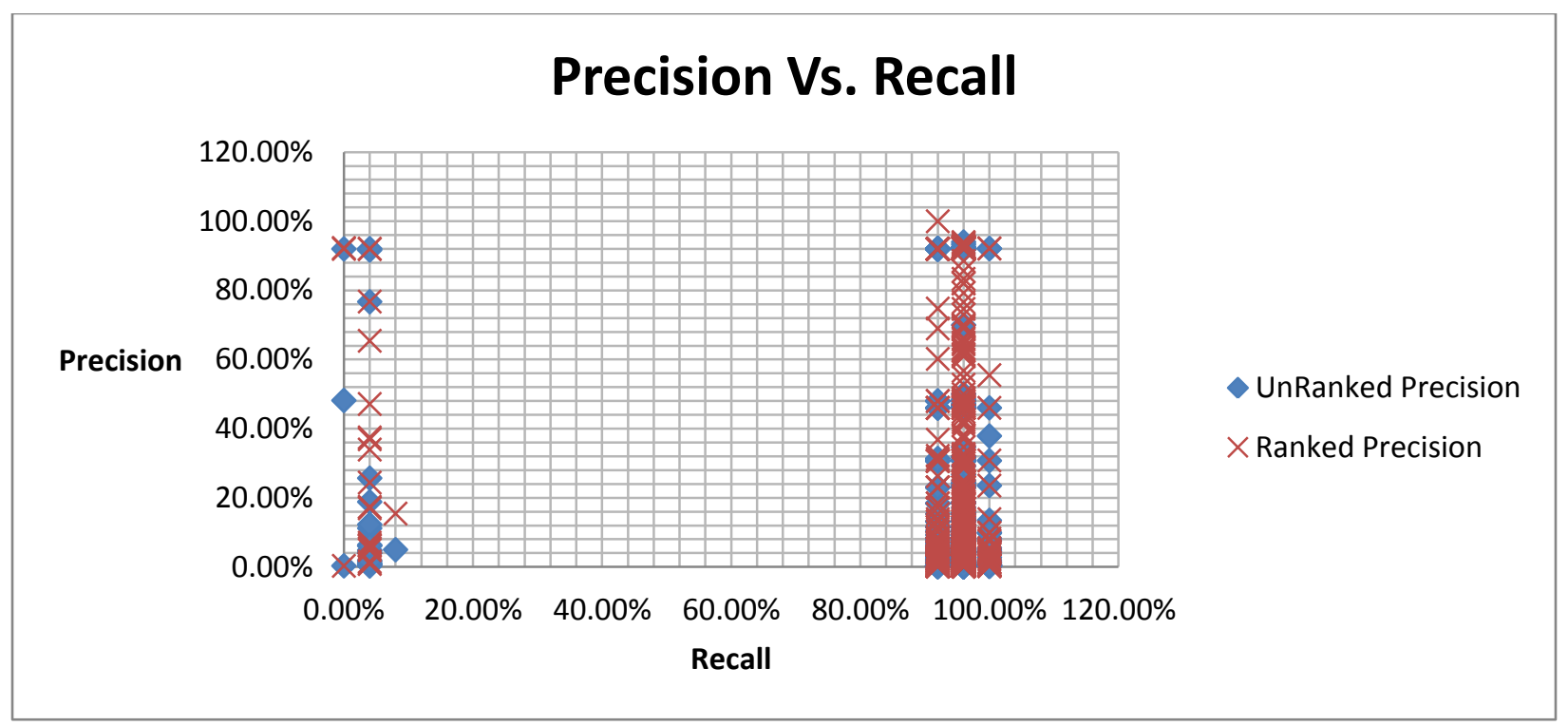

Figure 50: Precision vs. Recall

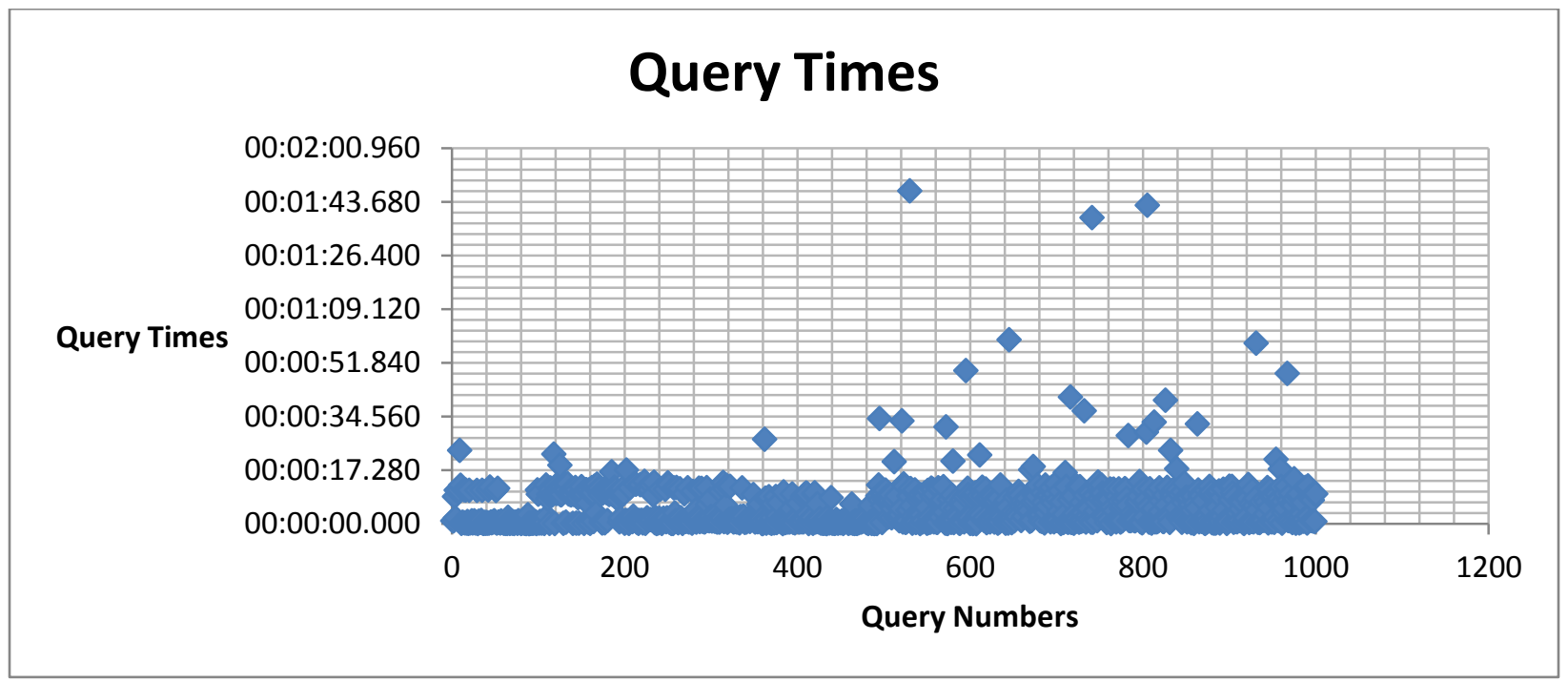

Figure 51: Query Times 


\section{Database Implementation with Best Match}

The following information is a summary of the database implementation combined using a Best Match 25 retrieval algorithm.

\begin{tabular}{|c|c|c|}
\hline & Average & Standard Deviation \\
\hline Average Query Time & $00: 00: 01.603$ & $\pm 00: 00: 03.636$ \\
\hline $\begin{array}{c}\text { Mean Average Unranked } \\
\text { Precision }\end{array}$ & $10.75 \%$ & $\pm 4.70 \%$ \\
\hline Mean Average Precision & $13.94 \%$ & $\pm 5.04 \%$ \\
\hline Mean Average Recall & $93.83 \%$ & $\pm 0.00 \%$ \\
\hline
\end{tabular}

Table 60: Database BM Summary

\begin{tabular}{|c|c|c|}
\hline Database & Relevant & Not Relevant \\
\hline Retrieved & 2 & 172 \\
\hline Not Retrieved & 9 & 9157 \\
\hline
\end{tabular}

Table 61: Confucius Matrix

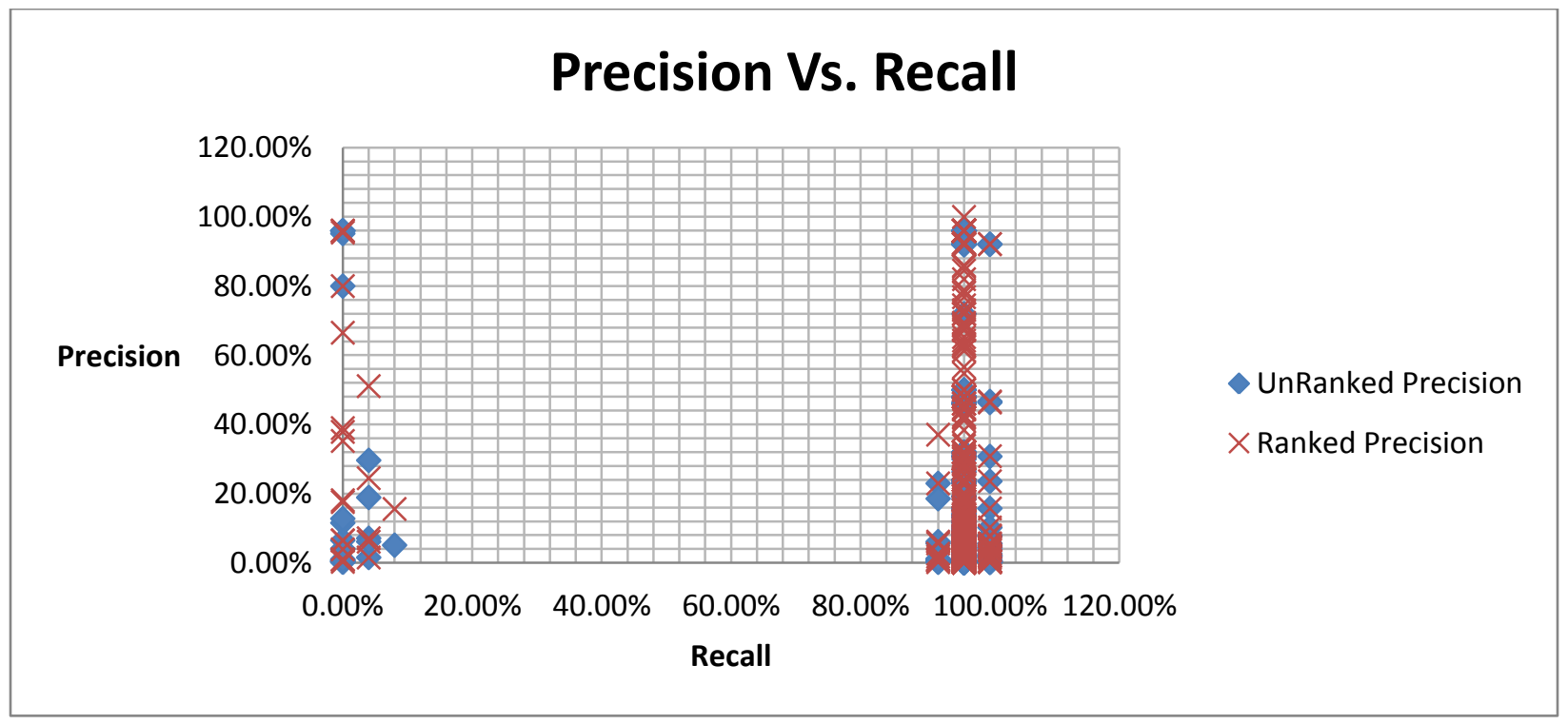

Figure 52: Precision vs. Recall 


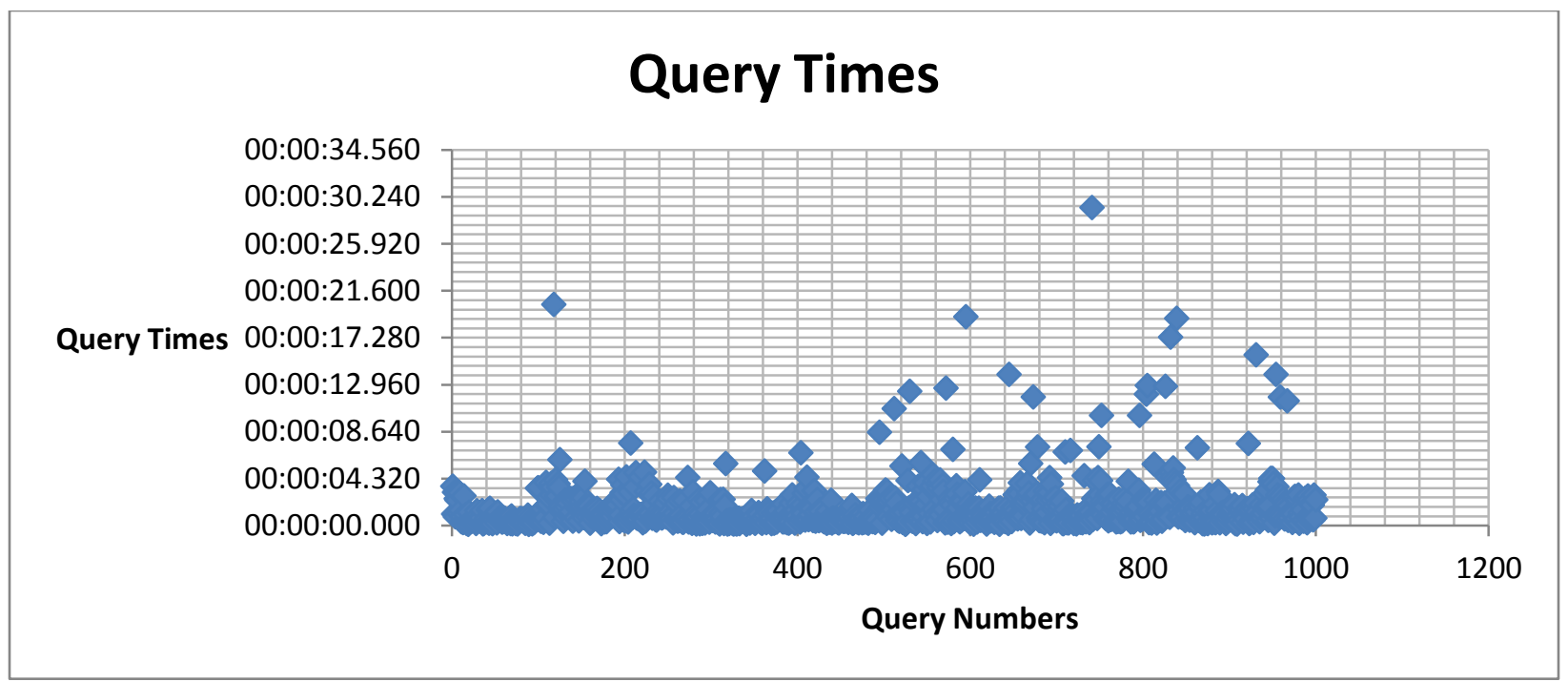

Figure 53: Query Times

\section{R.A.M. Implementation with Language Model}

The following summary is on the data collected for the RAM implementation using a Language model retrieval method.

\begin{tabular}{|c|c|c|}
\hline & Average & Standard Deviation \\
\hline Average Query Time & $00: 00: 00.094$ & $\pm 00: 00: 00.122$ \\
\hline $\begin{array}{c}\text { Mean Average Unranked } \\
\text { Precision }\end{array}$ & $11.01 \%$ & $\pm 7.65 \%$ \\
\hline Mean Average Precision & $14.23 \%$ & $\pm 8.10 \%$ \\
\hline Mean Average Recall & $97.54 \%$ & $\pm 0.00 \%$ \\
\hline
\end{tabular}

Table 62: RAM LM Summary

\begin{tabular}{|c|c|c|}
\hline RAM & Relevant & Not Relevant \\
\hline Retrieved & 2 & 180 \\
\hline Not Retrieved & 9 & 9149 \\
\hline
\end{tabular}

Table 63: Confucius Matrix RAM 


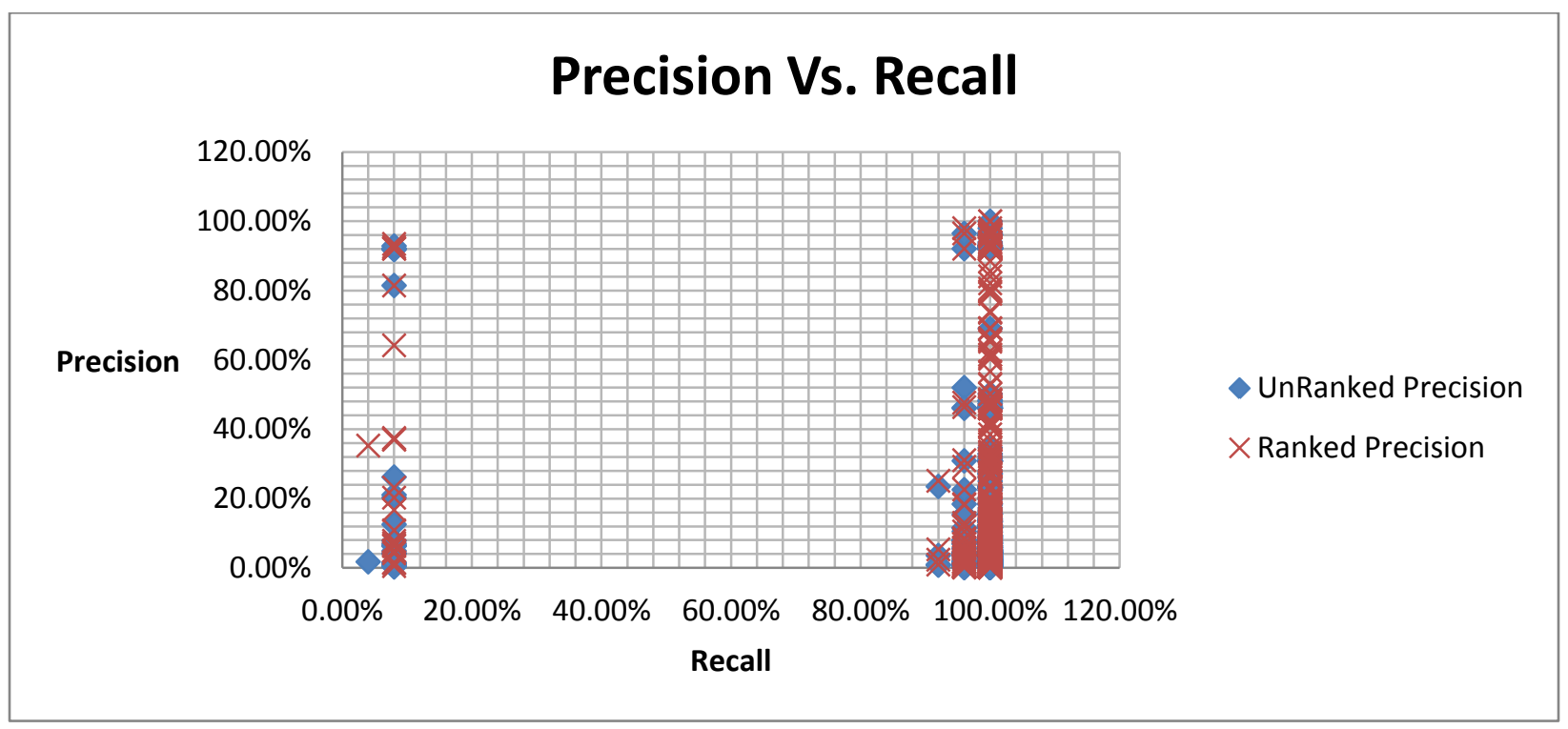

Figure 54: Precision vs. Recall

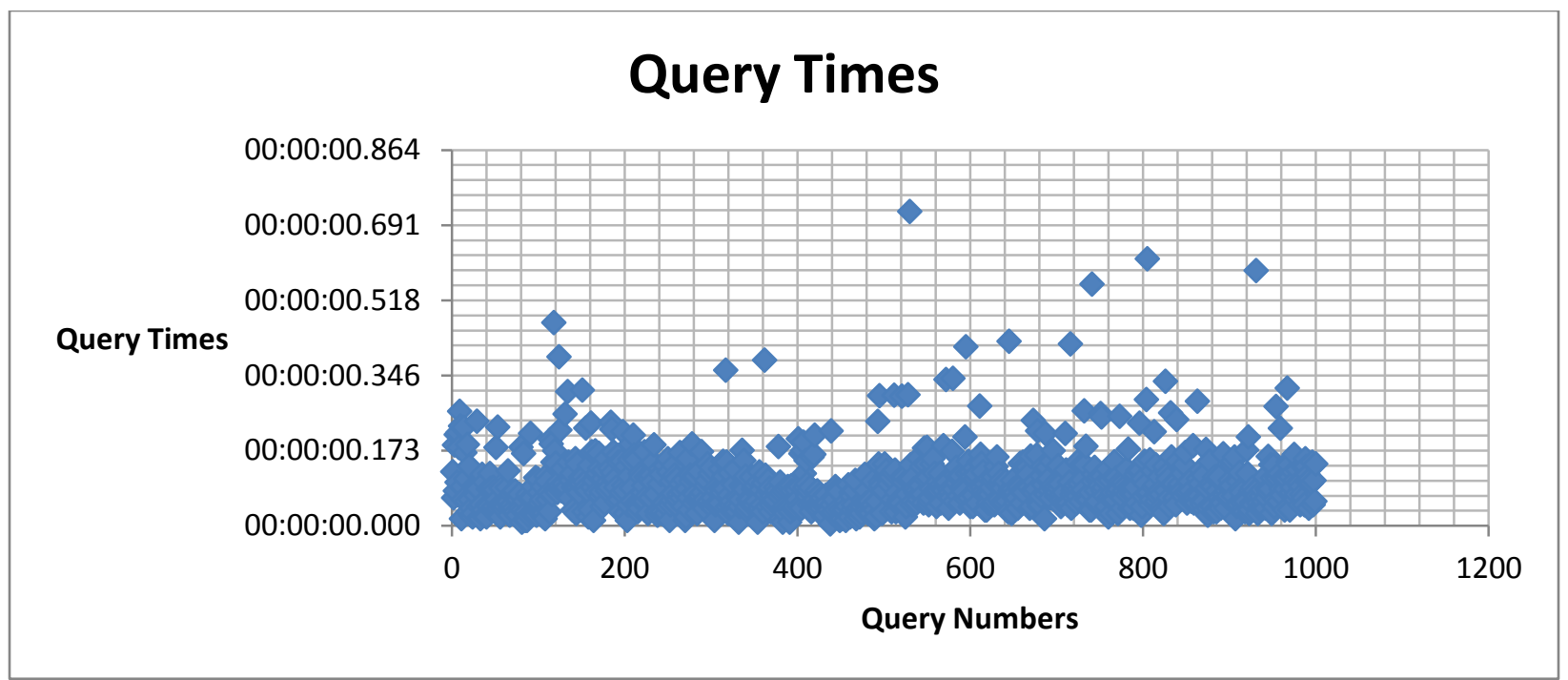

Figure 55: Query Times 


\section{File Implementation with Language Model}

The following data summarizes file implementation using a language model algorithm results:

\begin{tabular}{|c|c|c|}
\hline & Average & Standard Deviation \\
\hline Average Query Time & $00: 00: 02.772$ & $00: 00: 00.085$ \\
\hline $\begin{array}{c}\text { Mean Average Unranked } \\
\text { Precision }\end{array}$ & $10.10 \%$ & $0.00 \%$ \\
\hline Mean Average Precision & $13.45 \%$ & $0.00 \%$ \\
\hline Mean Average Recall & $93.60 \%$ & $0.00 \%$ \\
\hline
\end{tabular}

Table 64: File LM Summary

\begin{tabular}{|c|c|c|}
\hline RAM & Relevant & Not Relevant \\
\hline Retrieved & 2 & 162 \\
\hline Not Retrieved & 9 & 9168 \\
\hline
\end{tabular}




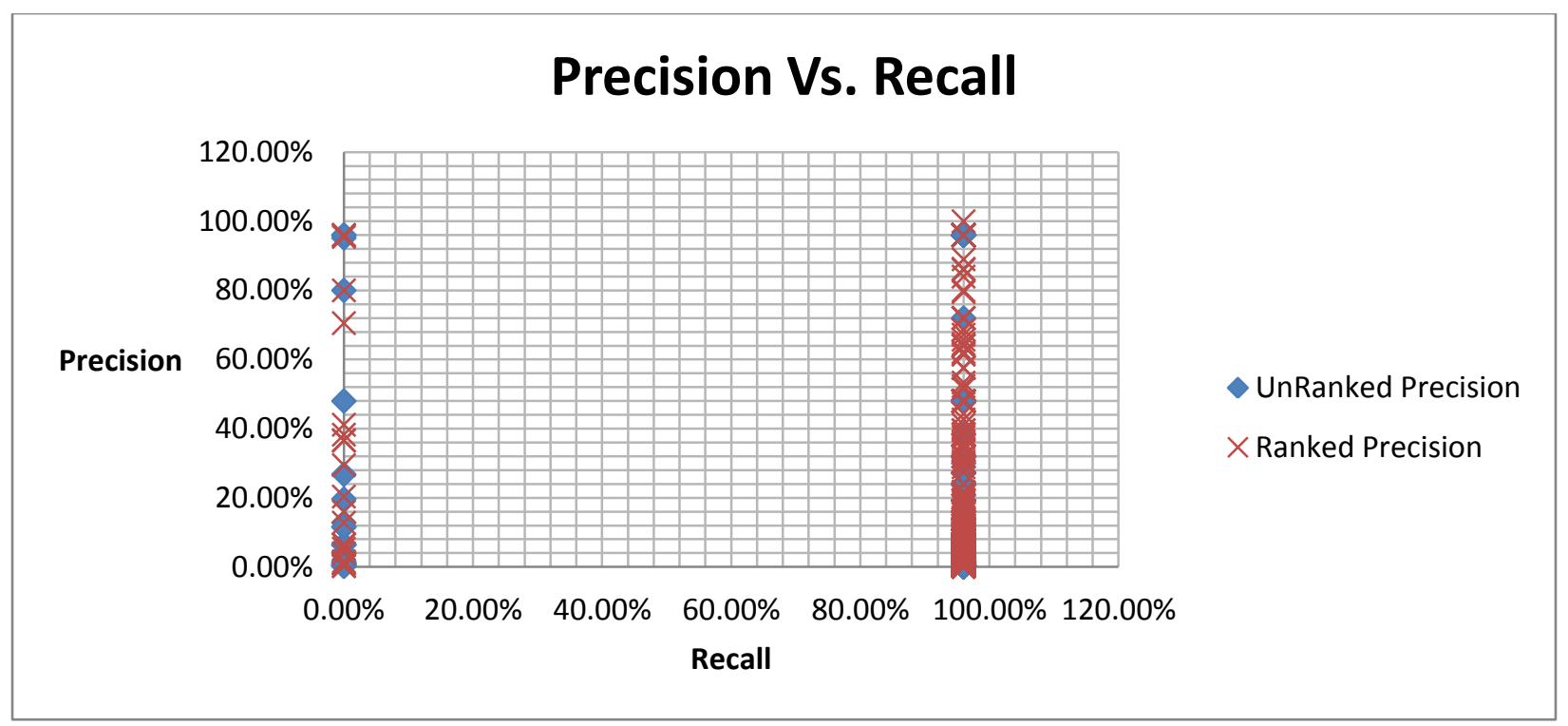

Figure 56: Precision vs. Recall

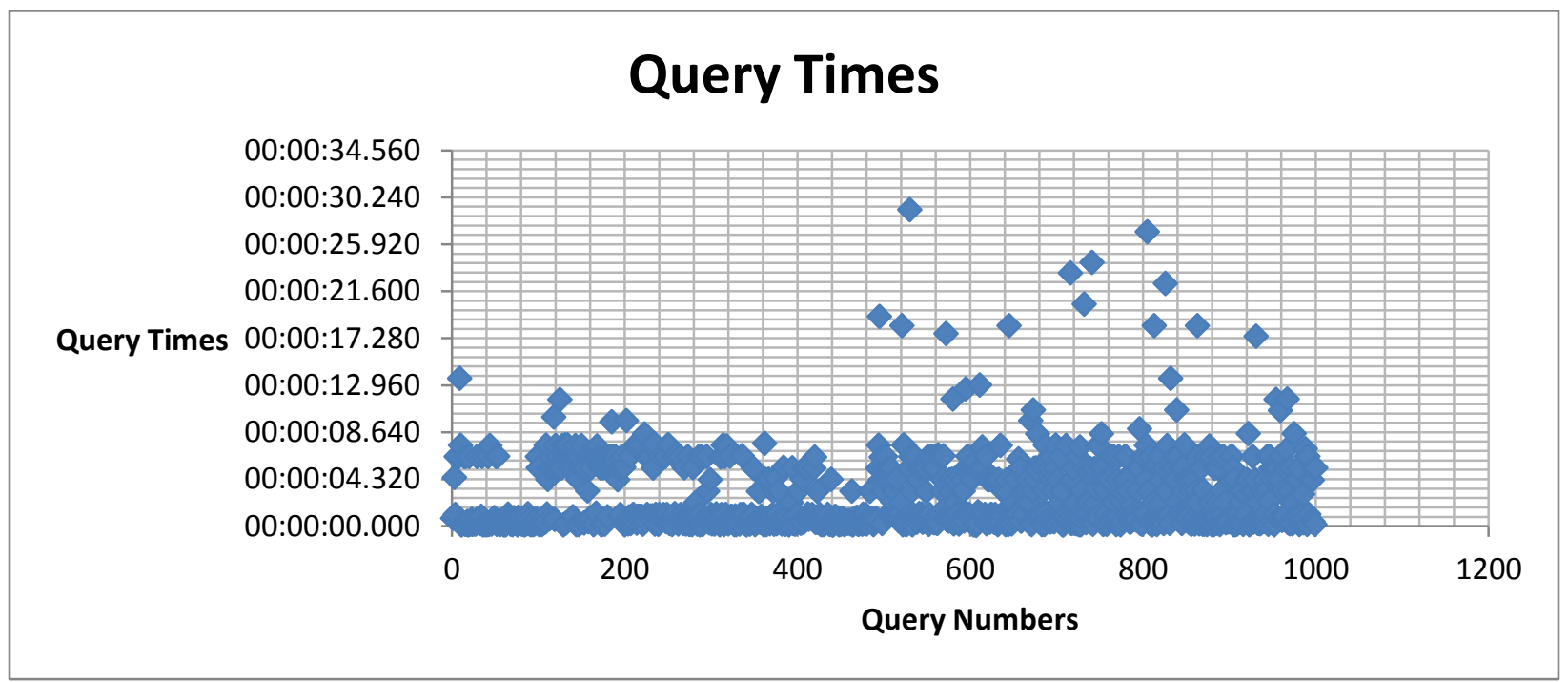

Figure 57: Query Times 


\section{Database Implementation with Language Model}

The following information summarizes language model retrieval algorithm with a database index implementation.

\begin{tabular}{|c|c|c|}
\hline & Average & Standard Deviation \\
\hline Average Query Time & $00: 00: 02.712$ & $\pm 00: 00: 06.117$ \\
\hline $\begin{array}{c}\text { Mean Average Unranked } \\
\text { Precision }\end{array}$ & $9.18 \%$ & $\pm 7.91 \%$ \\
\hline $\begin{array}{c}\text { Mean Average Precision } \\
\text { Mean Average Recall }\end{array}$ & $13.09 \%$ & $\pm 0.01 \%$ \\
\hline
\end{tabular}

Table 66: Database LM Summary

\begin{tabular}{|c|c|c|}
\hline Database & Relevant & Not Relevant \\
\hline Retrieved & 2 & 179 \\
\hline Not Retrieved & 9 & 9150 \\
\hline
\end{tabular}

Table 67: Confucius Matrix Database

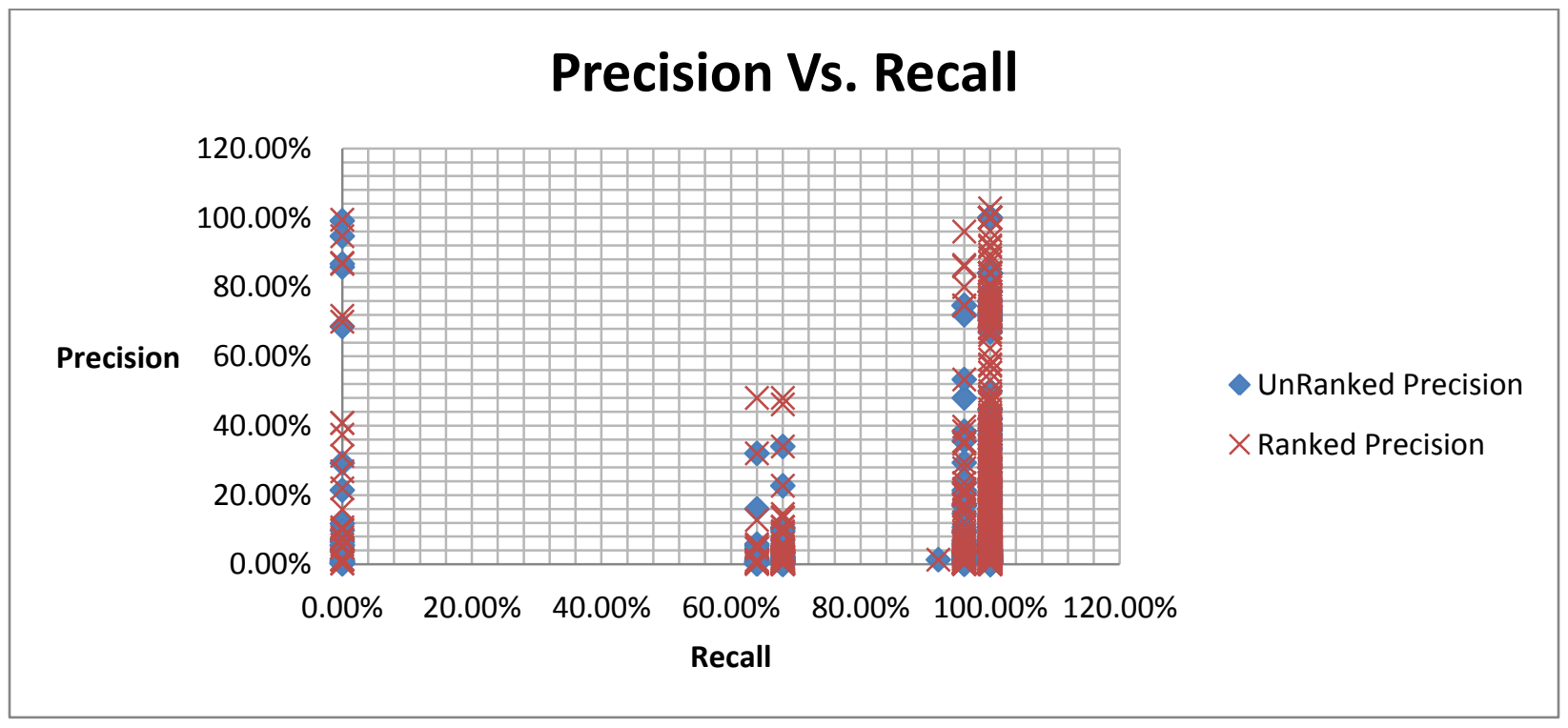

Figure 58: Precision vs. Recall 


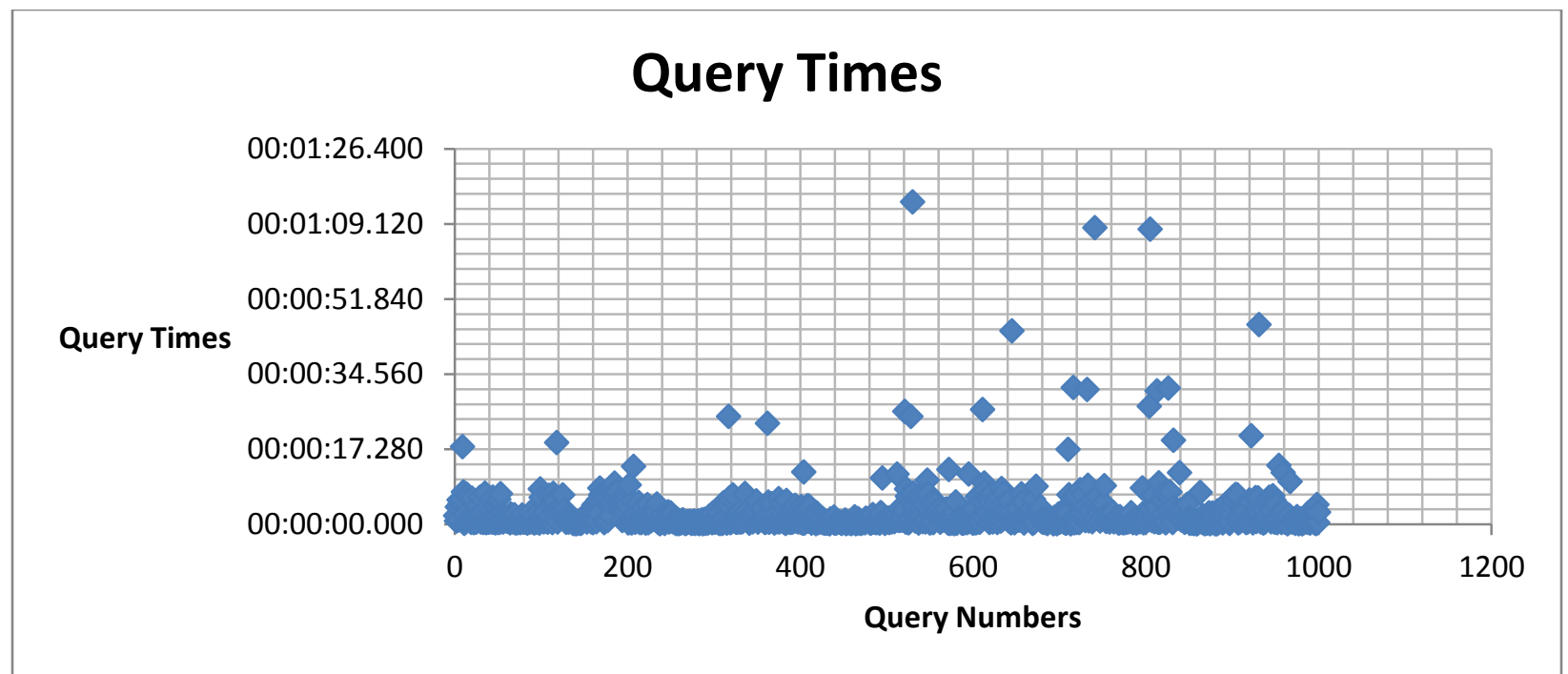

Figure 59: Query Times

\subsubsection{Vector Space Model Comparison}

The following section compares all vector space data collected for case study two. The tables below provide the averages recorded for each index implementation.

\begin{tabular}{|c|c|c|c|c|}
\hline Storage & $\begin{array}{c}\text { Average Query } \\
\text { Time }\end{array}$ & $\begin{array}{c}\text { Mean Average } \\
\text { Unranked Precision }\end{array}$ & $\begin{array}{c}\text { Mean Average } \\
\text { Ranked } \\
\text { Precision }\end{array}$ & $\begin{array}{c}\text { Average } \\
\text { Recall }\end{array}$ \\
\hline RAM & $00: 00: 00.810$ & $11.02 \%$ & $13.68 \%$ & $97.60 \%$ \\
\hline File & $00: 00: 04.682$ & $10.51 \%$ & $13.52 \%$ & $97.60 \%$ \\
\hline Database & $00: 00: 00.341$ & $6.71 \%$ & $11.89 \%$ & $97.60 \%$ \\
\hline
\end{tabular}




\begin{tabular}{|c|c|c|}
\hline RAM & Relevant & Not Relevant \\
\hline Retrieved & 2 & 180 \\
\hline Not Retrieved & 9 & 9149 \\
\hline
\end{tabular}

Table 69: Confucius Matrix for RAM

\begin{tabular}{|c|c|c|}
\hline File & Relevant & Not Relevant \\
\hline Retrieved & 2 & 180 \\
\hline Not Retrieved & 9 & 9149 \\
\hline
\end{tabular}

Table 70: Confucius Matrix for File

\begin{tabular}{|c|c|c|}
\hline Database & Relevant & Not Relevant \\
\hline Retrieved & 2 & 190 \\
\hline Not Retrieved & 9 & 9139 \\
\hline
\end{tabular}

Table 71: Confucius Matrix for Database

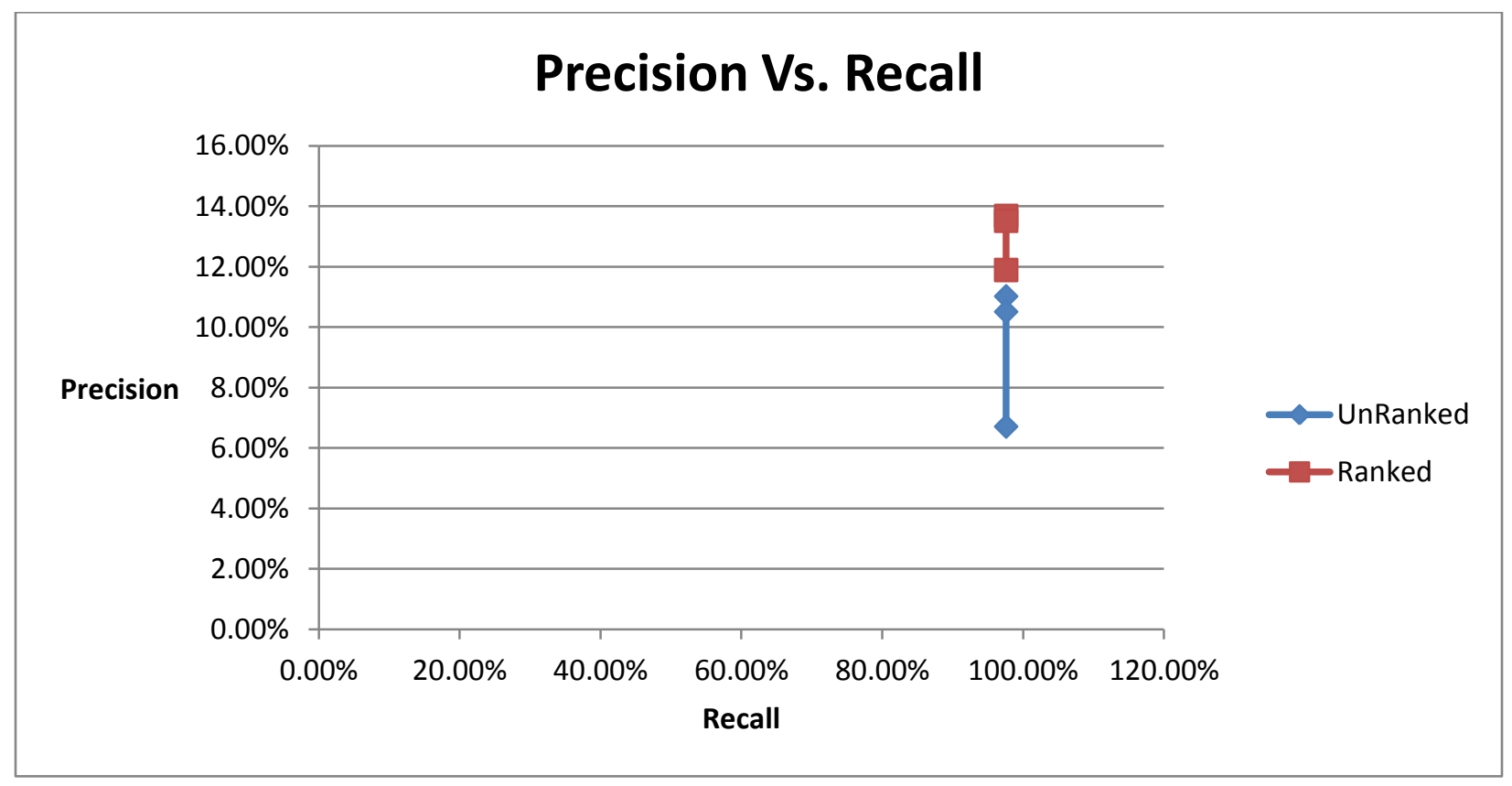

Figure 60: Precision vs. Recall Comparison 


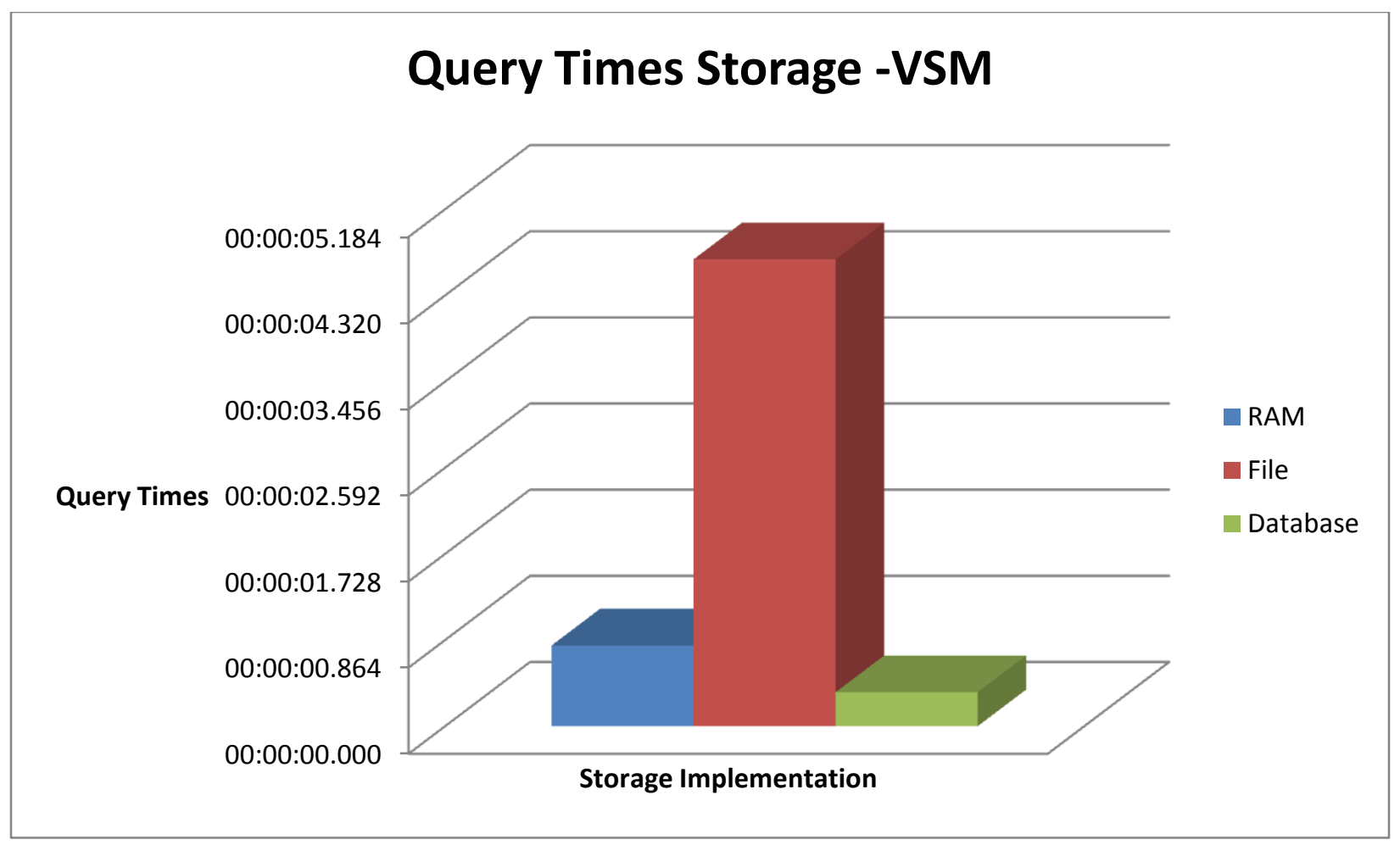

Figure 61: Query Time Comparison 


\subsubsection{Best Match Comparison}

This section covers the comparison of the Best Match 25 algorithm results with the different index implementations.

\begin{tabular}{|c|c|c|c|c|}
\hline Storage & $\begin{array}{c}\text { Average Query } \\
\text { Time }\end{array}$ & $\begin{array}{c}\text { Mean Average } \\
\text { Unranked Precision }\end{array}$ & $\begin{array}{c}\text { Mean } \\
\text { Average } \\
\text { Ranked } \\
\text { Precision }\end{array}$ & $\begin{array}{c}\text { Average } \\
\text { Recall }\end{array}$ \\
\hline RAM & $00: 00: 00.053$ & $10.56 \%$ & $13.77 \%$ & $93.24 \%$ \\
\hline File & $00: 00: 05.391$ & $10.09 \%$ & $13.74 \%$ & $93.24 \%$ \\
\hline Database & $00: 00: 01.603$ & $10.75 \%$ & $13.94 \%$ & $93.83 \%$ \\
\hline
\end{tabular}

Table 72: Best Match Comparison

The following tables are the confucius matrixes for each index implementation.

\begin{tabular}{|c|c|c|}
\hline RAM & Relevant & Not Relevant \\
\hline Retrieved & 2 & 172 \\
\hline Not Retrieved & 9 & 9157 \\
\hline
\end{tabular}

Table 73: Confucius Matrix for RAM

\begin{tabular}{|c|c|c|}
\hline File & Relevant & Not Relevant \\
\hline Retrieved & 2 & 172 \\
\hline Not Retrieved & 9 & 9157 \\
\hline
\end{tabular}

Table 74: Confucius Matrix for File

\begin{tabular}{|c|c|c|}
\hline Database & Relevant & Not Relevant \\
\hline Retrieved & 2 & 172 \\
\hline Not Retrieved & 9 & 9157 \\
\hline
\end{tabular}

Table 75: Confucius Matrix for Database 
The following graphs illustrate the trends that occurred during the investigation for precision and query times.

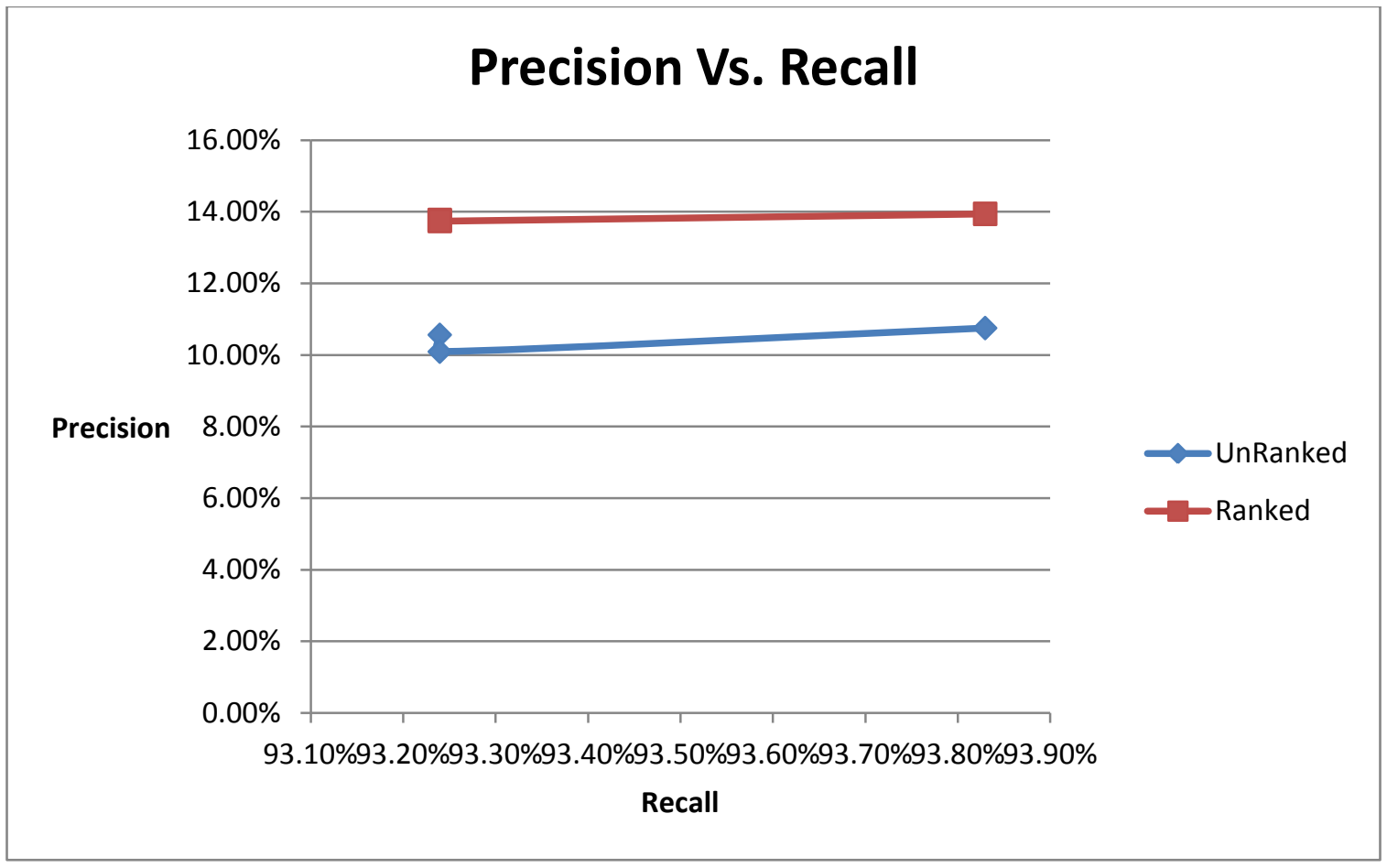

Figure 62: Precision vs. Recall

\section{Query Times Storage BM}

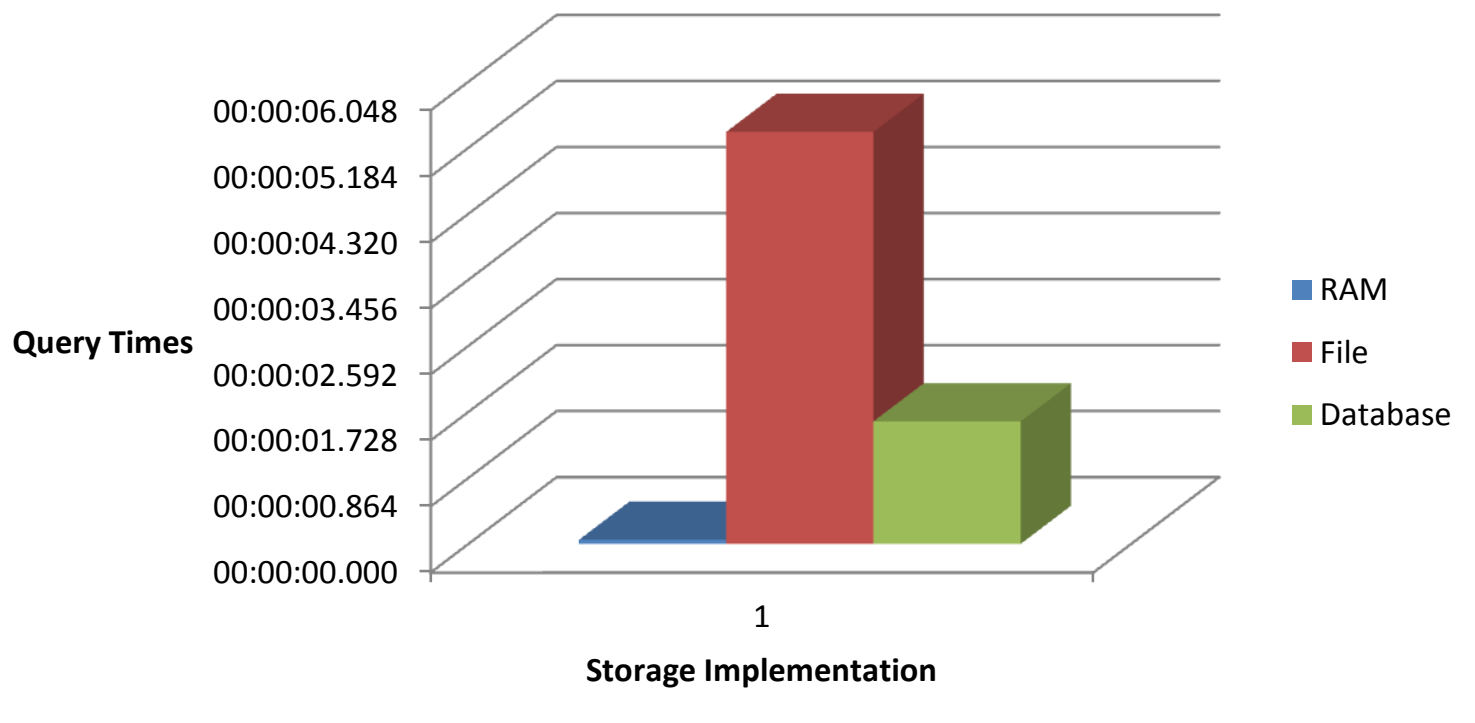

Figure 63: Query Times 


\subsubsection{Language Model Comparison}

The following section discusses the results obtained during the language model part of the case study two trials.

\begin{tabular}{|c|c|c|c|c|}
\hline Storage & $\begin{array}{c}\text { Average Query } \\
\text { Time }\end{array}$ & $\begin{array}{c}\text { Average } \\
\text { Unranked } \\
\text { Precision }\end{array}$ & $\begin{array}{c}\text { Mean } \\
\text { Average } \\
\text { Ranked } \\
\text { Precision }\end{array}$ & $\begin{array}{c}\text { Average } \\
\text { Recall }\end{array}$ \\
\hline RAM & $00: 00: 00.094$ & $11.01 \%$ & $14.23 \%$ & $97.54 \%$ \\
\hline File & $00: 00: 02.772$ & $10.01 \%$ & $13.45 \%$ & $93.60 \%$ \\
\hline Database & $00: 00: 02.712$ & $7.91 \%$ & $13.09 \%$ & $94.54 \%$ \\
\hline
\end{tabular}

Table 76: Language Model Comparison

\begin{tabular}{|c|c|c|}
\hline RAM & Relevant & Not Relevant \\
\hline Retrieved & 2 & 180 \\
\hline Not Retrieved & 9 & 9149 \\
\hline
\end{tabular}

Table 77: Confucius Matrix for RAM

\begin{tabular}{|c|c|c|}
\hline File & Relevant & Not Relevant \\
\hline Retrieved & 2 & 180 \\
\hline Not Retrieved & 9 & 9149 \\
\hline
\end{tabular}

\begin{tabular}{|c|c|c|}
\hline Database & Relevant & Not Relevant \\
\hline Retrieved & 2 & 179 \\
\hline Not Retrieved & 9 & 9150 \\
\hline
\end{tabular}

Table 79: Confucius Matrix for Database 


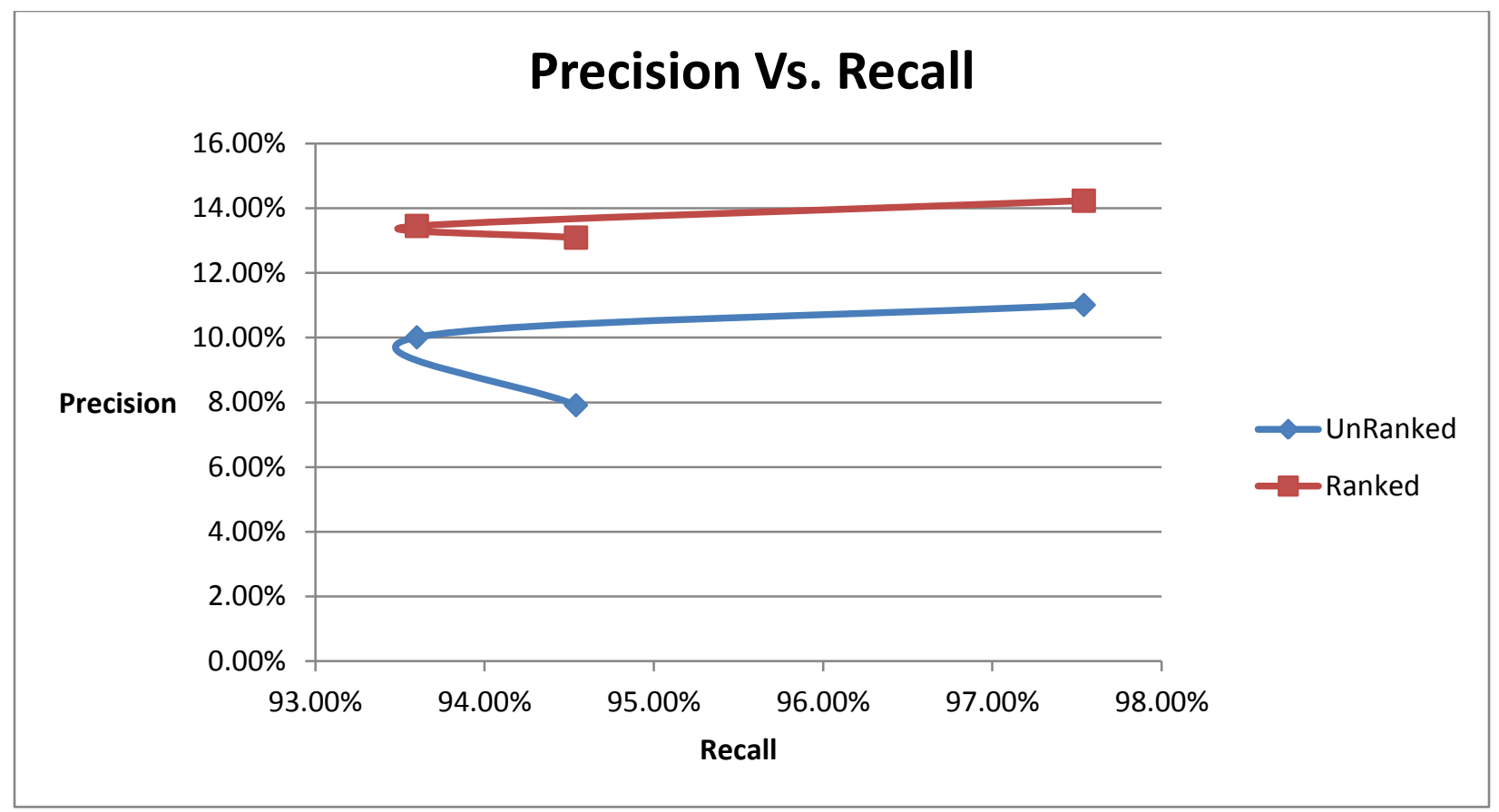

Figure 64: Precision vs. Recall

\section{Query Times Storage -LM}

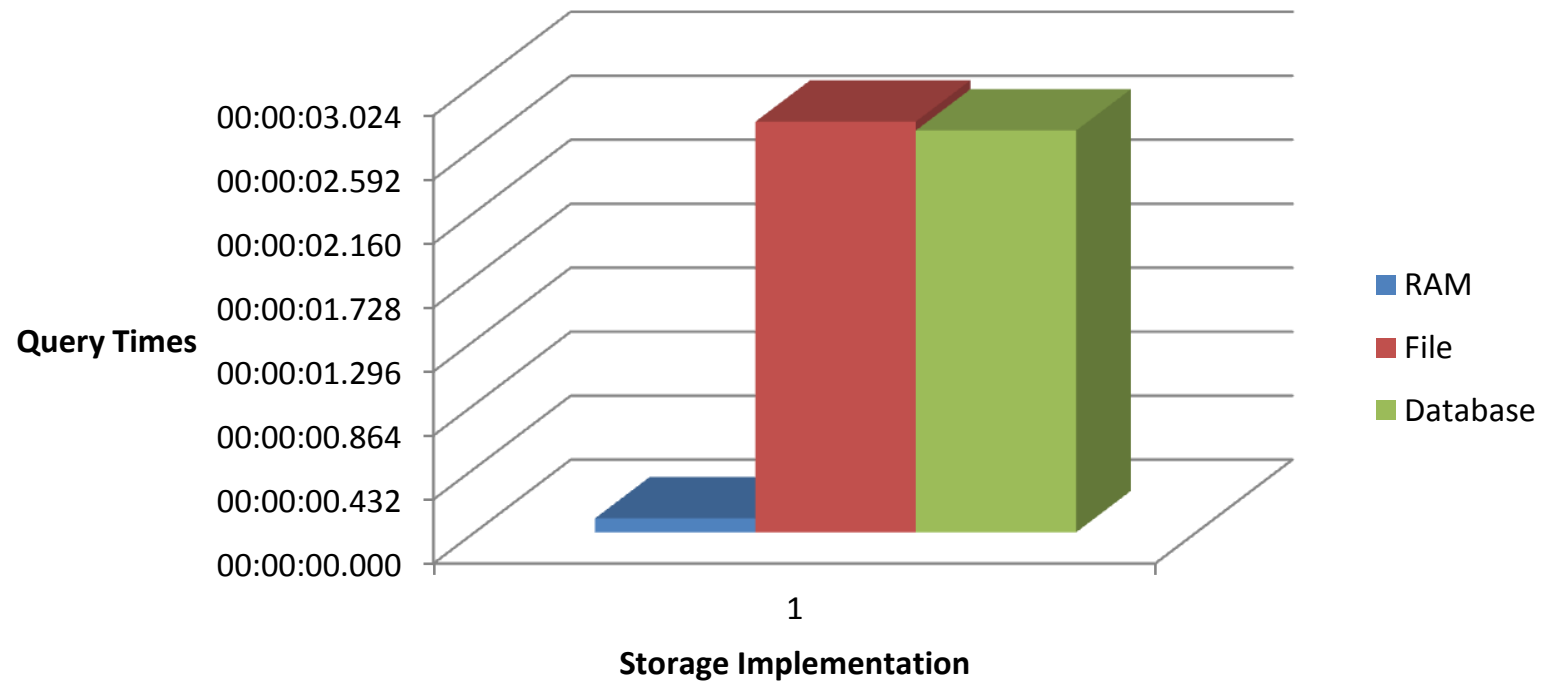

Figure 65: Query Times 


\subsubsection{Case Study Comparison}

This section combines all information into one summary for comparison purposes.

\begin{tabular}{|c|c|c|c|}
\hline Mean Avg. Precision & VSM & BM & LM \\
\hline RAM & $13.68 \%$ & $13.77 \%$ & $14.23 \%$ \\
\hline FILE & $13.52 \%$ & $13.74 \%$ & $13.45 \%$ \\
\hline Database & $11.89 \%$ & $13.94 \%$ & $13.09 \%$ \\
\hline
\end{tabular}

Table 80: MAP Case Study Two

\section{Mean Average Precision}

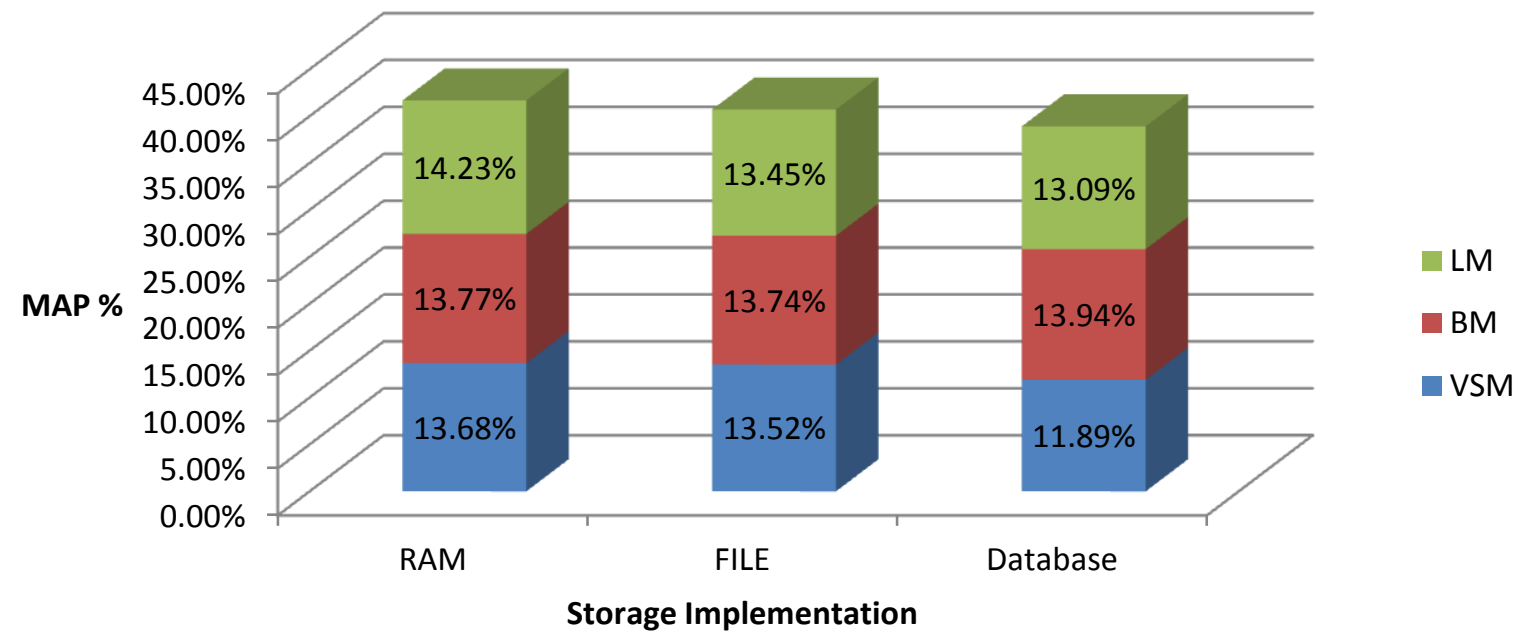

Figure 66: MAP Graph Comparison

\begin{tabular}{|c|c|c|c|}
\hline Mean Avg. Unranked Precision & VSM & BM & LM \\
\hline RAM & $11.02 \%$ & $10.56 \%$ & $11.01 \%$ \\
\hline FILE & $10.51 \%$ & $10.09 \%$ & $10.01 \%$ \\
\hline Database & $6.71 \%$ & $10.75 \%$ & $7.91 \%$ \\
\hline
\end{tabular}




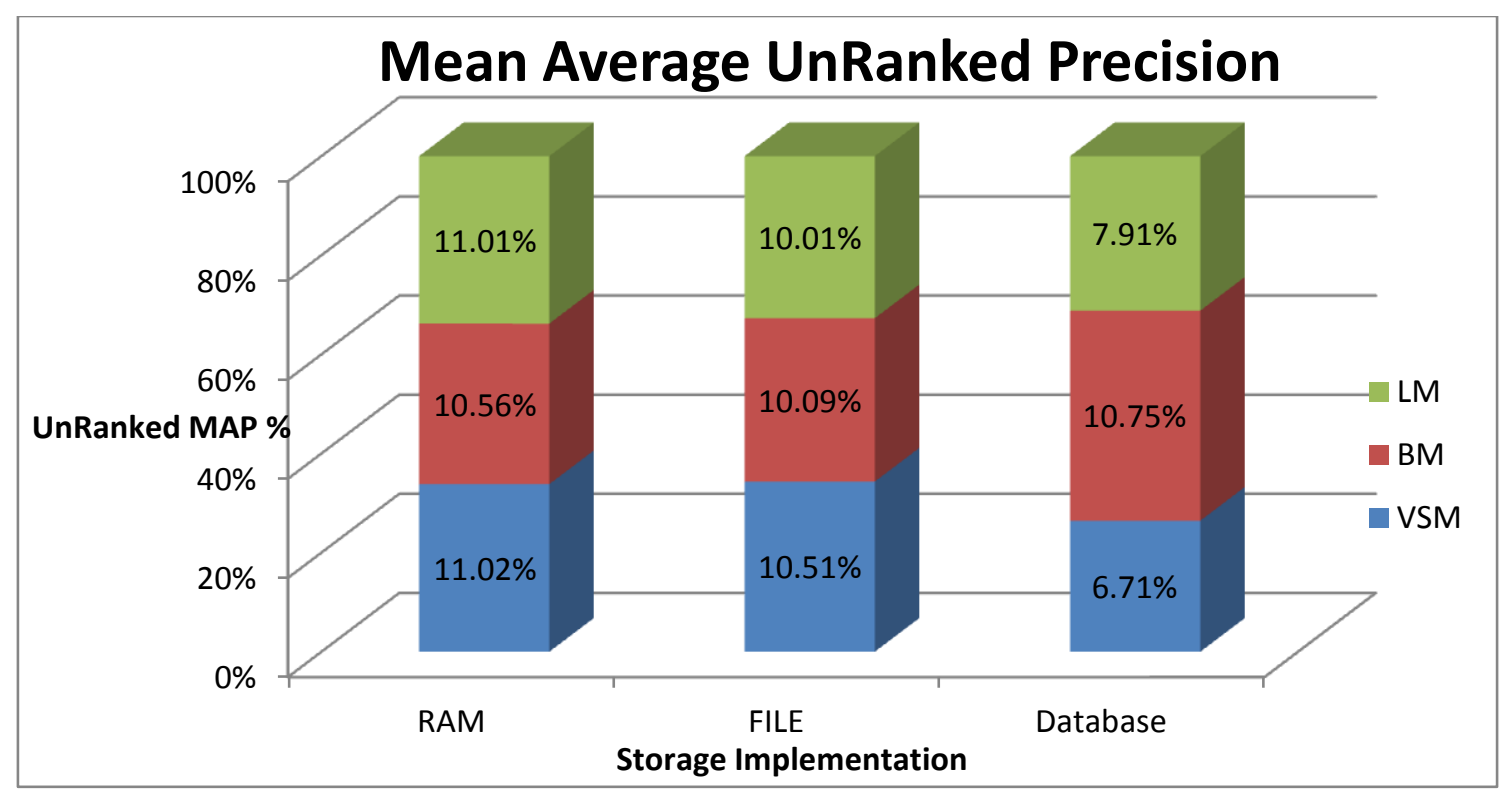

Figure 67: MAUP Graphical Comparison

\begin{tabular}{|c|c|c|c|}
\hline Recall & VSM & BM & LM \\
\hline RAM & $97.60 \%$ & $93.24 \%$ & $97.54 \%$ \\
\hline FILE & $97.60 \%$ & $93.24 \%$ & $93.60 \%$ \\
\hline Database & $97.60 \%$ & $93.83 \%$ & $94.54 \%$ \\
\hline
\end{tabular}

Table 82: Recall Comparison 


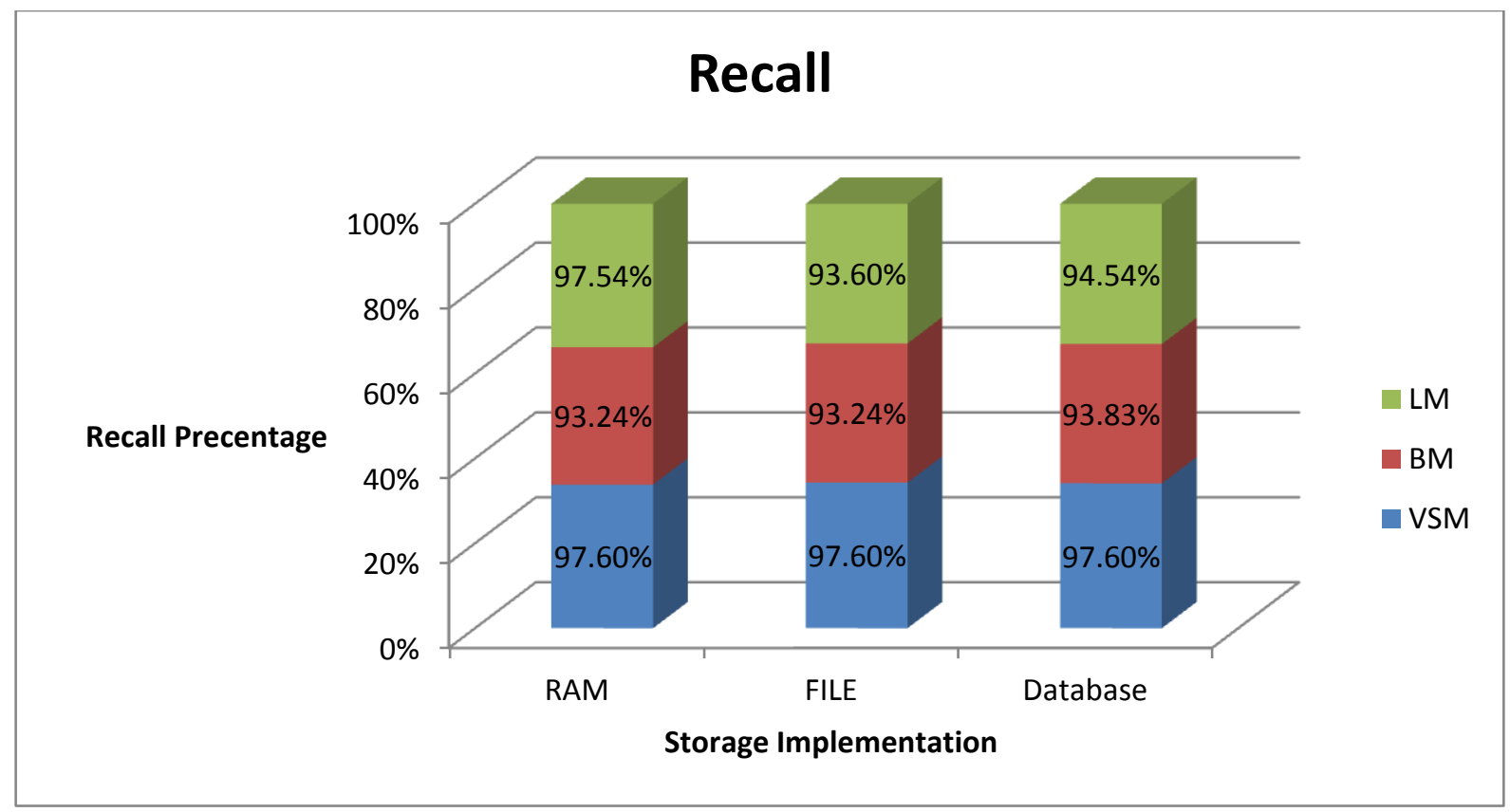

Figure 68: Recall Graphical Comparison

\begin{tabular}{|c|c|c|c|}
\hline Query Time & VSM & BM & LM \\
\hline RAM & $00: 00: 00.810$ & $00: 00: 00.053$ & $00: 00: 00.094$ \\
\hline FILE & $00: 00: 04.682$ & $00: 00: 05.391$ & $00: 00: 02.772$ \\
\hline Database & $00: 00: 00.341$ & $00: 00: 01.603$ & $00: 00: 02.712$ \\
\hline
\end{tabular}




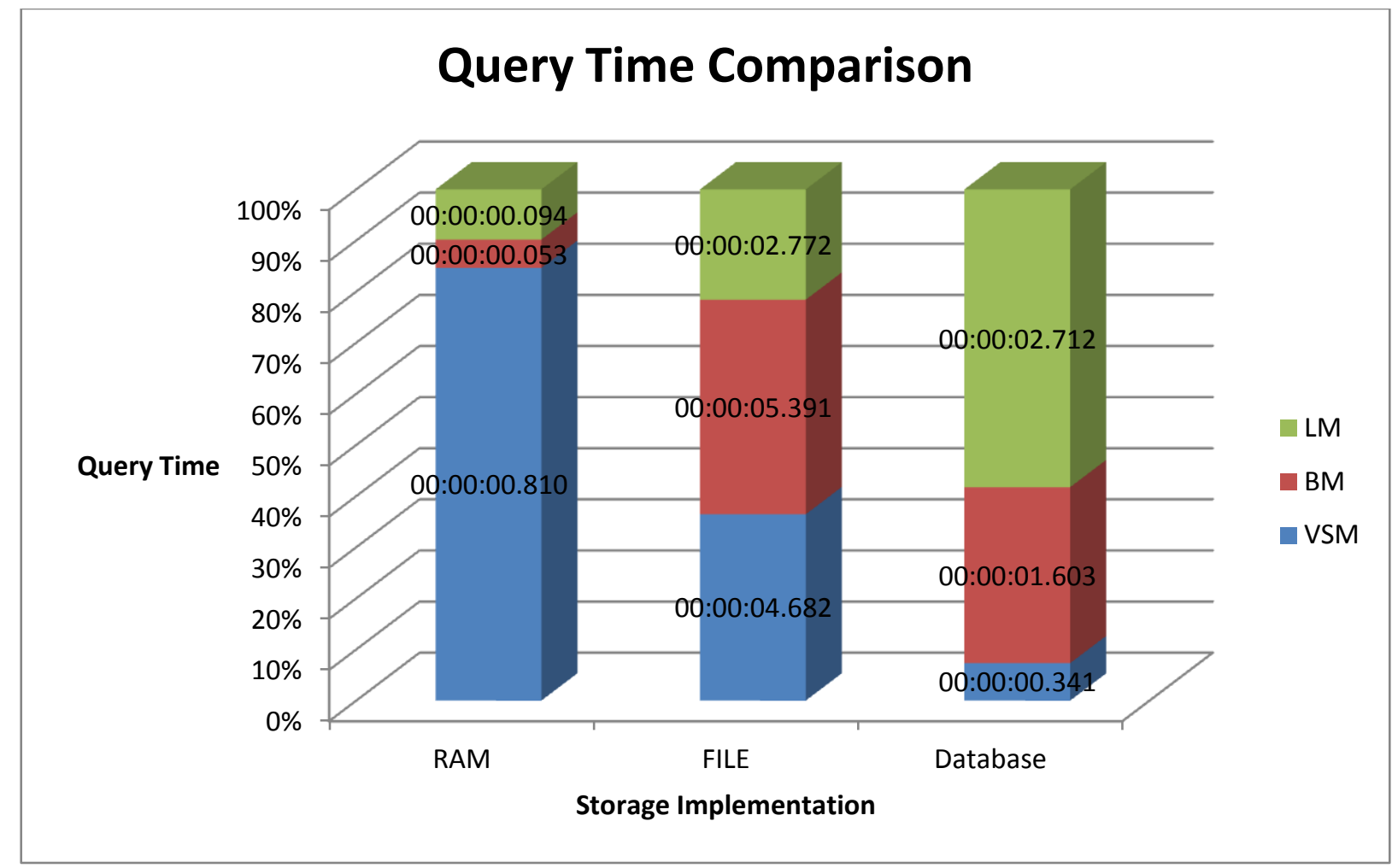

Figure 69: Query Times Comparison Graph

\begin{tabular}{|c|c|c|}
\hline VSM & Relevant & Not Relevant \\
\hline Retrieved & 2 & 183 \\
\hline Not Retrieved & 9 & 9146 \\
\hline \multicolumn{2}{|c}{} \\
\hline
\end{tabular}

\begin{tabular}{|c|c|c|}
\hline BM & Relevant & Not Relevant \\
\hline Retrieved & 2 & 172 \\
\hline Not Retrieved & 9 & 9157 \\
\hline
\end{tabular}

Table 85: Confucius Matrix BM

\begin{tabular}{|c|c|c|}
\hline LM & Relevant & Not Relevant \\
\hline Retrieved & 2 & 174 \\
\hline Not Retrieved & 9 & 9156 \\
\hline
\end{tabular}

Table 86: Confucius Matrix LM 


\subsection{Case Study Three}

The final case study is a 40000 collection subset of the Internet Movie Database (IMDB). The information below is a summary of the data collected during the case study three trials.

\section{R.A.M. Implementation with Vector Space}

The following information is the inverted index implemented in R.A.M. along using a vector space retrieval algorithm.

\begin{tabular}{|c|c|c|}
\hline & Average & Standard Deviation \\
\hline Average Query Time & $00: 00: 00.132$ & $\pm 00: 00: 00.029$ \\
\hline Mean Average Unranked Precision & $7.81 \%$ & $\pm 5.64 \%$ \\
\hline Mean Average Precision & $12.51 \%$ & $\pm 6.97 \%$ \\
\hline Mean Average Recall & $96.25 \%$ & $\pm 0.00 \%$ \\
\hline
\end{tabular}

Table 87: R.A.M. with Vector Space

\begin{tabular}{|c|c|c|}
\hline RAM & Relevant & Not Relevant \\
\hline Retrieved & 4 & 503 \\
\hline Not Retrieved & 0 & 39483 \\
\hline
\end{tabular}

Table 88: Confucius Matrix for R.A.M. 


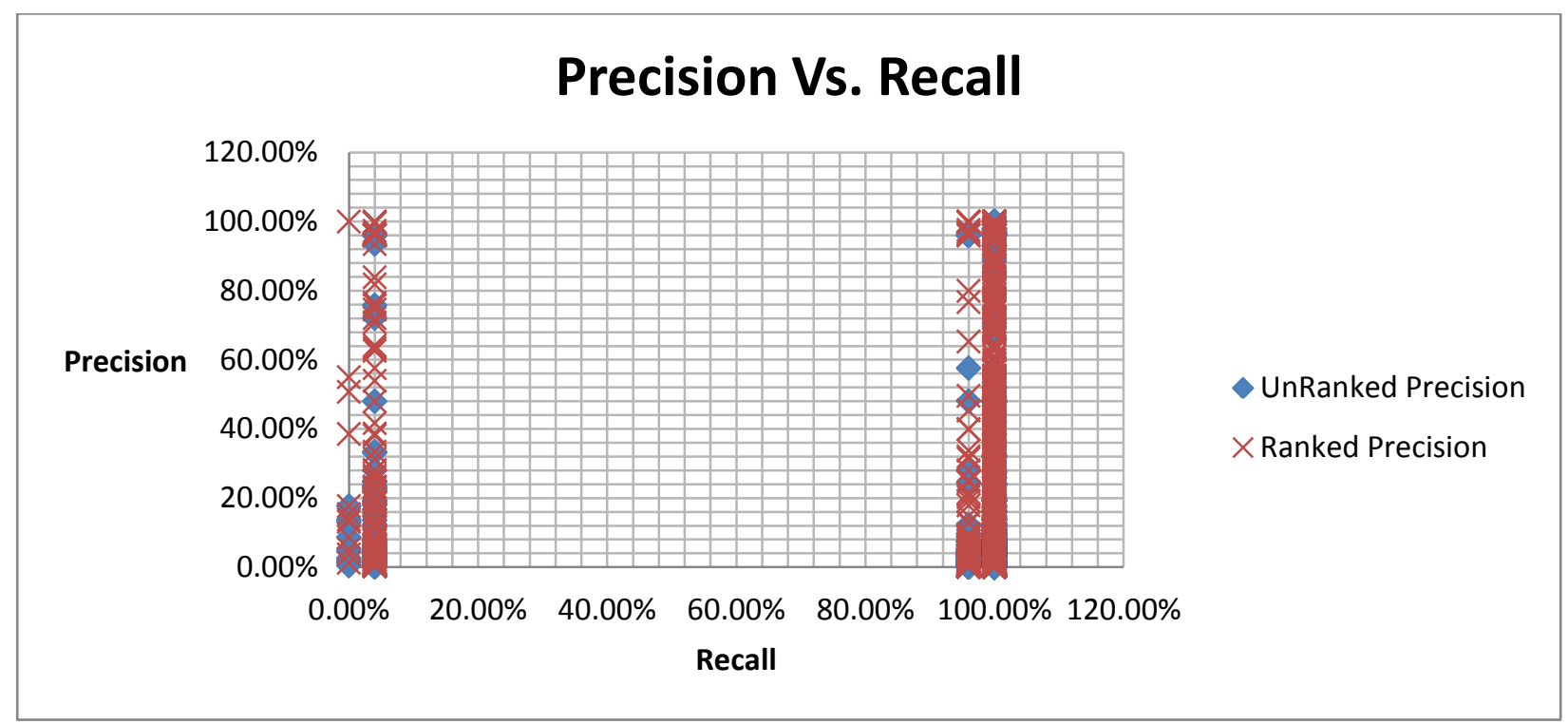

Figure 70: Precision vs. Recall

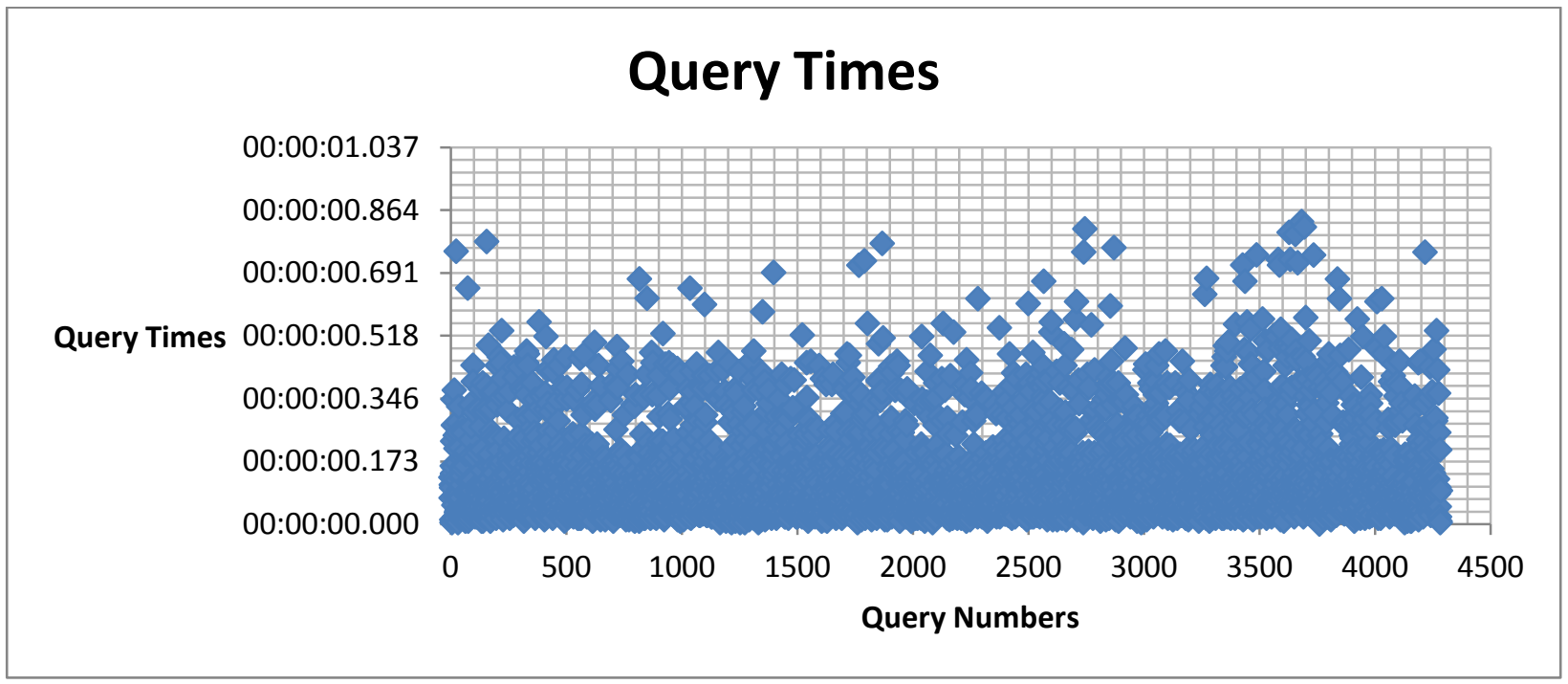

Figure 71: Query Time 


\section{File Implementation with Vector Space}

The data collected in this section summarizes the inverted index implemented in a file using a vector space retrieval strategy.

\begin{tabular}{|c|c|c|}
\hline & Average & Standard Deviation \\
\hline Average Query Time & $00: 00: 10.387$ & $\pm 00: 00: 07.039$ \\
\hline Mean Average Unranked Precision & $7.45 \%$ & $\pm 5.28 \%$ \\
\hline Mean Average Precision & $13.52 \%$ & $\pm 7.70 \%$ \\
\hline Mean Average Recall & $96.09 \%$ & $\pm 0.00 \%$ \\
\hline \multicolumn{2}{|c|}{ Table 89: File with Vector Space } \\
\hline
\end{tabular}

\begin{tabular}{|c|c|c|}
\hline File & Relevant & Not Relevant \\
\hline Retrieved & 4 & 390 \\
\hline Not Retrieved & 0 & 39596 \\
\hline
\end{tabular}

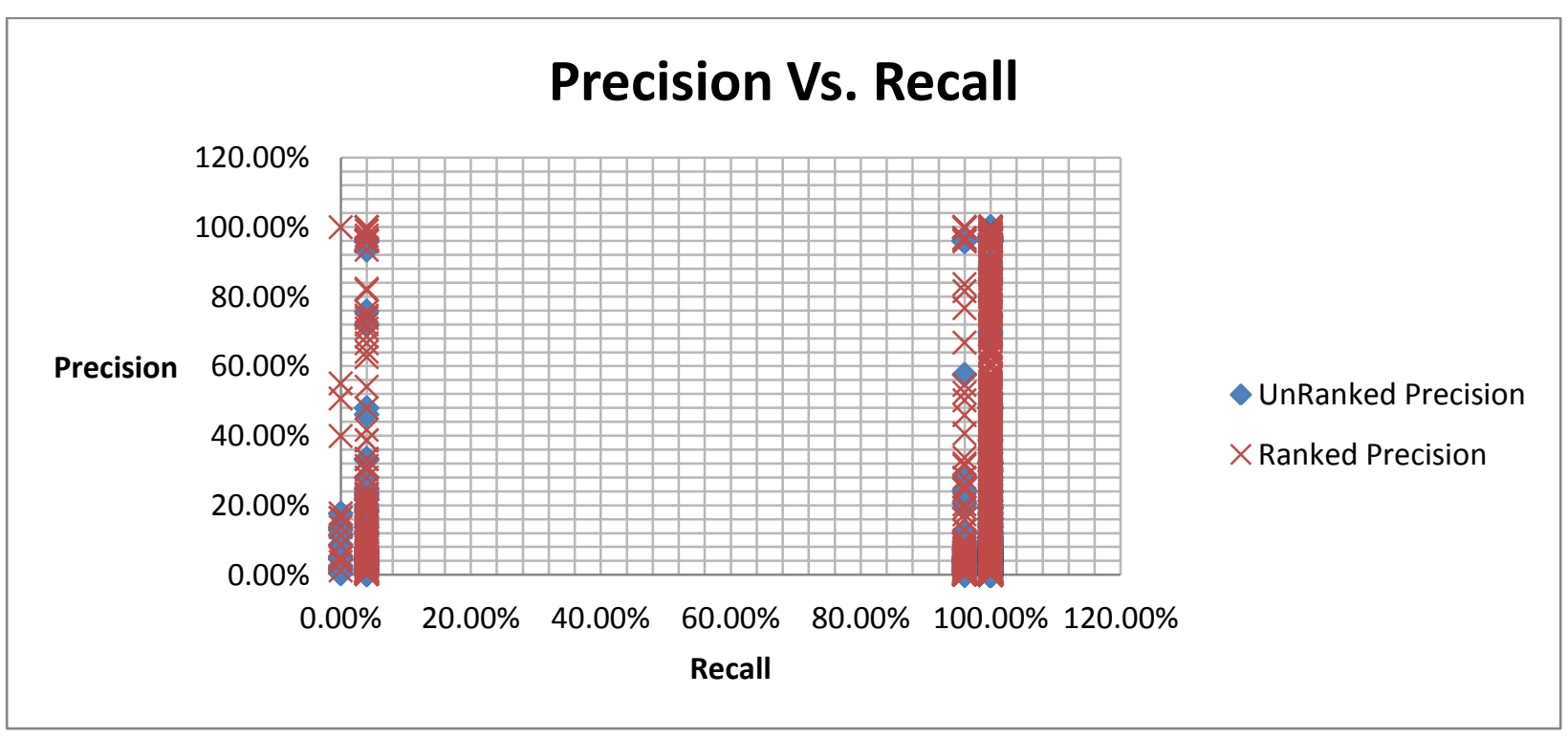

Figure 72: Precision vs. Recall 


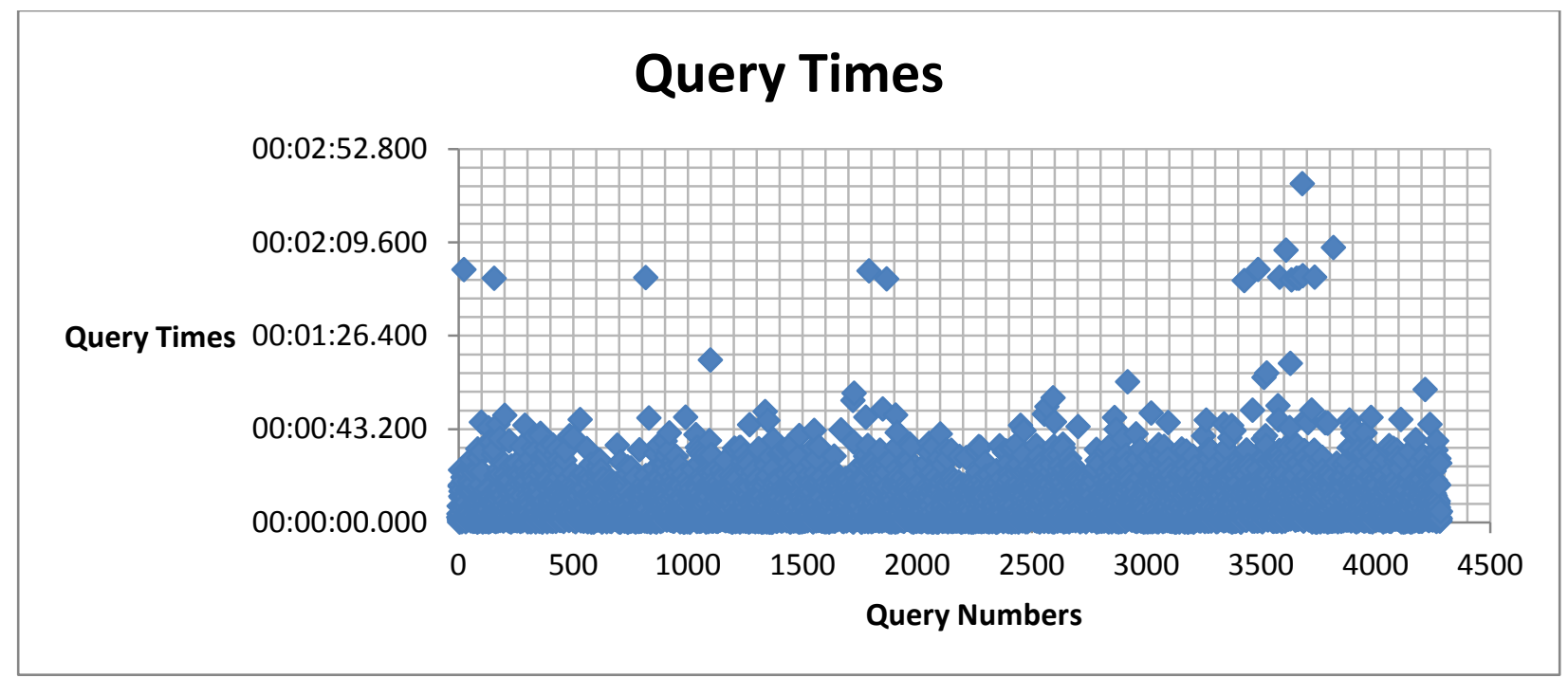

Figure 73: Query Times

\section{Database Implementation with Vector Space}

The data in this section summaries the inverted index implemented in a database using a vector space retrieval algorithm.

\begin{tabular}{|c|c|c|}
\hline & Average & Standard Deviation \\
\hline Average Query Time & $00: 00: 00.397$ & $\pm 00: 00: 00.114$ \\
\hline Mean Average Unranked Precision & $8.09 \%$ & $\pm 7.57 \%$ \\
\hline Mean Average Precision & $13.98 \%$ & $\pm 10.22 \%$ \\
\hline Mean Average Recall & $96.09 \%$ & $\pm 0.00 \%$ \\
\hline \multicolumn{2}{|r|}{ Table 91: Database with VSM } \\
\hline
\end{tabular}

\begin{tabular}{|c|c|c|}
\hline Database & Relevant & Not Relevant \\
\hline Retrieved & 4 & 390 \\
\hline Not Retrieved & 0 & 39596 \\
\hline
\end{tabular}




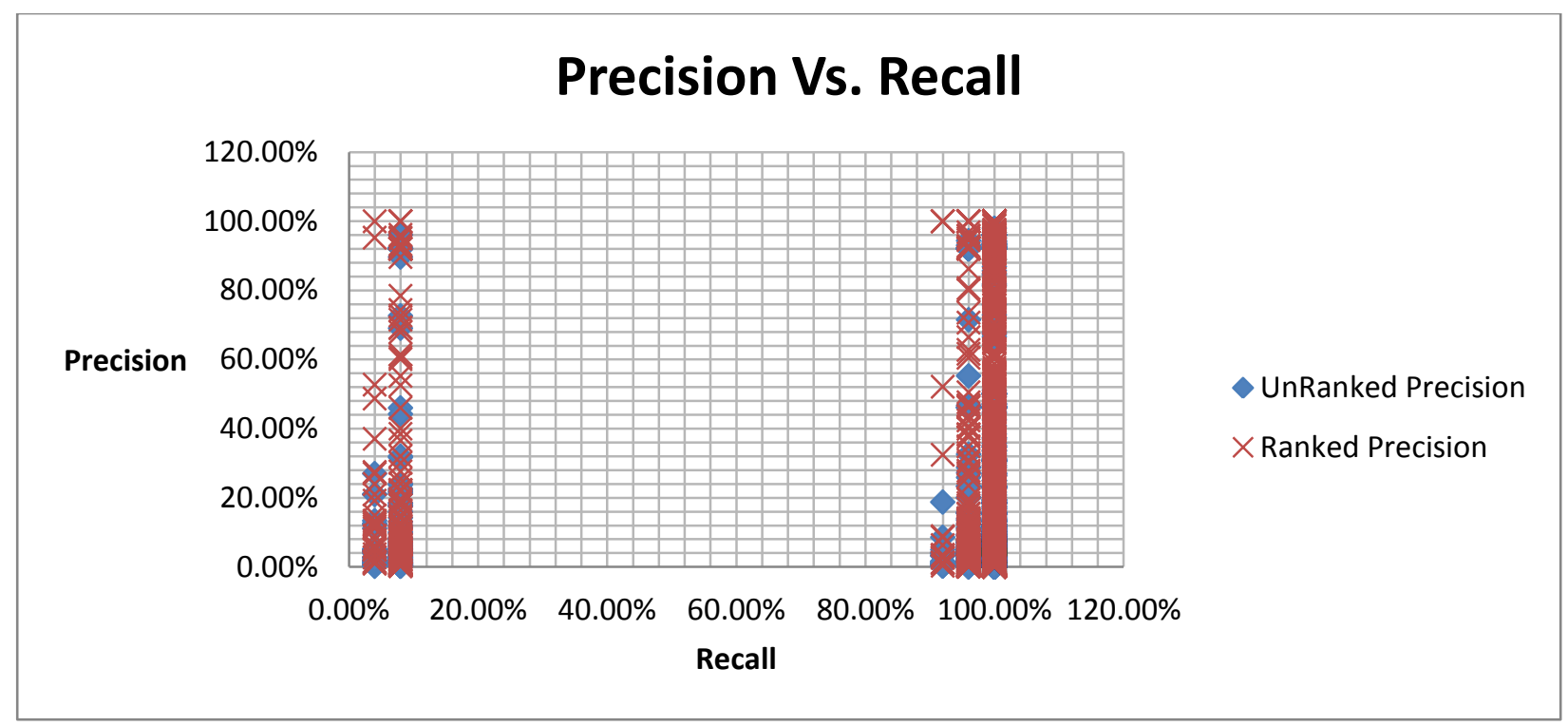

Figure 74: Precision vs. Recall

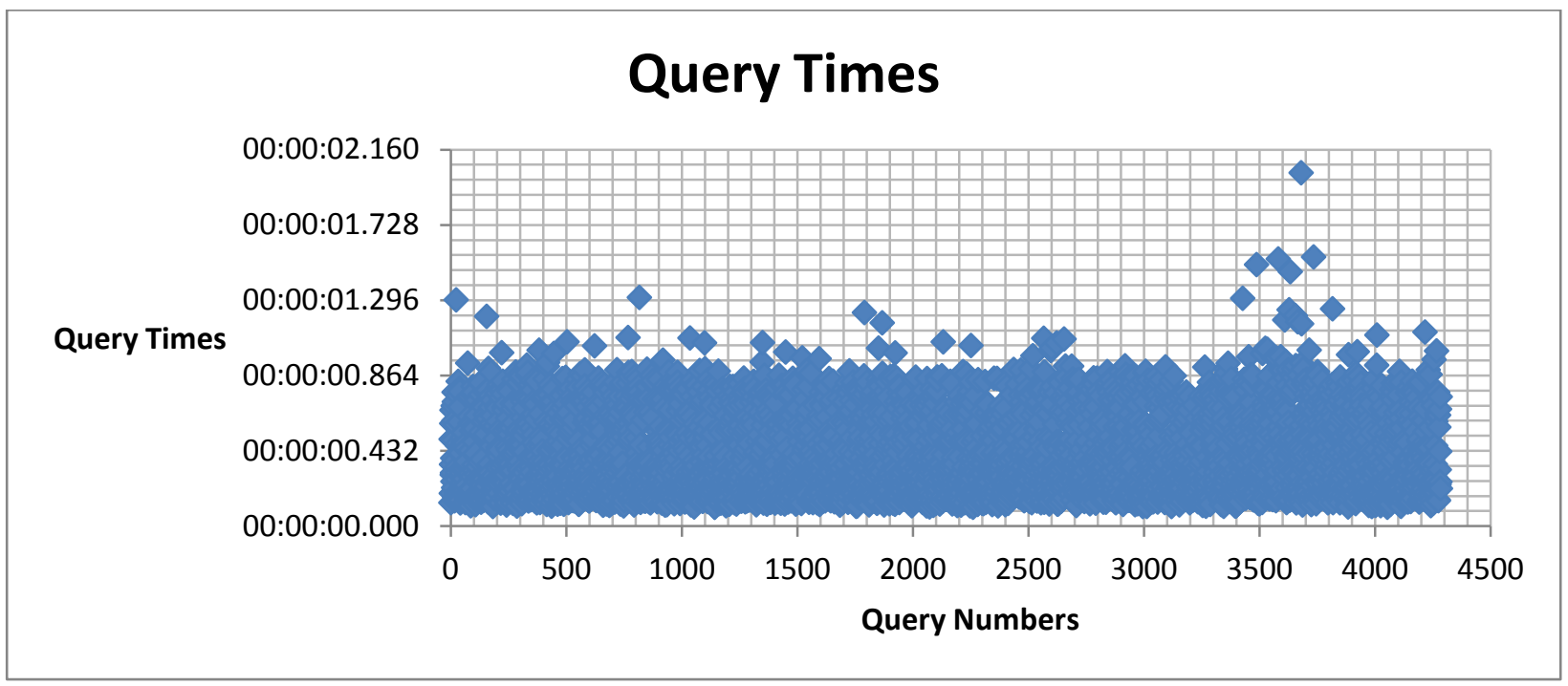

Figure 75: Query Times 


\section{R.A.M. Implementation with Best Match}

This section contains the data collected during the inverted index implemented in R.A.M. with the best match (BM) 25 algorithm.

\begin{tabular}{|c|c|c|}
\hline & Average & Standard Deviation \\
\hline Average Query Time & $00: 00: 00.083$ & $\pm 00: 00: 00.016$ \\
\hline Mean Average Unranked Precision & $8.09 \%$ & $\pm 5.65 \%$ \\
\hline Mean Average Precision & $13.57 \%$ & $\pm 7.21 \%$ \\
\hline Mean Average Recall & $96.09 \%$ & $\pm 0.00 \%$ \\
\hline
\end{tabular}

Table 93: R.A.M. with BM

\begin{tabular}{|c|c|c|}
\hline RAM & Relevant & Not Relevant \\
\hline Retrieved & 4 & 390 \\
\hline Not Retrieved & 0 & 39596 \\
\hline
\end{tabular}

Table 94: Confucius Matrix

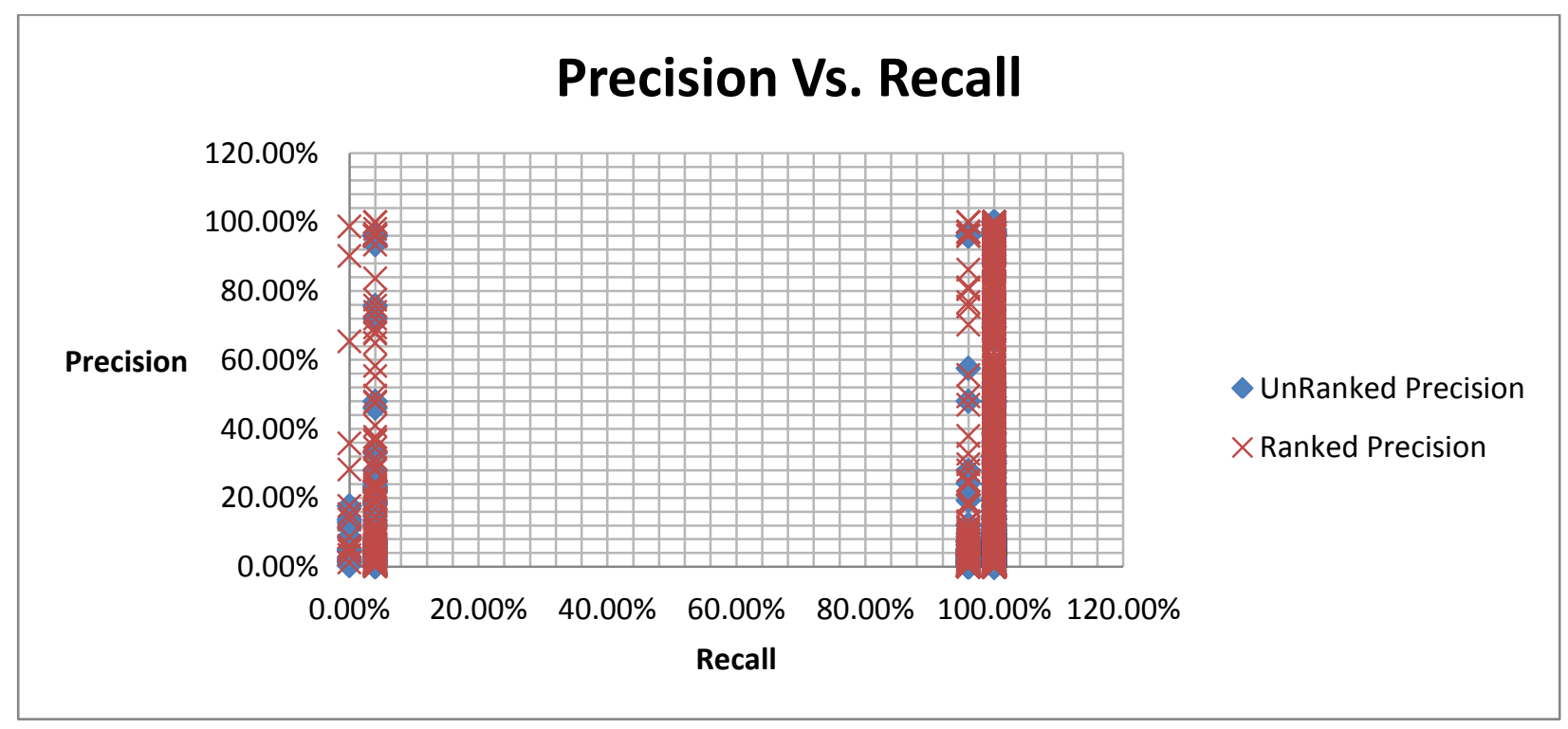

Figure 76: Precision vs. Recall 


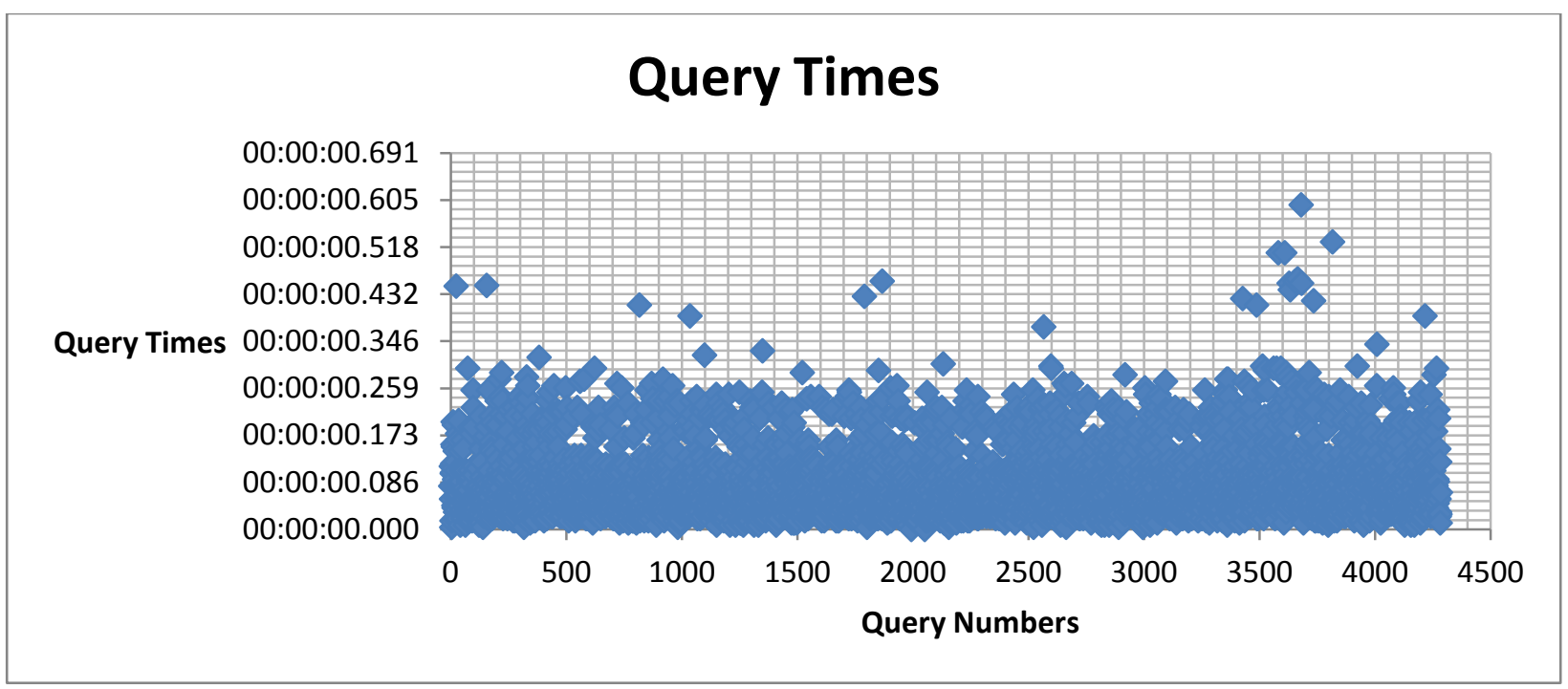

Figure 77: Precision vs. Recall

\section{File Implementation with Best Match}

The data collected in this section regards the inverted index implemented in a file with the Best Match 25 retrieval algorithm

\begin{tabular}{|c|c|c|}
\hline & Average & Standard Deviation \\
\hline Average Query Time & $00: 00: 09.151$ & $\pm 00: 00: 04.999$ \\
\hline Mean Average Unranked Precision & $7.78 \%$ & $\pm 5.44 \%$ \\
\hline Mean Average Precision & $13.39 \%$ & $\pm 7.13 \%$ \\
\hline Mean Average Recall & $96.09 \%$ & $\pm 0.00 \%$ \\
\hline
\end{tabular}

Table 95: File with BM

\begin{tabular}{|c|c|c|}
\hline File & Relevant & Not Relevant \\
\hline Retrieved & 4 & 390 \\
\hline Not Retrieved & 0 & 39596 \\
\hline
\end{tabular}




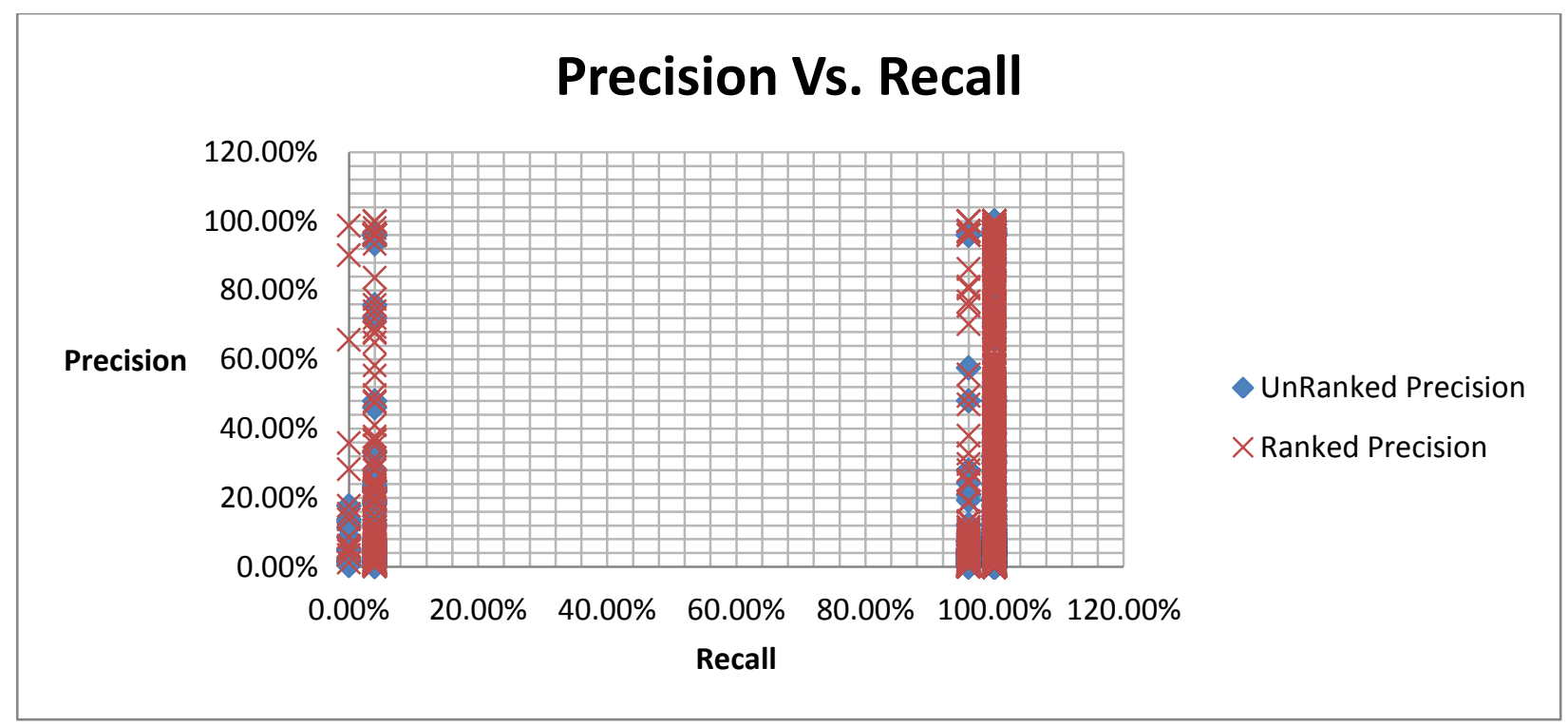

Figure 78: Precision vs. Recall

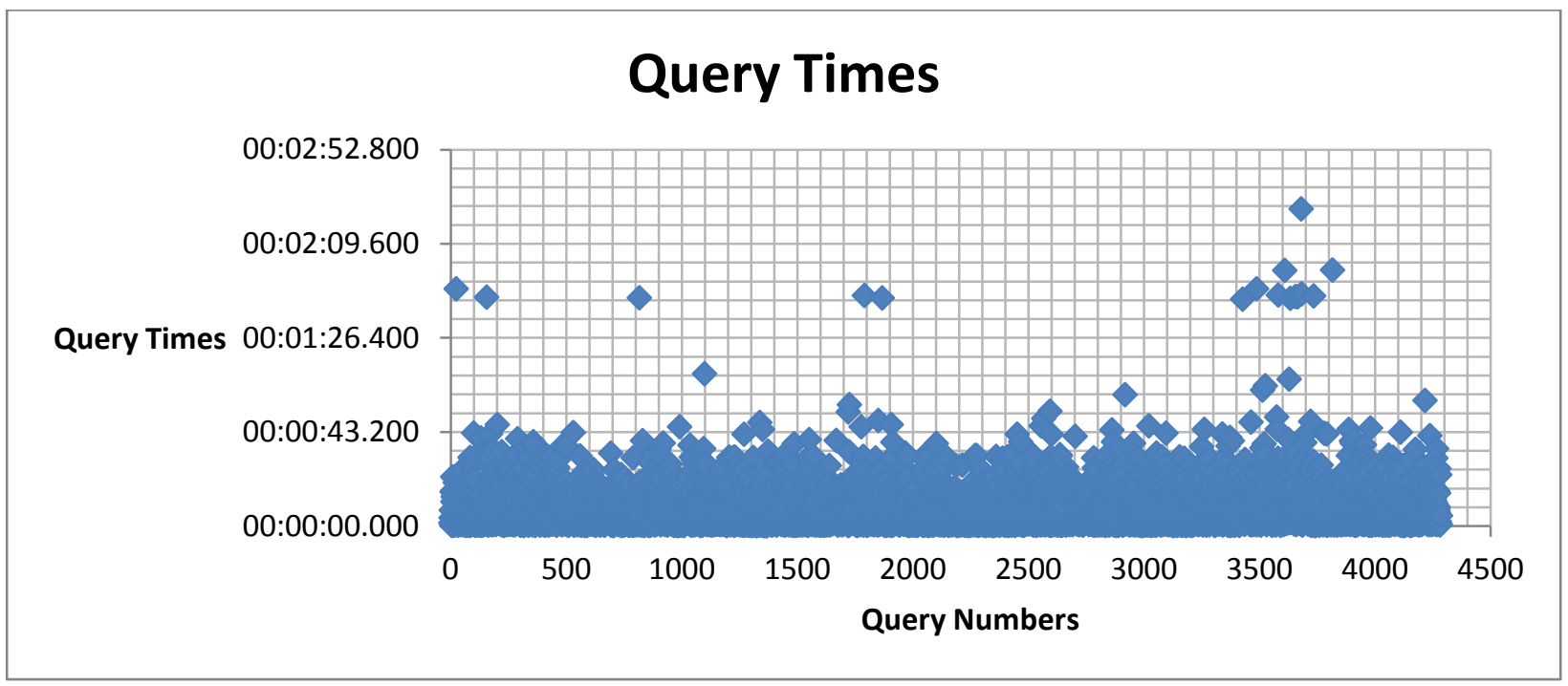

Figure 79: Query Times 


\section{Database Implementation with Best Match}

This section summarizes the data collected for an inverted index structure implemented as a table in a database with the Best Match 25 algorithm.

\begin{tabular}{|c|c|c|}
\hline & Average & Standard Deviation \\
\hline Average Query Time & $00: 00: 00.335$ & $\pm 00: 00: 00.073$ \\
\hline Mean Average Unranked Precision & $8.09 \%$ & $\pm 5.65 \%$ \\
\hline Mean Average Precision & $13.57 \%$ & $\pm 7.21 \%$ \\
\hline Mean Average Recall & $96.09 \%$ & $\pm 0.00 \%$ \\
\hline \multicolumn{2}{|c|}{ Table 97: Database with BM } & \\
\hline
\end{tabular}

\begin{tabular}{|c|c|c|}
\hline Database & Relevant & Not Relevant \\
\hline Retrieved & 4 & 390 \\
\hline Not Retrieved & 0 & 39596 \\
\hline
\end{tabular}

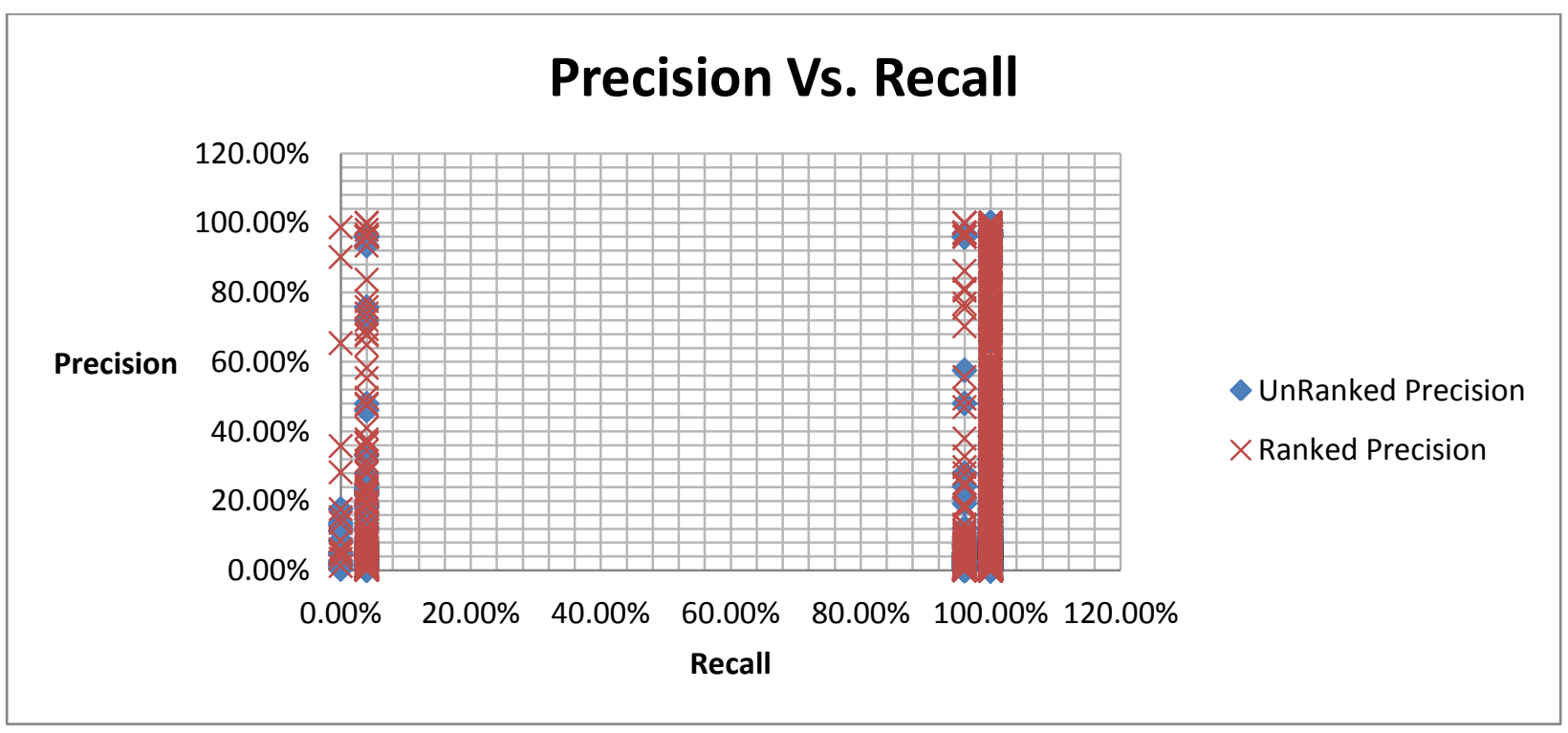

Figure 80: Precision vs. Recall 


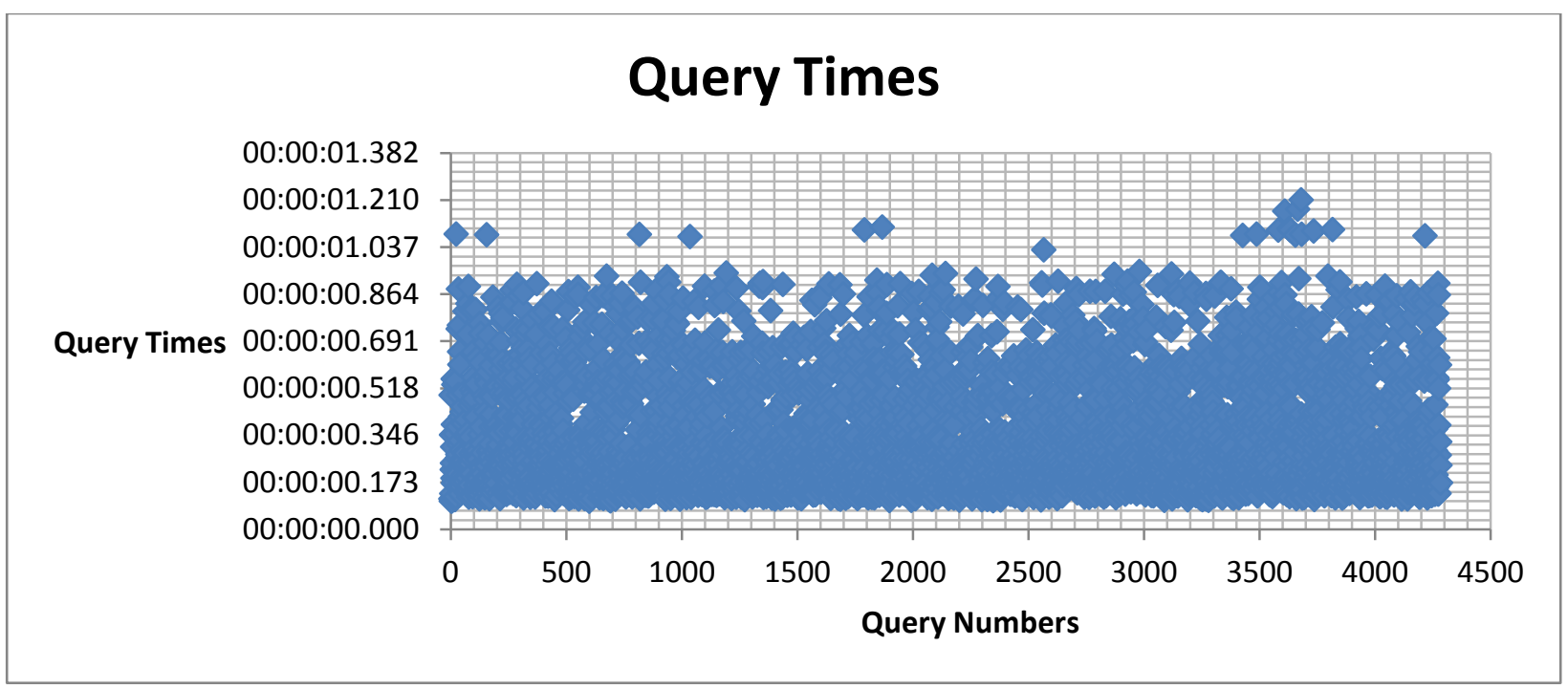

Figure 81: Query Times

\section{R.A.M. Implementation with Language Model}

The inverted index implemented in R.A.M. using a language model produced the following data.

\begin{tabular}{|c|c|c|}
\hline & Average & Standard Deviation \\
\hline Average Query Time & $00: 00: 00.115$ & $00: 00: 00.019$ \\
\hline Mean Average Unranked Precision & $8.10 \%$ & $0.00 \%$ \\
\hline Mean Average Precision & $13.52 \%$ & $0.00 \%$ \\
\hline Mean Average Recall & $96.10 \%$ & $0.00 \%$ \\
\hline
\end{tabular}

Table 99: R.A.M. with LM

\begin{tabular}{|c|c|c|}
\hline RAM & Relevant & Not Relevant \\
\hline Retrieved & 4 & 390 \\
\hline Not Retrieved & 0 & 39596 \\
\hline
\end{tabular}




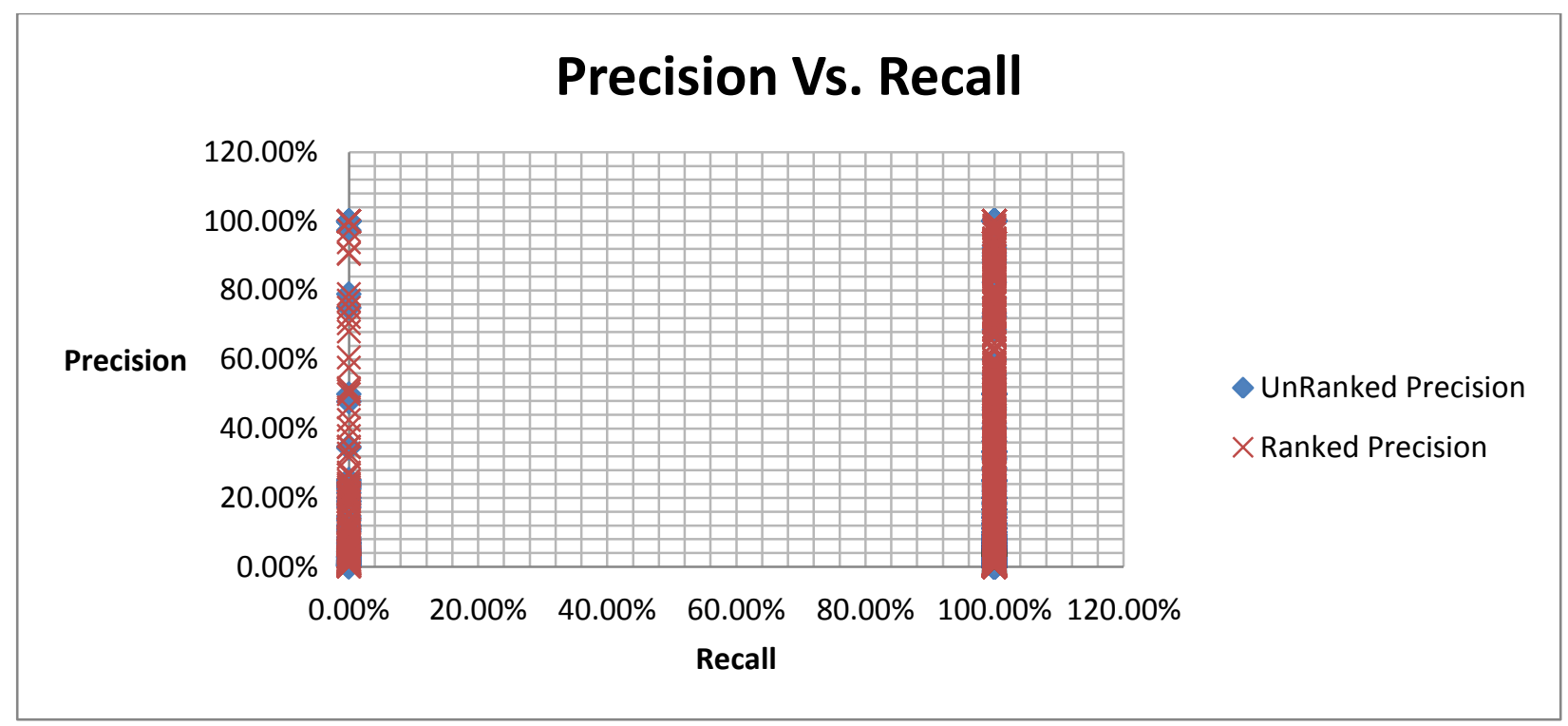

Figure 82: Precision vs. Recall

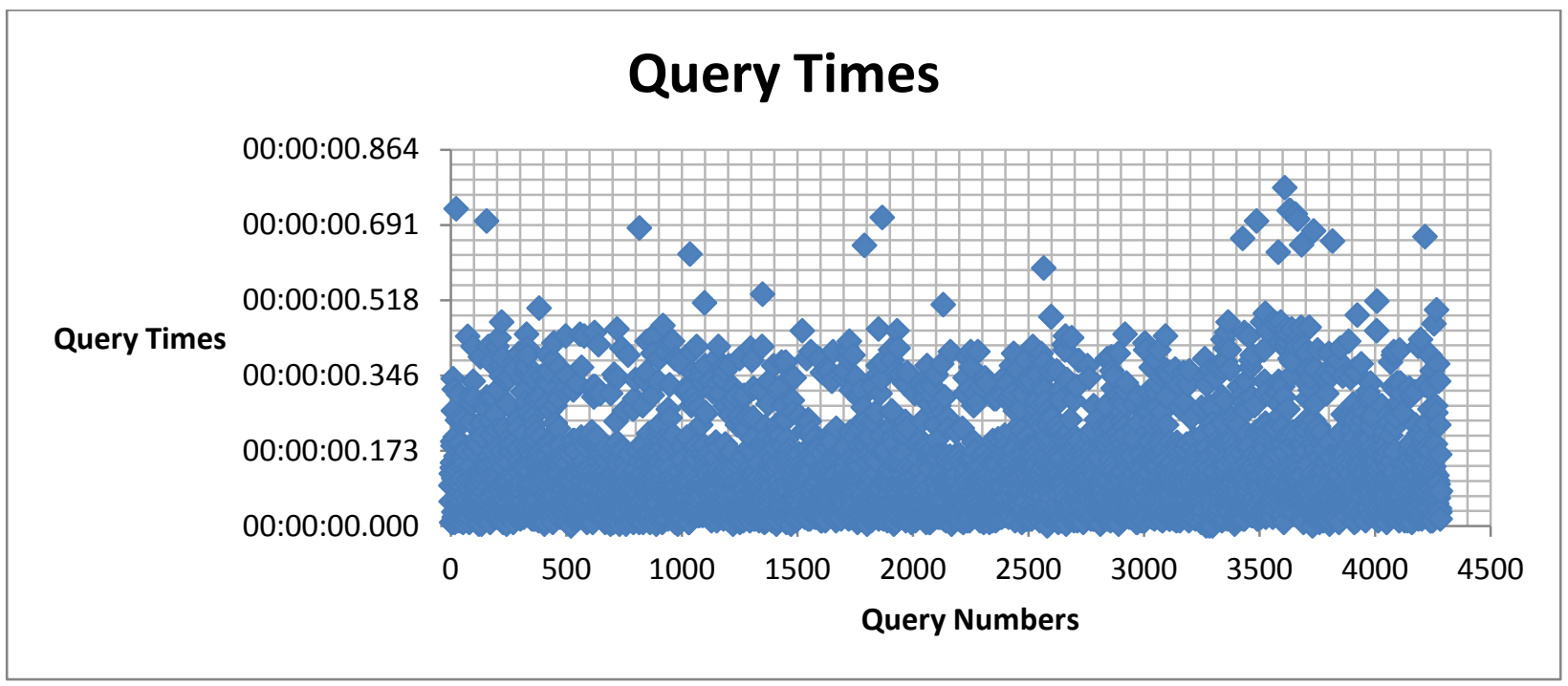

Figure 83: Query Times 


\section{File Implementation with Language Model}

The following data is a summary of the inverted index implemented in a file using a language model retrieval model.

\begin{tabular}{|c|c|c|}
\hline & Average & Standard Deviation \\
\hline Average Query Time & $00: 00: 16.348$ & $\pm 00: 00: 10.714$ \\
\hline Mean Average Unranked Precision & $7.78 \%$ & $\pm 0.00 \%$ \\
\hline Mean Average Precision & $13.25 \%$ & $\pm 0.00 \%$ \\
\hline Mean Average Recall & $96.10 \%$ & $\pm 0.00 \%$ \\
\hline
\end{tabular}

\begin{tabular}{|c|c|c|}
\hline File & Relevant & Not Relevant \\
\hline Retrieved & 4 & 390 \\
\hline Not Retrieved & 0 & 39596 \\
\hline
\end{tabular}

Table 102: Confucius Matrix

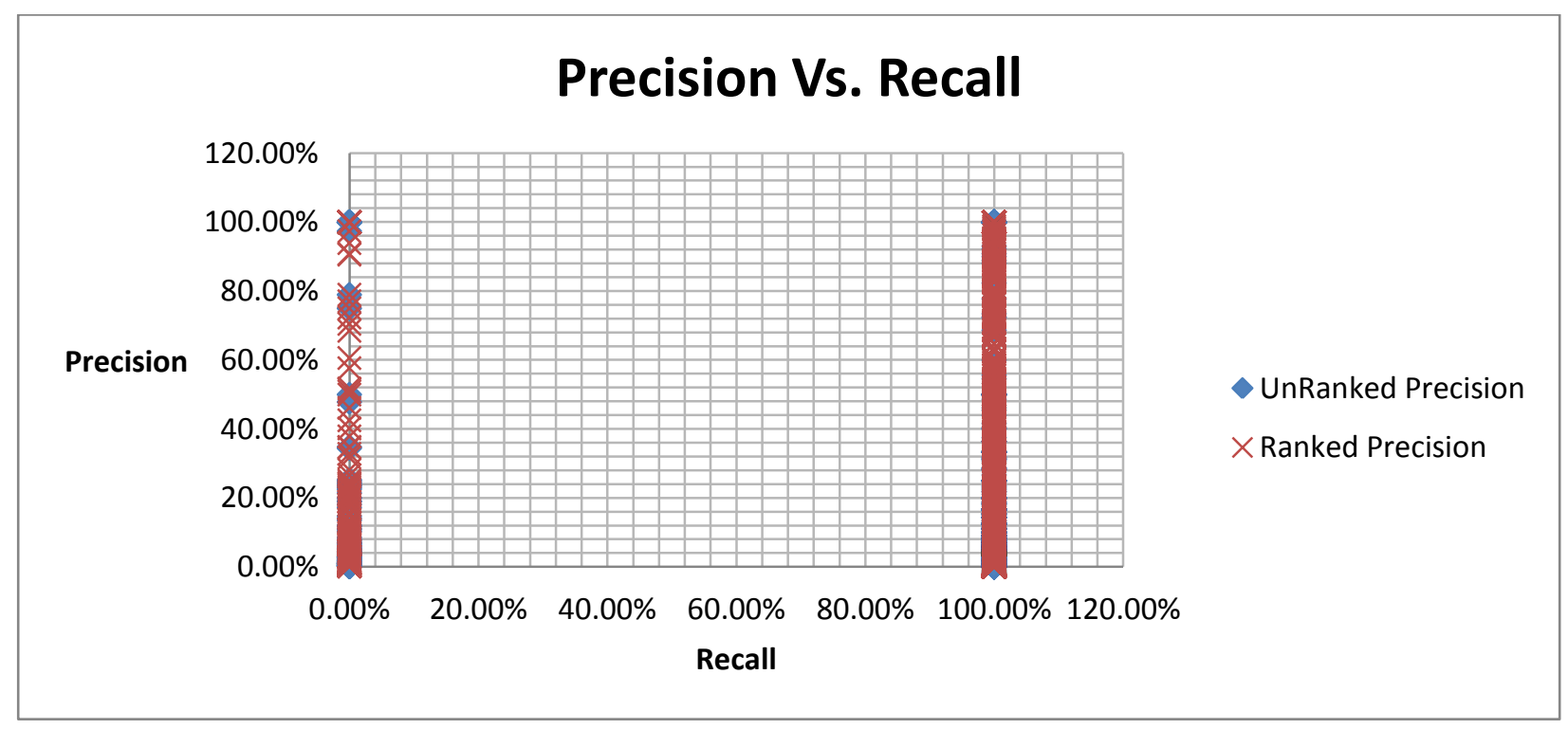

Figure 84: Precision vs. Recall 


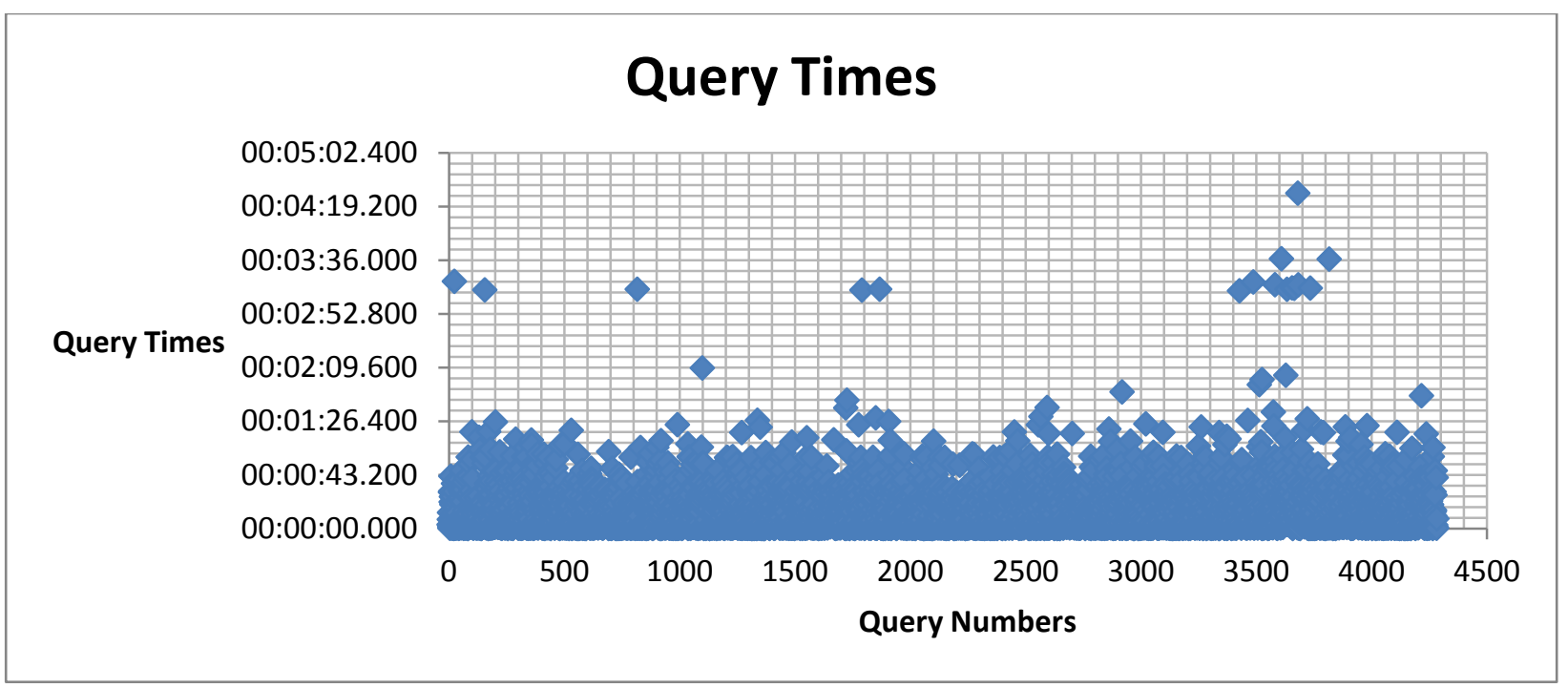

Figure 85: Query Times

\section{Database Implementation with Language Model}

The data in this section is a summary of the inverted index implemented in the database using a language model algorithm.

\begin{tabular}{|c|c|c|}
\hline & Average & Standard Deviation \\
\hline Average Query Time & $00: 00: 00.489$ & $\pm 00: 00: 00.130$ \\
\hline Mean Average Unranked Precision & $8.10 \%$ & $\pm 0.00 \%$ \\
\hline Mean Average Precision & $13.52 \%$ & $\pm 0.00 \%$ \\
\hline Mean Average Recall & $96.10 \%$ & $\pm 0.00 \%$ \\
\hline
\end{tabular}

\begin{tabular}{|c|c|c|}
\hline Database & Relevant & Not Relevant \\
\hline Retrieved & 4 & 390 \\
\hline Not Retrieved & 0 & 39596 \\
\hline
\end{tabular}




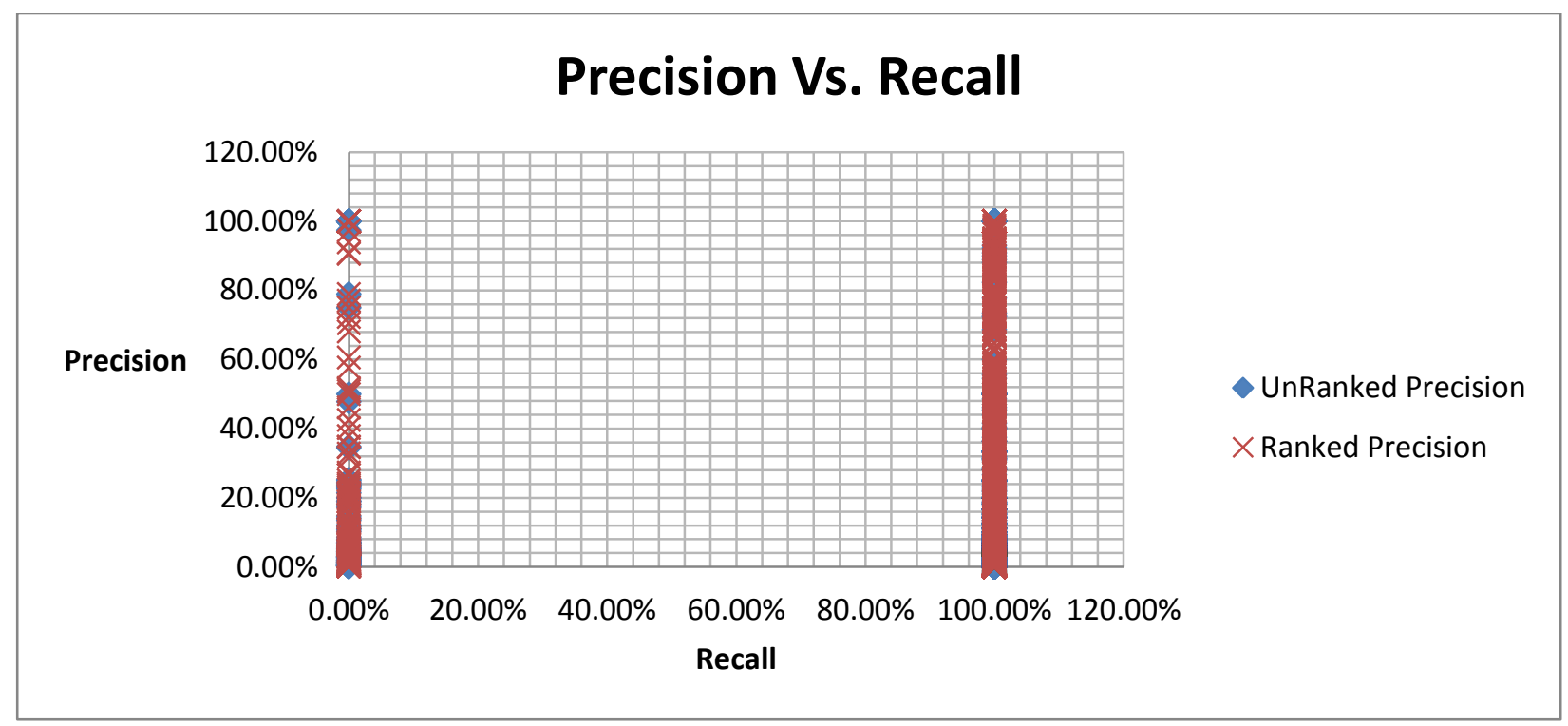

Figure 86: Precision vs. Recall

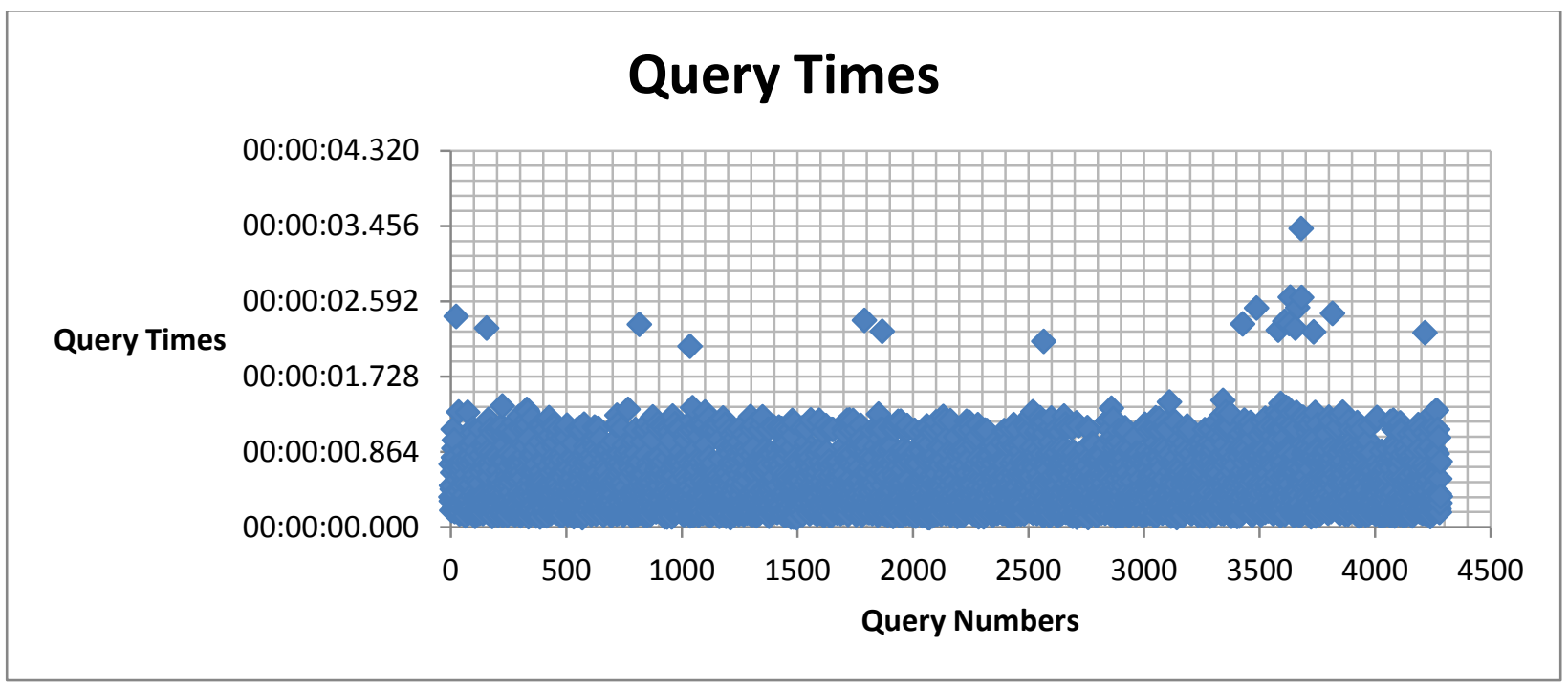

Figure 87: Query Times 


\subsubsection{Vector Space Comparison}

This section will compare all the vector space model data from case study three into one table.

\begin{tabular}{|c|c|c|c|c|}
\hline Storage & $\begin{array}{c}\text { Average Query } \\
\text { Time }\end{array}$ & $\begin{array}{c}\text { Mean Average } \\
\text { Unranked Precision }\end{array}$ & $\begin{array}{c}\text { Mean Average } \\
\text { Ranked } \\
\text { Precision }\end{array}$ & $\begin{array}{c}\text { Average } \\
\text { Recall }\end{array}$ \\
\hline RAM & $00: 00: 00.132$ & $7.81 \%$ & $12.51 \%$ & $96.25 \%$ \\
\hline File & $00: 00: 10.387$ & $7.45 \%$ & $13.52 \%$ & $96.09 \%$ \\
\hline Database & $00: 00: 00.397$ & $8.09 \%$ & $13.98 \%$ & $96.09 \%$ \\
\hline
\end{tabular}

\begin{tabular}{|c|c|c|}
\hline RAM & Relevant & Not Relevant \\
\hline Retrieved & 4 & 503 \\
\hline Not Retrieved & 0 & 39483 \\
\hline
\end{tabular}

\begin{tabular}{|c|c|c|}
\hline File & Relevant & Not Relevant \\
\hline Retrieved & 4 & 390 \\
\hline Not Retrieved & 0 & 39596 \\
\hline
\end{tabular}

\begin{tabular}{|c|c|c|}
\hline Database & Relevant & Not Relevant \\
\hline Retrieved & 4 & 390 \\
\hline Not Retrieved & 0 & 39596 \\
\hline
\end{tabular}

Table 108: Confucius Matrix Database 


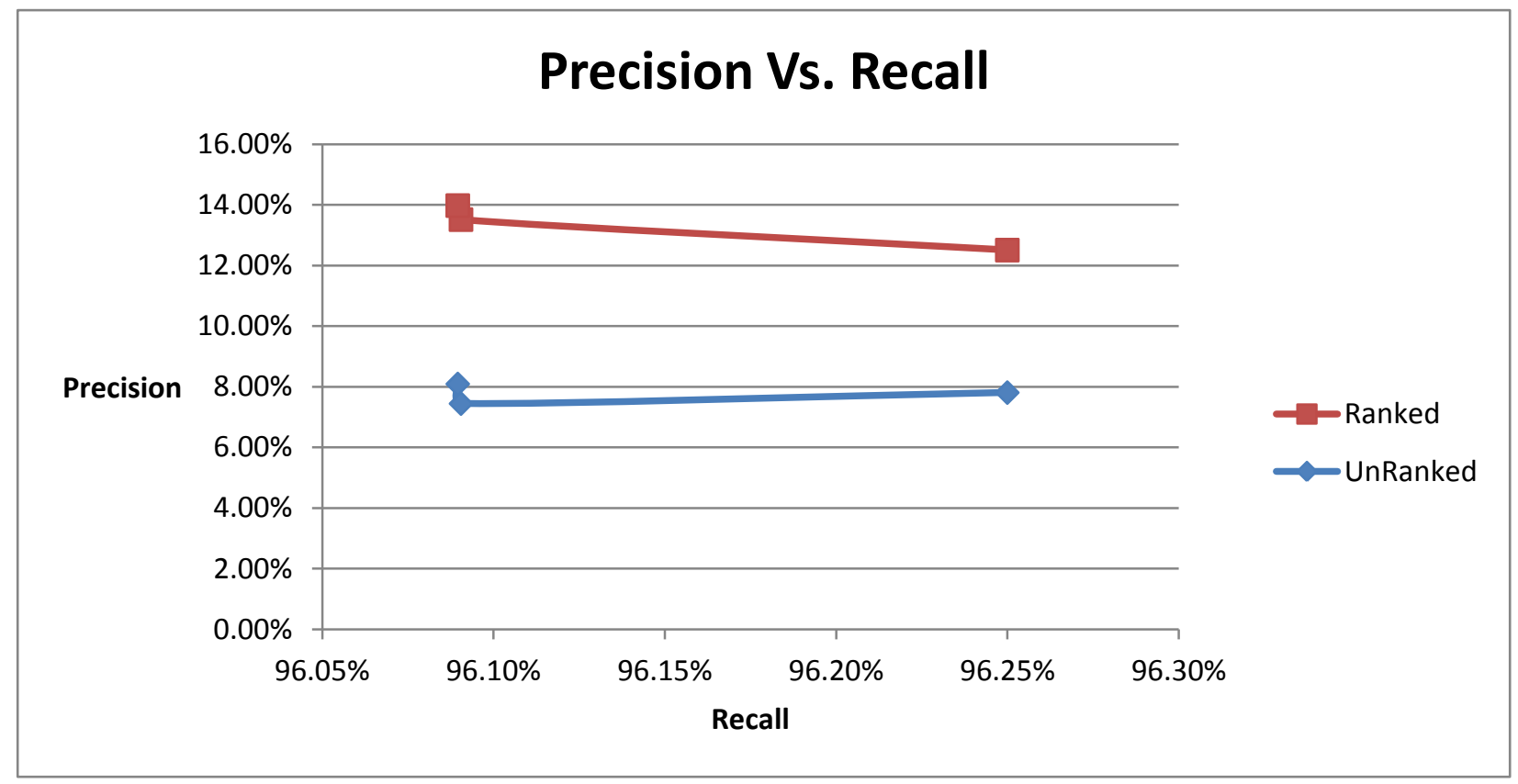

Figure 88: Precision vs. Recall

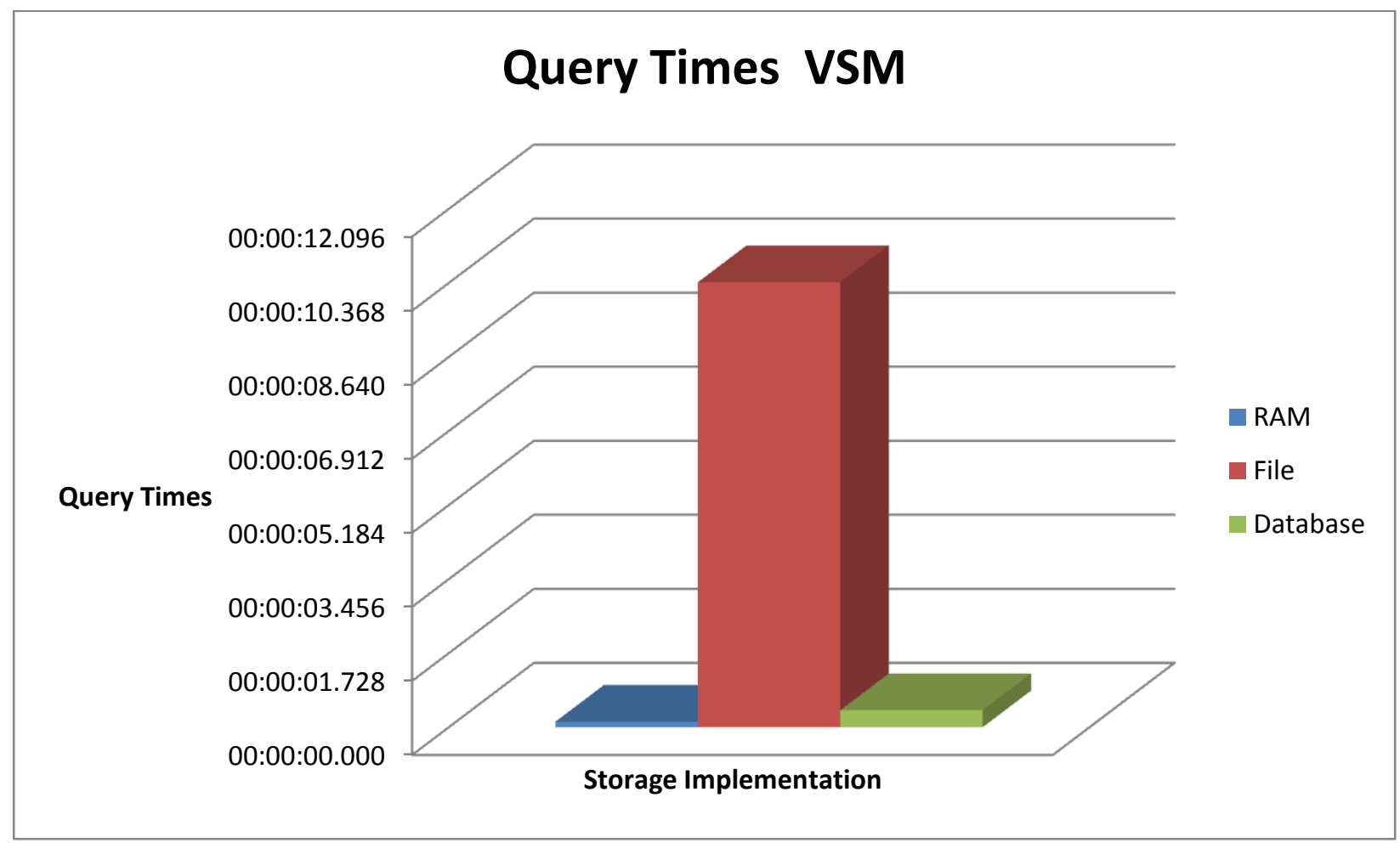

Figure 89: Query Times 


\subsubsection{Best Match Comparison}

This section compares the best match 25 results of case study three.

\begin{tabular}{|c|c|c|c|c|}
\hline Storage & Average Query Time & $\begin{array}{c}\text { Mean Average } \\
\text { Unranked } \\
\text { Precision }\end{array}$ & $\begin{array}{c}\text { Mean Average } \\
\text { Ranked Precision }\end{array}$ & $\begin{array}{c}\text { Average } \\
\text { Recall }\end{array}$ \\
\hline RAM & $00: 00: 00.083$ & $5.65 \%$ & $7.21 \%$ & $96.09 \%$ \\
\hline File & $00: 00: 09.151$ & $7.78 \%$ & $13.39 \%$ & $96.09 \%$ \\
\hline Database & $00: 00: 00.335$ & $8.09 \%$ & $13.57 \%$ & $96.09 \%$ \\
\hline \multicolumn{4}{|c|}{ Table 109: BM Summary } \\
\hline
\end{tabular}

\begin{tabular}{|c|c|c|}
\hline RAM & Relevant & Not Relevant \\
\hline Retrieved & 4 & 390 \\
\hline Not Retrieved & 0 & 39596 \\
\hline
\end{tabular}

\begin{tabular}{|c|c|c|}
\hline RAM & Relevant & Not Relevant \\
\hline Retrieved & 4 & 390 \\
\hline Not Retrieved & 0 & 39596 \\
\hline
\end{tabular}

\begin{tabular}{|c|c|c|}
\hline Database & Relevant & Not Relevant \\
\hline Retrieved & 4 & 390 \\
\hline Not Retrieved & 0 & 39596 \\
\hline
\end{tabular}

Table 112: Confucius Matrix 


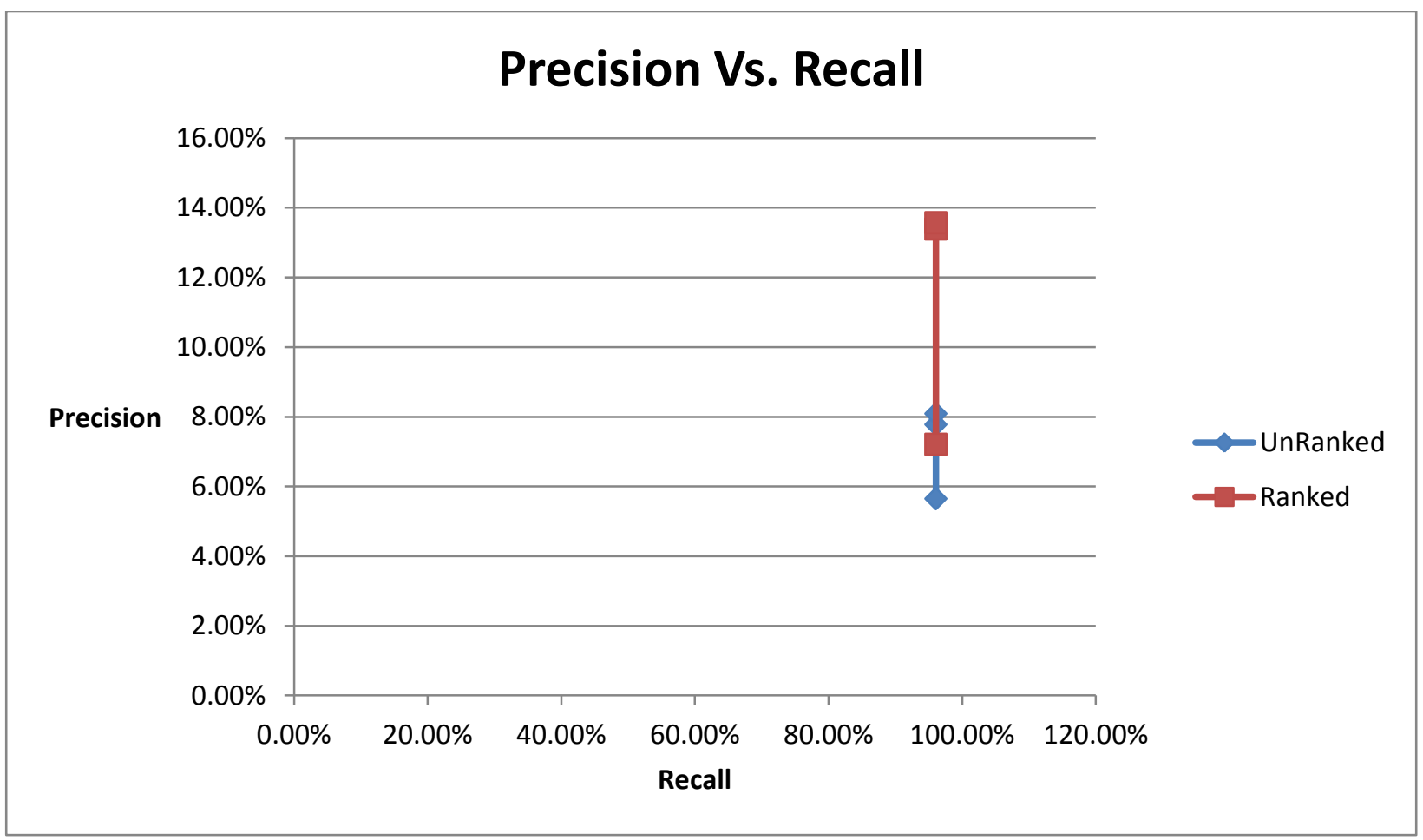

Figure 90: Precision vs. Recall

\section{Query Times BM}

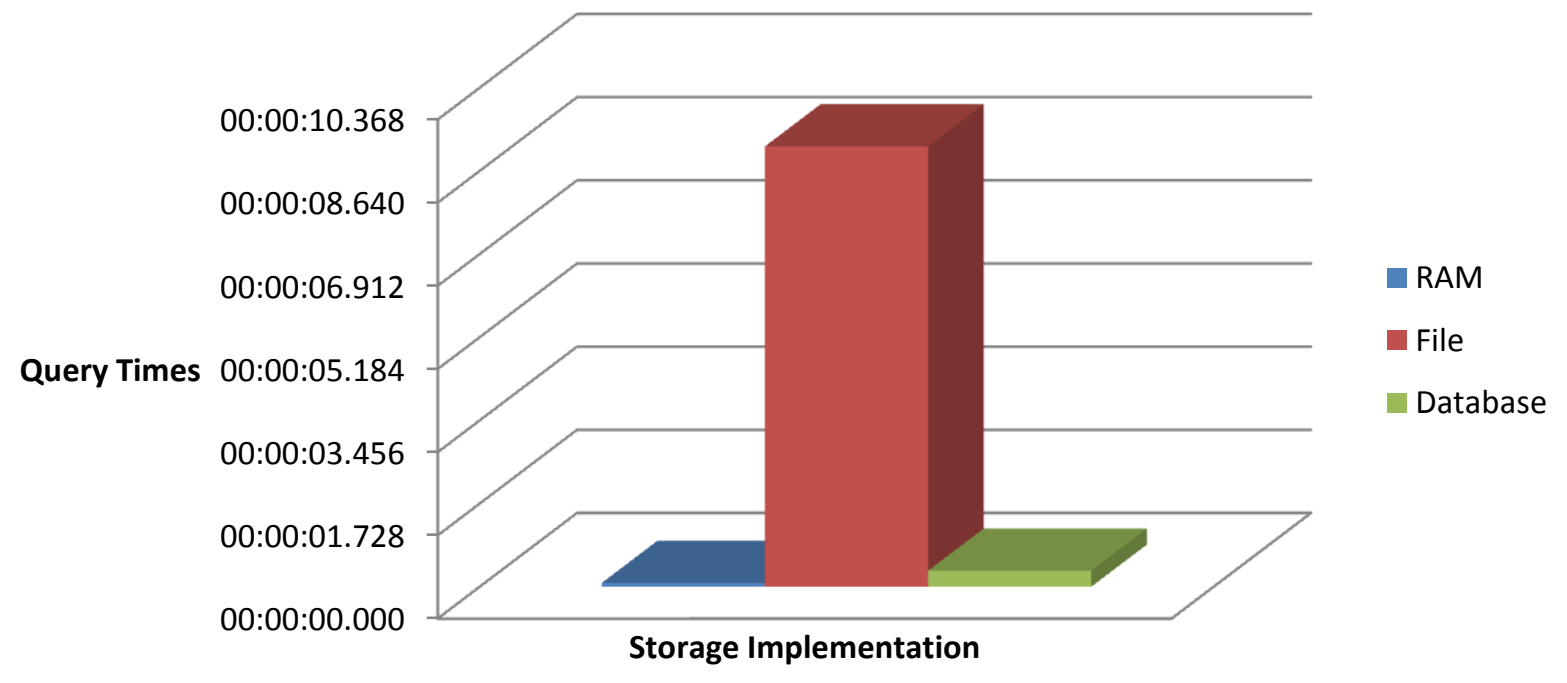

Figure 91: Query Times 


\subsubsection{Language Model Comparison}

This section compares all language model data collected during the case study three trials.

\begin{tabular}{|c|c|c|c|c|}
\hline Storage & $\begin{array}{c}\text { Average Query } \\
\text { Time }\end{array}$ & $\begin{array}{c}\text { Average } \\
\text { Unranked } \\
\text { Precision }\end{array}$ & $\begin{array}{c}\text { Mean } \\
\text { Average } \\
\text { Ranked } \\
\text { Precision }\end{array}$ & $\begin{array}{c}\text { Average } \\
\text { Recall }\end{array}$ \\
\hline RAM & $00: 00: 00.115$ & $8.10 \%$ & $13.52 \%$ & $96.10 \%$ \\
\hline File & $00: 00: 16.348$ & $7.78 \%$ & $13.25 \%$ & $96.10 \%$ \\
\hline Database & $00: 00: 00.489$ & $8.10 \%$ & $13.52 \%$ & $96.10 \%$ \\
\hline
\end{tabular}

\begin{tabular}{|c|c|c|}
\hline RAM & Relevant & Not Relevant \\
\hline Retrieved & 4 & 390 \\
\hline Not Retrieved & 0 & 39596 \\
\hline
\end{tabular}

Table 114: Confucius Matrix RAM

\begin{tabular}{|c|c|c|}
\hline File & Relevant & Not Relevant \\
\hline Retrieved & 4 & 390 \\
\hline Not Retrieved & 0 & 39596 \\
\hline
\end{tabular}

Table 115: Confucius Matrix File

\begin{tabular}{|c|c|c|}
\hline Database & Relevant & Not Relevant \\
\hline Retrieved & 4 & 390 \\
\hline Not Retrieved & 0 & 39596 \\
\hline
\end{tabular}

Table 116: Confucius Matrix Database 


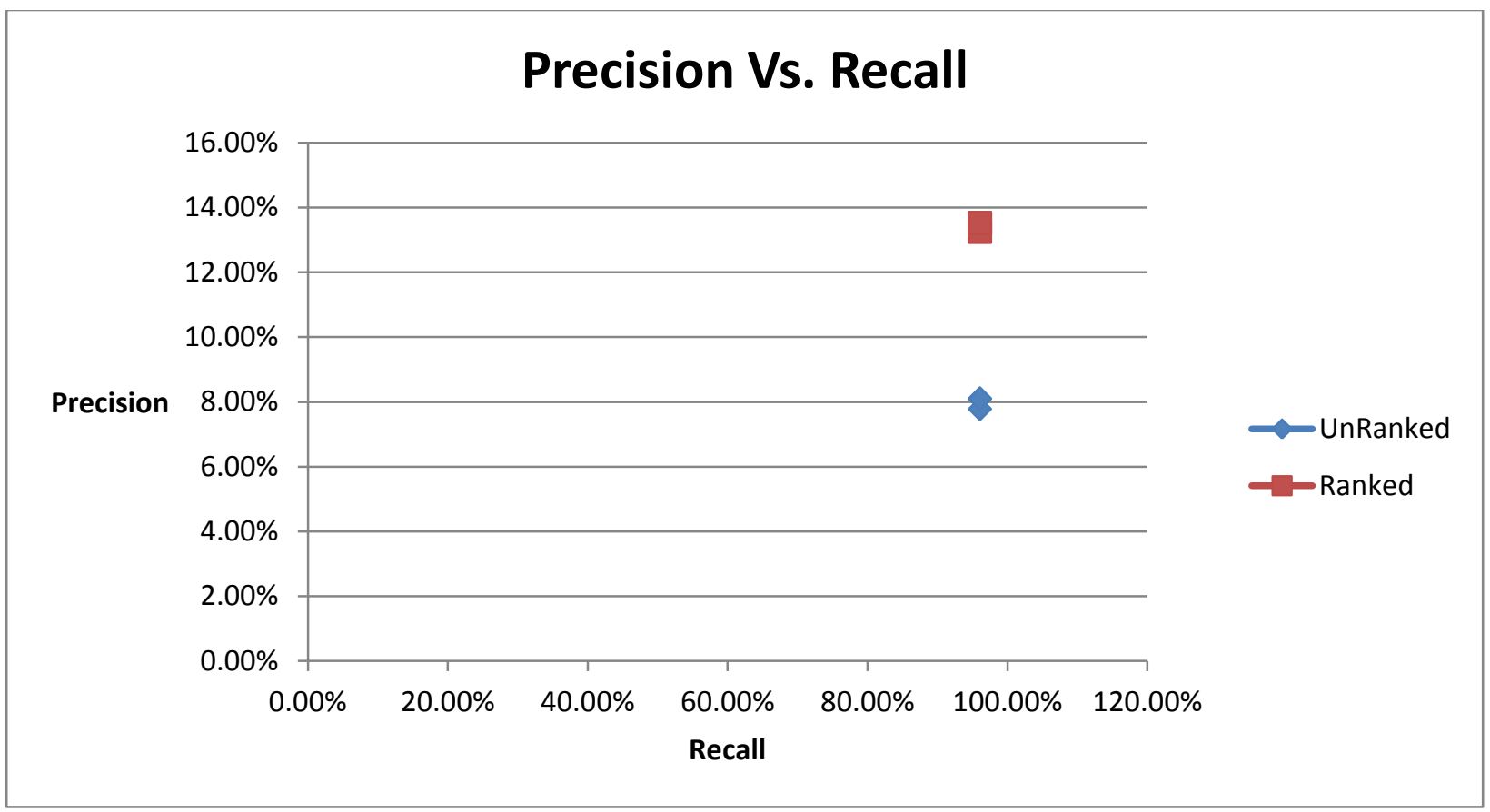

Figure 92: Precision vs. Recall

\section{Query Times LM}

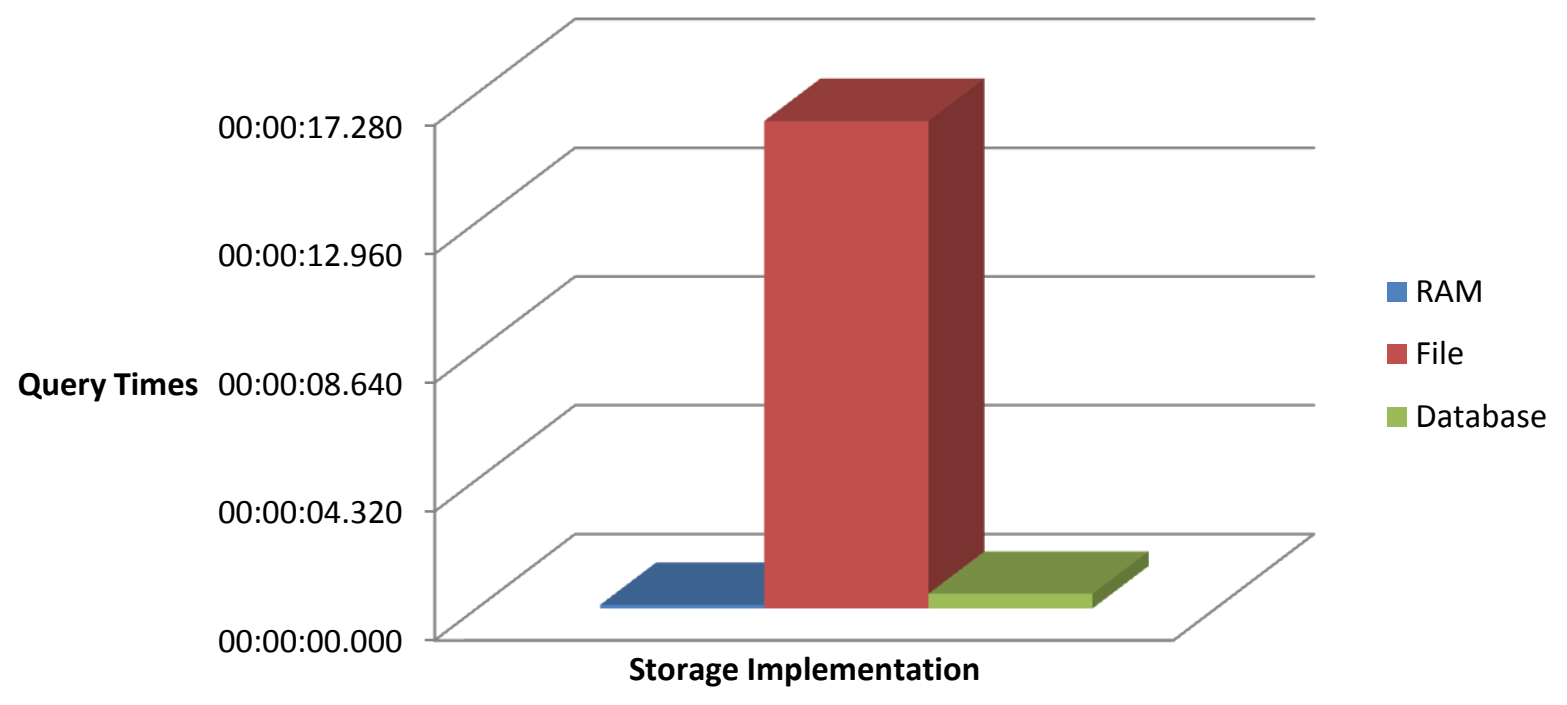

Figure 93: Query Times 


\subsubsection{Comparison}

This section will compare all case study three results together.

\begin{tabular}{|c|c|c|c|}
\hline Mean Avg. Precision & VSM & BM & LM \\
\hline RAM & $12.51 \%$ & $7.21 \%$ & $13.52 \%$ \\
\hline FILE & $13.52 \%$ & $13.39 \%$ & $13.25 \%$ \\
\hline Database & $13.98 \%$ & $13.57 \%$ & $13.52 \%$ \\
\hline
\end{tabular}

Table 117: MAP

\section{Mean Average Precision}

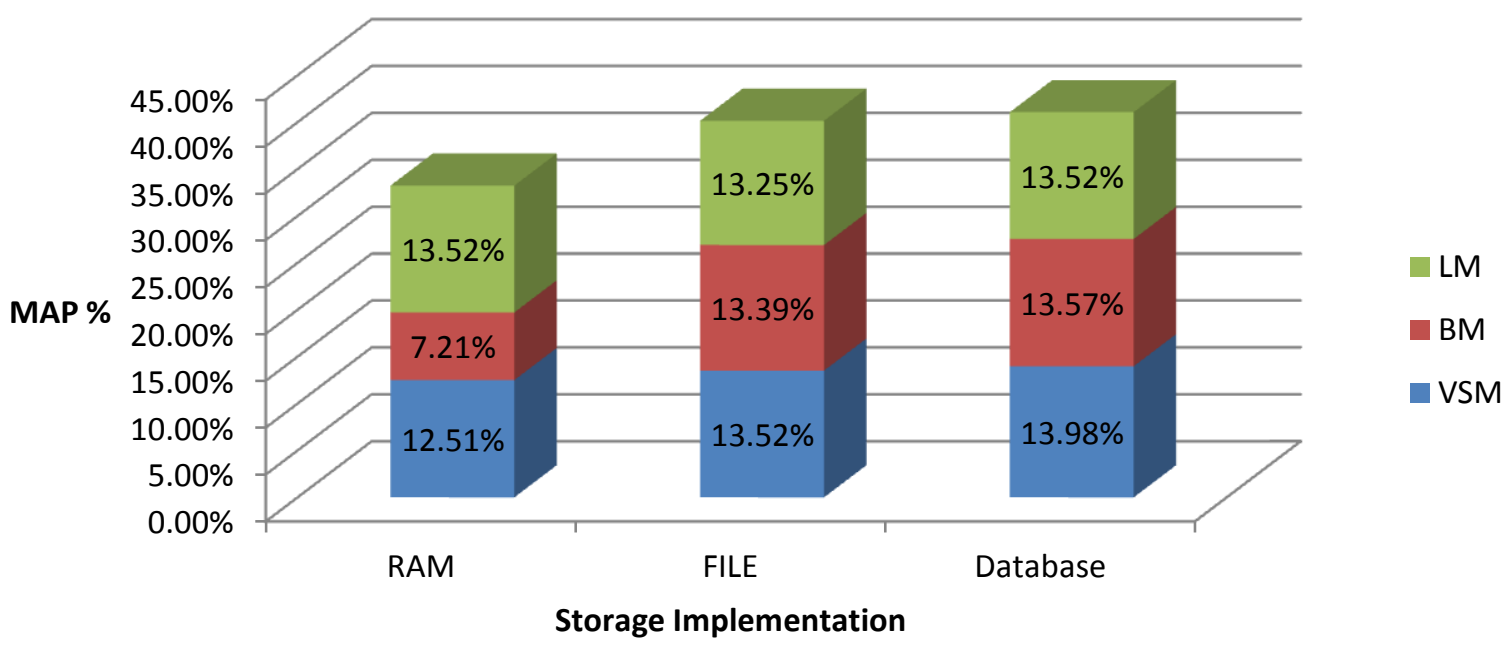

Figure 94: MAP

\begin{tabular}{|c|c|c|c|}
\hline Mean Avg. Unranked Precision & VSM & BM & LM \\
\hline RAM & $7.81 \%$ & $5.65 \%$ & $7.81 \%$ \\
\hline FILE & $7.45 \%$ & $7.78 \%$ & $7.45 \%$ \\
\hline Database & $8.09 \%$ & $8.09 \%$ & $8.09 \%$ \\
\hline
\end{tabular}

Table 118: Mean Avg. Unranked Precision 


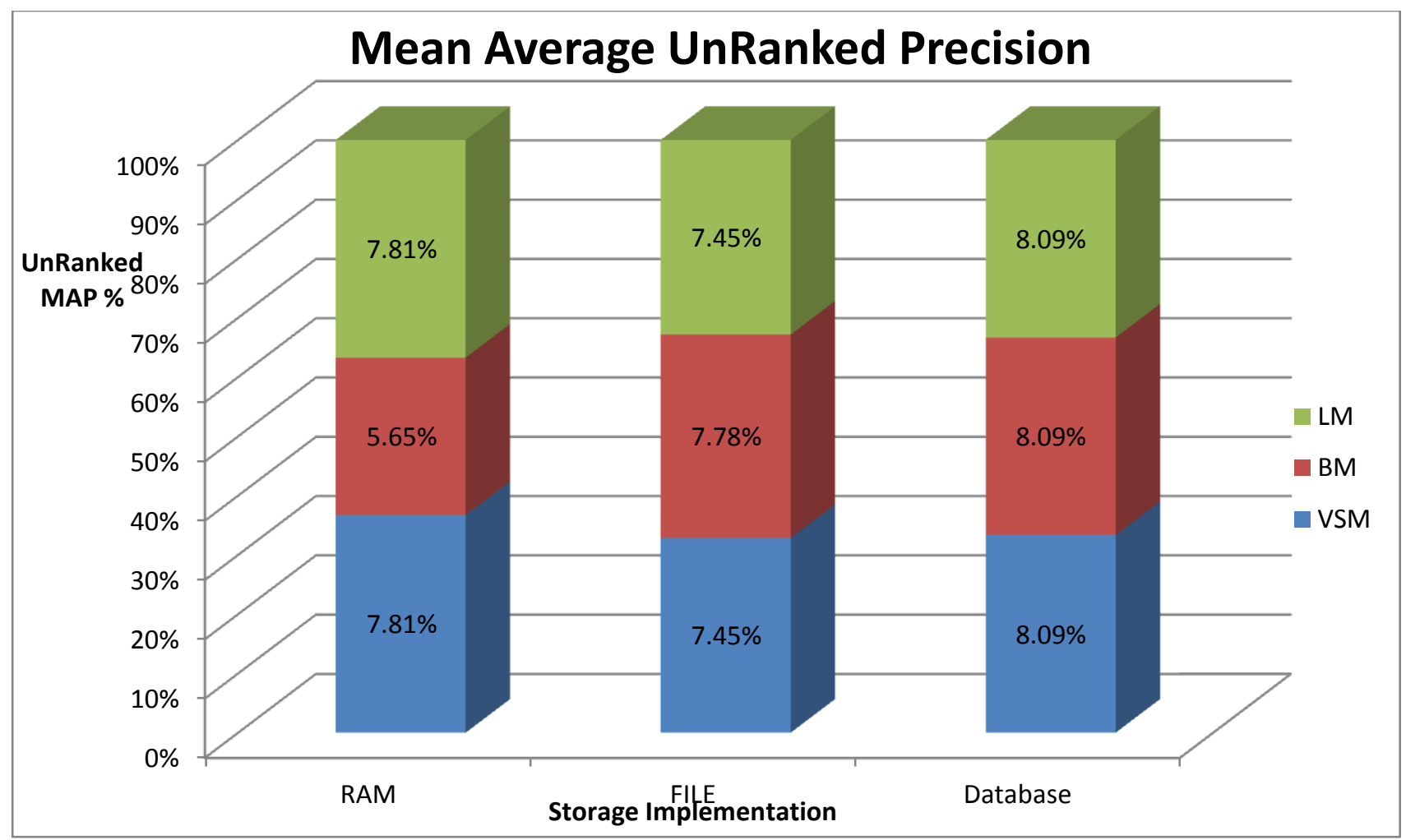

Figure 95: Mean Avg. Unranked Precision

\begin{tabular}{|c|c|c|c|}
\hline Recall & VSM & BM & LM \\
\hline RAM & $96.25 \%$ & $96.09 \%$ & $96.10 \%$ \\
\hline FILE & $96.09 \%$ & $96.09 \%$ & $96.10 \%$ \\
\hline Database & $96.09 \%$ & $96.09 \%$ & $96.10 \%$ \\
\hline
\end{tabular}




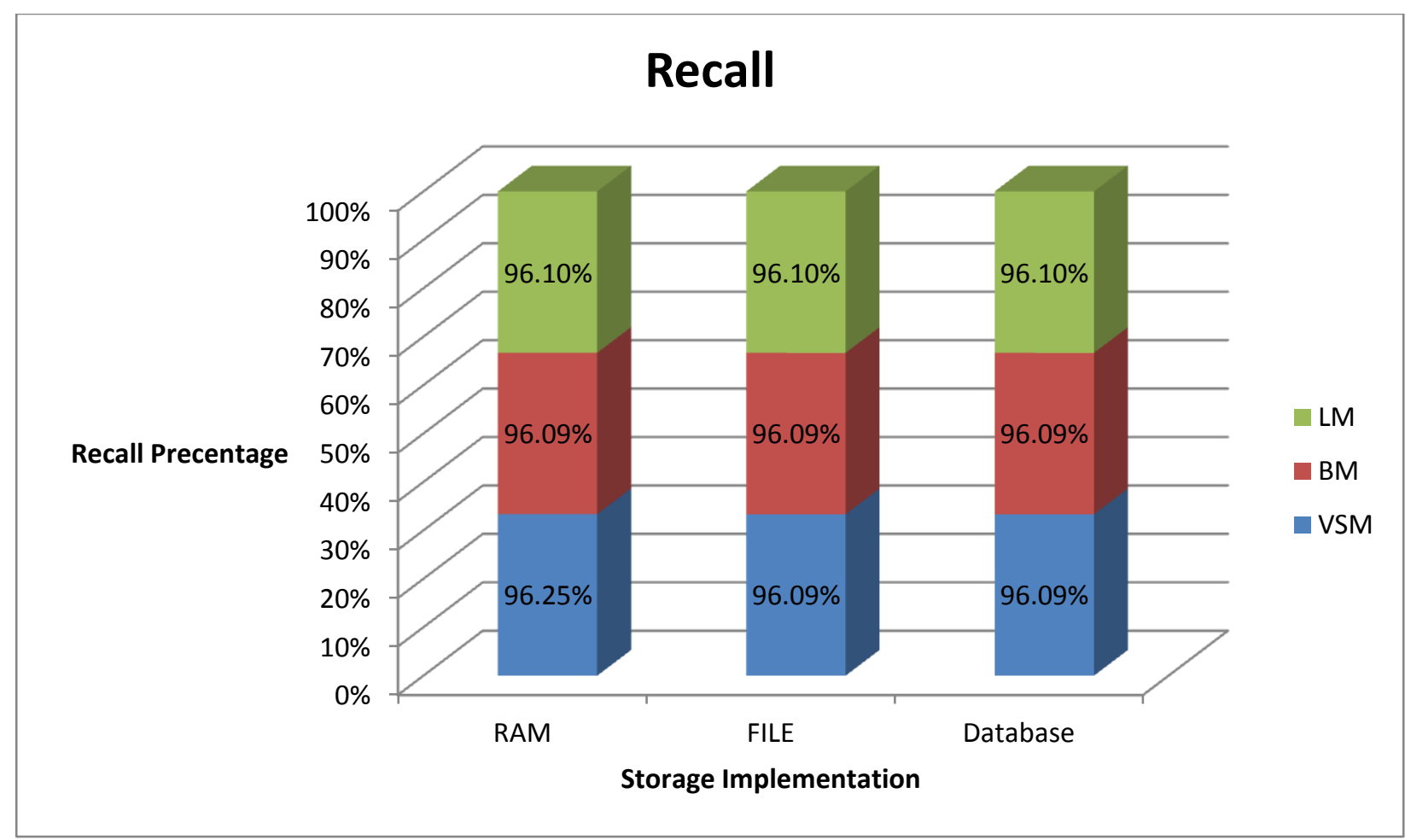

Figure 96: Recall

\begin{tabular}{|c|c|c|c|}
\hline Query Time & VSM & BM & LM \\
\hline RAM & $00: 00: 00.132$ & $00: 00: 00.083$ & $00: 00: 00.115$ \\
\hline FILE & $00: 00: 10.387$ & $00: 00: 09.151$ & $00: 00: 16.348$ \\
\hline Database & $00: 00: 00.397$ & $00: 00: 00.335$ & $00: 00: 00.489$ \\
\hline
\end{tabular}




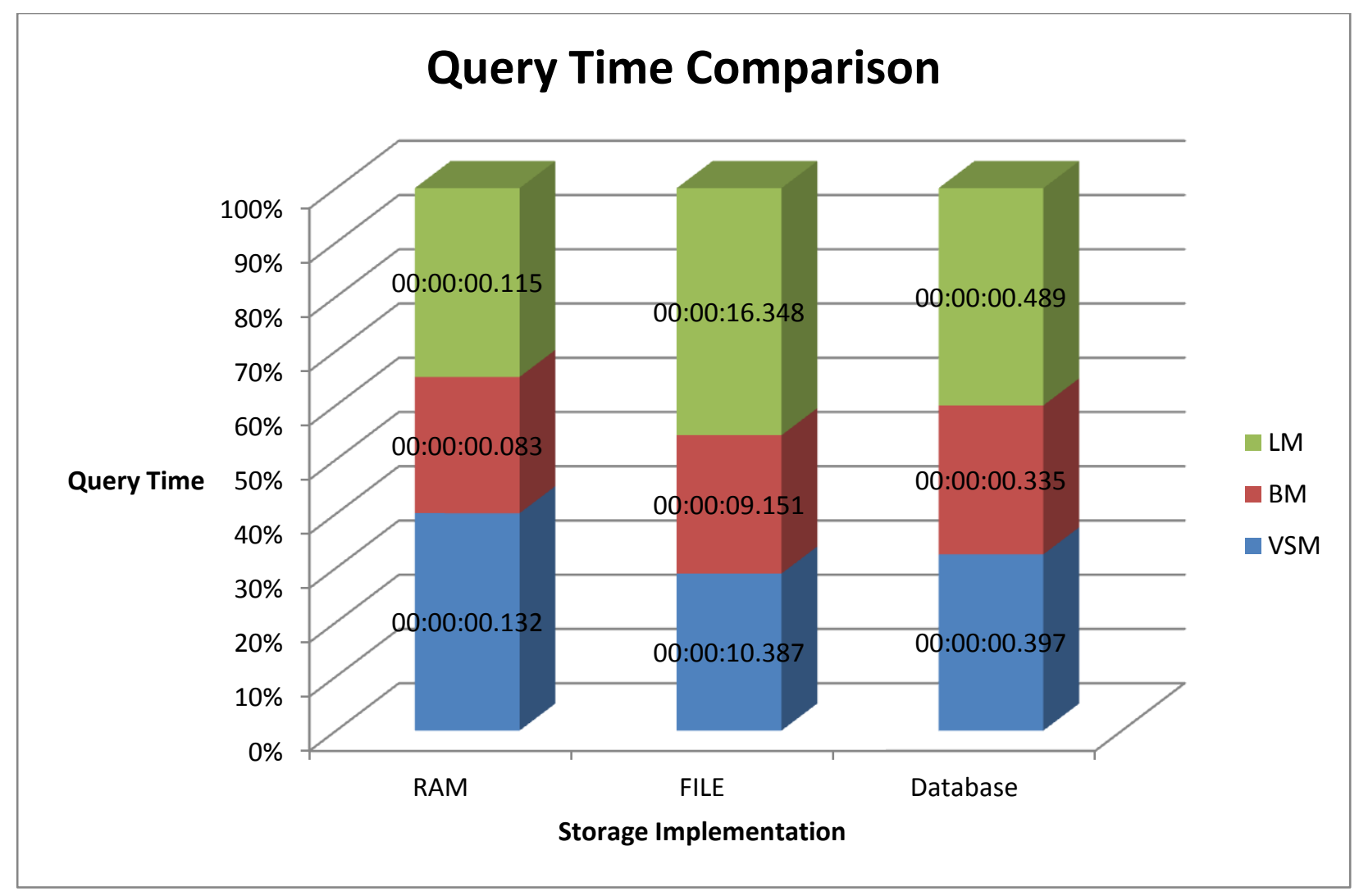

Figure 97: Query Times

\begin{tabular}{|c|c|c|}
\hline VSM & Relevant & Not Relevant \\
\hline Retrieved & 4 & 428 \\
\hline Not Retrieved & 0 & 39558 \\
\hline
\end{tabular}

Table 121: VSM Confucius Matrix

\begin{tabular}{|c|c|c|}
\hline BM & Relevant & Not Relevant \\
\hline Retrieved & 4 & 390 \\
\hline Not Retrieved & 0 & 39596 \\
\hline
\end{tabular}

\begin{tabular}{|c|c|c|}
\hline LM & Relevant & Not Relevant \\
\hline Retrieved & 4 & 390 \\
\hline Not Retrieved & 0 & 39596 \\
\hline
\end{tabular}

Table 123: LM Confucius Matrix 


\subsection{Comparison}

This section will compare all the data collected during this investigation. The data in the graphs is separated by case study.

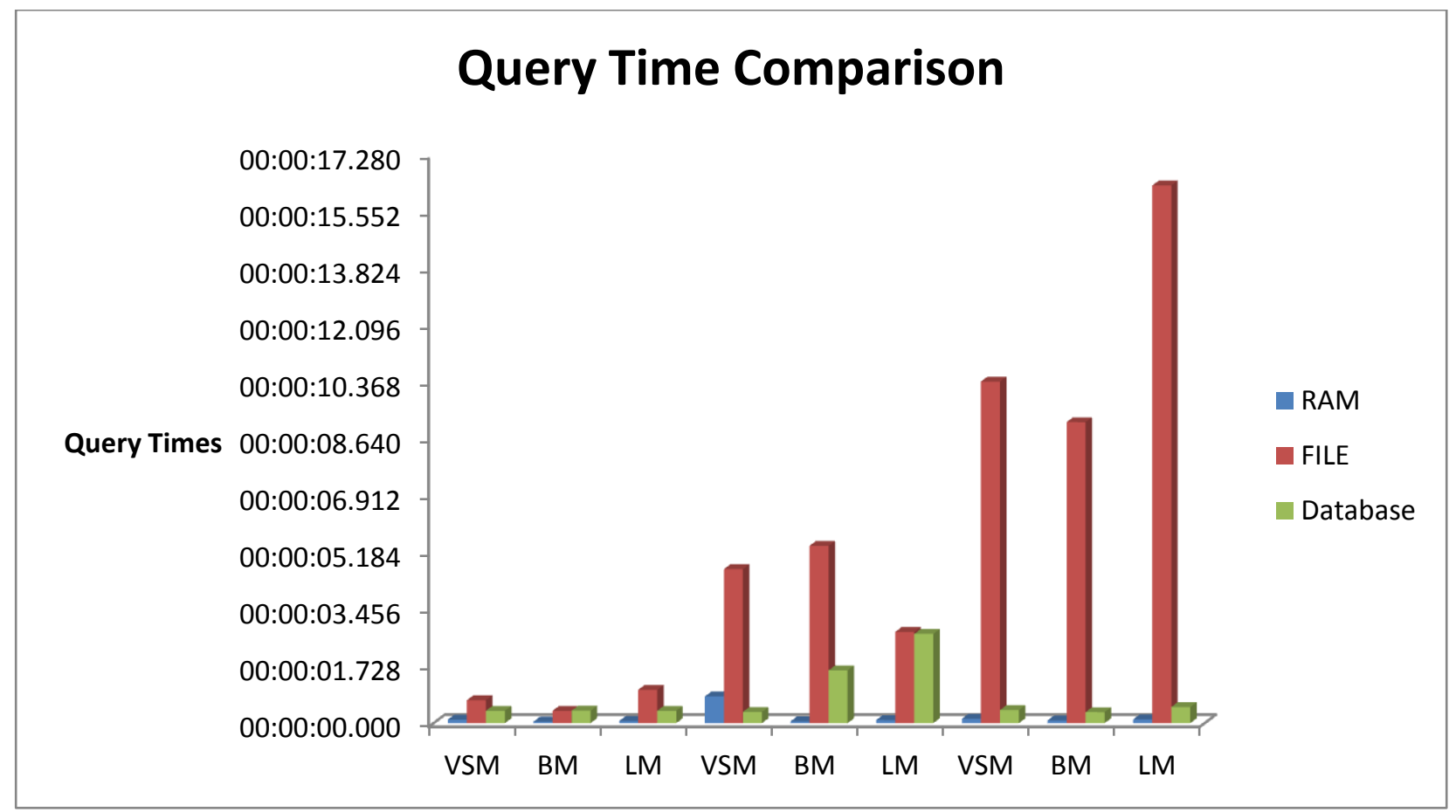

Figure 98: Query Time Comparison 


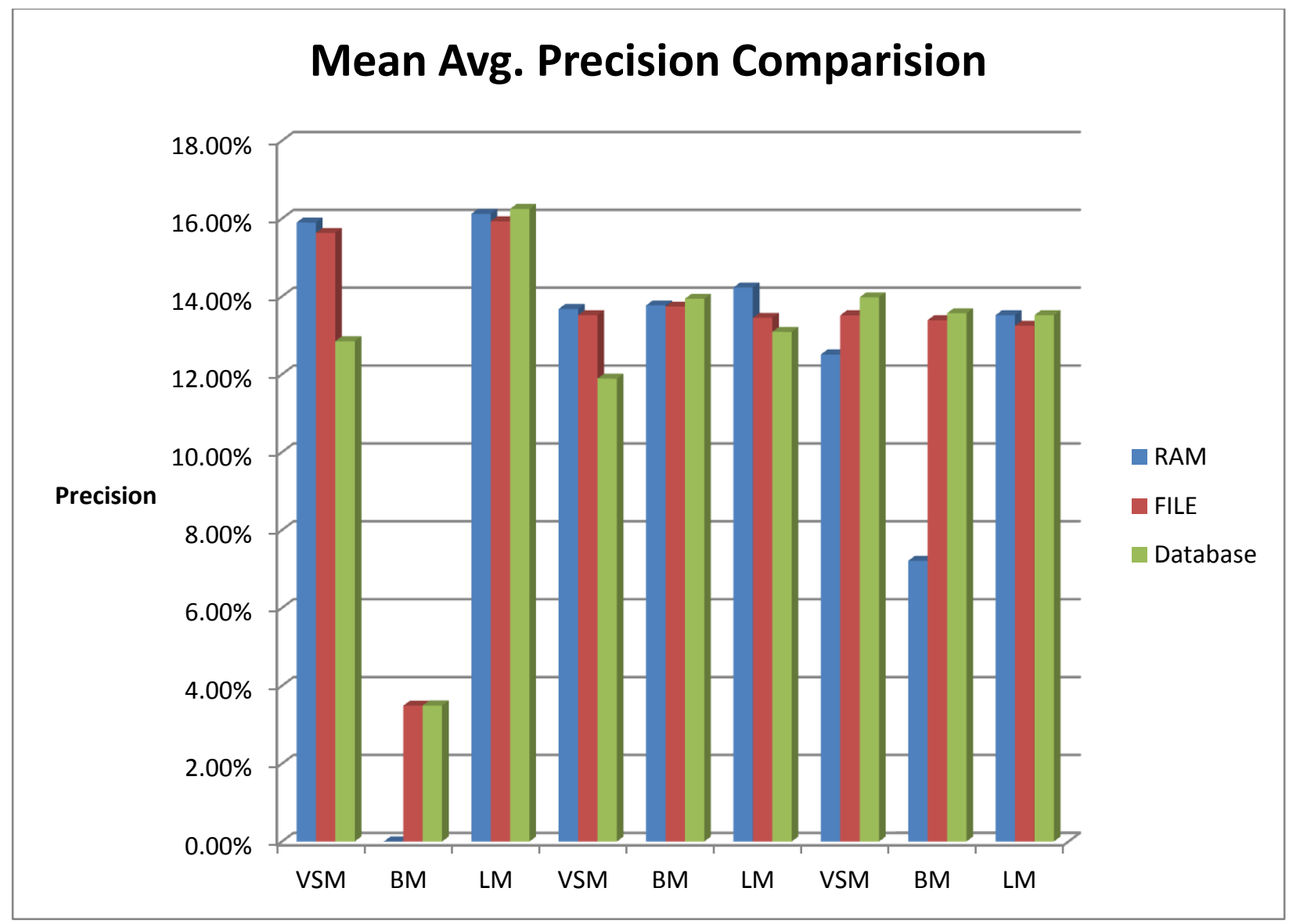

Figure 99: MAP Comparison 


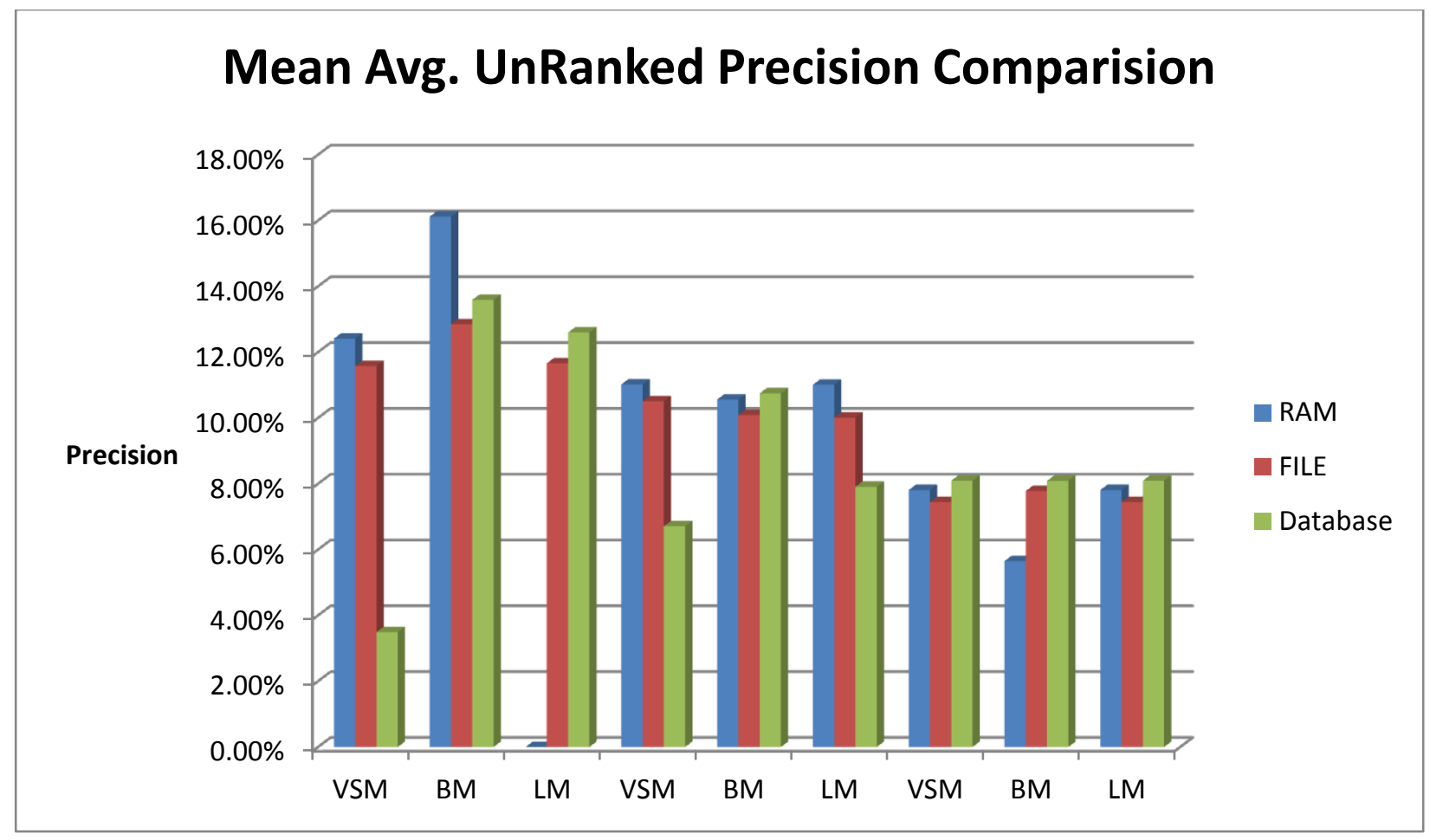

Figure 100: Mean Avg. Unranked Precision Comparison

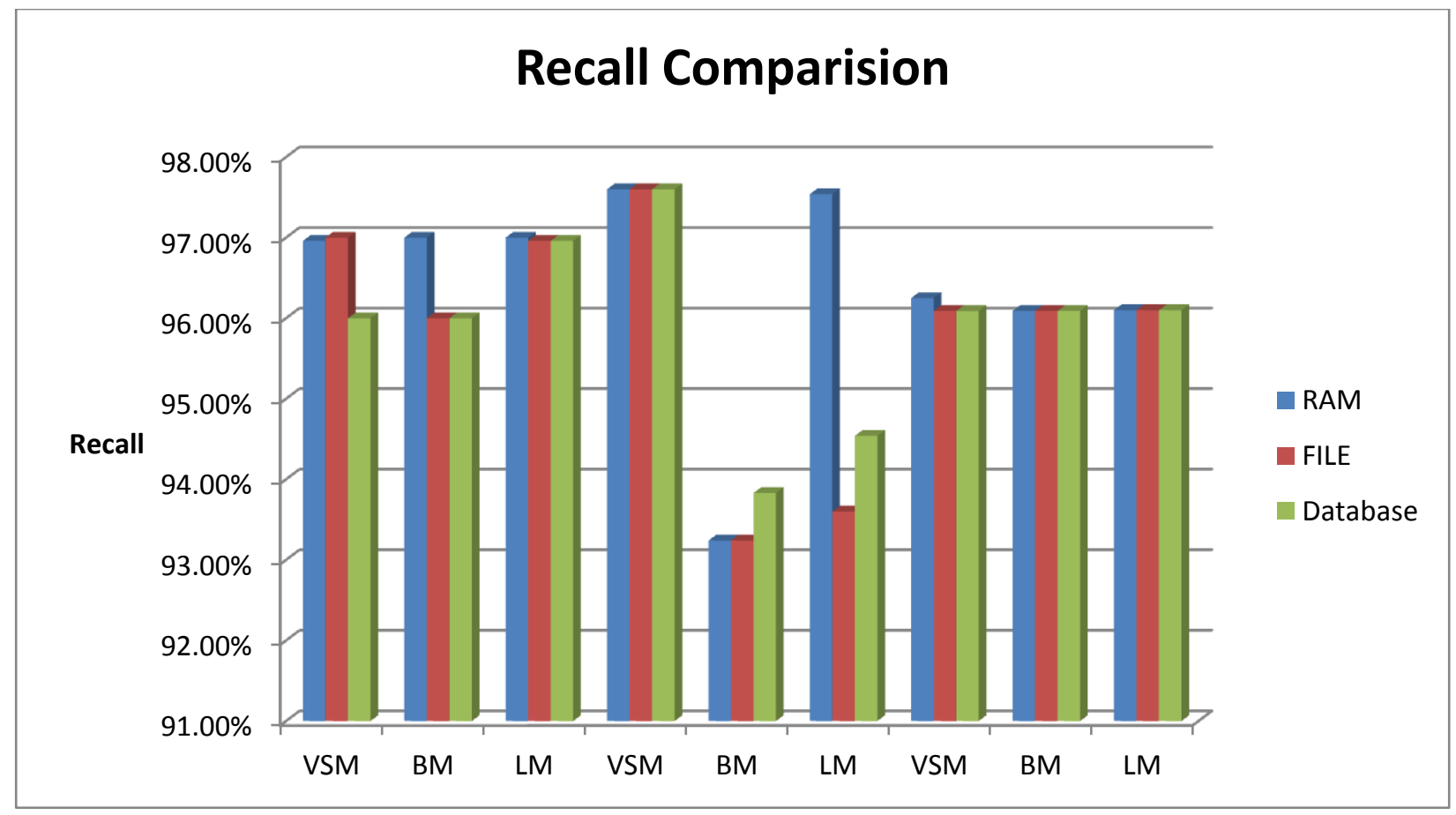

Figure 101: Recall Comparison 


\section{Chapter 6: Conclusion}

\subsection{Conclusion}

\subsubsection{Index Construction Conclusion}

One can conclude from the index construction phase of the investigation that the R.A.M. implemented index is constructed faster, while the database implemented index was the slowest. For each index implementation, the time needed for the index to be constructed grew. This is summed up in the following graph:

\section{Collection Size vs. Indexing Time}

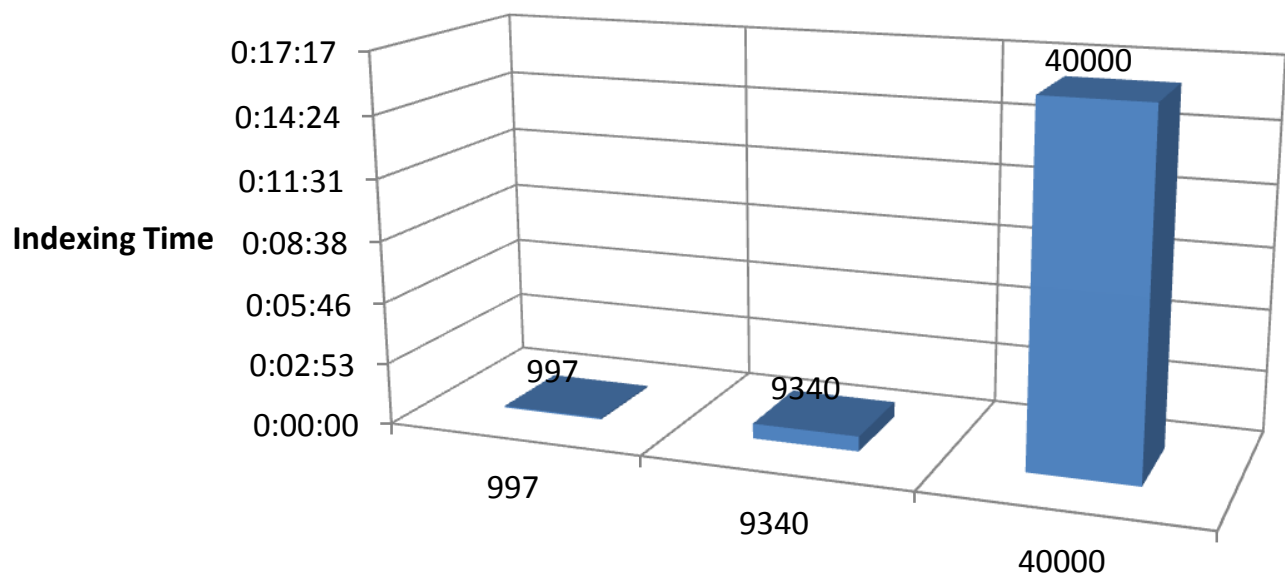

Collection Size

Figure 102: Index Construction Relationship 


\subsubsection{Query Time}

The query time phase of the investigation concluded that the equations derived in the theory section. The database implemented index implemented provides quicker response times than that of file. The following graph summarizes all the query times for all three case studies.

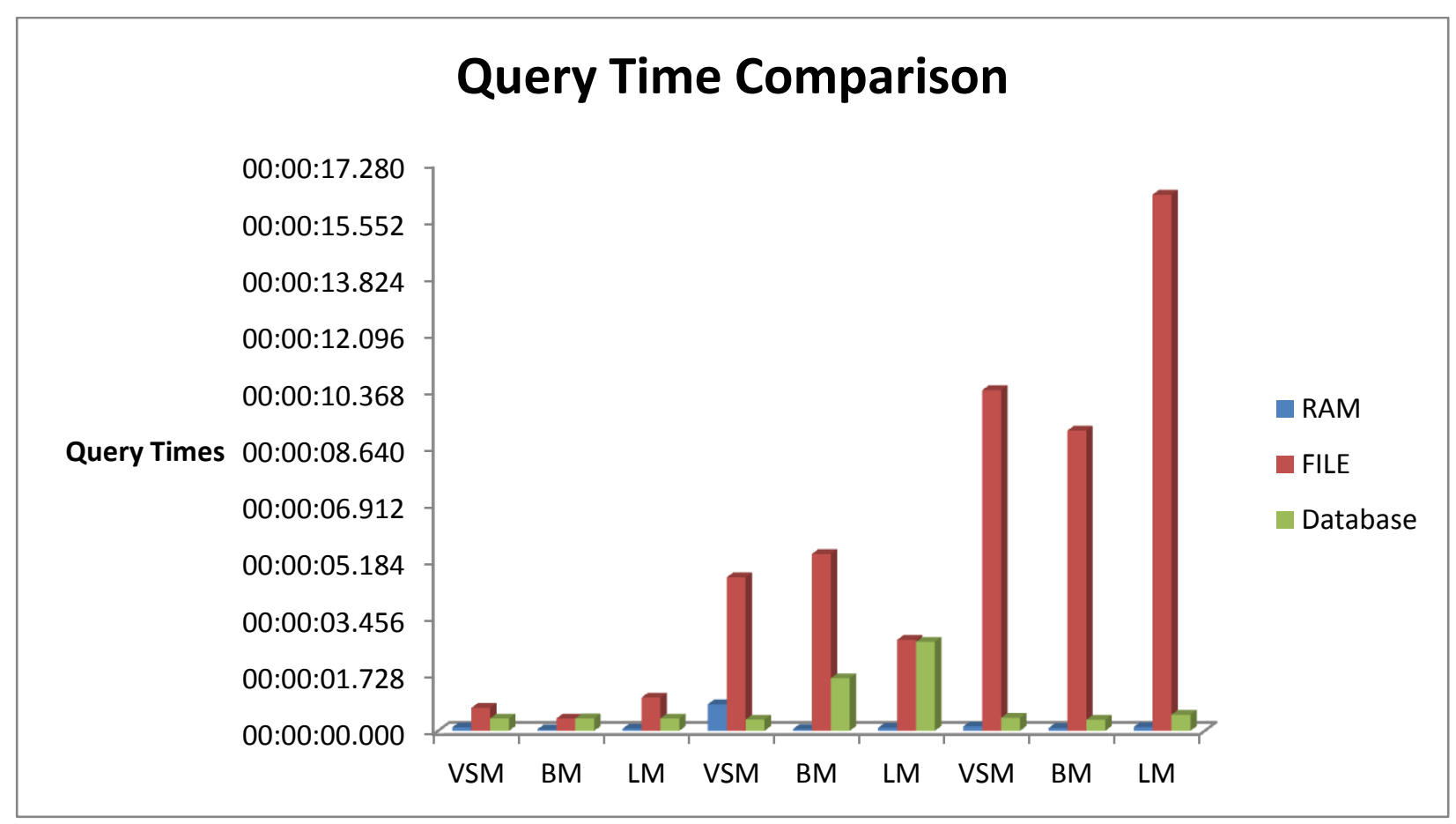

Figure 103: Query Time Summary

\subsubsection{Overall Conclusion}

The data collected in chapter five on the precision and recall showed that the Best Match 25 and the unigram language algorithm were the more consistent than the cosine similarity algorithm. Therefore, based on the tables in chapter 5, the optimal retrieval to index implementation would be either a unigram language algorithm or the best match 25 with an inverted index stored as a table in a database. This is assuming that the document collection does not fit entirely in RAM. 


\subsection{Future Work}

Now that the investigation has concluded, there are some extensions of this work that can be completed to enhance the information retrieval engines for a system. One area for future work is to implement a machine learning algorithm or a bigram language algorithm that develops a background to improve the precision to recall ratio. With this route, an interesting area of study is finding the minimal amount of time necessary for the system to develop a background which allows a precision percentage nearing 80 to $90 \%$ precision. In addition, one can look at the precision threshold that must be met before a background can stop being built. 


\section{Appendices}

\section{Appendix A: Case Studies Queries}

In this section are the queries that were run in the retrieval system and in the database during the investigation for each case study.

\section{A.1: Case Study One Query}

\begin{tabular}{|c|c|c|}
\hline Number & User Query & SQL Query \\
\hline 1 & who is hall cassidy? & Title: hall cassidy \\
\hline 2 & the legend of the jedi & Title: lengend jedi \\
\hline 3 & treatment of lies & Title: lies treatment \\
\hline 4 & where lost birds & Title: lost birds \\
\hline 5 & truman's lover & Title: lover truman \\
\hline 6 & what is a lovely jingle? & Title: lovely jingle \\
\hline 7 & the maiden home & Title: madien home \\
\hline 8 & peaches mask & Title: mask peaches \\
\hline 9 & what is mars & mars roman \\
\hline 10 & ace of millions & Title: millions roman desc: ace \\
\hline 11 & defeat robots in australia & Desc: defeat or robots or australia \\
\hline 12 & $\begin{array}{l}\text { database administrator } \\
\text { students }\end{array}$ & Desc: database or administrator or students \\
\hline 13 & tequila in the north! & Title: north tequila \\
\hline 14 & submarine october? & Title: october submarine \\
\hline 15 & movies with hunt & actor: hunt \\
\hline 16 & pirate with willis & Title: pirates roxanne Actor: willis \\
\hline 17 & secret agent stallone & Title: flatliners killer \\
\hline 18 & laura cabin & Title: cabin laura \\
\hline 19 & freedy's sister & Title: sister freedy \\
\hline 20 & suspect sleep & Title: sleep suspect \\
\hline 21 & voight park in tadpole & Title: tadpole park Actor: Voight \\
\hline 22 & kick the taxi & Title: taxi kick \\
\hline 23 & watch for texas & Title: texas watch \\
\hline 24 & ark town & Title: town ark \\
\hline 25 & hobbit & Title: hobbit alien \\
\hline 26 & butler in airplane & Title: airplane sierra Desc: Butler \\
\hline 27 & cat are alien & title: alien center Desc: cat \\
\hline
\end{tabular}




\begin{tabular}{|c|c|c|}
\hline 28 & braveheart & Title: breaveheart human \\
\hline 29 & berry bound & Title: bound cheaper Desc: Berry \\
\hline 30 & casper documentary & Title: casper dragonfly desc: Documentary \\
\hline 31 & julia desire & Title: cider desire actor: julia \\
\hline 32 & bob chef youth & Title: circus youth actor: bob Desc: chef \\
\hline 33 & sumo wrestler with angels & Title: clerks angels desc: sumo wrestler \\
\hline 34 & peck in paradise & Title: clockwork paradise desc: peck \\
\hline 35 & car robot in new orleans & desc: cat robot new orleans \\
\hline 36 & $\begin{array}{l}\text { wood in clueless bucket } \\
\text { desc: wood }\end{array}$ & Title: clueless bucket \\
\hline 37 & forensic psychologist japan & desc: forensic psychologist japan \\
\hline 38 & akroyd in candles & Title: confused candles actor: akroyd \\
\hline 39 & tom congeniality & Title: congeniality quest actor: tom \\
\hline 40 & conquerer nuts & Title: conquerer nuts \\
\hline 41 & chase cowboys & Title: cowboy doom desc: chase \\
\hline 42 & penelope suit & Title: core suit desc: penelope \\
\hline 43 & explorer car in manhattan & desc: explorer car manhattan \\
\hline 44 & home is crazy & Title: crazy home \\
\hline 45 & christopher kane & Title: creepers kane actor: chrisopher \\
\hline 46 & swank hunter in park & actor: swank desc: hunter park \\
\hline 47 & kilmer reese in breaking & desc: kilmer reese desc: breaking \\
\hline 48 & voight is sinner & Title: sinners cupboard actor: voight \\
\hline 49 & dancing fever & Title: dancing fever \\
\hline 50 & robot in pluto & Title: dares in pluto desc: robot \\
\hline 51 & forrester fawcett & Title: darn forrester actor: fawcett \\
\hline 52 & waitress in ancient china & desc: waitress ancient china \\
\hline 53 & zorror ark tandy & Title: zorro ark actor: tandy \\
\hline 54 & nicholson in wyoming? & Title: wyoming storm actor: nicholson \\
\hline 55 & is goldberg worst? & Title: worst banger actor: goldberg \\
\hline 56 & wonderland with cuba & Title: wonderland christmas actor: cuba \\
\hline 57 & documentary with u-boat & desc: documentary u-boat \\
\hline 58 & cruz with witches & Title: witches panic Actor: cruz \\
\hline 59 & $\begin{array}{l}\text { character study in a } \\
\text { monastery }\end{array}$ & dsc: character study monastery \\
\hline 60 & $\begin{array}{c}\text { madman psychologist } \\
\text { dench }\end{array}$ & desc: madman psychologist actor: dench \\
\hline 61 & hoffman with wife & Title: wife turn actor: hoffman \\
\hline 62 & guiness butler and husband & desc: guiness butler husband \\
\hline 63 & 2006 christian as teacher & release Year: 2006 actor: christian desc: teacher \\
\hline 64 & personal weekend & Title: weekend personal \\
\hline
\end{tabular}




\begin{tabular}{|c|c|c|}
\hline 65 & chase apollo tale & Title: wedding apollo desc: chase tale \\
\hline 66 & newton trip & Title: trip newton \\
\hline 67 & gurys are trapped & Title: trap guys \\
\hline 68 & sky of timberland & Title: timerland sky \\
\hline 69 & bergman asterminator & Title: terminator club actor: bergman \\
\hline 70 & $\begin{array}{c}\text { saga soviet georgia with } \\
\text { peck }\end{array}$ & desc: saga soviet georgia actor: peck \\
\hline 71 & streetcar in gulf of mexico & Title: streetcar intentions desc: guilf mexico \\
\hline 72 & kilmer in ridgemont & Title: streak ridgemont actor: kilmer \\
\hline 73 & santa in steel & Title: steel santa \\
\hline 74 & torn in wasteland & Title: state wasteland actor: torn \\
\hline 75 & tale of stallion & Title: stallion sundance desc: tale \\
\hline 76 & hoffman as spy & Title: spy mile desc: hoffman \\
\hline 77 & kilmer secret agent rockies & desc: secret agent rocket actor: kilmer \\
\hline 78 & zellweger as feminist & desc: feminist actor: zellweger \\
\hline 79 & bassinger in baloon & desc: baloon Title: bassinger \\
\hline 80 & bull candian rockies & Title: bull shawshank desc: candian rockies \\
\hline 81 & $\begin{array}{l}\text { woman vacation character } \\
\text { study }\end{array}$ & desc: woman vacation character study \\
\hline 82 & grape candles & Title: candles grapes \\
\hline 83 & helen voight in motions & Title: caper motions actor: helen voight \\
\hline 84 & $\begin{array}{l}\text { liberty caribbean with } \\
\text { willis }\end{array}$ & Tile: caribbean liberty actor: willis \\
\hline 85 & $\begin{array}{l}\text { casabalanca with akroyd } \\
2006\end{array}$ & $\begin{array}{l}\text { Title: casabalanca super release year: } 2006 \text { actor: } \\
\text { akroyd }\end{array}$ \\
\hline 86 & $\begin{array}{l}\text { wayne dinosaurs in } \\
\text { california }\end{array}$ & Title: center dinosaur actor: wayne desc: california \\
\hline 87 & $\begin{array}{l}\text { hackman chaplin with } \\
\text { license }\end{array}$ & Title: chaplin license actor: hackman \\
\hline 88 & hoffman student in chicago & Title: chicago north actor: hoffman desc: student \\
\hline 89 & gladiator with wood & Title: chinatown gladiator desc: wood \\
\hline 90 & swank harry action & Title: chocolat harry actor: swank \\
\hline 91 & clerks is angles & Title: clerk angels \\
\hline 92 & waitress squirrel jet boat & desc: waitress squirrel jet boat \\
\hline 93 & teacher feminist drama & desc: teacher feminist drama \\
\hline 94 & bergen enemy odds & Title: enemy odds actor: bergen \\
\hline 95 & brody sting & Title: exorcist sting actor: brody \\
\hline 96 & day-lewis as stallion & Title: expendable stallion actor: day-lewis \\
\hline 97 & fantasy with hopkins & Title: fantasy troopers actor: hopkins \\
\hline 98 & $\begin{array}{l}\text { goldberg feminist in } \\
\text { australia }\end{array}$ & actor: goldberg desc: feminist austrialia \\
\hline
\end{tabular}




\begin{tabular}{|c|c|c|}
99 & freddy mcqueen & Title: freddy storm actor: mcqueen \\
\hline 100 & chase head with frost & Title: frost head actor: chase \\
\hline
\end{tabular}




\section{A.2: Case Study Two Query}

\begin{tabular}{|c|c|c|}
\hline Number & User Query & SQL Query \\
\hline 1 & $\begin{array}{l}\text { The fall of the } \\
\text { night }\end{array}$ & Title: the fall of the night \\
\hline 2 & Legends of the Fall & Title: legends of the fall \\
\hline 3 & $\begin{array}{c}\text { War of the worlds } \\
\text { of } 2005\end{array}$ & Title: war of the worlds (2005) \\
\hline 4 & grumpy old men & Title:grumpier old men \\
\hline 5 & 1995 move Exhale & Title: Waiting to Exhale \\
\hline 6 & 18 Tom 1995 & Title: tom and huck(1995) Age Range 18-24 \\
\hline 7 & the president 1995 & Title: Amaerican President, The (1995) \\
\hline 8 & Dracula 1995 & Title: Dracula: Dead and Loving it (1995) \\
\hline 9 & $\begin{array}{c}\text { academic movie of } \\
\text { courage } 1995 \\
\end{array}$ & Title: Wings of Courage(1995) Occupation: ademic/educational \\
\hline 10 & $\begin{array}{l}\text { title is time and sea } \\
1995\end{array}$ & Title: Across the Sea of Time (1995) \\
\hline 11 & Babe & Title: Babe(1995) \\
\hline 12 & dead walking & Title: Dead man Walking (1995) \\
\hline 13 & beloved country & Title: Cry, the Beloved Country (1995) \\
\hline 14 & 1995 Richard & Title: Richard III (1995) \\
\hline 15 & $\begin{array}{c}3 \text { star mortal } \\
\text { kombat }\end{array}$ & Rating: 3 Title: Mortal Kombat \\
\hline 16 & $\begin{array}{c}\text { Ace Ventura } \\
(1995)\end{array}$ & Title: Ace Ventura: When Nature Calls (1995) \\
\hline 17 & $\begin{array}{l}\text { Sense and } \\
\text { sensibility }\end{array}$ & Title: Sense and Sensibility (1995) \\
\hline 18 & To Die & Title: To Die For (1995) \\
\hline 19 & Richard Nixon & Title: Nixon (1995) \\
\hline 20 & $\begin{array}{l}1995 \text { version of } \\
\text { Othello }\end{array}$ & Title: Othello (1995) \\
\hline 21 & cite of lost & Title: City of Lost Childern (Cite des enfants perdus, La) (1995) \\
\hline 22 & yao yao qiao & Title: Shanghai Triad (Yao a Yao Yao dao waipo qiao)(1995) \\
\hline 23 & Sudden Death & Title: Sudden Death (1995) \\
\hline 24 & carrington & Title: Carrington (1995) \\
\hline 25 & Jumanji & Title: Jumanji (1995) \\
\hline 26 & Monkeys 12 & Title: 12 monkeys (Twelve Monkeys) (1995) \\
\hline 27 & American Quilt & Title: How to make an American Quilt (1995) \\
\hline 28 & what is seven? & Title: Seven (a.k.a Se7en) (1995) \\
\hline
\end{tabular}




\begin{tabular}{|c|c|c|}
\hline 29 & $\begin{array}{l}\text { Pocahontas of } \\
1995\end{array}$ & Title: Pocahontas (1995) \\
\hline 30 & $\begin{array}{l}\text { Who are the Usual } \\
\text { Suspects? }\end{array}$ & Title: Usual Suspects, the (1995) \\
\hline 31 & les miserables & Title: Miserables, les (1995) \\
\hline 32 & Aphrodite & Title: Mighty Aphrodite (1995) \\
\hline 33 & Lamerica & Title: Lamerica (1994) \\
\hline 34 & Big Green & Title: Big Green, The (1995) \\
\hline 35 & Georgia of 1995 & Title: Georgia (1995) \\
\hline 36 & Round Table & Title: Kids of the Round Table (1995) \\
\hline 37 & $\begin{array}{l}\text { Cupboard with } \\
\text { indians }\end{array}$ & Title: Indian in the Cupboard, the (1995) \\
\hline 38 & Holidays and home & Title: Home for the Holidays (1995) \\
\hline 39 & Postman & Title: Postman, the (postino, ill) (1994) \\
\hline 40 & Confessional & Title: Confessional \\
\hline 41 & eye for a eye & Title: Eye for an Eye (1996) \\
\hline 42 & 1995 with Holland & Title: Mr. Holland's Opus (1995) \\
\hline 43 & Soun Central Hood & $\begin{array}{c}\text { Title: Don't be a Menace to South Central While Drinking your } \\
\text { juice in the hoold (1996) }\end{array}$ \\
\hline 44 & 2 by sea 1996 & Title: Two if by Sea (1996) \\
\hline 45 & Bio Dome & Title: Bio-Dome (1996) \\
\hline 46 & Gazon Maudit & Title: French Twist \\
\hline 47 & Dusk till dawn & Title: From Dusk Till Dawn (1996) \\
\hline 48 & Bed of rose & Title: Bed of Roses (1996) \\
\hline 49 & Big Bullies & Title: Big Bully (1996) \\
\hline 50 & Screamer under 18 & Title: Screamers (1995) Age_Range: under 18 \\
\hline 51 & Nico of 1995 & Title: Nico Icon (1995) \\
\hline 52 & $\begin{array}{l}\text { Crossing of the } \\
\text { Guard }\end{array}$ & Title: Corssing Guard, The (1995) \\
\hline 53 & Juror 1996 & Title: Juror, the (1996) \\
\hline 54 & Badkonake sefid & Title: White Ballon \\
\hline 55 & Dead in Denver & Title: Things to do in Denver When you're Dead (1995) \\
\hline 56 & Antonia & Title: Antonia's Line( Antonia) (1995) \\
\hline 57 & Once upon a time? & Title: Once upon a Time.. When we were collred (1995) \\
\hline 58 & $\begin{array}{l}\text { Summer at } \\
\text { Hamptons }\end{array}$ & Title: Last Summer in the Hamptons (1995) \\
\hline 59 & Insects and Angels & Title: Angels and Insects (1995) \\
\hline 60 & squall is White & Title: White Squall (1996) \\
\hline 61 & check in Duston & Title: Dustons Checks in (1996) \\
\hline 62 & sheep are black & Title: Black Sheep (1996) \\
\hline 63 & In the Nick of time & Title: Nick of Time (1995) \\
\hline
\end{tabular}




\begin{tabular}{|c|c|c|}
\hline 64 & $\begin{array}{c}\text { who is Mary } \\
\text { Reilly? }\end{array}$ & Title: Mary Reilly (1996) \\
\hline 65 & $\begin{array}{l}\text { August King's } \\
\text { Journey }\end{array}$ & Title: Journey of August King, the (1995) \\
\hline 66 & game was fair & Title: FairGame (1995) \\
\hline 67 & beyond cyberspace & Title: Lawnmower man 2: Beyond Cyberspace (1996) \\
\hline 68 & brooklyn Vampire & Title: Vampire in Brooklyn (1995) \\
\hline 69 & $\begin{array}{c}\text { that girl is } \\
\text { beautiful }\end{array}$ & Title: Beautiful Girls (1996) \\
\hline 70 & $\begin{array}{c}\text { The arrow is } \\
\text { broken }\end{array}$ & Title: Broken Arrow (1996) \\
\hline 71 & midwinter is bleak & Title: in the bleak midwinter (1995) \\
\hline 72 & I hate haine & Title: Hate (Haine, la) (1995) \\
\hline 73 & $\begin{array}{l}\text { muppet in treasure } \\
\text { island }\end{array}$ & Title: Muppet Treasure Island (1996) \\
\hline 74 & Gilmore is happy & Title: Happy Gilmore (1996) \\
\hline 75 & rocket in a bottle? & Title: Bottle Rocket (1996) \\
\hline 76 & Hollywood Fleiss & Title: Heidi Fleiss: hollywood madam \\
\hline 77 & hall of the city & Title: City Hall \\
\hline 78 & $\begin{array}{c}\text { who is } \mathrm{Mr} . \\
\text { Wrong? }\end{array}$ & Title: Mr. Wrong (1996) \\
\hline 79 & he is unforgettable! & Title: Unforgettable (1996) \\
\hline 80 & Keiner liebrt mich & Title: Nobody loves me (keiner liebt mich) (1994) \\
\hline 81 & catwalk & Title: Catwalk (1996) \\
\hline 82 & hont faan who? & Title: Rumble in the Brox \\
\hline 83 & $\begin{array}{c}\text { what is before and } \\
\text { after? }\end{array}$ & Title: Before and after (1996) \\
\hline 84 & $\begin{array}{c}\text { Museum } 4 \\
\text { margaret }\end{array}$ & Title: Margaret's Museum Rating: 4 \\
\hline 85 & Anne Frank & Title: Anne Frank Remembered \\
\hline 86 & $\begin{array}{l}\text { Posisoner's } \\
\text { Handbook }\end{array}$ & Title: Young Poisoner's Handbook, the (1995) \\
\hline 87 & lucy & Title: if Lucy Fell (1996) \\
\hline 88 & Stea big or little & Title Steal big, Steal little (1995) \\
\hline 89 & St. Vincent & Title: Boys of St. Vincent, the (1992) \\
\hline 90 & boomerang & Title: boomerang (1992) \\
\hline 91 & Maker of the Star & Title: Star Maker \\
\hline 92 & delle stelle & Title: Star Maker \\
\hline 93 & $\begin{array}{l}\text { Express of } \\
\text { Chungking }\end{array}$ & Title: Chungking Express \\
\hline 94 & Saimt el qusur & Title: Silence of the Palace \\
\hline 95 & Wife of Jupiter & Title: Jupiter's Wife \\
\hline
\end{tabular}




\begin{tabular}{|c|c|c|}
\hline 96 & who is angela & Title: Angela (1995) \\
\hline 97 & Nueba Yol! & Title: Nueba Yol \\
\hline 98 & outlaws! Sonic! & Title: Sonic Outlaws \\
\hline 99 & 1995 target & Title: target (1995) \\
\hline 100 & $\begin{array}{c}\text { Queen Margot } \\
1994\end{array}$ & Title: Queen Margot (La Reine Margot) (1994) \\
\hline 101 & 3 star gordy & Rating: 3 Title: Gordy (1995) \\
\hline 102 & 34th street miracle & Title: Miracle on 34th Street (1994) \\
\hline 103 & $\begin{array}{c}\text { vampire chronicles } \\
\text { interview }\end{array}$ & Interview with the Vampire: The Vampire Chronicles (1994) \\
\hline 104 & 2 star major payne & Rating: 2 Title: Major Payne (1995) \\
\hline 105 & pyromaniac love & Title: Pyromaniac's Love Story, A (1995) \\
\hline 106 & castle freak & Title: Castle Freak (1995) \\
\hline 107 & waterworld & Title: Waterworld (1995) \\
\hline 108 & dolores claiborne & Rating: 3 Title: Dolores Claiborne (1995) \\
\hline 109 & $\begin{array}{c}\text { goofy movie } \\
(1995)\end{array}$ & Title: Goofy Movie, A (1995) \\
\hline 110 & little women & Title: Little Women (1994) \\
\hline 111 & Bedlam (1993) & Title: Beyond Bedlam (1993) \\
\hline 112 & Quiz (1994) & Title: Quiz Show (1994) \\
\hline 113 & Poison Ivy & Title: Poison Ivy II (1996) \\
\hline 114 & $\begin{array}{l}\text { title is quick and } \\
\text { dead } 1995\end{array}$ & Title: Quick and the Dead, The (1995) \\
\hline 115 & 25 Isaiah 1995 & Title: Losing Isaiah (1995) Age Range: 25-34 \\
\hline 116 & Enfer 1994 & Title: Enfer, L' (1994) \\
\hline 117 & before rain 1994 & Title: Before the Rain (Pred dozhdot) (1994) \\
\hline 118 & $\begin{array}{l}\text { programmer movie } \\
\text { of man } 1995\end{array}$ & Title: Dream Man (1995) Occupation: programmer \\
\hline 119 & 56 unzipped & Title: Unzipped (1995) Age Range: 56+ \\
\hline 120 & side boys (1995) & Title: Boys on the Side (1995) \\
\hline 121 & hoop dreams 1994 & Title: Hoop Dreams (1994) \\
\hline 122 & 1995 hunted & Title: Hunted, The (1995) \\
\hline 123 & jefferson 1995 & Title: Jefferson in Paris (1995) \\
\hline 124 & $\begin{array}{c}\text { Nobodys Fool } \\
1994\end{array}$ & Title: Nobody's Fool (1994) \\
\hline 125 & $\begin{array}{l}\text { academic movie of } \\
\text { hands } 1992\end{array}$ & $\begin{array}{l}\text { Title: Pushing Hands (Tui shou) (1992) Occupation: } \\
\text { academic/educator }\end{array}$ \\
\hline 126 & nuts 1994 & Title: Mixed Nuts (1994) \\
\hline 127 & 1994 Eden & Title: Exit to Eden (1994) \\
\hline 128 & 18 Kiss 1995 & Title: French Kiss (1995) Age Range: 18-24 \\
\hline 129 & $\begin{array}{c}3 \text { star } \\
\text { Bushwhacked }\end{array}$ & Rating: 3 Title: Bushwhacked (1995) \\
\hline
\end{tabular}




\begin{tabular}{|c|c|c|}
\hline 130 & 25 Paris 1995 & Title: Forget Paris (1995) Age Range: 25-34 \\
\hline 131 & death kiss 1995 & Title: Kiss of Death (1995) \\
\hline 132 & Ladybird & Title: Ladybird Ladybird (1994) \\
\hline 133 & nemesis 1995 & Title: Nemesis 2: Nebula (1995) \\
\hline 134 & $\begin{array}{l}\text { King Arthur } \\
\text { (1995) }\end{array}$ & Title: Kid in King Arthur's Court, A (1995) \\
\hline 135 & 5 star Eat (1994) & Rating: 5 Title: Eat Drink Man Woman (Yin shi nan nu) (1994) \\
\hline 136 & Pulp (1994) & Title: Pulp Fiction (1994) \\
\hline 137 & $\begin{array}{c}\text { Madness of } \\
\text { George } 1994 \\
\end{array}$ & Title: Madness of King George, The (1994) \\
\hline 138 & IQ 1994 & Title: I.Q. (1994) \\
\hline 139 & $\begin{array}{c}3 \text { star Roommates } \\
1995 \\
\end{array}$ & Rating: 3 Title: Roommates (1995) \\
\hline 140 & Beloved Immortal & Title: Immortal Beloved (1994) \\
\hline 141 & 18 Drop 1994 & Title: Drop Zone (1994) Age Range: 18-24 \\
\hline 142 & $\begin{array}{c}\text { Bill Madison } \\
(1995)\end{array}$ & Title: Billy Madison (1995) \\
\hline 143 & 3 star family 1995 & Rating: 3 Title: My Family (1995) \\
\hline 144 & Nell & Title: Nell (1994) \\
\hline 145 & fluke 1995 & Title: Fluke (1995) \\
\hline 146 & mi vida loco 1993 & Title: My Crazy Life (Mi vida loca) (1993) \\
\hline 147 & 4 star Outbreak & Rating: 3 Title: Outbreak (1995) \\
\hline 148 & Dumb 1994 & Title: Dumb \& Dumber (1994) \\
\hline 149 & $\begin{array}{c}\text { Natural Killers } \\
1994\end{array}$ & Title: Natural Born Killers (1994) \\
\hline 150 & Wild Billy 1995 & Title: Wild Bill (1995) \\
\hline 151 & 25 Exotic 1994 & Title: Exotica (1994) Age Range: 25-34 \\
\hline 152 & franky 1994 & Title: Frankenstein (Mary Shelley's Frankenstein) (1994) \\
\hline 153 & Priest & Title: Priest (1994) \\
\hline 154 & 3 star Wear 1994 & Rating: 3 Title: Ready to Wear (Pret-A-Porter) (1994) \\
\hline 155 & 35 demarco 1995 & Title: Don Juan DeMarco (1995) Age Range: 35-44 \\
\hline 156 & Gumby 1995 & Title: Gumby: The Movie (1995) \\
\hline 157 & 25 house man & Title: Man of the House (1995) Age Range: 25-34 \\
\hline 158 & 35 houseguest & Title: Houseguest (1994) Age Range: 35-44 \\
\hline 159 & Babysitter (1995) & Title: Babysitter, The (1995) \\
\hline 160 & 18 Under Siege & Title: Under Siege 2: Dark Territory (1995) Age Range: 18-24 \\
\hline 161 & $\begin{array}{c}\text { title is shield and } \\
\text { glass } 1994\end{array}$ & Title: Glass Shield, The (1994) \\
\hline 162 & Princess 1995 & Title: Little Princess, A (1995) \\
\hline 163 & money milk 1994 & Title: Milk Money (1994) \\
\hline 164 & Hideaway & Title: Hideaway (1995) \\
\hline
\end{tabular}




\begin{tabular}{|c|c|c|}
\hline 165 & $\begin{array}{l}\text { first of Murder } \\
1995\end{array}$ & Title: Murder in the First (1995) \\
\hline 166 & $\begin{array}{l}\text { 1 star Destiny } \\
\text { Radio } 1995\end{array}$ & Rating: 1 Title: Destiny Turns on the Radio (1995) \\
\hline 167 & Star Wars 1977 & $\begin{array}{l}\text { Rating: } 5 \text { Title: Star Wars: Episode IV - A New Hope (a.k.a. } \\
\text { Star Wars) (1977) }\end{array}$ \\
\hline 168 & $\begin{array}{l}\text { title is home and } \\
\text { adventures } 1995\end{array}$ & Title: Far From Home: The Adventures of Yellow Dog (1995) \\
\hline 169 & 25 Love 1994 & Title: Love Affair (1994) Age Range: 25-34 \\
\hline 170 & $\begin{array}{l}\text { Death and Maiden } \\
\text { (1994) }\end{array}$ & Title: Death and the Maiden (1994) \\
\hline 171 & 3 star Heroes 1995 & Rating: 3 Title: Unstrung Heroes (1995) \\
\hline 172 & $\begin{array}{l}45 \text { Disclosure } \\
(1994)\end{array}$ & Title: Disclosure (1994) Age Range: 45-59 \\
\hline 173 & Panther & Title: Panther (1995) \\
\hline 174 & 4 star clerks & Rating: 4 Title: Clerks (1994) \\
\hline 175 & 25 Junior 1994 & Title: Junior (1994) Age Range: 25-34 \\
\hline 176 & $\begin{array}{c}4 \text { star The Perez } \\
1995\end{array}$ & Rating: 4 Title: Perez Family, The (1995) \\
\hline 177 & 4 star cure & Rating: 4 Title: Cure, The (1995) \\
\hline 178 & $\begin{array}{l}\text { title is just and } \\
\text { cause } 1995\end{array}$ & Title: Just Cause (1995) \\
\hline 179 & You (1994) & Title: Only You (1994) \\
\hline 180 & 3 star Jerky Boys & Rating: 3 Title: Jerky Boys, The (1994) \\
\hline 181 & Cloud Walk 1995 & Title: Walk in the Clouds, A (1995) \\
\hline 182 & 45 Ed 1994 & Title: Ed Wood (1994) Age Range: 45-59 \\
\hline 183 & $\begin{array}{l}3 \text { star Jersey Drive } \\
1995\end{array}$ & Rating: 3 Title: New Jersey Drive (1995) \\
\hline 184 & $\begin{array}{l}18 \text { Fall Legends } \\
1994\end{array}$ & Title: Legends of the Fall (1994) Age Range: 18-24 \\
\hline 185 & $\begin{array}{l}\text { title is burden and } \\
\text { man } 1995\end{array}$ & Title: White Man's Burden (1995) \\
\hline 186 & 18 Heavenly 1994 & Title: Heavenly Creatures (1994) Age Range: 18-24 \\
\hline 187 & 56 Love Nina 1994 & Title: Nina Takes a Lover (1994) Age Range: 56+ \\
\hline 188 & $\begin{array}{l}3 \text { star Friends } \\
(1995)\end{array}$ & Rating: 3 Title: Circle of Friends (1995) \\
\hline 189 & $\begin{array}{l}\text { heavy weights } \\
(1995)\end{array}$ & Title: Heavyweights (a.k.a. Heavy Weights) (1995) \\
\hline 190 & $\begin{array}{l}\text { the professional } \\
1994\end{array}$ & Title: Léon: The Professional (Léon) (Professional, The) (1994) \\
\hline 191 & sun burnt (1994) & Title: Burnt by the Sun (Utomlyonnye solntsem) (1994) \\
\hline 192 & $\begin{array}{l}\text { title is water and } \\
\text { chocolate } 1992\end{array}$ & $\begin{array}{l}\text { Title: Like Water for Chocolate (Como agua para chocolate) } \\
\text { (1992) }\end{array}$ \\
\hline
\end{tabular}




\begin{tabular}{|c|c|c|}
\hline 193 & $\begin{array}{c}3 \text { star Warriors } \\
(1994)\end{array}$ & Rating: 3 Title: Once Were Warriors (1994) \\
\hline 194 & 45 Before Sunrise & Title: Before Sunrise (1995) Age Range: $45-59$ \\
\hline 195 & Little Odessa & Title: Little Odessa (1994) \\
\hline 196 & $\begin{array}{c}25 \text { The Browning } \\
1994\end{array}$ & Title: Browning Version, The (1994) Age Range: 25-34 \\
\hline 197 & 3 star Miami 1995 & Rating: 3 Title: Miami Rhapsody (1995) \\
\hline 198 & $\begin{array}{c}5 \text { star castrato } \\
(1994)\end{array}$ & Rating: 5 Title: Farinelli: il castrato (1994) \\
\hline 199 & 25 Bride 1994 & Title: Picture Bride (Bijo photo) (1994) Age Range: 25-34 \\
\hline 200 & is periscope down? & Title: down Periscope (1996) \\
\hline 201 & Jean seberg & Title: From the jornals of jean seberg (1995) \\
\hline 202 & $\begin{array}{l}1995 \text { man of the } \\
\text { year }\end{array}$ & Title: Man of the Year (1995) \\
\hline 203 & The bible & Title: Neon Bible, the (1995) \\
\hline 204 & jour of belle & Title: Belle de jour (1967) \\
\hline 205 & 24-35 and up close & Title: up close and personal (1996) age range: $24-35$ \\
\hline 206 & $\begin{array}{l}1996 \text { version of } \\
\text { birdcage }\end{array}$ & Title: Birdcage (1996) \\
\hline 207 & cienie executive & Title: shadows (Cienie) (1988) Occupation: executive \\
\hline 208 & gospa movie? & Title: Gospa (1995) \\
\hline 209 & mcmullen family & Title: Brothers McMullen, the (1995) \\
\hline 210 & first bad boys & Title: Bad Boys (1995) \\
\hline 211 & $\begin{array}{l}\text { movie with panda } \\
\text { in it }\end{array}$ & Title: Amazing Panda Adventures, the (1995) \\
\hline 212 & $\begin{array}{c}\text { basketball movie } \\
\text { in } 1995\end{array}$ & Title: Basketball Diaries, the (1995) \\
\hline 213 & $\begin{array}{c}\text { Big adventure of } \\
1995\end{array}$ & Title: Awfully Big Adventure, An (1995) \\
\hline 214 & who is an Amateur & Title: Amateur (1994) \\
\hline 215 & Apollo movie & Title: Apollo 13 (1995) \\
\hline 216 & Robbie Roy & Title: Rob Roy (1995) \\
\hline 217 & addiction movie & Title: Addiction, the (1995) \\
\hline 218 & $\begin{array}{c}\text { batman movie of } \\
1995\end{array}$ & Title : Batman forever (1995) \\
\hline 219 & $\begin{array}{c}\text { canadian bacon } \\
\text { movie }\end{array}$ & Title: canadian Bacon (1995) \\
\hline 220 & The Casper movie & Title: Casper (1995) \\
\hline 221 & $\begin{array}{c}\text { Submarine } \\
\text { Crimson Tide }\end{array}$ & Title: Crimson Tide (1995) \\
\hline 222 & face is blue & Title: Blue in the face (1995) \\
\hline 223 & $\begin{array}{c}\text { Die hard } 1995 \\
\text { movie }\end{array}$ & Title: Die Hard: With a Vengeance (1995) \\
\hline
\end{tabular}




\begin{tabular}{|c|c|c|}
\hline 224 & $\begin{array}{c}\text { our generaton is } \\
\text { doom }\end{array}$ & Title: Doom generation, The (1995) \\
\hline 225 & $\begin{array}{l}1995 \text { version of } \\
\text { first knight }\end{array}$ & Title: First Knight (1995) \\
\hline 226 & $\begin{array}{c}\text { second Free Willy } \\
\text { movie }\end{array}$ & Title: Free Willy 2: The advanture home(1995) \\
\hline 227 & Hacker movie & Title: Hackers (1995) \\
\hline 228 & $\begin{array}{c}\text { Mnemonic } 1995 \\
\text { version }\end{array}$ & Title: Johnny Mnemonic (1995) \\
\hline 229 & 1995 Judge Dredd & Title: Judge Dredd (1995) \\
\hline 230 & movie with kids & Title: Kids (1995) \\
\hline 231 & $\begin{array}{c}\text { Oblivion } 1995 \\
\text { movie }\end{array}$ & Title: Living in Oblivion (1995) \\
\hline 232 & $\begin{array}{c}\text { under } 18 \text { movie } \\
\text { with Lord }\end{array}$ & Title: Lord of Illusions (1995) Age Range: under 18 \\
\hline 233 & $\begin{array}{l}1993 \text { movie with } \\
\text { human reminas }\end{array}$ & Title: Love \& Human Remains (1993) \\
\hline 234 & $\begin{array}{c}1995 \text { movie on } \\
\text { love }\end{array}$ & Title: Mad Love (1995) \\
\hline 235 & MallRats & Title: MallRats(1995) \\
\hline 236 & $\begin{array}{c}\text { Power Ranges } \\
\text { movie }\end{array}$ & Title: Mighty Morphin Power Rangers: The Movie (1995) \\
\hline 237 & $\begin{array}{l}\text { valentino or } \\
\text { moonlight }\end{array}$ & Title: moonlight and valentino (1995) \\
\hline 238 & witness is mute & Title: Mute Witness (1995) \\
\hline 239 & Nadja & Title: Nadja \\
\hline 240 & $\begin{array}{c}3 \text { star movie on the } \\
\text { net }\end{array}$ & Title: Net, the (1995) Rating: 3 \\
\hline 241 & $\begin{array}{c}1995 \text { on Nine } \\
\text { Months }\end{array}$ & Title: Nine Months (1995) \\
\hline 242 & $\begin{array}{c}\text { Girl who likes } \\
\text { parties }\end{array}$ & Title: Party Girl (1995) \\
\hline 243 & Prophecy & Title: Prophecy, The (1995) \\
\hline 244 & Reckless movie & Title: reckless (1995) \\
\hline 245 & 5 star movie Safe & Title: Safe (1995) Rating: 5 \\
\hline 246 & $\begin{array}{c}\text { Scarlet Letter } \\
\text { movie }\end{array}$ & Title: Scarlet Letter, the (1995) \\
\hline 247 & 1995 show & Title: Show, the (1995) \\
\hline 248 & Showgirls & Title: Showgirls (1995) \\
\hline 249 & $\begin{array}{c}\text { movie named } \\
\text { Smoke? }\end{array}$ & Title: Smoke (1995) \\
\hline 250 & 1995 talk movie & Title: Something to talk about (1995) \\
\hline 251 & $\begin{array}{c}1995 \text { species } \\
\text { movie }\end{array}$ & Title: Species (1995) \\
\hline
\end{tabular}




\begin{tabular}{|c|c|c|}
\hline 252 & henrietta & Title: Stars fell on Henrietta, the (1995) \\
\hline 253 & $\begin{array}{c}\text { these are stange } \\
\text { days }\end{array}$ & Title: Strange Days (1995) \\
\hline 254 & $\begin{array}{l}\text { Parapluies of } \\
\text { cherourg }\end{array}$ & $\begin{array}{l}\text { Title: Umbrellas of Cherbourg, The (Parapluies de Cherbourg, } \\
\text { Les) (1964) }\end{array}$ \\
\hline 255 & Show with a quiz? & Title: Quiz Show (1994) \\
\hline 256 & Bijo Picture & Title: Picture Bride (Bijo photo) (1994) \\
\hline 257 & $\begin{array}{l}1994 \text { start trek } \\
\text { movie }\end{array}$ & Title: Star Trek: Generations (1994) \\
\hline 258 & $\begin{array}{c}\text { movie with an } \\
\text { idiot boy named } \\
\text { Tommy }\end{array}$ & Title: Tommy Boy (1994) \\
\hline 259 & Gilbert Grape & Tile: What's Eating Gilbert Grape (1993) \\
\hline 260 & $\begin{array}{l}1994 \text { movie with } \\
\text { war }\end{array}$ & Tile: War, the (1994) \\
\hline 261 & $\begin{array}{c}\text { does happiness } \\
\text { double? }\end{array}$ & Title: Double happiness (1994) \\
\hline 262 & $\begin{array}{l}\text { muriel goes to a } \\
\text { wedding }\end{array}$ & Title: Muriel's Wedding (1994) \\
\hline 263 & $\begin{array}{c}\text { A club for baby } \\
\text { sitters }\end{array}$ & Title: Baby-Sitters Club, The (1995) \\
\hline 264 & $\begin{array}{c}\text { Pet Detective } \\
\text { movie from } 1994\end{array}$ & Title: Ace Ventura: Pet Detective (1994) \\
\hline 265 & $\begin{array}{l}\text { Priscilla Queen of } \\
\text { Desert movie }\end{array}$ & Title: Adventures of Priscilla, Queen of the Desert, The (1994) \\
\hline 266 & $\begin{array}{l}\text { under } 18 \text { with a } \\
\text { moon }\end{array}$ & Title: Bitter Moon (1992) Age Range: under 18 \\
\hline 267 & $\begin{array}{l}\text { Bullets on } \\
\text { Broadway }\end{array}$ & Title: Bullets Over BroadWay (1994) \\
\hline 268 & Danger is it clear? & Title: Clear and Present Danger (1994) \\
\hline 269 & $\begin{array}{l}1994 \text { version of } \\
\text { client }\end{array}$ & Title: Client, the (1994) \\
\hline 270 & corrina & Title: Corrina, Corrina (1994) \\
\hline 271 & Crooklyn & Title: Crooklyn (1994) \\
\hline 272 & $\begin{array}{c}3 \text { start movie on } \\
\text { Crow }\end{array}$ & Title: Crow, The (1994) Rating: 3 \\
\hline 273 & $\begin{array}{c}\text { Flinstones movie } \\
\text { of } 1994\end{array}$ & Title: Flinstones, the (1994) \\
\hline 274 & Forest Gump & Title: Forest Gump (1994) \\
\hline 275 & $\begin{array}{l}\text { weddings and } \\
\text { funerals }\end{array}$ & Title: Four Weddings and a Funeral (1994) \\
\hline 276 & $\begin{array}{l}\text { I like it like that I } \\
\text { don't know how to } \\
\text { act? }\end{array}$ & Title: I like it like that (1994) \\
\hline
\end{tabular}




\begin{tabular}{|c|c|c|}
\hline 277 & is love trouble? & Title: I love trouble (1994) \\
\hline 278 & $\begin{array}{l}\text { could it happen to } \\
\text { you? }\end{array}$ & Title: It could happen to you (1994) \\
\hline 279 & $\begin{array}{l}1994 \text { verion of the } \\
\text { Jungle book }\end{array}$ & Title: Jungle Book, the (1994) \\
\hline 280 & $\begin{array}{l}\text { Leni Riefenstahl } \\
\text { movie }\end{array}$ & $\begin{array}{l}\text { Title: Wonderful, Horrible Life of Leni Riefenstahl, The (Macht } \\
\text { der Bilder: Leni Riefenstahl, Die) (1993) }\end{array}$ \\
\hline 281 & Macht der Bilder & $\begin{array}{l}\text { Title: Wonderful, Horrible Life of Leni Riefenstahl, The (Macht } \\
\text { der Bilder: Leni Riefenstahl, Die) (1993) }\end{array}$ \\
\hline 282 & $\begin{array}{l}\text { first lion king } \\
\text { movie }\end{array}$ & Title: Lion King, the (1994) \\
\hline 283 & $\begin{array}{l}\text { small verion of } \\
\text { Buddha }\end{array}$ & Title: Little Buddha (1993) \\
\hline 284 & Wes Craven & Title: Wes Craven's New Nightmare (1994) \\
\hline 285 & green mask movie? & Title: Mask, The (1994) \\
\hline 286 & $\begin{array}{l}1994 \text { maverick } \\
\text { movie }\end{array}$ & Title: Maverick (1994) \\
\hline 287 & $\begin{array}{l}\text { Parker and vicious } \\
\text { circle }\end{array}$ & Title: Mrs. Parker and the Vicious Circle (1994) \\
\hline 288 & $\begin{array}{l}\text { the naked gun } \\
\text { movie }\end{array}$ & Title: Naked Gun 331 \\
\hline 289 & 1994 paper movie & Title: Paper, The (1994) \\
\hline 290 & $\begin{array}{l}\text { does reality really } \\
\text { bite? }\end{array}$ & Title: Reality Bites (1994) \\
\hline 291 & west has red rocks & Title: Red Rock West (1992) \\
\hline 292 & Title: Richie Rick & Title: Richie Rich (1994) \\
\hline 293 & $\begin{array}{l}\text { passage safe to } \\
\text { pass? }\end{array}$ & Title: Safe Passage (1994) \\
\hline 294 & is the river wild & Title: River Wild, the (1994) \\
\hline 295 & 1994 speed movie & Title: Speed (1994) \\
\hline 296 & $\begin{array}{l}\text { this man was left } \\
\text { speechless }\end{array}$ & Title: speechless (1994) \\
\hline 297 & title was timecop & Title: Timecop (1994) \\
\hline 298 & the lies were true & Title: true lies (1994) \\
\hline 299 & $\begin{array}{l}\text { romance: man } \\
\text { loves a woman }\end{array}$ & Title: When a man loves a woman (1994) \\
\hline 300 & $\begin{array}{l}\text { scifi movie of a } \\
\text { wolf }\end{array}$ & Title: wolf (1994) \\
\hline 301 & $\begin{array}{l}\text { who is Wyatt } \\
\text { Earp? }\end{array}$ & Title: Wyatt Earp (1994) \\
\hline 302 & $\begin{array}{c}\text { the company was } \\
\text { bad }\end{array}$ & Title: Bad Company (1995) \\
\hline 303 & $\begin{array}{c}\text { no importance } \\
\text { movie }\end{array}$ & Title: Man of No Importance, A (1994) \\
\hline
\end{tabular}




\begin{tabular}{|c|c|c|}
\hline 304 & S.F.W. & Title: S.F.W (1994) \\
\hline 305 & $\begin{array}{l}\text { title was dirty } \\
\text { shame }\end{array}$ & Title: Low Down Dirty Shame, A (1994) \\
\hline 306 & life of a boy & Title: Boys Life (1995) \\
\hline 307 & $\begin{array}{l}\text { colonel Chabert } \\
\text { was a character }\end{array}$ & Title: Colonel Chabert, Le (1994) \\
\hline 308 & $\begin{array}{l}\text { movie regarding } \\
\text { pussycats }\end{array}$ & Title: Faster Pussycat! Kill! Kill! (1965) \\
\hline 309 & lyrics by jason & Title: Jason's Lyric (1994) \\
\hline 310 & $\begin{array}{l}\text { tom thumb } \\
\text { adventrues }\end{array}$ & Title: Secret Adventures of Tom Thumb, The (1993) \\
\hline 311 & $\begin{array}{l}1994 \text { edition of } \\
\text { street fighter }\end{array}$ & Title: Street Fighter (1994) \\
\hline 312 & $\begin{array}{c}2 \text { star movie called } \\
\text { coldblooded }\end{array}$ & Title: Coldblooded rating; 2 \\
\hline 313 & time to fall & Title: Fall time (1995) \\
\hline 314 & $\begin{array}{l}1995 \text { movie with } \\
\text { fear in title }\end{array}$ & Title: fear, the (1995) \\
\hline 315 & $\begin{array}{l}\text { ollie and frank } \\
\text { were in it }\end{array}$ & Title: Frank and Ollie (1995) \\
\hline 316 & 1995 homage & Title: Homage (1995) \\
\hline 317 & $\begin{array}{l}\text { programmer movie } \\
\text { called mirage }\end{array}$ & Title: Mirage (1995) occupation: programmer \\
\hline 318 & 1996 open season & Title: open season (1996) \\
\hline 319 & Dos crimes & Title: Two Crimes \\
\hline 320 & Highlander III & $\begin{array}{l}\text { Title: Highlander III: The Sorcerer (a.k.a. Highlander: The } \\
\text { Final Dimension) (1994) }\end{array}$ \\
\hline 321 & $\begin{array}{c}\text { federal hill } \\
\text { baltimore moive }\end{array}$ & Title: Federal Hill (1994) \\
\hline 322 & madness of 1995 & Title: mouth of madness \\
\hline 323 & movie 8 seconds & Title: 8 seconds (1994) \\
\hline 324 & $\begin{array}{c}\text { above the rim } \\
\text { movie }\end{array}$ & Title: above the rim(1994) \\
\hline 325 & addams family & Title: Addams family values (1993) \\
\hline 326 & am I so crazy? & Title: You so crazy (1994) \\
\hline 327 & innocence age & Title: Age of Innocence, the (1993) \\
\hline 328 & $\begin{array}{l}\text { movie of idiot } \\
\text { airheads }\end{array}$ & Title: Airheads (1994) \\
\hline 329 & $\begin{array}{c}\text { basketball air up } \\
\text { there }\end{array}$ & Title: air up there, the (1994) \\
\hline 330 & $\begin{array}{l}\text { the second } \\
\text { stakeout }\end{array}$ & Title: another stakeout (1994) \\
\hline 331 & are the girls bad? & Title: Bad Girls (1994) \\
\hline
\end{tabular}




\begin{tabular}{|c|c|c|}
\hline 332 & barcelona & Title: Barcelona (1994) \\
\hline 333 & $\begin{array}{l}\text { its just being } \\
\text { human }\end{array}$ & Title: being human (1993) \\
\hline 334 & $\begin{array}{l}\text { beverly hillbillies } \\
\text { movies }\end{array}$ & Title: Beverly Hillbillies, The (1993) \\
\hline 335 & $\begin{array}{l}\text { Beverly hills cop } \\
\text { number III }\end{array}$ & Title: Beverly Hills Cop III (1994) \\
\hline 336 & $\begin{array}{l}1994 \text { black beauty } \\
\text { movie }\end{array}$ & Title: Black Beauty (1994) \\
\hline 337 & $\begin{array}{l}3 \text { star movie } \\
\text { named blink }\end{array}$ & Title: Blink (1994) Rating: 3 \\
\hline 338 & $\begin{array}{l}\text { movie that was } \\
\text { blown away }\end{array}$ & Title: Blown Away (1994) \\
\hline 339 & $\begin{array}{l}\text { are those chips } \\
\text { blue? }\end{array}$ & Title: Blue Chips (1994) \\
\hline 340 & $\begin{array}{l}\text { the snatchers are } \\
\text { taking bodies }\end{array}$ & Title: Body Snatchers (1993) \\
\hline 341 & helena boxing? & Title: Boxing Helena (1993) \\
\hline 342 & tale of the bronx & Title: Bronx Tale, A (1993) \\
\hline 343 & boy in a cabin & Title: Cabin Boy (1994) \\
\hline 344 & girl in the calendar & Title: Calendar Girl (1993) \\
\hline 345 & way of carlito & Title: Carlito's Way (1993) \\
\hline 346 & $\begin{array}{c}\text { second city } \\
\text { slickers }\end{array}$ & Title: City Slickers II: The Legend of Curly's Gold (1994) \\
\hline 347 & $\begin{array}{l}3 \text { star movie called } \\
\text { clean slate }\end{array}$ & Title: clean slate Rating: 3 \\
\hline 348 & $\begin{array}{l}\text { movie is a } \\
\text { cliffhanger? }\end{array}$ & Title: Cliffhanger (1993) \\
\hline 349 & $\begin{array}{l}1993 \text { coneheads } \\
\text { movie }\end{array}$ & Title: coneheads (1993) \\
\hline 350 & night colors & Title: color of night (1994) \\
\hline 351 & cops and robbers & Title: Cops and Robbersons (1994) \\
\hline 352 & $\begin{array}{l}\text { what is the cowboy } \\
\text { way? }\end{array}$ & Title: Cowboy Way, The (1994) \\
\hline 353 & $\begin{array}{l}\text { is this game } \\
\text { dangerous? }\end{array}$ & Title: Dangerous Game (1993) \\
\hline 354 & dave? & Title: Dave (1993) \\
\hline 355 & $\begin{array}{l}\text { a man is dazed and } \\
\text { confused }\end{array}$ & Title: Dazed and Confused (1993) \\
\hline 356 & $\begin{array}{l}\text { man who is the } \\
\text { demolition }\end{array}$ & Title: Demolition Man (1993) \\
\hline 357 & $\begin{array}{l}\text { the summer that } \\
\text { was endless }\end{array}$ & Title: endless summer 2 , the (1994) \\
\hline 358 & movie about & Title: Even Cowgirls Get the Blues (1993) \\
\hline
\end{tabular}




\begin{tabular}{|c|c|c|}
\hline & cowgirls & \\
\hline 359 & ba wang bie ji & Title: Farewell My Concubine (Ba wang bie ji) (1993) \\
\hline 360 & are you fearless? & Title: fearless (1993) \\
\hline 361 & black hat fear & Title: fear of a black hat (1994) \\
\hline 362 & $\begin{array}{l}\text { college movie with } \\
\text { honors }\end{array}$ & Title: with Honors (1994) \\
\hline 363 & $\begin{array}{c}\text { see flesh and bone } \\
(1993)\end{array}$ & Title: flesh and bone (1993) \\
\hline 364 & $\begin{array}{l}\text { peak with a } \\
\text { window }\end{array}$ & Title: windows' Peak (1994) \\
\hline 365 & love or money & Title: For Love or Money (1993) \\
\hline 366 & the firm of 1993 & Title: Firm, The (1993) \\
\hline 367 & Free willy of 1993 & Title: Free willy (1993) \\
\hline 368 & $\begin{array}{l}\text { fugitive } 3 \text { star } \\
\text { rating }\end{array}$ & Title: Fugitive, the (1993) Rating: 3 \\
\hline 369 & Geronimo movies & Title: Geronimo: An American Legend (1993) \\
\hline 370 & $\begin{array}{c}\text { getting back with } \\
\text { Dad }\end{array}$ & Title: Getting Even with Dad (1994) \\
\hline 371 & $\begin{array}{l}\text { movie about going } \\
\text { fishing }\end{array}$ & Title: go fish (1994) \\
\hline 372 & man in Africa & Title: Good man in Africa, A (1994) \\
\hline 373 & I'm guilty as sin & Title: Guilty as Sin \\
\hline 374 & this target is hard & Title: Hard Target (1993) \\
\hline 375 & $\begin{array}{c}\text { heavean and Earth } \\
\text { (1993) }\end{array}$ & Title: Heaven \& Earth (1993) \\
\hline 376 & Hot Shots part two & Title: Hot Shots! Part Deux (1993) \\
\hline 377 & Nude Girls LIVE! & Title: Live Nude Girls (1995) \\
\hline 378 & $\begin{array}{l}\text { man who went up } \\
\text { a hil and down a } \\
\text { mountain }\end{array}$ & $\begin{array}{l}\text { Title: Englishman Who Went Up a Hill But Came Down a } \\
\text { Mountain, The (1995) }\end{array}$ \\
\hline 379 & spirits of a house & Title: House of the Spirits, The (1993) \\
\hline 380 & $\begin{array}{c}3 \text { house parties are } \\
\text { enough }\end{array}$ & Title: House Party 3 (1994) \\
\hline 381 & Hudsucker Proxy & Title: Hudsucker Proxy, The (1994) \\
\hline 382 & I'll do anything? & Title: I'll Do Anything (1994) \\
\hline 383 & Army Now! & Title: In the Army Now (1994) \\
\hline 384 & line of fire (1994) & Title: In the Line of Fire (1993) \\
\hline 385 & name of the father & Title: In the Name of the Father (1993) \\
\hline 386 & inkwell & title: Inkwell, The (1994) \\
\hline 387 & $\begin{array}{c}\text { love got to do with } \\
\text { it? }\end{array}$ & Title: What's Love Got to Do with It? (1993) \\
\hline 388 & $\begin{array}{c}\text { jimmy in } \\
\text { hollywood! }\end{array}$ & Title: Jimmy Hollywood (1994) \\
\hline
\end{tabular}




\begin{tabular}{|c|c|c|}
\hline 389 & night of judgement & Title: Judgement Night (1993) \\
\hline 390 & jurrassic park & Title: Jurrassic Park (1993) \\
\hline 391 & kalifornia & Title: kalifornia (1993) \\
\hline 392 & zoe killing & Title: Killing Zoe (1994) \\
\hline 393 & $\begin{array}{l}\text { King of the hill } \\
\text { movie }\end{array}$ & Title: King of the Hill (1993) \\
\hline 394 & $\begin{array}{l}\text { Lassie } 1994 \\
\text { version }\end{array}$ & Title: Lassie (1994) \\
\hline 395 & the last action hero & Title: Last Action hero (1993) \\
\hline 396 & mikey life & Title: Life with Mikey (1993) \\
\hline 397 & jack and Lightning & Title: Lightnining Jack \\
\hline 398 & $\begin{array}{c}\text { butterfly movie } \\
\text { that is } 2 \text { stars }\end{array}$ & Title: M. Butterfly Rating: 2 \\
\hline 399 & $\begin{array}{c}\text { made in america } \\
\text { movie }\end{array}$ & Title: made in America (1993) \\
\hline 400 & $\begin{array}{c}25 \text { to } 35 \text { malice } \\
\text { movie }\end{array}$ & Title: Malice (1993) Age Range: 25-35 \\
\hline 401 & $\begin{array}{c}\text { does that man have } \\
\text { a face? }\end{array}$ & Title: Man without a Face, the (1993) \\
\hline 402 & $\begin{array}{l}\text { a murder in } \\
\text { manhattan }\end{array}$ & Title: Manhattan Murder Mystery (1993) \\
\hline 403 & meanace to society & Title: Menace II Society (1993) \\
\hline 404 & $\begin{array}{c}\text { decisions by the } \\
\text { executive }\end{array}$ & Title: Executive Decisions (1996) \\
\hline 405 & Ai no corrida & Title: In the Realm of the Senses (Ai no corrida) (1976) \\
\hline 406 & $\begin{array}{c}\text { much ado about } \\
\text { nothing } 1993\end{array}$ & Title: Much Ado About Nothing (1993) \\
\hline 407 & who is Mr. Jones? & Title: Mr. Jones (1993) \\
\hline 408 & $\begin{array}{l}1993 \text { movie with } \\
\text { wonderful in title }\end{array}$ & Title: Mr. Wonderful (1993) \\
\hline 409 & $\begin{array}{c}\text { a woman named } \\
\text { doubtfire }\end{array}$ & Title: Mrs. Doubtfire (1993) \\
\hline 410 & $\begin{array}{c}\text { Naked movie of } \\
1993\end{array}$ & Title: Naked (1993) \\
\hline 411 & 1994 Karate Kid & Title: next Karate Kid, The (1994) \\
\hline 412 & new age movie & Title: New Age, The (1994) \\
\hline 413 & There is no escape & Title: No Escape (1994) \\
\hline 414 & movie in Orlando & Title: Orlando (1992) \\
\hline 415 & $\begin{array}{l}\text { it's a perfect } \\
\text { world! }\end{array}$ & Title: Perfect World, A (1993) \\
\hline 416 & $\begin{array}{c}\text { movie in } \\
\text { Philadelphia }\end{array}$ & Title: Philadelphia (1993) \\
\hline 417 & 2 star movie on & Title: Piano, The (1993) Rating: 2 \\
\hline
\end{tabular}




\begin{tabular}{|c|c|c|}
\hline & Pianos & \\
\hline 418 & tis Poetic Justic & Title: Poetic Justic (1993) \\
\hline 419 & $\begin{array}{l}\text { Movie about a } \\
\text { football Program } \\
\text { in } 1993\end{array}$ & Title: Program, The (1993) \\
\hline 420 & $\begin{array}{c}\text { Puppet Masters of } \\
1994\end{array}$ & Title: Puppet Masters, The (1994) \\
\hline 421 & $\begin{array}{l}\text { murders in } \\
\text { radioland }\end{array}$ & Title: Radioland Murders (1994) \\
\hline 422 & Day remains & Title: Remains of the Day, The (1993) \\
\hline 423 & $\begin{array}{c}\text { man in renaissance } \\
\text { era }\end{array}$ & Title: Renaissance Man (1994) \\
\hline 424 & Sun Rises & Title: Rising Sun (1993) \\
\hline 425 & Wellville Road & Title: Road to Wellville, The (1994) \\
\hline 426 & $\begin{array}{c}3 \text { star RoboCop } \\
\text { Movie }\end{array}$ & Title: RoboCop 3 (1993) \\
\hline 427 & $\begin{array}{l}\text { robin hood in } \\
\text { tights }\end{array}$ & Title: Robin Hood: Men in Tights (1993) \\
\hline 428 & bleeding romeo & Title: Romeo is Bleeding (1993) \\
\hline 429 & 5 star romper & Title: Romper Stomper (1993) Rating: 5 \\
\hline 430 & $\begin{array}{l}\text { Paradise with } \\
\text { Ruby }\end{array}$ & Title: Ruby in Paradise \\
\hline 431 & fort Washington & Title: Saint of Fort Washington, The (1993) \\
\hline 432 & Nuits fauve & Title: Savage Nights (Nuits fauves, Les) (1992) \\
\hline 433 & list of schindler & Title: Schindler's List (1993) \\
\hline 434 & Bobby Fisher & Title: Searching for Bobby Fischer (1993) \\
\hline 435 & Second best movie & Title: Second Best (1994) \\
\hline 436 & secret garden & Title: Secret Garden, The (1993) \\
\hline 437 & mom is serial & Title: Serial Mom (1994) \\
\hline 438 & shadowlands & Title: Shadowlands (1993) \\
\hline 439 & Short cuts of 1993 & Title: Short Cuts (1993) \\
\hline 440 & twist of fate & Title: Simple Twist of Fate, A (1994) \\
\hline 441 & Sirens & Title: Sirens (1994) \\
\hline 442 & Six separations & Title: Six degress of separations (1993) \\
\hline 443 & $\begin{array}{c}\text { seattle is sleepless } \\
\text { place }\end{array}$ & Title: Sleepless in Seattle (1993) \\
\hline 444 & runner is on blades & Title: Blade Runner (1982) \\
\hline 445 & my son in law & Title: Son in Law (1993) \\
\hline 446 & $\begin{array}{c}\text { Axe Murderer I } \\
\text { Married }\end{array}$ & Title: So I married an Axe Murderer (1993) \\
\hline 447 & $\begin{array}{c}\text { in Striking } \\
\text { Distance }\end{array}$ & Title: Striking Distance (1993) \\
\hline
\end{tabular}




\begin{tabular}{|c|c|c|}
\hline 448 & super mario & Title: super mario bros. (1993) \\
\hline 449 & Angus & Title: Angus (1995) \\
\hline 450 & Daens 5 & Title: Daens (1992) Rating: 5 \\
\hline 451 & Face & Title: Faces (1968) \\
\hline 452 & Boy & Title: Boys (1996) \\
\hline 453 & The Quest & Title: Quest, The (1996) \\
\hline 454 & Cosi & Title: Cosi (1996) \\
\hline 455 & Park Sunset & Title: Sunset Park (1996) \\
\hline 456 & Fall Mulholland & Title: Mulholland Falls (1996) \\
\hline 457 & Truth about cats & Title: Truth About Cats \& Dogs, The (1996) \\
\hline 458 & Company Oliver & Title: Oliver \& Company (1988) \\
\hline 459 & Pride of Celtic & Title: Celtic Pride (1996) \\
\hline 460 & Flipper & Title: Flipper (1996) \\
\hline 461 & Captive & Title: Captives (1994) \\
\hline 462 & Shadows of Love & Title: Of Love and Shadows (1994) \\
\hline 463 & Man Dead & Title: Dead Man (1995) \\
\hline 464 & Roof on Horseman & $\begin{array}{l}\text { Title: Horseman on the Roof, The (Hussard sur le toit, Le) } \\
\text { (1995) }\end{array}$ \\
\hline 465 & $\begin{array}{c}\text { Sister of } \\
\text { Switchblades }\end{array}$ & Title: Switchblade Sisters (1975) \\
\hline 466 & Mouth Mouth 1 & Title: Mouth to Mouth Rating: 1 \\
\hline 467 & les visiteurs 45-59 & Title: Visitors, The (Les Visiteurs) (1993) Age Range: $45-59$ \\
\hline 468 & The Multiplicity & Title: Multiplicity (1996) \\
\hline 469 & $\begin{array}{l}\text { Wallace Gromit } \\
\text { Animation }\end{array}$ & $\begin{array}{l}\text { Title: Wallace \& Gromit: The Best of Aardman Animation } \\
\text { (1996) }\end{array}$ \\
\hline 470 & $\begin{array}{l}\text { Halfmoon Bowles } \\
3\end{array}$ & Title: Halfmoon (Paul Bowles - Halbmond) (1995) \\
\hline 471 & $\begin{array}{l}\text { Edwards haunted } \\
\text { Jr. wood }\end{array}$ & Title: Haunted World of Edward D. Wood Jr., The (1996) \\
\hline 472 & Friends two 3 & Title: Two Friends (1986) Rating: 3 \\
\hline 473 & Craft $18-24$ & Title: Craft, The (1996) Age Range: $18-24$ \\
\hline 474 & The Hype 1 & Title: Great White Hype, The (1996) Rating: 1 \\
\hline 475 & Dance Last other & Title: Last Dance (1996) Occupation: other \\
\hline 476 & Stories of War & Title: War Stories (1995) \\
\hline 477 & $\begin{array}{l}\text { Cold Farm of } \\
\text { Comfort }\end{array}$ & Title: Cold Comfort Farm (1995) \\
\hline 478 & $\begin{array}{l}\text { People Dream of } \\
\text { Benjamenta }\end{array}$ & $\begin{array}{c}\text { Title: Institute Benjamenta, or This Dream People Call Human } \\
\text { Life (1995) }\end{array}$ \\
\hline 479 & life low!!!! 1 & Title: Low Life (1994) Rating: 1 \\
\hline 480 & $\begin{array}{l}\text { Prisoner of Heaven } \\
35-44\end{array}$ & Title: Heaven's Prisoners (1996) Age Range: 35-44 \\
\hline 481 & gangsta original & Title: Original Gangstas (1996) \\
\hline
\end{tabular}




\begin{tabular}{|c|c|c|}
\hline 482 & Rock 3 & Title: Rock, The (1996) Rating: 3 \\
\hline 483 & Murder Away 3 & Title: Getting Away With Murder (1996) Rating: 3 \\
\hline 484 & dellamorte man & Title: Cemetery Man (Dellamorte Dellamore) (1994) \\
\hline 485 & Twister & Title: Twister (1996) \\
\hline 486 & Wire Barb 2 Other & Title: Barb Wire (1996) Rating: 2 Occupation: other \\
\hline 487 & Moon Honey & Title: Honey Moon (Honigmond) (1996) \\
\hline 488 & Shell Ghost & Title: Ghost in the Shell (1995) \\
\hline 489 & Thinner 3 & Title: Thinner (1996) Rating: 3 \\
\hline 490 & Hard Spy & Title: Spy Hard (1996) \\
\hline 491 & Trouble Brother & Title: Brothers in Trouble (1995) \\
\hline 492 & gromit wallace 5 & Title: Wallace \& Gromit: A Close Shave (1995) Rating: 5 \\
\hline 493 & $\begin{array}{l}\text { Behind Enemy } \\
2001 \\
\end{array}$ & title:Behind Enemy Lines (2001) \\
\hline 494 & 1999 King $25 ?$ & title:Anna and the King (1999) age range:25-34 \\
\hline 495 & $\begin{array}{l}1963 \text { Terror } \\
\text { clearical admin! }\end{array}$ & title:Terror The (1963) occupation:clearical/admin \\
\hline 496 & 2006 Puffy Chair? & title:Puffy Chair The (2006) \\
\hline 497 & bars Girls 1994? & title:Bar Girls (1994) \\
\hline 498 & Karenina 1935 & title:Anna Karenina (1935) \\
\hline 499 & walk Man! & title:Walk Like a Man (1987) \\
\hline 500 & $\begin{array}{l}\text { The puppet } 1994 \\
56 \\
\end{array}$ & title:Puppet Masters The (1994) age range:56+ \\
\hline 501 & 2004 Yao 3425. & title:Year of the Yao The (2004) age range:25-34 \\
\hline 502 & $\begin{array}{c}1995 \text { Nemesis } \\
\text { other! }\end{array}$ & title:Nemesis 2: Nebula (1995) occupation:other \\
\hline 503 & $\begin{array}{c}1983 \text { Staying Alive } \\
1983 .\end{array}$ & title:Staying Alive (1983) \\
\hline 504 & Pigs 20012534. & title:Disco Pigs (2001) age range:25-34 \\
\hline 505 & Rabid $19771824 ?$ & title:Rabid (1977) age range: $18-24$ \\
\hline 506 & Window 2004! & title:Secret Window (2004) \\
\hline 507 & $\begin{array}{l}2005 \text { Waiting } 24 \\
18 .\end{array}$ & title:Waiting... (2005) age range: $18-24$ \\
\hline 508 & 2000 Pane e Tulips & title:Bread and Tulips (Pane e Tulipani) (2000) \\
\hline 509 & bedknobs 1971 ! & title:Bedknobs and Broomsticks (1971) \\
\hline 510 & 1986 Beyond. & title:From Beyond (1986) \\
\hline 511 & $\begin{array}{l}1981 \text { Looney } \\
\text { Movie }\end{array}$ & title:Looney Looney Looney Bugs Bunny Movie The (1981) \\
\hline 512 & $\begin{array}{c}\text { the Booth } \\
\text { executive } \\
\text { managerial? }\end{array}$ & $\begin{array}{l}\text { title:Man in the Glass Booth The (1975) } \\
\text { occupation:executive/managerial }\end{array}$ \\
\hline 513 & $\begin{array}{c}\text { santa clause } 2006 \\
1 ?\end{array}$ & title:Santa Clause 3: The Escape Clause The (2006) rating:1 \\
\hline
\end{tabular}




\begin{tabular}{|c|c|c|}
\hline 514 & Walkabout 1971 & title:Walkabout (1971) title:Walkabout (1971) \\
\hline 515 & 2003 Hulk 24. & title:Hulk (2003) age range:18-24 \\
\hline 516 & $\begin{array}{l}1969 \text { salesman } \\
\text { other! }\end{array}$ & title:Salesman (1969) occupation:other \\
\hline 517 & bunny scientist & title:How to Draw a Bunny (2002) occupation:scientist \\
\hline 518 & Crazy 1941! & title:Love Crazy (1941) \\
\hline 519 & Moon 1973 & title:Paper Moon (1973) \\
\hline 520 & 1980 Heavenly 25. & title:Oh Heavenly Dog! (1980) age range:25-34 \\
\hline 521 & $\begin{array}{c}\text { Praise love } \\
\text { clearical admin! }\end{array}$ & $\begin{array}{c}\text { title:In Praise of Love ( } i_{i}^{1} / 2 \text { loge de L'amour) (2001) } \\
\text { occupation:clearical/admin }\end{array}$ \\
\hline 522 & $\begin{array}{l}\text { smartest guy } 34 \\
25 .\end{array}$ & $\begin{array}{l}\text { title:Enron: The Smartest Guys in the Room (2005) age } \\
\text { range:25-34 }\end{array}$ \\
\hline 523 & 1996 Day 4. & title:One Fine Day (1996) rating:4 \\
\hline 524 & sleep 19962418. & title:Innocent Sleep The (1996) age range:18-24 \\
\hline 525 & rocky 2 & title:Rocky II (1979) rating:2 \\
\hline 526 & Rising 24 ? & title:Deep Rising (1998) age range:18-24 \\
\hline 527 & Dance cat other! & title:Cats Don't Dance (1997) occupation:other \\
\hline 528 & $\begin{array}{c}\text { match } \\
\text { programmer! }\end{array}$ & title:Match The (1999) occupation:programmer \\
\hline 529 & 2000 debut44 35? & title:Debut The (2000) age range:35-44 \\
\hline 530 & $\begin{array}{c}\text { Dream } 2002 \\
\text { college grad } \\
\text { student } \\
\end{array}$ & title:Pipe Dream (2002) occupation:college/grad student \\
\hline 531 & 1998 Giant. & title:My Giant (1998) \\
\hline 532 & 1991 critters $2 ?$ & title:Critters 3 (1991) rating:2 \\
\hline 533 & Nessuno 4 star & $\begin{array}{c}\text { title:My Name Is Nobody (Il Mio nome } \ddot{i ̈}_{i}^{1 / 2} \text { Nessuno) (1973) } \\
\text { rating:4 }\end{array}$ \\
\hline 534 & $\begin{array}{l}\text { Mummy } 1959 \\
\text { other. }\end{array}$ & title:Mummy The (1959) occupation:other \\
\hline 535 & knight Tale 4. & title:Knight's Tale A (2001) rating:4 \\
\hline 536 & apollo 1995 other! & title:Apollo 13 (1995) occupation:other \\
\hline 537 & Fingered freddy! & title:Freddy Got Fingered (2001) \\
\hline 538 & Reloaded matrix 2. & title:Matrix Reloaded The (2003) rating: 2 \\
\hline 539 & 1993 bronx $4 ?$ & title:Bronx Tale A (1993) rating:4 \\
\hline 540 & 2001 Bandits 3. & title:Bandits (2001) rating:3 \\
\hline 541 & 1983 flashdance $3 !$ & title:Flashdance (1983) rating:3 \\
\hline 542 & snapper 35. & title:Snapper The (1993) age range:35-44 \\
\hline 543 & Crush 2002 other & title:Blue Crush (2002) occupation:other \\
\hline 544 & $\begin{array}{l}\text { Mountain } 1963 \\
\text { artist! }\end{array}$ & title:Spencer's Mountain (1963) occupation:artist \\
\hline 545 & Rich Wife 3 & title:Rich Man's Wife The (1996) rating:3 \\
\hline 546 & big Knife 24 ! & title:Big Knife The (1955) age range: $18-24$ \\
\hline
\end{tabular}




\begin{tabular}{|c|c|c|}
\hline 547 & News 2001? & title:Shipping News The (2001) \\
\hline 548 & 2002 Tadpole 4. & title:Tadpole (2002) rating:4 \\
\hline 549 & Psycho 1986 & title:Psycho III (1986) \\
\hline 550 & waterdance age 59. & title:Waterdance The (1992) age range:45-59 \\
\hline 551 & Powers 19993 ? & title:Austin Powers: The Spy Who Shagged Me (1999) rating:3 \\
\hline 552 & $\begin{array}{l}\text { Club emperor } \\
\text { other. }\end{array}$ & title:Emperor's Club The (2002) occupation:other \\
\hline 553 & Sherlock Life 4. & title:Private Life of Sherlock Holmes The (1970) rating:4 \\
\hline 554 & $\begin{array}{l}\text { born on the fourth } \\
3 .\end{array}$ & title:Born on the Fourth of July (1989) rating:3 \\
\hline 555 & Jingle 1996 other & title:Jingle All the Way (1996) occupation:other \\
\hline 556 & 2000 Goulds 25! & title:Joe Gould's Secret (2000) age range:25-34 \\
\hline 557 & Edie Pen 1997. & title:Edie \& Pen (1997) \\
\hline 558 & Fright Night other & title:Fright Night Part II (1989) occupation:other \\
\hline 559 & Moneky 19944. & title:Spanking the Monkey (1994) rating:4 \\
\hline 560 & $\begin{array}{l}\text { canterbury } 1944 \\
\text { homemaker. }\end{array}$ & title:Canterbury Tale A (1944) occupation:homemaker \\
\hline 561 & $\begin{array}{c}\text { Land Before } 5 \\
\text { star! }\end{array}$ & title:Land Before Time The (1988) rating:5 \\
\hline 562 & battle of sexes 4 & title:Battle of the Sexes The (1959) rating:4 \\
\hline 563 & silver 1996 other! & title:Forgotten Silver (1996) occupation:other \\
\hline 564 & 1998 siege $18 ?$ & title:Siege The (1998) age range:18-24 \\
\hline 565 & Lolita 19974. & title:Lolita (1997) rating:4 \\
\hline 566 & 1973 Shamus 4 & title:Shamus (1973) rating:4 \\
\hline 567 & 1987 cherry 3. & title:Cherry 2000 (1987) rating:3 \\
\hline 568 & 2007 blood other. & title:Blood and Chocolate (2007) occupation:other \\
\hline 569 & Romeo 1996 ! & title:William Shakespeare's Romeo + Juliet (1996) \\
\hline 570 & $\begin{array}{l}1983 \text { trading } \\
\text { places other. }\end{array}$ & title:Trading Places (1983) occupation:other \\
\hline 571 & $\begin{array}{c}\text { The grey zone } \\
\text { rating } 4 .\end{array}$ & title:Grey Zone The (2001) rating:4 \\
\hline 572 & $\begin{array}{l}\text { Zoom clearical } \\
\text { admin! }\end{array}$ & title:Zoom (2006) occupation:clearical/admin \\
\hline 573 & dumb rating 2 & title:Dumb \& Dumber (1994) rating:2 \\
\hline 574 & $\begin{array}{l}\text { the Bobby Soxer } \\
5 ?\end{array}$ & title:Bachelor and the Bobby-Soxer The (1947) rating:5 \\
\hline 575 & Patriot Games. & title:Patriot Games (1992) \\
\hline 576 & image 2004 other & title:Look at Me (Comme une image) (2004) occupation:other \\
\hline 577 & $\begin{array}{l}1981 \text { Out blow } \\
1981 \text { Out! }\end{array}$ & title:Blow Out (1981) \\
\hline 578 & $\begin{array}{l}\text { sommaren med } 18 \\
24 !\end{array}$ & $\begin{array}{l}\text { title:Summer With Monika (Sommaren med Monika) (1953) } \\
\text { age range:18-24 }\end{array}$ \\
\hline
\end{tabular}




\begin{tabular}{|c|c|c|}
\hline 579 & 2007 Die Hard 25. & title:Live Free or Die Hard (2007) age range:25-34 \\
\hline 580 & $\begin{array}{c}1990 \text { love death } \\
\text { academic } \\
\text { educator! }\end{array}$ & title:I Love You to Death (1990) occupation:academic/educator \\
\hline 581 & $\begin{array}{l}\text { The Christ of } \\
\text { passion! }\end{array}$ & title:Passion of the Christ The (2004) \\
\hline 582 & Secretos Heart 4 & $\begin{array}{c}\left.\text { title:Secrets of the Heart (Secretos del Corazï } i^{1 / 2 n}\right)(1997) \\
\text { rating:4 }\end{array}$ \\
\hline 583 & $\begin{array}{l}2008 \text { stars Wars } 18 \\
24 ?\end{array}$ & title:Star Wars: The Clone Wars (2008) age range:18-24 \\
\hline 584 & $\begin{array}{l}\text { Pick of destinyin } \\
2006\end{array}$ & title:Tenacious D in The Pick of Destiny (2006) \\
\hline 585 & big jake 1971 ! & title:Big Jake (1971) \\
\hline 586 & $\begin{array}{l}1957 \text { three faces } \\
\text { other? }\end{array}$ & title:Three Faces of Eve The (1957) occupation:other \\
\hline 587 & forever Young 3? & title:Forever Young (1992) rating:3 \\
\hline 588 & Club Vista other & title:Buena Vista Social Club (1999) occupation:other \\
\hline 589 & 1992 Baraka other. & title:Baraka (1992) occupation:other \\
\hline 590 & 1954 Them other. & title:Them! (1954) occupation:other \\
\hline 591 & $\begin{array}{l}\text { Gardener } 2005 \\
\text { other? }\end{array}$ & title:Constant Gardener The (2005) occupation:other \\
\hline 592 & $\begin{array}{l}1991 \text { Dead Again } \\
\text { other. }\end{array}$ & title:Dead Again (1991) occupation:other \\
\hline 593 & Hulk other? & title:Hulk (2003) occupation:other \\
\hline 594 & 2007 Young Heart & title:Young at Heart (a.k.a. Young@Heart) (2007) rating:1 \\
\hline 595 & $\begin{array}{l}\text { Day I Died college } \\
\text { grad }\end{array}$ & $\begin{array}{c}\text { title:I Woke Up Early the Day I Died (1998) } \\
\text { occupation:college/grad student }\end{array}$ \\
\hline 596 & $\begin{array}{l}\text { Robinson } 1960 \\
\text { other }\end{array}$ & title:Swiss Family Robinson (1960) occupation:other \\
\hline 597 & brid Prey 1996 & title:Bird of Prey (1996) \\
\hline 598 & $\begin{array}{l}\text { Rangers } 200135 \\
44\end{array}$ & title:Texas Rangers (2001) age range:35-44 \\
\hline 599 & 1967 Cougar $56 !$ & title:Charlie the Lonesome Cougar (1967) age range:56+ \\
\hline 600 & blade 3 . & title:Blade II (2002) rating:3 \\
\hline 601 & $\begin{array}{l}2001 \text { brothers } 25 \\
34 ?\end{array}$ & title:Brothers The (2001) age range:25-34 \\
\hline 602 & 2003 bunny other! & title:Brown Bunny The (2003) occupation:other \\
\hline 603 & The Cradle 4! & title:Hand That Rocks the Cradle The (1992) rating:4 \\
\hline 604 & Altered States. & title:Altered States (1980) \\
\hline 605 & $\begin{array}{c}\text { 2001 Joe } \\
\text { Somebody } 59 ! \\
\end{array}$ & title:Joe Somebody (2001) age range:45-59 \\
\hline 606 & $\begin{array}{l}1982 \text { Night Shift } \\
\text { 1982? }\end{array}$ & title:Night Shift (1982) \\
\hline
\end{tabular}




\begin{tabular}{|c|c|c|}
\hline 607 & The Brawl 3! & title:Big Brawl The (1980) rating:3 \\
\hline 608 & Rush 2 Hour & title:Rush Hour 2 (2001) title:Rush Hour 2 (2001) \\
\hline 609 & Places 1983 ? & title:Trading Places (1983) \\
\hline 610 & $\begin{array}{c}2002 \text { Showtime } 24 \\
18\end{array}$ & title:Showtime (2002) age range:18-24 \\
\hline 611 & $\begin{array}{c}1994 \text { Enfer L' } \\
\text { academic } \\
\text { educator? }\end{array}$ & title:Enfer L' (1994) occupation:academic/educator \\
\hline 612 & Eaten Alive 1976. & title:Eaten Alive (1976) \\
\hline 613 & 2001 Zo lawyer! & title:Zus \& Zo (2001) occupation:lawyer \\
\hline 614 & Lovely 1997. & title:She's So Lovely (1997) \\
\hline 615 & Myself 2000 other! & title:Me Myself I (2000) occupation:other \\
\hline 616 & big Fish 35. & title:Big Fish (2003) age range:35-44 \\
\hline 617 & King anna other. & title:Anna and the King (1999) occupation:other \\
\hline 618 & 1997 Hunt 24 & title:Mouse Hunt (1997) age range:18-24 \\
\hline 619 & Pleasure company? & title:Pleasure of Your Company The (2006) \\
\hline 620 & Rosenstrasse 2003 & title:Rosenstrasse (2003) \\
\hline 621 & Home Voyage Star & title:Star Trek IV: The Voyage Home (1986) \\
\hline 622 & Express 20042. & title:Secuestro Express (2004) rating:2 \\
\hline 623 & $\begin{array}{l}\text { Dinosaurs Back } \\
\text { other! }\end{array}$ & title:We're Back! A Dinosaur's Story (1993) occupation:other \\
\hline 624 & $\begin{array}{l}\text { the game } 1939 \\
\text { other }\end{array}$ & $\begin{array}{c}\text { title:Rules of the Game The (La Rï }{ }^{1 / 2} \text { gle du jeu) (1939) } \\
\text { occupation:other }\end{array}$ \\
\hline 625 & $\begin{array}{l}\text { marjorie } 195818 \\
24 .\end{array}$ & title:Marjorie Morningstar (1958) age range:18-24 \\
\hline 626 & $\begin{array}{l}2003 \text { Temptations } \\
\text { other? }\end{array}$ & title:Fighting Temptations The (2003) occupation:other \\
\hline 627 & $\begin{array}{l}\text { Man goes home } \\
1945 \text { other }\end{array}$ & title:Thin Man Goes Home The (1945) occupation:other \\
\hline 628 & band hand 2 ! & title:Band of the Hand (1986) rating:2 \\
\hline 629 & 1953 Heat & title:Big Heat The (1953) \\
\hline 630 & $\begin{array}{l}1987 \text { Prom Night } 3 \\
\text { star? }\end{array}$ & title:Hello Mary Lou: Prom Night II (1987) rating:3 \\
\hline 631 & $\begin{array}{l}1954 \text { window } 34 \\
25 .\end{array}$ & title:Rear Window (1954) age range:25-34 \\
\hline 632 & Have eye 4 & title:Hills Have Eyes The (2006) rating:4 \\
\hline 633 & Sight 2007 artist! & title:No End in Sight (2007) occupation:artist \\
\hline 634 & $\begin{array}{l}\text { Donors brain } \\
\text { other? }\end{array}$ & title:Brain Donors (1992) occupation:other \\
\hline 635 & man 2005 other! & title:Man The (2005) occupation:other \\
\hline 636 & $\begin{array}{l}\text { G.I. Joe doctor } \\
\text { health. }\end{array}$ & title:Story of G.I. Joe The (1945) occupation:doctor/health care \\
\hline 637 & Darling 1965! & title:Darling (1965) \\
\hline
\end{tabular}




\begin{tabular}{|c|c|c|}
\hline 638 & 1990 twister & title:Twister (1990) rating:2 \\
\hline 639 & 1982 soldier? & title:Soldier The (1982) rating:4 \\
\hline 640 & jason Lives other. & $\begin{array}{l}\text { title:Friday the 13th Part VI: Jason Lives (1986) } \\
\text { occupation:other }\end{array}$ \\
\hline 641 & Tigers 4 star & title:Go Tigers! (2001) rating:4 \\
\hline 642 & 2007 Halloween & title:Halloween (2007) \\
\hline 643 & $\begin{array}{l}1981 \text { mephisto } \\
\text { other! }\end{array}$ & title:Mephisto (1981) occupation:other \\
\hline 644 & get Real other? & title:Get Real (1998) occupation:other \\
\hline 645 & $\begin{array}{l}1998 \text { Century } \\
\text { college grad? }\end{array}$ & $\begin{array}{l}\text { title:Goodbye 20th Century (Zbogum na dvadesetiot vek) } \\
\text { (1998) occupation:college/grad student }\end{array}$ \\
\hline 646 & Wish 197434 & title:Death Wish (1974) age range:25-34 \\
\hline 647 & 1966 Harper. & title:Harper (1966) \\
\hline 648 & My voice 5! & title:Year My Voice Broke The (1987) rating:5 \\
\hline 649 & It Hot 1959 other! & title:Some Like It Hot (1959) occupation:other \\
\hline 650 & $\begin{array}{l}\text { Little woman } 1949 \\
24\end{array}$ & title:Little Women (1949) age range:18-24 \\
\hline 651 & $\begin{array}{l}\text { Don monde Le } \\
\text { Camillo? }\end{array}$ & $\begin{array}{l}\text { title:Little World of Don Camillo The (Le Petit monde de Don } \\
\text { Camillo) (1953) }\end{array}$ \\
\hline 652 & $\begin{array}{l}\text { Philadelphia } 1993 \\
\text { other! }\end{array}$ & title:Philadelphia Experiment II (1993) occupation:other \\
\hline 653 & Twice Live 4 ? & title:You Only Live Twice (1967) rating:4 \\
\hline 654 & 1971 apes rating $3 !$ & title:Escape from the Planet of the Apes (1971) rating:3 \\
\hline 655 & 1964 Lilith4 & title:Lilith (1964) rating:4 \\
\hline 656 & Heart 2000! & title:Where the Heart Is (2000) \\
\hline 657 & 2000 Lakeboat & title:Lakeboat (2000) \\
\hline 658 & 2000 turn up 35. & title:Turn It Up (2000) age range:35-44 \\
\hline 659 & Cannibal Ferox 2. & title:Make Them Die Slowly (Cannibal Ferox) (1981) rating:2 \\
\hline 660 & 1981 Tattoo & title:Tattoo (1981) \\
\hline 661 & 1987 wish 5945 ! & title:Wish You Were Here (1987) age range:45-59 \\
\hline 662 & Hooper 19783 ! & title:Hooper (1978) rating:3 \\
\hline 663 & Poison 1991. & title:Poison (1991) \\
\hline 664 & $\begin{array}{c}\text { Bad News Bears } \\
197634\end{array}$ & title:Bad News Bears The (1976) age range:25-34 \\
\hline 665 & $\begin{array}{l}\text { Man vegas age } 24 \\
18 ?\end{array}$ & title:I Shot a Man in Vegas (1995) age range:18-24 \\
\hline 666 & $\begin{array}{l}2006 \text { Calls } \\
\text { Stranger }\end{array}$ & title:When a Stranger Calls (2006) \\
\hline 667 & Time aka 34. & $\begin{array}{c}\text { title:Anything But Love (a.k.a. Standard Time) (2002) age } \\
\text { range:25-34 }\end{array}$ \\
\hline 668 & $\begin{array}{l}2008 \text { rating } 5 \\
\text { sleepwalking. }\end{array}$ & title:Sleepwalking (2008) rating:5 \\
\hline
\end{tabular}




\begin{tabular}{|c|c|c|}
\hline 669 & $\begin{array}{c}\text { The proprietor } 24 \\
18 .\end{array}$ & title:Proprietor The (1996) age range: $18-24$ \\
\hline 670 & $\begin{array}{c}1996 \text { Mans age } 44 \\
35 ?\end{array}$ & title:Rich Man's Wife The (1996) age range:35-44 \\
\hline 671 & $\begin{array}{l}\text { Quadrophenia } 4 \\
\text { stars! }\end{array}$ & title:Quadrophenia (1979) rating:4 \\
\hline 672 & 1987 Must 2534 & title:Surf Nazis Must Die (1987) age range:25-34 \\
\hline 673 & $\begin{array}{c}1990 \text { Condition } \\
\text { academic educator. }\end{array}$ & title:Heart Condition (1990) occupation:academic/educator \\
\hline 674 & 2000 contender! & title:Contender The (2000) \\
\hline 675 & $\begin{array}{c}2006 \text { sentinel } \\
\text { audience other? }\end{array}$ & title:Sentinel The (2006) occupation:other \\
\hline 676 & $\begin{array}{c}2000 \text { battlefield } \\
\text { Earth. }\end{array}$ & title:Battlefield Earth (2000) \\
\hline 677 & $\begin{array}{c}\text { Girls } 2003 \text { rating } \\
\text { of } 5 .\end{array}$ & title:Calendar Girls (2003) rating:5 \\
\hline 678 & $\begin{array}{c}\text { Oglala at academic } \\
\text { educator? }\end{array}$ & title:Incident at Oglala (1992) occupation:academic/educator \\
\hline 679 & $\begin{array}{c}\text { Year woman } 1942 \\
!\end{array}$ & title:Woman of the Year (1942) \\
\hline 680 & $\begin{array}{c}\text { treasure Island } \\
1950 \\
\end{array}$ & title:Treasure Island (1950) \\
\hline 681 & Heart rating of 5 ! & title:Angel Heart (1987) rating:5 \\
\hline 682 & 1957 face crowd $4 !$ & title:Face in the Crowd A (1957) rating:4 \\
\hline 683 & $\begin{array}{l}\text { heaven discovery } \\
\text { ages } 56\end{array}$ & title:Discovery of Heaven The (2001) age range:56+ \\
\hline 684 & 2001 News other? & title:Shipping News The (2001) occupation:other \\
\hline 685 & 1989 future 3 ! & title:Back to the Future Part II (1989) rating:3 \\
\hline 686 & $\begin{array}{l}\text { Henderson } \\
\text { Presents }\end{array}$ & title:Mrs. Henderson Presents (2005) \\
\hline 687 & $\begin{array}{l}1996 \text { love ages of } \\
3544 !\end{array}$ & title:Love and Other Catastrophes (1996) age range:35-44 \\
\hline 688 & losin' it $19832 ?$ & title:Losin' It (1983) rating:2 \\
\hline 689 & $\begin{array}{l}\text { Love got to do } \\
\text { other! }\end{array}$ & title:What's Love Got to Do with It? (1993) occupation:other \\
\hline 690 & gangster 20004. & title:Gangster No. 1 (2000) rating:4 \\
\hline 691 & $\begin{array}{c}2002 \text { orange } \\
\text { count! }\end{array}$ & title:Orange County (2002) \\
\hline 692 & 1995 Brooklyn & title:Vampire in Brooklyn (1995) \\
\hline 693 & The Crown 2418 & title:Thomas Crown Affair The (1999) age range:18-24 \\
\hline 694 & $\begin{array}{l}2001 \text { Dog Day } \\
\text { retired. }\end{array}$ & title:Dog Days (Hundstage) (2001) occupation:retired \\
\hline 695 & 2006 Queen 56! & title:Queen The (2006) age range:56+ \\
\hline 696 & 1988 The & title:Moderns The (1988) \\
\hline
\end{tabular}




\begin{tabular}{|c|c|c|}
\hline & moderns! & \\
\hline 697 & Mystic Pizza 3! & title:Mystic Pizza (1988) rating:3 \\
\hline 698 & Captives 19943. & title:Captives (1994) rating:3 \\
\hline 699 & 1996 Wild ride & $\begin{array}{l}\text { title:Mr. Toad's Wild Ride (a.k.a. The Wind in the Willows) } \\
\text { (1996) }\end{array}$ \\
\hline 700 & 1952 Ikiru 3425 & title:Ikiru (1952) age range:25-34 \\
\hline 701 & grudge 2004 other? & title:Grudge The (2004) occupation:other \\
\hline 702 & Die For ages $35 !$ & title:To Die For (1995) age range:35-44 \\
\hline 703 & $\begin{array}{l}1983 \text { version Class } \\
5 \text { star }\end{array}$ & title:Class (1983) rating:5 \\
\hline 704 & swiri 19993. & title:Shiri (Swiri) (1999) rating:3 \\
\hline 705 & the Shakes 5 ? & title:Wind That Shakes the Barley The (2006) rating:5 \\
\hline 706 & Game 19913 ! & title:Talent for the Game (1991) rating:3 \\
\hline 707 & coeur Roi 45! & title:King of Hearts (Le Roi de coeur) (1966) age range:45-59 \\
\hline 708 & $\begin{array}{c}\text { Michael Clayton } \\
\text { other! }\end{array}$ & title:Michael Clayton (2007) occupation:other \\
\hline 709 & 1983 Valor $18 !$ & title:Uncommon Valor (1983) age range: $18-24$ \\
\hline 710 & $\begin{array}{l}\text { Lighthouse } \\
\text { technician } \\
\text { engineer? }\end{array}$ & $\begin{array}{l}\text { title:Under the Lighthouse Dancing (1997) } \\
\text { occupation:technician/engineer }\end{array}$ \\
\hline 711 & $\begin{array}{l}\text { sudden death } 1995 \\
\text { other! }\end{array}$ & title:Sudden Death (1995) occupation:other \\
\hline 712 & Feng Moon 3 ? & title:Temptress Moon (Feng Yue) (1996) rating:3 \\
\hline 713 & Potter and other. & $\begin{array}{l}\text { title:Harry Potter and the Prisoner of Azkaban (2004) } \\
\text { occupation:other }\end{array}$ \\
\hline 714 & Qiu of si of! & title:Story of Qiu Ju The (Qiu Ju da guan si) (1992) \\
\hline 715 & 2005 are we there? & title:Are We There Yet? (2005) \\
\hline 716 & $\begin{array}{c}2000 \text { crew } \\
\text { clearical admin? }\end{array}$ & title:Crew The (2000) occupation:clearical/admin \\
\hline 717 & $\begin{array}{l}\text { league Gentlemen } \\
3 ?\end{array}$ & title:League of Extraordinary Gentlemen The (2003) rating:3 \\
\hline 718 & hunted 2003 & title:Hunted The (2003) \\
\hline 719 & 2002 Passionada & title:Passionada (2002) \\
\hline 720 & $\begin{array}{l}\text { beyond Borders } \\
\text { academiceducator }\end{array}$ & title:Beyond Borders (2003) occupation:academic/educator \\
\hline 721 & $\begin{array}{l}\text { "80" } 1983 \text { age: } 44 \\
35\end{array}$ & title:Star 80 (1983) age range:35-44 \\
\hline 722 & $\begin{array}{l}\text { Women femmes } \\
\text { ages: } 3425 \text {. }\end{array}$ & title: 8 Women ( 8 femmes) (2002) age range:25-34 \\
\hline 723 & Purple Rain other? & title:Purple Rain (1984) occupation:other \\
\hline 724 & $\begin{array}{c}1995 \text { walking dead } \\
\text { other? }\end{array}$ & title:Walking Dead The (1995) occupation:other \\
\hline 725 & swing Time 1936 & title:Swing Time (1936) \\
\hline
\end{tabular}




\begin{tabular}{|c|c|c|}
\hline 726 & $\begin{array}{l}\text { stelle maker } 4 \\
\quad \text { stars? }\end{array}$ & title:Star Maker The (L'Uomo delle stelle) (1995) rating:4 \\
\hline 727 & 1996 sunset park? & title:Sunset Park (1996) \\
\hline 728 & Gothic 3 stars & title:Gothic (1986) rating:3 \\
\hline 729 & $\begin{array}{l}1988 \text { Memories of } \\
\text { Me }\end{array}$ & title:Memories of Me (1988) \\
\hline 730 & 1944 Frankenstein & title:House of Frankenstein (1944) \\
\hline 731 & 1961 Devil 4! & title:Devil at 4 O'Clock The (1961) \\
\hline 732 & $\begin{array}{l}\text { Witchboard } 1986 \\
\text { clearical admin! }\end{array}$ & title:Witchboard (1986) occupation:clearical/admin \\
\hline 733 & $\begin{array}{l}\text { Doll yr } 20003 \\
\text { star? }\end{array}$ & title:Russian Doll (2000) rating:3 \\
\hline 734 & Beast beauty other. & $\begin{array}{l}\text { title:Beauty and the Beast (Belle et la bï } i^{1 / 2 \text { te La) }}(1946) \\
\text { occupation:other }\end{array}$ \\
\hline 735 & 1986 Liberty. & title:Sweet Liberty (1986) \\
\hline 736 & 1979 brood? & title:Brood The (1979) \\
\hline 737 & 1980 the other! & title:Raise the Titanic (1980) occupation:other \\
\hline 738 & 2001Nobody Baby & title:Nobody's Baby (2001) \\
\hline 739 & Dina 2002 & title:I Am Dina (2002) \\
\hline 740 & Sleuth 34 ! & title:Sleuth (1972) age range:25-34 \\
\hline 741 & $\begin{array}{c}\text { Musketeers } 1948 \\
\text { college grad } \\
\text { student? }\end{array}$ & $\begin{array}{c}\text { title:Three Musketeers The (1948) occupation:college/grad } \\
\text { student }\end{array}$ \\
\hline 742 & $\begin{array}{l}\text { Barrels smoke } \\
\quad \text { stars } 5 !\end{array}$ & title:Lock Stock \& Two Smoking Barrels (1998) rating:5 \\
\hline 743 & 1992 New Gun 44. & title:My New Gun (1992) age range:35-44 \\
\hline 744 & $\begin{array}{l}\text { The } 2001 \text { Wasnt } \\
\text { There. }\end{array}$ & title:Man Who Wasn't There The (2001) \\
\hline 745 & $\begin{array}{l}\text { The } 2008 \text { The } \\
\text { Chronicles? }\end{array}$ & $\begin{array}{c}\text { title:Spiderwick Chronicles The (2008) title:Spiderwick } \\
\text { Chronicles The (2008) }\end{array}$ \\
\hline 746 & 1981 Story 24. & title:Ghost Story (1981) age range: $18-24$ \\
\hline 747 & 1952 Not Married! & title:We're Not Married! (1952) \\
\hline 748 & Bad Things 1998 & title:Very Bad Things (1998) \\
\hline 749 & Le 19954 star? & title:Garï ${ }^{1} 1 / 2 u$ Le (1995) rating:4 \\
\hline 750 & 2004 secuestro & title:Secuestro Express (2004) \\
\hline 751 & $\begin{array}{l}\text { taxi year } 2000 \\
\text { ages } 3544 ?\end{array}$ & title:Taxi 2 (2000) age range:35-44 \\
\hline 752 & $\begin{array}{l}\text { Right One } \\
\text { academic } \\
\text { educator? }\end{array}$ & 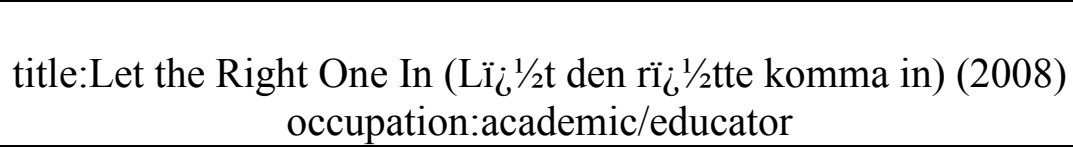 \\
\hline 753 & 1994 inoubliable 3 & $\begin{array}{l}\text { title:Unforgettable Summer An (Un } \ddot{i} i^{1 / 2} \text { tï } i^{1 / 2} \text { inoubliable) } \\
\text { (1994) rating:3 }\end{array}$ \\
\hline
\end{tabular}




\begin{tabular}{|c|c|c|}
\hline 754 & $\begin{array}{l}\text { Made for each } \\
\text { other stars: } 4 .\end{array}$ & title:Made for Each Other (1939) rating:4 \\
\hline 755 & $\begin{array}{c}\text { condemned } 2007 \\
24 .\end{array}$ & title:Condemned The (2007) age range:18-24 \\
\hline 756 & $\begin{array}{l}1987 \text { bedroom } \\
\text { Window! }\end{array}$ & title:Bedroom Window The (1987) \\
\hline 757 & $\begin{array}{c}1967 \\
\text { Collectionneuse59 } \\
45 ?\end{array}$ & title:Collectionneuse La (1967) age range:45-59 \\
\hline 758 & Moonstruck other! & title:Moonstruck (1987) occupation:other \\
\hline 759 & 2007 Hostel 3425 & title:Hostel: Part II (2007) age range:25-34 \\
\hline 760 & Wild Wild other! & title:Something Wild (1986) occupation:other \\
\hline 761 & Listener 200634. & title:Night Listener The (2006) age range:25-34 \\
\hline 762 & Radio 19951 star. & title:Destiny Turns on the Radio (1995) rating:1 \\
\hline 763 & Redl Oberst & title:Colonel Redl (Oberst Redl) (1985) \\
\hline 764 & The Lagoon 1980! & title:Blue Lagoon The (1980) \\
\hline 765 & Molly 19994 & title:Molly (1999) rating:4 \\
\hline 766 & $\begin{array}{c}1998 \text { Croupier } \\
\text { other. }\end{array}$ & title:Croupier (1998) occupation:other \\
\hline 767 & $\begin{array}{c}2008 \text { Minutes ages } \\
\text { of } 2534 ?\end{array}$ & title:88 Minutes (2008) age range:25-34 \\
\hline 768 & 1942 Tigers other & title:Flying Tigers (1942) occupation:other \\
\hline 769 & $\begin{array}{c}\text { Puppet attack } 18 \\
24 . \\
\end{array}$ & title:Attack of the Puppet People (1958) age range:18-24 \\
\hline 770 & Dream 20074. & title:Cassandra's Dream (2007) rating:4 \\
\hline 771 & $\begin{array}{c}1952 \text { crimson } \\
\text { pirate }\end{array}$ & title:Crimson Pirate The (1952) \\
\hline 772 & all Over me 1997. & title:All Over Me (1997) \\
\hline 773 & 1987 Laid Rosie & title:Sammy and Rosie Get Laid (1987) \\
\hline 774 & Juno 5 star. & title:Juno (2007) rating:5 \\
\hline 775 & 1982 Rambo 4 & title:First Blood (Rambo: First Blood) (1982) rating:4 \\
\hline 776 & bad santa & title:Bad Santa (2003) \\
\hline 777 & Hell $198044 ?$ & title:Motel Hell (1980) age range:35-44 \\
\hline 778 & $\begin{array}{c}2006 \text { Dreamgirls } \\
18 !\end{array}$ & title:Dreamgirls (2006) age range: $18-24$ \\
\hline 779 & 1995 Hood & title:Tales from the Hood (1995) \\
\hline 780 & 1998 The 34 ! & title:Babylon 5: The River of Souls (1998) age range:25-34 \\
\hline 781 & 2007 silk stars $2 !$ & title:Silk (2007) rating:2 \\
\hline 782 & man tomorrow 3 & title:Man Who Saw Tomorrow The (1980) rating:3 \\
\hline 783 & $\begin{array}{l}8 \text { butterfield } \\
\text { employed . }\end{array}$ & title:Butterfield 8 (1960) occupation:self-employed \\
\hline 784 & $\begin{array}{l}\text { Journey center } \\
\text { earth } 5 \text { stars }\end{array}$ & title:Journey to the Center of the Earth (2008) rating:5 \\
\hline
\end{tabular}




\begin{tabular}{|c|c|c|}
\hline 785 & Nuit for other! & 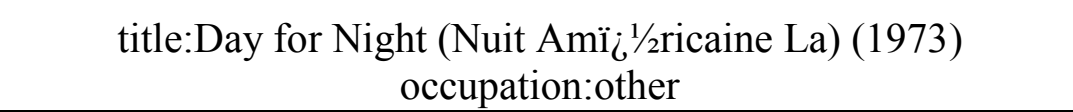 \\
\hline 786 & race Sun? & title:Race the Sun (1996) \\
\hline 787 & $\begin{array}{c}\text { big country } 1958 \\
3 ?\end{array}$ & title:Big Country The (1958) rating:3 \\
\hline 788 & evil Men 3425 & title:Evil That Men Do The (1984) age range:25-34 \\
\hline 789 & 1945 Expendable? & title:They Were Expendable (1945) \\
\hline 790 & fugitive $1947 ?$ & title:Fugitive The (1947) \\
\hline 791 & $\begin{array}{l}\text { body soul } 194724 \\
18\end{array}$ & title:Body and Soul (1947) age range:18-24 \\
\hline 792 & deseo La 4? & title:Law of Desire (La Ley del deseo) (1987) rating:4 \\
\hline 793 & $\begin{array}{c}1987 \text { Opera } 1987 \\
\text { Opera? }\end{array}$ & title:Opera (1987) title:Opera (1987) \\
\hline 794 & Pin 1988 stars $3 ?$ & title:Pin... (1988) rating:3 \\
\hline 795 & 2001 Glitter. & title:Glitter (2001) title:Glitter (2001) \\
\hline 796 & $\begin{array}{l}1939 \text { Frankenstein } \\
\text { academic educator. }\end{array}$ & title:Son of Frankenstein (1939) occupation:academic/educator \\
\hline 797 & $\begin{array}{l}\text { Flying saucers } \\
\text { rating } 2 ?\end{array}$ & title:Earth vs. the Flying Saucers (1956) rating:2 \\
\hline 798 & Maniac other. & title:Maniac (1980) occupation:other \\
\hline 799 & edge of heaven? & title:Edge of Heaven The (Auf der anderen Seite) (2007) \\
\hline 800 & Othello 199525 ! & title:Othello (1995) age range:25-34 \\
\hline 801 & 1995 last Supper! & title:Last Supper The (1995) \\
\hline 802 & 1960 Psycho other! & title:Psycho (1960) occupation:other \\
\hline 803 & down valley 2005 & title:Down in the Valley (2005) \\
\hline 804 & $\begin{array}{c}2007 \text { reservation } \\
\text { executive } \\
\text { managerial! }\end{array}$ & title:Reservation Road (2007) occupation:executive/managerial \\
\hline 805 & $\begin{array}{c}1981 \text { marleen } \\
\text { college grad } \\
\text { student! }\end{array}$ & title:Lili Marleen (1981) occupation:college/grad student \\
\hline 806 & panther Pink 1982 & title:Trail of the Pink Panther (1982) \\
\hline 807 & $\begin{array}{l}1938 \text { can't take it } \\
5 ?\end{array}$ & title:You Can't Take It with You (1938) rating:5 \\
\hline 808 & ugly $19973544 ?$ & title:Ugly The (1997) age range:35-44 \\
\hline 809 & Home own other & title:Home of Our Own A (1993) occupation:other \\
\hline 810 & IV Eagle 34 ! & title:Iron Eagle IV (1995) age range:25-34 \\
\hline 811 & $\begin{array}{l}\text { The conformist } \\
\text { stars of } 5\end{array}$ & title:Conformist The (Il Conformista) (1970) rating:5 \\
\hline 812 & $\begin{array}{c}\text { Snowman } 1985 \\
\text { other. }\end{array}$ & title:Falcon and the Snowman The (1985) occupation:other \\
\hline 813 & $\begin{array}{l}1954 \text { Jail bait } \\
\text { clearical admin. }\end{array}$ & title:Jail Bait (1954) occupation:clearical/admin \\
\hline
\end{tabular}




\begin{tabular}{|c|c|c|}
\hline 814 & MaCleane 5 & title:Plunkett \& MaCleane (1999) rating:5 \\
\hline 815 & $\begin{array}{l}\text { sweetest Thing } \\
20021 !\end{array}$ & title:Sweetest Thing The (2002) rating: 1 \\
\hline 816 & $\begin{array}{c}\text { Spinal Tap ages } 59 \\
45 !\end{array}$ & title:This Is Spinal Tap (1984) age range:45-59 \\
\hline 817 & $\begin{array}{c}\text { American Gigolo } \\
3425 !\end{array}$ & title:American Gigolo (1980) age range:25-34 \\
\hline 818 & 1950 DOA $18 ?$ & title:D.O.A. (1950) age range: $18-24$ \\
\hline 819 & $\begin{array}{c}1995 \text { frank ollie } 35 \\
44\end{array}$ & title:Frank and Ollie (1995) age range:35-44 \\
\hline 820 & $\begin{array}{l}\text { King new york } \\
\text { other! }\end{array}$ & title:King of New York (1990) occupation:other \\
\hline 821 & brylluppet 2006 & title:After the Wedding (Efter brylluppet) (2006) \\
\hline 822 & T-Men $1947 !$ & title:T-Men (1947) \\
\hline 823 & Anaconda 18. & title:Anaconda (1997) age range:18-24 \\
\hline 824 & Firewalker 1. & title:Firewalker (1986) rating:1 \\
\hline 825 & Creatures 20004. & title:Beautiful Creatures (2000) rating:4 \\
\hline 826 & $\begin{array}{c}2003 \text { blood } \\
\text { clearical admin }\end{array}$ & title:My Flesh and Blood (2003) occupation:clearical/admin \\
\hline 827 & People 19864 ! & title:Ruthless People (1986) rating:4 \\
\hline 828 & $\begin{array}{c}1995 \text { Love bye } \\
\text { other? }\end{array}$ & title:Bye Bye Love (1995) occupation:other \\
\hline 829 & 1966 Finie 1824. & $\begin{array}{c}\text { title:War Is Over The (La Guerre Est Finie) (1966) age } \\
\text { range: } 18-24\end{array}$ \\
\hline 830 & Moon 195044. & title:Destination Moon (1950) age range:35-44 \\
\hline 831 & Hollywood heidi? & title:Heidi Fleiss: Hollywood Madam (1995) \\
\hline 832 & $\begin{array}{l}1982 \text { Man } \\
\text { academic } \\
\text { educator? }\end{array}$ & title:Honkytonk Man (1982) occupation:academic/educator \\
\hline 833 & Gump 1994 other! & title:Forrest Gump (1994) occupation:other \\
\hline 834 & 2003 Japanese 34. & title:Japanese Story (2003) age range:25-34 \\
\hline 835 & $\begin{array}{l}\text { Serendipity } 2001 \\
2534 !\end{array}$ & title:Serendipity (2001) age range:25-34 \\
\hline 836 & $\begin{array}{l}1997 \text { Nightwatch } \\
2 .\end{array}$ & title:Nightwatch (1997) rating:2 \\
\hline 837 & Infamous $2006 ?$ & title:Infamous (2006) \\
\hline 838 & Barbarosa 1982! & title:Barbarosa (1982) \\
\hline 839 & $\begin{array}{l}\text { thin man academic } \\
\text { educator }\end{array}$ & $\begin{array}{l}\text { title:Song of the Thin Man (1947) } \\
\text { occupation:academic/educator }\end{array}$ \\
\hline 840 & 1994 New York & title:Naked in New York (1994) \\
\hline 841 & Heart wild 3425 & title:Wild at Heart (1990) age range:25-34 \\
\hline 842 & $\begin{array}{c}1965 \text { Battle of } \\
\text { Bulge? }\end{array}$ & title:Battle of the Bulge (1965) \\
\hline
\end{tabular}




\begin{tabular}{|c|c|c|}
\hline 843 & spy Kids 2001 & title:Spy Kids (2001) \\
\hline 844 & $\begin{array}{c}\text { I'm going } 19454 \\
\text { stars? }\end{array}$ & title:I Know Where I'm Going! (1945) rating:4 \\
\hline 845 & erin Brockovich & title:Erin Brockovich (2000) \\
\hline 846 & $\begin{array}{l}\text { Generations } 1994 \\
\text { other? }\end{array}$ & title:Star Trek: Generations (1994) occupation:other \\
\hline 847 & Majo 1989 other & $\begin{array}{l}\text { title:Kiki's Delivery Service (Majo no takkyï ¿1/2bin) (1989) } \\
\text { occupation:other }\end{array}$ \\
\hline 848 & $\begin{array}{l}\text { Man Year } 2006 \\
\text { other! }\end{array}$ & title:Man of the Year (2006) occupation:other \\
\hline 849 & Race death $3 ?$ & title:Death Race 2000 (1975) rating:3 \\
\hline 850 & $\begin{array}{l}1993 \text { Philadelphia } \\
18 .\end{array}$ & title:Philadelphia (1993) age range:18-24 \\
\hline 851 & advise Consent 4. & title:Advise and Consent (1962) rating:4 \\
\hline 852 & Yu-gi-oh 2004 & title:Yu-Gi-Oh! (2004) \\
\hline 853 & $\begin{array}{l}2007 \text { Dance ages } \\
50 ?\end{array}$ & title:War Dance (2007) age range:50-55 \\
\hline 854 & View room other! & title:Room with a View A (1986) occupation:other \\
\hline 855 & shadow man 24. & title:Shadow of the Thin Man (1941) age range:18-24 \\
\hline 856 & Choice sophie 3 ? & title:Sophie's Choice (1982) rating:3 \\
\hline 857 & $\begin{array}{l}1976 \text { President } \\
\text { men other? }\end{array}$ & title:All the President's Men (1976) occupation:other \\
\hline 858 & $\begin{array}{l}\text { eight men } 1988 \\
\text { other }\end{array}$ & title:Eight Men Out (1988) occupation:other \\
\hline 859 & $\begin{array}{l}\text { Teacher piano } \\
\text { other! }\end{array}$ & title:Piano Teacher The (La Pianiste) (2001) occupation:other \\
\hline 860 & $\begin{array}{c}\text { Jigureul Green } \\
\text { jikyeora! }\end{array}$ & title:Save The Green Planet! (Jigureul jikyeora!) (2003) \\
\hline 861 & 1989 Starr 24. & title:Brenda Starr (1989) age range:18-24 \\
\hline 862 & 1980 Scared? & title:Running Scared (1980) \\
\hline 863 & $\begin{array}{l}\text { Friend Best } \\
\text { clearical admin }\end{array}$ & title:Man's Best Friend (1993) occupation:clearical/admin \\
\hline 864 & $\begin{array}{l}1999 \text { Strange } \\
\text { Planet! }\end{array}$ & title:Strange Planet (1999) \\
\hline 865 & Lost Ark other! & $\begin{array}{c}\text { title:Raiders of the Lost Ark (Indiana Jones and the Raiders of } \\
\text { the Lost Ark) (1981) occupation:other }\end{array}$ \\
\hline 866 & 2000 Dead 56? & title:Legion of the Dead (2000) age range:56+ \\
\hline 867 & $\begin{array}{l}2001 \text { believer } \\
\text { other! }\end{array}$ & title:Believer The (2001) occupation:other \\
\hline 868 & $\begin{array}{c}\text { Continent atlantis } \\
\text { other. }\end{array}$ & title:Atlantis the Lost Continent (1961) occupation:other \\
\hline 869 & dance me! & title:Dance with Me (1998) \\
\hline 870 & The dead $1987 !$ & title:Dead The (1987) occupation:other \\
\hline
\end{tabular}




\begin{tabular}{|c|c|c|}
\hline 871 & land lotto! & title:Lotto Land (1995) \\
\hline 872 & $\begin{array}{l}\text { Contamination } 25 \\
34 ?\end{array}$ & title:Alien Contamination (1980) age range:25-34 \\
\hline 873 & $\begin{array}{l}\text { Nightwatch } 1997 \\
2534 ?\end{array}$ & title:Nightwatch (1997) age range:25-34 \\
\hline 874 & $\begin{array}{l}\text { batman Phantasm } \\
\text { other! }\end{array}$ & title:Batman: Mask of the Phantasm (1993) occupation:other \\
\hline 875 & $\begin{array}{l}\text { Unagi } \\
\text { clearicaladmin }\end{array}$ & title:Eel The (Unagi) (1997) occupation:clearical/admin \\
\hline 876 & Moon Rising 3? & title:Black Moon Rising (1986) rating:3 \\
\hline 877 & $\begin{array}{l}\text { Shadrach } 1998 \\
\text { artist! }\end{array}$ & title:Shadrach (1998) occupation:artist \\
\hline 878 & $\begin{array}{l}\text { dead Mantis } 1957 \\
1957 ?\end{array}$ & title:Deadly Mantis The (1957) \\
\hline 879 & Hill king 1993 & title:King of the Hill (1993) title:King of the Hill (1993) \\
\hline 880 & exorcist Prequel 1? & title:Dominion: Prequel to the Exorcist (2005) rating:1 \\
\hline 881 & $\begin{array}{l}\text { Number Wrong } \\
1948 ?\end{array}$ & title:Sorry Wrong Number (1948) \\
\hline 882 & Peter Pan 4? & title:Peter Pan (2003) rating:4 \\
\hline 883 & $\begin{array}{l}\text { Mandolin } 200159 \\
45 ?\end{array}$ & title:Captain Corelli's Mandolin (2001) age range:45-59 \\
\hline 884 & The reat Escape. & title:Great Escape The (1963) \\
\hline 885 & $\begin{array}{c}\text { Settebellezze } \\
\text { Beauties }\end{array}$ & $\begin{array}{l}\text { title:Seven Beauties (Pasqualino Settebellezze) (1976) } \\
\text { title:Seven Beauties (Pasqualino Settebellezze) (1976) }\end{array}$ \\
\hline 886 & $\begin{array}{l}\text { nine to } 5198050 \\
\quad 55 ?\end{array}$ & title:Nine to Five (a.k.a. 9 to 5) (1980) age range:50-55 \\
\hline 887 & $\begin{array}{l}2002 \text { Know } \\
\text { people? }\end{array}$ & title:People I Know (2002) \\
\hline 888 & $\begin{array}{l}\text { aka satisfaction } \\
19883 !\end{array}$ & title:Satisfaction (a.k.a. Girls of Summer) (1988) rating:3 \\
\hline 889 & $\begin{array}{l}1930 \text { western front } \\
\text { other? }\end{array}$ & title:All Quiet on the Western Front (1930) occupation:other \\
\hline 890 & Le Guard? & title:On Guard (Le Bossu) (1997) \\
\hline 891 & 1988 caddyshack? & title:Caddyshack II (1988) \\
\hline 892 & 1965 Ligeia. & $\begin{array}{l}\text { title:Tomb of Ligeia The (1965) title:Tomb of Ligeia The } \\
\qquad(1965)\end{array}$ \\
\hline 893 & Hood 19953 stars? & title:Tales from the Hood (1995) rating:3 \\
\hline 894 & class 19844 & title:Class of 1984 (1982) rating:4 \\
\hline 895 & Bilder Die 5. & $\begin{array}{l}\text { title: Wonderful Horrible Life of Leni Riefenstahl The (Macht } \\
\text { der Bilder: Leni Riefenstahl Die) (1993) rating:5 }\end{array}$ \\
\hline 896 & Adams 19351824 & title:Alice Adams (1935) age range: $18-24$ \\
\hline 897 & $\begin{array}{c}\text { Galaxy Guide } \\
\text { other! }\end{array}$ & $\begin{array}{l}\text { title:Hitchhiker's Guide to the Galaxy The (2005) } \\
\text { occupation:other }\end{array}$ \\
\hline 898 & Fauntleroy Lord & title:Little Lord Fauntleroy (1936) \\
\hline
\end{tabular}




\begin{tabular}{|c|c|c|}
\hline & 1936 & \\
\hline 899 & Shopping Night & title:Late Night Shopping (2001) \\
\hline 900 & $\begin{array}{c}2007 \text { Sukkar banat } \\
?\end{array}$ & title:Caramel (Sukkar banat) (2007) \\
\hline 901 & Daddy $2004 ?$ & title:My Baby's Daddy (2004) \\
\hline 902 & quest 19963 & title:Quest The (1996) rating:3 \\
\hline 903 & 1995 tough dead? & title:Tough and Deadly (1995) \\
\hline 904 & just a kiss 20021. & title:Just a Kiss (2002) rating:1 \\
\hline 905 & $\begin{array}{l}\text { down to You } 45- \\
59\end{array}$ & title:Down to You (2000) age range:45-59 \\
\hline 906 & $\begin{array}{l}\text { Misma La } 2007 \\
\text { Same? }\end{array}$ & title:Under the Same Moon (La Misma luna) (2007) \\
\hline 907 & $\begin{array}{l}\text { snowball Express } \\
4 ?\end{array}$ & title:Snowball Express (1972) rating:4 \\
\hline 908 & animal House! & title:Animal House (1978) \\
\hline 909 & $\begin{array}{l}\text { Away un Travolti } \\
\text { insolito? }\end{array}$ & $\begin{array}{c}\text { title:Swept Away (Travolti da un insolito destino nell'azzurro } \\
\text { mare d'Agosto) (1975) }\end{array}$ \\
\hline 910 & devil 19714435 & title:Devils The (1971) age range:35-44 \\
\hline 911 & moving 1988 ! & title:Moving (1988) \\
\hline 912 & $\begin{array}{l}\text { Sunday never stars } \\
4 ?\end{array}$ & title:Never on Sunday (Pote tin Kyriaki) (1960) rating:4 \\
\hline 913 & $\begin{array}{c}\text { Academy } 19841 \\
\text { star? }\end{array}$ & title:Police Academy (1984) rating:1 \\
\hline 914 & 1987 voice broke? & title:Year My Voice Broke The (1987) \\
\hline 915 & 19891989 other! & title:Creature Comforts (1989) occupation:other \\
\hline 916 & $\begin{array}{l}\text { theory flight } \\
\text { rating of } 4\end{array}$ & title:Theory of Flight The (1998) rating:4 \\
\hline 917 & Macao rating of $2 ?$ & title:Macao (1952) rating:2 \\
\hline 918 & Irene myself $1 ?$ & title:Me Myself and Irene (2000) rating: 1 \\
\hline 919 & 1957 Blood 5 & title:Throne of Blood (Kumonosu jï ¿1/2) (1957) rating:5 \\
\hline 920 & $\begin{array}{c}2004 \text { village } \\
\text { version } 3425 .\end{array}$ & title:Village The (2004) age range:25-34 \\
\hline 921 & $\begin{array}{l}2007 \text { Road } \\
\text { reservation? }\end{array}$ & title:Reservation Road (2007) \\
\hline 922 & $\begin{array}{l}\text { treat lady } \\
\text { academic } \\
\text { educator? }\end{array}$ & $\begin{array}{c}\text { title:No Way to Treat a Lady (1968) } \\
\text { occupation:academic/educator }\end{array}$ \\
\hline 923 & spring Forward! & title:Spring Forward (1999) \\
\hline 924 & autumn Spring 4. & title:Autumn Spring (Babï ${ }^{1} 1 / 2$ lï ${ }^{1 / 2}$ to) (2001) rating:4 \\
\hline 925 & tin Man. & title:Tin Man (2007) \\
\hline 926 & Hell 3 stars! & title:From Hell (2001) rating:3 \\
\hline 927 & $\begin{array}{c}\text { Screamers } 1995 \\
\text { other }\end{array}$ & title:Screamers (1995) occupation:other \\
\hline
\end{tabular}




\begin{tabular}{|c|c|c|}
\hline 928 & flight Dragons? & title:Flight of Dragons The (1982) \\
\hline 929 & hand 1981 & title:Hand The (1981) \\
\hline 930 & $\begin{array}{l}2006 \text { black } \\
\text { christmas }\end{array}$ & title:Black Christmas (2006) occupation:other \\
\hline 931 & $\begin{array}{l}25 \text { year } 2000 \\
\text { college grad! }\end{array}$ & $\begin{array}{l}\text { title:Jonah Who Will Be } 25 \text { in the Year } 2000 \text { (Jonas qui aura } 25 \\
\text { ans en l'an 2000) (1976) occupation:college/grad student }\end{array}$ \\
\hline 932 & splatter 198435 ! & title:Splatter University (1984) age range:35-44 \\
\hline 933 & Frenzy 4. & title:Frenzy (1972) rating:4 \\
\hline 934 & Dont Dance 5945 & title:Cats Don't Dance (1997) age range:45-59 \\
\hline 935 & terror Train. & title:Terror Train (1980) title:Terror Train (1980) \\
\hline 936 & $\begin{array}{l}\text { Volcano The } \\
\text { other? }\end{array}$ & title:Under The Volcano (1984) occupation:other \\
\hline 937 & Paperhouse 1988? & title:Paperhouse (1988) \\
\hline 938 & The Ripley? & title:Talented Mr. Ripley The (1999) \\
\hline 939 & Time Next $5 ?$ & title:Same Time Next Year (1978) rating:5 \\
\hline 940 & $\begin{array}{l}\text { evil dead Dawn } \\
\text { other }\end{array}$ & title:Evil Dead II (Dead by Dawn) (1987) occupation:other \\
\hline 941 & $\begin{array}{l}2004 \text { punisher } 34 \\
25\end{array}$ & title:Punisher The (2004) age range:25-34 \\
\hline 942 & $\begin{array}{l}1968 \text { shakiest gun } \\
5\end{array}$ & title:Shakiest Gun in the West The (1968) rating:5 \\
\hline 943 & $\begin{array}{l}1992 \text { Cradle } \\
\text { Rocks? }\end{array}$ & title:Hand That Rocks the Cradle The (1992) \\
\hline 944 & 1996 Fear other! & title:Fear (1996) occupation:other \\
\hline 945 & $\begin{array}{l}\text { invasion body } \\
\text { snatchers } 1 .\end{array}$ & title:Invasion of the Body Snatchers (1956) rating:1 \\
\hline 946 & 1982 Smiles other. & title:Savannah Smiles (1982) occupation:other \\
\hline 947 & doom 1995 other. & title:Doom Generation The (1995) occupation:other \\
\hline 948 & Softly 20023. & title:Killing Me Softly (2002) rating:3 \\
\hline 949 & Nora 1 & title:Nora (2000) rating: 1 \\
\hline 950 & $\begin{array}{l}2003 \text { Galactica } \\
\text { battlestar. }\end{array}$ & title:Battlestar Galactica (2003) \\
\hline 951 & Empire $2002 !$ & title:Empire (2002) \\
\hline 952 & Smiles savannah $5 !$ & title:Savannah Smiles (1982) rating:5 \\
\hline 953 & $\begin{array}{l}1959 \text { teenagers } \\
\text { space other? }\end{array}$ & title:Teenagers from Outer Space (1959) occupation:other \\
\hline 954 & $\begin{array}{c}2003 \text { Empty } \\
\text { academic educator }\end{array}$ & title:Big Empty The (2003) occupation:academic/educator \\
\hline 955 & $\begin{array}{l}2001 \text { don't say } \\
\text { Word! }\end{array}$ & title:Don't Say a Word (2001) \\
\hline 956 & 2007 stop yard 44 & title:Stomp the Yard (2007) age range:35-44 \\
\hline 957 & $\begin{array}{c}\text { Apache } 194824 \\
18 ?\end{array}$ & title:Fort Apache (1948) age range:18-24 \\
\hline
\end{tabular}




\begin{tabular}{|c|c|c|}
\hline 958 & $\begin{array}{l}2005 \text { Bride corpse } \\
4 !\end{array}$ & title:Corpse Bride (2005) rating:4 \\
\hline 959 & $\begin{array}{l}\text { Tex } 1982 \\
\text { academic } \\
\text { educator? }\end{array}$ & title:Tex (1982) occupation:academic/educator \\
\hline 960 & $\begin{array}{l}\text { Unforgettable } \\
1996 \text { other }\end{array}$ & title:Unforgettable (1996) occupation:other \\
\hline 961 & guyeok gyeongbi & $\begin{array}{l}\text { title:Joint Security Area (Gongdong gyeongbi guyeok JSA) } \\
\qquad(2000)\end{array}$ \\
\hline 962 & $\begin{array}{l}1988 \text { gonna git } 24 \\
18 .\end{array}$ & title:I'm Gonna Git You Sucka (1988) age range:18-24 \\
\hline 963 & 1951 river retired! & title:River The (1951) occupation:retired \\
\hline 964 & david gale 2 . & title:Life of David Gale The (2003) rating:2 \\
\hline 965 & $\begin{array}{l}\text { Everlasting } 2002 \\
\text { other! }\end{array}$ & title:Tuck Everlasting (2002) occupation:other \\
\hline 966 & $\begin{array}{l}1996 \text { revolution } \\
\text { other! }\end{array}$ & title:Children of the Revolution (1996) occupation:other \\
\hline 967 & $\begin{array}{l}\text { Ever Mozart } \\
\text { college grad! }\end{array}$ & title:For Ever Mozart (1996) occupation:college/grad student \\
\hline 968 & $\begin{array}{c}\text { empire ants stars } \\
\text { of } 1 !\end{array}$ & title:Empire of the Ants (1977) rating: 1 \\
\hline 969 & $\begin{array}{c}1990 \text { care of } \\
\text { business other? }\end{array}$ & title:Taking Care of Business (1990) occupation:other \\
\hline 970 & Firestarter $4 ?$ & title:Firestarter (1984) rating:4 \\
\hline 971 & $\begin{array}{l}1982 \text { Within beast } \\
1982 ?\end{array}$ & title:Beast Within The (1982) title:Beast Within The (1982) \\
\hline 972 & 1980 States 5 ! & title:Altered States (1980) rating:5 \\
\hline 973 & $\begin{array}{l}\text { fistful of dollars } 25 \\
34 !\end{array}$ & $\begin{array}{l}\text { title:Fistful of Dollars A (Per un pugno di dollari) (1964) age } \\
\text { range:25-34 }\end{array}$ \\
\hline 974 & $\begin{array}{l}1980 \text { circle of } \\
\text { friends } 34 .\end{array}$ & title:Small Circle of Friends A (1980) age range:25-34 \\
\hline 975 & $\begin{array}{l}\text { Man } 2000 \text { ages } 34 \\
25 ?\end{array}$ & title:Company Man (2000) age range:25-34 \\
\hline 976 & $\begin{array}{l}\text { Compass } 2007 \\
\text { other. }\end{array}$ & title:Golden Compass The (2007) occupation:other \\
\hline 977 & $\begin{array}{l}\text { the punisher } \\
\text { occupation other. }\end{array}$ & title:Punisher The (2004) occupation:other \\
\hline 978 & $\begin{array}{l}\text { Earthquake } 1974 \\
\text { other. }\end{array}$ & title:Earthquake (1974) occupation:other \\
\hline 979 & 2003 cat hat other. & title:Cat in the Hat The (2003) occupation:other \\
\hline 980 & 2001 gabriela $3 ?$ & title:Gabriela (2001) rating:3 \\
\hline 981 & $\begin{array}{c}\text { Knocks } \\
\text { opportunity } 44\end{array}$ & title:Opportunity Knocks (1990) age range:35-44 \\
\hline 982 & Detail last 1973 & title:Last Detail The (1973) occupation:other \\
\hline
\end{tabular}




\begin{tabular}{|c|c|c|}
\hline 983 & 1931 frankenstein . & title:Frankenstein (1931) \\
\hline 984 & $\begin{array}{c}\text { The } 1970 \text { Lovers } \\
\text { vampire }\end{array}$ & title:Vampire Lovers The (1970) \\
\hline 985 & victory 19815 & title:Victory (a.k.a. Escape to Victory) (1981) rating:5 \\
\hline 986 & hour wolf 2534 & title:Hour of the Wolf (Vargtimmen) (1968) age range:25-34 \\
\hline 987 & green Valley 1941 & title:How Green Was My Valley (1941) \\
\hline 988 & Lilies 19963544. & title:Lilies (1996) age range:35-44 \\
\hline 989 & $\begin{array}{c}1999 \text { dreams age } \\
\text { range } 3425 !\end{array}$ & title:In Dreams (1999) age range:25-34 \\
\hline 990 & million dollar other & title:Million Dollar Baby (2004) occupation:other \\
\hline 991 & 1998 Baked 34. & title:Half Baked (1998) age range:25-34 \\
\hline 992 & Maniacs $1964 ?$ & title:Two Thousand Maniacs! (1964) \\
\hline 993 & Saved 2004 other! & title:Saved! (2004) occupation:other \\
\hline 994 & 1988 LA alien! & title:Alien from L.A. (1988) \\
\hline 995 & Bugsy Malone 4 & title:Bugsy Malone (1976) rating:4 \\
\hline 996 & 2005 Doom other & title:Doom (2005) occupation:other \\
\hline 997 & Panic 2? & title:Panic (2000) rating:2 \\
\hline 998 & 20024 stars "dina" & title:I Am Dina (2002) rating:4 \\
\hline 999 & $\begin{array}{c}\text { Lowdown sweet } \\
25 .\end{array}$ & title:Sweet and Lowdown (1999) age range:25-34 \\
\hline 1000 & $\begin{array}{c}1994 \text { Barcelona } \\
59 .\end{array}$ & title:Barcelona (1994) age range:45-59 \\
\hline
\end{tabular}




\section{A.3: Case Study Three Query}

\begin{tabular}{|c|c|c|}
\hline Number & User Query & SQL Query \\
\hline 1 & coming Out Male. & movie: Coming Out \\
\hline 2 & Jason stafford & actor: Jason Stafford \\
\hline 3 & Baldwin 1946? & actor: Walter Baldwin year: 1946 \\
\hline 4 & het weer lente Male! & movie: Als het weer lente is \\
\hline 5 & Corsarios? & movie: Corsarios \\
\hline 6 & Charkh & movie: Charkh \\
\hline 7 & 1971 una reforma & year: 1971 movie: una reforma \\
\hline 8 & Hour 1912? & movie: At the Eleventh Hour year: 1912 \\
\hline 9 & bell year $1984 !$ & actor: Barry Bell year: 1984 \\
\hline 10 & 1976 blank generation? & Year: 1976 movie: Blank Generation \\
\hline 11 & BET aaliya 2001. & movie: BET tonight special: aaliyah year: 2001 \\
\hline 12 & Le bal $1897 ?$ & year: 1897 movie: aprs le bal \\
\hline 13 & 1970 cinico & year: 1970 movie: Cinico \\
\hline 14 & 3.7 Dawn. & movie: black dawn rank: 3.7 \\
\hline 15 & 2003golden globe & year: 2003 movie: 60 th annual golden globe \\
\hline 16 & 1984 senado el. & year: 1984 movie: Asesinato en el senado de la nacin \\
\hline 17 & 1931 Bunch Albany? & year: 1931 movie: Albany Bunch \\
\hline 18 & call remember & movie: call to remember \\
\hline 19 & 1926 Hewston & year: 1926 actor: Alfred Hewston \\
\hline 20 & 1915 shield & year: 1915 movie: blot on the shield \\
\hline 21 & clockwork & movie: clockwork \\
\hline 22 & Hall truman ward & actor: Donald Hall movie: truman's ward \\
\hline 23 & Morgan III Onion! & actor: Jack Morgan movie: andy nose his Onions \\
\hline 24 & carl sagan Himself & movie:carl sagan role: himself \\
\hline 25 & Abascal bonita & movie: bonita actor: Ramón Abascal \\
\hline 26 & 1964 lagim ng! & year: 1964 movie: Bata ng lagim \\
\hline 27 & casada Alegre & movie: Alegre casada \\
\hline 28 & Cala 1966! & movie: Cala naprzd year: 1966 \\
\hline 29 & bright shining & movie: Bright Shining Lie \\
\hline 30 & 1915 beauty distress & year: 1915 movie: beauty in distress \\
\hline 31 & 61941 blues night. & rank: 6 movie: blues in the night \\
\hline 32 & chudovishche & movie: chudovishche ili kto-to drugoj \\
\hline 33 & Jorge Arriaga destino & actor: Jorge Arriaga movie: cruel destino \\
\hline
\end{tabular}




\begin{tabular}{|c|c|c|}
\hline 34 & Patrick barr? & actor: Patrick Barr \\
\hline 35 & 2004 Astakhov? & year: 2004 actor: Sergei Astakhov \\
\hline 36 & Bob Tammy & role: Bob movie: Bob and Tammy's party to blow \\
\hline 37 & Card $1922 ?$ & movie: Card year: 1922 \\
\hline 38 & Bruce Brighton & role: ray Brighton \\
\hline 39 & $\begin{array}{l}\text { historia Laboriel } \\
\text { Johnny? }\end{array}$ & movie: angelica actor: Johnny Laboriel \\
\hline 40 & born to kill hwan & movie: Born to Kill actor: Kyeong-hwan Jo \\
\hline 41 & 1989 "1939"! & year: 1989 movie: 1939 \\
\hline 42 & cenerentola & movie: cenerentola \\
\hline 43 & Ismet in bir yrek satildi! & movie: bir yrek satildi actor: Ismet Ay \\
\hline 44 & Bretons rating: $6.6 ?$ & movie: Astrix chez les Bretons rank: 6.6 \\
\hline 45 & corrida goyesca & movie: corrida goyesca \\
\hline 46 & 1920 Luis! & year: 1920 actor: Luis Alberni \\
\hline 47 & Attila $6.7 !$ & role: Attila rank: 6.7 \\
\hline 48 & 1991 outlaw & year: 1991 movie: Dancing Outlaw \\
\hline 49 & Corman Baby Killer? & actor: Roger Corman movie: Cry Baby Killer \\
\hline 50 & Female lady delivery! & role: seafood delivery lady gender: $\mathrm{f}$ \\
\hline 51 & Compere Male! & role: Compere \\
\hline 52 & 1963 bu adam? & year: 1963 movie: bu adam kim \\
\hline 53 & 1913 bout-de-zan & year: 1913 movie: bout-de-zan s'amuse \\
\hline 54 & Popeye & movie: adventures of popeye \\
\hline 55 & 1997 Kitty. & year: 1997 role: Kitty \\
\hline 56 & april sometimes? & movie: ....Sometimes in April \\
\hline 57 & South Seas! & movie: Cast Adrift in the South Seas \\
\hline 58 & Emilio & actor: Andrés Emilio \\
\hline 59 & benya kirk & movie: benya krik \\
\hline 60 & George French! & actor: George B. French \\
\hline 61 & Accidental 1913. & year: 1913 movie: Accidental Dentist \\
\hline 62 & 1943 colt comrades & year: 1943 movie: colt comrades \\
\hline 63 & 5.2 rating $\mathrm{Bu}$ dao de & rank: 5.2 movie: Bu dao de de li wu \\
\hline 64 & Anja & movie: Anja \\
\hline 65 & Giampiero 1992! & actor: Giampiero Bianchi year: 1992 \\
\hline 66 & $\begin{array}{l}\text { andere Revolution } \\
\text { gefhrliche Peter }\end{array}$ & $\begin{array}{l}\text { movie: Revolution und andere gefhrliche Sachen actor: } \\
\text { Peter Buchholz }\end{array}$ \\
\hline 67 & Cannone batte si? & movie: Cretinetti si batte al cannone \\
\hline 68 & came dawn & movie: came the dawn \\
\hline 69 & Thursday Cannon. & movie: Big Thursday actor: Harold Cannon \\
\hline 70 & 1951 cadet & year: 1951 movie: cadet holiday \\
\hline 71 & Babo chilseongi & movie: Babo chilseongi \\
\hline
\end{tabular}




\begin{tabular}{|c|c|c|}
\hline 72 & Actress Doped! & movie: Case of a Doped Actress \\
\hline 73 & celui qui & movie: celui qui doit mourir \\
\hline 74 & $\begin{array}{l}2000 \text { Adventures } \\
\text { Trouble. }\end{array}$ & $\begin{array}{c}\text { year: } 2000 \text { movie: Born to Trouble: Adventures of } \\
\text { Huckleberry Finn }\end{array}$ \\
\hline 75 & barry mckenzie & movie: Adventures of Barry McKenzie \\
\hline 76 & Aaram? & movie: Aaram \\
\hline 77 & 1925 breakin' loose & year: 1925 movie: breakin' loose \\
\hline 78 & 6.7 do nieba mil & rank: 6.7 movie: 300 mil do nieba \\
\hline 79 & godina nesvrstanosti & movie: 20 godina nesvrstanosti \\
\hline 80 & carry a henry & movie: carry on henry \\
\hline 81 & alegra batalln & movie: alegra del batalln \\
\hline 82 & Zellen Bse! & movie: Bse Zellen \\
\hline 83 & Giga Chiranjeevi! & actor: Giga Chirakadze movie: Chiranjeevi \\
\hline 84 & Germano Haiut 1999? & actor: Germano Haiut year: 1999 \\
\hline 85 & 1996 Ama & year: 1996 movie: Ama \\
\hline 86 & Almost $1989 !$ & movie: Almost Beat year: 1989 \\
\hline 87 & Leon Ames Ambush & actor: Leon Ames movie: ambush \\
\hline 88 & bra breakers & movie: bra breakers vol. 1 \\
\hline 89 & Adorables $4.8 ?$ & movie: Adorables mentiras rank: 4.8 \\
\hline 90 & 1998 Kaufman? & $\begin{array}{c}\text { year: } 1998 \text { movie: Andy Kaufman: The E! True } \\
\text { Hollywood Story }\end{array}$ \\
\hline 91 & Casa manchada? & movie: Casa Manchada \\
\hline 92 & bizarre lust & movie: sexual deviant \\
\hline 93 & awful truth & movie: Awful Truth \\
\hline 94 & $\begin{array}{l}1954 \text { anemos tou } \\
\text { misous }\end{array}$ & year: 1954 movie: anemos tou misous \\
\hline 95 & Mick 7.1. & role: Mick rank: 7.1 \\
\hline 96 & Harvey Clark & actor: Harvey Clark \\
\hline 97 & 2003 Herb Andress. & year: 2003 actor: Herb Andress \\
\hline 98 & 2001 djurgrden & year: 2001 movie: Bsta frn Djurgrden i Allsvenskan 2001 \\
\hline 99 & casa vuelve & movie: Antoito vuelve a casa \\
\hline 100 & Albright, john & actor: John Albright \\
\hline 101 & $\begin{array}{l}\text { Barbarians Amongst } \\
\text { Jason David. }\end{array}$ & movie: Amongst Barbarians actor: David Jason \\
\hline 102 & Ben Aii anderson. & role: Ali Ben actor: Tom Anderson \\
\hline 103 & Amour fou & movie: amour fou \\
\hline 104 & Avenging Baxley. & movie: Avenging Angel actor: Craig Baxley Jr. \\
\hline 105 & Australian 100 & movie: 100 Years: The Australian Story \\
\hline 106 & Kya Bedard & movie: Bedard Zamana Kya Jaane \\
\hline 107 & de Santa Cruz & movie: Bonifacio: Un misterio llegado de Santa Cruz \\
\hline
\end{tabular}




\begin{tabular}{|c|c|c|}
\hline 108 & Hymn Battle. & movie: Battle Hymn \\
\hline 109 & Future backside? & movie: Backside to the Future II \\
\hline 110 & 1957 Cielo & year: 1957 movie: Cielo brucia \\
\hline 111 & Centeotl Miguel? & movie: Centeotl actor: Miguel Anorve \\
\hline 112 & Male Southerne. & gender: $\mathrm{m}$ role: Rufus \\
\hline 113 & kahapon Bakit! & movie: Bakit may kahapon pa? \\
\hline 114 & limpio Male? & movie: A tiro limpio gender: $\mathrm{m}$ \\
\hline 115 & $\begin{array}{l}\text { Nicolay Hals Alles } \\
\text { Mord }\end{array}$ & role: Nicolay movie: Alles auer Mord - Hals ber Kopf \\
\hline 116 & pico Antonio & movie: Cuatro bodas y pico actor: Antonio Casal \\
\hline 117 & B Francis! Boy & actor: Alec B. Francis movie: boy \\
\hline 118 & Aatma Nageshwara & $\begin{array}{c}\text { movie: Aatma Gowravam actor: Nageshwara Rao } \\
\text { Akkineni }\end{array}$ \\
\hline 119 & Aeon Male & gender: m movie: Aeon Flux \\
\hline 120 & 1983 Attention? & $\begin{array}{c}\text { year: } 1983 \text { movie: Attention! Une femme peut en cacher } \\
\text { une autre }\end{array}$ \\
\hline 121 & Pirates 1954 & year: 1954 movie: Black Pirates \\
\hline 122 & pole drama & movie: avstrijskoye pole genre: drama \\
\hline 123 & Prayer First & movie: Bad Man's First Prayer \\
\hline 124 & 7.4 Border & rank: 7.4 role: Border Officer \\
\hline 125 & 1952 peligro & year: 1952 movie: Almas en peligro \\
\hline 126 & Jennings ABC & actor: Peter Jennings movie: ABC 2000: The Millennium \\
\hline 127 & Max Wanne tante. & role: Max movie: Bij tante Wanne \\
\hline 128 & Guy 4.4 & role: Stuffy Old Guy rank: 4.4 \\
\hline 129 & Zirkusluft 2002 & year: 2002 movie: Bulle von Tlz - Zirkusluft \\
\hline 130 & Rafael Morte. & actor: Rafael Almeida movie: A um Pulo da Morte \\
\hline 131 & Francisco San Narrator & movie: Cavalcade of San Francisco role: Narrator \\
\hline 132 & Janny Contrabandista & movie: Contrabandista actor: Janny 'el Portugues' \\
\hline 133 & 1962 Go we will & year: 1962 movie: A-Haunting We Will Go \\
\hline 134 & Thor Male? & actor: Thor Bishopric gender: $\mathrm{m}$ \\
\hline 135 & 1920 Charles. & year: 1920 actor: Charles Angelo \\
\hline 136 & 1917 of Go. Lets & year: 1917 movie: Battle of 'Let's Go' \\
\hline 137 & Squidge Male! & role: Squidge gender: $\mathrm{m}$ \\
\hline 138 & Lab. Mind Deadly & role: Lab Man movie: Columbo: A Deadly State of Mind \\
\hline 139 & 1910? Adventure & year: 1910 movie: Adventures of a Cowpuncher \\
\hline 140 & Reasonable & movie: Be Reasonable \\
\hline 141 & Ringheim! Berre & actor: Nils Morten Ringheim movie: Berre ein hund \\
\hline 142 & Agguato 1958 & year: 1958 movie: Agguato a Tangeri \\
\hline 143 & 6.7 Chobizenesse & rank: 6.7 movie: Chobizenesse \\
\hline 144 & grsse viele & movie: viele Grsse \\
\hline
\end{tabular}




\begin{tabular}{|c|c|c|}
\hline 145 & 1999 A.W.O.L. & year: 1999 movie: A.W.O.L. (1999/I) \\
\hline 146 & 1967 marido! & year: 1967 movie: Cmo pescar marido \\
\hline 147 & Belli. millet & movie: Bir millet uyaniyor actor: Emin Belig Belli \\
\hline 148 & crazy Jimmy!!! & movie: Crazy House actor: Jimmy Aubrey \\
\hline 149 & E Johnson Baboona & actor: Martin E. Johnson movie: Baboona \\
\hline 150 & Male Leone! & gender: $m$ role: Leone \\
\hline 151 & 100 Affleck? & actor: Ben Affleck movie: AFI's 100 Years \\
\hline 152 & Male Eye L.E.D.! & gender: $\mathrm{m}$ role: L.E.D. Eye \\
\hline 153 & 1998? Influence & year: 1998 movie: Art of Influence \\
\hline 154 & 1961? Contra Armas & year: 1961 movie: Armas contra la ley \\
\hline 155 & Artists Walt Adventure & $\begin{array}{c}\text { movie: } 4 \text { Artists Paint } 1 \text { Tree: A Walt Disney 'Adventure in } \\
\text { Art' }\end{array}$ \\
\hline 156 & $\begin{array}{l}\text { Leontyne at with } \\
\text { Himself Guitarist }\end{array}$ & $\begin{array}{c}\text { movie: Christmas at Kennedy Center with Leontyne Price } \\
\text { role: Himself/Guitarist }\end{array}$ \\
\hline 157 & Asher 1999 & actor: Bill Asher year: 1999 \\
\hline 158 & Everready Male & actor: Tony Everready gender: $\mathrm{m}$ \\
\hline 159 & cop Craven & movie: Careless Cop actor: R.J. Craven \\
\hline 160 & Broken chambers & movie: Broken Law actor: Lyster Chambers \\
\hline 161 & Alexandra Manley & movie: Alexandra - prinsesse i tiden actor: Richard Manley \\
\hline 162 & Closer Henry? & movie: Closer and Closer actor: Henry Alessandroni \\
\hline 163 & $\begin{array}{l}\text { Yiorgos Economou } \\
\text { Adelfos? }\end{array}$ & actor: Yiorgos Economou movie: Adelfos Anna \\
\hline 164 & bio $100 \% 2003$. & movie: $100 \%$ bio year: 2003 \\
\hline 165 & 1980 no rajes! & year: 1980 movie: Ay Chihuahua no te rajes! \\
\hline 166 & 1966 amor & year: 1966 movie: Amor a ritmo de go go \\
\hline 167 & Generation Next 1988. & movie: Bonanza: The Next Generation year: 1988 \\
\hline 168 & 1945 Clarence? & year: 1945 actor: Clarence Nash \\
\hline 169 & Raymond 1949 ! & actor: Raymond Burr year: 1949 \\
\hline 170 & Rush Bums & movie: Bum's Rush \\
\hline 171 & Cheolindeul & movie: Cheolindeul \\
\hline 172 & Male Haworth. & gender: $\mathrm{m}$ actor: Peter Haworth \\
\hline 173 & John Archer. & actor: John Archer \\
\hline 174 & Boat 2002 & movie: Boat Trip year: 2002 \\
\hline 175 & king against Robert & movie: Conspiracy Against the King actor: Robert Brower \\
\hline 176 & Sleuth! Bailey & movie: Campus Sleuth actor: William Bailey \\
\hline 177 & 1996 Mick. & year: 1996 role: Mick Ross \\
\hline 178 & 1955 Carosello & year: 1955 movie: Carosello del variet \\
\hline 179 & Divorced! Gino & movie: Almost Divorced actor: Gino Corrado \\
\hline 180 & of Walls House Male! & movie: Behind the Walls of 'Full House' gender: $\mathrm{m}$ \\
\hline 181 & Travis Barker 1999! & actor: Travis Barker year: 1999 \\
\hline
\end{tabular}




\begin{tabular}{|c|c|c|}
\hline 182 & Female Debb Jeanett? & gender: $\mathrm{F}$ actor: Jeanett Debb \\
\hline 183 & Bruce Davison Male? & actor: Bruce Davison gender: $\mathrm{m}$ \\
\hline 184 & Tom Bay Male! & actor: Tom Bay gender: $\mathrm{m}$ \\
\hline 185 & Larry! 1967 & role: Larry year: 1967 \\
\hline 186 & $\begin{array}{l}\text { Grissemann Awards } \\
\text { Music? }\end{array}$ & $\begin{array}{c}\text { actor: Christoph Grissemann movie: Amadeus Austrian } \\
\text { Music Awards } 2003\end{array}$ \\
\hline 187 & 4.9 Charlie & rank: 4.9 movie: Charlie \& Louise - Das doppelte Lottchen \\
\hline 188 & Louie pandan & role: Louie movie: Buko pandan \\
\hline 189 & $\begin{array}{l}\text { Cassels JWS? Tonta } \\
\text { semana }\end{array}$ & $\begin{array}{c}\text { actor: J.W.S. Cassels movie: Busco tonta para fin de } \\
\text { semana }\end{array}$ \\
\hline 190 & 1917 Fisher! & year: 1917 actor: George Fisher \\
\hline 191 & Phil? Checkers & movie: Checkers \& Pogo: Remembered actor: Phil Arnone \\
\hline 192 & 1997 sueldo! & year: 1997 movie: Asesino a sueldo \\
\hline 193 & Jim Henson? & actor: Jim Henson \\
\hline 194 & 1984 Budgeting? & year: 1984 movie: Budgeting \\
\hline 195 & Vol. Covergirls & movie: Covergirls year: 2002 \\
\hline 196 & Pennington Male. & actor: Earl Pennington gender: $\mathrm{m}$ \\
\hline 197 & Male Cuccagna & gender: $\mathrm{m}$ movie: Cuccagna \\
\hline 198 & Male Carter Donal & gender: $\mathrm{m}$ actor: Donald Carter \\
\hline 199 & Infante ra 1963 & movie: As ra Pedro Infante year: 1963 \\
\hline 200 & Forlorn Male & gender: $\mathrm{m}$ movie: Cape Forlorn \\
\hline 201 & Male la de Cerca! & gender: $\mathrm{m}$ movie: Cerca de la frontera \\
\hline 202 & Blind John? & role: John movie: Blind Eye \\
\hline 203 & 1997 zouave & year: 1997 movie: Culotte d'une zouave \\
\hline 204 & Kumar Mohan Grover. & role: Mohan Kumar actor: Gulshan Grover \\
\hline 205 & $\begin{array}{l}3.8 \text { rating with role of } \\
\text { Po }\end{array}$ & rank: 3.8 role: $\mathrm{Po}$ \\
\hline 206 & bir garip & movie: bir garip adam \\
\hline 207 & Barella 1995. & actor: Jaime Barella year: 1995 \\
\hline 208 & 1958 cavaliere & year: 1958 movie: cavaliere \\
\hline 209 & Bradley as Frank & actor: David (X) Bradley role: Frank \\
\hline 210 & Henry Lucas 2001. & actor: Henry Lucas year: 2001 \\
\hline 211 & cradle song & movie: cradle song \\
\hline 212 & dewey $1899 ?$ & movie: admiral dewey year: 1899 \\
\hline 213 & Male Adaktepe? & gender: m movie: Adaktepe \\
\hline 214 & 1 Dog 4.2 huskeys & rank: 4.2 movie: ace of huskeys \\
\hline 215 & captive Audience Male. & movie: Captive Audience gender: $\mathrm{m}$ \\
\hline 216 & Jimmy Boone 6.7. & actor: John Boone rank: 6.7 movie: alias Jimmy Valentine \\
\hline 217 & Cretinetti 1910 ! & role: Cretinetti year: 1910 \\
\hline 218 & Combs sean 2002. & actor: Sean Combs year: 2002 \\
\hline
\end{tabular}




\begin{tabular}{|c|c|c|}
\hline 219 & 2001 dakota & year: 2001 movie: Dakota bound \\
\hline 220 & $\begin{array}{l}\text { Tango Mandoline } \\
\text { Gerd? }\end{array}$ & movie: amour tango mandoline actor: Gerd Frickhöffer \\
\hline 221 & Peter Graves 2000! & actor: Peter Graves year: 2000 \\
\hline 222 & Captain Kidd 6.2 & movie: Captain Kidd rank: 6.2 \\
\hline 223 & Male caradura & gender: $\mathrm{m}$ movie: caradura y la millonaria \\
\hline 224 & gold 2002 & movie: 03 from gold year: 2002 \\
\hline 225 & 1984 crnica malandros & year: 1984 movie: Crnica dos Bons Malandros \\
\hline 226 & Pale Clarke Clarke I. & movie: beyond the pale actor: Patrick Clarke \\
\hline 227 & Brivido & movie: Brivido \\
\hline 228 & 4.3 back in business? & rank: 4.3 movie: Back in business \\
\hline 229 & Jasper carrott sol & actor: Jasper Carrott movie: carrott del sol \\
\hline 230 & $\begin{array}{l}\text { Borxhliu Sejfulla } \\
\text { Myftari }\end{array}$ & movie: Borxhliu actor: Sejfulla Myftari \\
\hline 231 & 1996 cater & year: 2001 movie: carter \\
\hline 232 & House Bleak Joe? & movie: Bleak House role: Joe \\
\hline 233 & Bachelor Daddy & movie: Bachelor Daddy \\
\hline 234 & biao ge dao & movie: biao ge dao \\
\hline 235 & andrea & movie: andrea \\
\hline 236 & criminal expert & movie: criminal expert \\
\hline 237 & last merchant king & $\begin{array}{c}\text { movie: A\&E Biography: Neiman Marcus - The Last of the } \\
\text { Merchant Kings }\end{array}$ \\
\hline 238 & Seville Alvira Pablo. & movie: Barber of Seville actor: Pablo Alvira \\
\hline 239 & Pressure 2001 & movie: Cabin Pressure year: 2001 \\
\hline 240 & St. Mary 1937 ! & movie: Bells of St. Mary's year: 1937 \\
\hline 241 & 1912 Adopted & year: 1912 movie: Cowboy's Adopted Child \\
\hline 242 & Straight Up & movie: Colors Straight Up \\
\hline 243 & Certain Rand Joe? & movie: Certain Women role: Joe Rand \\
\hline 244 & Gables Green 1919 & movie: Anne of Green Gables year: 1919 \\
\hline 245 & Adalid Ricardo guilas & actor: Ricardo Adalid movie: Cementerio de las guilas \\
\hline 246 & Bodrogi Gyula semmi & actor: Gyula Bodrogi movie: csak semmi \\
\hline 247 & Billy 1989 & Movie: Billy the kid \\
\hline 248 & Carrie of 1993 & movie: Carrie year: 1994 \\
\hline 249 & alien facor brown & actor: William L. Brown movie: Alien Factor \\
\hline 250 & Cheap Thrill & movie: Cheap Thrill \\
\hline 251 & Male Ernesto Alterio? & gender: $\mathrm{m}$ actor: Ernesto Alterio \\
\hline 252 & 1912 Suicide a Suicide. & year: 1912 movie: Almost a Suicide \\
\hline 253 & Male Bee Deviled? & role: $\mathrm{m}$ movie: Bee-Deviled Bruin \\
\hline 254 & Pury 2002 & role: De Pury year: 2002 \\
\hline 255 & Dancer angels & role: Dancer movie: Angels \& Insects \\
\hline
\end{tabular}




\begin{tabular}{|c|c|c|}
\hline 256 & milsa Daeryukui & movie: Daeryukui milsa \\
\hline 257 & James Hans Almanzar & actor: James Almanzar role: Hans \\
\hline 258 & 1990 West, Randy & year: 1990 actor: Randy West \\
\hline 259 & fill cup rating of $7 ?$ & rank: 7 movie: come fill the cup \\
\hline 260 & 6.8 rating in year 1939 & rank: 6.8 year: 1939 \\
\hline 261 & Male Arnell Timmy? & gender: $\mathrm{m}$ actor: Timmy Arnell \\
\hline 262 & birke 1979. & movie: Birke da oben year: 1979 \\
\hline 263 & abschied Baker & movie: Abschied in den Tod actor: Stuart Baker \\
\hline 264 & attack of 1992. & movie: Attack year: 1992 \\
\hline 265 & $\begin{array}{c}\text { Chandelier Mireille } \\
\text { amateur }\end{array}$ & actor: Mireille Chandelier movie: amateur madness 40 \\
\hline 266 & Trilogy Alien Carter. & movie: Alien Trilogy actor: Carter Inskeep \\
\hline 267 & Male After Lifetime. & gender: $\mathrm{m}$ movie: After a Lifetime \\
\hline 268 & 4.3 rating named "priv" & rank: 4.3 movie: Cours priv \\
\hline 269 & movie "4 the people" & movie: 4 the People \\
\hline 270 & Niro's saluate scorsese & actor: Robert De Niro movie: Salute to Martin Scorsese \\
\hline 271 & Arrapaho & movie: Arrapaho \\
\hline 272 & $\begin{array}{c}\text { Female Brabant Anne } \\
\text { Sophie. }\end{array}$ & gender: F actor: Anne-Sophie Brabant \\
\hline 273 & Heo Bijeon & actor: Jang-kang Heo Title: Bijeon \\
\hline 274 & 2002 Baroness Pig? & year: 2002 movie: Baroness and the pig \\
\hline 275 & 1995 Baiser Suzanne & year: 1995 movie: Baiser \\
\hline 276 & Det. Sally Paul 1996. & role: Detective Paul Sally year: 1996 \\
\hline 277 & frei Abel? & movie: Anwalt Abel - Sprecht mir diesen Mrder frei \\
\hline 278 & asunto interno & movie: Asunto interno \\
\hline 279 & 1982 Late Never Than. & year: 1982 movie: Better Late Than Never \\
\hline 280 & $\begin{array}{l}\text { Achille Antonaglia } \\
\text { Ciao. }\end{array}$ & actor: Achille Antonaglia movie: Ciao maschio \\
\hline 281 & Ang Ambisyosa & movie: Ambisyosa \\
\hline 282 & Strong Austin woman? & role: Austin Strong movie: All Woman \\
\hline 283 & Bir bcek iek? & movie: Bir iek \\
\hline 284 & 1938 apariencias & year: 1938 movie: Apariencias engaan \\
\hline 285 & blueprint $1993 ?$ & movie: blueprint year: 1993 \\
\hline 286 & Captain Bletcher? & actor: Billy Bletcher movie: Captain's Pup \\
\hline 287 & 2005 svnyei fny! & year: 2005 movie: A fny svnyei \\
\hline 288 & $\begin{array}{l}\text { Crampton Howard } \\
\text { orchids }\end{array}$ & actor: Howard Crampton Movie: Black Orchids \\
\hline 289 & 1915 Country Parson. & year: 1915 movie: Country Parson \\
\hline 290 & $\begin{array}{c}\text { Beach Babies Kemper } \\
\text { Charles }\end{array}$ & movie: Beach Babies actor: Charles Kemper \\
\hline 291 & 1983 Bob burns. & year: 1983 role: Bob Burns \\
\hline
\end{tabular}




\begin{tabular}{|c|c|c|}
\hline 292 & confidence of 1909 & movie: confidence year: 1909 \\
\hline 293 & Franz Von Lovers! & role: Dr. Franz Von Leinsdorff movie: City Lovers \\
\hline 294 & 1992 Perez. & year: 1992 actor: Vincent Perez \\
\hline 295 & Jackson Ada! & role: Steve Jackson movie: Ada \\
\hline 296 & 2003 Slobodan & year: 2003 actor: Slobodan Alavanja \\
\hline 297 & 1922 carillon & year: 1922 movie: Carillon de minuit \\
\hline 298 & curse 1913 & year: 1913 movie: Curse \\
\hline 299 & Emil Lorenz Bergfhrer! & actor: Emil Gyr movie: Bergfhrer Lorenz \\
\hline 300 & $\begin{array}{l}\text { James Almanzar } \\
\text { Dobermans? }\end{array}$ & actor: James Almanzar movie: Amazing Dobermans \\
\hline 301 & Crusoes Child. & movie: Child Crusoes \\
\hline 302 & Animatrix flight? & movie: Animatrix: Final Flight of the Osiris \\
\hline 303 & Bahar Barkha & movie: Barkha Bahar \\
\hline 304 & Hood 2.7. & movie: Beverly Hood rank: 2.7 \\
\hline 305 & Corporal Law & role: Corporal movie: Beyond the Law \\
\hline 306 & 2002 sangre & year: 2002 movie: A sangre Fria \\
\hline 307 & 1984 violi pouli & year: 1984 movie: An itan to Violi Pouli \\
\hline 308 & Emery the Horn Battle? & $\begin{array}{c}\text { actor: Dick Emery movie: Case of the Mukkinese Battle } \\
\text { Horn }\end{array}$ \\
\hline 309 & SCI-FI Alien Body & movie: Alien Nation genre: Sci-Fi \\
\hline 310 & 2.8 rating Aquanoids! & rank: 2.8 Movie: aquanoids \\
\hline 311 & All Hands of 1940. & movie: All Hands year: 1940 \\
\hline 312 & Billy Gets Square 1913 & movie: Broncho Billy Gets Square year: 1913 \\
\hline 313 & c2 h5 oh & movie: $\mathrm{c} 2 \mathrm{~h} 5 \mathrm{oh}$ \\
\hline 314 & $\begin{array}{l}\text { The Feature Length } \\
\text { coronation street }\end{array}$ & movie: Coronation Street: The Feature Length Special \\
\hline 315 & Siebzehn Siebzehn die! & actor: Oliver Bröcker movie: Alles auf die Siebzehn \\
\hline 316 & 5 rating Bejleren & rank: 5 movie: Bejleren - en jydsk rverhistorie \\
\hline 317 & Binarius. & movie: Binarius \\
\hline 318 & Decker Cruise & Movie: Cruise of the Zaca actor: John Decker \\
\hline 319 & 1993 MTV movies & Movie: 1993 MTV Movie Awards \\
\hline 320 & Bofors Gun & Movie: Bofors Gun \\
\hline 321 & Goddess Clary & Actor: Charles Clary Movie: Blind Goddess \\
\hline 322 & $\begin{array}{l}6.5 \text { rating with } \\
\text { Customers }\end{array}$ & rank: 6.5 movie: Customers Wanted \\
\hline 323 & Louise de Casamento! & movie: Casamento de Louise \\
\hline 324 & Morrow Orchestra & $\begin{array}{c}\text { actor: Buddy Morrow Movie: Buddy Morrow and His } \\
\text { Orchestra }\end{array}$ \\
\hline 325 & 1999 Arrangement & year: 1999 movie: Arrangement \\
\hline 326 & Buffy Chaos Tom & $\begin{array}{c}\text { movie: Buffy the Vampire Slayer: Chaos Bleeds actor: } \\
\text { Tom Bourdon }\end{array}$ \\
\hline
\end{tabular}




\begin{tabular}{|c|c|c|}
\hline 327 & Dev P 2000 & actor: Rajan P. Dev year: 2000 \\
\hline 328 & Luigi 2001? & actor: Luigi Aycardi year: 2001 \\
\hline 329 & 2002 Todsnde Das? & year: 2002 movie: Todsnde: Das Toskana-Karussell \\
\hline 330 & $\begin{array}{l}2001 \text { Front Access } \\
\text { Live. }\end{array}$ & $\begin{array}{c}\text { year: } 2001 \text { movie: All Access: Front Row. Backstage. } \\
\text { Live! }\end{array}$ \\
\hline 331 & Berlin Sebastian! & movie: Berlin - Abschnitt 40 actor: Sebastian Bezzel \\
\hline 332 & Bob and Bill of 1920 & movie: Adventures of Bob and Bill year: 1920 \\
\hline 333 & Jonathan Adams 1998 & actor: Jonathan Adams year: 1998 \\
\hline 334 & Bug 2003 & movie: Bug year: 2003 \\
\hline 335 & celos 1940 & year: 1940 Movie: Celos de Cndida \\
\hline 336 & 5.4 rating with Ramiro. & rank: 5.4 actor: Ramiro Agüero \\
\hline 337 & congiura borgia & movie: Congiura dei Borgia \\
\hline 338 & 1999 Chittagong & movie: Dernire Escale year: 1999 \\
\hline 339 & Bizim kiz & movie: bizim kiz \\
\hline 340 & broncho billy & movie: broncho billy \\
\hline 341 & 2005 beauty shop & movie: Beauty Shop year: 2005 \\
\hline 342 & 1983 bog & year: 1983 movie: Bog \\
\hline 343 & $\begin{array}{l}\text { corrida Valencia } \\
\text { Belmont Willie! }\end{array}$ & movie: Corrida de toros en Valencia actor: Willie Belmont \\
\hline 344 & 195 Bailoretto & movie: Bailoretto year: 1985 \\
\hline 345 & burning land & movie: burning land \\
\hline 346 & 1919 Brat & year: 1919 movie: brat \\
\hline 347 & 1947 Carnegie & year: 1947 movie: Carnegie Hall \\
\hline 348 & 1921 Buried Treasure & movie: Buried Treasure year: 1921 \\
\hline 349 & English in Acid Test & Actor: James English movie: Acid Test \\
\hline 350 & $\begin{array}{l}\text { Bonamino Gustavo in } \\
\text { comedy }\end{array}$ & genre: comedy actor: Gustavo Brandau \\
\hline 351 & coward of 1913 & movie: Coward year: 1913 \\
\hline 352 & agnosti nychtas & movie: Agnosti tis nychtas \\
\hline 353 & 4.4 rating aroused & rank: 4.4 movie: aroused \\
\hline 354 & Indahouse of 2002 & movie: Ali G Indahouse year: 2002 \\
\hline 355 & smoke bellew & movie: Adventures of Smoke Bellew \\
\hline 356 & 1915 fate & year: 1915 movie: arrangement with Fate \\
\hline 357 & $\begin{array}{c}\text { Amorevolmente } \\
\text { Castaldo. }\end{array}$ & movie: Amorevolmente actor: Pippo Castaldo \\
\hline 358 & FitzPatrick Narrator & actor: James A. FitzPatrick role: Narrator \\
\hline 359 & $\begin{array}{l}1936 \text { version of thin } \\
\text { man }\end{array}$ & year: 1936 movie: After the Thin Man \\
\hline 360 & $\begin{array}{l}\text { Faithful Always } \\
\text { Davidson! }\end{array}$ & movie: Always Faithful actor: William B. Davidson \\
\hline 361 & 1930 Bandit & year: 1930 movie: Beau Bandit \\
\hline
\end{tabular}




\begin{tabular}{|c|c|c|}
\hline 362 & Bazaar $1997 ?$ & movie: Bazaar year: 1997 \\
\hline 363 & 1927 with Morgan & actor: Jack Morgan year: 1927 \\
\hline 364 & Bell Hank Devil & actor: Hank Bell movie: Brand of the Devil \\
\hline 365 & Bassan in Cinmaton & actor: Raphaël Bassan movie: cinmaton n 2050 \\
\hline 366 & Bsewichter Dran & movie: Bsewichter mssen dran \\
\hline 367 & 3.4 rating in $1998 ?$ & rank: 3.4 year: 1998 \\
\hline 368 & Snchez with Bofarull! & movie: Abel Snchez actor: Antonio Bofarull \\
\hline 369 & battle of who in 1913 & movie: Battle of Who Run year: 1913 \\
\hline 370 & Andy in Blackenstein & movie: Blackenstein actor: M Andy \\
\hline 371 & $\begin{array}{c}\text { Anderson in Captain } \\
\text { Kate }\end{array}$ & movie: Captain Kate actor: Tom Anderson \\
\hline 372 & $\begin{array}{l}\text { Vladimir Aukstikalnis } \\
\text { in poshchadu! }\end{array}$ & $\begin{array}{l}\text { actor: Vladimir Aukstikalnis movie: Bez Prava na } \\
\text { Poshchadu }\end{array}$ \\
\hline 373 & 7.5 rating napoletano & rank: 7.5 movie: Carosello Napoletano \\
\hline 374 & Adam Evil Arnfinson & movie: Adam \& Evil actor: Sean Arnfinson \\
\hline 375 & ftera Vasilis Kailas! & movie: Aggeloi horis ftera actor: Vasilis Kailas \\
\hline 376 & 1979 Body Human & year: 1979 movie: Body Human: The Magic Sense \\
\hline 377 & 1931 Edmund Breese & year: 1931 actor: Edmund Breese \\
\hline 378 & Body Busy $1967 !$ & movie: Busy Body year: 1967 \\
\hline 379 & cracked David Bowie & movie: Film About David Bowie \\
\hline 380 & revel $1921 !$ & movie: Beau Revel year: 1921 \\
\hline 381 & barbie nutcracker & movie: Barbie in the Nutcracker \\
\hline 382 & $\begin{array}{l}5.8 \text { rating commando } \\
\text { infierno } \\
\end{array}$ & rank: 5.8 movie: infierno \\
\hline 383 & 2003 Blue collar & year: 2003 movie: Blue collar comedy Tour: The movie \\
\hline 384 & brown in rating 6.2 & actor: Russ Brown rank: 6.2 \\
\hline 385 & Dr Burger Vlastimil & role: Dr. Burger actor: Vlastimil Brodský \\
\hline 386 & 1999 version choke & movie: choke year: 1999 \\
\hline 387 & Apna Apna? & movie: Andaz Apna Apna \\
\hline 388 & Dunham in Cowards & actor: Phil Dunham movie: Brave Cowards \\
\hline 389 & 1Bye Bye Brasil & movie: Bye Bye Brasil \\
\hline 390 & Bittere Larssen Robert? & movie: Bittere Unschuld role: Dr. Robert Larssen \\
\hline 391 & Down Earth & Movie: Down to Earth \\
\hline 392 & Adriana 1988 & movie: Adriana year: 1988 \\
\hline 393 & 6.4 Blondie & rank: 6.4 movie: Blondie Knows Best \\
\hline 394 & Koch $2002 !$ & role: Captain Koch year: 2002 \\
\hline 395 & 1989 Chanakyan & year: 1989 movie: Chanakyan \\
\hline 396 & $1989 \mathrm{BBB}$ & year: 1989 movie: BBB \\
\hline 397 & Butterfly Grey? & movie: Butterfly in Shades of Grey \\
\hline 398 & 1990 amingos? & year: 1990 movie: Buenos amigos \\
\hline
\end{tabular}




\begin{tabular}{|c|c|c|}
\hline 399 & 4.7 Angel Harp & rank: 4.7 movie: Angel Who Pawned Her Harp \\
\hline 400 & haengbok yeojaui 1979 . & movie: Bulhaenghan yeojaui haengbok year: 1979 \\
\hline 401 & 1975 window & year: 1975 movie: Coming Through the Window \\
\hline 402 & 1950 Dallas & year: 1950 movie: Dallas \\
\hline 403 & Cruces sobre & movie: Cruces Sobre el Yermo \\
\hline 404 & Amateur ecstasy & movie: All Amateur Ecstasy \\
\hline 405 & 8.4 rating in 1957 & rank: 8.4 year: 1957 \\
\hline 406 & Baranski & movie: Baranski \\
\hline 407 & love Blacksmiths. & movie: Blacksmith's Love \\
\hline 408 & $\begin{array}{l}\text { Finkenzeller Brunnen } \\
\text { dem }\end{array}$ & actor: Karl Finkenzeller movie: Am Brunnen vor dem Tore \\
\hline 409 & $\begin{array}{l}\text { Theodore Rossevelt } \\
\text { officers }\end{array}$ & movie: Col. Theodore Roosevelt and Officers of His Staff \\
\hline 410 & 2000 Comunidad & year: 2000 movie: Comunidad \\
\hline 411 & arirang & movie: arirang \\
\hline 412 & 1998 Estrella Alberto? & year: 1998 actor: Alberto Estrella \\
\hline 413 & adult $100 \%$ girls & movie: $100 \%$ girls genre: adult \\
\hline 414 & 1924 Flirt Dangerous? & year: 1924 movie: Dangerous Flirt \\
\hline 415 & 6.4 rating Kane. & rank: 6.4 role: Kane \\
\hline 416 & Palos Bonnin? & role: Palos actor: Bernard Bonnin \\
\hline 417 & 6.4 day valley & rank: 6.4 movie: 2 Days in the Valley \\
\hline 418 & 1982 ciao! & year: 1982 movie: Ciao Nemico \\
\hline 419 & Springsteen 2003 & $\begin{array}{l}\text { year: } 2003 \text { movie: Bruce Springsteen \& the E Street Band: } \\
\text { Live in Barcelona }\end{array}$ \\
\hline 420 & 2004 Beyond Bath and & movie: bird, bath and beyond year: 2004 \\
\hline 421 & California Kevin James & movie: California Reaming actor: Kevin James \\
\hline 422 & close eyes young & movie: Close my eyes role: Young Man \\
\hline 423 & 1962 Cappadocce & year: 1962 movie: cappadocce \\
\hline 424 & Boris 1994. & actor: Boris Andrusevic year: 1994 \\
\hline 425 & Harvey in skyline & movie: Beyond the Skyline actor: Harvey Keitel \\
\hline 426 & soaped up 2002 & year: 2002 movie: All Soaped Up \\
\hline 427 & 1989 scratch fever & year: 1989 movie: Cat Scratch Fever \\
\hline 428 & Banquet Yves! & movie: Banquet des fraudeurs actor: Yves Deniaud \\
\hline 429 & $\begin{array}{l}\text { Graustark Max } \\
\text { Barwyn! }\end{array}$ & movie: Beverly of Graustark actor: Max Barwyn \\
\hline 430 & 1963 Aqua Duck & year: 1963 movie: Aqua Duck \\
\hline 431 & 1999 Villarama & year: 1999 movie: Abel Villarama: Armado \\
\hline 432 & $\begin{array}{l}\text { Little Wolfs Harner } \\
\text { Butler? }\end{array}$ & movie: 3 Little Wolfs actor: Jason Butler Harner \\
\hline 433 & cupid Policeman & movie: Cupid and the cook role: The Policeman \\
\hline 434 & Agnes psihes & movie: Agnes psihes \\
\hline
\end{tabular}




\begin{tabular}{|c|c|c|}
\hline 435 & Beery bombs & actor: Wallace Beery movie: Bombs and Banknotes \\
\hline 436 & 1910 Childs Message & year: 1910 movie: Child's Message to Heaven \\
\hline 437 & 1923 Bumps & year: 1923 movie: Bumps \\
\hline 438 & Jack in Charming & Actor: Jack Connolly Movie: Charming Villain \\
\hline 439 & Cotton King & movie: Cotton King \\
\hline 440 & Black 2 valley & movie: Black Valley Girls 2 \\
\hline 441 & corsetry 101 & movie: corsetry 101 \\
\hline 442 & beartooth & movie: beartooth \\
\hline 443 & 2004 Bafta awards & year: 2004 movie: BAFTA TV Awards 2004 \\
\hline 444 & 1980 bouzoukia & year: 1989 movie: Apo ta Thrania sta bouzoukia \\
\hline 445 & Event Blessed 7? & movie: Blessed Event rank: 7 \\
\hline 446 & carry on 1970 & movie: Carry it On year: 1970 \\
\hline 447 & 2000 Alexander. & year: 2000 actor: Marc Alexander \\
\hline 448 & Melvin Chen 15 ! & actor: Melvin Chen movie: 15 \\
\hline 449 & All this 1940 & year: 1940 movie: All this \\
\hline 450 & 1928 correspondence & year: 1928 movie: By correspondence \\
\hline 451 & Busty babes 5 & movie: Busty Babes in Heat 5 \\
\hline 452 & 2004 cudownie & year: 2004 movie: cudownie ocalony \\
\hline 453 & Black Brown Syd? & movie: Black Christmas actor: Syd Brown \\
\hline 454 & Beck Buzzy & actor: Jackson Beck role: Buzzy \\
\hline 455 & tiempos cuatro tiempos & movie: Amor en cuatro tiempos \\
\hline 456 & Frank Capra Salute? & movie: American Film Institute Salute to Frank Capra \\
\hline 457 & 5.6 Lester Lister? & rank: 5.6 role: Lester Lister \\
\hline 458 & Lin in china & role: Lin Cho movie: china \\
\hline 459 & call Yukon & movie: Call of the Yukon \\
\hline 460 & pornografo 1989 & movie: Aprendiz de pornografo year: 1989 \\
\hline 461 & burglar of 1916 & movie: the Burglar year: 1916 \\
\hline 462 & 1946 Roger Pigaut. & year: 1946 actor: Roger Pigaut \\
\hline 463 & Grandon Affair heart & actor: Francis J. Grandon movie: Affair of Hearts \\
\hline 464 & $\begin{array}{l}\text { Bakalyan Richard blair } \\
\text { witch }\end{array}$ & $\begin{array}{c}\text { actor: Richard Bakalyan movie: Blair Witch Mountain } \\
\text { Project }\end{array}$ \\
\hline 465 & Account 2004 ! & movie: Account year: 2004 \\
\hline 466 & 1913 Eddie Lyons & year: 1913 actor: Eddie Lyons \\
\hline 467 & 6.3 rating chain gang! & rank: 6.3 movie: Chain Gang \\
\hline 468 & $\begin{array}{l}\text { Beautiful Gambler } \\
1921\end{array}$ & movie: Beautiful Gambler year: 1921 \\
\hline 469 & Glory 2000 Soloist! & movie: Christmas Glory 2000 role: Soloist \\
\hline 470 & 5.9 Palace Htel & rank: 5.9 movie: Bunker Palace Htel \\
\hline 471 & 1916 Calamity & year: 1916 movie: Calamity Anne \\
\hline 472 & Hillbillies Dean Paul & movie: Cannibal Hillbillies actor: Dean Paul \\
\hline
\end{tabular}




\begin{tabular}{|c|c|c|}
\hline 473 & 1958 Mr Scruby. & year: 1958 role: Mr. Scruby \\
\hline 474 & Waldemar Brem? & actor: Rudolf Waldemar Brem \\
\hline 475 & Currie in Bawn & actor: Clive Currie movie: Colleen Bawn \\
\hline 476 & 1989 Petrovna & year: 1989 movie: Anna Petrovna \\
\hline 477 & Sam and Porky Lonney & $\begin{array}{c}\text { role: Bugs Bunny/Yosemite Sam/Porky movie: bugs } \\
\text { bunny's Looney Christmas Tales }\end{array}$ \\
\hline 478 & 1929 Alte Kleider? & year: 1929 movie: Alte Kleider \\
\hline 479 & Copyright 1993 & movie: Copyright year: 1993 \\
\hline 480 & 8.3 Cudotvorni? & rank: 8.3 movie: Cudotvorni mac \\
\hline 481 & Bambi Justice Hunting & movie: American Justice: Hunting Bambi \\
\hline 482 & aksam gnesi & movie: aksam gnesi \\
\hline 483 & 1961 challenge ideas & year: 1961 movie: Challenge of Ideas \\
\hline 484 & cit des enfants. & movie: Cit des enfants perdus \\
\hline 485 & Guard Prison 1999 & role: Prison Guard year: 1999 \\
\hline 486 & Cabeleira 1963. & movie: Cabeleira year: 1963 \\
\hline 487 & fin mar & movie: fin, el mar \\
\hline 488 & 1902 dames & year: 1902 movie: Bonsoir m'sieurs dames \\
\hline 489 & Juan est Aguilar & movie: Aqu est Juan Colorado actor: Luis Aguilar \\
\hline 490 & $\begin{array}{l}\text { Columbo Brainerd } \\
\text { Chris }\end{array}$ & role: Columbo actor: Chris Brainerd \\
\hline 491 & Mel Blanc 6.4? & actor: Mel Blanc rank: 6.4 \\
\hline 492 & Adams in battlers & actor: Steve Adams movie: Battlers \\
\hline 493 & colpo rovente & movie: Colpo rovente \\
\hline 494 & Acord Battle life & actor: Art Acord movie: Battle of Life \\
\hline 495 & aires Bret & actor: Bert movie: Buenos Aires, here \\
\hline 496 & biggest wish 2004 & movie: Biggest Wish year: 2004 \\
\hline 497 & $\begin{array}{l}\text { Mornar Gainsbourg } \\
\text { Serge? }\end{array}$ & role: Mornar actor: Serge Gainsbourg \\
\hline 498 & aprs tout 2003 & movie: Aprs Tout year: 2003 \\
\hline 499 & chills or fever? & movie: Chills and Fever \\
\hline 500 & automatic 1994 & movie: Automatic year: 1994 \\
\hline 501 & movies from 1985. & year: 1985 \\
\hline 502 & china black 1992 & movie: China Black year: 1992 \\
\hline 503 & Baradan kaagiyim & actor: Hüseyin Baradan movie: Ben bir kanun kaagiyim \\
\hline 504 & Phil 2001? & role: Phil year: 2001 \\
\hline 505 & Campus Flirt & movie: Campus Flirt \\
\hline 506 & City Raiders Joe. & movie: Carson City Raiders role: Joe \\
\hline 507 & Baker Dusty & actor: Bob Baker role: Dusty \\
\hline 508 & Avellana Mari 1983? & actor: Joe Mari Avellana year: 1983 \\
\hline 509 & 72 artetur & movie: Artetur - 72 \\
\hline
\end{tabular}




\begin{tabular}{|c|c|c|}
\hline 510 & Dennis Akayama 3.9. & actor: Dennis Akayama rank: 3.9 \\
\hline 511 & Maguire Dalekmani & actor: Josh Maguire movie: Dalekmani \\
\hline 512 & Abel Hassan & role: Ablard 'Abel' Hassan de Chiri \\
\hline 513 & Bell in Boxer & actor: Tom Bell movie: Boxer \\
\hline 514 & Burning rage Albert & movie: burning rage actor: Eddie Albert \\
\hline 515 & Schnhauser Ecke 1957! & movie: Berlin - Ecke Schnhauser year: 1957 \\
\hline 516 & 1972 Chaudhuri & year: 1972 actor: Nirad C. Chaudhuri \\
\hline 517 & 1914 broadway? & movie: Back to Broadway year: 1914 \\
\hline 518 & 1974 Amudo & year: 1974 movie: Amudo eobseotdeon yeoleum \\
\hline 519 & 1986 Aguilar & year: 1986 actor: Luis Aguilar \\
\hline 520 & $\begin{array}{c}\text { Wano Bastion } \\
\text { Lawrence Campbell? }\end{array}$ & role: Te Wano Bastion actor: Lawrence Campbell \\
\hline 521 & DDR schriftsteller & movie: Bln. DDR und ein Schriftsteller \\
\hline 522 & 6.9 Claire & rank: 6.9 movie: Claire makes it big \\
\hline 523 & Castle Ranch & movie: Castle Ranch \\
\hline 524 & 1935 dames & year: 1935 movie: Champion de ces dames \\
\hline 525 & Alex Complain & actor: Alex Matthews movie: Complain \\
\hline 526 & 1971 hormona & year: 1971 movie: Aunque la hormona se vista \\
\hline 527 & 1973 Zapper & year: 1973 movie: big Zapper \\
\hline 528 & 3.9 rating Pierce Stack! & rank: 3.9 actor: Stack Pierce \\
\hline 529 & $\begin{array}{c}\text { bartender Frank Blake } \\
\text { Oliver! }\end{array}$ & role: Frank (bartender) actor: Oliver Blake \\
\hline 530 & Clayton Alice & actor: John Clayton movie: Alice to Nowhere \\
\hline 531 & John wayne A E & $\begin{array}{c}\text { movie: A\&E Biography: John Wayne Gacy: A Monster in } \\
\text { Disguise }\end{array}$ \\
\hline 532 & hie chun & movie: chun-hie \\
\hline 533 & cible Bacri Bacri & actor: Jean-Pierre Bacri movie: $7 \mathrm{me}$ cible \\
\hline 534 & Alerme in Bicho & actor: André Alerme movie: Bichon \\
\hline 535 & 7.3 Bourne identify & rank: 7.3 movie: Bourne Identity \\
\hline 536 & billy kid texas & movie: Billy the kid in texas \\
\hline 537 & over world & movie: All over the world \\
\hline 538 & Higgins Beach Party! & role: Dean Higgins movie: Cheerleaders' Beach Party \\
\hline 539 & Alden Bob 5.5 & actor: Bob Alden rank: 5.5 \\
\hline 540 & Haenjga Buin & movie: Buin Haenjga \\
\hline 541 & 1966 christmas tree & year: 1966 movie: Christmas Tree \\
\hline 542 & addio abbot & movie: Addio Mim! actor: John Abbott \\
\hline 543 & Martino beverly blones & actor: Tony Martino movie: Beverly Hills Blondes 2 \\
\hline 544 & 80 Simon Hamlin & movie: 80 's ending actor: Simon Hamlin \\
\hline 545 & 5.9 champion? & rank: 5.9 movie: champion \\
\hline 546 & bien pagada & movie: Bien pagada \\
\hline
\end{tabular}




\begin{tabular}{|c|c|c|}
\hline 547 & 6.3 bridal path & rank: 6.3 movie: Bridal path \\
\hline 548 & Justo nguez 2000. & actor: Justo Domínguez year: 2000 \\
\hline 549 & Mel blanc 1953. & actor: Mel Blanc year: 1953 \\
\hline 550 & ainalaiyn & movie: Ainalaiyn \\
\hline 551 & brian Wilson Tribute & movie: All-Star Tribute to Brian Wilson \\
\hline 552 & Berlin German Film! & movie: Berlin Metropolis: Jews in Early German Film \\
\hline 553 & $\begin{array}{l}\text { Byeongmu cheongchun } \\
\text { daehak. }\end{array}$ & $\begin{array}{l}\text { role: Byeong-mu movie: Chumchuneun cheongchun } \\
\text { daehak }\end{array}$ \\
\hline 554 & 1934 Squadron. & year: 1934 movie: Blue Squadron \\
\hline 555 & Interlude Catalina & movie: Catalina Interlude \\
\hline 556 & All over 1976 & movie: all over year: 1976 \\
\hline 557 & caudillo & movie: Caudillo \\
\hline 558 & 8th screen actors & movie: 8th Annual Screen Actors Guild Awards \\
\hline 559 & $\begin{array}{l}\begin{array}{l}\text { Ehrenfreund Heinz } \\
\text { adieu }\end{array} \\
\end{array}$ & actor: Heinz Ehrenfreund movie: Adieu \\
\hline 560 & $\begin{array}{l}\text { Peter BlochHansen } \\
\text { 2001! }\end{array}$ & actor: Peter Bloch-Hansen year: 2001 \\
\hline 561 & Shots Deputy & movie: Big Shots role: Deputy \\
\hline 562 & Alpenglhn Dirndlrock & gender: $\mathrm{m}$ movie: Alpenglhn im Dirndlrock \\
\hline 563 & Blackjack Buchanan. & movie: Blackjack: Ace Point Game role: Buchanan \\
\hline 564 & Penance Blackwell & actor: Carlyle Blackwell movie: Bell of Penance \\
\hline 565 & $\begin{array}{c}1992 \text { chiller theatre vol. } \\
1\end{array}$ & year: 1992 movie: Chiller Theatre Expo Video Vol. 1 \\
\hline 566 & $\begin{array}{c}\text { Angelescu Moreni } \\
\text { Blauvogel }\end{array}$ & actor: Ion Angelescu-Moreni movie: Blauvogel \\
\hline 567 & 2004 24th day & year: 2004 movie: 24th Day \\
\hline 568 & arthur West Side & actor: Arthur Acuña movie: Batang West Side \\
\hline 569 & $\begin{array}{c}\text { Brodthagen Viggo med } \\
\text { glde }\end{array}$ & actor: Viggo Brodthagen movie: Betal din skat med glde \\
\hline 570 & maden ikke & movie: Bag om filmen 'Manden som ikke ville d' \\
\hline 571 & 1994 sangrienta & movie: Caceria Sangrienta year: 1994 \\
\hline 572 & Long ga choi yau. & role: Long movie: 97 ga yau choi si \\
\hline 573 & 5.4 chambers horrors & rank: 5.4 movie: Chamber of Horrors \\
\hline 574 & Body library $1984 ?$ & movie: Body in the Library year: 1984 \\
\hline 575 & 1971 aggittorok & year: 1971 movie: Agittorok \\
\hline 576 & 70th sugar bowl & movie: 70th Annual Nokia Sugar Bowl \\
\hline 577 & 1959 sangre. & year: 1959 movie: A sangre fra \\
\hline 578 & 2004 Blue Rose & year: 2004 movie: Blue Rose \\
\hline 579 & Murders Doll! & movie: baby doll murders \\
\hline 580 & 5up 2down & movie: 5up 2down \\
\hline 581 & Arlette 4.4 & movie: Arlette rank: 4.4 \\
\hline
\end{tabular}




\begin{tabular}{|c|c|c|}
\hline 582 & Black white Jeffrey? & movie: Black or white actor: Jeffrey Anderson-Gunter \\
\hline 583 & Da She & movie: Da she \\
\hline 584 & Bailey chem 102 & movie: chem 102 actor: Jason Bailey \\
\hline 585 & Policeman Motorist? & role: Motorist movie: Ask a Policeman \\
\hline 586 & Philly 4? & role: Philly rank: 4 \\
\hline 587 & schwabing 1971 & movie: Cream - Schwabing-Report year: 1971 \\
\hline 588 & Calle Mayor. & movie: Calle Mayor \\
\hline 589 & circle death & movie: Circle of Death \\
\hline 590 & Amsal jiryeong? & movie: Amsal jiryeong \\
\hline 591 & 1962 Baradan & year: 1962 actor: Hüseyin Baradan \\
\hline 592 & Dangerous Females & movie: Dangerous females \\
\hline 593 & 1976 Compadre & year: 1976 movie: Compadre ms padre \\
\hline 594 & Afterward 1985 & movie: Afterward year: 1985 \\
\hline 595 & Chinbind shjin. & movie: Chinbind shjin \\
\hline 596 & 5.9 Comedians! & rank: 5.9 movie: Comedians \\
\hline 597 & 1955 Alunno! & year: 1955 role: Alunno \\
\hline 598 & Billie and Buster? & movie: Buster and Billie \\
\hline 599 & Christmas lilies field & movie: Christmas Lilies of the Field \\
\hline 600 & L di Peccati singrossa? & movie: Affare s'ingrossa: Peccati di culo 2 \\
\hline 601 & cabin lake Blake & movie: cabin by the lake role: Blake \\
\hline 602 & 100 Proof & movie: 100 Proof \\
\hline 603 & 1954 hombres & year: 1954 movie: Crisol de hombres \\
\hline 604 & Giselle April & movie: April in Bondage actor: Giselle Apricot \\
\hline 605 & Hansen rating 5.3 & actor: Paul Hansen rank: 5.3 \\
\hline 606 & ana hua hia? & movie: Ana wa hua wa hia \\
\hline 607 & Anderson crossplot & movie: Crossplot actor: Leslie Anderson \\
\hline 608 & 1915 zan l'embusqu & movie: Bout de Zan et l'embusqu year: 1915 \\
\hline 609 & mario antofagasta & actor: Mario Guzmám movie: Antofagasta \\
\hline 610 & 1915 Revenge & year: 1915 movie: Christmas Revenge \\
\hline 611 & 1985 Blue? & year: 1985 movie: Blue Yonder \\
\hline 612 & Cardcaptor $8 ?$ & movie: Cardcaptor Sakura rank: 8 \\
\hline 613 & Abbott with two yanks & movie: Abroad with Two Yanks actor: John Abbott \\
\hline 614 & 1979 Strikes again & movie: Contest kid Strikes again year: 1979 \\
\hline 615 & Brody & movie: Brody \\
\hline 616 & delito Eduardo! & actor: Eduardo Alcaraz movie: Cuerpazo del delito \\
\hline 617 & Robert big premiere & actor: Robert Blake movie: Big Premiere \\
\hline 618 & Antonio Anelli 1972! & actor: Antonio Anelli year: 1972 \\
\hline 619 & 52 Mail California & rank: 5.2 movie: California Mail \\
\hline 620 & billy big stick & movie: Billy and the Big Stick \\
\hline
\end{tabular}




\begin{tabular}{|c|c|c|}
\hline 621 & cais seafish Pexelim & movie: cais do sodr role: Pexelim (sea-fish) \\
\hline 622 & $19954.6 ?$ & year: 1995 rank: 4.6 \\
\hline 623 & Monty Arnold 2003! & actor: Monty Arnold year: 2003 \\
\hline 624 & Colgecen Feridun & actor: Feridun Colgecen movie: bar kizi \\
\hline 625 & 2004 Blindgnger & year: 2004 movie: Blindgnger \\
\hline 626 & Jacques Boudet ventre? & actor: Jacques Boudet movie: conte du ventre plein \\
\hline 627 & 1981 Bukit Kepong. & year: 1981 movie: Bukit Kepong \\
\hline 628 & damaged goods & movie: damaged goods \\
\hline 629 & 2004 beyond ordinary & $\begin{array}{c}\text { year: } 2004 \text { movie: Beyond Ordinary: The Making of 'An } \\
\text { Ordinary Killer' }\end{array}$ \\
\hline 630 & $\begin{array}{l}\text { call kids role } \\
\text { Buckwheat! }\end{array}$ & movie: Calling All Kids role: Buckwheat \\
\hline 631 & William Clifford Trail. & actor: William Clifford movie: Blazed Trail \\
\hline 632 & $\begin{array}{l}\text { Blackbeard Vendor } \\
\text { Popcorn. }\end{array}$ & movie: Blackbeard's Ghost role: Popcorn Vendor \\
\hline 633 & Buru Eisei Amamoto? & movie: Buru kurisumasu actor: Eisei Amamoto \\
\hline 634 & Bridges Robert! & movie: Bridges Burned actor: Robert Broderick \\
\hline 635 & agente lei & movie: Agente da Lei \\
\hline 636 & 1976 Atybaty? & year: 1976 movie: Aty-baty \\
\hline 637 & arsonist 2004 & Movie: Arsonist year: 2004 \\
\hline 638 & Heads Buddha? & movie: Buddha Heads \\
\hline 639 & Blonde Stayed. & movie: Blonde stayed on \\
\hline 640 & 2003 Casual & year: 2003 movie: Casual Friday \\
\hline 641 & 1979 babushkin? & year: 1979 movie: Babushkin vnuk \\
\hline 642 & Wow Bow? & movie: Bow Wow \\
\hline 643 & Capital Prize & movie: Capital Prize \\
\hline 644 & 1983 brussels Night. & year: 1983 movie: Brussels by Night \\
\hline 645 & coming ice & movie: Coming Out of the Ice \\
\hline 646 & 1918 Fox! & year: 1918 role: The Fox \\
\hline 647 & clandestina Eliazar & movie: Avioneta clandestina actor: Eliazar García Jr. \\
\hline 648 & alla 2004 nazimova & movie: Alla Nazimova and Rudolph Valentino year: 2000 \\
\hline 649 & Baron Baron & movie: Baron \\
\hline 650 & busty babes 8 & movie: Busty babes in heat 8 \\
\hline 651 & 1986 Jangmok! & year: 1986 actor: Jang-mok Kim \\
\hline 652 & balladen Carl & movie: Balladen om Carl-Henning \\
\hline 653 & Condemned shot & movie: Condemned to Be Shot \\
\hline 654 & 4.7 bruce fingers? & rank: 4.7 movie: Bruce's Fingers \\
\hline 655 & $\begin{array}{c}\text { Atuma Pascal } \\
\text { Accidental Life. }\end{array}$ & actor: Pascal Atuma movie: Accidental Life \\
\hline 656 & hero in Chaddian di & movie: Chaddian Di Doli role: Hero \\
\hline
\end{tabular}




\begin{tabular}{|c|c|c|}
\hline 657 & $\begin{array}{l}6.7 \text { rating of } \\
\text { Brigadoon? }\end{array}$ & rank: 6.7 movie: Brigadoon \\
\hline 658 & 1985 California & movie: California Girls year: 1985 \\
\hline 659 & 1993 Malik! & year: 1993 role: Malik \\
\hline 660 & Benson IV Pike & actor: Chris (IV) Benson movie: Pike Street \\
\hline 661 & 4.9 rating Attia. & rank: 4.9 actor: Karim Attia \\
\hline 662 & Goldfish Blinding & movie: Blinding Goldfish \\
\hline 663 & dr. christian Baldwin & actor: Robert Baldwin movie: Courageous Dr. Christian \\
\hline 664 & Julien of colombe. & role: Julien movie: Colombe \\
\hline 665 & Adalist of Command? & actor: Jack Adalist movie: Chain of Command \\
\hline 666 & brother frontier & movie: brothers of the frontier \\
\hline 667 & boruneo tokero & movie: Boruneo taisho: Akamichi ni tokero \\
\hline 668 & color of justice & movie: Color of Justice \\
\hline 669 & Ammoru? & movie: Ammoru \\
\hline 670 & Adams Custer & actor: Ted Adams movie: Custer's Last Stand (1936/II) \\
\hline 671 & Boireau Deed & movie: Boireau Magistrat actor: André Deed \\
\hline 672 & 1987 British Cinema & year: 1987 movie: Best of British Cinema \\
\hline 673 & 5.3 Asphaltflimmern! & rank: 5.3 movie: Asphaltflimmern \\
\hline 674 & Abbott keystone & movie: Abbott and Costello Meet the Keystone Kops \\
\hline 675 & Baker clive Freakz & actor: Clive Barker movie: Clive Barker's Freakz \\
\hline 676 & Baby Clothes 1926 & movie: Baby Clothes year: 1926 \\
\hline 677 & 5.2 Blame rio & movie: Blame it on Rio rank: 5.2 \\
\hline 678 & baby clown & movie: Clown on the farm role: Baby Huey \\
\hline 679 & Freedom of Story & movie: Born In Freedom: The Story of Colonel Drake \\
\hline 680 & Wilderness challenge & movie: Challenge the Wilderness \\
\hline 681 & $\begin{array}{c}\text { Shorts Hollywood } \\
\text { attraction }\end{array}$ & movie: Added Attractions: The Hollywood Shorts Story \\
\hline 682 & 1977 Beyond & year: 1977 movie: Beyond Reason \\
\hline 683 & 1973 chabelo & year: 1973 movie: Chabelo y Pepito contra los monstruos \\
\hline 684 & $\begin{array}{c}\text { Bump Takes chump } \\
1939 . \\
\end{array}$ & movie: Chump Takes a Bump year: 1939 \\
\hline 685 & Wiedersehen Auf? & movie: Auf Wiedersehen \\
\hline 686 & Epstine Story & movie: Brian Epstein Story \\
\hline 687 & Vidur 1921. & movie: Bhakta Vidur year: 1921 \\
\hline 688 & Cult Live Cult 1976! & movie: Blue yster Cult: Live 1976 year: 1976 \\
\hline 689 & $\begin{array}{c}\text { Walter Cronkite } \\
\text { apartheid }\end{array}$ & actor: Walter Cronkite movie: Children of Apartheid \\
\hline 690 & castful condamnatilor & movie: Castelul Condamnatilor \\
\hline 691 & 1970 barbra & year: 1970 movie: Barbra: Yesterday \\
\hline 692 & compartment & movie: compartment \\
\hline
\end{tabular}




\begin{tabular}{|c|c|c|}
\hline 693 & bad business 1997 & year: 1997 movie: Bad Business \\
\hline 694 & Buchi rating 5.1 & movie: Buchi neri rank: 5.1 \\
\hline 695 & 1960 Bahana! & movie: Bahana year: 1960 \\
\hline 696 & atraco gran & movie: Atraco en la gran final \\
\hline 697 & 6.9 rating ruth? & rank: 6.9 movie: Citizen Ruth \\
\hline 698 & Almeida in Brasil & actor: Fernando Almeida movie: Churrascaria Brasil \\
\hline 699 & rivera caballero & actor: Jorge Rivera movie: Caballero de La Habana \\
\hline 700 & agent todd bad guys & role: Special Agent Todd movie: Bad Guys \\
\hline 701 & Edgar Border River! & actor: Edgar Jones movie: Border River \\
\hline 702 & $\begin{array}{c}\text { Concessionaire Grove } \\
\text { Cocoanut }\end{array}$ & role: Concessionaire movie: Cocoanut Grove \\
\hline 703 & Annie Your Gun 1957! & movie: Annie Get Your Gun year: 1957 \\
\hline 704 & Bimilgaek & movie: Bimilgaek 2 \\
\hline 705 & almohadon 1990 & year: 1990 movie: Almohadon \\
\hline 706 & 8.5 owaranai? & rank: 8.5 movie: Asobi no jikan wa owaranai \\
\hline 707 & father Akaki 1947! & role: Father of Akaki year: 1947 \\
\hline 708 & $\begin{array}{c}\text { movie } 5.3 \text { in year } \\
1994 !\end{array}$ & rank: 5.3 year: 1994 \\
\hline 709 & 48 somos celedonio & rank: 4.8 movie: Celedonio y yo somos as \\
\hline 710 & Daniel Leonardo fuego & actor: Leonardo Daniel movie: Con fuego en la sangre \\
\hline 711 & Toulon complexe & movie: Complexe de Toulon \\
\hline 712 & art possibly? & movie: Art of the Possible \\
\hline 713 & Bellhop 1940? & role: Bellhop year: 1940 \\
\hline 714 & 2002 Anansi & year: 2002 movie: Anansi \\
\hline 715 & born Kill 1947! & movie: Born to Kill year: 1947 \\
\hline 716 & 19896.4 rating & year: 1989 rank: 6.4 \\
\hline 717 & aztec $1988 ?$ & movie: Aztec Two Step 15th Anniversary Show year: 1988 \\
\hline 718 & Barfly Bar? & role: Barfly movie: Bar Girls \\
\hline 719 & Flight Mystery crash & movie: Crash: The Mystery of Flight 1501 \\
\hline 720 & April $20006.8 !$ & movie: 1 . April 2000 rank: 6.8 \\
\hline 721 & adventures Dr. Felatio & movie: Blowjob Adventures of Dr. Fellatio 24 \\
\hline 722 & Chiltern Hundreds & movie: Chiltern Hundreds \\
\hline 723 & Uri Myles 2001. & actor: Uri Myles year: 2001 \\
\hline 724 & Brian death & actor: Brian Boe movie: Courier of Death \\
\hline 725 & Raymond Queneau & movie: Cit Raymond Queneau \\
\hline 726 & 1986 sons & year: 1986 movie: All my Sons \\
\hline 727 & baja marea? & movie: Cuando baja la marea actor: Alejandro Cobo \\
\hline 728 & Great in City & movie: Adrift in a Great City \\
\hline 729 & 1963 Nelson Sheridon. & year: 1963 actor: Sheridon Nelson \\
\hline 730 & eurovision finale & movie: Concours eurovision de la chanson - finale \\
\hline
\end{tabular}




\begin{tabular}{|c|c|c|}
\hline & nationale & nationale \\
\hline 731 & Brisket & movie: Brisket \\
\hline 732 & Jay Ilagan bondying & actor: Jay Ilagan movie: Ato ti Bondying \\
\hline 733 & 1989 Astrix coup & year: 1989 movie: Astrix et le coup du menhir \\
\hline 734 & Country $6.3 !$ & movie: Country Doctor rank: 6.3 \\
\hline 735 & Ajax Danny Blind. & $\begin{array}{c}\text { movie: Ajax: Daar Hoorden Zij engelen zingen actor: } \\
\text { Danny Blind }\end{array}$ \\
\hline 736 & $\begin{array}{l}\text { Fisher M Bideau Jean- } \\
\text { Luc! }\end{array}$ & role: M. Fisher actor: Jean-Luc Bideau \\
\hline 737 & sister blues 1948 & movie: Big Sister Blues year: 1948 \\
\hline 738 & Black heart & movie: Black Heart \\
\hline 739 & misbehavin Cheerleader & movie: Cheerleaders Misbehavin' 2 role: Cheerleader \\
\hline 740 & blind musician & movie: Blind Musician \\
\hline 741 & 2004 andy love & movie: Andy in love year: 2004 \\
\hline 742 & Brother Cruze! & role: Bob's Brother actor: James Cruze \\
\hline 743 & $\begin{array}{l}\text { Eduardo Alcaraz } \\
\text { Cuatro? }\end{array}$ & actor: Eduardo Alcaraz movie: Cuatro dedos \\
\hline 744 & Chief Annapolis & movie: Annapolis Salute role: Chief Martin \\
\hline 745 & Brunton William Alibi & actor: William Brunton movie: Alibi \\
\hline 746 & ark time & movie: Ark of Time \\
\hline 747 & cardinal's edict & movie: Cardinal's Edict \\
\hline 748 & $\begin{array}{l}\text { Mitsuo Hamada } \\
\text { mitsumete }\end{array}$ & actor: Mitsuo Hamada movie: Ai to shi o mitsumete \\
\hline 749 & movie: Children labor & movie: Children who labor \\
\hline 750 & $\begin{array}{c}\text { Babendererde Brian } \\
\text { and Stupidity Butthead! }\end{array}$ & $\begin{array}{c}\text { actor: Brian Babendererde movie: Beavis and Butt-head in } \\
\text { Virtual Stupidity }\end{array}$ \\
\hline 751 & Katnip tamale & role: Katnip movie: Cat Tam \\
\hline 752 & 1916 Billy Deusen & year: 1916 movie: Billy van Deusen and the Vampire \\
\hline 753 & Nate as Alexander & role: Nate actor: Richard Alexander \\
\hline 754 & Garber in movie 44 & role: Darío Garber movie: 44 \\
\hline 755 & A\&E Anna and King & $\begin{array}{c}\text { movie: A\&E Biography: Anna and the King - The Real } \\
\text { Story of Anna Leonowens }\end{array}$ \\
\hline 756 & 5.9 chance Heaven! & rank: 5.9 movie: Chance at Heaven \\
\hline 757 & $\begin{array}{l}\text { Francisco Cantalejo } \\
\text { palmeras? }\end{array}$ & actor: Francisco Cantalejo movie: Dama y las palmeras \\
\hline 758 & army one & movie: Army of One \\
\hline 759 & Bambole! & movie: Bambole \\
\hline 760 & 1966 Ancermo? & year: 1966 actor: Ancermo Fukuda \\
\hline 761 & Broken Vase & movie: Broken Vase \\
\hline 762 & $\begin{array}{c}\text { Doug Abrahams in } \\
\text { convictions }\end{array}$ & actor: Doug Abrahams movie: Convictions \\
\hline 763 & cinmaton 1978 & year: 1978 movie: Cinmaton n 41 \\
\hline
\end{tabular}




\begin{tabular}{|c|c|c|}
\hline 764 & 1967 Bitgwa & year: 1967 movie: Bitgwa geurimja \\
\hline 765 & Blind DateFlorey & movie: Blind Date actor: François Florey \\
\hline 766 & Blithe 1956 & movie: Blithe Spirit year: 1956 \\
\hline 767 & 5.5 rating Baberellas & movie: Baberellas rank: 5.5 \\
\hline 768 & La Cruz & movie: la Cruz \\
\hline 769 & Bagatelle? & movie: Bagatelle \\
\hline 770 & Archer Anne Carnival & actor: Anne Archer movie: Carnival in Rio \\
\hline 771 & 1915 Colomba & year: 1915 movie: Colomba \\
\hline 772 & Bingham Junior & movie: Captain Junior actor: S.J. Bingham \\
\hline 773 & Appa annyeong! & movie: Appa annyeong \\
\hline 774 & bu tang ma & movie: $\mathrm{Bu}$ yi tang de ma ma \\
\hline 775 & Chad in business & actor: Dominic Chad movie: Closed for Business \\
\hline 776 & Policeman Banks. & role: Policeman actor: Howard Banks \\
\hline 777 & Culpepper cattle & movie: Culpepper Cattle Company \\
\hline 778 & 5.2 rating Asansor? & rank: 5.2 movie: Asansor \\
\hline 779 & The Boer War & movie: Boer War \\
\hline 780 & O Dias Dias? & movie: Antnio Dias: O Pas Inventado \\
\hline 781 & Saturday Night & movie: Another Saturday Night \\
\hline 782 & martnez 1971. & year: 1971 movie: Casa de los Martnez \\
\hline 783 & $\begin{array}{c}\text { after dark with } \\
\text { Hutchison? }\end{array}$ & movie: After Dark (1924/I) actor: Charles Hutchison \\
\hline 784 & 8.8 Balls Deep? & rank: 8.8 movie: Balls Deep \\
\hline 785 & Oliver Hardy in Fire & actor: Oliver Hardy movie: Building a Fire \\
\hline 786 & Augusta 5.1 & actor: Karel Augusta rank: 5.1 \\
\hline 787 & automobile thieves & movie: Automobile Thieves \\
\hline 788 & $\begin{array}{c}\text { baset with Elwon } \\
\text { Bakly. }\end{array}$ & actor: Elwon Bakly movie: Basket \\
\hline 789 & about moreau & movie: About Jeanne Moreau \\
\hline 790 & 1999 Asansor & movie: Asansor year: 1999 \\
\hline 791 & Bharosa & movie: Bharosa \\
\hline 792 & $2.61970 !$ & rank: 2.6 year: 1970 \\
\hline 793 & 1943 belaya & year: 1943 movie: Belaya Roza \\
\hline 794 & Because love & movie: Because he loved her \\
\hline 795 & 1966 Dag iegi & year: 1966 movie: Dag iegi \\
\hline 796 & Hellboy $2004 ?$ & movie: 'Hellboy': The Seeds of Creation year: 2004 \\
\hline 797 & kaagiyim Baradan & movie: Ben bir kanun Kaagiyim actor: Hüseyin Baradan \\
\hline 798 & baisers with rating 7.6 & movie: Baisers vols rank: 7.6 \\
\hline 799 & secon delay 5.5 & rank: 5.5 movie: 4 second delay \\
\hline 800 & Bathroom 2002 & year: 2002 movie: Bathroom \\
\hline 801 & movie with 5.9 Rabbit. & rank: 5.9 role: Rabbit \\
\hline
\end{tabular}




\begin{tabular}{|c|c|c|}
\hline 802 & 1916 Call Unborn & year: 1916 movie: Call of the Unborn \\
\hline 803 & Luis Buscona & actor: Luis Alarcón movie: Buscona \\
\hline 804 & Contraband? & movie: Contraband \\
\hline 805 & Alec Jordan Spikes? & actor: Jordan Alec role: Spike's Posse \\
\hline 806 & Dunn 1923. & actor: Bobby Dunn year: 1923 \\
\hline 807 & 1982 Annette & year: 1982 movie: All About Annette \\
\hline 808 & crash donovan & movie: Crash Donovan \\
\hline 809 & 6. 5 Jimmy & rank: 6.5 actor: Jimmy Aubrey \\
\hline 810 & 1914 aftermath & year: 1914 movie: Aftermath (1914/II) \\
\hline 811 & 1983 Bestia & year: 1983 movie: Bestia y la espada mgica \\
\hline 812 & bryan home & movie: Bryan at Home \\
\hline 813 & abenteuer knigs & movie: Abenteuer des Knigs Pausole \\
\hline 814 & Temple 1976. & movie: Blazing Temple year: 1976 \\
\hline 815 & Allerseelen Max! & movie: Allerseelen role: Max \\
\hline 816 & babochki & movie: Babochki \\
\hline 817 & Himself Moreau. & role: Himself movie: About Jeanne Moreau \\
\hline 818 & Clint Edwards in love! & actor: Clint Edwards movie: Cycle of Love \\
\hline 819 & Eddie Robbitt with soul & actor: Eddie Rabbitt movie: Body and Soul \\
\hline 820 & svet Forda & movie: Cudesan svet Horasa Forda \\
\hline 821 & Cabaret Aguirre & movie: Cabaret Shangai actor: José Luis Aguirre \\
\hline 822 & Barnyard 1923 & movie: Barnyard year: 1923 \\
\hline 823 & Reunion abducted & movie: Abducted II: The Reunion \\
\hline 824 & cost kiss & movie: Cost of a Kiss \\
\hline 825 & Haworth eggs & actor: Peter Haworth movie: Case of Eggs: Episode 4 \\
\hline 826 & Bill with Bushman? & movie: Bill Bumper's Bargain actor: Francis Bushman \\
\hline 827 & Rider Rodeo bronco & role: Rodeo Rider movie: Bronco Buster \\
\hline 828 & $\begin{array}{c}\text { Buried Alive William } \\
\text { Atherton. }\end{array}$ & movie: Buried Alive (1990/II) actor: William Atherton \\
\hline 829 & ciel Back Yvon. & movie: Ciel d'asile actor: Yvon Back \\
\hline 830 & breakin rules & movie: Breakin' All the Rules \\
\hline 831 & 1997 anything baby & year: 1997 movie: Anything for my Baby \\
\hline 832 & American mystic 1975. & movie: Charles Fillmore: American Mystic year: 1975 \\
\hline 833 & 18Eighteen Xtra & movie: 18Eighteen Xtra 2 \\
\hline 834 & 1981 Cabeza & year: 1981 movie: Andjar y su virgen de la Cabeza \\
\hline 835 & 1962 Bunnies & movie: Bunnies Abundant year: 1962 \\
\hline 836 & Desires with Sebastien & actor: Sebastien Guy movie: Burning Desires \\
\hline 837 & 2001 Anandam! & year: 2001 movie: Anandam \\
\hline 838 & Chamula Reynoso! & movie: Chamula actor: Jorge Reynoso \\
\hline 839 & Lecouvreur Adrienne! & movie: Adrienne Lecouvreur \\
\hline 840 & 1991 blindada & year: 1991 movie: Blazer blindada \\
\hline
\end{tabular}




\begin{tabular}{|c|c|c|}
\hline 841 & Giachetti addio & actor: Fosco Giachetti movie: Addio \\
\hline 842 & Hayward Fischer & actor: David Hayward role: Fischer Lourdes \\
\hline 843 & Accused 7.1 & movie: Accused rank: 7.1 \\
\hline 844 & 6.3 Beauty & rank: 6.3 movie: Beauty and the Boss \\
\hline 845 & Cologne Guettler & movie: Cologne actor: 'Old Man' Guettler \\
\hline 846 & 2000 bukas mamahalin & year: 2000 movie: Bukas na lang kita mamahalin \\
\hline 847 & 1978 convention & movie: Convention Girls year: 1978 \\
\hline 848 & 5.6 Casper Ghost & rank: 5.6 movie: Casper the Friendly Ghost \\
\hline 849 & Ballroom Romance & movie: Ballroom of Romance \\
\hline 850 & Animal in us 2003 & movie: Animal in Us All year: 2003 \\
\hline 851 & Cao Xa! & gender: $\mathrm{m}$ movie: Cao-Xa \\
\hline 852 & Hockey Goalie Clark. & movie: clark, Canadian hockey goalie \\
\hline 853 & 1965 Accidente & year: 1965 movie: Accidente \\
\hline 854 & amour 1922 & year: 1922 movie: amour \\
\hline 855 & IAllen Paper the Suit! & actor: Steve Allen role: Gentleman in the Paper Suit \\
\hline 856 & Behirah v'hagoral & movie: Behirah V'Hagoral \\
\hline 857 & 1996 Dalziel & year: 1996 movie: Dalziel and Pascoe: An Autumn Shroud \\
\hline 858 & AFI's 100 years & movie: AFI's 100 Years \\
\hline 859 & Ace hearts movie & movie: Ace of Hearts \\
\hline 860 & 11th hour & movie: 11th Hour \\
\hline 861 & Cinderella man & movie: Cinderella Man \\
\hline 862 & 1897 Siam & year: 1897 movie: Berne: Arrive du roi de Siam \\
\hline 863 & tikhiye tikhiye zdes . & movie: A zori zdes tikhiye \\
\hline 864 & Alisik Anadolu & actor: Sadri Alisik movie: Anadolu \\
\hline 865 & Barbosa Barulho! & actor: Antônio Sá Barbosa movie: Caula do Barulho \\
\hline 866 & Arthur Allardt brother? & actor: Arthur Allardt movie: Brother for Brother \\
\hline 867 & 1976 Tennyson & $\begin{array}{c}\text { movie: Anyone for Tennyson? The Master Poets } \\
\text { Collection year: } 1976\end{array}$ \\
\hline 868 & combustion Gregory & movie: Combustion actor: Michael Gregory \\
\hline 869 & George MoranTrails & role: George Moran movie: Crossed Trails \\
\hline 870 & 2003 Att barn & movie: Att dda ett barn year: 2003 \\
\hline 871 & 6.6 Dresser? & rank: 6.6 role: The Dresser \\
\hline 872 & Eddie battle carne? & actor: Eddie Lyons movie: Battle for Chili-Con-Carne \\
\hline 873 & 1998 art influence & year: 1998 movie: Art of Influence \\
\hline 874 & Burning of Annie? & movie: Burning Annie \\
\hline 875 & bonheur fim of 2003 & movie: Bonheur ne tient qu' un film year: 2003 \\
\hline 876 & $\begin{array}{l}\text { Virginia Davis in } \\
\text { stung? }\end{array}$ & movie: Alice gets Stung actor: Virginia Davis \\
\hline 877 & Charlie Champagne? & movie: Champagne Charlie \\
\hline 878 & Figols Carlos Bala & actor: Carlos Figols movie: bala \\
\hline
\end{tabular}




\begin{tabular}{|c|c|c|}
\hline 879 & 1955 nuestras? & year: 1955 movie: Nuestras Banderas \\
\hline 880 & licena luta 1986 & movie: Com Licena year: 1986 \\
\hline 881 & 1923 Boston blackie & year: 1923 role: Boston Blackie \\
\hline 882 & 6. 8 Rahi. & rank: 6.8 role: Rahi \\
\hline 883 & 1917 death brand & year: 1917 movie: brand of Death \\
\hline 884 & Csnom Palk & movie: Csnom Palk \\
\hline 885 & $\begin{array}{c}\text { Angels and Imams } \\
\text { Gibba }\end{array}$ & movie: Angels and Imams role: Caramo Gibba \\
\hline 886 & $\begin{array}{c}\text { Eyes are Brown } \\
\text { Operator! }\end{array}$ & movie: Big Brown Eyes role: Elevator Operator \\
\hline 887 & dawn cold night & movie: cold Night into Dawn \\
\hline 888 & blinks elevates & movie: Binks Elevates the Stage \\
\hline 889 & buster Frame with dog & movie: Buster's Frame up actor: Pete the Dog \\
\hline 890 & Asturias & movie: Asturias \\
\hline 891 & Rico Richard Bakalyan & actor: Richard Bakalyan movie: Brothers Rico \\
\hline 892 & 1980 Aasha & year: 1980 movie: Aasha \\
\hline 893 & Alfredo Asesinato & movie: Asesinato por Encargo actor: Alfredo Gutiérrez \\
\hline 894 & Father cry childern & movie: Cry of the Children role: The working father \\
\hline 895 & $\begin{array}{l}\text { Lloyd Harold in } \\
\text { clippings }\end{array}$ & actor: Harold Lloyd movie: Close-Cropped Clippings \\
\hline 896 & 1990 gris Camioneta. & year: 1990 movie: Camioneta gris \\
\hline 897 & Bulle Gerd Anthoff & movie: Bulle von Tlz actor: Gerd Anthoff \\
\hline 898 & 2001 with Leif! & year: 2001 actor: Leif Andrée \\
\hline 899 & 1996 vediamo & year: 1996 movie: $\mathrm{Ci}$ vediamo in tribunale \\
\hline 900 & $\begin{array}{c}\text { danger ahead with } \\
\text { Harry. }\end{array}$ & movie: Danger Ahead actor: Harry Depp \\
\hline 901 & 2003 Animatrix & year: 2003 movie: Animatrix \\
\hline 902 & $\begin{array}{c}1961 \text { Salutes Jack } \\
\text { Benny }\end{array}$ & year: 1961 movie: Carnegie Hall Salutes Jack Benny \\
\hline 903 & 1997 noord & movie: 78 noord year: 1997 \\
\hline 904 & Orgy London Boy! & movie: Aaron's London Boy Orgy \\
\hline 905 & Jean in Appartement & actor: Jean-François Calvé movie: Appartement des filles \\
\hline 906 & Paul Antony in Benfit & actor: Paul Antony-Barber movie: Benefit of the Doubt \\
\hline 907 & Canada 1982. & movie: Canada Vignettes: Ice year: 1982 \\
\hline 908 & Michaelabnormal man & actor: Michael Cooper movie: Abnormal Man \\
\hline 909 & Garry in Bindle & movie: Bindle actor: Charles Garry \\
\hline 910 & arme eva & movie: Arme kleine Eva \\
\hline 911 & 1970 wonderland & movie: Alex in Wonderland year: 1970 \\
\hline 912 & Bartender role 5.6. & role: Bartender rank: 5.6 \\
\hline 913 & black sheep of 1909 & movie: Black Sheep year: 1909 \\
\hline 914 & Ariane 1931 & movie: Ariane year: 1931 \\
\hline
\end{tabular}




\begin{tabular}{|c|c|c|}
\hline 915 & Isidoro Almirante? & role: Isidoro Janosky actor: Ernesto Almirante \\
\hline 916 & bangkero & movie: Bangkero \\
\hline 917 & Wet 1926 ! & movie: All Wet year: 1926 \\
\hline 918 & billy kid wanted & movie: Billy the kid wanted \\
\hline 919 & August Black 2004! & movie: Black August year: 2004 \\
\hline 920 & Checkpoint Narrator & movie: Checkpoint role: Narrator \\
\hline 921 & asquith orbit & movie: Asquith in Orbit \\
\hline 922 & comte ory & movie: Comte Ory \\
\hline 923 & claude lorrain & movie: Art of Claude Lorrain \\
\hline 924 & Bohme! & movie: Bohme \\
\hline 925 & 1910 Brother & year: 1910 movie: Brother \\
\hline 926 & Jay Ashley in Dinner & actor: Jay Ashley movie: All Night Diner \\
\hline 927 & Almost Elvis? & movie: Almost Elvis \\
\hline 928 & beto rockfeller & movie: Beto Rockfeller \\
\hline 929 & 1927 Carnevale venezia & year: 1927 movie: Carnevale di Venezia \\
\hline 930 & 2002 with Dating Game & year: 2002 role: Dating Game Director \\
\hline 931 & Timoushin 1977. & actor: Alec Timoushin year: 1977 \\
\hline 932 & affair skin 1963 ! & year: 1963 movie: Affair of the Skin \\
\hline 933 & 1992 abgetrieben & movie: Abgetrieben year: 1992 \\
\hline 934 & Batalla flores & movie: Batalla de flores \\
\hline 935 & bed tales & movie: Bed Tales \\
\hline 936 & 7.5 Cyrano Bergerac & rank: 7.5 movie: Cyrano de Bergerac \\
\hline 937 & $\begin{array}{l}\text { 7.8 Arrivano } \\
\text { Margherito }\end{array}$ & rank: 7.8 movie: Arrivano Joe e Margherito \\
\hline 938 & Guise Tom 1918! & actor: Tom Guise year: 1918 \\
\hline 939 & Cyrus rating $8.1 !$ & actor: Cyrus Elias rank: 8.1 \\
\hline 940 & 1913 blot sky & year: 1913 movie: Bolt from the Sky \\
\hline 941 & $\begin{array}{c}\text { Fraser Brendan } \\
\text { husbanb }\end{array}$ & actor: Brendan Fraser movie: Accidental Husban \\
\hline 942 & Country Girl 1915. & movie: Country Girl year: 1915 \\
\hline 943 & Aru Callgirl 1976? & movie: Aru Callgirl no shogen: roshutsu year: 1976 \\
\hline 944 & $\begin{array}{l}4.6 \text { rating with } \\
\text { gunfighter }\end{array}$ & rank: 4.6 movie: Ballad of a Gunfighter \\
\hline 945 & 2003 Denise bouch & year: 2003 movie: Best of Denise La Bouch \\
\hline 946 & Ford in centerfold & actor: Luke Ford movie: Centerfold Babylon \\
\hline 947 & charlie Campout & movie: Charlie's Campout \\
\hline 948 & barbie 2001 & movie: Barbie in the Nutcracker year: 2001 \\
\hline 949 & Rickman closet land & movie: Closet land actor: Alan Rickman \\
\hline 950 & dog yukon 1997 & movie: Call of the Wild: Dog of the Yukon year: 1997 \\
\hline 951 & Amie America & role: Amie movie: America 3000 \\
\hline
\end{tabular}




\begin{tabular}{|c|c|c|}
\hline 952 & Evans Davies Elfed! & actor: Daniel Evans role: Elfed Davies \\
\hline 953 & 36 Daniel? & movie: 36 actor: Daniel Auteuil \\
\hline 954 & Herricht Rolf 1978! & actor: Rolf Herricht year: 1978 \\
\hline 955 & artist dream 1913 & movie: Artist's Dreams year: 1913 \\
\hline 956 & melodiya Brazilska & movie: Brazilska melodiya \\
\hline 957 & $\begin{array}{c}\text { gadaprena Chichinadze } \\
\text { Amiran! }\end{array}$ & movie: Angelozebis gadaprena actor: Amiran Chichinadze \\
\hline 958 & bust babes 15 & movie: Big Bust Babes 15 \\
\hline 959 & 1922sam allen & year: 1922 actor: Sam Allen \\
\hline 960 & $\begin{array}{l}\text { South American } \\
\text { Maisner }\end{array}$ & $\begin{array}{l}\text { role: South-American movie: Byl jednou jeden polda II - } \\
\text { Major Maisner opet Zasahuje! }\end{array}$ \\
\hline 961 & 2003 flight osiris & year: 2003 movie: Animatrix: Final Flight of the Osiris \\
\hline 962 & gonul Aci! & movie: Aci gonul \\
\hline 963 & 1922 Insp Richardson. & year: 1922 role: Insp. Richardson \\
\hline 964 & death part Chang & movie: 'Til Death Do Us Part actor: Tommy Chang \\
\hline 965 & Johnson. Black heart & actor: Emory Johnson movie: Black Heart \\
\hline 966 & Mona Kelly XVI! & role: Mona Lisa actor: John (XVI) Kelly \\
\hline 967 & Katz Josef & movie: Bernhardiner \& Katz actor: Josef Koepp \\
\hline 968 & 1925 Cactus & year: 1925 movie: Cactus Trails \\
\hline 969 & Bakayar Andoh Kazuo! & $\begin{array}{c}\text { movie: Bakayar! 2: Shiawase ni naritai actor: Kazuo } \\
\text { Andoh }\end{array}$ \\
\hline 970 & Voss Fatty automaniacs & actor: Fatty Voss movie: Automaniacs \\
\hline 971 & 2003 Chelyabumbiya & year: 2003 movie: Chelyabumbiya \\
\hline 972 & Corkscrew & movie: Corkscrew \\
\hline 973 & Dolne libert & actor: N. Dolne movie: Carillon de la libert \\
\hline 974 & 1979 aragosta & year: 1979 movie: Aragosta a colazione \\
\hline 975 & concours Henry? & movie: Concours eurovision actor: Henry Des \\
\hline 976 & Burton Pal Blackies! & actor: John Burton movie: Boston Blackie's Little Pal \\
\hline 977 & $\begin{array}{c}\text { Calypso Cowboy } \\
\text { Vocalist Accordionist }\end{array}$ & movie: Cowboy Calypso role: Vocalist-Accordionist \\
\hline 978 & peligrosas 1950 ! & movie: Curvas Peligrosas year: 1950 \\
\hline 979 & Monkey rating 5.1 & role: Monkey rank: 5.1 \\
\hline 980 & citti 1962. & movie: Citt prigioniera year: 1962 \\
\hline 981 & 1993 Frenetico El! & year: 1993 movie: Adventures of El Frenetico and Go Girl \\
\hline 982 & 1718 Nasty! & movie: 18 and Nasty 17 \\
\hline 983 & avariya & movie: Avariya \\
\hline 984 & Burlesxxx Female & movie: Burlesxxx gender: $\mathrm{f}$ \\
\hline 985 & Bye Bye Tiger & movie: Bye Bye Tiger \\
\hline 986 & segretissimi agenti? & movie: 002 agenti segretissimi \\
\hline 987 & wives trusted? & movie: Can wives Be Trusted \\
\hline
\end{tabular}




\begin{tabular}{|c|c|c|}
\hline 988 & Tobacco Buckley? & $\begin{array}{c}\text { movie: Big Leaf Tobacco Company, The actor: Andrew } \\
\text { Buckley }\end{array}$ \\
\hline 989 & red Barn? & movie: Barn Red \\
\hline 990 & $\begin{array}{c}\text { Bella Bella Bella } \\
\text { Morales! }\end{array}$ & movie: Bella! Bella! Bella! actor: Henry J. Morales \\
\hline 991 & Anderson 1998 & actor: Kenneth R. Anderson year: 1998 \\
\hline 992 & bronc stomper & movie: Bronc Stomper \\
\hline 993 & 1929 campus knights & year: 1929 movie: Campus Knights \\
\hline 994 & Cuddler? & movie: Cuddler \\
\hline 995 & bullet 1985 & year: 1985 movie: Bullet \\
\hline 996 & monde cration & movie: Cration du monde \\
\hline 997 & buck naked 21 st & movie: Buck Naked in the 21 st Century \\
\hline 998 & Chocolat 6.3 & movie: Chocolat rank: 6.3 \\
\hline 999 & araguaya conspiraro & movie: Araguaya - A Conspirao do Silncio \\
\hline 1000 & 1961 Dangai & year: 1961 movie: Dangai \\
\hline 1001 & Bob Benny $1962 ?$ & actor: Bob Benny year: 1962 \\
\hline 1002 & $\begin{array}{l}\text { Adam Hubert } \\
\text { Backroads }\end{array}$ & actor: Adam Hubert movie: Backroads \\
\hline 1003 & Gene crazy love & actor: Gene Bervoets movie: Crazy Love \\
\hline 1004 & 6.6 vida & rank: 6.6 movie: As es la vida \\
\hline 1005 & Bernal cuestin & actor: Agustín Bernal movie: Cuestin de fe \\
\hline 1006 & 1946 Buitres & year: 1946 movie: Buitres sobre el tejado \\
\hline 1007 & Blood Doug! & movie: Blood (2000/II) role: Doug \\
\hline 1008 & Donatas Beethoven! & $\begin{array}{c}\text { actor: Donatas Banionis movie: Beethoven - Tage aus } \\
\text { einem Leben }\end{array}$ \\
\hline 1009 & Daws Chilly Willy? & movie: Airlift a la Carte role: Chilly Willy \\
\hline 1010 & 3.6 lavventura & rank: 3.6 movie: dura l'avventura \\
\hline 1011 & Dev Anand afsar & actor: Dev Anand movie: Afsar \\
\hline 1012 & Jean Asociacin & $\begin{array}{l}\text { actor: Jean Pierre Reguerraz movie: Asociacin Argentina } \\
\text { de actores }\end{array}$ \\
\hline 1013 & 1940 Dancing & year: 1940 movie: Dancing on a Dime \\
\hline 1014 & Varga kronika Blznova! & role: Varga movie: Blznova kronika \\
\hline 1015 & 1918 Accusing Toe & year: 1918 movie: Accusing Toe \\
\hline 1016 & 2004 British & year: 2004 movie: Brilliantly British \\
\hline 1017 & 2004 Cum! & year: 2004 movie: Cum Filled Throats 7 \\
\hline 1018 & Laurence Criner Moon! & actor: Laurence Criner movie: Black Moon \\
\hline 1019 & Bettys 1911 & year: 1911 movie: Betty's Buttons \\
\hline 1020 & 24 Blowjobs Michiko & movie: $100 \%$ Blowjobs 24 actor: Michiko Ari \\
\hline 1021 & 1952 Bounce & year: 1952 movie: Caught on the Bounce \\
\hline 1022 & Harrison 1970 ! & role: Thomas L. Harrison year: 1970 \\
\hline 1023 & 1996 Azzurro. & year: 1996 movie: Azzurro \\
\hline
\end{tabular}




\begin{tabular}{|c|c|c|}
\hline 1024 & Kim Dalimyeon & actor: Hie-gab Kim movie: Dalimyeon eoddae \\
\hline 1025 & 1971 Abel? & year: 1971 movie: Abel \\
\hline 1026 & $\begin{array}{l}\text { Gewandhaus Cho } \\
\text { Beethoven }\end{array}$ & $\begin{array}{l}\text { role: Chorus Master (Gewandhaus Cho movie: Beethoven: } \\
\text { Symphony No. } 9 \text { Op. } 125\end{array}$ \\
\hline 1027 & $\begin{array}{c}1920 \text { Cigarette } \\
\text { Romance }\end{array}$ & year: 1920 movie: Cigarette Maker's Romance \\
\hline 1028 & 2004 Blowjobs 24 & year: 2004 movie: $100 \%$ Blowjobs 24 \\
\hline 1029 & Durst Face & actor: Eric Durst movie: About Face \\
\hline 1030 & Male Brick. & gender: $\mathrm{m}$ movie: Brick Lane \\
\hline 1031 & Moore Coryphee & actor: Matt Moore movie: Coryphee \\
\hline 1032 & Estes Amonasro? & actor: Simon Estes movie: Aida (1981/II) \\
\hline 1033 & 1902 Flypaper & year: 1902 movie: Comedian and the Flypaper \\
\hline 1034 & Paul Hell & actor: Paul Birchard movie: Absolute Hell \\
\hline 1035 & Julio extrao & actor: Julio Alemán movie: Amor extrao \\
\hline 1036 & 1999 la msica & year: 1999 movie: Con la msica por dentro \\
\hline 1037 & Cortex Fallon! & movie: Cortex actor: Ian Fallon \\
\hline 1038 & 1966 yavrum & year: 1966 movie: Allahaismarladik yavrum \\
\hline 1039 & Cirkus Sven! & movie: Cirkus Casablanca actor: Sven Bay \\
\hline 1040 & Suraj Dahej! & role: Suraj movie: Dahej \\
\hline 1041 & Hank Fred Bustin. & actor: Fred Hank movie: Bustin' Thru (1925/II) \\
\hline 1042 & 1999 Blast! & year: 1999 movie: Blast from the Past \\
\hline 1043 & 2003 chango & year: 2003 movie: Cara de chango \\
\hline 1044 & Aida 5.5? & movie: Aida rank: 5.5 \\
\hline 1045 & $\begin{array}{l}\text { Revenge Pumpkinheads } \\
\text { Clinton! }\end{array}$ & $\begin{array}{l}\text { movie: Bloodwings: Pumpkinhead's Revenge actor: Roger } \\
\text { Clinton }\end{array}$ \\
\hline 1046 & 2000 Bloomers & year: 2000 movie: Auntie's Golden Bloomers \\
\hline 1047 & Clinch 1999! & movie: Clinch year: 1999 \\
\hline 1048 & Potel Bell-Hop & actor: Victor Potel movie: Bell-Hop \\
\hline 1049 & Bitter Richard & movie: Bitter Jester actor: Richard Belzer \\
\hline 1050 & 5.9 Badding & rank: 5.9 movie: Badding \\
\hline 1051 & $\begin{array}{l}\text { Armando Aventura al } \\
\text { centro? }\end{array}$ & $\begin{array}{l}\text { actor: Armando Acosta movie: Aventura al centro de la } \\
\text { tierra }\end{array}$ \\
\hline 1052 & Calwell Blood & role: Insp. Calwell movie: Bad Blood \\
\hline 1053 & $\begin{array}{c}\text { Arroyo Dindo } \\
\text { Chickboy? }\end{array}$ & actor: Dindo Arroyo movie: Chickboy \\
\hline 1054 & Carril 1941? & actor: Hugo del Carril year: 1941 \\
\hline 1055 & Boy $1939 ?$ & role: Boy year: 1939 \\
\hline 1056 & Billi Pinocchio & actor: Riccardo Billi movie: Avventure di Pinocchio \\
\hline 1057 & Bob Hope 1994 & movie: Bob Hope: Happy 91st Birthday year: 1994 \\
\hline 1058 & The Cowboy Plains & role: The Cowboy movie: Across the Plains \\
\hline 1059 & Pepe Adosados & actor: Pepe Alcázar movie: Adosados \\
\hline
\end{tabular}




\begin{tabular}{|c|c|c|}
\hline 1060 & 1943 Alfred & year: 1943 role: Alfred Johnson \\
\hline 1061 & Conway Animals & actor: Paul (IV) Conway movie: Animals \\
\hline 1062 & 1993 Batimam & year: 1993 movie: Batimam e Robim \\
\hline 1063 & The Nun 1993 & role: The Nun year: 1993 \\
\hline 1064 & eurovision 1956 & movie: Concours eurovision year: 1956 \\
\hline 1065 & Blake Poopler 2002? & movie: Blake Poopler year: 2002 \\
\hline 1066 & Bartender 40. & role: Bartender movie: 40 \\
\hline 1067 & Streets 5.9? & movie: Cry from the Streets rank: 5.9 \\
\hline 1068 & 1900 England & $\begin{array}{c}\text { year: } 1900 \text { movie: Children of the Royal Family of } \\
\text { England (1900/I) }\end{array}$ \\
\hline 1069 & Farm 7.1 & movie: Animal Farm rank: 7.1 \\
\hline 1070 & Moledet 1956 & movie: B'Ein Moledet year: 1956 \\
\hline 1071 & minouches Barbey? & movie: Balanoire actor: Michel Barbey \\
\hline 1072 & Akcija 1965? & movie: Akcija epej year: 1965 \\
\hline 1073 & Wet Charley Chase? & movie: All Wet actor: Charley Chase \\
\hline 1074 & 1963 Anotimpuri. & year: 1963 movie: Anotimpuri \\
\hline 1075 & Bravo 1998! & movie: Bravo year: 1998 \\
\hline 1076 & $\begin{array}{c}\text { Giuseppe } \\
\text { Appuntamento. }\end{array}$ & $\begin{array}{l}\text { actor: Giuseppe Addobbati movie: Appuntamento col } \\
\text { disonore }\end{array}$ \\
\hline 1077 & Xander Belt & actor: Xander Berkeley movie: Below the Belt \\
\hline 1078 & 2002 Brushstroke & year: 2002 movie: Brushstroke \\
\hline 1079 & Champ 1949! & movie: Alias the Champ year: 1949 \\
\hline 1080 & Dechent Calentito & actor: Antonio Dechent movie: Calentito \\
\hline 1081 & Athens 6.8 ! & movie: Athens rank: 6.8 \\
\hline 1082 & 1969 Alive! & year: 1969 movie: 'It's Alive!' \\
\hline 1083 & Harry 1986. & role: Harry year: 1986 \\
\hline 1084 & ruka 1968 & movie: Brilliantovaya ruka year: 1968 \\
\hline 1085 & Tyrone All of Me? & role: Tyrone Wattell movie: All of Me \\
\hline 1086 & 2000 Feng & year: 2000 actor: Feng Ku \\
\hline 1087 & Alton Bang. & actor: John Alton movie: Big Bang \\
\hline 1088 & Careful $1926 ?$ & movie: Be Careful year: 1926 \\
\hline 1089 & Vagabond 1915. & movie: Beloved Vagabond year: 1915 \\
\hline 1090 & 1980 Hevel! & year: 1980 movie: Al Hevel Dak \\
\hline 1091 & Dutt Paraye? & actor: Utpal Dutt movie: Apne Paraye \\
\hline 1092 & 2000 livet & year: 2000 movie: Bnd p livet \\
\hline 1093 & Addams 1991! & movie: Addams Family year: 1991 \\
\hline 1094 & 1969 Babysitter? & year: 1969 movie: Babysitter \\
\hline 1095 & 1997 Georg! & year: 1997 role: Georg \\
\hline 1096 & 1995 Frhliche & year: 1995 movie: Ach du Frhliche \\
\hline 1097 & 8.3 Male! & rank: 8.3 role: $\mathrm{m}$ \\
\hline
\end{tabular}




\begin{tabular}{|c|c|c|}
\hline 1098 & 1989 Izgnaniye. & year: 1989 movie: Aleksandr Galich. Izgnaniye \\
\hline 1099 & A John 1998 & role: A John year: 1998 \\
\hline 1100 & 1987 infierno & year: 1987 movie: Camino al infierno \\
\hline 1101 & Bayit 2003. & movie: Bayit Shel Simcha year: 2003 \\
\hline 1102 & Black Fox 1962? & $\begin{array}{l}\text { movie: Black Fox: The True Story of Adolf Hitler year: } \\
\qquad 1962\end{array}$ \\
\hline 1103 & 1983 Through the Night & year: 1983 movie: Chase Through the Night \\
\hline 1104 & $\begin{array}{l}\text { Cirkusrevyen } 1998 \\
\text { Olsen } \\
\end{array}$ & $\begin{array}{c}\text { movie: Cirkusrevyen } 1998 \text { - Langt ude i skoven actor: } \\
\text { Niels Olsen }\end{array}$ \\
\hline 1105 & Bunnyman Torben! & movie: Benny and the Bunnyman actor: Torben Rebild \\
\hline 1106 & Dale Ring! & actor: Dale Cook movie: Blood Ring 2 \\
\hline 1107 & 1985 Crveni & year: 1985 movie: Crveni i crni \\
\hline 1108 & Bridesmaids Hamilton! & movie: Bridesmaids actor: Hamilton Camp \\
\hline 1109 & Carlos Blanco! & actor: Carlos East movie: Blanco \\
\hline 1110 & 1908 Monte & year: 1908 movie: Count of Monte Cristo \\
\hline 1111 & Witwen Horst & movie: Club der grnen Witwen actor: Horst Buchholz \\
\hline 1112 & Zaoua $7.3 !$ & movie: Ali Zaoua rank: 7.3 \\
\hline 1113 & Connecticut Brunner & movie: Connecticut Kid actor: Jason Brunner \\
\hline 1114 & Baba-It 1987 & movie: Baba-It year: 1987 \\
\hline 1115 & Baillargeon Infinite! & actor: Paul Baillargeon movie: Anna to the Infinite Power \\
\hline 1116 & Barastoff 1915 & movie: Confession of Madame Barastoff year: 1915 \\
\hline 1117 & Dutch Buckaroo? & actor: Dutch Maley movie: Bonanza Buckaroo \\
\hline 1118 & Alcatraz 2002 & $\begin{array}{l}\text { movie: Creed \& the Wallflowers: Live at Alcatraz year: } \\
\qquad 2002\end{array}$ \\
\hline 1119 & Contact Stevens? & movie: Contact actor: Marc Stevens \\
\hline 1120 & Carlos Corazn! & actor: Juan Carlos Barbieri movie: Corazn \\
\hline 1121 & 1991 Fantasy? & year: 1991 movie: Cosmic Fantasy 2 \\
\hline 1122 & 2002 krlighed! & year: 2002 movie: Brndende krlighed \\
\hline 1123 & 1958 Calavera. & year: 1958 movie: Calavera \\
\hline 1124 & Call Caple Shaw & movie: Call actor: Andrew Caple-Shaw \\
\hline 1125 & Svullo 6.2 & movie: Angne \& Svullo 'Hr och nu!' rank: 6.2 \\
\hline 1126 & 1936 Custer $(1936 / \mathrm{II})$ & year: 1936 movie: Custer's Last Stand (1936/II) \\
\hline 1127 & Arizona 1928 & movie: Arizona Days year: 1928 \\
\hline 1128 & Olympics Boitano & $\begin{array}{c}\text { movie: } 1994 \text { Winter Olympics Figure Skating Competition } \\
\text { and Exhibition Highlights actor: Brian Boitano }\end{array}$ \\
\hline 1129 & Bourgeois Abandon & actor: Cyril Bourgeois movie: Abandon \\
\hline 1130 & Cinderdrift Davila? & movie: Cinderdrift actor: Angel Davila \\
\hline 1131 & Imscher Chaal! & actor: Fred Imscher movie: Chaal Baaz \\
\hline 1132 & 1987 Star-Club & year: 1987 movie: Bye Bye Star-Club \\
\hline 1133 & Francisco Blablabl & actor: Francisco Arruda movie: Blablabl \\
\hline
\end{tabular}




\begin{tabular}{|c|c|c|}
\hline 1134 & 8.2 Alyonka! & rank: 8.2 movie: Alyonka \\
\hline 1135 & 2000 Clarilandia? & year: 2000 movie: Clarilandia \\
\hline 1136 & Bhalevadivi Dragan! & actor: Dragan Balkovic movie: Bhalevadivi Basu \\
\hline 1137 & 1990 Shots & year: 1990 movie: Best Shots \\
\hline 1138 & 1970 Bhai! & year: 1970 movie: Bhai Bhai \\
\hline 1139 & 1968 sevmeyecegim & year: 1968 movie: Artik sevmeyecegim \\
\hline 1140 & bandonen 1935 ! & movie: Alma de bandonen year: 1935 \\
\hline 1141 & Dicamillo CKY & actor: Brandon Dicamillo movie: CKY 4 Latest \& Greatest \\
\hline 1142 & 1943 Amricas? & year: 1943 movie: Canto a las Amricas \\
\hline 1143 & Butler Champ! & actor: Daws Butler movie: Chump Champ \\
\hline 1144 & Baby $6.6 !$ & movie: Baby (2002/I) rank: 6.6 \\
\hline 1145 & Americano 1916 & movie: Americano year: 1916 \\
\hline 1146 & Company Capobianco & movie: Bad Company actor: Jorge Capobianco \\
\hline 1147 & 1912 Rocks Last Stand & year: 1912 movie: Big Rock's Last Stand \\
\hline 1148 & asesinadita 1955 & movie: Caso de la mujer asesinadita year: 1955 \\
\hline 1149 & Jefferson 1995! & $\begin{array}{c}\text { movie: A\&E Biography: Thomas Jefferson - Philosopher } \\
\text { of Freedom year: } 1995\end{array}$ \\
\hline 1150 & 2003 Heimkehr. & year: 2003 movie: Annas Heimkehr \\
\hline 1151 & Biberti Harmonists! & actor: Robert Biberti movie: Comedian Harmonists \\
\hline 1152 & Scratch Dough! & movie: Cat Scratch Fever actor: Jon Dough \\
\hline 1153 & 1958 Superpup? & year: 1958 movie: Adventures of Superpup \\
\hline 1154 & 2002 Cigarrettes! & year: 2002 movie: Cigarettes \\
\hline 1155 & Calvario Emilio! & movie: Calvario actor: Emilio Guerrero \\
\hline 1156 & Agios 1969 & year: 1969 movie: Agios Nektarios \\
\hline 1157 & 5.3 Divorcement & rank: 5.3 movie: Bill of Divorcement \\
\hline 1158 & Williams Eleven & actor: William A. Williams movie: At the Hour of Eleven \\
\hline 1159 & 2003 Closed Doors? & year: 2003 movie: Behind Closed Doors (2003/I) \\
\hline 1160 & Carless 1915. & movie: Careless Anarchist year: 1915 \\
\hline 1161 & Apartheid Mikels & $\begin{array}{l}\text { movie: Apartheid Slave Woman's Justice actor: Ted V. } \\
\text { Mikels }\end{array}$ \\
\hline 1162 & 1970 lleg & year: 1970 movie: Con ella lleg el amor \\
\hline 1163 & Charwoman Drainie? & movie: Charwoman actor: John Drainie \\
\hline 1164 & 1914 Billys Jealousy. & year: 1914 movie: Broncho Billy's Jealousy \\
\hline 1165 & Graves Reformer! & $\begin{array}{c}\text { actor: Peter Graves movie: A\&E Biography: Peter the } \\
\text { Great - The Tyrant Reformer }\end{array}$ \\
\hline 1166 & Belasco Caravan & actor: Jay Belasco movie: Caravan \\
\hline 1167 & Buckham Comfortably & actor: Jason Buckham movie: Comfortably Numb (2004/I) \\
\hline 1168 & 1964 Cinma & year: 1964 movie: Cinma de notre temps: Luis Buuel \\
\hline 1169 & Allister Charleys Aunt? & actor: Claud Allister movie: Charley's Aunt \\
\hline 1170 & Christmas Fearon & movie: Christmas Carol actor: Ray Fearon \\
\hline
\end{tabular}




\begin{tabular}{|c|c|c|}
\hline 1171 & Dancing Edward? & movie: Dancing Co-Ed actor: Edward Arnold Jr. \\
\hline 1172 & 1985 Medicine & year: 1985 movie: Bad Medicine \\
\hline 1173 & Cruz Padilla & movie: Cara y Cruz actor: Dennis Padilla \\
\hline 1174 & Peace Payton & movie: City at Peace actor: Rickey Payton Sr. \\
\hline 1175 & 1902 Coronation First! & $\begin{array}{c}\text { year: } 1902 \text { movie: Coronation of Their Majesties King } \\
\text { Edward VII and Queen Alexandra }\end{array}$ \\
\hline 1176 & 1957 Bugsy! & year: 1957 movie: Bugsy and Mugsy \\
\hline 1177 & 2003 Johannja? & year: 2003 movie: 78 -as szent Johannja \\
\hline 1178 & Aufair Caviar & actor: Maurice Aufair movie: Caviar rouge \\
\hline 1179 & 2000 Baliktaran! & year: 2000 movie: Baliktaran \\
\hline 1180 & Beeny Child's Play & actor: Christopher Beeny movie: Child's Play \\
\hline 1181 & Alibi Ackland. & movie: Alibi actor: Rodney Ackland \\
\hline 1182 & Edwin Vagabond & actor: Edwin Arden movie: Beloved Vagabond \\
\hline 1183 & Sanchez Honor! & $\begin{array}{l}\text { role: Commandante Sanchez movie: Columbo: A Matter of } \\
\text { Honor }\end{array}$ \\
\hline 1184 & Guillermo amor. & actor: Guillermo Cramer movie: Cita de amor, Una \\
\hline 1185 & 1980 Aki! & year: 1980 movie: Aki toori-ame \\
\hline 1186 & paloma 1936 & movie: Conventillo de la paloma year: 1936 \\
\hline 1187 & Cohen Brotherly. & role: Dr. Marvin Cohen movie: Brotherly Love \\
\hline 1188 & 2002 Aprs & year: 2002 movie: Aprs l'enfance \\
\hline 1189 & 1994 Bermuda! & year: 1994 movie: Bermuda Grace \\
\hline 1190 & A list $2004 !$ & movie: A-List year: 2004 \\
\hline 1191 & 1957 Stroke of Nine. & year: 1957 movie: At the Stroke of Nine \\
\hline 1192 & musume fujiwara & movie: sono musume actor: Kamatari Fujiwara \\
\hline 1193 & afueras 7.4 . & movie: Casa en las afueras, Una rank: 7.4 \\
\hline 1194 & Byoinzaka 1979? & movie: Byoinzaka no kubikukuri no ie year: 1979 \\
\hline 1195 & Nate Castlerock! & role: Nate movie: Castlerock \\
\hline 1196 & 6.5 Ayyam! & rank: 6.5 movie: Ayyam al-tawila, al- \\
\hline 1197 & 1954 Biyenang. & year: 1954 movie: Biyenang hindi tumatawa \\
\hline 1198 & alazan Capetillo & movie: Cuando corrio el alazan actor: Manuel Capetillo \\
\hline 1199 & 1993 kadin? & year: 1993 movie: Bir kadin yz \\
\hline 1200 & 1982 Broadway. & year: 1982 movie: Broadway Plays Washington \\
\hline 1201 & 1972 guerra & year: 1972 movie: Correo de guerra \\
\hline 1202 & Alvaro Bagong! & actor: Dan Alvaro movie: Bagong hari \\
\hline 1203 & Baker Belong to heart? & $\begin{array}{l}\text { actor: Don (IX) Baker movie: Community Sing: You } \\
\text { Belong to My Heart } \\
\end{array}$ \\
\hline 1204 & $\begin{array}{l}\text { American Girls Dream } \\
1994\end{array}$ & movie: American Dream Girls year: 1994 \\
\hline 1205 & 1965 Brillante & year: 1965 movie: Brillante porvenir \\
\hline 1206 & $\begin{array}{l}\text { 1941 Canadian } \\
\text { Landscape }\end{array}$ & year: 1941 movie: Canadian LandScape \\
\hline
\end{tabular}




\begin{tabular}{|c|c|c|}
\hline 1207 & Brown Judd 1920! & role: Judd Brown year: 1920 \\
\hline 1208 & 1999 Cement? & movie: Cement year: 1999 \\
\hline 1209 & baekhwa & movie: 5 baekhwa \\
\hline 1210 & walls full house & movie: Behind the Walls of 'Full House' \\
\hline 1211 & Avengers Alien & movie: Alien Avengers \\
\hline 1212 & 1989 Luna & year: 1989 movie: Anna Luna \\
\hline 1213 & 1988 vietnam & year: 1988 movie: Bye Bye Vietnam (1988/I) \\
\hline 1214 & $\begin{array}{c}\text { cobra Sorapong } \\
\text { Chatree? }\end{array}$ & movie: Cobra Thunderbolt actor: Sorapong Chatree \\
\hline 1215 & 1954 Armando & year: 1954 actor: Armando Arriola \\
\hline 1216 & akiniyeo jiokhaeng & movie: Akiniyeo jiokhaeng geubhaeng yeoljareul tara \\
\hline 1217 & 2004 Axensprung & year: 2004 movie: Axensprung \\
\hline 1218 & Holt claw & actor: Jack Holt movie: Claw \\
\hline 1219 & Bourne truth movie? & $\begin{array}{c}\text { actor: Lindsay Bourne movie: Accident: A Moment of } \\
\text { Truth Movie }\end{array}$ \\
\hline 1220 & Daffy $1980 ?$ & role: Daffy Duck year: 1980 \\
\hline 1221 & Josh Brolin Danny? & actor: Josh Brolin role: Danny \\
\hline 1222 & Bajan. & movie: Bajan \\
\hline 1223 & crazy contest & movie: Contest Crazy \\
\hline 1224 & Path Albertson. & movie: Crooked Path actor: Arthur Albertson \\
\hline 1225 & 1933 ange gardien & year: 1933 movie: Ange gardien \\
\hline 1226 & 1999 Emberson. & year: 1999 actor: Dana Emberson \\
\hline 1227 & cage of Evil 56 ! & movie: Cage of Evil rank: 5.6 \\
\hline 1228 & Revisited Blerta Blerta! & movie: Blerta Revisited \\
\hline 1229 & 1974 comprador & movie: Comprador de Fazendas year: 1974 \\
\hline 1230 & Chacales Alvarado! & movie: Chacales actor: Crox Alvarado \\
\hline 1231 & $\begin{array}{l}1999 \text { Claudio } \\
\text { Santamaria }\end{array}$ & year: 1999 actor: Claudio Santamaria \\
\hline 1232 & Ford in Crash & actor: Ford Sterling movie: Crash \\
\hline 1233 & Aanch & movie: Aanch \\
\hline 1234 & babes good 1934 & year: 1934 movie: Babes in the Goods \\
\hline 1235 & 2001 Contranatura & year: 2001 movie: Contranatura \\
\hline 1236 & captain table & movie: Captain's Table \\
\hline 1237 & $\begin{array}{l}\text { Crucifijo Fernando } \\
\text { Casanova? }\end{array}$ & actor: Fernando Casanova movie: Crucifijo de piedra \\
\hline 1238 & 1914 Blotted. & year: 1914 movie: Blotted Page \\
\hline 1239 & thumbelina with boyd & $\begin{array}{c}\text { actor: Alex Boyd movie: Adventures of Tom Thumb and } \\
\text { Thumbelina }\end{array}$ \\
\hline 1240 & aunt hallie & movie: Aunt Hallie \\
\hline 1241 & Kirk 1997? & actor: Kirk Anderson year: 1997 \\
\hline 1242 & Bala 2.8? & movie: Bala marcada rank: 2.8 \\
\hline
\end{tabular}




\begin{tabular}{|c|c|c|}
\hline 1243 & Anand Baadbaan. & actor: Dev Anand movie: Baadbaan \\
\hline 1244 & Chesney washington & actor: Kenny Chesney movie: Christmas in Washington \\
\hline 1245 & $\begin{array}{l}\text { Christensen West } \\
\text { South? }\end{array}$ & actor: M. Christensen movie: 2612 South Ave. West \\
\hline 1246 & luna querida querida & movie: Adis querida luna \\
\hline 1247 & 1945 Andaluz. & year: 1945 role: Andaluz \\
\hline 1248 & clueless 1998 ! & movie: Clueless year: 1998 \\
\hline 1249 & Amour tenace. & movie: Amour tenace \\
\hline 1250 & Christian Bale 2000. & actor: Christian Bale year: 2000 \\
\hline 1251 & Aires 1922. & movie: Buenos Aires year: 1922 \\
\hline 1252 & Sjefen 3 & role: Sjefen movie: 3 \\
\hline 1253 & bitumes & movie: Bitumes \\
\hline 1254 & Costello bella & actor: Maurice Costello movie: Bella's Elopement \\
\hline 1255 & Baba Yaga & movie: Baba Yaga \\
\hline 1256 & Acting Business! & movie: Acting Business \\
\hline 1257 & closin Jack? & movie: Closin' in role: Jack Bradon \\
\hline 1258 & Boreo Emile 1947! & actor: Emile Boreo year: 1947 \\
\hline 1259 & Wolo Czawienko 4.9! & role: Wolo Czawienko rank: 4.9 \\
\hline 1260 & begurebis $1980 !$ & movie: Begurebis gadaprena year: 1980 \\
\hline 1261 & 1994 bed breakfast & year: 1994 movie: Bed \& Breakfast \\
\hline 1262 & sa kinabuhi & movie: Badlis sa kinabuhi \\
\hline 1263 & convoi de femmes! & movie: convoi de femmes \\
\hline 1264 & 1997 Priest & year: 1997 role: Priest \\
\hline 1265 & Angel Balas & movie: 800 Balas actor: Ángel López \\
\hline 1266 & 1912 version of Billie & movie: Billie year: 1912 \\
\hline 1267 & bindyuzhnik korol & movie: Bindyuzhnik i korol \\
\hline 1268 & Alice Carol 1969 & movie: Bob \& Carol \& Ted \& Alice year: 1969 \\
\hline 1269 & $\begin{array}{l}\text { Thompson Jerry } 8.7 \\
\text { rating }\end{array}$ & role: Jerry Thompson rank: 8.7 \\
\hline 1270 & Leigh John bradman & actor: John Leigh movie: Bradman \\
\hline 1271 & $\begin{array}{l}\text { Private Eye Biography } \\
\text { Pinkerton } \\
\end{array}$ & $\begin{array}{c}\text { movie: A\&E Biography: Allan Pinkerton - The Original } \\
\text { Private Eye }\end{array}$ \\
\hline 1272 & 2.4 banzai & movie: Banzai rank: 2.4 \\
\hline 1273 & BUK & movie: BUK! gender: $\mathrm{m}$ \\
\hline 1274 & carson badge bravery & actor: Ted Carson movie: Badge of Bravery \\
\hline 1275 & Howes Reed 1925? & actor: Reed Howes year: 1925 \\
\hline 1276 & collins circus $\# 9$ & actor: Gary Collins movie: Circus of the stars $\# 9$ \\
\hline 1277 & police in aftermath & role: Police Officer movie: aftermath \\
\hline 1278 & Beitar 5? & movie: Beitar Provence rank: 5 \\
\hline 1279 & 1991 bugs bunny & year: 1991 movie: Bugs Bunny's Overtures to Disaster \\
\hline
\end{tabular}




\begin{tabular}{|c|c|c|}
\hline 1280 & Babylon Vista Akop. & movie: Babylon Vista role: Akop \\
\hline 1281 & Cheyenne Sheriff & movie: Cheyenne Tornado role: Sheriff \\
\hline 1282 & 1922 accidents happen? & year: 1922 movie: Accidents will Happen \\
\hline 1283 & McCullough in bulge & $\begin{array}{l}\text { actor: David McCullough movie: Battle of the Bulge: } \\
\text { World War II's Deadliest Battle }\end{array}$ \\
\hline 1284 & 1970I Breakout Pipes. & movie: Breakout (1970/I) role: Pipes \\
\hline 1285 & course taureaux & movie: Course de taureaux \\
\hline 1286 & billy strikes oil? & movie: Billy Strikes Oil \\
\hline 1287 & Grefe William 1962. & actor: William Grefe year: 1962 \\
\hline 1288 & cactus with Thurston. & movie: Code of the Cactus role: Thurston \\
\hline 1289 & Christian eventyr! & actor: Christian Arhoff movie: Baby p eventyr \\
\hline 1290 & $\begin{array}{l}\text { Dancer in Dance in the } \\
\text { sun? }\end{array}$ & movie: Dance in the Sun role: Dancer \\
\hline 1291 & Benny Clancy & role: Benny movie: Clancy Street Boys \\
\hline 1292 & chambre & movie: Chambre \\
\hline 1293 & Bicicletas verano & movie: Bicicletas son para el verano \\
\hline 1294 & 1922 circus clowns & year: 1922 movie: circus clowns \\
\hline 1295 & Beograde Beograde? & movie: Beograde \\
\hline 1296 & 1973 Brannen & year: 1973 movie: Brannen \\
\hline 1297 & 1977 Askwith Robin. & year: 1977 actor: Robin Askwith \\
\hline 1298 & 2000 alcohol years & year: 2000 movie: alcohol years \\
\hline 1299 & $\begin{array}{l}1936 \text { Abrahamsson } \\
\text { Eric! }\end{array}$ & year: 1936 actor: Eric Abrahamsson \\
\hline 1300 & builders castles & movie: builders of castles \\
\hline 1301 & $\begin{array}{c}\text { Tingvad Aschengreen } \\
\text { Mand DSB. }\end{array}$ & actor: Anders Tingvad Aschengreen role: DSB Mand \\
\hline 1302 & Fred of blood money & role: Fred movie: Blood Money \\
\hline 1303 & Peter Ashmore Jungle & actor: Peter Ashmore movie: Beauty Jungle \\
\hline 1304 & Wilson Tribute 2001 & movie: All-Star Tribute to Brian Wilson year: 2001 \\
\hline 1305 & Big Game 1926 & movie: Big Game year: 1926 \\
\hline 1306 & Xander in $3 \mathrm{AM}$ & movie: 3 AM actor: Xander Chauncey \\
\hline 1307 & 1919 Foss! & year: 1919 actor: Kenelm Foss \\
\hline 1308 & $\begin{array}{l}1960 \text { Diamonds } \\
\text { Canadian! }\end{array}$ & year: 1960 movie: Canadian Diamonds \\
\hline 1309 & Avena Element Sue! & movie: Avena X-tra Edition 4 actor: Sue Element \\
\hline 1310 & 1999 Almost & year: 1999 movie: Almost \\
\hline 1311 & 2000 mandamiento & year: 2000 movie: $11 *$ mandamiento \\
\hline 1312 & Crime blossoms & movie: Crime at Blossoms \\
\hline 1313 & Bandalbaaz! & movie: Bandalbaaz \\
\hline 1314 & butler romance & movie: Butler's Busted Romance \\
\hline 1315 & 1922 version of & year: 1922 movie: Clarence \\
\hline
\end{tabular}




\begin{tabular}{|c|c|c|}
\hline & Clarence & \\
\hline 1316 & $\begin{array}{l}\text { loop De Loop in } \\
\text { Bearly! }\end{array}$ & role: Loopy De Loop movie: Bearly Able \\
\hline 1317 & Thimios Astero & role: Thimios movie: Astero \\
\hline 1318 & Clover Syd & movie: Crimson and Clover role: Syd Hilliard \\
\hline 1319 & Camp Cheerleader. & movie: Cheerleader Camp \\
\hline 1320 & Claymation 1987 & movie: Claymation Christmas Celebration year: 1987 \\
\hline 1321 & Shin Bong Taegyu & role: Shin actor: Tae-gyu Bong \\
\hline 1322 & 1933 Dancing & movie: Dancing year: 1933 \\
\hline 1323 & Fairen gringo? & actor: Juan Fairen movie: Attento Gringo \\
\hline 1324 & 1952 Cennet yolculari? & year: 1952 movie: Cennet yolculari \\
\hline 1325 & $\begin{array}{l}\text { Camoiras Francisco } \\
1981 !\end{array}$ & actor: Francisco Camoiras year: 1981 \\
\hline 1326 & Aeon Csokas Marton! & movie: Aeon Flux actor: Marton Csokas \\
\hline 1327 & 7.1 Bal gouverneur & rank: 7.1 movie: Bal du gouverneur \\
\hline 1328 & 1972 Albin. & year: 1972 actor: Andy Albin \\
\hline 1329 & cohens Albertson & $\begin{array}{c}\text { actor: Frank Albertson movie: Cohens and Kellys in } \\
\text { Trouble }\end{array}$ \\
\hline 1330 & 6.6 minutter & rank: 6.6 movie: 2 minutter \\
\hline 1331 & druppels water twee! & movie: Als twee druppels water \\
\hline 1332 & Aanslag & movie: Aanslag \\
\hline 1333 & $\begin{array}{l}\text { Steven Brigham } \\
\text { Karrow }\end{array}$ & actor: Steven Brigham role: Karrow \\
\hline 1334 & cretinetti del solito & movie: Cretinetti pi del solito \\
\hline 1335 & bergtagen & movie: Bergtagen \\
\hline 1336 & Kiss Brothers & movie: Brother's Kiss \\
\hline 1337 & 1930 Nachtigall & year: 1930 movie: Blonde Nachtigall \\
\hline 1338 & 1941 Bagni John. & year: 1941 actor: John Bagni \\
\hline 1339 & bali beautiful & movie: Beautiful Bali \\
\hline 1340 & 5.9 Charleston & rank: 5.9 movie: Charleston \\
\hline 1341 & 1920 chance & year: 1920 movie: Blind Chance \\
\hline 1342 & levantar 1000 & movie: Cmo levantar 1000 kilos \\
\hline 1343 & Anderson $1955 !$ & actor: James Anderson year: 1955 \\
\hline 1344 & Crooks Dishonest & movie: Are Crooks Dishonest \\
\hline 1345 & Bill Apperson! & movie: Bill Apperson's Boy \\
\hline 1346 & Amor Mara & movie: Amor de Mara Isabel \\
\hline 1347 & $\begin{array}{l}\text { Myeongjung } \mathrm{Ha} \\
\text { Bicheun }\end{array}$ & actor: Myeong-jung Ha movie: Bicheun eodie \\
\hline 1348 & all lust 2003 & movie: All for Lust year: 2003 \\
\hline 1349 & 1995 babewatch & year: 1995 movie: Black Babewatch \\
\hline 1350 & Charles Chaplin 2003 & actor: Charles Chaplin year: 2003 \\
\hline
\end{tabular}




\begin{tabular}{|c|c|c|}
\hline 1351 & Tommy Bacon & role: Tommy actor: Tony Bacon \\
\hline 1352 & Bathing Beauty! & movie: Bathing Beauty \\
\hline 1353 & huwag humirit & movie: Carta alas: Huwag ka nang humirit \\
\hline 1354 & 1950 Chitterson & year: 1950 actor: Herman Chitterson \\
\hline 1355 & 1983 carpool & year: 1983 movie: carpool \\
\hline 1356 & Childern wind & movie: children of the wind \\
\hline 1357 & Ayos ko pare! & movie: Ayos lang pare ko \\
\hline 1358 & Damier & movie: Damier \\
\hline 1359 & Adult European Friend & genre: Adult movie: Aaron's European Friend \\
\hline 1360 & Blue 1992. & movie: Blue year: 1992 \\
\hline 1361 & bertie strategem & movie: Bertie's Stratagem \\
\hline 1362 & 1916 Caprice & year: 1916 movie: Caprice of the Mountains \\
\hline 1363 & 1958 Akvarel & year: 1958 movie: Akvarel \\
\hline 1364 & automrchen & movie: Automrchen \\
\hline 1365 & canto tierra & movie: Canto a mi tierra \\
\hline 1366 & covek sesir & movie: Covek I sesir \\
\hline 1367 & babanin evlatlari & movie: Babanin Evlatlari \\
\hline 1368 & Askin gelince & movie: Askin saati gelince \\
\hline 1369 & drama compensation & movie: Compensation genre: drama \\
\hline 1370 & Geng Ashima & actor: Geng Baosheng movie: Ashima \\
\hline 1371 & Lost Billy Crystal & $\begin{array}{c}\text { movie: Billy Crystal: Don't Get Me Started - The Lost } \\
\text { Minutes }\end{array}$ \\
\hline 1372 & 1913 joe? & year: 1913 movie: Absent Minded Abe \\
\hline 1373 & 5.8 Bohme? & rank: 5.8 movie: Bohme \\
\hline 1374 & $\begin{array}{l}1913 \text { Anderson } \\
\text { Broncho? }\end{array}$ & year: 1913 actor: Gilbert Anderson \\
\hline 1375 & sac Male? & movie: Cul de sac \\
\hline 1376 & 2002 Zalam! & movie: Amir El Zalam year: 2002 \\
\hline 1377 & 19456.1. & year: 1945 rank: 6.1 \\
\hline 1378 & Bride for Knight & movie: Bride for a Knight \\
\hline 1379 & sheep 1956. & movie: Are People Sheep year: 1956 \\
\hline 1380 & que aguante que 1980 ! & movie: As no hay cama que aguante year: 1980 \\
\hline 1381 & annas dag & movie: Annas dag \\
\hline 1382 & cage $1991 ?$ & movie: Cage/Cunningham year: 1991 \\
\hline 1383 & $19792.4 !$ & year: 1979 rank: 2.4 \\
\hline 1384 & Cetrdeset 1988 & movie: Cetrdeset osma - Zavera ili izdaja year: 1988 \\
\hline 1385 & Bill 1921 & actor: Bill Bradbury year: 1921 \\
\hline 1386 & Carter Mario Breakin! & actor: Mario Carter movie: Breakin' All the Rules \\
\hline 1387 & Agostino 5.9! & movie: Agostino rank: 5.9 \\
\hline 1388 & Athletes Oldman & movie: Battle Athletes role: Grant Oldman \\
\hline
\end{tabular}




\begin{tabular}{|c|c|c|}
\hline & Grant? & \\
\hline 1389 & veinard Rigadin! & movie: Ce veinard de Rigadin role: Rigadin \\
\hline 1390 & Rathore Ajit Bhagat? & role: Insp. Rathore actor: Ajit Bhagat \\
\hline 1391 & Norwood Almost? & role: Norwood movie: Almost Beat \\
\hline 1392 & Battling Butler 7.2. & movie: Battling Butler rank: 7.2 \\
\hline 1393 & Caught a Colonel. & movie: Boy Who Caught a Crook role: The Colonel \\
\hline 1394 & ce soir soir? & movie: Au thtre ce soir: Colinette \\
\hline 1395 & Cagney Lacy & movie: Cagney \& Lacey: The Return \\
\hline 1396 & 1921 Broken Road! & year: 1921 movie: Broken Road \\
\hline 1397 & 6.5 amore & rank: 6.5 movie: Amore \\
\hline 1398 & 2000 Adult & year: 2000 genre: Adult \\
\hline 1399 & Cave man & movie: Cave Man \\
\hline 1400 & Adams bad songs? & actor: Bryan Adams movie: 50 most Awesomely Bad \\
\hline 1401 & Bolivar Yves & movie: Bolivar 63-29 actor: Yves Brainville \\
\hline 1402 & 1989 Satirat segment. & year: 1989 role: Petar (segment Satirat) \\
\hline 1403 & 1978 Baraka Amiri! & year: 1978 actor: Amiri Baraka \\
\hline 1404 & 1953 Ashore & year: 1953 movie: All Ashore \\
\hline 1405 & Asian Street & movie: Asian Street \\
\hline 1406 & Bolvar Castillo & actor: Carlos Castillo movie: Bolvar \\
\hline 1407 & Cactus invades & movie: Cactus Jack Invades ECW \\
\hline 1408 & Saeed $4.9 !$ & actor: Saeed Jaffrey rank: 4.9 \\
\hline 1409 & Cheek Cheek & movie: Cheek to Cheek \\
\hline 1410 & BusyBody & movie: BusyBody's Busy Day \\
\hline 1411 & Bluff truffe & movie: Bluff Storia di truffe e di imbroglioni \\
\hline 1412 & Charlot 1998. & actor: Kawena Charlot year: 1998 \\
\hline 1413 & 1998 Archive & year: 1998 movie: Archive \\
\hline 1414 & argo Balogh. & movie: argo actor: Ádám Balogh \\
\hline 1415 & seduction Ackroyd. & $\begin{array}{c}\text { movie: Cocaine: One Man's Seduction actor: David } \\
\text { Ackroyd }\end{array}$ \\
\hline 1416 & 1985 Criminal & year: 1985 movie: Criminal \\
\hline 1417 & 300.000 peng $1937 ?$ & movie: 300.000 peng az utcn year: 1937 \\
\hline 1418 & cream of clapton & movie: Cream of Eric Clapton \\
\hline 1419 & 9 Ball & rank: 9 movie: Butterfly Ball \\
\hline 1420 & 1928 Barney! & year: 1928 actor: Barney Hellum \\
\hline 1421 & 2001 Asphalt & year: 2001 movie: Asphalt Zahov \\
\hline 1422 & Curse Gore Garner? & movie: Curse of the Talisman actor: Max Garner Gore \\
\hline 1423 & Con el puro & movie: Con el ms puro amor \\
\hline 1424 & 2.8 City & movie: city Limits rank: 2.8 \\
\hline 1425 & Missouri 1951 & movie: Across the Wide Missouri year: 1951 \\
\hline 1426 & Cartas 1948 & movie: Cartas marcadas year: 1948 \\
\hline
\end{tabular}




\begin{tabular}{|c|c|c|}
\hline 1427 & big wheel of 1949 & movie: Big Wheel year: 1949 \\
\hline 1428 & 1930 Anglais & movie: Anglais tel qu'on le parle year: 1930 \\
\hline 1429 & 1924 District Attorney? & year: 1924 role: District Attorney \\
\hline 1430 & Boss of Call me & movie: Call me role: Boss \\
\hline 1431 & 7.8 Abbey Street & rank: 7.8 movie: Angel on Abbey Street \\
\hline 1432 & Aile cuisse 6.8 & movie: Aile ou la cuisse rank: 6.8 \\
\hline 1433 & $20016.1 !$ & year: 2001 rank: 6.1 \\
\hline 1434 & 1995 poporul & year: 1995 movie: $\mathrm{Cu}$ poporul \\
\hline 1435 & corrs: unplugged & movie: Corrs: Unplugged \\
\hline 1436 & cinderella 1925 & movie: Cinderella year: 1925 \\
\hline 1437 & 1988 Chocolat & movie: Chocolat year: 1988 \\
\hline 1438 & 1948 Bamdoda & year: 1948 movie: Bandida \\
\hline 1439 & 1999 Collegians & movie: Collegians Are Go!! year: 1999 \\
\hline 1440 & Lambert uncle sol & actor: Eddie Lambert movie: Ask Uncle sol \\
\hline 1441 & 1999 Argument & year: 1999 movie: Argument \\
\hline 1442 & $19978.6 ?$ & year: 1997 rank: 8.6 \\
\hline 1443 & Plus Almanah & movie: Almanah Plus \\
\hline 1444 & 1913 Coward! & year: 1913 movie: Coward \\
\hline 1445 & Brotherhood 1913 & movie: Brotherhood year: 1913 \\
\hline 1446 & Comets Randle! & movie: Comets actor: Randle Ayrton \\
\hline 1447 & 1967 Ana & movie: Ana (1967/II) \\
\hline 1448 & Things Broken & movie: Broken Things \\
\hline 1449 & Hiranandani $6 ?$ & role: Hiranandani rank: 6 \\
\hline 1450 & 2000 chemin & year: 2000 movie: Chemin de Traverse \\
\hline 1451 & Mammoth Vignettes & movie: Canada Vignettes: Woolly Mammoth \\
\hline 1452 & 1997 Father & year: 1997 role: Heather's Father \\
\hline 1453 & 2 Throats Filled & movie: Cum Filled Throats 2 \\
\hline 1454 & Bachelor of art Durti. & movie: Bachelor of Arts role: Miklos Durti \\
\hline 1455 & 1915 Club & year: 1915 movie: Club Man \\
\hline 1456 & 4.8 cool ones & rank: 4.8 movie: Cool Ones \\
\hline 1457 & 1920 bubbles & year: 1920 movie: Broken bubbles \\
\hline 1458 & 1966 Necati & year: 1966 actor: Necati Er \\
\hline 1459 & billy Stormy & movie: Billy's Stormy Courtship role: Billy \\
\hline 1460 & Rafael 1970 & actor: Rafael Albaicín year: 1970 \\
\hline 1461 & The Years Horror 100 & movie: 100 Years of Horror: The Evil Unseeable \\
\hline 1462 & Anuncio cinco & movie: Anuncio y cinco cartas \\
\hline 1463 & 1992 Hara. & year: 1992 actor: Mitch Hara \\
\hline 1464 & 7.73 Fed! & rank: 7.7 role: Fed $\# 3$ \\
\hline 1465 & cream coffee Waiter! & movie: Cream in My Coffee role: Waiter \\
\hline
\end{tabular}




\begin{tabular}{|c|c|c|}
\hline 1466 & Alma Mater 1969 & movie: Alma Mater \\
\hline 1467 & $\begin{array}{l}1990 \text { filmlerinin } \\
\text { unutulmaz yonetmeni. }\end{array}$ & year: 1990 movie: Ask filmlerinin unutulmaz yonetmeni \\
\hline 1468 & Carpenter 1913 & movie: Carpenter year: 1913 \\
\hline 1469 & 1917 Lee & year: 1917 actor: Frankie Lee \\
\hline 1470 & Romeo Gribbon! & movie: Battling Romeo actor: Eddie Gribbon \\
\hline 1471 & Allan Ernest Bliss & $\begin{array}{l}\text { actor: John R. Allan movie: Amazing Quest of Mr. Ernest } \\
\text { Bliss }\end{array}$ \\
\hline 1472 & Bad Apple 2004 & movie: Bad Apple year: 2004 \\
\hline 1473 & Erbil. & $\begin{array}{l}\text { movie: Allah cezani versin Osman Bey actor: Sadettin } \\
\text { Erbil }\end{array}$ \\
\hline 1474 & Anni idin & movie: Anni Tahtoo idin \\
\hline 1475 & comete obligado & movie: Comte Obligado \\
\hline 1476 & Aldrich Phil? & actor: Frank Aldrich role: Phil \\
\hline 1477 & Bodyguard Estrada & movie: Bodyguard actor: Joseph Estrada \\
\hline 1478 & Versa Vice Aires? & movie: Buenos Aires Vice Versa \\
\hline 1479 & $\begin{array}{l}\text { Christmas Billys Billy } \\
\text { Broncho M. }\end{array}$ & $\begin{array}{l}\text { movie: Broncho Billy's Christmas Deed actor: Gilbert } \\
\text { Anderson }\end{array}$ \\
\hline 1480 & 7.1 Brandenburg Otto & rank: 7.1 actor: Otto Brandenburg \\
\hline 1481 & 2004 Jayasuriva. & year: 2004 actor: Saranapala Jayasuriva \\
\hline 1482 & 1912 Country! & year: 1912 movie: Church and Country \\
\hline 1483 & Croft Clash Night & role: Insp. Croft movie: Clash by Night \\
\hline 1484 & chiflando loma & movie: Chiflando en la loma \\
\hline 1485 & 1974 karenina & year: 1974 movie: Anna Karenina (1974/I) \\
\hline 1486 & 8.7 Boca Ouro & rank: 8.7 movie: Boca de Ouro \\
\hline 1487 & Black Pearl 1915 & movie: Black Pearl year: 1915 \\
\hline 1488 & BenKamal Airwolf. & role: Maj. Mufta Ben-Kamal movie: Airwolf \\
\hline 1489 & Airport Central & movie: Central Airport \\
\hline 1490 & 6.6 Amnesia & rank: 6.6 movie: Amnesia \\
\hline 1491 & 8.4 bus stop & rank: 8.4 movie: Bus Stop (1998/II) \\
\hline 1492 & Ates Roscoe 5.9 rating! & actor: Roscoe Ates rank: 5.9 \\
\hline 1493 & Billi Pinocchio. & actor: Riccardo Billi movie: Avventure di Pinocchio, Le \\
\hline 1494 & Baker Tip Vengeance & actor: Silver Tip Baker movie: Border Vengeance \\
\hline 1495 & De Niro Wegen & movie: Alles Wegen Robert De Niro \\
\hline 1496 & Banner moons & actor: John Banner movie: Crash of Moons \\
\hline 1497 & 1967 Doug? & year: 1967 role: Doug \\
\hline 1498 & Noriega & movie: Caida de Noriega \\
\hline 1499 & Bagdadskij & movie: Bagdadskij vor \\
\hline 1500 & raffaella lane & movie: Acquiring a Taste for Raffaella actor: Craig Lane \\
\hline 1501 & Panda Garden & movie: Andy Panda's Victory Garden \\
\hline
\end{tabular}




\begin{tabular}{|c|c|c|}
\hline 1502 & Biho 1978 & movie: Biho Sangjaeng year: 1978 \\
\hline 1503 & Blame love & movie: Blame it on Love \\
\hline 1504 & Beccerra 6.8 & movie: Beccerra rank: 6.8 \\
\hline 1505 & Anytime Joey Airdo & actor: Joey Airdo movie: Anytime \\
\hline 1506 & born Maid & movie: Born to Be Maid \\
\hline 1507 & 3.3 Cyclone & rank: 3.3 movie: Cyclone \\
\hline 1508 & Antarctica 1991 & movie: Antarctica year: 1991 \\
\hline 1509 & $\begin{array}{l}\text { vida por vida Herself } \\
\text { Presenter? }\end{array}$ & movie: Concierto por la vida role: Herself (Presenter) \\
\hline 1510 & Dan Big 1923 & movie: Big Dan year: 1923 \\
\hline 1511 & Das 3 Reich 1953 & $\begin{array}{l}\text { movie: Bis } 5 \text { nach } 12 \text { - Adolf Hitler und das 3. Reich year: } \\
1953\end{array}$ \\
\hline 1512 & $\begin{array}{l}\text { Diddy Combs and } \\
\text { Snoop Dogg }\end{array}$ & $\begin{array}{l}\text { actor: Sean Combs movie: Bigg Snoop Dogg Raw N Uncut } \\
\text { Vol. } 1\end{array}$ \\
\hline 1513 & AFI's Heros & movie: AFI's 100 Years... 100 Heroes \& Villains \\
\hline 1514 & cedrus 1975 & movie: Cedrus year: 1975 \\
\hline 1515 & Baisur Joe Dracula & actor: Joe Baisur movie: Countess Dracula's Orgy of Blood \\
\hline 1516 & Mariano Madrid es? & role: Mariano movie: As es Madrid \\
\hline 1517 & clair femme & movie: Clair de femme \\
\hline 1518 & 1926 Bonehead. & year: 1926 movie: Bonehead Bobby \\
\hline 1519 & crook wolf $1963 ?$ & movie: Crook who Cried Wolf year: 1963 \\
\hline 1520 & Bertin force & actor: Pierre Bertin movie: Carte Force \\
\hline 1521 & daughter arrowmaker & movie: Arrowmaker's Daughter \\
\hline 1522 & $\begin{array}{c}\text { Las tumbas } 2 \text { Fernando } \\
\text { Casanova. }\end{array}$ & movie: 3 tumbas parte 2 actor: Fernando Casanova \\
\hline 1523 & $\begin{array}{c}\text { muzikant Chuy } \\
\text { zvezdite! }\end{array}$ & role: Slepiyat muzikant movie: Chuy zvezdite \\
\hline 1524 & 1959 bunny & year: 1959 movie: Bonanza Bunny \\
\hline 1525 & 1960 Brune & movie: Brune que voil year: 1960 \\
\hline 1526 & covek pojeo 1981. & movie: Covek koji je pojeo vuka year: 1981 \\
\hline 1527 & Attrazione 1987 & movie: Attrazione year: 1987 \\
\hline 1528 & $\begin{array}{c}\text { Berger Pablo } 73 \text { hizo } \\
\text { Torremolinos? }\end{array}$ & actor: Pablo Berger movie:'As se hizo' - Torremolinos 73 \\
\hline 1529 & Anderson Prospector & actor: Gilbert Anderson movie: Crazy Prospector \\
\hline 1530 & Ahn Philip Holliday! & actor: Philip Ahn movie: Amazing Mrs. Holliday \\
\hline 1531 & Ory Comte 1995 & movie: Comte Ory year: 1995 \\
\hline 1532 & Asesino embarca & movie: Asesino se embarca \\
\hline 1533 & Byline & movie: Byline \\
\hline 1534 & Rafael 1974? & actor: Rafael Albaicín year: 1974 \\
\hline 1535 & Smith 1900? & actor: Jacob H. Smith year: 1900 \\
\hline 1536 & 1922 boss camp & year: 1922 movie: Boss of Camp Four \\
\hline
\end{tabular}




\begin{tabular}{|c|c|c|}
\hline 1537 & Agente Ps 6.1 & role: Agente P.s. rank: 6.1 \\
\hline 1538 & House PaynesCynthia & movie: Cynthia Payne's House of Cyn \\
\hline 1539 & 1997 biological & year: 1997 movie: Biological Maintenance Department \\
\hline 1540 & Adams 1986! & actor: Don Adams year: 1986 \\
\hline 1541 & Alpha Omega 2003 & movie: Alpha \& Omega year: 2003 \\
\hline 1542 & Babysitting adventure & movie: Adventures in Babysitting \\
\hline 1543 & Bulldog Custer Bob! & movie: Bulldog Pluck actor: Bob Custer \\
\hline 1544 & aside 35. & movie: 35 Aside \\
\hline 1545 & Adlon big show & actor: Louis Adlon movie: Big Show-Off \\
\hline 1546 & Dancer collection '92 & movie: Captivated '92: The video collection role: Dancer \\
\hline 1547 & Ruum Apparatspot & movie: Apparatspott - Gerangel in Ruum un Tied \\
\hline 1548 & 1991 Alarc ̃̃n Luis. & year: 1991 actor: Luis Alarcón \\
\hline 1549 & Eric $1954 ?$ & actor: Eric Rohmer year: 1954 \\
\hline 1550 & Careless Lady & movie: Careless Lady \\
\hline 1551 & Aqu Colorado 1946 & movie: Aqu est Juan Colorado year: 1946 \\
\hline 1552 & $\begin{array}{l}\text { Narrator in Banned } \\
\text { America }\end{array}$ & role: Narrator Movie: Banned! In America III \\
\hline 1553 & benny place & movie: Benny's Place \\
\hline 1554 & cuentas de Agust Ãn & movie: Ajuste de cuentas actor: Agustín Bernal \\
\hline 1555 & alczar cinema & movie: Cinema Alczar \\
\hline 1556 & avenging hand & movie: Avenging Hand \\
\hline 1557 & captain baby & movie: Captain Barnacle's Baby \\
\hline 1558 & 4.31989 & rank: 4.3 year: 1989 \\
\hline 1559 & Bennett 2003. & actor: Jeff Bennett year: 2003 \\
\hline 1560 & $\begin{array}{l}1985 \text { Eduardo } \\
\text { Coutinho! }\end{array}$ & year: 1985 actor: Eduardo Coutinho \\
\hline 1561 & clue oak leave & movie: Clue of the Oak Leaf \\
\hline 1562 & Edwards boden boy & actor: Henry Edwards movie: Boden's Boy \\
\hline 1563 & Carpenter christmas & movie: Carpenters: A Christmas Portrait \\
\hline 1564 & $\begin{array}{l}\text { A Heroes Muhammad } \\
\text { Ali! }\end{array}$ & $\begin{array}{l}\text { movie: America: A Tribute to Heroes actor: Muhammad } \\
\text { Ali }\end{array}$ \\
\hline 1565 & 1991 Ruth & year: 1991 movie: Babe Ruth \\
\hline 1566 & agdasarian Jr Wolfman & $\begin{array}{l}\text { actor: Ross Bagdasarian Jr. movie: Alvin and the } \\
\text { Chipmunks Meet the Wolfman }\end{array}$ \\
\hline 1567 & $\begin{array}{c}\text { Central Thanxgiveaway } \\
\text { XBox } \\
\end{array}$ & movie: Comedy Central XBox Thanxgiveaway \\
\hline 1568 & Captain tennille & movie: Captain and Tennille in Hawaii \\
\hline 1569 & alraune 1918 & movie: Alraune (1918/I) year: 1918 \\
\hline 1570 & Up Khyber 6.9. & movie: Carry On... Up the Khyber rank: 6.9 \\
\hline 1571 & Carnival sadie & role: Carnival Boss movie: Charlie and Sadie \\
\hline 1572 & Achard Olivier Oblix & actor: Olivier Achard movie: Astrix et Oblix contre Csar \\
\hline
\end{tabular}




\begin{tabular}{|c|c|c|}
\hline 1573 & 1937 Saunders Jack? & year: 1937 role: Jack Saunders \\
\hline 1574 & Jimmy Barton alias & movie: Alias Jimmy Barton \\
\hline 1575 & Harry Bowen Count & actor: Harry Bowen movie: Count takes the Count \\
\hline 1576 & Actress Drake Walter. & actor: Walter Drake movie: Case of a Doped Actress \\
\hline 1577 & Kidnapping Sinatra. & movie: American Justice: The Sinatra Kidnapping \\
\hline 1578 & Andrea Camilla & role: Andrea Rossetti movie: Camilla \\
\hline 1579 & Sam Bright light & actor: Sam Ash movie: Bright Lights \\
\hline 1580 & 1915 Protg & year: 1915 movie: Broncho Billy's Protg \\
\hline 1581 & Waters Bread & movie: Bread Upon the Waters \\
\hline 1582 & Amir Zalam. & movie: Amir El Zalam \\
\hline 1583 & $\begin{array}{c}\text { Aleksandr } \\
\text { Parkhomenko. }\end{array}$ & movie: Aleksandr Parkhomenko \\
\hline 1584 & 1917 Birmingham & year: 1917 movie: Birmingham Girl's Last Hope \\
\hline 1585 & Blaue Heinz Emigholz! & movie: Blaue Distanz actor: Heinz Emigholz \\
\hline 1586 & buen mam? & movie: Buen da mam, buen da \\
\hline 1587 & $\begin{array}{c}\text { Anemos Desnitsky } \\
\text { Sergei? }\end{array}$ & movie: Anemos Stin Poli actor: Sergei Desnitsky \\
\hline 1588 & 1990 casualty war? & year: 1990 movie: Casualty of War \\
\hline 1589 & Crooked Shadow! & movie: Chase a Crooked Shadow \\
\hline 1590 & Edwin Aunt Huldah & actor: Edwin R. Phillips movie: Aunt Huldah \\
\hline 1591 & cinaga AndrÃ $\bigodot s ?$ & movie: Cinaga actor: Ángel López \\
\hline 1592 & $\begin{array}{l}\text { Alexander Racheal's } \\
\text { attic }\end{array}$ & $\begin{array}{l}\text { actor: Alex Alexander movie: Behind the Attic Door: The } \\
\text { Making of 'Rachel's Attic' }\end{array}$ \\
\hline 1593 & $\begin{array}{c}\text { Tomas Arana } \\
\text { bodyguard }\end{array}$ & actor: Tomas Arana movie: Bodyguard \\
\hline 1594 & society Bucking & movie: Bucking Society \\
\hline 1595 & McKenna 2003! & actor: Terence McKenna year: 2003 \\
\hline 1596 & materialaktion otto & movie: 6/64: Mama und Papa (Materialaktion Otto Mhl) \\
\hline 1597 & Anthony Career? & actor: Anthony Franciosa movie: Career \\
\hline 1598 & bride red & movie: Bride Wore Red \\
\hline 1599 & Amor Martin Space & actor: Martin Amor movie: 2001: A Space Odyssey \\
\hline 1600 & austria Como. & movie: Christmas in Austria actor: Perry Como \\
\hline 1601 & $\begin{array}{l}\text { Redding Franklin } \\
\text { Batie? }\end{array}$ & role: Jim Redding actor: Franklin Batie \\
\hline 1602 & $\begin{array}{l}\text { Gustavo Abriqueta } \\
\text { MartÃn? }\end{array}$ & role: Gustavo actor: Martín Abriqueta \\
\hline 1603 & Antychryst Maciuk. & movie: Antychryst actor: Dariusz Maciuk \\
\hline 1604 & 1957 colonies & year: 1957 movie: Colonies Look Ahead \\
\hline 1605 & bubba Bannister Reggie & movie: bubba Ho-tep actor: Reggie Bannister \\
\hline 1606 & Arimpara & movie: Arimpara \\
\hline 1607 & 1912 brains? & movie: Brains and Brawn year: 1912 \\
\hline
\end{tabular}




\begin{tabular}{|c|c|c|}
\hline 1608 & mistress 1992. & role: Dungeon mistress year: 1992 \\
\hline 1609 & Rene Omen II & $\begin{array}{c}\text { actor: Rene Auberjonois movie: Blood Omen II: Legacy of } \\
\text { Kain }\end{array}$ \\
\hline 1610 & 1952 Brief City. & year: 1952 movie: Brief City \\
\hline 1611 & brujo Amor & movie: Amor brujo \\
\hline 1612 & ChaalBaaz 6.3? & movie: ChaalBaaz rank: 6.3 \\
\hline 1613 & elegy Bop yotaro & movie: Bee Bop highschool; Koko yotaro elegy \\
\hline 1614 & Uncle Walked & movie: And in Walked Uncle \\
\hline 1615 & $\begin{array}{c}\text { Auclair Michel bonnes } \\
!\end{array}$ & actor: Michel Auclair movie: Bonnes tuer \\
\hline 1616 & Blazer Butt. & movie: Butt Blazer \\
\hline 1617 & Badmen $6 ?$ & movie: Best of the Badmen rank: 6 \\
\hline 1618 & The Party Birthday. & movie: Birthday Party \\
\hline 1619 & Adams Monty. & role: Monty Adams actor: Monty Banks \\
\hline 1620 & $\begin{array}{l}\text { cartomante Ivan } \\
\text { CÃ } \notin \text { ndido! }\end{array}$ & movie: Cartomante actor: Ivan Cândido \\
\hline 1621 & Arriba Quint Ãn? & movie: Arriba las Manos Texan actor: Quintín Bulnes \\
\hline 1622 & Arianas bondage & movie: Ariana's Bondage \\
\hline 1623 & barber Bolam James & actor: James Bolam movie: Barber \\
\hline 1624 & Hart Frost Blake & actor: William S. Hart movie: Conversion of Frosty Blake \\
\hline 1625 & 2002 Tyce? & year: 2002 actor: Tyce Bune \\
\hline 1626 & 5.6 women & rank: 5.6 movie: 8 Women \\
\hline 1627 & Bella Martha Doctor. & movie: Bella Martha role: Doctor \\
\hline 1628 & All Esther Dave Brake & movie: Call Esther All actor: Dave Brake \\
\hline 1629 & jeg igen $\mathrm{fr}$ & movie: Alt hvad jeg ejer \\
\hline 1630 & 1989 silk secrets. & year: 1989 movie: Black Silk Secrets \\
\hline 1631 & $\begin{array}{l}1922 \text { dangerous } \\
\text { pastime }\end{array}$ & year: 1922 movie: Dangerous Pastime \\
\hline 1632 & $\begin{array}{l}\text { Rene Rupnik } \\
\text { Busenfreund }\end{array}$ & actor: Rene Rupnik movie: Busenfreund \\
\hline 1633 & 1920 cuando patria & movie: Cuando la patria lo mande year: 1920 \\
\hline 1634 & $1993 \mathrm{LA}^{3}$ pez de. & year: 1993 actor: Ángel de Andrés López \\
\hline 1635 & 1987 Brad Armacost? & year: 1987 actor: Brad Armacost \\
\hline 1636 & Bruiser 5.6! & role: Bruiser rank: 5.6 \\
\hline 1637 & Alien Kevin Alber. & movie: Alien Terminator actor: Kevin Alber \\
\hline 1638 & 2001 Jon Bon! & year: 2001 actor: Jon Bon Jovi \\
\hline 1639 & 1990 Image Awards. & year: 1990 movie: 22nd NAACP Image Awards \\
\hline 1640 & Sergeant Woody 5.5? & role: Sergeant Woody rank: 5.5 \\
\hline 1641 & Curse Desert & movie: Curse of the Desert \\
\hline 1642 & $\begin{array}{l}\text { Ren } \tilde{A} \subset \text { Poyen } \\
\text { l'embusqu }\end{array}$ & actor: René Poyen movie: Bout de Zan et l'embusqu \\
\hline
\end{tabular}




\begin{tabular}{|c|c|c|}
\hline 1643 & Gregorio Asesino & actor: Gregorio Casal movie: Asesino nocturno \\
\hline 1644 & days of glory 1985 . & movie: 16 Days of Glory year: 1985 \\
\hline 1645 & Aragn 1937! & movie: Aragn 1937 \\
\hline 1646 & Cinderella Creek & movie: cripple creek cinderella \\
\hline 1647 & 1994 ailes & year: 1994 movie: Ailes du Plaisir \\
\hline 1648 & mujer seducir & movie: Cmo seducir a una mujer \\
\hline 1649 & avenger Sidney! & actor: Sidney Bracey movie: Avenger \\
\hline 1650 & 2000 Aazad & year: 2000 movie: Aazad \\
\hline 1651 & $\begin{array}{c}\text { Donald Briggs All } \\
\text { American }\end{array}$ & actor: Donald Briggs movie: All-American Sweetheart \\
\hline 1652 & 1978 tiempo? & movie: All lejos y hace tiempo year: 1978 \\
\hline 1653 & 1971 munte mare! & movie: munte si la mare year: 1971 \\
\hline 1654 & $\begin{array}{c}\text { Anticasanova Relja } \\
\text { Basic. }\end{array}$ & movie: Anticasanova actor: Relja Basic \\
\hline 1655 & Eli Altonio 2002 & actor: Eli Altonio year: 2002 \\
\hline 1656 & Julian Cheung amoeba & actor: Julian Cheung movie: Ai qing amoeba \\
\hline 1657 & 1953 arena & year: 1953 movie: arena \\
\hline 1658 & Blinky 1992 & movie: Blinky Bill year: 1992 \\
\hline 1659 & Andel 1988. & movie: Andel svd dbla year: 1988 \\
\hline 1660 & Alisik 1966. & actor: Sadri Alisik year: 1966 \\
\hline 1661 & Dancing Queen Ticket? & movie: Dancing Queen role: Ticket Collector \\
\hline 1662 & Barcroft Roy 1945 & actor: Roy Barcroft year: 1945 \\
\hline 1663 & $\begin{array}{l}\text { Worlds Commercials } \\
\text { The Host }\end{array}$ & $\begin{array}{c}\text { movie: Banned in America: The World's Sexiest } \\
\text { Commercials role: Host }\end{array}$ \\
\hline 1664 & Mel Blanc Pains & actor: Mel Blanc movie: Crowing Pains \\
\hline 1665 & 1955 MacDonald & year: 1955 actor: James MacDonald \\
\hline 1666 & Larait Brennan & movie: Cupid's Larait actor: John Brennan \\
\hline 1667 & Anthony comedy & actor: Anthony Hopkins genre: Comedy \\
\hline 1668 & Almada Archivos & actor: Mário Almada movie: Archivos policiacos \\
\hline 1669 & $\begin{array}{l}\text { Narrator Terzieff } \\
\text { Laurent. }\end{array}$ & role: Narrator actor: Laurent Terzieff \\
\hline 1670 & Buffalo Hunting . & movie: Buffalo Hunting \\
\hline 1671 & $\begin{array}{l}\text { soupe Bonne GÃđCrard } \\
\text { Blain. }\end{array}$ & movie: Bonne soupe actor: Gérard Blain \\
\hline 1672 & Joseph i Aulisi Lucy & $\begin{array}{l}\text { actor: Joseph G. Aulisi movie: Angelic Attire: Dressing } \\
\text { Cameron, Drew \& Lucy }\end{array}$ \\
\hline 1673 & cais Sodr & movie: Cais do Sodr \\
\hline 1674 & 1981 Bingjeom! & year: 1981 movie: Bingjeom '81 \\
\hline 1675 & course Collision? & movie: Collision Course \\
\hline 1676 & 1989 Dont Shoot & year: 1989 movie: Blackmailers Don't Shoot \\
\hline 1677 & ausente de $C \tilde{A}^{3}$ rdova & actor: Arturo de Córdova movie: Ausente \\
\hline
\end{tabular}




\begin{tabular}{|c|c|c|}
\hline 1678 & Cactus Capers & movie: Cactus Capers \\
\hline 1679 & Barton Crayoluv & actor: Daniel Barton movie: Crayoluv \\
\hline 1680 & Burnzy's call & movie: Burnzy's Last Call \\
\hline 1681 & 1913 Ike Alkali? & year: 1913 role: Alkali Ike \\
\hline 1682 & Broken Cop? & movie: Broken Vessels role: Cop \\
\hline 1683 & aadavallu Chirakadze & movie: Aadavallu Meeku Joharulu actor: Giga Chirakadze \\
\hline 1684 & 1918 bully & year: 1918 movie: Bully \\
\hline 1685 & rockefeller christmas & movie: Christmas in Rockefeller center \\
\hline 1686 & Brunelli Tenente $3.2 ?$ & role: Tenente Brunelli rank: 3.2 \\
\hline 1687 & Player in Brian's Song & role: Veteran Player movie: Brian's song \\
\hline 1688 & absolution anthony & movie: Absolution of Anthony \\
\hline 1689 & diablo $1993 ?$ & movie: Aliento del diablo year: 1993 \\
\hline 1690 & chica autostop & movie: Chica del autostop \\
\hline 1691 & cage 1975 & movie: Cage year: 1975 \\
\hline 1692 & Acosta Bad Men & actor: Rodolfo Acosta movie: City of Bad Men \\
\hline 1693 & Rum Hicks Omelet. & movie: Chimmie Hicks and the Rum Omelet \\
\hline 1694 & 1977 Cann Baln? & year: 1977 movie: Baln Cann \\
\hline 1695 & 1963 Orhan GÃ¹/4nsiray & year: 1963 actor: Orhan Günsiray \\
\hline 1696 & Love Spencer. & movie: Art of Love role: Spencer \\
\hline 1697 & Family Wagon Hooper & movie: Chicken Wagon Family actor: Hooper Atchley \\
\hline 1698 & Project Forbin 7? & movie: Colossus: The Forbin Project rank: 7 \\
\hline 1699 & Foxe Earle shy & actor: Earle Foxe movie: Car Shy \\
\hline 1700 & charlie chinatown & movie: Chinatown Charlie \\
\hline 1701 & 1988 sorriso & movie: Casa del sorriso year: 1988 \\
\hline 1702 & $5.71966 ?$ & rank: 5.7 year: 1966 \\
\hline 1703 & Kane Kane Kane 2002 & role: Tom Berendo/Charlie Kane year: 2002 \\
\hline 1704 & Heern van Heern 2004 & role: Mr. van Heern year: 2004 \\
\hline 1705 & $\begin{array}{c}1950 \text { You Ready } \\
\text { marriage? }\end{array}$ & year: 1950 movie: Are You Ready for Marriage? \\
\hline 1706 & $\begin{array}{l}\text { contra } \begin{array}{l}\text { Alc } \tilde{A} \\
\text { ctorar VÃ }\end{array} \\
\text { ctor. }\end{array}$ & movie: contra la pared actor: Víctor Alcázar \\
\hline 1707 & 6.6 Parade comedy & movie: Big Parade of Comedy rank: 6.6 \\
\hline 1708 & 1976 conto chiuso & year: 1976 movie: Conto chiuso, Il \\
\hline 1709 & Marcus saint or singer? & actor: Marcus Bonnée movie: Bob Geldof: Saint or Singer \\
\hline 1710 & hope vietnam christmas & $\begin{array}{c}\text { actor: Bob Hope movie: Bob Hope Vietnam Christmas } \\
\text { Show }\end{array}$ \\
\hline 1711 & $\begin{array}{l}\text { Tomiyama Takashi } \\
\text { paradise? }\end{array}$ & actor: Takashi Tomiyama movie: $1+2=$ Paradise \\
\hline 1712 & $\begin{array}{l}\text { Accidental Clue with } \\
\text { brown? }\end{array}$ & movie: Accidental Clue actor: William H. Brown \\
\hline 1713 & Anzaldo Matthew & actor: Sebastian Anzaldo III role: Matthew Stoney \\
\hline
\end{tabular}




\begin{tabular}{|c|c|c|}
\hline & Stoney. & \\
\hline 1714 & 1976 sainte Anne & year: 1976 movie: Anne sainte \\
\hline 1715 & nella 1984 & movie: Cuori nella tormenta year: 1984 \\
\hline 1716 & ID Caller 2003 & movie: Caller ID year: 2003 \\
\hline 1717 & Tayo crepe! & actor: Masashi Tayo movie: Crepe \\
\hline 1718 & 1981 campineiro! & year: 1981 movie: Campineiro \\
\hline 1719 & crown of Indoensia & movie: Crown jewel of indonesia \\
\hline 1720 & chantaje torero & movie: Chantaje a un torero \\
\hline 1721 & John Aprea 1974! & actor: John Aprea year: 1974 \\
\hline 1722 & 1925 comedy & year: 1925 genre: comedy \\
\hline 1723 & Telegraphist Atlantik & role: Telegraphist movie: Atlantik \\
\hline 1724 & movie 19997.2 & year: 1999 rank: 7.2 \\
\hline 1725 & Romeo Cumpson! & actor: John R. Cumpson movie: Bumptious as Romeo \\
\hline 1726 & John petersen 1986 ! & actor: John Hahn-Petersen year: 1986 \\
\hline 1727 & 1968 Brahmachari & year: 1968 movie: Brahmachari (1968/I) \\
\hline 1728 & behind mask & movie: Behind the Mask \\
\hline 1729 & $\begin{array}{l}\text { Coxen Edward } \\
\text { Butterfly! }\end{array}$ & actor: Edward Coxen movie: Butterfly \\
\hline 1730 & 1918 Bonne & year: 1918 movie: Chambre de la bonne \\
\hline 1731 & Billy Lowell Bobby & $\begin{array}{l}\text { role: Billy Lowell movie: Billy \& Bobby: The Hollywood } \\
\text { Years }\end{array}$ \\
\hline 1732 & bullwhip Adams I & actor: Peter Adams movie: Bullwhip \\
\hline 1733 & 2004 Allentoff Jason. & year: 2004 actor: Jason Allentoff \\
\hline 1734 & Colmena 1982? & movie: Colmena year: 1982 \\
\hline 1735 & Malta. & movie: Cavalieri di Malta \\
\hline 1736 & Alf $1915 ?$ & role: Alf Sloppy year: 1915 \\
\hline 1737 & 1983 Frightened Lady! & year: 1983 movie: Case of the Frightened Lady \\
\hline 1738 & calzonazos & movie: Calzonazos \\
\hline 1739 & colleens corner & movie: Corner in Colleens \\
\hline 1740 & cantor 1958 & movie: Cantor e o Milionrio year: 1958 \\
\hline 1741 & $\begin{array}{l}\text { Bertolucci Bernardo } \\
\text { Limelight Today }\end{array}$ & $\begin{array}{l}\text { actor: Bernardo Bertolucci movie: Chaplin Today: } \\
\text { Limelight }\end{array}$ \\
\hline 1742 & McCarthy 2003 & actor: Paul Boyington-McCarthy Jr. year: 2003 \\
\hline 1743 & $\begin{array}{l}\text { Hooper with Rex and } \\
\text { Rinty }\end{array}$ & $\begin{array}{l}\text { actor: Hooper Atchley movie: Adventures of Rex and } \\
\text { Rinty }\end{array}$ \\
\hline 1744 & 1958 Bobby & year: 1958 movie: Bobby \\
\hline 1745 & Fear Lince! & movie: Bond of Fear actor: John Lince \\
\hline 1746 & James Friends & movie: Between Friends actor: James Bearden \\
\hline 1747 & 1926 Juliet & year: 1926 movie: Bromo and Juliet \\
\hline 1748 & Catalano George love & actor: George Catalano movie: Crazy in Love \\
\hline
\end{tabular}




\begin{tabular}{|c|c|c|}
\hline 1749 & 1918 Desmond & year: 1918 actor: William Desmond \\
\hline 1750 & 1913 deadline & year: 1913 movie: Below the Deadline \\
\hline 1751 & Oleg 7.9? & actor: Oleg Anofriyev rank: 7.9 \\
\hline 1752 & Manager 1981. & role: Manager year: 1981 \\
\hline 1753 & caf chinos & movie: Caf de chinos \\
\hline 1754 & Antonio Dilsa Marshall & actor: Antonio Dilsa role: Dr. Rawlins Marshall \\
\hline 1755 & simpton curfew & movie: Curfew at Simpton Center \\
\hline 1756 & 2002 cathedral & year: 2002 movie: Cathedral (2002/I) \\
\hline 1757 & 1916 Plumes! & year: 1916 movie: Borrowed Plumes (1916/I) \\
\hline 1758 & Katsura Yasuki? & movie: Aizen Katsura role: Yasuki \\
\hline 1759 & Enklaar AmnesiA & actor: Cas Enklaar movie: AmnesiA \\
\hline 1760 & Command 1985 & movie: Command 5 year: 1985 \\
\hline 1761 & Auroville Euroville & movie: Auroville-Euroville \\
\hline 1762 & babam degildi & movie: Babam katil degildi \\
\hline 1763 & 1910 brothers & movie: Brothers year: 1910 \\
\hline 1764 & Annes 807.1 ! & movie: Annes 80 rank: 7.1 \\
\hline 1765 & $\begin{array}{l}\text { 1974 Lombard } \\
\text { Lombard I }\end{array}$ & year: 1974 actor: Robert Lombard \\
\hline 1766 & 1990 Arabia & year: 1990 movie: Dangerous Man: Lawrence After Arabia \\
\hline 1767 & Le Crime damour? & movie: Crime d'amour \\
\hline 1768 & Avlonitis comedy & actor: Vasilis Avlonitis genre: Comedy \\
\hline 1769 & $\begin{array}{c}\text { IV RodrÃguez } \tilde{A} \bullet n \text { gel } \\
\text { Atrapados }\end{array}$ & actor: Miguel Rodríguez movie: Atrapados \\
\hline 1770 & 1988 Oklahoma & year: 1988 movie: Baja Oklahoma \\
\hline 1771 & 2004 Odyssey & year: 2004 movie: 2004: A Light Knight's Odyssey \\
\hline 1772 & $1997 \mathrm{KC}$ & year: 1997 role: K.C. \\
\hline 1773 & 5.2 Bruch & rank: 5.2 movie: Bruch \\
\hline 1774 & 1958 Mexico? & movie: Cuando Mexico canta year: 1958 \\
\hline 1775 & 2000 Conteuse & movie: Conteuse year: 2000 \\
\hline 1776 & bigger man & movie: Bigger man \\
\hline 1777 & country John Lithgow? & actor: John Lithgow movie: Country Mouse \\
\hline 1778 & Camas & movie: Camas \\
\hline 1779 & angel with Trumpet & movie: Angel with the Trumpet \\
\hline 1780 & 7.8 Aviv & rank: 7.8 movie: Aviv \\
\hline 1781 & 1998 Worker! & year: 1998 role: The Worker \\
\hline 1782 & 1938 Annabel & year: 1938 movie: Affairs of Annabel \\
\hline 1783 & Fantasies and Gould & movie: Cher... and Other Fantasies actor: Elliott Gould \\
\hline 1784 & Fedaileri 1966. & movie: Cennet Fedaileri year: 1966 \\
\hline 1785 & Victor Death & actor: Victor Banerjee movie: Dadah Is Death \\
\hline 1786 & 1999 casualty & year: 1999 movie: Casualty \\
\hline
\end{tabular}




\begin{tabular}{|c|c|c|}
\hline 1787 & tge Daibosatsu & movie: Dai-bosatsu tge \\
\hline 1788 & kaputt berlin & movie: Berlin Kaputt \\
\hline 1789 & Amanat 4.7 & movie: Amanat rank: 4.7 \\
\hline 1790 & 3.7 American & movie: American Anthem rank: 3.7 \\
\hline 1791 & Hjorth as Himself & actor: Peter Hjorth role: Himself \\
\hline 1792 & 1966 Luis Galiardo? & year: 1966 actor: Juan Luis Galiardo \\
\hline 1793 & As Armas Vento? & role: Pé-de-Vento movie: As Armas \\
\hline 1794 & Kyle Ryan? & role: Kyle actor: Ryan Bumcrot \\
\hline 1795 & 1995 preuishchen & $\begin{array}{c}\text { year: } 1995 \text { movie: Anekdote aus dem letzten preuischen } \\
\text { kriege }\end{array}$ \\
\hline 1796 & 6.6 Barnes. & rank: 6.6 actor: Julian Barnes \\
\hline 1797 & Chilling Blanchard. & actor: Rick Blanchard movie: Chilling \\
\hline 1798 & Adams ted movie & actor: Ted Adams \\
\hline 1799 & carters conversation & movie: Carters: A Conversation \\
\hline 1800 & 199520 years $\mathrm{HBO}$ & year: 1995 movie: 20 years of comedy on $\mathrm{HBO}$ \\
\hline 1801 & Men Arnold Reudi! & movie: Boys to Men actor: Reudi Arnold \\
\hline 1802 & Alarma! & movie: Alarma \\
\hline 1803 & bathroom disco in 7.8 ! & role: Guy in disco bathroom laughin rank: 7.8 \\
\hline 1804 & $\begin{array}{l}\text { Fairlie Frederick Crime } \\
\text { Dark? }\end{array}$ & role: Frederick Fairlie movie: Crimes at the Dark House \\
\hline 1805 & 1989 Churchill? & year: 1989 actor: Winston Churchill \\
\hline 1806 & Stallion Axton Hoyt! & movie: Black Stallion actor: Hoyt Axton \\
\hline 1807 & 5.1 Bedside & rank: 5.1 movie: Bedside Manner \\
\hline 1808 & Iskade Alice 1989 & movie: Alla lskade Alice year: 1989 \\
\hline 1809 & Bond Albert Austin! & movie: Bond actor: Albert Austin \\
\hline 1810 & $\begin{array}{l}\text { Edgar McInnis! } \\
\text { Colonies? }\end{array}$ & actor: Edgar McInnis movie: Colonies Look Ahead \\
\hline 1811 & Athlte 1932 ! & movie: Athlte incomplet year: 1932 \\
\hline 1812 & Male bitter Harvest! & gender: $\mathrm{m}$ movie: Bitter Harvest \\
\hline 1813 & Sexiest Male video & gender: $\mathrm{m}$ movie: 50 Sexiest Video Moments \\
\hline 1814 & Hercules?? Biography & movie: A\&E Biography: Hercules - Power of the Gods \\
\hline 1815 & Cape 1996. & movie: Cape year: 1996 \\
\hline 1816 & 1967 salvajes & year: 1967 movie: Cuatro salvajes \\
\hline 1817 & Count Countless & movie: Countless Count \\
\hline 1818 & Male Woody Allen. & gender: $\mathrm{m}$ actor: Woody Allen \\
\hline 1819 & Male Sunset? & gender: $\mathrm{m}$ movie: Calm at Sunset \\
\hline 1820 & Tony Male. & role: Tony gender: $\mathrm{m}$ \\
\hline 1821 & chapeaux dames & movie: Ces dames aux chapeaux verts \\
\hline 1822 & 2084 & movie: 2084 \\
\hline 1823 & Hopkins Anthony & actor: Anthony Hopkins movie: Behind the Scenes: \\
\hline
\end{tabular}




\begin{tabular}{|c|c|c|}
\hline & Scenes & Hannibal \\
\hline 1824 & 2001 Muse & year: 2001 movie: Blood for the Muse \\
\hline 1825 & Donald Champions & actor: Donald Brittain movie: Champions \\
\hline 1826 & 1915 Inspector Police. & year: 1915 role: Police Inspector \\
\hline 1827 & 1993 Penetration & year: 1993 movie: Best of Double Penetration \\
\hline 1828 & Queen Kate? & movie: Bondage Queen Kate \\
\hline 1829 & Squall. After & movie: After the Squall \\
\hline 1830 & sarkisi Akdeniz & movie: Akdeniz sarkisi \\
\hline 1831 & Male Celebration sur & gender: m movie: Celebration at Big Sur \\
\hline 1832 & 1990 Extra & year: 1990 movie: Busen Extra 2 \\
\hline 1833 & 5.8 Aftermath! & rank: 5.8 movie: Aftermath \\
\hline 1834 & Harufa Brown! & role: Harufa actor: Alfred Brown \\
\hline 1835 & Champakali Male? & movie: Champakali gender: $\mathrm{m}$ \\
\hline 1836 & Joys Boys Surveyor? & movie: Boys Will Be Joys role: Surveyor \\
\hline 1837 & Astounding. 1957 & movie: Astounding She-Monster year: 1957 \\
\hline 1838 & Battman 1994? & movie: Battman year: 1994 \\
\hline 1839 & 1999 Babewatch & year: 1999 movie: Babewatch \\
\hline 1840 & Appel Cyril! & movie: Appel d'air actor: Cyril Brière \\
\hline 1841 & 1916 Heinie & year: 1916 role: Heinie \\
\hline 1842 & 1986 Schriftsteller? & year: 1986 movie: Bln. DDR und ein Schriftsteller \\
\hline 1843 & 1990 buin? & year: 1990 movie: Aema buin 4 \\
\hline 1844 & Arson Brophy & actor: Edward Brophy movie: Arson \\
\hline 1845 & 4.4? Cinderella & movie: Cinderella Story rank: 4.4 \\
\hline 1846 & Jihun mucho Besame. & role: Han Ji-hun movie: Besame mucho \\
\hline 1847 & 5.5 Mr Henshow. & rank: 5.5 role: Mr. Henshow \\
\hline 1848 & $\begin{array}{l}\text { hacer txilindrn maneras } \\
\text { pollo! }\end{array}$ & movie: Cien maneras de hacer el pollo al txilindrn \\
\hline 1849 & Alexanderplatz 1931! & movie: Berlin - Alexanderplatz year: 1931 \\
\hline 1850 & 2000 Jay! & role: Jay year: 2000 \\
\hline 1851 & Ardizoni John 1934 & actor: John Ardizoni year: 1934 \\
\hline 1852 & 2003 American & year: 2003 movie: American Idol Christmas \\
\hline 1853 & Arashi otoko & movie: Arashi o yobu otoko \\
\hline 1854 & Champions Louis & actor: Louis de Funès movie: Champions Juniors \\
\hline 1855 & Fukuda shnen! & $\begin{array}{l}\text { role: Fukuda Clorde movie: Ch-Bakumatsu shnen seiki } \\
\text { takamaru }\end{array}$ \\
\hline 1856 & J Aberwood 1.3? & actor: Lawrence J. Aberwood rank: 1.3 \\
\hline 1857 & Guy Quarrie Male! & role: Maj. Guy Quarrie gender: $\mathrm{m}$ \\
\hline 1858 & $\begin{array}{l}\text { Bochkaryov Vasili } \\
\text { Akseleratka . }\end{array}$ & actor: Vasili Bochkaryov movie: Akseleratka \\
\hline 1859 & men 2004 & movie: Big Men year: 2004 \\
\hline
\end{tabular}




\begin{tabular}{|c|c|c|}
\hline 1860 & Captain wife & role: Captain Wolf movie: Another Man's Wife \\
\hline 1861 & 1997 Marquis & $\begin{array}{c}\text { year: } 1997 \text { movie: A\&E Biography: Marquis de Sade - The } \\
\text { Depraved Aristocrat }\end{array}$ \\
\hline 1862 & Paquito Diaz Kasunod & actor: Paquito Diaz movie: Ayos na ang Kasunod \\
\hline 1863 & 2002 Zirkusluft & year: 2002 movie: Bulle von Tlz - Zirkusluft \\
\hline 1864 & The Ackroyd Ackroyd? & movie: Backyard actor: Jack Ackroyd \\
\hline 1865 & $\begin{array}{c}\text { Bellman Summer } \\
\text { Aloha. }\end{array}$ & role: Bellman movie: Aloha Summer \\
\hline 1866 & storm 1915 & movie: After the Storm (1915/II) \\
\hline 1867 & cars Paris & movie: Cars That Ate Paris \\
\hline 1868 & Himself as Cobbs! & role: Himself actor: Bill Cobbs \\
\hline 1869 & Altman Own 2000 & movie: Altman on His Own Terms year: 2000 \\
\hline 1870 & BabySitters Club & movie: Baby-Sitters Club \\
\hline 1871 & Vernon the Woods & actor: Vernon Dent movie: Back to the Woods \\
\hline 1872 & 1918 crime & year: 1918 genre: Crime \\
\hline 1873 & Lukas Mourir & role: Lukas movie: Celui qui doit Mourir \\
\hline 1874 & kisobran crini & movie: Crni kisobran \\
\hline 1875 & Sorority Mort! & movie: Confessions of a Sorority Girl role: Mort \\
\hline 1876 & 1942 Crumb? & year: 1942 role: $\mathrm{Mr}$. Crumb \\
\hline 1877 & $\begin{array}{l}\text { Besos prohibidos } \\
\text { Aguilar! }\end{array}$ & movie: Besos prohibidos actor: Luis Aguilar \\
\hline 1878 & Inocente cinema & movie: Cinema Inocente \\
\hline 1879 & leoni nella & movie: Cretinetti nella gabbia dei leoni \\
\hline 1880 & columbo bird & movie: Columbo: A bird in the hand \\
\hline 1881 & i mladi byahme 1961 & movie: A byahme mladi year: 1961 \\
\hline 1882 & 1990 forest & year: 1990 movie: Circles in a Forest \\
\hline 1883 & Basmaci Fiisi! & movie: Barut Fiisi actor: Aziz Basmaci \\
\hline 1884 & 1981 Cleste! & year: 1981 movie: Cleste \\
\hline 1885 & 1983 szigete. & year: 1983 movie: Bke szigete \\
\hline 1886 & T.T Audition & actor: T.T. Boy movie: Audition \\
\hline 1887 & Eric Cremators & actor: Eric Allison movie: Cremators \\
\hline 1888 & Beautiful Walker! & actor: Johnnie Walker movie: Beautiful Impostor \\
\hline 1889 & Anna 1951 & movie: Anna year: 1951 \\
\hline 1890 & Atlanten & movie: Atlanten \\
\hline 1891 & babes illustrated & movie: Babes Illustrated 2 \\
\hline 1892 & veli in Tamer! & movie: Ali ile veli actor: Tamer Balci \\
\hline 1893 & cheated Turner & actor: Bowditch Turner movie: Cheated love \\
\hline 1894 & 1978 Bikov. & year: 1978 actor: Kosta Bikov \\
\hline 1895 & Farc AbrahÃ£o 1973! & actor: Abrahão Farc year: 1973 \\
\hline 1896 & crimson 1916 & year: 1916 movie: Crimson Trail \\
\hline
\end{tabular}




\begin{tabular}{|c|c|c|}
\hline 1897 & Avery $6.2 !$ & actor: James Avery rank: 6.2 \\
\hline 1898 & Suphi Kaner 1959 & actor: Suphi Kaner year: 1959 \\
\hline 1899 & Sherwood 4.9? & rank: 4.9 movie: Arciere di Sherwood \\
\hline 1900 & cavalcade Dick! & movie: Cavalcade of Stars actor: Dick Rambone \\
\hline 1901 & blunders trips & movie: Blunders of Mr. Butterbun: Trips and Tribunals \\
\hline 1902 & Baliktaran Fernandez & movie: Baliktaran actor: Dan Fernandez \\
\hline 1903 & city under sea & movie: City under the sea \\
\hline 1904 & Trumper Freddie 2003? & role: Freddie Trumper year: 2003 \\
\hline 1905 & 5.61990. & rank: 5.6 year: 1990 \\
\hline 1906 & buscando Perico & movie: Buscando a Perico \\
\hline 1907 & 1961 Narrator! & year: 1961 role: Narrator \\
\hline 1908 & colorado Douglas & movie: Conquering the Colorado actor: Paul Douglas \\
\hline 1909 & Bakken collar & movie: Blue Collar America actor: Tom Bakken \\
\hline 1910 & 1994 blue & year: 1994 movie: Bunny Bleu: A Gang Bang Fantasy \\
\hline 1911 & 2004 Antonio Banderas & year: 2004 actor: Antonio Banderas \\
\hline 1912 & tsumi Fujimaki Jun. & movie: Bib ni tsumi ari actor: Jun Fujimaki \\
\hline 1913 & Mel 1976 & actor: Mel Blanc year: 1976 \\
\hline 1914 & Reyes Narrator & movie: Alfonso Reyes role: Narrator \\
\hline 1915 & Liam Catholics! & actor: Liam Burk movie: Catholics \\
\hline 1916 & $\begin{array}{c}1970 \text { Giuseppe } \\
\text { Addobbati! }\end{array}$ & year: 1970 actor: Giuseppe Addobbati \\
\hline 1917 & black cherry 15 & movie: Black Cherry Coeds 15 \\
\hline 1918 & blutsbrder & movie: Blutsbrder \\
\hline 1919 & 7.6 Applause & movie: Applause rank: 7.6 \\
\hline 1920 & 8.6 sidan Andra? & rank: 8.6 movie: Andra sidan \\
\hline 1921 & Callboy Allan & movie: Callboy role: Allan \\
\hline 1922 & Patsy $1914 ?$ & role: Patsy year: 1914 \\
\hline 1923 & News breaking & movie: Breaking the News \\
\hline 1924 & Patsy $2002 !$ & role: Patsy year: 1914 \\
\hline 1925 & Andere & movie: Andere \\
\hline 1926 & 1988 Astronomy & year: 1988 movie: Astronomy \\
\hline 1927 & Metal Grammy & actor: Jeff Metal movie: 34th Annual Grammy Awards \\
\hline 1928 & Devon alive & actor: Tony Devon movie: As long as You're Alive \\
\hline 1929 & 5.8 Wong Joseph. & rank: 5.8 role: Joseph Wong \\
\hline 1930 & $\begin{array}{c}\text { Chronique danoise } \\
\text { Marc? }\end{array}$ & movie: Chronique danoise role: Marc \\
\hline 1931 & Coast Guard? & movie: Coast Guard \\
\hline 1932 & 2002 Piano Guy! & year: 2002 role: The Piano Guy \\
\hline 1933 & Evil Deputy. & movie: 976-Evil role: Deputy \\
\hline 1934 & Canzone 1956 ! & movie: Canzone Proibita year: 1956 \\
\hline
\end{tabular}




\begin{tabular}{|c|c|c|}
\hline 1935 & $\begin{array}{l}\text { Stowaway Circus } \\
\text { Marie? }\end{array}$ & movie: Circus Stowaway actor: Marie Eline \\
\hline 1936 & Chant Parisien & movie: Chant de Guerre Parisien \\
\hline 1937 & Cupps 2003 & actor: Cindy Cupps year: 2003 \\
\hline 1938 & 1993 rating 4.3 & year: 1993 rank: 4.3 \\
\hline 1939 & Walter 1934. & actor: Walter Brennan year: 1934 \\
\hline 1940 & No! Kazem? & movie: No! actor: Kazem Rastgoftar \\
\hline 1941 & 1947 blonde! & year: 1947 movie: Borrowed Blonde \\
\hline 1942 & all norte & movie: All en el Norte \\
\hline 1943 & Ball in Bit & actor: Vincent Ball movie: Bit Part \\
\hline 1944 & $\$ 50,000$ reward & movie: $\$ 50,000$ Reward \\
\hline 1945 & $\begin{array}{c}\text { Babchak Jim rating of } \\
5.1 !\end{array}$ & actor: Jim Babchak rank: 5.1 \\
\hline 1946 & Blanc yeux! & movie: Blanc des yeux \\
\hline 1947 & $6.41956 !$ & rank: 6.4 year: 1956 \\
\hline 1948 & cleo 2002 & movie: Cleo Bachelor 2002: Real Men Revealed \\
\hline 1949 & 1982 Antonieta & year: 1982 movie: Antonieta \\
\hline 1950 & 19738.2 rating? & year: 1973 rank: 8.2 \\
\hline 1951 & 1984 champ? & year: 1984 movie: Champ \\
\hline 1952 & 6.4 Damned Cry & rank: 6.4 movie: Damned Don't Cry \\
\hline 1953 & Beck Soldiers & movie: Buffalo Soldiers actor: John Beck \\
\hline 1954 & Between Lines 1977 & movie: Between the Lines year: 1977 \\
\hline 1955 & sangre $1997 ?$ & movie: Con fuego en la sangre year: 1997 \\
\hline 1956 & Dama muerte 1. & movie: Dama de la muerte \\
\hline 1957 & Bells 1926 & movie: Bells year: 1926 \\
\hline 1958 & police Kapoor & actor: Shakti Kapoor movie: Chor Police \\
\hline 1959 & baby Soldier Killed & $\begin{array}{c}\text { movie: Baby: Secret of the Lost Legend role: Killed } \\
\text { Soldier }\end{array}$ \\
\hline 1960 & boost 1988 & year: 1988 movie: Boost \\
\hline 1961 & $\begin{array}{l}\text { all stars Bellach } \\
\text { Francine! }\end{array}$ & movie: Barely Legal All stars 2 actor: Francine Bellach \\
\hline 1962 & braun cheveux & actor: Victor Braun movie: Cheveux de ma mre \\
\hline 1963 & weird Al No & movie: Weird Al' Yankovic: (There's No) \\
\hline 1964 & Aazad 2000? & year: 2000 movie: Aazad \\
\hline 1965 & guard SS Bent. & role: SS guard movie: Bent \\
\hline 1966 & 1996 Dennis & year: 1996 actor: Dennis Boutsikaris \\
\hline 1967 & sun went down & movie: Sun Went Down \\
\hline 1968 & 1994 MTV Awards & year: 1994 movie: 1994 MTV Movie Awards \\
\hline 1969 & blue christmas & movie: Baby Blues Christmas Special \\
\hline 1970 & biandan & movie: Biandan \\
\hline
\end{tabular}




\begin{tabular}{|c|c|c|}
\hline 1971 & advertising did & movie: Advertising Did it \\
\hline 1972 & Byoinzaka 1979 & movie: Byoinzaka no kubikukuri no ie year: 1979 \\
\hline 1973 & Aera 1972 ! & movie: Aera! Aera! Aera! year: 1972 \\
\hline 1974 & $\begin{array}{c}\text { Arnold Rick Black } \\
\text { Whip }\end{array}$ & actor: Rick Arnold movie: Black Whip \\
\hline 1975 & 1995 boys side & year: 1995 movie: Boys on the Side \\
\hline 1976 & 2002 Chatroom & year: 2002 movie: Chatroom \\
\hline 1977 & comme doigts & movie: Comme les doigts de la main \\
\hline 1978 & 1921 Cameron? & year: 1921 actor: Cameron Coffey \\
\hline 1979 & detectress dog watch & movie: Bess the Detectress in the Dog Watch \\
\hline 1980 & ABCTeam 1983? & role: Himself (ABC Team contestant0 year: 1983 \\
\hline 1981 & cazador asesinos & movie: Cazador de asesinos \\
\hline 1982 & Caribbean Mystery! & movie: Caribbean Mystery \\
\hline 1983 & Atraco 6.6? & movie: Atraco rank: 6.6 \\
\hline 1984 & Club Hades. & movie: Club Hades \\
\hline 1985 & $\begin{array}{c}\text { Behjat Mohamadi } \\
\text { Firouz Booye! }\end{array}$ & actor: Firouz Behjat-Mohamadi movie: Booye kafoor \\
\hline 1986 & 1933 crook. & year: 1933 movie: Crook's Tour \\
\hline 1987 & Engine borrowed & movie: Borrowed Engine \\
\hline 1988 & crystal $1916 ?$ & year: 1916 movie: Crystal's Warning \\
\hline 1989 & Krishna Kallu & actor: Krishna Ghattamaneni movie: Ave Kallu \\
\hline 1990 & volume 53 Jake & movie: afro-centrix volume 53: hooked on jake steed \\
\hline 1991 & butterfly Cody Lew. & actor: Lew Cody movie: Butterfly Man \\
\hline 1992 & Lands Bad & movie: Bad Lands \\
\hline 1993 & bulldog Postman & movie: Bulldog Drummond at Bay role: Postman \\
\hline 1994 & comancheros & movie: Comancheros \\
\hline 1995 & 1938 Conary & year: 1938 movie: Canary Comes Across \\
\hline 1996 & carson kid? & movie: Carson City Kid \\
\hline 1997 & 1908 Gebhardt & year: 1908 actor: George Gebhardt \\
\hline 1998 & msica con Clnica & movie: Clnica con msica \\
\hline 1999 & cook 1923 & actor: Clyde Cook year: 1923 \\
\hline 2000 & 1987 Benji? & year: 1987 movie: Benji the Hunted \\
\hline 2001 & 1946 Alice! & year: 1946 movie: Alice \\
\hline 2002 & 1936 Roylott & year: 1936 movie: Anonima Roylott \\
\hline 2003 & Fightin 1961. & movie: D' Fightin' Ones year: 1961 \\
\hline 2004 & Avalanche Carleton & movie: Avalanche actor: William T. Carleton \\
\hline 2005 & Bedouins Clark! & movie: Captured by Bedouins actor: Jack J. Clark \\
\hline 2006 & Aaron Bookworms & role: Aaron movie: Bookworms \\
\hline 2007 & Daniels Jinks' & actor: Frank Daniels movie: Captain Jinks' Cure \\
\hline 2008 & 2001 Brogma & year: 2001 movie: Brogma 2 - Como no se seleccion \\
\hline
\end{tabular}




\begin{tabular}{|c|c|c|}
\hline 2009 & Colerider Krugh 3 & actor: Kyle Colerider-Krugh movie: 3 Minutes \\
\hline 2010 & Margaret Scopes! & $\begin{array}{c}\text { movie: Choices of the Heart: The Margaret Sanger Story } \\
\text { role: Arnold Scopes }\end{array}$ \\
\hline 2011 & Espaa 1970 & movie: Compre usted en Espaa year: 1970 \\
\hline 2012 & sfarmata Atanasiu. & movie: Citadela sfarmata actor: Nicky Atanasiu \\
\hline 2013 & 4.4 Avventuriero & rank: 4.4 movie: Avventuriero \\
\hline 2014 & Blood 1934! & movie: Colonel Blood year: 1934 \\
\hline 2015 & $\begin{array}{c}\text { Dameshek } \\
\text { Mohammed? }\end{array}$ & $\begin{array}{c}\text { movie: B'Yom Bahir Ro'im et Dameshek actor: } \\
\text { Mohammed Bakri }\end{array}$ \\
\hline 2016 & Mars Barcroft? & movie: D-Day on Mars actor: Roy Barcroft \\
\hline 2017 & $\begin{array}{l}\text { Receiving the } \\
\text { Washington } 3.7\end{array}$ & $\begin{array}{l}\text { movie: Admiral Dewey Receiving the Washington and } \\
\text { New York Committees rank: } 3.7\end{array}$ \\
\hline 2018 & Annushka Ivanovich & movie: Annushka role: Ivan Ivanovich \\
\hline 2019 & 1964 Tannenbaum & year: 1964 movie: 9/64: O Tannenbaum \\
\hline 2020 & Colorado Kid Adams & movie: Colorado Kid actor: Ernie Adams \\
\hline 2021 & Bolondos Andriescu & movie: Bolondos vakci actor: Virgil Andriescu \\
\hline 2022 & Amante Fregois? & movie: Amante Latino actor: Roger Fregois \\
\hline 2023 & 1981 Bobine 2e partie & year: 1981 movie: Champions \\
\hline 2024 & Darkfeather Spider? & actor: Mona Darkfeather movie: Bottled Spider \\
\hline 2025 & 9.1 Bachelor & rank: 9.1 movie: Bachelor Tom Peeping \\
\hline 2026 & Adamson Cowboy & actor: Victor Adamson movie: Boss Cowboy \\
\hline 2027 & Samurai Bowen Cliff. & movie: Black Samurai actor: Cliff Bowen \\
\hline 2028 & Amato seconde! & actor: Nicolas Amato movie: Cent francs par seconde \\
\hline 2029 & Caballero $1954 !$ & movie: Caballero andaluz year: 1954 \\
\hline 2030 & 1976 Blizkaya! & year: 1976 movie: Blizkaya dal' \\
\hline 2031 & Clyde Bears & actor: Andy Clyde movie: Bulls and Bears \\
\hline 2032 & $\begin{array}{c}\text { Cover Girl } \\
\text { Abatemarco? }\end{array}$ & movie: Cover Girl and the Cop actor: Tony Abatemarco \\
\hline 2033 & 6.8 Mendoza? & rank: 6.8 movie: Compadre Mendoza \\
\hline 2034 & 1984 guerra! & year: 1984 movie: Chicos de la guerra \\
\hline 2035 & Wolves 6.5 & movie: Clash of the Wolves rank: 6.5 \\
\hline 2036 & Benjamin Win & actor: Paul Benjamin movie: Born to Win \\
\hline 2037 & 199129 th Street! & year: 1991 movie: 29th Street \\
\hline 2038 & Sebastin Rey. & movie: As es San Sebastin actor: Miguel Rey \\
\hline 2039 & 1915 Adventure. & year: 1915 movie: Adventure Hunter \\
\hline 2040 & Alcaraz Chucho. & actor: Eduardo Alcaraz movie: Captura de Chucho el Roto \\
\hline 2041 & 2001 Holden. & year: 2001 movie: Chasing Holden \\
\hline 2042 & 3:15 Achorn! & movie: $3: 15$ actor: John Achorn \\
\hline 2043 & Caminhos 1949. & movie: Caminhos do Sul year: 1949 \\
\hline 2044 & 5.8 disfrutarlo & rank: 5.8 movie: Cmo ser infeliz y disfrutarlo \\
\hline
\end{tabular}




\begin{tabular}{|c|c|c|}
\hline 2045 & 2004 Carrot? & year: 2004 movie: Carrot or the Stick \\
\hline 2046 & Adolscentes 1968 ! & movie: Adolescentes year: 1968 \\
\hline 2047 & Sweeties 4.1 & movie: Dancing Sweeties rank: 4.1 \\
\hline 2048 & Bleed Robert Simpson! & movie: Bleed role: Det. Robert Simpson \\
\hline 2049 & 1926 Kimono? & year: 1926 movie: Cow's Kimono \\
\hline 2050 & Romance 1934. & movie: Crimson Romance year: 1934 \\
\hline 2051 & Tommy Bogan Airmail. & role: Tommy Bogan movie: Airmail \\
\hline 2052 & Apo Bebek? & role: Apo movie: Bebek \\
\hline 2053 & Cargo 1999. & movie: Cargo year: 1999 \\
\hline 2054 & 1996 Bob Hope & $\begin{array}{l}\text { year: } 1996 \text { movie: Bob Hope: Laughing with the } \\
\text { Presidents }\end{array}$ \\
\hline 2055 & Bentinho Otrios! & role: Bentinho movie: Acabaram-se os Otrios \\
\hline 2056 & 1966 Winchester & year: 1966 movie: 3 colpi di Winchester per Ringo \\
\hline 2057 & Boutique 1995 & movie: Boutique del llanto year: 1995 \\
\hline 2058 & Bodine Yankovic & $\begin{array}{c}\text { role: Jethro Bodine movie: 'Weird Al' Yankovic: The } \\
\text { Videos }\end{array}$ \\
\hline 2059 & 1935 Scandal. & year: 1935 movie: College Scandal \\
\hline 2060 & Cobrador Segura! & $\begin{array}{c}\text { movie: Cobrador del gas slo llama una vez actor: Santiago } \\
\text { Segura }\end{array}$ \\
\hline 2061 & 2001 Texana & year: 2001 movie: Dama de la Texana 1000x \\
\hline 2062 & 612001 & movie: $61 *$ year: 2001 \\
\hline 2063 & Cash Dylan & $\begin{array}{c}\text { actor: Johnny Cash movie: Bob Dylan: 30th Anniversary } \\
\text { Concert Celebration }\end{array}$ \\
\hline 2064 & Asghar Lagne & actor: Ali Asghar movie: Aap Mujhe Achche Lagne Lage \\
\hline 2065 & Blood 1971 & movie: Beast of Blood year: 1971 \\
\hline 2066 & Botschaft Shatner & movie: Botschaft der Gtter actor: William Shatner \\
\hline 2067 & Beery Curing? & actor: Wallace Beery movie: Curing a Husband \\
\hline 2068 & Williams Genie & actor: Alex Williams movie: Bi Dream of Genie \\
\hline 2069 & 1987 Allnighter & year: 1987 movie: Allnighter \\
\hline 2070 & Afta 7.5 & movie: Afta rank: 7.5 \\
\hline 2071 & 1999 Sofies & year: 1999 movie: Bak Sofies verden \\
\hline 2072 & Ajibade Safari & actor: Yemi Ajibade movie: Black Safari \\
\hline 2073 & 7.5 Avventura & rank: 7.5 movie: Avventura \\
\hline 2074 & Acapulco 1983 & movie: Acapulco year: 1983 \\
\hline 2075 & Athos Crespi & movie: Athos actor: Emerval Crespi \\
\hline 2076 & Holder Crimes & actor: Philip Holder movie: Crimes of Fashion \\
\hline 2077 & Corbett Fight & actor: James J. Corbett movie: Corbett-Fitzsimmons Fight \\
\hline 2078 & Corrigan Town & actor: D'Arcy Corrigan movie: All Over Town \\
\hline 2079 & 4.7 Reef & rank: 4.7 movie: Beyond the Reef \\
\hline 2080 & 1991 Sheriff & year: 1991 movie: Acting Sheriff \\
\hline
\end{tabular}




\begin{tabular}{|c|c|c|}
\hline 2081 & Morris Bouquet & actor: J. Morris Foster movie: Bridal Bouquet \\
\hline 2082 & 2001 Milis & year: 2001 movie: Caca Milis \\
\hline 2083 & Adrei Saver & actor: Damir Andrei movie: Child Saver \\
\hline 2084 & Barro 1929 & movie: Barro Humano year: 1929 \\
\hline 2085 & Calendar 1992 & movie: Calendar Girl year: 1992 \\
\hline 2086 & Rajan Desh. & actor: Rajan Haksar movie: Apna Desh \\
\hline 2087 & Berserker 2001! & movie: Berserker year: 2001 \\
\hline 2088 & Gypsy Ernest Bango! & role: Gypsy actor: Ernest Bango \\
\hline 2089 & Chastity Latex & actor: Sweet Chastity movie: Bound in Latex \\
\hline 2090 & Aquest Fuentes & movie: Aquest Actor: Manel Fuentes \\
\hline 2091 & 1989 Bakayar & year: 1989 movie: Bakayar! 2: Shiawase ni naritai \\
\hline 2092 & Davidson Faithful & actor: William B. Davidson movie: Always Faithful \\
\hline 2093 & Buttafuoco Ever & actor: Joey Buttafuoco movie: Better Than Ever \\
\hline 2094 & 1963 Badem! & year: 1963 movie: Badem sekeri \\
\hline 2095 & Bomba Belasco & movie: Bomba and the Hidden City actor: Leon Belasco \\
\hline 2096 & Joel 1997 & movie: Billy Joel: Greatest Hits Volume III year: 1997 \\
\hline 2097 & Vrana Vignettes & actor: Vlasta Vrana movie: Canada Vignettes: The Visitor \\
\hline 2098 & Dalheim Heijden. & movie: Dalheim actor: Hein van der Heijden \\
\hline 2099 & 2002 Mythology! & year: 2002 movie: Age of Mythology \\
\hline 2100 & Broncho Pal & role: Broncho Billy movie: Broncho Billy's Pal \\
\hline 2101 & 2001 Condenado. & year: 2001 movie: Condenado Liberdade \\
\hline 2102 & Bavaria Narrator! & movie: Beautiful Bavaria role: Narrator \\
\hline 2103 & Barnes Angels & actor: Jonas Barnes movie: Charlie's Angels: Full Throttle \\
\hline 2104 & 1993 Bloodlines & year: 1993 movie: Bloodlines: Murder in the Family \\
\hline 2105 & Verrazzano 4.3 & movie: Commissario Verrazzano rank: 4.3 \\
\hline 2106 & Arnold Civility & actor: Tom Arnold movie: Civility \\
\hline 2107 & Cowboy Darro & movie: Cowboy Cop actor: Frankie Darro \\
\hline 2108 & 1991 Catatan & year: 1991 movie: Catatan si boy 5 \\
\hline 2109 & Awards Brar & $\begin{array}{l}\text { movie: } 2003 \text { Much Music Video Music Awards actor: } \\
\text { Jeevan Brar }\end{array}$ \\
\hline 2110 & Fellows Clinton & actor: Don Fellows movie: Clinton: His Struggle with Dirt \\
\hline 2111 & 1927 Busters & year: 1927 movie: Buster's Handicap \\
\hline 2112 & Cristo Delbo & movie: Comte de Monte Cristo actor: Jean-Jacques Delbo \\
\hline 2113 & 1996 Girls & year: 1996 movie: Career Girls \\
\hline 2114 & 2.3 Car 54 & rank: 2.3 movie: Car 54 \\
\hline 2115 & Benninger Hero? & actor: Otto Benninger movie: Almost a Hero \\
\hline 2116 & $\begin{array}{l}\text { Wednesday Willie } \\
\text { Nutter }\end{array}$ & movie: Any Given Wednesday role: Willie Nutter \\
\hline 2117 & 1945 Wives? & year: 1945 movie: Army Wives \\
\hline 2118 & Wife Sammy! & movie: Be My Wife actor: Sammy Brooks \\
\hline
\end{tabular}




\begin{tabular}{|c|c|c|}
\hline 2119 & Alvarado tema! & actor: Crox Alvarado movie: Cada loco con su tema \\
\hline 2120 & Abbott Edge & actor: Fredric Abbott movie: City's Edge \\
\hline 2121 & 8.4 Cordobs & rank: 8.4 movie: Cordobs \\
\hline 2122 & Billingslea Armed & actor: Beau Billingslea movie: Armed and Innocent \\
\hline 2123 & dames 4.3 & movie: Bru et ces dames rank: 4.3 \\
\hline 2124 & 1971 Lace & year: 1971 movie: Blood and Lace \\
\hline 2125 & Castle Mirror! & actor: Max Castle movie: Carol's Mirror \\
\hline 2126 & Louis 2000 & movie: A\&E Biography: Louis Lepke year: 2000 \\
\hline 2127 & Broadway 6.1 & movie: Broadway Bad rank: 6.1 \\
\hline 2128 & Americanos 1999! & movie: Americanos Concert year: 1999 \\
\hline 2129 & Brugger Continental! & actor: George Brugger movie: Continental Girl \\
\hline 2130 & 1990 sangre! & year: 1990 movie: Con el fuego en la sangre \\
\hline 2131 & Plata 1960. & movie: Bala de Plata year: 1960 \\
\hline 2132 & 2003 Big? & $\begin{array}{l}\text { year: } 2003 \text { movie: Big, Fat and Tacky: A Trip to } \\
\text { Karloffornia }\end{array}$ \\
\hline 2133 & Kevin Erogenous & actor: Kevin Conroy movie: Battle In the Erogenous Zone \\
\hline 2134 & 1983 Amada? & year: 1983 movie: Amada (1983/I) \\
\hline 2135 & Champa Champagne & actor: Jo Champa movie: Champagne Bound at Home \\
\hline 2136 & $\begin{array}{c}\text { Valentin } \\
\text { Antennendraht? }\end{array}$ & actor: Karl Valentin movie: Antennendraht \\
\hline 2137 & 1965 Avariya. & year: 1965 movie: Avariya \\
\hline 2138 & Chance 1917 & movie: Car of Chance year: 1917 \\
\hline 2139 & 2004 War. & year: 2004 movie: Actors in War \\
\hline 2140 & Columbo Braverman & $\begin{array}{l}\text { movie: Columbo: A Case of Immunity actor: Bart } \\
\text { Braverman }\end{array}$ \\
\hline 2141 & Bear 9.2! & movie: Bear Country rank: 9.2 \\
\hline 2142 & 1932 Mxico? & year: 1932 movie: Alma de Mxico \\
\hline 2143 & Eurovision 1968. & movie: Concours Eurovision year: 1968 \\
\hline 2144 & Lecocq Catherine! & role: Lecocq movie: Catherine the Great \\
\hline 2145 & Police 1990? & movie: AD Police Files year: 1990 \\
\hline 2146 & Chevalier toujours! & actor: Pierre Chevalier movie: Court toujours: L'inconnu \\
\hline 2147 & Boys Albano & movie: Boys Behind the Desk actor: Lou Albano \\
\hline 2148 & 7.3 Buffalo & rank: 7.3 movie: Brawl in Buffalo: UFC VII \\
\hline 2149 & Umanath Shravana & actor: Umanath Bhattacharya movie: Baishey Shravana \\
\hline 2150 & 1999 Palestine! & year: 1999 movie: Cyber Palestine \\
\hline 2151 & Selena Kurtis. & $\begin{array}{l}\text { movie: American Justice: Selena - Murder of a Star actor: } \\
\text { Bill Kurtis }\end{array}$ \\
\hline 2152 & $\begin{array}{l}\text { Reynolds Steve } \\
\text { Raiders. }\end{array}$ & role: Steve Reynolds movie: Cattle Raiders \\
\hline 2153 & 1993 Brothers. & year: 1993 movie: Best of the Blues Brothers \\
\hline 2154 & Hawaii 1983 & movie: Blomman frn Hawaii year: 1983 \\
\hline
\end{tabular}




\begin{tabular}{|c|c|c|}
\hline 2155 & Revol brumes! & actor: Jean-Luc Revol movie: Chant des brumes \\
\hline 2156 & Marriage Ballard & movie: Consolation Marriage actor: Elmer Ballard \\
\hline 2157 & 1971 Waiting & year: 1971 movie: Columbo: Lady in Waiting \\
\hline 2158 & Hench Conversation. & actor: John Hench movie: Conversation with John Hench \\
\hline 2159 & mortels Arnault & movie: Coma des mortels actor: Yves Arnault \\
\hline 2160 & 1976 Andariego! & year: 1976 movie: Andariego \\
\hline 2161 & 1988 destino! & year: 1988 movie: Cita con el destino \\
\hline 2162 & Bas Betrayer? & actor: Philippe Bas movie: Betrayer \\
\hline 2163 & 2003 Eileen. & year: 2003 movie: 12 Stories About Eileen \\
\hline 2164 & Conlon Days. & actor: Chris Conlon movie: 4 More Days \\
\hline 2165 & Beauty Bakula! & $\begin{array}{c}\text { movie: American Beauty: Look Closer... actor: Scott } \\
\text { Bakula }\end{array}$ \\
\hline 2166 & Awful 6.4 & movie: Awful Normal rank: 6.4 \\
\hline 2167 & Panic Call! & $\begin{array}{l}\text { movie: Baywatch: Panic at Malibu Pier actor: Brandon } \\
\text { Call }\end{array}$ \\
\hline 2168 & Hubler pique? & actor: Franz Hubler movie: Dame de pique \\
\hline 2169 & 1975 Chagiga. & year: 1975 movie: Chagiga L'Enayim \\
\hline 2170 & Brotherhood 1971 & movie: Brotherhood of Satan year: 1971 \\
\hline 2171 & Armstrong 3.8 & movie: Armstrong rank: 3.8 \\
\hline 2172 & Cuteys Flora. & movie: Cutey's Vacation actor: Flora Finch \\
\hline 2173 & 1962 Thieves? & year: 1962 movie: Band of Thieves \\
\hline 2174 & 5.4 Chappan? & rank: 5.4 movie: Ab Tak Chappan \\
\hline 2175 & Barton Crayoluv & actor: Daniel Barton movie: Crayoluv \\
\hline 2176 & Roland Weekend & role: Dr. Roland movie: 300 Year Weekend \\
\hline 2177 & 1939 Crime! & year: 1939 movie: Crime Rave \\
\hline 2178 & Washington Blood! & actor: Isaiah Washington movie: Blood on a Happy Face \\
\hline 2179 & Claudia 6.6. & movie: Claudia and David rank: 6.6 \\
\hline 2180 & Death 1984 ! & movie: Courier of Death year: 1984 \\
\hline 2181 & Big Batie! & movie: Big Boy actor: Franklin Batie \\
\hline 2182 & 1991 Heat. & year: 1991 movie: Country Girls in Heat \\
\hline 2183 & Albright Smith. & actor: Wally Albright movie: Case of Lena Smith \\
\hline 2184 & Tonic Leif. & movie: Air Tonic actor: Leif Erickson \\
\hline 2185 & 6.2 Blondies. & rank: 6.2 movie: Blondie's Blessed Event \\
\hline 2186 & $1998 \mathrm{G}$ & year: 1998 movie: $(\mathrm{G})$ \\
\hline 2187 & Luis Barraqueta & actor: Luis Bori movie: Barraqueta del Nano \\
\hline 2188 & Christians 1920. & movie: Burnt Wings year: 1920 \\
\hline 2189 & Alejar Amang? & $\begin{array}{c}\text { actor: Toby Alejar movie: Amang Capulong - Anak ng } \\
\text { Tondo II }\end{array}$ \\
\hline 2190 & 4.9 Cappuccino. & rank: 4.9 movie: Cappuccino (1989/I) \\
\hline 2191 & 1921 Corey. & year: 1921 actor: Jim Corey \\
\hline
\end{tabular}




\begin{tabular}{|c|c|c|}
\hline 2192 & Screenwriting 1964? & movie: Child's Guide to Screenwriting year: 1964 \\
\hline 2193 & Bharadwaj Amaanat! & actor: Bhram Bharadwaj movie: Amaanat \\
\hline 2194 & 1985 Friday? & year: 1985 movie: Came a Hot Friday \\
\hline 2195 & Cornelius Beauty. & actor: Cornelius Booth movie: Beauty \\
\hline 2196 & Brevity 1938 & movie: Broadway Brevity: Zero Girl year: 1938 \\
\hline 2197 & Bergkatze 7.1 & movie: Bergkatze rank: 7.1 \\
\hline 2198 & 1942 limn! & year: 1942 movie: A la lima y al limn \\
\hline 2199 & Casanova Zirkusluft! & actor: Tristano Casanova movie: Bulle von Tlz - Zirkusluft \\
\hline 2200 & BelgiTurkije Achmed. & movie: Belgi-Turkije actor: Achmed Oussihmin \\
\hline 2201 & 6.2 American & rank: 6.2 movie: American Tragedy \\
\hline 2202 & Records 1927 & movie: Breaking Records year: 1927 \\
\hline 2203 & Jackson Corner & actor: Maxwell Jackson movie: Dangerous Corner \\
\hline 2204 & Belle Francis! & movie: Belle verte actor: Francis Bouc \\
\hline 2205 & 1990 Sawyer. & $\begin{array}{c}\text { year: } 1990 \text { movie: Back to Hannibal: The Return of Tom } \\
\text { Sawyer and Huckleberry Finn }\end{array}$ \\
\hline 2206 & Contranatura Ren! & movie: Contranatura role: Ren \\
\hline 2207 & 2.1 Challenge? & rank: 2.1 movie: Challenge \\
\hline 2208 & Ladyfingers Elliott. & movie: Alias Ladyfingers actor: Frank Elliott \\
\hline 2209 & $\begin{array}{c}\text { Bellantoni } \\
\text { Circumstances. }\end{array}$ & actor: Natino Bellantoni movie: Circumstances Unknown \\
\hline 2210 & Inexpression Quentin. & $\begin{array}{l}\text { movie: Anxiety of Inexpression and the Otherness } \\
\text { Machine actor: Quentin Lee }\end{array}$ \\
\hline 2211 & neke 7.2 & movie: Bolondok neke rank: 7.2 \\
\hline 2212 & 5.8 poliziotto? & rank: 5.8 movie: Bambino e il poliziotto \\
\hline 2213 & Lee Light & actor: Duke R. Lee movie: Canyon of Light \\
\hline 2214 & Keyhole 1933. & movie: Broadway Through a Keyhole year: 1933 \\
\hline 2215 & 1997 Death & year: 1997 movie: Cause of Death \\
\hline 2216 & Altar 1985 & movie: Altar year: 1985 \\
\hline 2217 & Abbott Boxing? & actor: David (VI) Abbott movie: Celebrity Boxing \\
\hline 2218 & Mike Lover & role: Mike movie: Alien Lover \\
\hline 2219 & bombarder Nebojsa & movie: Crni bombarder actor: Nebojsa Bakocevic \\
\hline 2220 & Alexandre Chica & actor: Manuel Alexandre movie: Chica y un seor \\
\hline 2221 & Ayrilik $1972 ?$ & movie: Ayrilik year: 1972 \\
\hline 2222 & Mohammed Sky & actor: Ahmed Ben Mohammed movie: Another Sky \\
\hline 2223 & 1971 geondaldeul. & year: 1971 movie: 5inui geondaldeul \\
\hline 2224 & Abomination EBB? & movie: Abomination: Evil Maker 2 actor: Warren E.B.B. \\
\hline 2225 & $20025 !$ & year: 2002 movie: 5 \\
\hline 2226 & 3.8 cinta. & rank: 3.8 movie: 30 hari mencari cinta \\
\hline 2227 & 2000 Resistance! & year: 2000 movie: C-12: Final Resistance \\
\hline 2228 & Arbeit Luna & movie: Alle fr Arbeit actor: Tim Luna \\
\hline
\end{tabular}




\begin{tabular}{|c|c|c|}
\hline 2229 & Abbott Frankenstein? & $\begin{array}{c}\text { actor: Bud Abbott movie: Bud Abbott Lou Costello Meet } \\
\text { Frankenstein }\end{array}$ \\
\hline 2230 & Cheolbuji 1985. & movie: Cheolbuji year: 1985 \\
\hline 2231 & 1984 Bounty & year: 1984 movie: Bounty \\
\hline 2232 & Backyard Ackroyd & movie: Backyard actor: Jack Ackroyd \\
\hline 2233 & 2003 Cinta? & year: 2003 movie: Cinta 24 karat \\
\hline 2234 & Balarama Babu & movie: Balarama Krishnulu actor: Jagapathi Babu \\
\hline 2235 & Barlow Born! & actor: Jeff Barlow movie: As He Was Born \\
\hline 2236 & Bis Adolf & movie: Bis fnf nach zwlf actor: Adolf Hitler \\
\hline 2237 & 1910 camlias? & year: 1910 movie: Dame aux camlias \\
\hline 2238 & Babykram Elvis? & movie: Babykram ist Mnnersache actor: Elvis Grabbe \\
\hline 2239 & 1970 guardarla. & year: 1970 movie: Basta guardarla \\
\hline 2240 & Atacadas Albizu? & movie: Atacadas actor: Fernando Albizu \\
\hline 2241 & 1988 Superclash! & year: 1988 movie: AWA Superclash III \\
\hline 2242 & Benben Comfort? & actor: Brian Benben movie: Comfort \\
\hline 2243 & Buffalo 1961 & movie: Buffalo Gun year: 1961 \\
\hline 2244 & 1991 Asalto! & year: 1991 movie: Asalto \\
\hline 2245 & Arcencl 2000? & movie: Arc-en-CL year: 2000 \\
\hline 2246 & Yeonggyun Agineun? & actor: Yeong-gyun Shin movie: Agineun eobda \\
\hline 2247 & 7.3 Backroads? & rank: 7.3 movie: Backroads \\
\hline 2248 & Alzaga limpio & actor: Jorge Alzaga movie: A tiro limpio \\
\hline 2249 & Cruel Warwick? & movie: Cruel Sea actor: Warwick Ashton \\
\hline 2250 & 1.9 Comeback & rank: 1.9 movie: Comeback \\
\hline 2251 & Orange 2003 & movie: Christmas Orange year: 2003 \\
\hline 2252 & 2002 Break. & year: 2002 movie: Break \\
\hline 2253 & Beer Janssen. & movie: Beer actor: Marc Janssen \\
\hline 2254 & Chenault Crimson? & actor: Lawrence Chenault movie: Crimson Skull \\
\hline 2255 & BAFTA Caine & $\begin{array}{l}\text { movie: BBC and the BAFTA Tribute to Michael Caine } \\
\text { actor: Michael Caine }\end{array}$ \\
\hline 2256 & Calzavara Carmela! & actor: Flavio Calzavara movie: Carmela \\
\hline 2257 & 1939 Serenade! & year: 1939 movie: Broadway Serenade \\
\hline 2258 & Angel 7.2! & movie: Black Angel rank: 7.2 \\
\hline 2259 & Edge Miller & movie: Beyond the Edge actor: Warren Miller \\
\hline 2260 & 7.2 Bombay? & rank: 7.2 movie: Bombay \\
\hline 2261 & Appointment $4.7 !$ & movie: Appointment rank: 4.7 \\
\hline 2262 & 1969 Boskovic? & year: 1969 actor: Bogic Boskovic \\
\hline 2263 & Shane Death! & actor: Shane Aaron movie: 18 Fingers of Death! \\
\hline 2264 & meilleur 1999 & movie: C'est bien meilleur le dimanche year: 1999 \\
\hline 2265 & 1994 Wer Gewalt? & year: 1994 movie: Alles auer Mord - Wer Gewalt st \\
\hline 2266 & Cuneo Love? & actor: Lester Cuneo movie: Between Love and the Law \\
\hline
\end{tabular}




\begin{tabular}{|c|c|c|}
\hline 2267 & 5.6 Castagne! & rank: 5.6 movie: Castagne sono buone \\
\hline 2268 & Avvoltoio 1991! & movie: Avvoltoio pu attendere year: 1991 \\
\hline 2269 & 1932 Coffret? & year: 1932 movie: Coffret de laque \\
\hline 2270 & Cream 1999 & movie: Cream of Comedy year: 1999 \\
\hline 2271 & Bridges Climb! & actor: Todd Bridges movie: Climb \\
\hline 2272 & 4.8 Chastnyj? & rank: 4.8 movie: Chastnyj detektiv \\
\hline 2273 & Canvas 1.9. & movie: 5 Dead on the Crimson Canvas rank: 1.9 \\
\hline 2274 & Africa 4.7 & movie: Africa Express rank: 4.7 \\
\hline 2275 & Bates Doll! & actor: Les Bates movie: Broken Doll \\
\hline 2276 & 1975 Coonskin & year: 1975 movie: Coonskin \\
\hline 2277 & Boyfriend 1999. & movie: Boyfriend kong pari, Ang year: 1999 \\
\hline 2278 & Terzieff Araya. & actor: Laurent Terzieff movie: Araya \\
\hline 2279 & Aldea $1942 !$ & movie: Aldea maldita year: 1942 \\
\hline 2280 & 5.4 heonbeob & rank: 5.4 movie: Daehanminguk heonbeob je 1 jo \\
\hline 2281 & Duarte Chillona! & actor: Jose Duarte movie: Chillona \\
\hline 2282 & 2003 Animals! & year: 2003 movie: Animals \\
\hline 2283 & Byeongcheol pungseon & actor: Byeong-cheol Choi movie: Dallyeola pungseon \\
\hline 2284 & daidardo 8.7 & movie: Ar daidardo rank: 8.7 \\
\hline 2285 & Bejshenaliyev Cherez! & actor: Bolot Bejshenaliyev movie: Cherez Gobi i Khingan \\
\hline 2286 & 1989 tanj! & $\begin{array}{c}\text { year: } 1989 \text { movie: Chjin densetsu Urotsukidji 1: Chjin tanj } \\
\text { hen }\end{array}$ \\
\hline 2287 & Cuando 1933 & movie: Cuando el amor re year: 1933 \\
\hline 2288 & Belasi 1976? & movie: Bas belasi year: 1976 \\
\hline 2289 & 4.5 Cat & rank: 4.5 movie: Cat Girl \\
\hline 2290 & Ambroses 1919 & movie: Ambrose's Day Off year: 1919 \\
\hline 2291 & Barkoulis Alexandros. & actor: Andreas Barkoulis movie: Alexandros kai Aiche \\
\hline 2292 & Ciel 6.7 & movie: Ciel est vous, Le rank: 6.7 \\
\hline 2293 & Buza 1989 & movie: Buza year: 1989 \\
\hline 2294 & 1961 Vengeance! & year: 1961 movie: Cry Vengeance! \\
\hline 2295 & Grammy 1979 & movie: 21st Annual Grammy Awards year: 1979 \\
\hline 2296 & Contact Adnurn & movie: Contact (2002/III) actor: Glenn Adnurn \\
\hline 2297 & Sergio Pain. & role: Sergio movie: City of Pain \\
\hline 2298 & 5.9 Boys III? & rank: 5.9 movie: Boys III, Les \\
\hline 2299 & Akasya 1940 & movie: Akasya palas year: 1940 \\
\hline 2300 & Gaylord Lit. & actor: Gaylord Lloyd movie: All Lit Up \\
\hline 2301 & 1915 Shackles. & year: 1915 movie: Breaking the Shackles \\
\hline 2302 & Apacible Check inn & actor: John Apacible movie: Check-Inn \\
\hline 2304 & Concours Roland? & movie: Concours eurovision actor: Jo Roland \\
\hline 2305 & 4.3 Champion & rank: 4.3 movie: Carman: The Champion \\
\hline 2306 & 2 plus 1 Doran & movie: $2+1$ actor: Harris Doran \\
\hline
\end{tabular}




\begin{tabular}{|c|c|c|}
\hline 2307 & Abero Tragedy. & actor: Seth C. Abero movie: American Tragedy \\
\hline 2308 & Autopsia Electron! & movie: Autopsia de un fantasma role: Electron \\
\hline 2309 & Aquarians Casey! & movie: Aquarians actor: Lawrence P. Casey \\
\hline 2310 & Brown North & actor: Milton Brown movie: Call of the North \\
\hline 2311 & 1934 Arizona! & year: 1934 movie: Arizona Cyclone \\
\hline 2312 & 7.3 Card & rank: 7.3 movie: Card \\
\hline 2313 & Adelin Chlo. & actor: Jean-Claude Adelin movie: Chlo \\
\hline 2314 & 2002 Bloody. & year: 2002 movie: Bloody Roar Extreme \\
\hline 2315 & FortyNiner 2.7 & movie: Curse of the Forty-Niner rank: 2.7 \\
\hline 2316 & Alvaro lalagot. & actor: Dan Alvaro movie: Ako ang lalagot sa hininga mo \\
\hline 2317 & Funt No $4 ?$ & actor: Allen Funt movie: Candid Microphone: Series 4 \\
\hline 2318 & Croisade 1995? & movie: Croisade d'Anne Buridan year: 1995 \\
\hline 2319 & 1962 alan & year: 1962 movie: Ati alan skdar'i geti \\
\hline 2320 & Girard Shanghai! & actor: Joseph W. Girard movie: Back from Shanghai \\
\hline 2321 & rapadas Gustavo! & movie: Cabezas rapadas actor: Gustavo Aguilar \\
\hline 2322 & Bitterzoet 2000? & movie: Bitterzoet year: 2000 \\
\hline 2323 & 1917 Argyle! & year: 1917 movie: Argyle Case \\
\hline 2324 & Isamu 725 ! & actor: Isamu Hyuga movie: 7/25 Nana-Ni-Go \\
\hline 2325 & Alibaba Bose! & movie: Alibaba actor: Madhu Bose \\
\hline 2326 & Cummings Beasts! & actor: Richard Cummings movie: Beast Within \\
\hline 2327 & Cern 1988 & movie: Cern puncocha year: 1988 \\
\hline 2328 & 1963 Criminale & year: 1963 movie: Criminale \\
\hline 2329 & Cantor 1940. & movie: Cantor del circo year: 1940 \\
\hline 2330 & 3.1 Carambola? & rank: 3.1 movie: Carambola \\
\hline 2331 & 1970 scaten & year: 1970 movie: Arizona si scaten... e li fece fuori tutti \\
\hline 2332 & Bew Alien & actor: Kieran Bew movie: AVP: Alien Vs. Predator \\
\hline 2333 & Pentjak Tracker! & role: Pentjak \#2 movie: Bounty Tracker \\
\hline 2334 & Beery Bavu? & actor: Wallace Beery movie: Bavu \\
\hline 2335 & 1959 ariyorum & year: 1959 movie: Annemi ariyorum \\
\hline 2336 & Betimi 1953! & $\begin{array}{l}\text { movie: Betimi i popullit shqiptar para Stalinit t madh year: } \\
\qquad 1953\end{array}$ \\
\hline 2337 & Birthday 8 ! & movie: Birthday Blues rank: 8 \\
\hline 2338 & 6.3 Bonjour. & rank: 6.3 movie: Bonjour l'angoisse \\
\hline 2339 & Murray Parade. & $\begin{array}{c}\text { actor: Murray Gendzel movie: Community Sing: The } \\
\text { Parade of Hits }\end{array}$ \\
\hline 2340 & Bandit Back. & role: Bandit \#2 movie: Back to Back \\
\hline 2341 & Kamen Burnett. & actor: Milt Kamen movie: Carol Burnett Show \\
\hline 2342 & 1942 Caballera? & year: 1942 movie: Caballera del imperio \\
\hline 2343 & Chase Chump? & actor: Charley Chase movie: Chump Takes a Bump \\
\hline 2344 & Lantern 6.8 ! & movie: Aladdin's Lantern rank: 6.8 \\
\hline
\end{tabular}




\begin{tabular}{|c|c|c|}
\hline 2345 & Culte 1931 & movie: Culte de beaut year: 1931 \\
\hline 2346 & 1989 Lepetic & $\begin{array}{l}\text { year: } 1989 \text { movie: Covjek koji je znao gdje je sjever, a gdje } \\
\text { jug }\end{array}$ \\
\hline 2347 & Airman's Payne & movie: Airman's Children actor: Douglas Payne \\
\hline 2348 & 1996 Burlesxxx. & year: 1996 movie: Burlesxxx \\
\hline 2349 & Bologna Danger! & $\begin{array}{c}\text { actor: Joseph Bologna movie: Danger of Love: The } \\
\text { Carolyn Warmus Story }\end{array}$ \\
\hline 2350 & Freddie Clown & actor: Freddie Chapman movie: Clown Princes \\
\hline 2351 & Blazeland 1992 & movie: Blazeland year: 1992 \\
\hline 2352 & Ferniot clinique! & actor: Vincent Ferniot movie: Cas clinique \\
\hline 2353 & 2004 Annabelle. & year: 2004 movie: Annabelle \\
\hline 2354 & Gironella 1965. & movie: Alberto Gironella year: 1965 \\
\hline 2355 & 4.3 Big! & rank: 4.3 movie: Big Day \\
\hline 2356 & 1988 Crackdown? & year: 1988 movie: Crackdown (1988/I) \\
\hline 2357 & Bubble Gaillard. & movie: Broadway Bubble actor: Robert Gaillard \\
\hline 2358 & Amercian 1985 & movie: American Carrott year: 1985 \\
\hline 2359 & 7.9 Csar & rank: 7.9 movie: Csar \\
\hline 2360 & Dirt Beyond. & role: Dirt movie: Beyond the Law \\
\hline 2361 & Kerrigan Another. & actor: J. Warren Kerrigan movie: Another Man's Wife \\
\hline 2362 & Curiosity $1999 ?$ & movie: Curiosity \& the Cat year: 1999 \\
\hline 2363 & 1956 Aan & year: 1956 movie: Aan Baan \\
\hline 2364 & Blanco salvaje! & actor: Victorio Blanco movie: Amor salvaje \\
\hline 2365 & 1970 Body? & year: 1970 movie: Body \\
\hline 2366 & $2.8 \mathrm{Bug} !$ & rank: 2.8 movie: Bug Off! \\
\hline 2367 & Astaire 1959 & movie: Another Evening with Fred Astaire year: 1959 \\
\hline 2368 & Nancie pujaan. & actor: Nancie Foo movie: Bintang pujaan \\
\hline 2369 & Bombalera 1945 ! & movie: Bombalera year: 1945 \\
\hline 2370 & Accidents 4.7 & movie: Accidents rank: 4.7 \\
\hline 2371 & 1938 Boy & year: 1938 movie: Boy Meets Girl \\
\hline 2372 & Craig Conan. & actor: Andrew Craig movie: Conan: The Adventurer \\
\hline 2373 & Prudence 1920. & movie: Away Goes Prudence year: 1920 \\
\hline 2374 & 1926 Bindle. & year: 1926 movie: Bindle in Charge \\
\hline 2375 & Born Jaeckel! & movie: Born Innocent actor: Richard Jaeckel \\
\hline 2376 & 5.9 Crime & rank: 5.9 movie: Crime et chtiment \\
\hline 2377 & Amrish Chhatrabhang & actor: Amrish Puri movie: Chhatrabhang \\
\hline 2378 & Dandin Adelin! & movie: Dandin actor: Jean-Claude Adelin \\
\hline 2379 & Aas 22 & actor: Knut Aas movie: 22 \\
\hline 2380 & Dalle Dreams? & actor: Peter Dalle movie: Bombay Dreams \\
\hline 2381 & 1939 Abuna & year: 1939 movie: Abuna Messias \\
\hline 2382 & Clark Heart. & actor: Frank Clark movie: Craven Heart \\
\hline
\end{tabular}




\begin{tabular}{|c|c|c|}
\hline 2383 & Jack Victor? & movie: Adventures of Texas Jack actor: Victor Adamson \\
\hline 2384 & Baer CIA? & actor: Robert Baer movie: CIA: Guerres secrtes \\
\hline 2385 & 1956 Amor. & year: 1956 movie: Amor a primera vista \\
\hline 2386 & Enrique Chiquilines! & actor: Enrique Alonso movie: Chiquilines \\
\hline 2387 & Bataillard Flame & actor: Stephan Bataillard movie: Blue Flame \\
\hline 2388 & 1954 Command. & year: 1954 movie: Command \\
\hline 2389 & Censor $6.9 ?$ & movie: Censor rank: 6.9 \\
\hline 2390 & 1999 Apberget. & year: 1999 movie: Apberget \\
\hline 2391 & Zeugin Mario. & movie: Anwalt Abel - Ihre Zeugin actor: Mario Andersen \\
\hline 2392 & Angels Drew? & movie: Angels actor: Drew Abrams \\
\hline 2393 & Claudio 5.2 & movie: Cos come sei rank: 5.2 \\
\hline 2394 & 1936 Stevens. & year: 1936 movie: Crown Vs. Stevens \\
\hline 2395 & Evening 4.9 & movie: Color of Evening rank: 4.9 \\
\hline 2396 & Albert 1985. & role: Albert Herring year: 1985 \\
\hline 2397 & 2000 Argentina! & year: 2000 movie: Asociacin Argentina de actores \\
\hline 2398 & Huntley Brother? & actor: Huntley Gordon movie: Brother of the Bear \\
\hline 2399 & Blues 1987. & movie: Big Ape Blues year: 1987 \\
\hline 2400 & Zhang shuang. & actor: Xiaoqing Zhang movie: Bi shui shuang hun \\
\hline 2401 & Bagar stena! & actor: Andrej Bagar movie: Certova stena \\
\hline 2402 & 1927 Brunettes & year: 1927 movie: Are Brunettes Safe? \\
\hline 2403 & Bashung 1987! & actor: Alain Bashung year: 1987 \\
\hline 2404 & Simon $5.7 ?$ & actor: Simon Brooke rank: 5.7 \\
\hline 2405 & Salvador Philip 1988 & actor: Philip Salvador year: 1988 \\
\hline 2406 & 1982 Baawri! & movie: Baawri year: 1982 \\
\hline 2407 & Augustus Balfour1912! & actor: Augustus Balfour year: 1912 \\
\hline 2408 & Cuneo Lester 1914 & actor: Lester Cuneo year: 1914 \\
\hline 2409 & Cicatrices 2001 & movie: Cicatrices year: 2001 \\
\hline 2410 & 1912 West & movie: Caleb West year: 1912 \\
\hline 2411 & amber paris 2 & movie: Amber \& Sharon Do Paris 2 \\
\hline 2412 & 1999 Appointment! & year: 1999 movie: Appointment \\
\hline 2413 & 1973 agamemmon & year: 1973 movie: Agamemnon \\
\hline 2414 & carabina Flavio Bucci? & actor: Flavio Bucci movie: Carabina \\
\hline 2415 & fence Circular? & movie: Circular Fence \\
\hline 2416 & Bluff Balmer! & movie: Bluff actor: Jean-François Balmer \\
\hline 2417 & 1965 Mariano. & actor: Mariano Azaña year: 1965 \\
\hline 2418 & $\begin{array}{c}\text { Conspiracy Alcaldes } \\
1912 ?\end{array}$ & movie: Alcalde's Conspiracy year: 1912 \\
\hline 2419 & George Seeger! & actor: Götz George role: Hannes Seeger \\
\hline 2420 & $\begin{array}{l}\text { Romance movies of } \\
1998\end{array}$ & Genre: Romance year: 1998 \\
\hline
\end{tabular}




\begin{tabular}{|c|c|c|}
\hline 2421 & Mt. Ariat $1900 ?$ & movie: Battle of Mt. Ariat year: 1900 \\
\hline 2422 & Round Table Corbett & movie: At the Round Table actor: James J. Corbett \\
\hline 2423 & Coors Biography & movie: A\&E Biography: The Coors - A Family Brew \\
\hline 2424 & Cmicos Mais 1971 & movie: Cmicos e Mais Cmicos year: 1971 \\
\hline 2425 & andrej 1990 & actor: Andrej Scharonow year: 1990 \\
\hline 2426 & Behar Kip 1963. & year: 1963 actor: Kip Behar \\
\hline 2427 & cylinder 1912 & movie: Cylinder's Secret year: 1912 \\
\hline 2428 & Ave 2000 & movie: Ave Brank year: 2000 \\
\hline 2429 & Bestiario Carlos! & movie: Bestiario actor: Carlos Bardem \\
\hline 2430 & Eigoro Hichry shinku? & $\begin{array}{l}\text { actor: Eigoro Onoe movie: Akad Suzunosuke: Hichry } \\
\text { shinku giri }\end{array}$ \\
\hline 2431 & Alles Mord & movie: Alles auer Mord - Marion Nr. 5 \\
\hline 2432 & 1963 Law Wife & movie: Common Law Wife year: 1963 \\
\hline 2433 & bellas noche & movie: Bellas de noche \\
\hline 2434 & Prison Guard 1991? & role: Prison Guard year: 1991 \\
\hline 2435 & Almada 1999 & actor: Mário Almada year: 1999 \\
\hline 2436 & Bosko's Fountains & movie: Bosko's Soda Fountain \\
\hline 2437 & Cinders 1913 & movie: Cinders year: 1913 \\
\hline 2438 & 2000 Butler & year: 2000 actor: Henry Butler \\
\hline 2439 & crisantem branco & movie: Crisantemi per un branco di carogne \\
\hline 2440 & moive act necessity & movie: Act of Necessity \\
\hline 2441 & Campbell 64! & actor: John Lyle Campbell rank: 6.4 \\
\hline 2442 & asesino of $1966 ?$ & movie: Chantaje a un asesino year: 1966 \\
\hline 2443 & pricne barnes & actor: Justus D. Barnes movie: Arabella Prince \\
\hline 2444 & childern Nesbitt & movie: City of Children actor: John Nesbitt \\
\hline 2445 & $3.41997 !$ & rank: 3.4 year: 1997 \\
\hline 2446 & crips in 1913 & actor: Donald Crisp year: 1913 \\
\hline 2447 & count of ten & movie: Count of Ten \\
\hline 2448 & 5.2 rating German. & rank: 5.2 role: German Doctor \\
\hline 2449 & 1938 Tommy Devlin & year: 1938 role: Tommy Devlin \\
\hline 2450 & 1982 Misbehavin' & year: 1982 movie: Ain't Misbehavin' \\
\hline 2451 & Sheehan 1916 & year: 1916 actor: John Sheehan \\
\hline 2452 & thriller 1973 & genre: Thriller year: 1973 \\
\hline 2453 & John Cassavetes 7.3? & actor: John Cassavetes rating 7.3 \\
\hline 2454 & Arauz 1985 & actor: Ricardo Arauz year: 1985 \\
\hline 2455 & chasing ones 16 & movie: Chasing the Big Ones 16 \\
\hline 2456 & 7.3 Blystone. & rank: 7.3 actor: Stanley Blystone \\
\hline 2457 & $\begin{array}{c}\text { broadway with } 7.4 \\
\text { rating }\end{array}$ & movie: Bullets Over Broadway rank: 7.4 \\
\hline 2458 & Arias and Joy & actor: José Arias movie: Comfort and Joy \\
\hline
\end{tabular}




\begin{tabular}{|c|c|c|}
\hline 2459 & Cash Cash 1967 & movie: Cash? Cash! year: 1967 \\
\hline 2460 & 1914 Jones & year: 1914 actor: Edgar Jones \\
\hline 2461 & 5.2 Misael & rank: 5.2 role: Misael \\
\hline 2462 & salvador 1963 & actor: Lou Salvador year: 1962 \\
\hline 2463 & 1954 Basil Appleby. & year: 1954 actor: Basil Appleby \\
\hline 2464 & Delchambre 1998 & actor: Laurent Delchambre year: 1998 \\
\hline 2465 & 1980 Daniel Young! & year: 1980 role: Daniel Young \\
\hline 2466 & 1921 bourdelle movie? & year: 1921 actor: Thomy Bourdelle \\
\hline 2467 & childern eve movie? & movie: Children of Eve \\
\hline 2468 & 1923 Bad man? & year: 1923 movie: Bad Man \\
\hline 2469 & $\begin{array}{c}\text { drinving confessions } \\
\text { rating } 3.5 \\
\end{array}$ & movie: Confessions of a Driving Instructor rank: 3.5 \\
\hline 2470 & Girl Meets boys! & movie: Boy Meets Girl \\
\hline 2471 & 1971 carpenters & movie: Carpenters 1971 BBC Television Special \\
\hline 2472 & Mark crossing Floor & actor: Mark Benton movie: Crossing the Floor \\
\hline 2473 & Archangel Worshipper & movie: Archangel role: Worshipper \\
\hline 2474 & 1987 Binart! & year: 1987 movie: Binart \\
\hline 2475 & Boinng Aberwood & movie: Boin-n-g actor: Lawrence J. Aberwood \\
\hline 2476 & Hart in 1915 & year: 1915 actor: William S. Hart \\
\hline 2477 & Cathy is special? & movie: Cathy Jones Gets a Special \\
\hline 2478 & creature in $2004 !$ & role: Creature year: 2004 \\
\hline 2479 & 1914 Combs & year: 1914 Actor: Guy Coombs \\
\hline 2480 & $\begin{array}{c}\text { Alex Aitchison rating } \\
6.3 \text {. }\end{array}$ & actor: Alex Aitchison rank: 6.3 \\
\hline 2481 & 1938 Samuel & year: 1938 role: Samuel Coleridge \\
\hline 2482 & cheaters situations & movie: Cheaters: Sticky Situations \\
\hline 2483 & Neto Contos & movie: Contos de Balnerio actor: Moisés Neto \\
\hline 2484 & Audrey Hepburn & movie: Audrey Hepburn: In her Own Words \\
\hline 2485 & sean 1992. & movie: Aah Sean year: 1992 \\
\hline 2486 & Bill Leslie! & role: Bill actor: Leslie Bradley \\
\hline 2487 & Hostess Hall & movie: Dance Hall Hostess \\
\hline 2488 & Carmen 1918 & movie: Carmen year: 1918 \\
\hline 2489 & $\begin{array}{c}\text { Franco Acampora } \\
\text { Avanti }\end{array}$ & actor: Franco Acampora movie: Avanti! \\
\hline 2490 & 3 klaphoeden? & movie: 3 klaphoeden \\
\hline 2491 & bullock 2003 & year: 2003 actor: S. Scott Bullock \\
\hline 2492 & Inamdar 1986? & year: 1986 actor: Shafi Inamdar \\
\hline 2493 & Ernesto in 1995 & year: 1995 actor: Ernesto Alterio \\
\hline 2494 & Costello 2000 & actor: Frank Costello year: 2000 \\
\hline 2495 & attiki & movie: attiki \\
\hline
\end{tabular}




\begin{tabular}{|c|c|c|}
\hline 2496 & 1914 Hurst & year: 1914 actor: Paul Hurst \\
\hline 2497 & 1921 Concert & year: 1921 movie: Concert \\
\hline 2498 & $\begin{array}{c}\text { Brennan in Black } \\
\text { Butterfly! }\end{array}$ & actor: Edward Brennan movie: Black Butterfly \\
\hline 2499 & Heinie and 1916. & year: 1916 role: Heinie \\
\hline 2500 & 1964 blood! & year: 1964 actor: William Blood \\
\hline 2501 & rose documentary & actor: Norman Rose genre: Documentary \\
\hline 2502 & Concours eurovision & movie: Concours eurovision \\
\hline 2503 & 1915 and Donaldson & actor: Arthur Donaldson year: 1915 \\
\hline 2504 & 2000 Kennedy & year: 2000 actor: John F. Kennedy \\
\hline 2505 & 1982 Zazerkalye. & year: 1982 movie: Alisa v Zazerkalye \\
\hline 2506 & 1925 Hardy & year: 1925 actor: Oliver Hardy \\
\hline 2507 & terror of 2001 & movie: Blind Terror year: 2001 \\
\hline 2508 & dirk Coldrick Paul & role: dirk Rogers/Man Screaming actor: Paul Coldrick \\
\hline 2509 & Daughter Bankers? & movie: Banker's Daughter \\
\hline 2510 & 5.4 Affleck Ben. & rank: 5.4 actor: Ben Affleck \\
\hline 2511 & alarm 2001 & movie: Alarm year: 2001 \\
\hline 2512 & 1992 Rosario. & year: 1992 actor: Monsour Del Rosario \\
\hline 2513 & arrested billy? & movie: Billy Gets Arrested \\
\hline 2514 & autry show & actor: Gene Autry movie: Big Show \\
\hline 2515 & 1915 connely & year: 1915 actor: Bobby Connelly \\
\hline 2516 & Junction Flapjack & movie: Autocrat of Flapjack Junction \\
\hline 2517 & Jolitorax 6.6 . & role: Jolitorax rank: 6.6 \\
\hline 2518 & akutagawa musical & actor: Eiji Akutagawa genre: musical \\
\hline 2519 & Hiegab 1966 & actor: Hie-gab Kim year: 1966 \\
\hline 2520 & 1995 Hackett! & year: 1995 actor: Jonathan Hackett \\
\hline 2521 & 2001 Peter. & year: 2001 role: Peter \\
\hline 2522 & dashti aghchike dashti? & movie: Araratyan dashti aghchike \\
\hline 2523 & Travers angel & actor: Richard Travers movie: Angel Unaware \\
\hline 2524 & Bamui yujeong & movie: Bamui yujeong \\
\hline 2525 & tiger bengal is? & movie: Bengal Tiger \\
\hline 2526 & cabbie Year $1996 ?$ & movie: Cabbie of the Year year: 1996 \\
\hline 2527 & dream Door 1989 & movie: Beyond Dream's Door year: 1989 \\
\hline 2528 & who is blinn in 1916 & year: 1916 actor: Holbrook Blinn \\
\hline 2529 & Aris 1994 & actor: Aris Gounaris year: 1994 \\
\hline 2530 & 1958 Rajen Kapoor & year: 1958 actor: Rajen Kapoor \\
\hline 2531 & who is crazy richard & movie: Crazy Richard \\
\hline 2532 & Baby 1940 & movie: baby year: 1940 \\
\hline 2533 & 1988 with rating 5.9 & year: 1988 rank: 5.9 \\
\hline 2534 & His Harmonica and Leo & movie: Borrah Minnevitch and His Harmonica School \\
\hline
\end{tabular}




\begin{tabular}{|c|c|c|}
\hline & Diamond? & actor: Leo Diamond \\
\hline 2535 & chapman Bunting & actor: Edward chapman role: Mr. Bunting \\
\hline 2536 & siriyala bhakta & movie: Bhakta Siriyala \\
\hline 2537 & $2004240 !$ & year: 2004 movie: 240 Monroe \\
\hline 2538 & Phone guy plans & role: Phone Guy No. 1 movie: Best Laid Plans \\
\hline 2539 & 1979Costa Ã・vi la. & year: 1979 actor: Ávila Costa \\
\hline 2540 & $4.41996 ?$ & year: 1996 rank: 4.4 \\
\hline 2541 & dag blijde movie? & movie: Blijde dag \\
\hline 2542 & brine with rating 5.6 & rank: 5.6 actor: Adrian Brine \\
\hline 2543 & chien Le 1985. & role: Le chien year: 1985 \\
\hline 2544 & Haven best & movie: Best of Haven \\
\hline 2545 & affair & movie: Affair \\
\hline 2546 & 1992 Ariel & year: 1992 movie: Ariel \\
\hline 2547 & Carcies Jordi. & movie: Carcies actor: Jordi Cercos \\
\hline 2548 & 1958 Wang jie & year: 1958 role: Wang jie \\
\hline 2549 & 1967 Luis Coll? & year: 1967 actor: José Luis Coll \\
\hline 2550 & Minho Kang 1966 & actor: Min-ho Kang year: 1966 \\
\hline 2551 & argonavty & movie: Argonavty \\
\hline 2552 & Nizhalgal Ravi 2000? & actor: Nizhalgal Ravi year: 2000 \\
\hline 2553 & 1957 short & year: 1957 genre: Short \\
\hline 2554 & 1989 Martha & year: 1989 actor: Martha Bamattre \\
\hline 2555 & 1984 Olivier. & actor: Franck Olivier year: 1984 \\
\hline 2556 & chavito rating 6.9 ! & role: El Chavito rank: 6.9 \\
\hline 2557 & anderson in 1998 & actor: Brent Anderson year: 1998 \\
\hline 2558 & 1993 with Ron Jeremy & actor: Ron Jeremy year: 1993 \\
\hline 2559 & $\begin{array}{c}\text { movie with blackbelt } \\
2.4\end{array}$ & rank: 2.4 movie: Blackbelt II \\
\hline 2560 & Czardasfrstin! & movie: Czardasfrstin \\
\hline 2561 & Bound 1979! & movie: Bound year: 1979 \\
\hline 2562 & Blake Down River. & actor: Larry J. Blake movie: Cruisin' Down the River \\
\hline 2563 & Friebe 2000 & actor: Paulo Friebe year: 2000 \\
\hline 2564 & Head Nurses & movie: Black Head Nurses \\
\hline 2565 & year 1973 drama & year: 1973 genre: Drama \\
\hline 2566 & Vessago Baptism & role: Vessago movie: Cauldron: Baptism of Blood \\
\hline 2567 & bergmann crime & actor: Rudolf Bergmann genre: Crime \\
\hline 2568 & man changed in $2003 ?$ & movie: Changed Man year: 2003 \\
\hline 2569 & homme ordures $2004 ?$ & role: L'homme-ordures year: 2004 \\
\hline 2570 & Hawthorne $1924 ?$ & year: 1924 actor: David Hawthorne \\
\hline 2571 & 200023 & year: 2000 movie: 23 \\
\hline 2572 & Diva 2004! & movie: Con Diva year: 2004 \\
\hline
\end{tabular}




\begin{tabular}{|c|c|c|}
\hline 2573 & 1976 utan akt? & year: 1976 movie: Akt utan ord II \\
\hline 2574 & alila Ilan Appel. & movie: Alila actor: Ilan Appel \\
\hline 2575 & 1990 Jeffrey? & year: 1990 actor: Jeffrey Arbaugh \\
\hline 2576 & Cappadocce short? & movie: Cappadocce genre: short \\
\hline 2577 & 5.8 Interviewer. & rank: 5.8 role: TV Interviewer \\
\hline 2578 & Among Believers? & movie: Believers Among Us \\
\hline 2579 & 1970 Dong hwi Jang? & year: 1970 actor: Dong-hwi Jang \\
\hline 2580 & Male Friendly? & gender: $\mathrm{m}$ role: Friendly \\
\hline 2581 & 1991 Up 35. & year: 1991 movie: $35 \mathrm{Up}$ \\
\hline 2582 & Artur Frantisek. & movie: Artur a Leontna actor: Frantisek Beranský \\
\hline 2583 & Biaklar! & movie: Biaklar fora \\
\hline 2584 & all for Jim 1912 & movie: All for Jim year: 1912 \\
\hline 2585 & bluffs fan $1944 !$ & role: Council Bluffs fan year: 1944 \\
\hline 2586 & Farention Frankie & actor: James Farentino role: Frankie \\
\hline 2587 & Cousin 1998 & movie: Cousin year: 1998 \\
\hline 2588 & Jorge 1995 ! & actor: José Jorge year: 1995 \\
\hline 2589 & Amor ciego & movie: Amor no es ciego \\
\hline 2590 & 1935 rating 5.8 & year: 1934 rank: 5.8 \\
\hline 2591 & Hamed Si 1933! & role: Si Hamed year: 1933 \\
\hline 2592 & Babycakes Olympia & movie: Babycakes actor: Olympia Dukakis \\
\hline 2593 & Barragan Canica. & actor: Hector Barragan movie: Canica \\
\hline 2594 & 4.5 Parts of Body & rank: 4.5 movie: Body Parts \\
\hline 2595 & Jack 1998. & role: Jack year: 1998 \\
\hline 2596 & Airplane 7.7 & movie: Airplane! rank: 7.7 \\
\hline 2597 & 2000 Agustoni & year: 2000 actor: Luis Agustoni \\
\hline 2598 & 1999 Porembski. & year: 1999 actor: Jan Porembski \\
\hline 2599 & Grammy Awards 2003 & movie: 45th Annual Grammy Awards year: 2003 \\
\hline 2600 & Barbaros Pasa & movie: Barbaros Hayrettin Pasa \\
\hline 2601 & 2002 David Mandineau. & year: 2002 actor: David Mandineau \\
\hline 2602 & hate the bride & movie: Bride of Hate \\
\hline 2603 & Brian Bye Child? & actor: Brian Devlin movie: Bye-Child \\
\hline 2604 & 1993 rating 5.9 & year: 1993 rank: 5.9 \\
\hline 2605 & Abgeschminkt & movie: Abgeschminkt! \\
\hline 2606 & 1964 Forlani. & actor: Rémo Forlani year: 1964 \\
\hline 2607 & jinks half & movie: Captain Jinks' Better Half \\
\hline 2608 & 1989 Stefano & actor: Marco Di Stefano year: 1989 \\
\hline 2609 & 19407.5 rating? & year: 1940 rank: 7.5 \\
\hline 2610 & 1981 Ryan Barcroft. & actor: Ryan Barcroft year: 1981 \\
\hline 2611 & 1977 beda! & year: 1977 movie: Beda \\
\hline
\end{tabular}




\begin{tabular}{|c|c|c|}
\hline 2612 & Adhbhuta $1957 !$ & movie: Allauddin Adhbhuta Deepam year: 1957 \\
\hline 2613 & 4.4 Close Dangerously. & rank: 4.4 movie: Dangerously Close \\
\hline 2614 & Fixer Leroy! & movie: Bab the Fixer role: Leroy Scott \\
\hline 2615 & 19892.5 rating! & year: 1989 rank: 2.5 \\
\hline 2616 & beneath sea & movie: Beneath the Sea \\
\hline 2617 & $\begin{array}{c}\text { family movie from } \\
1980\end{array}$ & year: 1980 genre: family \\
\hline 2618 & 1995 rating 5 & year: 1996 rank: 5 \\
\hline 2619 & Brown $1936 ?$ & movie: Brown Wallet year: 1936 \\
\hline 2620 & Anne Green Drainie. & movie: Anne of Green Gables actor: John Drainie \\
\hline 2621 & bailey 6.3 & rank: 6.3 actor: leslie Bailey \\
\hline 2622 & veken 1969 & actor: Bob Van der Veken year: 1969 \\
\hline 2623 & $\begin{array}{c}\text { administration Charles } \\
\text { Clary. }\end{array}$ & actor: Charles Clary movie: Change of Administration \\
\hline 2624 & 2003 Beckford & year: 2003 actor: Tyson Beckford \\
\hline 2625 & Ball Tony of 1912. & actor: Tony Ball year: 1912 \\
\hline 2626 & Bridesmaids! & movie: Bridesmaids \\
\hline 2627 & the Pacific Across & movie: Across the Pacific \\
\hline 2628 & 1976 rating of 4.8 & year: 1976 rank: 4.8 \\
\hline 2629 & ancel as wedding guest & actor: Jordan Ancel role: Wedding Guest \\
\hline 2630 & bond with 7.8 ! & actor: Tommy Bond rank: 7.8 \\
\hline 2631 & Atrapado & movie: Atrapado \\
\hline 2632 & 1936 Charlie Circus. & year: 1936 movie: Charlie Chan at the Circus \\
\hline 2633 & 5.7 Cid Leyenda? & rank: 5.7 movie: Cid: La leyenda \\
\hline 2634 & barry red as marshal. & actor: don 'red' barry role: marshal Karnin \\
\hline 2635 & mujeres fu fa & movie: A m las mujeres ni fu ni fa \\
\hline 2636 & 1919 victor linsky? & actor: Victor De Linsky year: 1919 \\
\hline 2637 & 1912 Blackwell! & year: 1912 actor: Carlyle Blackwell Sr. \\
\hline 2638 & 1999 Dangerous & year: 1999 movie: Charmed \& Dangerous \\
\hline 2639 & alkimista & movie: Alkimista s a szz \\
\hline 2640 & tequila Amigo 1999! & movie: Amigo tequila year: 1999 \\
\hline 2641 & bunker & movie: bunker \\
\hline 2642 & 1915 broken pledge & year: 1915 movie: Broken Pledge \\
\hline 2643 & oglu daglarin & movie: Daglarin oglu \\
\hline 2644 & 19815.4 & year: 1981 rank: 5.4 \\
\hline 2645 & 1988 blanc & actor: mel blanc yar: 1988 \\
\hline 2646 & 1916 cahill & year: 1916 actor: Johnny Cahill \\
\hline 2647 & andrejka & movie: Andrejka \\
\hline 2648 & Reinout Bertus! & actor: Reinout Bussemaker role: Bertus \\
\hline 2649 & Desailly $1962 !$ & actor: Jean Desailly year: 1962 \\
\hline
\end{tabular}




\begin{tabular}{|c|c|c|}
\hline 2650 & Taguchi 1967 & actor: Kazuya Taguchi year: 1967 \\
\hline 2651 & alaren & movie: alarcn \\
\hline 2652 & 1916 Rossin & year: 1916 actor: Richard Rosson \\
\hline 2653 & Hideki $1967 ?$ & year: 1967 role: Hideki \\
\hline 2654 & 1949 Doctor & year: 1949 role: Doctor \\
\hline 2655 & Asalto Tijuana. & movie: Asalto en Tijuana \\
\hline 2656 & Gelu in 2003 movie & role: Gelu year: 2003 \\
\hline 2657 & bill bradbury movies & actor: Bill Bradbury \\
\hline 2658 & 1945 comedy & Year: 1945 genre: Comedy \\
\hline 2659 & gentleman amateur & movie: amateur gentleman \\
\hline 2660 & 2003 air & year: 2003 movie: air \\
\hline 2661 & 1994 arditi & year: 1994 actor: Pierre arditi \\
\hline 2662 & 2003 comedys & year: 2003 genre: comedy \\
\hline 2663 & beverly hills ninja? & movie: Beverly Hills Ninja \\
\hline 2664 & Casares Francisco $3.4 ?$ & actor: Francisco Casares rank: 3.4 \\
\hline 2665 & Baekbaekgyo . & movie: Baekbaekgyo \\
\hline 2666 & Bubbles Kiddies & movie: Bubbles actor: The Vitaphone Kiddies \\
\hline 2667 & Tom Santschi 1911 & actor: Tom Santschi year: 1911 \\
\hline 2668 & 1991 Patrick Bauchau? & year: 1991 actor: Patrick Bauchau \\
\hline 2669 & documentaries of 2001 & year: 2001 genre: documentary \\
\hline 2670 & Guido Authentizitt! & actor: Guido Zimmermann movie: Authentizitt \\
\hline 2671 & Witch Maisie Congo & role: Third Witch Doctor movie: Congo Maisie \\
\hline 2672 & Swenson Kari 1987 & movie: Abduction of Kari Swenson year: 1987 \\
\hline 2673 & Nassim Dakwar movies & actor: Nassim Dakwar \\
\hline 2674 & shorts of 1996. & genre: short year: 1996 \\
\hline 2675 & 6.8 movie with Barreiro & rank: 6.8 actor: Luis G. Barreiro \\
\hline 2676 & animations of 1992. & year: 1992 \\
\hline 2677 & 1996 bisschen & movie: Bisschen Glck year: 1996 \\
\hline 2678 & hamilton as Bradley? & movie: Alexander Hamilton actor: Bradley Barker \\
\hline 2679 & circus aces? & movie: Circus Ace \\
\hline 2680 & behind a counter? & movie: Behind the Counter \\
\hline 2681 & garret's body & movie: Body by Garret \\
\hline 2682 & tom bliss movies? & actor: Tom Bliss \\
\hline 2683 & Jenkins Allen Billie? & actor: Derrell Allen Jenkins movie: Billie \\
\hline 2684 & 1985 Aviator. & year: 1985 movie: Aviator \\
\hline 2685 & breakfast day movie? & movie: All Day Breakfast \\
\hline 2686 & 1995 Svankmajer & year: 1995 actor: Jan Svankmajer \\
\hline 2687 & $\mathrm{Bj} \tilde{\mathrm{A}} \boldsymbol{\mathrm { rn }} 1994$ & actor: Björn Cordes year: 1994 \\
\hline 2688 & Years Heartbeat! & movie: 10 Years of Heartbeat \\
\hline
\end{tabular}




\begin{tabular}{|c|c|c|}
\hline 2689 & 2003 Jay Brazeau & year: 2003 actor: Jay Brazeau \\
\hline 2690 & bredal in comedy & actor: Peter Bredal genre: comedy \\
\hline 2691 & cellar and tree? & movie: Cellar and the Almond Tree \\
\hline 2692 & Mr Loomis Mame & role: Mr. Loomis movie: Auntie Mame \\
\hline 2693 & bric compagnie & movie: Brac et compagnie \\
\hline 2694 & movies with Sungkee! & actor: Sung-kee Ahn \\
\hline 2695 & dame de l'aube? & movie: Dame de l'aube \\
\hline 2696 & Emilio 1969? & role: Emilio year: 1969 \\
\hline 2697 & Camera 1987? & movie: Candid Candid Camera Volume 6 year: 1987 \\
\hline 2698 & $\begin{array}{c}\text { Adams Gregory Boy } \\
\text { Orphan. }\end{array}$ & actor: Gregory Adams role: Orphan Boy \\
\hline 2699 & Turner Spike! & actor: Jared Turner role: Spike \\
\hline 2700 & heinrich blaue? & movie: Blaue Heinrich \\
\hline 2701 & Rival 1914 & movie: Canine Rival year: 1914 \\
\hline 2702 & 19854.4. & year: 1985 rank: 4.4 \\
\hline 2703 & 1964 caballero & year: 1964 movie: Casi un caballero \\
\hline 2704 & Bear Narrator & movie: Bear Country role: Narrator \\
\hline 2705 & crime mala 1928 ! & movie: Crime da Mala year: 1928 \\
\hline 2706 & barretts wimpole street & movie: Barretts of Wimpole Street \\
\hline 2707 & 1914 comedy? & genre: comedy year: 1914 \\
\hline 2708 & 1959 Cip! & year: 1959 role: Cip \\
\hline 2709 & 1925 bustin' & year: 1925 movie: Bustin' Thru (1925/II) \\
\hline 2710 & 2004 music & year: 2004 genre: music \\
\hline 2711 & Blast 'Em movie? & movie: Blast 'Em \\
\hline 2712 & L'Auberge rouge & movie: Auberge rouge \\
\hline 2713 & corsa in discesa & movie: Corsa in discesa \\
\hline 2714 & 1993 falcone & year: 1993 role: Det. Falcone \\
\hline 2715 & steve perry movies? & actor: Steve (V) Perry \\
\hline 2716 & $\begin{array}{l}1922 \text { movies with } \\
\text { Walter miller }\end{array}$ & year: 1922 actor: Walter Miller \\
\hline 2717 & Rick of cherry bomb & role: Rick movie: Cherry Bomb \\
\hline 2718 & Fly Paloma Flores? & role: Paloma Fly actor: Carlos (XI) Flores \\
\hline 2719 & caza diablo 2001 & movie: Caza del diablo year: 2001 \\
\hline 2720 & closas Alberto movies? & actor: Alberto Closas \\
\hline 2721 & fisk in family movies? & genre: family actor: Eliot Fisk \\
\hline 2722 & 6.4 brothers karamazov & rank: 6.4 movie: Brothers Karamazov \\
\hline 2723 & clin d'oeil? & movie: Clin d'oeil \\
\hline 2724 & Biktima? & movie: Biktima \\
\hline 2725 & chefredaktuer jordan? & role: Chefredaktuer Paul Jordan \\
\hline 2726 & shy soldier moive? & role: Shy Soldier \\
\hline
\end{tabular}




\begin{tabular}{|c|c|c|}
\hline 2727 & rio adriana is? & movie: Adriana del rio \\
\hline 2728 & 1971 short? & year: 1971 genre: short \\
\hline 2729 & 1992 bonnel ! & year: 1992 actor: Patrick Bonnel \\
\hline 2730 & 1916 Male? & year: 1916 gender: $\mathrm{m}$ \\
\hline 2731 & crazed 1982 & movie: Crazed year: 1982 \\
\hline 2732 & $\begin{array}{l}\text { Babilonia Bertone } \\
\text { Ricardo. }\end{array}$ & movie: Babilonia actor: Ricardo Bertone \\
\hline 2733 & $\begin{array}{c}\text { Aurvaag } \\
\text { Andreaskorset! }\end{array}$ & actor: Trond Fausa Aurvaag movie: Andreaskorset \\
\hline 2734 & Big Kahuna & movie: Big Kahuna \\
\hline 2735 & Seok-gu kim movies! & actor: Seok-gu Kim \\
\hline 2736 & bebek $1979 ?$ & movie: Bebek year: 1979 \\
\hline 2737 & 1990 Dawn 83! & year: 1990 movie: 83 Hours 'Til Dawn \\
\hline 2738 & Theory Conspiracy? & movie: Conspiracy Theory \\
\hline 2739 & Computercide. & movie: Computercide \\
\hline 2740 & Offiseren 1969. & role: Offiseren year: 1969 \\
\hline 2741 & barrera documentary & actor: Antonia barrera genre: documentary \\
\hline 2742 & Girl Cabaret. & movie: Cabaret Girl \\
\hline 2743 & Dr.Mas infierno & role: Dr.Mas movie: Camino del infierno \\
\hline 2744 & 1924 Lyons & year: 1924 actor: Eddie Lyons \\
\hline 2745 & what year is bambola? & movie: Bambola \\
\hline 2746 & 1989 action movies & year: 1989 genre: action \\
\hline 2747 & movies with Dantes & actor: Tony Dantes \\
\hline 2748 & big town moive? & movie: Big Town Round-up \\
\hline 2749 & Alexandra 1902 & $\begin{array}{c}\text { movie: Crowning of King Edward and Queen Alexandra } \\
\text { year: } 1902\end{array}$ \\
\hline 2750 & movie with sammy lee & role: Dr. Sammy Lee \\
\hline 2751 & Conductor 1987 & role: Himself (Conductor) year: 1987 \\
\hline 2752 & casa troya $1936 !$ & year: 1936 movie: Casa de la Troya \\
\hline 2753 & 1960 Bezzashchitnoye & year: 1960 movie: Bezzashchitnoye sushchestvo \\
\hline 2754 & Bucko Ralph. & actor: Ralph Bucko \\
\hline 2755 & 1975 colegio muerte & year: 1975 movie: Colegio de la muerte \\
\hline 2756 & mcClure 1974 & actor: Doug McClure year: 1974 \\
\hline 2757 & 2003 sirven & year: 2003 actor: Sirven Auduc \\
\hline 2758 & caine in movies & role: $\mathrm{m}$ actor: Michael Caine \\
\hline 2759 & Parade Beauty! & movie: Beauty Parade \\
\hline 2760 & rita amalu & gender: f actor: Rita Amalu \\
\hline 2761 & 1985 Donga! & year: 1985 movie: Adavi Donga \\
\hline 2762 & Blue 5.7? & movie: Blue Eagle rank: 5.7 \\
\hline 2763 & cattivo is cuore? & movie: Cuore cattivo \\
\hline
\end{tabular}




\begin{tabular}{|c|c|c|}
\hline 2764 & 2002 The Perspective! & year: 2002 movie: 9-11: The Teens' Perspective \\
\hline 2765 & Sweet Bone Mel. & movie: Bone Sweet Bone actor: Mel Blanc \\
\hline 2766 & $\begin{array}{c}\text { Augustus Plays the } \\
\text { devil }\end{array}$ & actor: Augustus Carney movie: Alkali Ike Plays the Devil \\
\hline 2767 & 1919 Parson & year: 1919 role: Parson Toogowd \\
\hline 2768 & $\begin{array}{c}\text { Alem } \tilde{A}_{j n} \text { Anastasio } \\
\text { arte? }\end{array}$ & actor: Anastasio Alemán movie: Arte de vivir \\
\hline 2769 & Ride Sleigh busters & movie: Buster's Sleigh Ride \\
\hline 2770 & 7.4 rating martin & rank: 7.4 actor: john Marin \\
\hline 2771 & auf Naxos Naxos & movie: Ariadne auf Naxos \\
\hline 2772 & cuidado com & movie: Cuidado com o co \\
\hline 2773 & caso para & movie: Caso para dos \\
\hline 2774 & adult $1994 ?$ & genre: adult year: 1994 \\
\hline 2775 & $\begin{array}{l}1991 \text { Cannone Jean- } \\
\text { Michel! }\end{array}$ & year: 1991 actor: Jean-Michel Cannone \\
\hline 2776 & Auction Mart & movie: Auction Mart \\
\hline 2777 & Driss role in movie? & role: Driss \\
\hline 2778 & George 1917 & actor: George George year: 1917 \\
\hline 2779 & Victor di branco. & actor: Victor Cavallo movie: Compagni di branco \\
\hline 2780 & 1942 anderson & year: 1942 actor: Rick Anderson \\
\hline 2781 & cerro locos & movie: Cerro de los locos \\
\hline 2782 & 1000 kivika & movie: 1000 kivika trela \\
\hline 2783 & 1928 Hamilton Lloyd & year: 1928 actor: Lloyd Hamilton \\
\hline 2784 & citizen 1999. & year: 1999 movie: Citizen \\
\hline 2785 & blagajnica more & movie: Blagajnica hoce ici na more \\
\hline 2786 & 7.6 Buchli & rank: 7.6 actor: James Buchli \\
\hline 2787 & jack Drumier movies! & actor: Jack Drumier \\
\hline 2788 & $\begin{array}{l}\text { Male Stefano in } \\
\text { adventures! }\end{array}$ & actor: Stefano Oppedisano genre: Adventure \\
\hline 2789 & Guard Marty Antonini & role: Guard \#1 actor: Marty Antonini \\
\hline 2790 & 1959 Drama & year: 1959 genre: Drama \\
\hline 2791 & ati glleri Baharin 1961. & movie: Baharin glleri ati year: 1961 \\
\hline 2792 & cretinetti AndrãđC. & movie: Cretinetti sa tutto e fa tutto actor: André Deed \\
\hline 2793 & 4.6 rating Ben Ãtez . & actor: Andrés Benítez rank: 4.6 \\
\hline 2794 & guard Coast? & movie: Coast Guard \\
\hline 2795 & como gotas & movie: Como dos gotas de agua \\
\hline 2796 & 1984 frank & year: 1967 actor: Frank oliver \\
\hline 2797 & $\mathrm{ab}$ in Sden! & movie: $\mathrm{Ab}$ in den Sden \\
\hline 2798 & Gitchy $1965 ?$ & movie: Camp Gitchy Gloomy year: 1965 \\
\hline 2799 & college grad Buck! & role: College Grad \#2 actor: Buck Bucholtz \\
\hline
\end{tabular}




\begin{tabular}{|c|c|c|}
\hline 2800 & $19646 !$ & year: 1964 rank: 6 \\
\hline 2801 & Place Est Ainda! & movie: Barbara Parkins Ainda Est em 'Peyton Place' \\
\hline 2802 & Fabrizi Franco Amiche. & actor: Franco Fabrizi movie: Amiche \\
\hline 2803 & Paul broken? & role: Paul movie: Broken \\
\hline 2804 & Sun-jae dabachyeo. & actor: Sun-jae Lee movie: Cheongchuneul dabachyeo \\
\hline 2805 & 6.7 Canterville & rank: 6.7 movie: Canterville Ghost \\
\hline 2806 & Ricki Big! & role: Ricki movie: Big Al \\
\hline 2807 & Awkat azima & movie: Awkat azima \\
\hline 2808 & cato bill movie & actor: Bill Cato \\
\hline 2809 & 1931 comedies & year: 1931 genre: Comedy \\
\hline 2810 & captain's paradise & movie: Captain's Paradise \\
\hline 2811 & 1962 drama & genre: drama year: 1962 \\
\hline 2812 & campfire tales & movie: Campfire Tales \\
\hline 2813 & Atcha & movie: Atcha \\
\hline 2814 & 1979 Dada & year: 1979 movie: Dada \\
\hline 2815 & $\begin{array}{l}\text { Ugo Gracci de } \\
\text { Maxims? }\end{array}$ & actor: Ugo Gracci movie: Dama de Chez Maxim's \\
\hline 2816 & Antoine Artemis 1992 & actor: Artemis Antoine year: 1992 \\
\hline 2817 & 1928 Cobb. & year: 1928 actor: Edmund Cobb \\
\hline 2818 & again broke! & movie: broke again \\
\hline 2819 & $\begin{array}{c}\text { Lyubomir Farkov } \\
\text { movies }\end{array}$ & actor: Lyubomir Farkov \\
\hline 2820 & son anybody? & movie: Anybody's son will do \\
\hline 2821 & 1917 barrier & year: 1917 movie: Barrier \\
\hline 2822 & $\begin{array}{c}\text { Barry Raymond } \mathrm{Mr} \\
\text { Grand? }\end{array}$ & actor: Raymond Barry role: Mr. Grand \\
\hline 2823 & tensin Alta? & movie: Alta tensin \\
\hline 2824 & Bradley of 1934 & actor: Harry C. Bradley year: 1934 \\
\hline 2825 & Mingus Charles movies & actor: Charles Mingus \\
\hline 2826 & chases pimple street & movie: Chases of Pimple Street \\
\hline 2827 & $\max$ of 1963. & role: Max year: 1963 \\
\hline 2828 & lady beautiful & movie: Beautiful Lady \\
\hline 2829 & Beautiful 1938! & movie: Beautiful year: 1938 \\
\hline 2830 & Romance of 1915 ! & genre: Romance year: 1915 \\
\hline 2831 & Alceste. & movie: Alceste \\
\hline 2832 & frank daniels movies? & actor: Frank Daniels \\
\hline 2833 & Hardy 1917. & actor: Oliver Hardy year: 1917 \\
\hline 2834 & Berggren 1945. & actor: Ulf Berggren year: 1945 \\
\hline 2835 & 1909 short & Year: 1909 genre: Short \\
\hline 2836 & 1999 Jesper! & year: 1999 actor: Jesper Asholt \\
\hline
\end{tabular}




\begin{tabular}{|c|c|c|}
\hline 2837 & chacales & movie: Chacales \\
\hline 2838 & 1961 boue? & year: 1961 movie: Ciel et la boue \\
\hline 2839 & 1985 crimewave! & year: 1985 movie: Crimewave (1985/I) \\
\hline 2840 & bottom up Series $8 ?$ & movie: Bottoms Up! Series 8 \\
\hline 2841 & 1913 drama movie* & year: 1913 genre: Drama \\
\hline 2842 & Good damage & movie: Damaged Good \\
\hline 2843 & 2003 click & year: 2003 movie: Click (2003/II) \\
\hline 2844 & Beisbol & movie: Beisbol \\
\hline 2845 & bedelia bluff & movie: Bedelia's Bluff \\
\hline 2846 & 1975 cheng! & movie: Da ge Cheng year: 1975 \\
\hline 2847 & 2003 bio! & year: 2003 movie: $100 \%$ bio \\
\hline 2848 & bums mystery & movie: Bums genre: mystery \\
\hline 2849 & $\begin{array}{l}1912 \text { Accident } \\
\text { Christmas. }\end{array}$ & year: 1912 movie: Christmas Accident \\
\hline 2850 & Apple 1995 & movie: Apple year: 1995 \\
\hline 2851 & Beanpole & movie: Beanpole \\
\hline 2852 & 2002 crossroads & year: 2002 movie: crossroads \\
\hline 2853 & david berkowitz & movie: A\&E Biography: David Berkowitz - Son of Sam \\
\hline 2854 & journey life? & movie: As we journey through life \\
\hline 2855 & $6.21946 ?$ & rank: 6.2 year: 1946 \\
\hline 2856 & 1995 adult movies & year: 1995 genre: adult \\
\hline 2857 & sinemalarim Benim & movie: Benim sinemalarim \\
\hline 2858 & Car Borrow 1970! & movie: Dad, Can I Borrow the Car? year: 1970 \\
\hline 2859 & acosta movie & actor: Armando Acosta \\
\hline 2860 & algo mat alguien & movie: Alguien mat algo \\
\hline 2861 & 2003 Andresito? & year: 2003 role: Andresito \\
\hline 2862 & 1979 Laci. & year: 1979 role: Perjés Laci \\
\hline 2863 & 1982 young man role & year: 1982 role: young man \\
\hline 2864 & brooklyn angle & movie: Angel comes to Brooklyn \\
\hline 2865 & Bohme Ellero & movie: Bohme actor: Francesco Ellero d'Artegna \\
\hline 2866 & Jeweler $5.4 ?$ & role: Jeweler rank: 5.4 \\
\hline 2867 & Adrians Hole & movie: Adrian's Hole \\
\hline 2868 & $2004 \operatorname{stan}$ & role: Stan year: 2004 \\
\hline 2869 & 2000 Perkins & year: 2000 actor: Jack Perkins \\
\hline 2870 & Arkelope Narrator & movie: Arkelope! role: Narrator \\
\hline 2871 & ausfahrt leben & movie: Ausfahrt Ost... ins Leben von Nico \\
\hline 2872 & 6.1 action movie & genre: action rank: 6.1 \\
\hline 2873 & Battle Virginia? & movie: Battle in the Virginia Hills \\
\hline 2874 & Bigfoot of 1979. & movie: Capture of Bigfoot year: 1979 \\
\hline 2875 & The cardinal movie? & movie: Cardinal \\
\hline
\end{tabular}




\begin{tabular}{|c|c|c|}
\hline 2876 & Banerjee Victor $1953 ?$ & actor: Victor Banerjee year: 1953 \\
\hline 2877 & 1922 Blaze & year: 1922 movie: Blaze Away \\
\hline 2878 & Toomas movies? & actor: Toomas Hussar \\
\hline 2879 & Maa Aavide. & movie: Aavida Maa Aavide \\
\hline 2880 & Akins Claude movies? & actor: Claude Akins \\
\hline 2881 & 78-as szent & movie: 78 -as szent johannja \\
\hline 2882 & chet anekwe & actor: Chet Anekwe \\
\hline 2883 & cavalcade broadway & movie: Cavalcade of Broadway: Leon and Eddie's \\
\hline 2884 & Ajil Abdellah & actor: Abdellah Ajil \\
\hline 2885 & comedies of 1991 & year: 1991 genre: comedy \\
\hline 2886 & Cavazza Adrian? & actor: Sebastian Cavazza movie: Adrian \\
\hline 2887 & Liar the amateur & movie: Amateur Liar \\
\hline 2888 & 1996 talkers? & year: 1996 movie: Belly Talkers \\
\hline 2889 & lyubov ...Pervaya & movie: Pervaya lyubov \\
\hline 2890 & 2004 documentary & year: 2004 genre: documentary \\
\hline 2891 & Casarsa Christine 2000. & actor: Christine Casarsa year: 2000 \\
\hline 2892 & Adjutant & role: Adjutant \\
\hline 2893 & 5.9 Bonnes causes? & rank: 5.9 movie: Bonnes causes \\
\hline 2894 & Bhanja Samit movie. & actor: Samit Bhanja \\
\hline 2895 & costa brava & movie: Costa Brava \\
\hline 2896 & cover me movie & movie: Cover Me \\
\hline 2897 & 1997 rating 4.6 & year: 1997 rank: 4.6 \\
\hline 2898 & $\begin{array}{l}\text { movies with chase } \\
\text { Charley. }\end{array}$ & actor: Charley Chase \\
\hline 2899 & 1920 Eldridge Charles? & year: 1920 actor: Charles Eldridge \\
\hline 2900 & Blake Gold Rush? & actor: Robert Blake movie: California Gold Rush \\
\hline 2901 & 1982 Barr. & year: 1982 actor: Douglas Barr \\
\hline 2902 & master barker? & role: Master field actor: kenneth Barker \\
\hline 2903 & 1997 amant & year: 1997 movie: Amant de Maman \\
\hline 2904 & Backstage 1988 & movie: Backstage (1988/II) year: 1988 \\
\hline 2905 & Bully 1976! & role: Bully 2 year: 1976 \\
\hline 2906 & Party 1967 & role: Party Secretary year: 1967 \\
\hline 2907 & drama of $1997 !$ & genre: Drama year: 1997 \\
\hline 2908 & $\begin{array}{c}1972 \text { Bang-jawa } \\
\text { Hyang-dani. }\end{array}$ & year: 1972 movie: Bang-jawa Hyang-dani \\
\hline 2909 & baisers tarzan & movie: Bons Baisers de Tarzan \\
\hline 2910 & Kaisanlahti Viljo? & actor: Viljo Kaisanlahti \\
\hline 2911 & corsican brothers & movie: The Corsican Brothers \\
\hline 2912 & member at party Frank! & role: Band member \#1 at party actor: Frank Beard \\
\hline 2913 & Bottomless pit movie & movie: Bottomless Pit \\
\hline
\end{tabular}




\begin{tabular}{|c|c|c|}
\hline 2914 & 1998 balkanska & year: 1998 movie: Balkanska ruleta \\
\hline 2915 & black madam sadista & movie: Black madam sadista \\
\hline 2916 & 2000 sci-fi & genre: sci-fi year: 2000 \\
\hline 2917 & Bob 1988! & actor: Bob Skid year: 1988 \\
\hline 2918 & book movie & movie: Book \\
\hline 2919 & 5.1 big buses & rank: 5.1 movie: Big Bus \\
\hline 2920 & John Cassavetes Black & actor: John Cassavetes movie: Columbo: tude in Black \\
\hline 2921 & sasanudu chanda & movie: Chanda Sasanudu \\
\hline 2922 & Marty Beecroft! & actor: Marty Beecroft \\
\hline 2923 & Hollywood 1989 & movie: Backdoor to Hollywood 9 year: 1989 \\
\hline 2924 & $\begin{array}{c}\text { mortal Ambicin } \\
\text { Roberto. }\end{array}$ & movie: Ambicin mortal actor: Roberto Guinar \\
\hline 2925 & Joe Bleak! & role: Joe movie: Bleak House \\
\hline 2926 & 2001 caretaker & year: 2001 movie: Caretaker \\
\hline 2927 & yeonin Adeulgwa Lee! & movie: Adeulgwa yeonin actor: Jae-ryong Lee \\
\hline 2928 & Black Snatch & movie: Black Snatch \\
\hline 2929 & 1912 brooke & actor: Van Dyke Brooke year: 1912 \\
\hline 2930 & Johnny Junior & role: Johnny movie: Absorbing Junior \\
\hline 2931 & Manuel Arvide & actor: Manuel Arvide \\
\hline 2932 & 1956 short & year: 1956 genre: Short \\
\hline 2933 & cherfitness attitude & movie: Cherfitness: A new Attitude \\
\hline 2934 & movies with Girard & actor: Joseph W. Girard \\
\hline 2935 & bombardovao 1976 & movie: Covek koji je bombardovao Beograd year: 1976 \\
\hline 2936 & 1938 Beach! & year: 1938 actor: John Beach \\
\hline 2937 & 5.8 afsporet & rank: 5.8 movie: Afsporet \\
\hline 2938 & Patton Bill Battlin? & actor: Bill Patton movie: Battlin' Buckaroo \\
\hline 2939 & Johnny arthur movies & role: actor: Johnny Arthur \\
\hline 2940 & $4.427 \mathrm{~A} ?$ & rank: 4.4 movie: $27 \mathrm{~A}$ \\
\hline 2941 & Allende movies & actor: Fernando Allende \\
\hline 2942 & movie Deo Ramesh? & actor: Ramesh Deo \\
\hline 2943 & marudai baka & movie: Baka Marudai \\
\hline 2944 & love addicted movies? & movie: Addicted to Love \\
\hline 2945 & The Yaklich Story? & movie: Cries Unheard: The Donna Yaklich Story \\
\hline 2946 & Beaulieu Isaac. & actor: Isaac Beaulieu \\
\hline 2947 & careron smith movies & actor: Cameron Smith \\
\hline 2948 & 1961 Arzu & movie: Arzu year: 1961 \\
\hline 2949 & $\begin{array}{l}\text { guy say no with Antin } \\
\text { Steve. }\end{array}$ & movie: Can a guy say no? actor: Steve Antin \\
\hline 2950 & jan budar movies? & actor: Jan Budar \\
\hline 2951 & 1992 Nej! & year: 1992 actor: Nej Adamson \\
\hline
\end{tabular}




\begin{tabular}{|c|c|c|}
\hline 2952 & 1957 Sea Lord? & year: 1957 role: First Sea Lord \\
\hline 2953 & Juan Fairen mvoies & actor: Juan Fairen \\
\hline 2954 & 6.2 cartographers & rank: 6.2 movie: Cartographer's Girlfriend \\
\hline 2955 & Beyond Patrick. & movie: Beyond Bob actor: Patrick Coyle \\
\hline 2956 & $5.61992 ?$ & rank: 5.6 year: 1992 \\
\hline 2957 & Narrator 7.9. & role: Narrator rank: 7.9 \\
\hline 2958 & $\begin{array}{l}\text { Abercrombie Ian Blood } \\
\text { Beach. }\end{array}$ & actor: Ian Abercrombie movie: Blood Beach \\
\hline 2959 & drama 1971? & year: 1971 genre: Drama \\
\hline 2960 & Tienan 4.6 & role: Tienan rank: 4.6 \\
\hline 2961 & $\begin{array}{l}\text { Billy Broncho } \\
\text { Anderson }\end{array}$ & role: Broncho Billy actor: Gilbert Anderson \\
\hline 2962 & 7 rating year 1957 & year: 1957 rank: 7 \\
\hline 2963 & 1948 Always together. & year: 1948 movie: Always Together \\
\hline 2964 & desnudo barrio & movie: Barrio 13 al desnudo \\
\hline 2965 & $\begin{array}{c}\text { Treasure Island Island } \\
\text { Treasure Treasure } \\
\text { Treasure. }\end{array}$ & movie: American Tail: The Treasure of Manhattan Island \\
\hline 2966 & Diet Bruce! & movie: Bruce Diet \\
\hline 2967 & asia nostra & movie: Asia cosa Nostra \\
\hline 2968 & Almost guys $8.9 !$ & movie: Almost Guys rank: 8.9 \\
\hline 2969 & movies with Scardon? & actor: Paul Scardon \\
\hline 2970 & l'innocent Aveux! & movie: Aveux de l'innocent \\
\hline 2971 & belgi leven & movie: Ah! t'Is zo fijn in Belgi te leven \\
\hline 2972 & agnoula & movie: Agnoula \\
\hline 2973 & 11 Harrowhouse & movie: 11 harrowhouse \\
\hline 2974 & 1930 Brinley. & year: 1930 actor: Charles Brinley \\
\hline 2975 & 1989 cover girl! & year: 1989 movie: Cover Girl and the Cop \\
\hline 2976 & Adamson Bright. & actor: James Adamson movie: Bright Leaf \\
\hline 2977 & $\begin{array}{l}\text { clark gable Handsome } \\
1996 . \\
\end{array}$ & movie: clark gable: Tall, Dark and Handsome year: 1996 \\
\hline 2978 & Progresso no Guerra & movie: Angola na Guerra e no Progresso \\
\hline 2979 & 8.2 Friedmans? & rank: 8.2 movie: Capturing the Friedmans \\
\hline 2980 & baat raat 8.2 . & movie: Baat Ek Raat Ki rank: 8.2 \\
\hline 2981 & cinma arabe movie & movie: Cinma arabe - tat des lieux \\
\hline 2982 & 2005 with Cutter & year: 2005 role: cutter \\
\hline 2983 & 1938 bennett & year: 1938 actor: Holland Bennett \\
\hline 2984 & Anniversary & movie: Anniversary \\
\hline 2985 & $\begin{array}{l}\text { Christopher Crotty } \\
\text { wiley World? }\end{array}$ & $\begin{array}{l}\text { actor: Christopher Crotty movie: A.K.A.: It's a Wiley } \\
\text { World! }\end{array}$ \\
\hline 2986 & Bradley Barker Brent & actor: Bradley Barker role:'Handsome Harry' Brent \\
\hline
\end{tabular}




\begin{tabular}{|c|c|c|}
\hline & Harry. & \\
\hline 2987 & 1965 Fete! & year: 1965 movie: Crow's Fete \\
\hline 2988 & Barrel Organ & movie: Barrel Organ \\
\hline 2989 & 1946 Lake Louise & year: 1946 movie: Beautiful Banff and Lake Louise \\
\hline 2990 & Bunar. & movie: Bunar \\
\hline 2991 & 1918 butler's blunder! & year: 1918 movie: Butler's Blunder \\
\hline 2992 & huey long A\&E & movie: A\&E Biography: Huey Long - The King Fish \\
\hline 2993 & Alfredo diestro movie & actor: Alfredo del Diestro \\
\hline 2994 & 6.1 cadence & rank: 6.1 movie: Cadence \\
\hline 2995 & 1990 Gmex? & year: 1990 movie: Chelo Gmez, detective privado \\
\hline 2996 & $\begin{array}{c}\text { Atajo Terranova } \\
\text { Osvaldo }\end{array}$ & movie: Atajo actor: Osvaldo Terranova \\
\hline 2997 & Borderlines & movie: Borderlines \\
\hline 2998 & Crettex in Montagne & actor: Emile Crettex movie: Appel de la montagne \\
\hline 2999 & Edmund breon movies & actor: Edmund Breon \\
\hline 3000 & inmortal bolero & movie: Bolero inmortal \\
\hline 3001 & Burns 1939 & actor: Bob Burns year: 1939 \\
\hline 3002 & Beregi csacsi. & actor: Péter Beregi movie: Csapd le csacsi \\
\hline 3003 & $\begin{array}{l}1980 \text { Ferruccio } \\
\text { Amendola! }\end{array}$ & year: 1980 actor: Ferruccio Amendola \\
\hline 3004 & Biloxi $6.5 ?$ & movie: Biloxi Blues rank: 6.5 \\
\hline 3005 & beqabu & movie: Beqabu \\
\hline 3006 & Karl 2003! & actor: Karl Bille year: 2003 \\
\hline 3007 & jim cactus $1915 ?$ & movie: Cactus Jim's Shop Girl year: 1915 \\
\hline 3008 & show Mousie Garner. & movie: After the Show actor: Paul 'Mousie' Garner \\
\hline 3009 & $6.12000 ?$ & rank: 6.1 year: 2000 \\
\hline 3010 & Augustus business & role: Augustus movie: Big Business \\
\hline 3011 & $\begin{array}{c}\text { Andrei } \\
\text { Barilosolnechnoj? }\end{array}$ & actor: Andrei Barilo movie: Beg po solnechnoj storone \\
\hline 3012 & 4.2 rating volta? & movie: C'era una volta la legge rank: 4.2 \\
\hline 3013 & cachito Marcelo? & role: Marcelo movie: Cachito mo \\
\hline 3014 & Doulas Universe & actor: Hugh Douglas movie: Are we Alone in the Universe \\
\hline 3015 & Jean Marc 6.3? & actor: Jean-Marc Bory rank: 6.3 \\
\hline 3016 & 1918 romance movie & year: 1918 genre: Romance \\
\hline 3017 & carr valet & movie: Carr de valets \\
\hline 3018 & 1998 catherine & year: 1998 movie: Catherine's Story \\
\hline 3019 & $\begin{array}{c}\text { Alphonso Alexander } \\
\text { Bustin' }\end{array}$ & actor: Alphonso Alexander movie: Bustin' loose \\
\hline 3020 & Battaglia Rik bomber & actor: Rik Battaglia movie: Bomber \\
\hline 3021 & comedy show 2004 & movie: All Star Comedy Show year: 2004 \\
\hline
\end{tabular}




\begin{tabular}{|c|c|c|}
\hline 3022 & Midnight $1994 !$ & movie: Caroline at Midnight year: 1994 \\
\hline 3023 & Narrator with Gilbert & role: Narrator actor: Richard Gilbert \\
\hline 3024 & woddy candid camera & $\begin{array}{l}\text { movie: Candid Camera's 50th Anniversary actor: Woody } \\
\text { Allen }\end{array}$ \\
\hline 3025 & Adams Trawler Rusty & actor: Stanley Adams role: Rusty Trawler \\
\hline 3026 & 1985 Fischer & year: 1985 actor: Oscar Fischer \\
\hline 3027 & 1981 Musical & year: 1981 genre: Musical \\
\hline 3028 & amlcar cabral & movie: Amlcar Cabral \\
\hline 3029 & 1991 Canada & year: 1991 actor: kevin Canada \\
\hline 3030 & 1955 rating of 6.8 & year: 1955 rank: 6.8 \\
\hline 3031 & 1965 Billie & movie: Billie year: 1965 \\
\hline 3032 & His Fathers $1917 !$ & movie: Blood of His Fathers year: 1917 \\
\hline 3033 & 2000 cord & year: 2000 movie: Cord \\
\hline 3034 & rating of 6 ruggenti & movie: Anni Ruggenti rank: 6 \\
\hline 3035 & 6.1 Toby! & rank: 6.1 role: Toby \\
\hline 3036 & Chouans Chouans & movie: Chouans! \\
\hline 3037 & 1914 Cricket & year: 1914 movie: Cricket on the Hearth \\
\hline 3038 & $\begin{array}{l}\text { Florescu Radu The } \\
\text { Dracula? }\end{array}$ & $\begin{array}{c}\text { actor: Radu Florescu movie: Bloodlines: The Dracula } \\
\text { Family Tree }\end{array}$ \\
\hline 3039 & 1912 Brand? & year: 1912 movie: Brand Blotter \\
\hline 3040 & celui qui ldit! & movie: Celui qui l'dit \\
\hline 3041 & Backstage 1988 & movie: Backstage (1988/I) \\
\hline 3042 & After Ewing Ted & movie: After Five role: Ted Ewing \\
\hline 3043 & $\begin{array}{c}\text { Bolwieser } \\
\text { Bayrhammer! }\end{array}$ & movie: Bolwieser actor: Gustl Bayrhammer \\
\hline 3044 & witness American & movie: American Justice: Eyewitness \\
\hline 3045 & Steed Flows with Jake? & $\begin{array}{l}\text { actor: Jake Steed movie: Chillin' with Jake Steed's Freaks } \\
\text { Whoes \& Flows } 16\end{array}$ \\
\hline 3046 & como vai & movie: Como Vai \\
\hline 3047 & cando terra & movie: Cano da Terra \\
\hline 3048 & Lennie Lazenby! & role: Lennie Lazenby \\
\hline 3049 & Seth Anderson movies? & actor: Seth Anderson \\
\hline 3050 & $5.81996 ?$ & rank: 5.8 year: 1996 \\
\hline 3051 & $7.41999 ?$ & rank: 7.4 year: 1999 \\
\hline 3052 & Azizi 1999 & actor: Rahi Azizi year: 1999 \\
\hline 3053 & 2002 Insp! & year: 2002 role: Insp. Rathore \\
\hline 3054 & AC Paramount . & actor: A.C. Lyles role: Himself - Paramount Pictures \\
\hline 3055 & chant des brumes? & movie: Chant des brumes \\
\hline 3056 & 1998 Camilo Michel & year: 1998 actor: Michel Camilo \\
\hline 3057 & $\begin{array}{l}\text { Billboard Awards } \\
\text { Music Jones? }\end{array}$ & movie: 1997 Billboard Music Awards actor: Davy Jones \\
\hline
\end{tabular}




\begin{tabular}{|c|c|c|}
\hline 3058 & $\begin{array}{l}\text { casualties love loita } \\
\text { story }\end{array}$ & movie: Casualties of Love: The Long Island Lolita Story \\
\hline 3059 & Absent Minded & gender: $m$ movie: Absent Minded \\
\hline 3060 & crimen pirindola & movie: Crimen de la pirindola \\
\hline 3061 & Matthew $3.8 ?$ & actor: Matthew Crosby rank: 3.8 \\
\hline 3062 & 2000 Abusada & year: 2000 movie: Drogada e Abusada \\
\hline 3063 & 1976 Mariano! & year: 1976 actor: Mariano Brancaccio \\
\hline 3064 & documentary 1960 & genre: Documentary year: 1960 \\
\hline 3065 & 1989 buddy system & year: 1989 movie: Buddy System \\
\hline 3066 & expansiva $451996 ?$ & movie: 45 expansiva year: 1996 \\
\hline 3067 & 200013 Nasty? & year: 2000 movie: 18 and Nasty 13 \\
\hline 3068 & Milicien movies & role: Milicien \\
\hline 3069 & 1945 Michael. & year: 1945 role: Michael \\
\hline 3070 & jack morgan moves & actor: Jack Morgan \\
\hline 3071 & $\begin{array}{l}\text { movies with Richard } \\
\text { Alexander }\end{array}$ & actor: Richard Alexander \\
\hline 3072 & Bologna Two! & actor: Joseph Bologna movie: Chapter Two \\
\hline 3073 & 1911 Cold? & year: 1911 movie: Cup of Cold Water \\
\hline 3074 & $\begin{array}{l}\text { Chavalanthrope } \\
\text { Jacques. }\end{array}$ & movie: Chavalanthrope actor: Jacques Dufilho \\
\hline 3075 & Dream 2001? & movie: 112th Dream year: 2001 \\
\hline 3076 & 7 Bhavum & rank: 7 movie: Bhavum \\
\hline 3077 & 1994 Badlands & year: 1994 movie: Badlands \\
\hline 3078 & coliers 6.7 & movie: Chemin des coliers rank: 6.7 \\
\hline 3079 & Black 1987! & movie: Black Tower year: 1987 \\
\hline 3080 & 4.8 polithrona & rank: 4.8 movie: Agapisa mia polithrona \\
\hline 3081 & Wilfred Deadline? & actor: Wilfred Lucas movie: Across the Deadline \\
\hline 3082 & Bhagi Kasam. & actor: Kuldeep Bhagi movie: Chambal Ki Kasam \\
\hline 3083 & 1987 Cannon! & $\begin{array}{c}\text { year: } 1987 \text { movie: Cannon Movie Tales: Beauty and the } \\
\text { Beast }\end{array}$ \\
\hline 3084 & 5.4 Dates & rank: 5.4 movie: 20 Dates \\
\hline 3085 & 1988 Bloodsport & year: 1988 movie: Bloodsport \\
\hline 3086 & Lloyd Lit & actor: Gaylord Lloyd movie: All Lit Up \\
\hline 3087 & 1971 Balgan & year: 1971 movie: Balgan maskui yeoin \\
\hline 3088 & William Avenging? & actor: William J. Butler movie: Avenging Sea \\
\hline 3089 & Sherwood 1992 & $\begin{array}{l}\text { movie: Child Lost Forever: The Jerry Sherwood Story } \\
\text { actor: Lewis Arquette }\end{array}$ \\
\hline 3090 & Gilbert Honeymoon & actor: Gilbert Ely movie: Belated Honeymoon \\
\hline 3091 & Bronx Barbs Ousmana & movie: Bronx-Barbs actor: Ousmana Bakayoko \\
\hline 3092 & 1920 Bitten. & year: 1920 movie: Bitten Biter \\
\hline 3093 & Crainquebille 7.3 ! & movie: Crainquebille rank: 7.3 \\
\hline
\end{tabular}




\begin{tabular}{|c|c|c|}
\hline 3094 & Castellaneta Simpsons & $\begin{array}{l}\text { actor: Dan Castellaneta movie: Bart Wars, the Simpsons } \\
\text { Strike Back }\end{array}$ \\
\hline 3095 & 2003 Awards? & year: 2003 movie: British Style Awards 2003 \\
\hline 3096 & Conquista 2002. & movie: Conquista year: 2002 \\
\hline 3097 & John Buntings & actor: John East movie: Bunting's Blink \\
\hline 3098 & $\begin{array}{l}\text { Byeoljubu King } \\
\text { Dragon! }\end{array}$ & movie: Byeoljubu hero role: Dragon King \\
\hline 3099 & Buddy $7.1 !$ & movie: Buddy rank: 7.1 \\
\hline 3100 & 1996 African! & year: 1966 movie: African Gold \\
\hline 3101 & Baan Dutt! & movie: Aan Baan actor: Som Dutt \\
\hline 3102 & Coronation $2000 !$ & $\begin{array}{c}\text { movie: 40th Anniversary Celebration of Coronation Street } \\
\text { year: } 2000\end{array}$ \\
\hline 3103 & Armetta Cheating & actor: Henry Armetta movie: Cheating Cheaters \\
\hline 3104 & 1958 Territory & year: 1958 movie: Apache Territory \\
\hline 3105 & Bessmertnyj Anatoli. & movie: Bessmertnyj garnizon actor: Anatoli Chemodurov \\
\hline 3106 & McCoy Tom Apostle & role: Tom McCoy movie: Apostle of Vengeance \\
\hline 3107 & 4.6 Mail? & rank: 4.6 movie: Check Is in the Mail... \\
\hline 3108 & Ernesto 1976. & actor: Ernesto Alonso movie: Coronacin \\
\hline 3109 & 1940 Blomstertid. & year: 1940 movie: Blomstertid... \\
\hline 3110 & Musikladen Tom & movie: Best of Musikladen Vol. 02 actor: Tom Evans \\
\hline 3111 & Cherian Arrangement & actor: Tina Cherian movie: Arrangement \\
\hline 3112 & 2000 Project. & year: 2000 movie: Bunk Witch Project \\
\hline 3113 & 5 Coolie & rank: 5 movie: Coolie No. 1 \\
\hline 3114 & Cinma Agoumi & $\begin{array}{l}\text { movie: Cinma arabe - tat des lieux actor: Sid Ahmed } \\
\text { Agoumi }\end{array}$ \\
\hline 3115 & $7.2 \mathrm{Big}$ & rank: 7.2 movie: Big \\
\hline 3116 & Ariella $1980 ?$ & movie: Ariella year: 1980 \\
\hline 3117 & Suitable Frank Simpson & $\begin{array}{l}\text { movie: Columbo: Suitable for Framing role: Frank } \\
\text { Simpson }\end{array}$ \\
\hline 3118 & Michiko Blowjobs 27 & actor: Michiko Ari movie: 100\% Blowjobs 27 \\
\hline 3119 & 1910 Dawsons. & year: 1910 movie: Angel of Dawson's Claim \\
\hline 3120 & Caballo 1961. & movie: Caballo blanco year: 1961 \\
\hline 3121 & 4.6 Couloirs. & rank: 4.6 movie: Couloirs du temps: Les visiteurs \\
\hline 3122 & Gharat 1991 & movie: Aamchya Gharat Gharoba year: 1991 \\
\hline 3123 & 1987 Candid & year: 1987 movie: Candid Candid Camera Volume 6 \\
\hline 3124 & indiavolata Ughetto! & movie: Bruna indiavolata actor: Ughetto Bertucci \\
\hline 3125 & 1988 Asesino? & year: 1988 movie: Asesino anda suelto \\
\hline 3126 & 6.4 Pigeons. & rank: 6.4 movie: Clay Pigeons \\
\hline 3127 & Anarkali 8.6 & movie: Anarkali rank: 8.6 \\
\hline 3128 & 1951 Cavalry. & year: 1951 movie: Cavalry Scout \\
\hline 3129 & fiammeggianti 6.6 & movie: Anime fiammeggianti rank: 6.6 \\
\hline
\end{tabular}




\begin{tabular}{|c|c|c|}
\hline 3130 & 1979 Doberman & year: 1979 movie: Alex and the Doberman Gang \\
\hline 3131 & 4.6 Climb. & rank: 4.6 movie: Climb \\
\hline 3132 & Hermanovic Callosum! & actor: Greg Hermanovic movie: *Corpus Callosum \\
\hline 3133 & Murder 3.6? & movie: .com for Murder rank: 3.6 \\
\hline 3134 & Baat 1962 & movie: Baat Ek Raat Ki year: 1962 \\
\hline 3135 & Soul Combs. & $\begin{array}{l}\text { movie: 16th Annual Soul Train Music Awards actor: Sean } \\
\text { Combs }\end{array}$ \\
\hline 3136 & Smith IQ & actor: Pete Smith movie:'What's Your 'I.Q.'?' Number Two \\
\hline 3137 & 2000 Pup & year: 2000 movie: Air Bud: World Pup \\
\hline 3138 & Ortiz Conmocin? & actor: Aquiles Ortiz movie: Conmocin y pavor \\
\hline 3139 & 1912 Scutcheon & year: 1912 movie: Blot on the 'Scutcheon \\
\hline 3140 & Tito crimen. & actor: Tito Alonso movie: Camino al crimen \\
\hline 3141 & Pieces $1985 ?$ & movie: Bits and Pieces year: 1985 \\
\hline 3142 & Kristina Cheerleader! & actor: Kristina Black movie: Cheerleader Diaries 4 \\
\hline 3143 & 1974 Kasam. & year: 1974 movie: Aap Ki Kasam \\
\hline 3144 & Ajay Bedardi & actor: Ajay Devgan movie: Bedardi \\
\hline 3145 & 1994 Marjorie & $\begin{array}{c}\text { year: } 1994 \text { movie: Acme Rooms and Sweet Marjorie } \\
\text { Russell }\end{array}$ \\
\hline 3146 & Ingersoll Academic. & actor: Chris Ingersoll movie: Academic Advances \\
\hline 3147 & Daughter 1915. & movie: Corporal's Daughter year: 1915 \\
\hline 3148 & King By Impulse! & actor: Henry King movie: By Impulse \\
\hline 3149 & 1921 Chef? & year: 1921 movie: Chef \\
\hline 3150 & 2004 Escapes. & year: 2004 movie: Dalziel and Pascoe: Great Escapes \\
\hline 3151 & Godfrey Ocean. & $\begin{array}{l}\text { actor: Arthur Godfrey movie: America: The Ocean } \\
\text { Frontier }\end{array}$ \\
\hline 3152 & 1911 president. & $\begin{array}{c}\text { year: } 1911 \text { movie: Bezoek van president Fallires aan } \\
\text { Brussel. Mei } 1911\end{array}$ \\
\hline 3153 & Crowe ANZACs & actor: Russell Crowe movie: Colour of War: The ANZACs \\
\hline 3154 & 6.4 Wash. & rank: 6.4 movie: Big Wash \\
\hline 3155 & Kaitlyn Come & actor: Kaitlyn Ashley movie: Come On \\
\hline 3156 & 1995 Blue! & year: 1995 movie: Blue River \\
\hline 3157 & Accused Mandela. & $\begin{array}{c}\text { movie: Accused \#1: Nelson Mandela actor: Nelson } \\
\text { Mandela }\end{array}$ \\
\hline 3158 & Burglar 1915! & movie: Bachelor's Burglar year: 1915 \\
\hline 3159 & Armetta Country. & actor: Henry Armetta movie: Cross Country Cruise \\
\hline 3160 & 5.9 Lupin? & rank: 5.9 movie: Arsne Lupin contre Arsne Lupin \\
\hline 3161 & 1985 Smiler. & year: 1985 movie: Bright Smiler \\
\hline 3162 & Montesano Bernegal! & actor: Sam Montesano movie: Bernegal \\
\hline 3163 & $\begin{array}{c}\text { Armando } \\
\text { Dalaguinding! }\end{array}$ & actor: Armando Goyena movie: Dalaguinding \\
\hline 3164 & Corvette $1943 ?$ & movie: Corvette K-225 year: 1943 \\
\hline
\end{tabular}




\begin{tabular}{|c|c|c|}
\hline 3165 & Symon Corner & role: Symon movie: Corner of the Eye \\
\hline 3166 & Georgi 1977. & actor: Georgi Burkov year: 1977 \\
\hline 3167 & 1979 Canned. & year: 1979 movie: Canned Laughter \\
\hline 3168 & Crime Sewell. & movie: Crime Story actor: George Sewell \\
\hline 3169 & verde $6 !$ & movie: Cuori al verde rank: 6 \\
\hline 3170 & 1977 unjeonsa. & year: 1977 movie: Byeolmyeongbuteun unjeonsa \\
\hline 3171 & sebation eyes & actor: Malcolm Sebastian movie: Angel Eyes \\
\hline 3172 & Rock Benatar? & $\begin{array}{l}\text { movie: } 100 \text { Greatest Women of Rock \& Roll actor: Pat } \\
\text { Benatar }\end{array}$ \\
\hline 3173 & 2000 South! & year: 2000 movie: Christmas in South Park \\
\hline 3174 & Craven 1912 ! & movie: Craven year: 1912 \\
\hline 3175 & Baldwin Drill. & actor: Frank Baldwin movie: Band Drill \\
\hline 3176 & 1999 Haggard! & year: 1999 movie: Best of Merle Haggard \\
\hline 3177 & Michael Anniversary. & actor: Michael G. Carroll movie: Anniversary Party \\
\hline 3178 & Avenging 1915 & movie: Avenging Hand year: 1915 \\
\hline 3179 & 1985 primavera & year: 1985 movie: Con la primavera en el bolsillo \\
\hline 3180 & Ivan Chuzhiye & actor: Ivan Bortnik movie: Chuzhiye pisma \\
\hline 3181 & Citizen Kuan & movie: Citizen Shaw actor: Kuan Tai Chen \\
\hline 3182 & 1952 African. & year: 1952 movie: African Treasure \\
\hline 3183 & Clues Gibson. & movie: Crossed Clues actor: Hoot Gibson \\
\hline 3184 & 7.1 Hero & rank: 7.1 movie: Bruce the Super Hero \\
\hline 3185 & Mograbi Erution & $\begin{array}{l}\text { actor: Avi Mograbi movie: August: A Moment Before the } \\
\text { Eruption }\end{array}$ \\
\hline 3186 & Snow $3.1 ?$ & movie: Black Snow rank: 3.1 \\
\hline 3187 & Robert Sun? & actor: Robert Desiderio movie: At Night the Sun Shines \\
\hline 3188 & Beneath Fowler. & movie: Beneath the Sea actor: Charles Fowler \\
\hline 3189 & Riata $2000 ?$ & movie: Corrido de la Riata de Michoacn year: 2000 \\
\hline 3190 & 5 Bullet! & rank: 5 movie: Bullet \\
\hline 3191 & 1934 Borneo. & year: 1934 movie: Beast of Borneo \\
\hline 3192 & Holland Bosworth & movie: Barge Man of Old Holland actor: Hobart Bosworth \\
\hline 3193 & 2001 Scared & year: 2001 movie: Bare Wench Project 2: Scared Topless \\
\hline 3194 & Mohnish Aag! & actor: Mohnish Bahl movie: Aag \\
\hline 3195 & 1959 Brls! & year: 1959 movie: Brls \\
\hline 3196 & Alcaraz parchada. & actor: Eduardo Alcaraz movie: Cara parchada \\
\hline 3197 & 6.8 BigLove! & rank: 6.8 movie: BigLove \\
\hline 3198 & Batas 2002 & movie: Batas ng lansangan year: 2002 \\
\hline 3199 & Bobby Andaa. & actor: Bobby Bedi movie: Andaaz \\
\hline 3200 & Orioles 1924. & movie: Battling Orioles year: 1924 \\
\hline 3201 & Seymour Wife? & actor: Seymour Hicks movie: Always Tell Your Wife \\
\hline 3202 & 1938 Paris. & year: 1938 movie: Caf de Paris \\
\hline
\end{tabular}




\begin{tabular}{|c|c|c|}
\hline 3203 & Blues Callaghan & movie: Blues actor: Barry Callaghan \\
\hline 3204 & Arquette Behind. & actor: David Arquette movie: Behind the 'Scream' \\
\hline 3205 & 1998 Cosmotic! & year: 1998 movie: Captain Cosmotic \\
\hline 3206 & 5.32069 & rank: 5.3 movie: 2069 A.D. \\
\hline 3207 & 1980 Body. & year: 1980 movie: Body Human: The Body Beautiful \\
\hline 3208 & Dario Immagini & $\begin{array}{c}\text { actor: Dario Argento movie: Bellissimo: Immagini del } \\
\text { cinema italiano }\end{array}$ \\
\hline 3209 & bestias 1.4 ! & movie: Carnaval de las bestias rank: 1.4 \\
\hline 3210 & 1990 Dead & year: 1990 movie: Brain Dead \\
\hline 3211 & Harvest Anderson & $\begin{array}{l}\text { movie: American Harvest actor: John Anderson gender: } \\
\text { Mal }\end{array}$ \\
\hline 3212 & kizuna 1969 & movie: Ai no kizuna year: 1969 \\
\hline 3213 & 1951 Burlada? & year: 1951 movie: Burlada \\
\hline 3214 & Blood Frank & movie: Blood Will Tell actor: Frank Borzage \\
\hline 3215 & Blossoms 1933 & movie: Crime at Blossoms year: 1933 \\
\hline 3216 & 8.9 chudes. & rank: 8.9 movie: Alisa v strane chudes \\
\hline 3217 & 1914 Baked & year: 1914 movie: Can of Baked Beans \\
\hline 3218 & Veronica Conrad? & movie: Biohazard Code: Veronica actor: Conrad Coates \\
\hline 3219 & Affair Bloom. & actor: John Bloom movie: Cartier Affair \\
\hline 3220 & Butter 1994! & movie: Butter year: 1994 \\
\hline 3221 & Lee Argument? & actor: Lee Montague movie: Argument \\
\hline 3222 & 1972 coeur! & year: 1972 movie: Avec le coeur \\
\hline 3223 & 7.5 Bodies & rank: 7.5 movie: Busy Bodies \\
\hline 3224 & Ahmed Bamboleho? & role: Ahmed movie: Bamboleho \\
\hline 3225 & Afro Centrix DiSilva & $\begin{array}{l}\text { movie: Afro-Centrix Volume 48: Just Say Blow \#2 actor: } \\
\text { Guy DiSilva }\end{array}$ \\
\hline 3226 & 1914 Brewerytown & year: 1914 movie: Brewerytown Romance \\
\hline 3227 & Bathala 1958. & movie: Anino ni Bathala year: 1958 \\
\hline 3228 & 1967 Chain & year: 1967 movie: Chain of Command \\
\hline 3229 & Joe 6.5 & movie: Crime Against Joe rank: 6.5 \\
\hline 3230 & Jeff Heston violenta! & role: Jeff Heston movie: Citt violenta \\
\hline 3231 & Dunne 1913 & movie: Capturing of David Dunne year: 1913 \\
\hline 3232 & European Lawrence & movie: Aaron's European Friend actor: Aaron Lawrence \\
\hline 3233 & 1974 Corpse & year: 1974 movie: Corpse Eaters \\
\hline 3234 & Earheart 48 & actor: Amy Earhart movie: 48 Film Hour \\
\hline 3235 & Arise 7.2 & movie: Arise rank: 7.2 \\
\hline 3236 & plage Laarbi & movie: Caf de la plage (2001/II) actor: Laarbi Dahbi \\
\hline 3237 & 1987 Fever. & year: 1987 movie: Beach Fever \\
\hline 3238 & 5.9 Bind! & rank: 5.9 movie: Blind Alley \\
\hline 3239 & rosse 1 & movie: Bugie rosse rank: 1 \\
\hline
\end{tabular}




\begin{tabular}{|c|c|c|}
\hline 3240 & 1949 veleni & year: 1949 movie: Amori e veleni \\
\hline 3241 & Doctor 1936. & movie: Country Doctor year: 1936 \\
\hline 3242 & Joseph Belmont! & $\begin{array}{c}\text { actor: Joseph Belmont movie: Baldy Belmont Lands a } \\
\text { Society Job }\end{array}$ \\
\hline 3243 & Whoes Flows 20? & $\begin{array}{l}\text { movie: Chillin' with Jake Steed's Freaks Whoes \& Flows } \\
\qquad 20\end{array}$ \\
\hline 3244 & Griffith $1914 ?$ & actor: Henry Griffith year: 1914 \\
\hline 3245 & Igor in drama? & role: Igor genre: Drama \\
\hline 3246 & $\begin{array}{l}\text { Drew Sidney } \\
\text { resemblance }\end{array}$ & actor: Sidney Drew movie:: A Close Resemblance \\
\hline 3247 & B.B. King in Africa & movie: B.B. King: Live in Africa \\
\hline 3248 & Sawamura Sonojo 1986 & role: Sonojo Sawamura year: 1986 \\
\hline 3249 & Bogarde Dirk Daddy! & actor: Dirk Bogarde role: Daddy \\
\hline 3250 & bir insan-Ayhan & movie: Bir yudum insan-Ayhan Isik \\
\hline 3251 & 1915 Buss Harry? & year: 1915 actor: Harry Buss \\
\hline 3252 & branded & movie: Branded \\
\hline 3253 & $\begin{array}{l}\text { Bentley Lamont } \\
\text { movies! }\end{array}$ & actor: Lamont Bentley \\
\hline 3254 & beyond 1996 call & movie: Beyond the Call year: 1996 \\
\hline 3255 & Miller Chicken Night. & actor: Joshua Miller movie: Chicken Night \\
\hline 3256 & Bluffing Bluffers & movie: Bluffing Bluffers \\
\hline 3257 & $\begin{array}{l}\text { Begolli Faruk udar } \\
\text { Crveni! }\end{array}$ & actor: Faruk Begolli movie: Crveni udar \\
\hline 3258 & $\begin{array}{l}\text { Abascal Petra lgrimas } \\
\text { von! }\end{array}$ & $\begin{array}{c}\text { actor: Silvia Abascal movie: Amargas lgrimas de Petra von } \\
\text { Kant }\end{array}$ \\
\hline 3259 & $\begin{array}{l}\text { Asrami Ramchana Aap } \\
\text { Beeti. }\end{array}$ & actor: Ramchana Asrami movie: Aap Beeti \\
\hline 3260 & arvo movies? & actor: Arvo Pärt \\
\hline 3261 & Foxx Tanya movies & actor: Tanya Foxx \\
\hline 3262 & 1917 Jock & year: 1917 actor: Jock Preston \\
\hline 3263 & john Bayliss movies? & actor: John Bayliss \\
\hline 3264 & 1961 night long & year: 1961 movie: All Night long \\
\hline 3265 & $\begin{array}{l}2000 \text { factories of } \\
\text { animal }\end{array}$ & year: 2000 movie: Animal Factory \\
\hline 3266 & Aiza 1968! & actor: Gonzalo Aiza year: 1968 \\
\hline 3267 & short of 1919 & year: 1919 genre: Short \\
\hline 3268 & animations of 1946 & year: 1946 genre: Animation \\
\hline 3269 & 1946 bruce lee & year: 1946 actor: bruce lee \\
\hline 3270 & $\begin{array}{c}\text { Pascoe The British } \\
\text { Alex Baillie-Hamilton! }\end{array}$ & $\begin{array}{l}\text { movie: Dalziel and Pascoe: The British Grenadier actor: } \\
\text { Alex Baillie-Hamilton }\end{array}$ \\
\hline 3271 & bhabhi & movie: Bhabhi \\
\hline 3272 & Esters brother & role: Ester's brother \\
\hline
\end{tabular}




\begin{tabular}{|c|c|c|}
\hline 3273 & adult genre of 1999 & year: 1999 genre: Adult \\
\hline 3274 & $\begin{array}{l}\text { movies with David } \\
\text { Fenner? }\end{array}$ & actor: David Fenner \\
\hline 3275 & 55 nulya & movie: 55 gradusov nizhe nulya \\
\hline 3276 & $\begin{array}{l}\text { bachelor and baby } \\
\text { movie? }\end{array}$ & movie: Bachelor and the Baby \\
\hline 3277 & 1975 Baretta? & year: 1975 movie: Baretta \\
\hline 3278 & jack as Jin & role: Jin actor: jack john \\
\hline 3279 & Hero Super bruce? & movie: Bruce the Super Hero \\
\hline 3280 & 1975 alises & year: 1975 actor: Adolfo Alises \\
\hline 3281 & $\begin{array}{l}\text { voglio ti bene Cioni } \\
\text { Mario. }\end{array}$ & movie: Berlinguer ti voglio bene role: Cioni Mario \\
\hline 3282 & Henry Dawes babs? & role: Henry Dawes movie: Babs \\
\hline 3283 & 1985 carolina & movie: Carolina dice year: 1985 \\
\hline 3284 & 1999 Brinkman! & year: 1999 actor: Bo Brinkman \\
\hline 3285 & Ametralladora & movie: Ametralladora \\
\hline 3286 & 1936 Broadcast? & movie: Big Broadcast of 1936 \\
\hline 3287 & imposible amor & movie: amor imposible \\
\hline 3288 & bettenstudent mdchen & $\begin{array}{l}\text { movie: Bettenstudent oder Was mach' ich mit den } \\
\text { Mdchen? }\end{array}$ \\
\hline 3289 & Perkins 2000. & actor: Jack Perkins year: 2000 \\
\hline 3290 & Aguilar correo norte & actor: Luis Aguilar movie: Correo del norte \\
\hline 3291 & Chinese 2002! & movie: Chinese muur year: 2002 \\
\hline 3292 & Black 5.6 & movie: Black Lash rank: 5.6 \\
\hline 3293 & prisoner of America & movie: American prisoner \\
\hline 3294 & sam allen movie & actor: Sam Allen \\
\hline 3295 & Antico Virgilio movies & actor: Virgilio Antico \\
\hline 3296 & movies with Panchik & actor: N. Panchik \\
\hline 3297 & gough comedy & actor: John Gough genre: comedy \\
\hline 3298 & Avgat. & movie: Avgat \\
\hline 3299 & george boy & actor: Boy George \\
\hline 3300 & $\begin{array}{l}1995 \text { Conductors of } \\
\text { Great. }\end{array}$ & $\begin{array}{c}\text { year: } 1995 \text { movie: Art of Conducting: Great Conductors of } \\
\text { the Past }\end{array}$ \\
\hline 3301 & 1935 Fury Black & year: 1935 movie: Black Fury \\
\hline 3302 & ice champions & movie: Champions on Ice \\
\hline 3303 & 1964 alta & year: 1964 movie: Alta infedelt \\
\hline 3304 & Rui Guilherme Miguel & role: Rui actor: Miguel Guilherme \\
\hline 3305 & 63 bolivar & movie: Bolivar 63-29 \\
\hline 3306 & karasi Ailenin & movie: Ailenin yz Karasi \\
\hline 3307 & ancel birdcage & actor: Jordan Ancel movie: Birdcage \\
\hline 3308 & texas kid ambush & movie: Adventures of the Texas Kid: Border Ambush \\
\hline
\end{tabular}




\begin{tabular}{|c|c|c|}
\hline 3309 & carney in 1976 & actor: Art Carney year: 1976 \\
\hline 3310 & 1976 Attack! & year: 1976 movie: Alien Attack \\
\hline 3311 & 2000 elliot & year: 2000 movie: Billy Elliot \\
\hline 3312 & boundary rider & movie: Boundary Rider \\
\hline 3313 & donnell \& bong & movie: Bong \& Donnell \\
\hline 3314 & Richters Ken & actor: Ken Richters \\
\hline 3315 & Gino Baghetti? & actor: Gino Baghetti \\
\hline 3316 & Billy Quirk? & actor: Billy Quirk \\
\hline 3317 & $\begin{array}{l}\text { lectures illimites les } \\
1981 .\end{array}$ & movie: Agatha et les lectures illimites year: 1981 \\
\hline 3318 & Enrique Amores? & actor: Enrique Amores \\
\hline 3319 & chapeau-claque $1909 ?$ & movie: Chapeau-claque year: 1909 \\
\hline 3320 & 1991 BÃ $₫$ cker Ingemar & year: 1991 actor: Ingemar Böcker \\
\hline 3321 & $1955 \mathrm{Rao} !$ & year: 1955 actor: Nageshwara Rao Akkineni \\
\hline 3322 & Abraham Murray 1990? & actor: F. Murray Abraham year: 1990 \\
\hline 3323 & Black Waters & movie: Black Waters \\
\hline 3324 & lechner of 1995 & year: 1995 actor: andreas lechner \\
\hline 3325 & $24 / 7$ and 10 & movie: $24 / 710$ gender: $\mathrm{m}$ \\
\hline 3326 & blue marine baby & movie: Baby blue marine \\
\hline 3327 & dramas of 1961 & year: 1961 genre: drama \\
\hline 3328 & artun $1954 ?$ & actor: sevki artun year: 1954 \\
\hline 3329 & werner in short & actor: Bob Werner genre: Short \\
\hline 3330 & blast Silence 1961. & movie: Blast of Silence year: 1961 \\
\hline 3331 & $\begin{array}{c}\text { Justice All Bates } \\
\text { Marvin! }\end{array}$ & movie: ...And Justice for All role: Marvin Bates \\
\hline 3332 & comedy of 8.3 rating & rank: 8.3 genre: comedy \\
\hline 3333 & $\begin{array}{l}\text { Abercrombie Ian } \\
\text { movies? }\end{array}$ & actor: Ian Abercrombie \\
\hline 3334 & Alessandro Daniel & role: Alessandro actor: Daniel Briquet \\
\hline 3335 & Alsace 1936. & actor: Gene Alsace year: 1936 \\
\hline 3336 & Bookworm Battling! & movie: Battling Bookworm \\
\hline 3337 & 2000 Manola & $\begin{array}{c}\text { year: } 2000 \text { movie: Comedy Central Presents: Pablo } \\
\text { Francisco }\end{array}$ \\
\hline 3338 & gunmen of Dangerous & movie: Dangerous role: Gunmen \\
\hline 3339 & Bademeister? & movie: Bademeister-Report \\
\hline 3340 & 2003 drama & genre: Drama year: 2003 \\
\hline 3341 & John $7.6 ?$ & actor: John Allmond rank: 7.6 \\
\hline 3342 & krds szerelemrl & movie: 7 krds a szerelemrl \\
\hline 3343 & Chino $1998 ?$ & movie: Buscando al Chino year: 1998 \\
\hline 3344 & 2002 Anthony & year: 2002 role: Anthony \\
\hline
\end{tabular}




\begin{tabular}{|c|c|c|}
\hline 3345 & are sheep black? & movie: Black Sheep \\
\hline 3346 & Carlos Portugus! & $\begin{array}{c}\text { actor: Antônio Carlos movie: Aventuras de Um Detetive } \\
\text { Portugus }\end{array}$ \\
\hline 3347 & Barreled? & movie: Barreled \\
\hline 3348 & 1987 Babettes? & year: 1987 movie: Babettes gstebud \\
\hline 3349 & thrillers of 1999 & year: 1999 genre: Thriller \\
\hline 3350 & 4.8 Victor. & rank: 4.8 role: Victor \\
\hline 3351 & 1990 Scott Cummins & year: 1990 actor: Gregory Scott Cummins \\
\hline 3352 & daughter of armorer & movie: Armorer's Daughter \\
\hline 3353 & 1989 horner movie & year: 1989 actor: Mike Horner \\
\hline 3354 & Max Stern role 2001 & role: Max Stern year: 2001 \\
\hline 3355 & Jari 2003! & actor: Jari Abusuis year: 2003 \\
\hline 3356 & $\begin{array}{l}\text { Alex Bolano Max } \\
\text { Young. }\end{array}$ & actor: Alex Bolano role: Young Max \\
\hline 3357 & 1943 pedro movie & year: 1943 actor: Pedro Calderón \\
\hline 3358 & 1938 On Come. & year: 1938 movie: Come On \\
\hline 3359 & Agenzia di Lodolini? & movie: Agenzia matrimoniale role: Zio di Lodolini \\
\hline 3360 & 2003 Mario & year: 2003 role: Mario \\
\hline 3361 & 6.2 Cimarron? & rank: 6.2 movie: Cimarron Kid \\
\hline 3362 & akmyeong & movie: Akmyeong \\
\hline 3363 & clubbable woman & movie: Dalziel and Pascoe: A Clubbable Woman \\
\hline 3364 & Raymond Bayad? & actor: Raymond Bagatsing movie: Bayad puri \\
\hline 3365 & $\begin{array}{l}\text { Cannon Raymond } \\
\text { Kathlyn }\end{array}$ & actor: Raymond Cannon movie: Adventures of Kathlyn \\
\hline 3366 & $\begin{array}{l}2001 \text { Michael } \\
\text { Bertenshaw }\end{array}$ & year: 2001 actor: Michael Bertenshaw \\
\hline 3367 & $\begin{array}{l}\text { Chinese Commandos } \\
\text { NA }\end{array}$ & movie: Chinese Commandos \\
\hline 3368 & 1946 Vernon Dent. & year: 1946 actor: Vernon Dent \\
\hline 3369 & Alamo Thirteen? & movie: Alamo: Thirteen Days to Glory \\
\hline 3370 & Pedro Amor-Love? & role: Pedro movie: Amor-Love \\
\hline 3371 & 2002 short in spanish & year: 2002 genre: Short \\
\hline 3372 & 1992 chaplin puzzle & year: 1992 movie: Chaplin Puzzle \\
\hline 3373 & evil maker $2 ?$ & movie: Abomination: Evil Maker 2 \\
\hline 3374 & 1996 Black. & year: 1996 movie: Black Kites \\
\hline 3375 & zhong Beijing! & movie: Beijing za zhong \\
\hline 3376 & colorado conquering & movie: conquering the colorado \\
\hline 3377 & buin baeksa & movie: Baeksa buin \\
\hline 3378 & 1960 sci fi & year: 1960 genre: Sci-Fi \\
\hline 3379 & Afta $7.5 !$ & movie: Afta rank: 7.5 \\
\hline 3380 & carol movies? & actor: John Carol \\
\hline
\end{tabular}




\begin{tabular}{|c|c|c|}
\hline 3381 & 1995 dramas?. & year: 1995 genre: Drama \\
\hline 3382 & $\begin{array}{c}\text { Louis Desfontaines } \\
\text { movie }\end{array}$ & role: Louis Desfontaines \\
\hline 3383 & Air Ross & movie: Air Hostess actor: Ross Ford \\
\hline 3384 & jack edwards movies? & actor: Jack (V) Edwards \\
\hline 3385 & Akasya 1974. & actor: Zeki Alasya year: 1974 \\
\hline 3386 & samuel jackson movies & actor: Samuel L. Jackson \\
\hline 3387 & music awards. & movie: American Music Awards \\
\hline 3388 & $\begin{array}{l}\text { Alimony Arthur } \\
\text { Allardt. }\end{array}$ & movie: Alimony actor: Arthur Allardt \\
\hline 3389 & 7.1 BillettkontrollÃ ,r! & rank: 7.1 role: Billettkontrollør \\
\hline 3390 & bonne mesure? & movie: Bonne mesure des distances \\
\hline 3391 & Johnny 1969. & role: Johnny year: 1969 \\
\hline 3392 & 1987 dark bros & year: 1987 movie: Best of the Dark Bros. \\
\hline 3393 & $\begin{array}{c}\text { broncho claim jumpers } \\
\text { of } 1915 .\end{array}$ & movie: Broncho Billy and the Claim Jumpers year: 1915 \\
\hline 3394 & Amangeldy Kapan. & movie: Amangeldy actor: Kapan Badyrov \\
\hline 3395 & Mirza Bahu. & role: Nawab Sikandar Mirza movie: Bahu Begum \\
\hline 3396 & abuelo & movie: Abuelo \\
\hline 3397 & 1955 drama movies & year: 1955 genre: Drama \\
\hline 3398 & 1974 crime movies? & year: 1974 genre: Crime \\
\hline 3399 & Comeback Comeback! & movie: Comeback \\
\hline 3400 & abduction club movies & movie: Abduction Club \\
\hline 3401 & $\mathrm{G} \tilde{\mathrm{A}}^{3} \mathrm{mez} 4.8$ & actor: Carmelo Gómez rank: 4.8 \\
\hline 3402 & Abrego Fidel 1990. & actor: Fidel Abrego year: 1990 \\
\hline 3403 & Albornoz 1921! & actor: Agustín Carrillo de Albornoz year: 1921 \\
\hline 3404 & blanc animation movies & genre: Animation actor: Mel Blanc \\
\hline 3405 & ciclone lento sutil? & movie: Ciclone Lento E Sutil \\
\hline 3406 & Squeeze Big? & movie: Big Squeeze \\
\hline 3407 & Craps James & movie: Craps actor: James Coburn \\
\hline 3408 & entrar de antes & movie: Antes de entrar \\
\hline 3409 & alma 1997. & movie: Alma year: 1997 \\
\hline 3410 & Monty and 1.8. & role: Monty rank: 1.8 \\
\hline 3411 & arnal rating 5.3 & actor: François Arnal rank: 5.3 \\
\hline 3412 & 1927 with Tige & year: 1927 role: Tige \\
\hline 3413 & Lmpada a maravilhosa & movie: Aladim e a Lmpada Maravilhosa \\
\hline 3414 & 1996 rating 6.2 & year: 1996 rank: 6.2 \\
\hline 3415 & inmortal Comedia? & movie: Comedia inmortal \\
\hline 3416 & abbayi America & movie: America abbayi \\
\hline 3417 & boss of $1911 ?$ & actor: Yale Boss year: 1911 \\
\hline
\end{tabular}




\begin{tabular}{|c|c|c|}
\hline 3418 & blank as downs & actor: Harry Blank role: Frank Downs \\
\hline 3419 & boys and girls $\mathrm{Al}$ cooke & movie: Boys Will Be Girls actor: Al Cooke \\
\hline 3420 & 1963 Nelson & year: 1963 actor: Sheridon Nelson \\
\hline 3421 & Pietro movies & actor: Pietro Biondi \\
\hline 3422 & 7.9 rating in 1954 & year: 1954 rank: 7.9 \\
\hline 3423 & Clerk in The 1913 & role: The Clerk year: 1913 \\
\hline 3424 & autres et les Bronzs. & movie: Bronzs, le pre Nol, papy et les autres, Les \\
\hline 3425 & 1971 borge movie & year: 1971 actor: Victor Borge \\
\hline 3426 & Female ari. & gender: f actor: michiko Ari \\
\hline 3427 & 6 Shelby Bacon. & rank: 6 actor: Shelby Bacon \\
\hline 3428 & movies with Ed clark & actor: Ed Clark \\
\hline 3429 & Hickman as Himself & actor: Bill Hickman role: Himself \\
\hline 3430 & Charley Accident. & role: Charley movie: Accident \\
\hline 3431 & Brave & movie: Brave \\
\hline 3432 & $\begin{array}{l}7.4 \text { rating with male } \\
\text { actor }\end{array}$ & rank: 7.4 gender: $\mathrm{m}$ \\
\hline 3433 & Siddall Cory movie. & actor: Cory Siddall \\
\hline 3434 & Bob of $1996 !$ & role: Bob year: 1966 \\
\hline 3435 & Dirk Writing & actor: Dirk Bogarde role: Himself - Presenter: Writing \\
\hline 3436 & 2003 certain justice & year: 2003 movie: Certain Justice \\
\hline 3437 & bicheongwon & movie: Bicheongwon \\
\hline 3438 & cielo rojo & movie: cielo rojo \\
\hline 3439 & $\begin{array}{l}\text { adult } 1993 \text { Video } \\
\text { Awards. }\end{array}$ & genre: Adult movie: Adult Video News Awards 1993 \\
\hline 3440 & Carlos Careqa & actor: Carlos Careqa \\
\hline 3441 & Martin Blind Lead. & actor: Martin Bedouim movie: Blind Lead \\
\hline 3442 & Arthur Herbie! & actor: Robert Arthur role: Herbie Cook \\
\hline 3443 & Crossover 2000? & movie: Crossover year: 2000 \\
\hline 3444 & 1982 student & year: 1982 role: student \\
\hline 3445 & 1984 City killer? & year: 1984 movie: City Killer \\
\hline 3446 & stanley adams of 1974 & actor: Stanley Adams year: 1974 \\
\hline 3447 & $\begin{array}{l}\text { hepburn story Adams I } \\
\text { I }\end{array}$ & movie: Audrey Hepburn Story actor: Steve Adams \\
\hline 3448 & action movies of 1993 & genre: Action year: 1993 \\
\hline 3449 & $\begin{array}{l}\text { buster and priates } \\
1912 ?\end{array}$ & movie: Buster and the Pirates year: 1912 \\
\hline 3450 & laurie annie bonnie & movie: Bonnie Annie Laurie \\
\hline 3451 & 1985 con frente polvo & year: 1985 movie: Con la frente en el polvo \\
\hline 3452 & ryan of backseat driver & movie: Backseat Driver 13 role: Ryan \\
\hline 3453 & $\begin{array}{l}\text { Reinette et Auffray } \\
\text { Jacques. }\end{array}$ & $\begin{array}{l}\text { movie: } 4 \text { aventures de Reinette et Mirabelle actor: Jacques } \\
\text { Auffray }\end{array}$ \\
\hline
\end{tabular}




\begin{tabular}{|c|c|c|}
\hline 3454 & 1959 Manuel? & year: 1959 actor: Manuel Arbó \\
\hline 3455 & Captain 2003. & role: Captain Kram year: 2003 \\
\hline 3456 & cmo se repite ciudad & movie: Cmo se repite una ciudad \\
\hline 3457 & 1985 rating 5.1 & year: 1985 rank: 5.1 \\
\hline 3458 & 5.1 Waiter & rank: 5.1 role: Skyland Cafe Waiter \\
\hline 3459 & romain cercle & movie: Cercle Romain \\
\hline 3460 & adventure with Chanoc! & genre:adventure role: Chanoc \\
\hline 3461 & 1931 Air! & year: 1931 movie: Air Eagles \\
\hline 3462 & western 1989 & genre: western year: 1989 \\
\hline 3463 & thriller of 2002 & year: 2002 genre: Thriller \\
\hline 3464 & onesti degli! & movie: Banda degli onesti \\
\hline 3465 & jack little in 1995 & actor: jack little year: 1995 \\
\hline 3466 & movies with Josef Bek & actor: Josef Bek \\
\hline 3467 & Receptionist carl & role: Receptionist movie: D-dag - carl \\
\hline 3468 & 2004 Takeo. & year: 2004 actor: Takeo Nakahara \\
\hline 3469 & Hongkong 2 Masters? & movie: Dancing Masters 2 (Hongkong) \\
\hline 3470 & 2003 rambold & year: 2003 role: Rambold \\
\hline 3471 & cowboys and Indians & movie: Cowboys and Indians \\
\hline 3472 & chao feng chao? & movie: Dan feng chao yang \\
\hline 3473 & Budenje Male? & movie: Budenje gender: $\mathrm{m}$ \\
\hline 3474 & cuando araas & movie: Cuando tejen las araas \\
\hline 3475 & bagen of 1985 & year: 1985 movie: Bagets 2 \\
\hline 3476 & anima sayula & movie: Anima de Sayula \\
\hline 3477 & arena Ayala Vicente! & movie: Bajo la arena actor: Vicente Ayala \\
\hline 3478 & documentary 1991? & year: 1991 genre: Documentary \\
\hline 3479 & terry clark as Performer & actor: Terry Clark role: Performer \\
\hline 3480 & tout le Treton Gilles. & movie: Comme tout le monde actor: Gilles Treton \\
\hline 3481 & Air Guard! & movie: Con Air role: Guard Garner \\
\hline 3482 & Bohm 1979! & actor: Hark Bohm year: 1979 \\
\hline 3483 & 1917 Lyons & year: 1917 actor: Eddie Lyons \\
\hline 3484 & 1979 short & year: 1979 genre: short \\
\hline 3485 & 1973 Buron & year: 1973 actor: Jacques Buron \\
\hline 3486 & 6.6 insaeng & rank: 6 movie: Ahobsal insaeng \\
\hline 3487 & 1994 romance movies & year: 1994 genre: Romance \\
\hline 3488 & $\begin{array}{c}\text { movies with Roberto } \\
\text { Alrean! }\end{array}$ & actor: Roberto Alrean \\
\hline 3489 & $\begin{array}{c}\text { Jason Allentoff as } \\
\text { Himself? }\end{array}$ & actor: Jason Allentoff role: Himself \\
\hline 3490 & Blood 1923 & movie: Blood Test year: 1923 \\
\hline 3491 & Villa Pancho & movie: Cabeza de Pancho Villa \\
\hline
\end{tabular}




\begin{tabular}{|c|c|c|}
\hline 3492 & movies with Wheaton & actor: Loyd Wheaton \\
\hline 3493 & Canadiens conformes? & movie: Canadiens conformes \\
\hline 3494 & awakening of candra & movie: Awakening of Candra \\
\hline 3495 & Angelo 1984 & actor: Paul Angelo year: 1984 \\
\hline 3496 & Jules 1921? & actor: Jules Raucourt year: 1921 \\
\hline 3497 & 19875.7 & year: 1987 rank: 5.7 \\
\hline 3498 & $\begin{array}{l}\text { Nights } 639+1 \text { with } \\
\text { Male actor! }\end{array}$ & movie: $639+1$ Nights gender: $m$ \\
\hline 3499 & Buster Brown 1926 & role: Buster Brown year: 1926 \\
\hline 3500 & mythologies American. & movie: American Mythologies \\
\hline 3501 & never late of 1922 & year: 1922 movie: Better Late Than Never \\
\hline 3502 & Rufus Beck Ole. & actor: Rufus Beck role: Ole \\
\hline 3503 & Blacksmith and art & movie: Art and Mystery of the Blacksmith \\
\hline 3504 & 5.4 and runaway Boy. & rank: 5.4 role: Runaway Boy \#1 \\
\hline 3505 & bubble of 1988 movie? & year: 1988 role: Bubble \\
\hline 3506 & 1963 bodas pico & year: 1963 movie: Cuatro bodas y pico \\
\hline 3507 & sheriff movie rating 5.3 & rank: 5.3 role: Sheriff \\
\hline 3508 & ama prjimo & movie: ama a tu prjimo \\
\hline 3509 & Jeemy of 1921 & role: Jeemy year: 1921 \\
\hline 3510 & $\begin{array}{l}1985 \text { Fernando } \\
\text { ArÃ Cbalo }\end{array}$ & year: 1985 actor: Fernando Arébalo \\
\hline 3511 & 8.4 Len? & rank: 8.4 actor: Len Cariou \\
\hline 3512 & 1985 Michael? & year: 1985 actor: Michael Bacall \\
\hline 3513 & carmen movie? & movie: Carmen \\
\hline 3514 & $\begin{array}{l}\text { manchester united } \\
\text { movie }\end{array}$ & $\begin{array}{c}\text { movie: Champions 2: Manchester United Official Review } \\
\text { of the 93/94 Season }\end{array}$ \\
\hline 3515 & 1994 John Bennes & year: 1994 actor: John Bennes \\
\hline 3516 & Crowd Comedy & movie: Comedy Crowd \\
\hline 3517 & Charles Ye sow & actor: Charles Dungan movie: As Ye Sow \\
\hline 3518 & 19772 Bimilgaek! & year: 1977 movie: Bimilgaek 2 \\
\hline 3519 & movies with Ramiro & actor: Ramiro Gómez \\
\hline 3520 & contrabando huevos & movie: Contrabando en los huevos \\
\hline 3521 & capulina las momias! & movie: Capulina contra las momia \\
\hline 3522 & Charles $1963 ?$ & actor: Charles Horvath year: 1963 \\
\hline 3523 & Ryan $1988 ?$ & actor: Ryan Chu year: 1988 \\
\hline 3524 & Axel 2001 & actor: Axel Monjé year: 2001 \\
\hline 3525 & beat of 1999 & year: 1999 movie: Beat \\
\hline 3526 & Cazador. & movie: Cazador \\
\hline 3527 & John 1996. & actor: John Agar year: 1996 \\
\hline 3528 & 1912 with rating of 6.5 & year: 1912 rank: 6.5 \\
\hline
\end{tabular}




\begin{tabular}{|c|c|c|}
\hline 3529 & hopp allez & movie: Allez Hopp \\
\hline 3530 & Fernando in 1995 & year: 1995 actor: Don Fernando \\
\hline 3531 & 1921 Eddie & year: 1921 actor: Eddie Polo \\
\hline 3532 & ritz childern & movie: Children of the Ritz \\
\hline 3533 & $51980 ?$ & rank: 5 year: 1980 \\
\hline 3534 & Ed Peter? & actor: Ed Amatrudo role: Peter \\
\hline 3535 & $\begin{array}{l}\text { Disney with Roy } \\
\text { Disney Edward }\end{array}$ & $\begin{array}{c}\text { movie: Conversation with Roy Disney actor: Roy Edward } \\
\text { Disney }\end{array}$ \\
\hline 3536 & $\max 2003 !$ & role: Max year: 2003 \\
\hline 3537 & william gaynor movies & actor: William J. Gaynor \\
\hline 3538 & $\begin{array}{l}\text { family movie from } \\
1990\end{array}$ & genre: Family year: 1990 \\
\hline 3539 & cita vida & movie: Cita con la vida \\
\hline 3540 & arizona broadway & movie: Broadway arizona \\
\hline 3541 & $\begin{array}{l}\text { Martin alderdice } \\
\text { movies }\end{array}$ & actor: Martin Alderdice \\
\hline 3542 & Romeo Arizona Harvey & movie: Arizona Romeo actor: Harvey Clark \\
\hline 3543 & Jordan Sid is in? & actor: Sid Jordan \\
\hline 3544 & Hotel 5.6? & role: Hotel Tenants rank: 5.6 \\
\hline 3545 & comedy of 1931 & genre: comedy year: 1931 \\
\hline 3546 & $\begin{array}{l}1932 \text { after tomorrow } \\
\text { verision }\end{array}$ & year: 1932 movie: After Tomorrow \\
\hline 3547 & $\begin{array}{l}\text { movies of Plesa } \\
\text { Branko. }\end{array}$ & actor: Branko Plesa \\
\hline 3548 & conscience movie & movie: Conscience \\
\hline 3549 & $\begin{array}{l}\text { movies with a } \\
\text { Belyavsky! }\end{array}$ & actor: Aleksandr Belyavsky \\
\hline 3550 & 1989 manaloto & year: 1989 actor: Smokey Manaloto \\
\hline 3551 & Campaign 1997? & movie: Campaign year: 1997 \\
\hline 3552 & anerkej vasku & movie: alisa v strane chudes \\
\hline 3553 & Bharathan Paravoor & actor: Paravoor Bharathan \\
\hline 3554 & shane citizen & movie: Citizen shane \\
\hline 3555 & dancing mothers 7.3 & rank: 7.3 movie: Dancing mothers \\
\hline 3556 & Rabelo Bloqueio! & actor: Fernando Rabelo movie: Bloqueio \\
\hline 3557 & $\begin{array}{l}\text { Broncho Anderson } \\
\text { Billy }\end{array}$ & actor: Gilbert Anderson \\
\hline 3558 & pallone Allenatore & movie: Allenatore nel pallone \\
\hline 3559 & Pino Ammendola & actor: Pino Ammendola \\
\hline 3560 & Andreu 5.5. & actor: Simón Andreu rank: 5.5 \\
\hline 3561 & 1945 Bliznetsy & year: 1945 movie: Bliznetsy \\
\hline 3562 & Teacher School 8.3? & role: School Teacher rank: 8.3 \\
\hline
\end{tabular}




\begin{tabular}{|c|c|c|}
\hline 3563 & Algara en las nubes. & actor: Alejandro Algara movie: Amor en las nubes \\
\hline 3564 & Devlin J.G. movies & actor: J.G. Devlin \\
\hline 3565 & martin in Cat & actor: Martin Head movie: Cat \\
\hline 3566 & Claudia adkins movies? & actor: Claudia Adkins \\
\hline 3567 & $6.31989 ?$ & rank: 6.3 year: 1989 \\
\hline 3568 & 6.3 bigamist? & rank: 6.2 movie: Bigamist \\
\hline 3569 & trouble courtin & movie: Courtin' Trouble \\
\hline 3570 & jack horner movies? & gender: $\mathrm{m}$ actor: Jack Horner \\
\hline 3571 & 6.8 Silence Angry! & rank: 6.8 movie: Angry Silence \\
\hline 3572 & 1976 Giuseppe & year: 1976 actor: Giuseppe Anatrelli \\
\hline 3573 & Botijo 1948! & movie: Botijo year: 1948 \\
\hline 3574 & $\begin{array}{l}\text { Begins Desmarteau } \\
\text { Raymond. }\end{array}$ & $\begin{array}{c}\text { movie: Beauty Begins Inside: The 'P' Syndrome actor: } \\
\text { Raymond Desmarteau }\end{array}$ \\
\hline 3575 & cowards of 1915 & year: 1915 movie: Coward \\
\hline 3576 & 1929 John Adams & year: 1928 role: John Adams \\
\hline 3577 & 1924 Broken Barriers? & movie: Broken Barriers year: 1924 \\
\hline 3578 & John Alredge movies? & actor: John Arledge \\
\hline 3579 & $\begin{array}{l}\text { movies named } \\
\text { ascension }\end{array}$ & movie: Ascension \\
\hline 3580 & cohen in 1917 & actor: Joe Cohen year: 1917 \\
\hline 3581 & 1954 Canyon Horse? & year: 1954 movie: Black Horse Canyon \\
\hline 3582 & 1999 conflict & movie: Conflict (1999/II) \\
\hline 3583 & Al Capone as himself & role: Himself actor: Al Capone \\
\hline 3584 & coupe de ville movie & movie: Coupe de Ville \\
\hline 3585 & 2001 cumpleaos & year: 2001 movie: Cumpleaos de Carlos \\
\hline 3586 & 1998 Raider? & year: 1998 role: Raider \\
\hline 3587 & 1926 adorable deceiver & year: 1926 movie: Adorable Deceiver \\
\hline 3588 & $\begin{array}{c}\text { Almada in action } \\
\text { movies } \\
\end{array}$ & actor: Horácio Almada genre: Action \\
\hline 3589 & 1997 alive tribe & year: 1997 movie: Alive Tribe \\
\hline 3590 & Cheik and Alligator? & actor: Cheik Doukouré movie: Alligator \\
\hline 3591 & 2000 cozart & year: 2000 actor: Cylk Cozart \\
\hline 3592 & 20027.1 rating & year: 2002 rank: 7.1 \\
\hline 3593 & iyagi bikiniseom & movie: Bikiniseom iyagi \\
\hline 3594 & 6.6 year 2000 & rank: 6.6 year: 2000 \\
\hline 3595 & 9.1 bagoliini movie & rank: 9.1 actor: Silvio Bagolini \\
\hline 3596 & Coltrane movies & actor: Robbie Coltrane \\
\hline 3597 & 1943 Eliot? & year: 1943 role: Charles Eliot \\
\hline 3598 & chaning guard $1936 ?$ & year: 1936 movie: Changing of the Guard \\
\hline 3599 & bette midler red hair & movie: Bette Midler: Ol' Red Hair Is Back \\
\hline
\end{tabular}




\begin{tabular}{|c|c|c|}
\hline 3600 & 1996 Fire courage & year: 1996 movie: Courage Under Fire \\
\hline 3601 & black cat & movie: Black Cat \\
\hline 3602 & Commencement 1995 & movie: Commencement year: 1995 \\
\hline 3603 & Mathieu Demy 2001? & actor: Mathieu Demy year: 2001 \\
\hline 3604 & Queen Cabbage & movie: Cabbage Queen \\
\hline 3605 & Azul Joseph. & role: Azul actor: Joseph Accerelli \\
\hline 3606 & Francis Samuel movie & actor: Samuel Francis \\
\hline 3607 & Bruidskist! & movie: Bruidskist \\
\hline 3608 & 2004 Gaetano! & year: 2004 role: Gaetano \\
\hline 3609 & $5.91975 !$ & rank: 5.9 year: 1975 \\
\hline 3610 & $\begin{array}{c}1919 \text { movie with } \\
\text { sammy brooks }\end{array}$ & actor: Sammy Brooks year: 1919 \\
\hline 3611 & Tim Allen as Himself & actor: Tim Allen role: Himself \\
\hline 3612 & 1923 Glendon & year: 1923 actor: Pat Lafani \\
\hline 3613 & 2003 Cuddler & year: 2003 movie: Cuddler \\
\hline 3614 & Almond tree 1970 & movie: Cellar and the Almond Tree year: 1970 \\
\hline 3615 & Jack Mercer $1936 ?$ & actor: Jack Mercer year: 1936 \\
\hline 3616 & Work Blood 2002 & movie: Blood Work year: 2002 \\
\hline 3617 & Hedlund Samuel & role: Samuel Hedlund \\
\hline 3618 & Akkineni Rao movie & actor: Nageshwara Rao Akkineni \\
\hline 3619 & 7.4 with Anand & rank: 7.4 actor: Balu Anand \\
\hline 3620 & Juan Castro movies & actor: Juan (VI) Castro \\
\hline 3621 & cup cold water? & movie: Cup of Cold Water \\
\hline 3622 & Dormer movie in 2000 & year: 2000 actor: Richard Dormer \\
\hline 3623 & Ashida 1964? & actor: Yusuke Ashida year: 1964 \\
\hline 3624 & $\begin{array}{c}\text { Archdale Alexander } \\
1979 ?\end{array}$ & actor: Alexander Archdale year: 1979 \\
\hline 3625 & 1994 with rating 6.8 & year: 1994 rank: 6.8 \\
\hline 3626 & abla hanim movie & movie: Bitirimsin hanim abla \\
\hline 3627 & 1999 Buck Adams & year: 1998 actor: Buck Adams \\
\hline 3628 & Luis Alberni movie & actor: Luis Alberni \\
\hline 3629 & seung-Ho in 1996 & actor: Jung-Yul Kim year: 1996 \\
\hline 3630 & 2003 john baker & year: 2003 actor: John Baker \\
\hline 3631 & Dress Suit Bills & movie: Bungling Bill's Dress Suit \\
\hline 3632 & 1936 with ode & actor: Erik Ode year: 1936 \\
\hline 3633 & year 1918 with 6.9 & year: 1918 rank: 6.9 \\
\hline 3634 & 2002 Cameron? & year: 2002 role: Cameron \\
\hline 3635 & hitler as Himself? & actor: Adolf Hitler role: Himself \\
\hline 3636 & 1954 adventure movie & year: 1954 genre: Adventure \\
\hline 3637 & denise in 2001 & year: 2001 actor: Nikita Denise \\
\hline
\end{tabular}




\begin{tabular}{|c|c|c|}
\hline 3638 & Ashes Angela! & movie: Angela's Ashes \\
\hline 3639 & carry on Cruising & movie: Carry On Cruising \\
\hline 3640 & $\begin{array}{l}2005 \text { movie with } \\
\text { Lennon }\end{array}$ & year: 2005 actor: Thomas Lennon \\
\hline 3641 & 2003 dim dik tin! & year: 2003 movie: Dai yat dim dik tin hung \\
\hline 3642 & bardem $2002 ?$ & actor: Carlos Bardem year: 2002 \\
\hline 3643 & Ashmore jay movies & actor: Jay Ashmore \\
\hline 3644 & 1995 Briggs & year: 1995 actor: Ali Briggs \\
\hline 3645 & $\begin{array}{l}\text { Federico Luciano } \\
\text { movie }\end{array}$ & actor: Luciano Federico \\
\hline 3646 & 1967 Herbert? & year: 1967 actor: Herbert Bötticher \\
\hline 3647 & 1956 birds bees & year: 1956 movie: Birds and the Bees \\
\hline 3648 & Cohen Leonard Angel & actor: Leonard Cohen movie: Angel \\
\hline 3649 & Cosmocrator Thomas & movie: Cosmocrator actor: Thomas Badek \\
\hline 3650 & who is Hans Bille. & actor: Hans Bille \\
\hline 3651 & $\begin{array}{c}\text { Edmondson William } \\
1934 .\end{array}$ & actor: William Edmondson year: 1934 \\
\hline 3652 & bei gao shangang & movie: Bei gao Shangang ye \\
\hline 3653 & hawk air $1924 ?$ & movie: Air Hawk year: 1924 \\
\hline 3654 & 1987 Akhanov & year: 1987 actor: Boris Akhanov \\
\hline 3655 & Curupira movie & movie: Curupira \\
\hline 3656 & billy and Navajo Maid & movie: Broncho Billy and the Navajo Maid \\
\hline 3657 & $\begin{array}{c}\text { mariano abud as } \\
\text { himself }\end{array}$ & actor: Mariano Abud role: Himself \\
\hline 3658 & Douzous comedy & actor: Teta Douzou genre: Comedy \\
\hline 3659 & 2004 Tom role? & year: 2004 role: Tom \\
\hline 3660 & $\begin{array}{l}\text { Amzic Jakup Saitovic } \\
\text { Sajin? }\end{array}$ & actor: Jakup Amzic role: Sajin Saitovic \\
\hline 3661 & 1933 Alberni & year: 1933 actor: Luis Alberni \\
\hline 3662 & 2001 rating of 7.1 & year: 2001 rank: 7.3 \\
\hline 3663 & $\begin{array}{c}5.2 \\
\text { Chromiumblue.com. }\end{array}$ & rank: 5.2 movie: Chromiumblue.com \\
\hline 3664 & 1993 detective movie & role: Detective year: 1993 \\
\hline 3665 & chiavi casa 6.9 & rank: 6.9 movie: Chiavi di casa \\
\hline 3666 & Julio in 1975 & actor: Julio Alemán year: 1975 \\
\hline 3667 & Himself Steve Bisley & role: Himself actor: Steve Bisley \\
\hline 3668 & 5.1 Pierre & rank: 5.1 role: Pierre \\
\hline 3669 & Girls Calendar 7 rating. & movie: Calendar Girls rank: 7 \\
\hline 3670 & paradise 1930 & movie: Dangerous Paradise year: 1930 \\
\hline 3671 & $\begin{array}{l}\text { Hayden character } \\
\text { building life }\end{array}$ & $\begin{array}{c}\text { actor: Hayden Christensen movie: Character Building: } \\
\text { Inside 'Life as a House' }\end{array}$ \\
\hline
\end{tabular}




\begin{tabular}{|c|c|c|}
\hline 3672 & 1925 with Booker & year: 1925 actor: John Bowers \\
\hline 3673 & ricky martin audience & movie: Audience with Ricky Martin \\
\hline 3674 & $\begin{array}{l}\text { Henry Merriman } \\
\text { Daniel. }\end{array}$ & role: Henry actor: Daniel Merriman \\
\hline 3675 & $\begin{array}{l}\text { shooting party } \\
\text { adventure }\end{array}$ & movie: Adventure of the Shooting Party \\
\hline 3676 & $\begin{array}{c}\text { Dialogues Pascoe and } \\
\text { Dalziel }\end{array}$ & movie: Dalziel and Pascoe: Dialogues of the Dead \\
\hline 3677 & Pigaut 1962. & actor: Roger Pigaut year: 1962 \\
\hline 3678 & george-martin 1994. & actor: Georg-Martin Bode year: 1994 \\
\hline 3679 & Ivan Sergeevich Puhov & role: Ivan Sergeevich Puhov \\
\hline 3680 & $\begin{array}{c}\text { Peterson } \\
\text { Handelsrejsende }\end{array}$ & actor: Kjeld Petersen role: Handelsrejsende \\
\hline 3681 & cable guy movie & movie: Cable Guy \\
\hline 3682 & $\begin{array}{c}\text { John Deacon as } \\
\text { Himself }\end{array}$ & role: Himself actor: John Deacon \\
\hline 3683 & Raid Sea Raid & movie: Black Sea Raid \\
\hline 3684 & Himself Pancho San. & role: Himself movie: Atltico San Pancho \\
\hline 3685 & 2003 Coeurs & year: 2003 movie: Coeurs d'athltes \\
\hline 3686 & $\begin{array}{l}1984 \text { Almonds and } \\
\text { Raisins! }\end{array}$ & year: 1984 movie: Almonds and Raisins \\
\hline 3687 & $\begin{array}{l}\text { Albel Villarama } \\
\text { Armado }\end{array}$ & movie: Abel Villarama: Armado \\
\hline 3688 & 1898 Sampson & year: 1898 actor: William T. Sampson \\
\hline 3689 & NachtItalian Berlin? & $\begin{array}{l}\text { movie: Aus der Waldbhne Berlin - Italienische } \\
\text { Nacht/Italian Night }\end{array}$ \\
\hline 3690 & bergman movies 1991 & actor: Jeff Bergman year: 1991 \\
\hline 3691 & Peter Lohmeyer 2004 & actor: Peter Lohmeyer year: 2004 \\
\hline 3692 & Bulyeowoo & movie: Bulyeowoo \\
\hline 3693 & Amok movie info? & movie: Amok \\
\hline 3694 & $\begin{array}{l}\text { Red Door Barfoot } \\
\text { Mack }\end{array}$ & movie: Behind the Red Door actor: Mack Barfoot \\
\hline 3695 & Jorge Aldama movies. & actor: Jorge Aldama \\
\hline 3696 & 1978 action movies & year: 1978 genre: Action \\
\hline 3697 & snake kommer movie & movie: Big Fat Snake kommer til byen \\
\hline 3698 & 1920 Eldridge Charles? & year: 1920 actor: Charles Eldridge \\
\hline 3699 & check gun 4.5 & movie: Check Your Guns rank: 4.5 \\
\hline 3700 & $\begin{array}{l}\text { beatles Chuck } \\
\text { Ardezzone. }\end{array}$ & actor: Chuck Ardezzone movie: Beatle Fan \\
\hline 3701 & Christina 2002 & role: Christina year: 2002 \\
\hline 3702 & adult movies of 1988 & year: 1988 genre: adult \\
\hline 3703 & 1962 All fall down & year: 1962 movie: All Fall down \\
\hline
\end{tabular}




\begin{tabular}{|c|c|c|}
\hline & version & \\
\hline 3704 & 2000 Louis Lepke & year: 2000 movie: A\&E biography: Louis Lepke \\
\hline 3705 & 1937 contre Arsne & year: 1937 movie: Arsne Lupin contre Arsne Lupin \\
\hline 3706 & $\begin{array}{l}\text { movies with Mort } \\
\text { Ransen }\end{array}$ & actor: Mort Ransen \\
\hline 3707 & almost Man 1912! & movie: Almost a Man year: 1912 \\
\hline 3708 & 1997 of ShadowBorg & year: 1997 movie: Curse of the ShadowBorg \\
\hline 3709 & Solomzi Bisholo 1985? & actor: Solomzi Bisholo year: 1985 \\
\hline 3710 & marconi in 2000 & year: 2000 actor: Guglielmo Marconi \\
\hline 3711 & Bao Fumin 74. & actor: Fumin Bao rank: 7.4 \\
\hline 3712 & 1934 City Limits. & year: 1934 movie: City Limits \\
\hline 3713 & 2004 Baileys! & year: 2004 movie: Bailey's Billion\$ \\
\hline 3714 & Power Coming? & movie: Coming Power \\
\hline 3715 & movie named betrayal & movie: Betrayal \\
\hline 3716 & mujer Anta Adolfo & movie: Anta mujer actor: Adolfo Morán \\
\hline 3717 & $6.52003 !$ & rank: 6.5 year: 2003 \\
\hline 3718 & Mark Party! & actor: Mark Dold movie: Charlie's Party \\
\hline 3719 & alien Class Appleby. & role: 3rd Class Alien actor: Mike Appleby \\
\hline 3720 & 1997 Linda. & year: 1997 actor: Linda Ellerbee \\
\hline 3721 & $\begin{array}{c}\text { Chirakadze Giga } \\
\text { Challenge. }\end{array}$ & actor: Giga Chirakadze movie: Challenge \\
\hline 3722 & Lucas 2004 & actor: Lucas Bryant year: 2004 \\
\hline 3723 & 1988 Ben david? & year: 1988 role: Chiko Ben David \\
\hline 3724 & $\begin{array}{c}\text { Frederic Etherlinck } \\
\text { movies }\end{array}$ & actor: Frederic Etherlinck \\
\hline 3725 & monumental Cceres & movie: Cceres la Monumental \\
\hline 3726 & farrell rating 4.6 & role: Jim Farrell rank: 4.6 \\
\hline 3727 & $\begin{array}{c}\text { Nageshwara Akkineni } \\
\text { movies }\end{array}$ & actor: Nageshwara Rao Akkineni \\
\hline 3728 & 8.21941 & rank: 8.2 year: 1941 \\
\hline 3729 & $\begin{array}{c}1974 \text { Brazilska } \\
\text { melodiya. }\end{array}$ & year: 1974 movie: Brazilska melodiya \\
\hline 3730 & Crimebroker Bach & movie: Crimebroker actor: John Bach \\
\hline 3731 & Chermeni & movie: Chermeni \\
\hline 3732 & Treasure buried movie & movie: Buried Treasure \\
\hline 3733 & 1987 Berger Theo & actor: Theo Berger year: 1987 \\
\hline 3734 & Blind Justice 1994. & movie: Blind Justice year: 1994 \\
\hline 3735 & Altalena movie? & movie: Altalena \\
\hline 3736 & Astaire as Himself? & actor: Fred Astaire role: Himself \\
\hline 3737 & 2002 Reggie. & year: 2002 actor: Reggie Bannister \\
\hline 3738 & 19386.7 movie & year: 1938 rank: 6.7 \\
\hline
\end{tabular}




\begin{tabular}{|c|c|c|}
\hline 3739 & Norwood Almost? & role: Norwood movie: Almost Beat \\
\hline 3740 & 1991 movie rating 5.7 & year: 1991 rank: 5.7 \\
\hline 3741 & Aleksandr? & actor: Aleksandr Berda \\
\hline 3742 & 1920 kotya role & role: Kotya year: 2002 \\
\hline 3743 & salani 2003! & actor: corso Salani year: 2003 \\
\hline 3744 & 1990 Aizpurua. & year: 1990 actor: Soledad Aizpurua \\
\hline 3745 & Orestes dalampasigan? & actor: Orestes Ojeda movie: Babae sa dalampasigan \\
\hline 3746 & after Death movie & movie: After Death \\
\hline 3747 & mongol complot & movie: Complot mongol \\
\hline 3748 & Aimez-vous autres & movie: Aimez-vous les uns les autres... mais pas trop \\
\hline 3749 & $19896.6 !$ & year: 1989 rank: 6.6 \\
\hline 3750 & movies with bulnes? & actor: Quintín Bulnes \\
\hline 3751 & gnesi aksam & movie: Aksam gnesi \\
\hline 3752 & 1980 Agreement & year: 1980 movie: Agreement \\
\hline 3753 & 2003 phillip & year: 2003 role: Phillip \\
\hline 3754 & $\begin{array}{c}\text { roles with Charlie } \\
\text { Barlett }\end{array}$ & actor: Anthony Barlett \\
\hline 3755 & Bodies Warren? & movie: Bodies actor: Warren Burton \\
\hline 3756 & Bankrotri & movie: Bankrotri \\
\hline 3757 & Langlais movies & actor: José María Langlais \\
\hline 3758 & Coroners 1997 & movie: Coroner's Camera year: 1997 \\
\hline 3759 & allen in body shop & actor: Chris Allen movie: Body Shop \\
\hline 3760 & judge it Skeet! & role: Skeet Judge \\
\hline 3761 & Chakmeh! & movie: Chakmeh \\
\hline 3762 & Cretinetti Male! & role: Cretinetti \\
\hline 3763 & Badgers Uncredited & movie: Badger's Green role: Uncredited \\
\hline 3764 & 4.51977. & rank: 4.5 year: 1977 \\
\hline 3765 & 1917 Cameron! & year: 1917 actor: Tom Cameron \\
\hline 3766 & 1928 musicals & genre: musical year: 1928 \\
\hline 3767 & 1992 rating of 3.9 & year: 1992 rank: 3.9 \\
\hline 3768 & Don Brian! & role: Don José actor: Brian Boitano \\
\hline 3769 & clean-up man & movie: Clean-Up Man \\
\hline 3770 & hanks in 1995 ! & actor: Tom Hanks year: 1995 \\
\hline 3771 & anny roman & movie: Anny - en gatepiges roman \\
\hline 3772 & best things had & movie: Best Thing You Ever Had \\
\hline 3773 & $\begin{array}{c}\text { Avant-premire } \\
\text { eurovision Male! }\end{array}$ & movie: Avant-premire eurovision gender: $\mathrm{m}$ \\
\hline 3774 & $\begin{array}{c}\text { Edwin Avedissian } \\
\text { movies }\end{array}$ & actor: Edwin Avedissian \\
\hline 3775 & Struggle with clinton & movie: Clinton: His Struggle with Dirt \\
\hline
\end{tabular}




\begin{tabular}{|c|c|c|}
\hline 3776 & cielo Luis. & movie: Cinco gallinas y el cielo actor: Luis Arata \\
\hline 3777 & Episode Hayashi 3 & role: Raizo Hayashi (Episode 3) \\
\hline 3778 & Laudator & role: Laudator \\
\hline 3779 & 1971 documentary & year: 1971 genre: Documentary \\
\hline 3780 & All Dick crockett? & movie: All Ashore actor: Dick Crockett \\
\hline 3781 & 1993 shorts & year: 1993 genre: short \\
\hline 3782 & Anderson 1996 movie & actor: Mario Andersen year: 1996 \\
\hline 3783 & storonu dollarov & movie: 1000 dollarov v odnu storonu \\
\hline 3784 & is Empresario a male? & role: Empresario De Carpa gender: $\mathrm{m}$ \\
\hline 3785 & Sugarman movie & actor: Jesse Sugarman \\
\hline 3786 & $\begin{array}{c}\text { Hauboff Joachim } \\
\text { movies }\end{array}$ & actor: Joachim Hauboff \\
\hline 3787 & $\begin{array}{c}\text { commentarist bosque } \\
\text { lmpara? }\end{array}$ & role: commentarist movie: Bajo la lmpara de bosque \\
\hline 3788 & movies with Arashi? & actor: Kanjuro Arashi \\
\hline 3789 & Murayama 1991! & actor: Noé Murayama year: 1991 \\
\hline 3790 & 2004 Linder movie & Actor: Emilio Linder year: 2004 \\
\hline 3791 & wilderness family & movie: Adventures of the Wilderness Family \\
\hline 3792 & Narutoshi Hayashi. & actor: Narutoshi Hayashi \\
\hline 3793 & John Cossar virtue! & actor: John Cossar movie: Blindness of Virtue \\
\hline 3794 & Brotherhood movies? & movie: Brotherhood \\
\hline 3795 & College Cuties & movie: College Cuties \\
\hline 3796 & service Dating? & movie: D-Cup Dating Service role: \\
\hline 3797 & Essentials Ed? & movie: Bare Essentials actor: Ed Amatrudo \\
\hline 3798 & $\begin{array}{c}\text { stevens in adult } \\
\text { movies? }\end{array}$ & genre: Adult actor: Marc Stevens \\
\hline 3799 & Brontosaurus & movie: Brontosaurus \\
\hline 3800 & Dennis 1990 & role: Dennis year: 1990 \\
\hline 3801 & Pouliot movies 1989 & actor: Carlos F. Pouliot year: 1989 \\
\hline 3802 & Bailey's 2001 movies & movie: Bailey's Mistake year: 2001 \\
\hline 3803 & Abraham as Price & actor: Jake Abraham role: Paul Price \\
\hline 3804 & carlos cruz movies & actor: Carlos Cruz \\
\hline 3805 & bloko & movie: Bloko \\
\hline 3806 & Albero Adamo & movie: Albero di Adamo \\
\hline 3807 & Allen with Pest & actor: Dayton Allen movie: Boy Pest with Osh \\
\hline 3808 & 1991 Irons & year: 1991 actor: Jeremy Irons \\
\hline 3809 & Ghost Crimson $7.1 ?$ & movie: Crimson Ghost rank: 7.1 \\
\hline 3810 & day out for buddy & movie: Buddy's Day Out \\
\hline 3811 & blaue & movie: Blaue \\
\hline 3812 & 2002 Between & movie: Between Us (2002/I) year: 2002 \\
\hline
\end{tabular}




\begin{tabular}{|c|c|c|}
\hline 3813 & verden rystede & movie: $10 \mathrm{r}$ der rystede verden \\
\hline 3814 & 1990 Diaz & year: 1990 actor: Julio Diaz \\
\hline 3815 & 1993 Sci-Fi & year: 1993 genre: Sci-Fi \\
\hline 3816 & Battleaxe & movie: Battleaxe \\
\hline 3817 & $\begin{array}{l}\text { wingham Granville } \\
\text { movies? }\end{array}$ & role: Granville Wingham \\
\hline 3818 & $\begin{array}{c}\text { Frank Sinatra as } \\
\text { Himself }\end{array}$ & role: Himself actor: Frank Sinatra \\
\hline 3819 & Bildt Anna & actor: Paul Bildt movie: Anna Favetti \\
\hline 3820 & 1931 Ariane & year: 1931 movie: Ariane \\
\hline 3821 & Badel in movies? & actor: Alan Badel \\
\hline 3822 & 19808.9. & year: 1980 rank: 8.9 \\
\hline 3823 & propias con manos & movie: Con mis propias manos \\
\hline 3824 & 1974 blume & year: 1974 actor: Ricardo Blume \\
\hline 3825 & Klavens Jeremy. & actor: Jeremy Klavens \\
\hline 3826 & Nils with bitter tea & actor: Nils Asther movie: Bitter Tea of General Yen \\
\hline 3827 & Der mit mdchen & $\begin{array}{l}\text { movie: Bettenstudent oder Was mach' ich mit den } \\
\text { Mdchen? }\end{array}$ \\
\hline 3828 & bussen movie & movie: Bussen \\
\hline 3829 & 5.6 Abrolat! & rank: 5.6 actor: Werner Abrolat \\
\hline 3830 & $\begin{array}{l}\text { Male actor named ron } \\
\text { Jeremy! }\end{array}$ & gender: $\mathrm{m}$ actor: Ron Jeremy \\
\hline 3831 & holland in 200 & year: 2000 actor: Steve Holland \\
\hline 3832 & Ingolf Gabold 2000? & actor: Ingolf Gabold year: 2000 \\
\hline 3833 & bill walker movies & actor: Bill (VII) Walker \\
\hline 3834 & aucells piscina & movie: Aucells de la piscina \\
\hline 3835 & cooper 5.5 & actor: George A. Cooper rank: 5.5 \\
\hline 3836 & L'officier de marine & role: L'officier de marine \\
\hline 3837 & 9.5 Deacon. & rank: 9.5 actor: John Deacon \\
\hline 3838 & onna Aru Kibe & movie: Aru onna role: Kibe \\
\hline 3839 & $\begin{array}{l}\text { badalamenti } \\
\text { documentary }\end{array}$ & actor: Angelo Badalamenti gener: Documentary \\
\hline 3840 & $\begin{array}{l}\text { Kleinert Volkmar } \\
\text { movies! }\end{array}$ & actor: Volkmar Kleinert \\
\hline 3841 & Castor of Castor 2002? & role: Children of Castor Fan year: 2002 \\
\hline 3842 & $\begin{array}{c}\text { movies with Phyllisha } \\
\text { Anne! }\end{array}$ & gender: f actor: Phyllisha Anne \\
\hline 3843 & 1933 captured movies & movie: Captured! year: 1933 \\
\hline 3844 & 1927 Hughes Lloyd? & year: 1927 actor: Lloyd Hughes \\
\hline 3845 & Buriano Atilio 1991 & actor: Atilio Buriano year: 1991 \\
\hline 3846 & Catene $9 ?$ & movie: Catene rank: 9 \\
\hline
\end{tabular}




\begin{tabular}{|c|c|c|}
\hline 3847 & final resistance movie & movie: C-12: Final Resistance \\
\hline 3848 & adult movies of 1992 & year: 1992 genre: Adult \\
\hline 3849 & amityville movie & movie: Amityville Horror \\
\hline 3850 & Prince Igor movie & $\begin{array}{c}\text { movie: CinemaScope Special: Polovetzian Dances From } \\
\text { 'Prince Igor' }\end{array}$ \\
\hline 3851 & 2003 Country Another & year: 2003 movie: Another Country \\
\hline 3852 & 6.8 intimes & rank: 6.8 movie: Confidences trop intimes \\
\hline 3853 & 1913 Atheist & year: 1913 movie: Atheist \\
\hline 3854 & $\begin{array}{l}\text { Chambliss Woody to } \\
\text { Yuma? }\end{array}$ & actor: Woody Chambliss movie: 3:10 to Yuma \\
\hline 3855 & Emilio 1985? & actor: Emilio Disi year: 1985 \\
\hline 3856 & attack of foot monster? & movie: Attack of the 50 Foot Monster Mania \\
\hline 3857 & Broken Fetters Dyer. & movie: Broken Fetters actor: William Dyer \\
\hline 3858 & Rodrigo movies! & actor: Rodrigo Aragón \\
\hline 3859 & malcolm as conductor & role: Himself - Conductor actor: Malcolm Arnold \\
\hline 3860 & Enchanted beauty beast & movie: Beauty and the Beast: The Enchanted Christmas \\
\hline 3861 & corsarios chip & movie: Corsarios del chip \\
\hline 3862 & farber in 2001 & year: 2001 actor: Stacey Farber \\
\hline 3863 & 1989 comedy & genre: Comedy year: 1989 \\
\hline 3864 & Clarke robert movie & actor: Robert Clarke \\
\hline 3865 & Maycock $1993 !$ & actor: Ger Maycock year: 1993 \\
\hline 3866 & $5.11976 ?$ & year: 1976 rank: 5.1 \\
\hline 3867 & about adam movie & movie: About Adam \\
\hline 3868 & 1933 Leonard & actor: Leonard Carey year: 1933 \\
\hline 3869 & Bhagyanath Master? & actor: Master Bhagyanath \\
\hline 3870 & $\begin{array}{l}\text { moive with Ray } \\
\text { Mondo }\end{array}$ & role: Mondo Ray \\
\hline 3871 & 1964 Amitis & year: 1964 movie: Amitis Particulires \\
\hline 3872 & $\begin{array}{l}\text { nero appuntamento } \\
\text { movie }\end{array}$ & movie: Appuntamento in Nero \\
\hline 3873 & $\begin{array}{l}\text { Forward son and } \\
\text { Daughter }\end{array}$ & movie: Backward Sons and Forward Daughters \\
\hline 3874 & 8 Badoni Guglielmo & rank: 8 actor: Guglielmo Badoni \\
\hline 3875 & $\begin{array}{c}\text { And the band played on } \\
\text { ? }\end{array}$ & movie: And the Band Played On \\
\hline 3876 & Luis 1951 & actor: Luis Beristáin year: 1951 \\
\hline 3877 & Myeong-jung movies ! & actor: Myeong-jung Ha role: \\
\hline 3878 & $\begin{array}{c}\text { William Hinckley } \\
\text { burned hand }\end{array}$ & actor: William Hinckley movie: Burned Hand \\
\hline 3879 & Zhixing Liu Zhenling! & role: Zhixing actor: Zhenling Liu \\
\hline 3880 & booker in the brain? & actor: Harry Booker movie: Brain \\
\hline
\end{tabular}




\begin{tabular}{|c|c|c|}
\hline 3881 & titani 5.1 & movie: Arrivano i titani rank: 5.1 \\
\hline 3882 & Dad 7.6 & role: Dad rank: 7.6 \\
\hline 3883 & 1969 Cinta MarÃa. & year: 1969 actor: María Cinta \\
\hline 3884 & 1932 Bosko & year: 1932 role: Bosko \\
\hline 3885 & $\begin{array}{l}\text { movie with camping } \\
\text { gas }\end{array}$ & movie: Camping Gas \\
\hline 3886 & 1985 North Peter? & year: 1985 actor: Peter North \\
\hline 3887 & $\begin{array}{l}\text { Comin Time Stills Nash } \\
\text { Long! }\end{array}$ & movie: Stills \& Nash: Long Time Comin \\
\hline 3888 & $\begin{array}{l}\text { movie with John } \\
\text { Alexander }\end{array}$ & actor: John Alexander \\
\hline 3889 & ANZACs war!! & movie: Colour of War: The ANZACs \\
\hline 3890 & $\begin{array}{c}1969 \text { movie with } \\
\text { Friend? }\end{array}$ & year: 1969 role: Friend \\
\hline 3891 & Coeur de coq $1947 !$ & movie: Coeur de coq year: 1947 \\
\hline 3892 & $\begin{array}{l}\text { cattle queen in } \\
\text { Montana! }\end{array}$ & movie: Cattle Queen of Montana \\
\hline 3893 & $\begin{array}{l}\text { blondes and danger } \\
\text { movie? }\end{array}$ & movie: Blondes for Danger \\
\hline 3894 & Beaumont Harry 1913 & actor: Harry Beaumont year: 1913 \\
\hline 3895 & Carradine Keith 5! & actor: Keith Carradine rank: 5 \\
\hline 3896 & Briolet 2000? & year: 2000 actor: Frédéric Briolet \\
\hline 3897 & masqu Bal 5.4 & movie: Bal masqu rank: 5.4 \\
\hline 3898 & american Civ? & movie: American Civ. -1 \\
\hline 3899 & $\begin{array}{c}\text { cooper man with no } \\
\text { name? }\end{array}$ & actor: Darin Cooper role: Man with No Name \\
\hline 3900 & Narrator as Dukakis & role: Narrator actor: Olympia Dukakis \\
\hline 3901 & Forrest 1914 & actor: Allan Forrest year: 1914 \\
\hline 3902 & acabar que gozar. & movie: A gozar, a gozar, que el mundo se va acabar \\
\hline 3903 & Narrator 8.1 . & role: Narrator rank: 8.1 \\
\hline 3904 & burnett reunion & movie: Carol Burnett Show: A Reunion \\
\hline 3905 & 1936 big noise movie? & year: 1936 movie: Big Noise \\
\hline 3906 & Tuer as Larry! & movie: Bonnes tuer role: Larry \\
\hline 3907 & $\begin{array}{c}\text { Brendas Beecher } \\
\text { Robert. }\end{array}$ & role: Brenda's Father actor: Robert Beecher \\
\hline 3908 & 2004 is aloft & year: 2004 movie: Aloft \\
\hline 3909 & Goes Anything & movie: Anything Goes \\
\hline 3910 & 1957 Abarkoroff & year: 1957 actor: Rabadan Abarkoroff \\
\hline 3911 & 1982 Charles Ned. & year: 1982 role: Ned Charles \\
\hline 3912 & Ablaze & movie: Ablaze \\
\hline 3913 & argention Yeshi? & actor: Yeshi Amao movie: Argentino en New York \\
\hline 3914 & Vishwanath Ron? & role: Vishwanath actor: Ron Kashin \\
\hline
\end{tabular}




\begin{tabular}{|c|c|c|}
\hline 3915 & $\begin{array}{c}\text { Vadim Lyubshin } \\
\text { movie? }\end{array}$ & actor: Vadim Lyubshin \\
\hline 3916 & Barr Jean-Marc 2004? & actor: Jean-Marc Barr year: 2004 \\
\hline 3917 & 1982 All star comedies & year: 1982 movie: All Star Comedy \\
\hline 3918 & allu allou rasa & movie: Allou papas allou ta rasa tou \\
\hline 3919 & 1933 carroll movie & year: 1933 actor: Earl Carroll \\
\hline 3920 & 2002 short & year: 2002 genre: short \\
\hline 3921 & Pedro is a bandit? & actor: Pedro Armendáriz Jr. movie: Bandits \\
\hline 3922 & byzance & movie: Byzance \\
\hline 3923 & 1923 battle love & year: 1923 movie: Battle of love \\
\hline 3924 & Man-Kin chow movies? & actor: Michael Chow Man-Kin \\
\hline 3925 & 2002 best bowie movie & year: 2002 Movie: Best of Bowie \\
\hline 3926 & sweet as it can be? & movie: As Sweet as Can Be \\
\hline 3927 & laciar as tito. & role: Tito actor: Elio Laciar \\
\hline 3928 & $\begin{array}{c}1985 \text { Yevgeni } \\
\text { Matveyev? }\end{array}$ & year: 1985 actor: Yevgeni Matveyev \\
\hline 3929 & The War Chocolate. & movie: Chocolate War \\
\hline 3930 & $6.21968 ?$ & rank: 6.2 year: 1968 \\
\hline 3931 & sadri alisik movie & actor: Sadri Alisik \\
\hline 3932 & movie with bookworm & movie: Bookworm \\
\hline 3933 & Short 1913 & genre: Short year: 1913 \\
\hline 3934 & Alibi 1916 & movie: Alibi year: 1916 \\
\hline 3935 & $\begin{array}{c}\text { American Farmers in } \\
\text { russia? }\end{array}$ & movie: American Farmers Visit Russia \\
\hline 3936 & 1950 drama & year: 1950 genre: Drama \\
\hline 3937 & Ni-ta ah movies . & actor: $\mathrm{Ni}$-ta $\mathrm{Ah}$ \\
\hline 3938 & chavo Banda & movie: Chavo banda \\
\hline 3939 & 5.2 Brooklyn Boys? & rank: 5.2 movie: Brooklyn Boys \\
\hline 3940 & Castle Black & movie: Black Castle \\
\hline 3941 & Cry Childs Guardian & movie: Child's Cry role: Guardian \\
\hline 3942 & Jim Grollman 2004! & actor: Jim Grollman year: 2004 \\
\hline 3943 & movie named Binari & movie: Binari \\
\hline 3944 & Ches rating 6.2 & role: Ches rank: 6.2 \\
\hline 3945 & Claquage tirements & movie: Claquage aprs tirements \\
\hline 3946 & Daniel 1994? & movie: Daniel year: 1994 \\
\hline 3947 & 1998 NA? & year: 1998 genre: Adult \\
\hline 3948 & coda di Besnehard! & movie: Colpo di coda actor: Dominique Besnehard \\
\hline 3949 & 1984 adult movie & year: 1984 genre: Adult \\
\hline 3950 & 1985 Chorus! & year: 1985 movie: Chorus Line \\
\hline 3951 & 5.1 Hopalong & rank: 5.1 role: Hopalong Cassidy \\
\hline
\end{tabular}




\begin{tabular}{|c|c|c|}
\hline 3952 & cocaine seduction & movie: Cocaine: One Man's Seduction \\
\hline 3953 & max baer jr movies & actor: Max Baer Jr. \\
\hline 3954 & Al's lads? & movie: Al's Lads \\
\hline 3955 & dance of love? & movie: Dance of Love \\
\hline 3956 & james in 1990. & actor: Frank James year: 1990 \\
\hline 3957 & Walk Angel & movie: Angel Walk \\
\hline 3958 & Logan Alambrado? & role: Juan Logan movie: Alambrado \\
\hline 3959 & Arizona Kid 6.3! & movie: Arizona Kid rank: 6.3 \\
\hline 3960 & Angela Baron & actor: Angela Baron \\
\hline 3961 & kardesler Acar & movie: Acar kardesler \\
\hline 3962 & 19937.4 rating & year: 1993 rank: 7.4 \\
\hline 3963 & girl in basement movie? & movie: Basement Girl \\
\hline 3964 & canada Natural film clo & $\begin{array}{l}\text { movie: Canada Naturally: The Film (Part One) role: } \\
\text { Himself/Proprietor of the clo }\end{array}$ \\
\hline 3965 & Alderman movie? & actor: Ralph J. Alderman \\
\hline 3966 & 7.4 Buckaroo bugs? & rank: 7.4 movie: Buckaroo Bugs \\
\hline 3967 & edward Fox $1972 ?$ & actor: Edward Fox year: 1972 \\
\hline 3968 & Graffiti American & movie: American Graffiti \\
\hline 3969 & 2004 Fordwood & year: 2004 actor: Nicola Fordwood \\
\hline 3970 & Daniel Dancer? & actor: Daniel Negrin role: Dancer \\
\hline 3971 & $19993.9 ?$ & year: 1999 rank: 3.9 \\
\hline 3972 & $\begin{array}{l}4.5 \text { Detectives } \\
\text { Clubhouse? }\end{array}$ & rank: 4.5 movie: Clubhouse Detectives \\
\hline 3973 & Branco vigliacchi & movie: Branco di vigliacchi \\
\hline 3974 & Choo Choo Swing & movie: Choo Choo Swing \\
\hline 3975 & Ribas 1933? & actor: Pery Ribas year: 1933 \\
\hline 3976 & Biscot Georges movies! & actor: Georges Biscot \\
\hline 3977 & 1920 Hank mann movie & year: 1920 actor: Hank Mann \\
\hline 3978 & Giono Onorato & movie: Ami Giono: Onorato \\
\hline 3979 & 1934 Merab. & year: 1934 actor: Merab Beburishvili \\
\hline 3980 & Baillot Pierre Blind! & actor: Pierre Baillot movie: Blind Alley \\
\hline 3981 & Civic Bruce Robert? & movie: Civic Duty actor: Bruce Robert Cole \\
\hline 3982 & 1998 Jean. & year: 1998 role: Jean \\
\hline 3983 & 1990 bruce lee movie & year: 1990 movie: Bruce Lee - Best of the Best \\
\hline 3984 & 1970 Norman movie & year: 1970 role: Norman \\
\hline 3985 & 1997 que Gritava? & year: 1997 movie: Cego que Gritava Luz \\
\hline 3986 & Coqueta $1949 ?$ & movie: Coqueta year: 1949 \\
\hline 3987 & chin Aau 7.2 & movie: Aau chin rank: 7.2 \\
\hline 3988 & Frog Milhouse. & role: Frog Milhouse \\
\hline 3989 & Felipe 1993! & actor: Felipe Ahedo year: 1993 \\
\hline
\end{tabular}




\begin{tabular}{|c|c|c|}
\hline 3990 & $\begin{array}{c}\text { movie with Rey } \\
\text { Fernando? }\end{array}$ & actor: Fernando Rey \\
\hline 3991 & Apfel Oscar 6.7. & actor: Oscar Apfel rank: 6.7 \\
\hline 3992 & Girl 1940? & role: Girl year: 1940 \\
\hline 3993 & 1973 Brazdat! & year: 1973 movie: Brazdat \\
\hline 3994 & 1969 Alexander. & year: 1969 role: Alexander Propritsjin \\
\hline 3995 & Fritz Hubert movie. & actor: Fritz Hubert \\
\hline 3996 & buy a gun movie? & role: movie: Buying a Gun \\
\hline 3997 & Viviens Father 1940. & role: Vivien's Father year: 1940 \\
\hline 3998 & Benoit 2005? & role: Benoit year: 2005 \\
\hline 3999 & 1957 Amre? & year: 1957 movie: Amre victoire \\
\hline 4000 & 6.6 Rober Alexander & rank: 6.6 actor: Robert Alexander \\
\hline 4001 & 1938 with rating 6.4 & year: 1938 rank: 6.4 \\
\hline 4002 & Charles Cross? & actor: Charles Brandt movie: Cross Bearer \\
\hline 4003 & 5.11957 & rank: 5.1 year: 1957 \\
\hline 4004 & itan to violi pouli & movie: An itan to violi pouli \\
\hline 4005 & Burns Neal in comedy & actor: Neal Burns genre: comedy \\
\hline 4006 & 1990 McCarty & year: 1990 actor: Conan McCarty \\
\hline 4007 & 1970 Cordoba & year: 1970 movie: Cannon for Cordoba \\
\hline 4008 & 1913 with role Boireau & year: 1913 role: Boireau \\
\hline 4009 & 2003 rating 8.3 & year: 2003 rank: 8.3 \\
\hline 4010 & sangre la fuego $1990 ?$ & movie: Con el fuego en la sangre year: 1990 \\
\hline 4011 & Choi Min movies & actor: Min Choi \\
\hline 4012 & 1969 Champagne! & year: 1969 movie: Champagne galoppen \\
\hline 4013 & Bin Amatsu & actor: Bin Amatsu \\
\hline 4014 & 1969 Hiroyuki Nagato. & year: 1969 actor: Hiroyuki Nagato \\
\hline 4015 & Croquette & movie: Croquette \\
\hline 4016 & $\begin{array}{l}\text { Hollywood and Duck } \\
\text { Daffy. }\end{array}$ & movie: Daffy Duck in Hollywood \\
\hline 4017 & Bedroom Dee Pamela? & movie: Bedroom Bondage actor: Pamela Dee \\
\hline 4018 & coleman 1933 & year: 1933 actor: Charles Coleman \\
\hline 4019 & Alec Baldwin movies & actor: Alec Baldwin \\
\hline 4020 & chief Zulu. & role: Zulu chief \\
\hline 4021 & Erticas Cangaceiras & movie: Cangaceiras Erticas \\
\hline 4022 & Aloha 1975. & year: 1975 movie: Aloha \\
\hline 4023 & 1939 Career? & year: 1939 movie: Career \\
\hline 4024 & Adler 30 days & movie: 30 Days actor: Jerry Adler \\
\hline 4025 & $\begin{array}{l}\text { blue blood and yellow } \\
\text { backs }\end{array}$ & movie: Blue Blood and Yellow Backs \\
\hline 4026 & Alimony & movie: Alimony \\
\hline
\end{tabular}




\begin{tabular}{|c|c|c|}
\hline 4027 & movie named Bochorno & movie: Bochorno \\
\hline 4028 & 6 Dissel Werner. & rank: 6 actor: Werner Dissel \\
\hline 4029 & Barnes 5.9. & actor: Rayford Barnes rank: 5.9 \\
\hline 4030 & Victoria $1952 ?$ & role: Victoria Driver year: 1952 \\
\hline 4031 & adult movies of 1996 & genre: Adult year: 1996 \\
\hline 4032 & aqua babes movies & movie: Aqua Babes \\
\hline 4033 & $\begin{array}{c}\text { movies with Anderson } \\
\text { Hillyard }\end{array}$ & actor: Hillyard Anderson \\
\hline 4034 & $\begin{array}{l}\text { America works when } \\
\text { works }\end{array}$ & movie: America Works When America Works \\
\hline 4035 & Hendrik Arnst! & actor: Hendrik Arnst \\
\hline 4036 & Cheyenne Alvin? & movie: Cheyenne actor: John Alvin \\
\hline 4037 & Butter Again 1981 & movie: Butter Again year: 1918 \\
\hline 4038 & $\begin{array}{l}\text { adventure Clancy } \\
\text { Brown! }\end{array}$ & genre: Adventure actor: Clancy Brown \\
\hline 4039 & 1988 Edwin Newman & year: 1988 actor: Edwin Newman \\
\hline 4040 & Diamond 1942! & actor: Leo Diamond year: 1942 \\
\hline 4041 & McDowell 1927? & actor: Nelson McDowell year: 1927 \\
\hline 4042 & ben adams music & genre: music actor: Ben Adams \\
\hline 4043 & $\begin{array}{l}\text { Oe Aloha Frank } \\
\text { Borzage! }\end{array}$ & movie: Aloha Oe actor: Frank Borzage \\
\hline 4044 & $\begin{array}{c}\text { Tom Guise Sam } \\
\text { Manson }\end{array}$ & actor: Tom Guise role: Sam Mason \\
\hline 4045 & $\begin{array}{c}\text { movie called Daam } \\
\text { autos? }\end{array}$ & movie: Daam autos \\
\hline 4046 & $\begin{array}{c}\text { Nikolic Marko } \\
\text { Buntovnik. }\end{array}$ & actor: Marko Nikolic movie: Buntovnik \\
\hline 4047 & Collen Henri & actor: Henri Collen \\
\hline 4048 & Model Hand of choke & role: Hand Model movie: Choke \\
\hline 4049 & Hart 5.9 movie & actor: William S. Hart rank: 5.9 \\
\hline 4050 & conway 1988 & actor: Dan Conway year: 1988 \\
\hline 4051 & desire a blonde? & movie: Blonde Desire \\
\hline 4052 & ncarcata atmosfera & movie: Atmosfera Ncarcata \\
\hline 4053 & Ivan Christy 1919 & actor: Ivan Christy year: 1919 \\
\hline 4054 & 7.2 Biette Jean-Claude. & rank: 7.2 actor: Jean-Claude Biette \\
\hline 4055 & $\begin{array}{l}\text { Doctor Justice } \\
\text { Questionable! }\end{array}$ & movie: American Justice: A Questionable Doctor \\
\hline 4056 & Suspicion beyond & movie: Beyond Suspicion \\
\hline 4057 & coeur Tokyo 117 ! & movie: Atout coeur Tokyo pour O.S.S. 117 \\
\hline 4058 & 1982 rating 3.2 & year: 1982 rank: 3.6 \\
\hline 4059 & 1999 O'Hurley! & year: 1999 actor: John O'Hurley \\
\hline 4060 & 1993 Boludas? & year: 1993 movie: Boludas \\
\hline
\end{tabular}




\begin{tabular}{|c|c|c|}
\hline 4061 & $\begin{array}{c}\text { Mans Wife Kerrigan } \\
\text { Warren }\end{array}$ & movie: Another Man's Wife actor: J. Warren Kerrigan \\
\hline 4062 & $\begin{array}{c}\text { weiter Bohr } \\
\text { Gmeinwieser Leopold. }\end{array}$ & movie: Bohr weiter actor: Leopold Gmeinwieser \\
\hline 4063 & 1957 Venancio Moreno & year: 1957 actor: Venancio Moreno \\
\hline 4064 & 1952 Bannon & year: 1952 actor: Jim Bannon \\
\hline 4065 & chelovek nevidimka & movie: Chelovek-nevidimka \\
\hline 4066 & 1925 Ackroyd & year: 1925 actor: Jack Ackroyd \\
\hline 4067 & 1925 Brooks & year: 1925 actor: Sammy Brooks \\
\hline 4068 & creating rem lezar & movie: Creating Rem Lezar \\
\hline 4069 & Maurice 2002! & actor: Maurice Béjart year: 2002 \\
\hline 4070 & 1996 affliction! & year: 1996 movie: Affliction \\
\hline 4071 & talkie bombay & movie: Bombay Talkie \\
\hline 4072 & Halle Alpine & actor: Roy Halle movie: Alpine Rendezvous \\
\hline 4073 & Frank Union Calamari. & role: Frank movie: Calamari Union \\
\hline 4074 & Cukic D-dag & actor: Dejan Cukic movie: D-dag - Lise \\
\hline 4075 & 1970 fedtefadet! & year: 1970 movie: fedtefadet, De \\
\hline 4076 & 19647.8. & year: 1964 rank: 7.8 \\
\hline 4077 & $\begin{array}{l}1910 \text { Chantecler } \\
\text { Atraioado. }\end{array}$ & year: 1910 movie: Chantecler Atraioado \\
\hline 4078 & Intrigue Bull Harding & movie: Border Intrigue role: Bull Harding \\
\hline 4079 & $\begin{array}{l}\text { Taylor Donovan } \\
\text { Officer? }\end{array}$ & actor: Michael Taylor Donovan role: Security Officer \#1 \\
\hline 4080 & $\begin{array}{c}\text { Age Emperor } \\
\text { Biography } 1997\end{array}$ & $\begin{array}{c}\text { movie: A\&E Biography: Hadrian - Emperor of the Golden } \\
\text { Age year: } 1997\end{array}$ \\
\hline 4081 & $7.52000 ?$ & rank: 7.5 year: 2000 \\
\hline 4082 & 19818.6 & year: 1981 rank: 8.6 \\
\hline 4083 & 2002 Chip Albers. & year: 2002 actor: Chip Albers \\
\hline 4084 & $\begin{array}{l}\text { Fernando Bonfante } \\
\text { movie }\end{array}$ & actor: Fernando Bonfante \\
\hline 4085 & who is Austin howard? & actor: Austin Howard \\
\hline 4086 & cheng 1988 & actor: Kent Cheng year: 1988 \\
\hline 4087 & $\begin{array}{l}\text { Barbora Bozdech } \\
\text { Bedrich }\end{array}$ & movie: Barbora rd actor: Bedrich Bozdech \\
\hline 4088 & Sproggs role in a movie & role: Sproggs \\
\hline 4089 & big beat '64 & movie: Big Beat '64 \\
\hline 4090 & 1997 bara prata & movie: Bara prata lite year: 1997 \\
\hline 4091 & Bagland movie & movie: Bagland \\
\hline 4092 & Patron Garage 2004. & role: Parking Garage Patron year: 2004 \\
\hline 4093 & $\begin{array}{l}\text { Travesti Aquebeque } \\
\text { Jaime }\end{array}$ & role: Travesti 'Perrito' 1 actor: Jaime Aquebeque \\
\hline 4094 & 1998 siffredi & year: 1998 actor: Rocco Siffredi \\
\hline
\end{tabular}




\begin{tabular}{|c|c|c|}
\hline 4095 & 4.3 Yates Sgt. & rank: 4.3 role: Sgt. Yates \\
\hline 4096 & Sylvan Lee. & actor: Sylvan Lee \\
\hline 4097 & 2002 ablution & year: 2002 movie: Ablution \\
\hline 4098 & $\begin{array}{l}\text { Ankudinov Andrei } \\
1997\end{array}$ & actor: Andrei Ankudinov year: 1997 \\
\hline 4099 & Teacher $4.8 ?$ & role: Teacher rank: 4.8 \\
\hline 4100 & 5 Hass Christensen! & rank: 5 actor: Emil Hass Christensen \\
\hline 4101 & Frontaura Rafael 1943 & actor: Rafael Frontaura year: 1943 \\
\hline 4102 & 1933 Boyer & year: 1933 actor: Charles Boyer \\
\hline 4103 & 16-sai Aiko & movie: Aiko 16-sai \\
\hline 4104 & 4.9 Jahr & movie: Brgschaft fr ein Jahr rank: 4.9 \\
\hline 4105 & Male Concours. & gender: $\mathrm{m}$ movie: Concours Eurovision \\
\hline 4106 & Male Caretaker's. & gender: m movie: Caretaker's Dilemma \\
\hline 4107 & $\begin{array}{c}\text { Branciaroli Franco? } \\
\text { Cos } \\
\end{array}$ & actor: Franco Branciaroli movie: Cos' l'amore \\
\hline 4108 & Blind 2003? & year: 2003 movie: Blind Spot \\
\hline 4109 & Male ich Schler! & gender: m movie: Auch ich war nur ein mittelmiger Schler \\
\hline 4110 & salida un Callejn & movie: Callejn sin salida \\
\hline 4111 & Aiken Clay $2004 ?$ & actor: Clay Aiken year: 2004 \\
\hline 4112 & Lennon John Bed-In. & actor: John Lennon movie: Bed-In \\
\hline 4113 & $\begin{array}{c}1975 \\
\text { Champagnegalopp }\end{array}$ & year: 1975 movie: Champagnegalopp \\
\hline 4114 & Aksel besg! & movie: Aksel Schitz p besg \\
\hline 4115 & Avgat 2001! & movie: Avgat year: 2001 \\
\hline 4116 & Oscar Apfel Sighs & actor: Oscar Apfel movie: Bridge of Sighs \\
\hline 4117 & Card 1942 & actor: Ken Card year: 1942 \\
\hline 4118 & 1958 Roma Avenida! & year: 1958 movie: Avenida Roma \\
\hline 4119 & 194634 Luna & year: 1946 movie: Albergo Luna, camera 34 \\
\hline 4120 & Better 1997? & movie: Better Place year: 1997 \\
\hline 4121 & Lisboa Cano & movie: Cano de Lisboa \\
\hline 4122 & 7 States Marshal? & rank: 7 role: United States Marshal \\
\hline 4123 & $\begin{array}{c}\text { Adharvam! Haasan } \\
\text { Charu }\end{array}$ & actor: Charu Haasan movie: Adharvam \\
\hline 4124 & Mikalovitch Yankel Al! & actor: Yankel Mikalovitch movie: Al Tafhidunu \\
\hline 4125 & Game Crucial 1972. & movie: Columbo: The Most Crucial Game year: 1972 \\
\hline 4126 & Deluxe Sharif & movie: Bridge Deluxe with Omar Sharif \\
\hline 4127 & nuda Africa. & movie: Africa nuda \\
\hline 4128 & Carolina plata!? & movie: Carolina, la nia de plata \\
\hline 4129 & Fausto 1960. & actor: Fausto Coppi year: 1960 \\
\hline 4130 & Caresses & movie: Caresses d'Oshun \\
\hline
\end{tabular}




\begin{tabular}{|c|c|c|}
\hline 4131 & Director 1984. & role: Director year: 1984 \\
\hline 4132 & $\begin{array}{l}\text { Mark Patton Dean } \\
\text { Jimmy! }\end{array}$ & $\begin{array}{l}\text { actor: Mark Patton movie: Come Back to the Five and } \\
\text { Dime, Jimmy Dean }\end{array}$ \\
\hline 4133 & Spring Brings Sesame & movie: Big Bird Brings Spring to Sesame Street \\
\hline 4134 & Leon Ames $1945 ?$ & actor: Leon Ames year: 1945 \\
\hline 4135 & 1987 Club! & year: 1987 movie: Bye Bye Star-Club \\
\hline 4136 & Brder Agnes & movie: Agnes und seine Brder \\
\hline 4137 & Romance Bachelor's & movie: Bachelor's Romance \\
\hline 4138 & 1946 Colegialas & year: 1946 movie: Colegialas, Las \\
\hline 4139 & Driver Danger? & role: Driver movie: Appointment with Danger \\
\hline 4140 & Sadri yudum Isik & actor: Sadri Alisik movie: Bir yudum insan-Ayhan Isik \\
\hline 4141 & British Ross. & actor: Jonathan Ross movie: British Comedy Awards 1991 \\
\hline 4142 & Csaldi & movie: Csaldi tzfszek \\
\hline 4143 & Male Norman. & gender: $m$ actor: Norman Doxat-Pratt \\
\hline 4144 & 1931 Bargain & year: 1931 movie: Bargain Day \\
\hline 4145 & Male Dijkstra Perry. & gender: $\mathrm{m}$ actor: Perry Dijkstra \\
\hline 4146 & Cowboy Sullivan! & actor: Patrick Sullivan Burke movie: Castaway Cowboy \\
\hline 4147 & Banduk! & movie: Banduk \\
\hline 4148 & 1988 Orhan Alkan & year: 1988 actor: Orhan Alkan \\
\hline 4149 & 1914 Arrowhead & year: 1914 movie: Arrowhead Romance \\
\hline 4150 & Dama palmeras & movie: Dama de Elche y las palmeras \\
\hline 4151 & 1998 Moon York? & year: 1998 movie: Agro \& York: Moon Monkeys \\
\hline 4152 & 1994 Brunner? & year: 1994 actor: Michael Brunner \\
\hline 4153 & Law Against! & movie: Against the Law \\
\hline 4154 & Segura Santiago! & actor: Santiago Segura \\
\hline 4155 & od pesama? & movie: Biseri od pesama \\
\hline 4156 & Patrol 1943 & movie: Border Patrol year: 1943 \\
\hline 4157 & Bose! & movie: Bose \\
\hline 4158 & Panopea 9? & actor: Panopea Abrupta rank: 9 \\
\hline 4159 & Bet $2004 !$ & movie: Bet Runner year: 2004 \\
\hline 4160 & Ayyam elhob! & movie: Ayyam el-hob \\
\hline 4161 & Camerieri! & movie: Camerieri \\
\hline 4162 & Mannen ansikte! & movie: Beck - Mannen utan ansikte \\
\hline 4163 & at Sea Smith. & movie: All at Sea actor: Sidney Smith \\
\hline 4164 & 1968 Guzmn & movie: Cristina Guzmn year: 1968 \\
\hline 4165 & Monty Banks 1923. & actor: Monty Banks year: 1923 \\
\hline 4166 & park 1934 ! & movie: City Park year: 1934 \\
\hline 4167 & Dameshek Bahir Male! & movie: B'Yom Bahir Ro'im et Dameshek gender: m \\
\hline 4168 & 1991 Bongbatnaguene & year: 1991 movie: Bongbatnaguene \\
\hline 4169 & Male Homeless! & role: homeless man and gender: $\mathrm{m}$ \\
\hline
\end{tabular}




\begin{tabular}{|c|c|c|}
\hline 4170 & Male Jarvis Alec? & role: alec jarvis and gender: $\mathrm{m}$ \\
\hline 4171 & 1979 Americathon! & year: 1979 movie: Americathon \\
\hline 4172 & obesenih & movie: Balada obesenih \\
\hline 4173 & 4.7 Barzellette! & rank: 4.7 movie: Barzellette \\
\hline 4174 & 2001 Fekry? & year: 2001 actor: Fekry Abaza \\
\hline 4175 & 1946 Fingers! & year: 1946 movie: Beast with Five Fingers \\
\hline 4176 & Forest Part Bagtry. & movie: Another Part of the Forest role: Bagtry \\
\hline 4177 & 1936 Man Thin! & year: 1936 movie: After the Thin Man \\
\hline 4178 & Detective Boy & movie: Boy Detective \\
\hline 4179 & alojamiento crimen & movie: Crimen en el hotel alojamiento \\
\hline 4180 & Male talkh! & movie: chay-e talkh gender: $\mathrm{m}$ \\
\hline 4181 & 1914 Vacation Bill! & year: 1914 movie: Bill Spoils a Vacation \\
\hline 4182 & Live To Male? & $\begin{array}{l}\text { movie: counterfeit world: making 'to live and die in 1.a. } \\
\text { gender: } \mathrm{m}\end{array}$ \\
\hline 4183 & 1993 Christie & year: 1993 actor: Dick Christie \\
\hline 4184 & Parade! Lake. & movie: Beauty Parade actor: Arthur Lake \\
\hline 4185 & Male Biglang? & movie: biglang liko gender: $\mathrm{m}$ \\
\hline 4186 & Devils Male. & movie: china's little devils gender: $\mathrm{m}$ \\
\hline 4187 & Frank Men & actor: Frank Albertson movie: City of Silent Men \\
\hline 4188 & Eduardo 6.7. & role: Eduardo rank: 6.7 \\
\hline 4189 & 2003 Andrej. & year: 2003 role: Andrej \\
\hline 4190 & yznden sigara? & movie: Bir sigara yznden \\
\hline 4191 & 1993 Tony? & year: 1993 actor: Tony Devon \\
\hline 4192 & Pijan Vachuda & role: Pijan Vachuda \\
\hline 4193 & 7.3 poche & rank: 7.3 movie: Argent de poche \\
\hline 4194 & $\begin{array}{c}\text { Asrami Ramchana } \\
\text { lawyer? }\end{array}$ & role: on's lawyer actor: ramchana asrami \\
\hline 4195 & 1929? Bosko & movie: Bosko the Talk-Ink Kid year: 1929 \\
\hline 4196 & $\begin{array}{l}\text { Crossplot guest } \\
\text { Wedding? }\end{array}$ & movie: crossplot role: wedding guest \\
\hline 4197 & Male Baby & movie: Baby Talks gender: $\mathrm{m}$ \\
\hline 4198 & Male Sjefen & gender: $m$ role: Sjefen \\
\hline 4199 & Abdullah Hisham 2003 & actor: Hisham Abdullah year: 2003 \\
\hline 4200 & $7.2 \mathrm{Big}$ & rank: 7.2 movie: Big \\
\hline 4201 & Tricycle Lover Male. & movie: Basta Tricycle Driver... Sweet Lover gender: $\mathrm{m}$ \\
\hline 4202 & Akkineni Rao 1965! & actor: Nageshwara Rao Akkineni year: 1965 \\
\hline 4203 & Robert Fischer movies & actor: Robert Fischer \\
\hline 4204 & Elio Germano & actor: Elio Germano \\
\hline 4205 & Fuentes Manel & actor: Manel Fuentes \\
\hline 4206 & 5.5 Harrington $\mathrm{Jr}$ ! & rank: 5.5 actor: Pat Harrington Jr. \\
\hline
\end{tabular}




\begin{tabular}{|c|c|c|}
\hline 4207 & 1956 horror movie & year: 1956 genre: Horror \\
\hline 4208 & 1946 Because of Him & year: 1946 movie: Because of Him \\
\hline 4209 & serio en Asesino! & movie: Asesino en serio \\
\hline 4210 & Goyri sergio. & actor: Sergio Goyri \\
\hline 4211 & robert culp brimmer & actor: robert culp role: investigator brimmer \\
\hline 4212 & Jones Cinderella 1946. & movie: Cinderella Jones year: 1946 \\
\hline 4213 & 1916 Edward Arnold & year: 1916 actor: Edward Arnold \\
\hline 4214 & Brooks in $1982 ?$ & actor: Mel Brooks year: 1982 \\
\hline 4215 & NA Male. & year: 2001 movie: All Over Again \\
\hline 4216 & 1995 Fox Peace & year: 1995 movie: Black Fox: The Price of Peace \\
\hline 4217 & 1985 Laredo & year: 1985 movie: Carcel de Laredo \\
\hline 4218 & Banfield John 2003. & actor: John Banfield year: 2003 \\
\hline 4219 & Mario burlesk & role: Mario movie: Burlesk King \\
\hline 4220 & $\begin{array}{l}\text { this experiment is } \\
\text { Dangerous! }\end{array}$ & movie: Dangerous Experiment \\
\hline 4221 & 19572.5 & year: 1957 rank: 2.5 \\
\hline 4222 & booker 1911 ! & actor: John I. Booker year: 1911 \\
\hline 4223 & Bonde Martin 1991? & actor: Poul Martin Bonde year: 1991 \\
\hline 4224 & 36 William Atherton. & rank: 3.6 actor: William Atherton \\
\hline 4225 & Ray Grey 1924 & actor: Ray Grey year: 1924 \\
\hline 4226 & $\begin{array}{l}\text { Reiches dritten des } \\
\text { Auge }\end{array}$ & movie: Auge des dritten Reiches \\
\hline 4227 & 1916 bridesmaids & year: 1916 movie: Bridesmaid's Secret \\
\hline 4228 & Carrell Kim movies & actor: Kim Carrell \\
\hline 4229 & Barrett Allan 1917 & role: Allan Barrett year: 1917 \\
\hline 4230 & 1912 Prior Herbert & actor: Herbert Prior year: 1912 \\
\hline 4231 & Cherchez l'idole & movie: Cherchez l'idole \\
\hline 4232 & Cartoonist 4.7 & role: Cartoonist rank: 4.7 \\
\hline 4233 & Bentley Wes 8.5 & actor: Wes Bentley rank: 8.5 \\
\hline 4234 & Carradine Keith 5! & actor: Keith Carradine rank: 5 \\
\hline 4235 & 6.1 Kings book & rank: 6.1 movie: Book of Kings \\
\hline 4236 & tez miller of 2004 & year: 2004 role: Tez Miller \\
\hline 4237 & $\begin{array}{c}\text { movies with Bob } \\
\text { Balaban }\end{array}$ & actor: Bob Balaban \\
\hline 4238 & 1993 Aprile. & year: 1993 actor: Luigi Aprile \\
\hline 4239 & 1992 Richard. & year: 1992 actor: Richard Beymer \\
\hline 4240 & 2000 Host & year: 2000 role: Host \\
\hline 4241 & klhanli derler. & movie: Bana derler klhanli \\
\hline 4242 & 1983 odio piel! & year: 1983 movie: Con el odio en la piel \\
\hline 4243 & Deichmann Erica & actor: Erica Deichmann \\
\hline
\end{tabular}




\begin{tabular}{|c|c|c|}
\hline 4244 & NA $1938 ?$ & year: 1938 \\
\hline 4245 & Carmen Maxwell 1933 & actor: Carmen Maxwell year: 1933 \\
\hline 4246 & $\begin{array}{c}\text { movies with Miguel } \\
\text { Ayones }\end{array}$ & actor: Miguel Ayones \\
\hline 4247 & Carmen $4.2 ?$ & movie: Carmen nue rank: 4.2 \\
\hline 4248 & 19336.3 & year: 1933 rank: 6.3 \\
\hline 4249 & Anita 2002. & role: Anita year: 2002 \\
\hline 4250 & Demon Cipriano? & role: Demon movie: Cipriano \\
\hline 4251 & Timothy Will & role: Timothy Clancy actor: Will Armstrong \\
\hline 4252 & 1949 Alonso Ernesto. & year: 1949 actor: Ernesto Alonso \\
\hline 4253 & Driscoll $2005 ?$ & role: Lee Driscoll year: 2005 \\
\hline 4254 & 3.8 concorde airport & rank: 3.8 movie: Concorde: Airport '79 \\
\hline 4255 & $19584.6 ?$ & year: 1958 rank: 4.6 \\
\hline 4256 & $\begin{array}{c}\text { Atkinson Rowan } \\
\text { Christmas Carol } \\
\text { Christmas. } \\
\end{array}$ & $\begin{array}{c}\text { actor: Rowan Atkinson movie: Blackadder's Christmas } \\
\text { Carol }\end{array}$ \\
\hline 4257 & Hoffman Nick 2003 & actor: Nick Hoffman year: 2003 \\
\hline 4258 & Ashley 1968 & actor: John Ashley year: 1968 \\
\hline 4259 & 1980 Fyodorov? & year: 1980 actor: Oleg Fyodorov \\
\hline 4260 & 5.9 Cheats. & rank: 5.9 movie: Cheats \\
\hline 4261 & $81961 ?$ & rank: 8 year: 1961 \\
\hline 4262 & 1979 CHOMPS? & year: 1979 movie: C.H.O.M.P.S. \\
\hline 4263 & 1990 offen Alles? & year: 1990 movie: Alles offen \\
\hline 4264 & $\begin{array}{l}1995 \text { cent simon } \\
\text { cinma! }\end{array}$ & year: 1995 movie: Cent et une nuits de Simon Cinma \\
\hline 4265 & Black 1987? & movie: Black Sensations year: 1987 \\
\hline 4266 & 1950 Bud? & year: 1950 actor: Bud Abbott \\
\hline 4267 & $\begin{array}{l}\text { Righteousness of } \\
\text { Loyalty }\end{array}$ & movie: Boxers of Loyalty and Righteousness \\
\hline 4268 & 2004 Leon Black. & year: 2004 actor: Leon Black \\
\hline 4269 & Browe York Report? & $\begin{array}{c}\text { actor: Roger M. Browe movie: } 1964 \text { New York World's } \\
\text { Fair Report }\end{array}$ \\
\hline 4270 & Cook Andrew benge & role: Andrew Cook actor: Wilson Benge \\
\hline 4271 & Tit Country. & movie: Big Tit Country \\
\hline 4272 & 6.4 Akpinar Metin & rank: 6.4 actor: Metin Akpinar \\
\hline 4273 & 2002 Fortunato Bruno. & year: 2002 actor: Bruno Fortunato \\
\hline 4274 & $\begin{array}{c}\text { movies with Ted } \\
\text { Casablanca }\end{array}$ & actor: Ted Casablanca \\
\hline 4275 & 1954 bandido genereso & year: 1954 movie: Bandido generoso \\
\hline 4276 & 1945 Wilfred Kittredge & year: 1945 role: Wilfred Kittredge \\
\hline 4277 & Healy Pat 2004. & actor: Pat Healy year: 2004 \\
\hline
\end{tabular}




\begin{tabular}{|c|c|c|}
\hline 4278 & 1997 Neiges & year: 1997 movie: Cabaret Neiges Noires \\
\hline 4279 & 6.9 Chambre! & rank: 6.9 movie: Chambre verte \\
\hline 4280 & Einstein Foster? & movie: Breakfast with Einstein actor: Ben Foster \\
\hline 4281 & Nasty 18 & movie: 18 and Nasty 23 \\
\hline 4282 & 1921 Harry Handsome? & year: 1921 role: 'Handsome Harry' Brent \\
\hline 4283 & Dawn At. & movie: At Dawn \\
\hline 4284 & Chukichi & movie: Chukichi wa kaetta \\
\hline 4285 & Bernard 2005. & actor: Bernard Campan year: 2005 \\
\hline
\end{tabular}




\section{Bibliography}

[1] "Metasearch engine," [Online]. Available: http://en.wikipedia.org/wiki/Metasearch_engine. [Accessed 2001 12].

[2] "Dogpile," [Online]. Available: http://en.wikipedia.org/wiki/Dogpile. [Accessed 2001 2012].

[3] Roy Goldman, Narayanan Shivakumar, Suresh Venkatasubramanian, and Hector Garcia-Molina, "Proximity Search in Databases," In Proceedings of the 24rd International Conference on Very Large Data Bases (VLDB '98), pp. 26-37, 1998.

[4] Khine, P.T.T.; Win, H.P.P.; Tun, K.N.N., "Keyword searching and browsing system over relational databases," 2011 Sixth International Conference on Digital Information Management (ICDIM), pp. 121-126, 2011.

[5] Jeffrey Xu Yu, Lu Qin, Lijun Chang, Keyword search in Databases, Morgan \& Claypool Publishers, 2010.

[6] "Steiner Tree Problem," [Online]. Available: http://en.wikipedia.org/wiki/Steiner_tree_problem. [Accessed 1806 2012].

[7] "Graph Database," [Online]. Available: http://en.wikipedia.org/wiki/Graph_database. [Accessed 18 06 2012].

[8] Jeffrey Xu Yu, Lu Qin, Lijun Chang, "Keyword Search in Relational Databases: A Survey," IEEE Data Engineering Bulletin, pp. 67-78, 2010.

[9] Fang Liu, Clement Yu, Weiyi Meng, and Abdur Chowdhury, "Effective keyword search in relational databases," In Proceedings of the 2006 ACM SIGMOD international conference on Management of data (SIGMOD '06), pp. 563-574, 2006.

[10] Joel Coffman and Alfred C. Weaver, "Learning to rank results in relational keyword search," In Proceedings of the 20th ACM international conference on Information and knowledge management (CIKM '11), pp. 1689-1698, 2011.

[11] Phyo Thu Thu Khine; Htwe Pa Pa Win; Khin Nwe Ni Tun, "Efficient relational keyword search system," 2011 IEEE International Conference on Intelligent Computer Communication and Processing (ICCP), pp. 65-70, 2011.

[12] Veli Bicer, Thanh Tran, and Radoslav Nedkov, "Ranking support for keyword search on structured data using relevance models," In Proceedings of the 20th ACM international conference on 
Information and knowledge management (CIKM '11), pp. 1669-1678, 2011.

[13] Microsoft Research, "DBXplorer: A System for Keyword-Based Search over Relational Databases," In Proceedings of the 18th International Conference on Data Engineering (ICDE '02), pp. 5-15, 2002.

[14] G. B. S. C. A. H. C. N. P. P. a. S. S. B. Aditya, "BANKS: browsing and keyword searching in relational databases," In Proceedings of the 28th international conference on Very Large Data Bases (VLDB '02), pp. 1083-1086, 2002.

[15] A. H. a. C. Nakhe, "Keyword Searching and Browsing in Databases using BANKS," In Proceedings of the 18th International Conference on Data Engineering (ICDE '02), pp. 431-441, 2002.

[16] V. H. a. Y. Papakonstantinou, "Discover: keyword search in relational databases," In Proceedings of the 28th international conference on Very Large Data Bases (VLDB '02), pp. 670-681, 2002.

[17] A. Markowetz, Keyword Search over Relational Database, Hong Kong: HKUST Library, 2008.

[18] D. Suelmann, Keyword-based Search in Relational Database, Groningen, Amsterdamn: University of Groningen, 2009.

[19] "Dependency Injection," [Online]. Available: http://en.wikipedia.org/wiki/Dependency_injection. [Accessed 266 2012].

[20] D. A. Grossman, Information Retrieval: Algorithms and heuristics, Dordrecht: Springer, 2004.

[21] S. Buttcher, C. Clarke and G. Cormack, Information Retrieval: Implementing and Evaluating Search Engines, London: The MIT Press, 2010.

[22] E. Hatcher, O. Gospodnetic and M. McCandless, Lucene in Action, Stamford: Manning, 2009.

[23] "Elastic Search," [Online]. Available: http://www.elasticsearch.org/. [Accessed 23 June 2012].

[24] "DBsight," DBSight Inc , [Online]. Available: http://www.dbsight.net. [Accessed 23 June 2012].

[25] "Hibernate," JBOSS Community, [Online]. Available: http://www.hibernate.org/. [Accessed 23 June 2012].

[26] "LuSQL," CISTI Lab, 17 November 2008. [Online]. Available: http://lab.cisti-icist.nrccnrc.gc.ca/cistilabswiki/index.php/Lusql_v09_usage. [Accessed 23 June 2012].

[27] D. Carmel, D. Cohen, R. Fagin, E. Farchi, M. Herscovici, Y. Maarek and A. Soffer, "Static Index Pruning for Information Retrieval Systems," SIGIR '01, pp. 43-50, 2001. 
[28] B. Huang and X. Zenglin, "Allocating Inverted Index into Flash Memory for Search Engine," WWW 2011, pp. 61-62, 2011.

[29] C. Manning, P. Raghavan and H. Schutze, Introduction to Information Retrieval, New York: Cambridge University Press, 2008.

[30] S. Robertson, H. Zaragoza and M. Taylor, "Simple BM25 Extension to Multiple Weighted Field," CIKM'04, pp. 42-49, 2004.

[31] A. Honkela, "Dirichlet Distribution," 30 April 2001. [Online]. Available: https://www.hiit.fi/u/ahonkela/dippa/node95.html. [Accessed 24 August 2012].

[32] M. Smucker and J. Allan, "An Investigation of Dirichlet Prior Smoothing's," 12 December 2007. [Online]. Available: http://www.mansci.uwaterloo.ca/ msmucker/publications/SmuckerAllanSmoothing-IR548.pdf. [Accessed 24 August 2012].

[33] L. Azzopardi, M. Girolami and C. Rijsbergen, "Topic Based Language Model for ad hoc Information Retrieval," IEE, pp. 3281-3286, 2004.

[34] S. Javanmardi, J. Gao and K. Wang, "Optimizing Two-Stage Bigram Language Models for IR," WWW2010, pp. 1125-1126, 2010.

[35] C. Zhai, "Statistical Language Models for Information," Foundations and Trends in Information Retrieval, vol. 02, no. 3, pp. 137-213, 2008.

[36] M. Sperious and T. Tashiro, "Comparison of Okapi BM25 and Language Modeling Algorithms for NTCIR-6," 2007.

[37] "B-Tree," [Online]. Available: http://en.wikipedia.org/wiki/B-tree. [Accessed 3 September 2012].

[38] Saelee, J.; Boonjing, V., "A Metadata Search Approach with Branch and Bound Algorithm to Keyword Query in Relational Databases," ICCIT '09. Fourth International Conference on Computer Sciences and Convergence Information Technology, pp. 653-658, 2009.

[39] Li, Peng, Zhu, Qing, Wang, Shan, "Advanced Web and Network Technologies, and Applications: The Research on the Algorithms of Keyword Search in Relational Database," in Lecture Notes in Computer Science, Berlin / Heidelberg, Springer Berlin / Heidelberg, 2008, pp. 134-143.

[40] R. Baeza-yates and R.-N. Berthier, Modern Information retrieval: The concepts and technology behind search, New York: Addison Wesley, 2011.

[41] "Compass Project," [Online]. Available: http://www.compass-project.org/. [Accessed 23 June 2012]. 
[42] Y. Hao, D. Shuai and T. Suel, "Inverted Index Compression and Query Processing with Optimized Document Ordering," WWW 2009, pp. 401-410, 2009.

[43] R. Y. L. Song, J.-R. Wen and H.-W. Hon, "A Proximity Probabilistic Model for Information Retrieval," Microsoft Research, 2011.

[44] "Lesson 4: An Introduction to Database Management Systems," Penn State, [Online]. Available: http://www.personal.psu.edu/glh10/ist110/topic/topic07/topic07_05.html. [Accessed 3108 2012].

[45] T. Menzies, A. Dekhtyar, J. Distefano and J. Greenwald, "'Problems with Precision: A Response to "Comments on 'Data Mining Static Code Attributes to Learn Defect Predictors'"," IEEE Transactions on Software Engineering, vol. 33, no. 9, pp. 637-640, 2007.

[46] B. Schwartz, "Sample datasets for benchmarking and testing," 1 February 2011. [Online]. Available: http://www.mysqlperformanceblog.com/2011/02/01/sample-datasets-for-benchmarking-andtesting/. [Accessed 10 August 2012].

[47] "imdb.sql," webstepbook, [Online]. Available: http://www.webstepbook.com/supplements/databases/imdb.sql. [Accessed 20 August 2012]. 


\section{Index}

A

and merge-based, 14

Apache Solr, 10

Average Query Time, 49, 51, 53, 55, 57, 58, 60, 62, 63, $65,67,70,76,78,79,81,82,84,85,87,89,90,93,95$, $101,103,104,106,107,109,110,112,113,115,117$, 119

\section{B}

backward edge, 5

Backwards Expanding Search, 5

Banks, 4, 5, 6, 190, 213, 282

batch, 15

Bayes' Rule, 20

Best Match 25, 2, 21, 33, 55, 56, 58, 84, 93, 107, 109, 129

bigram language algorithm, 130

bigram language model, 23

binary independence model, 20

binary tree, 27

Bloom filter, 11

BM25. See Best Match 25

BM25F, 22

Boolean Retrieval method, 10

b-tree, 27, 30, 33, 36

\section{C}

candidate network, 7, 9

cascaded merge operation, 14

Case Studies Queries, v, 131

$\mathrm{CN}$. See candidate networks, See candidate networks

commit, 30

compression, 14, 16

Confucius Matrix, vi, vii, viii, 50, 52, 54, 55, 57, 59, 60, 62,

$64,65,66,68,70,75,76,78,79,81,82,84,85,87,89$,

$91,93,95,100,101,103,104,106,107,109,110,112$,

$113,115,117,119,124$

cosine similarity, 18, 31, 129

cross product, 27

cutoff value, 16

data rate, 29

Database, vi, vii, viii, 4, 26, 30, 33, 37, 41, 42, 45, 46, 48, $53,54,58,59,60,63,64,65,66,67,68,70,72,73,74$, $79,84,89,90,91,93,95,97,98,99,101,104,109,113$, $115,117,119,121,122,123,287,288,290$
DBSight, 4, 10, 288

DBXplorer, 4, 7, 8, 9, 288

Dijkstra, 5, 282

Dirchlet, 24

directed data graph, 4

Discover, 4, 9, 288

DocID index, 12

document vector, 18

\section{E}

Edge Weight, ix, 6

Elastic Search, 4, 10, 288

$\mathbf{F}$

File, vi, vii, viii, 32, 41, 45, 46, 47, 51, 52, 53, 56, 57, 60, 62, $63,65,67,68,70,78,82,87,90,91,93,95,103,107$, $112,115,117,119$

finite automata, 22

foreign key, 5, 7

foreign keys, 4, 26

foreign language, 42, 43

Forward index, 11

frequency index, 12

\section{G}

graph-based, 4, 6

H

hard disk, 29

HashMap, 8, 28, 29

IMDB database, 43

incremental, 15

Index Construction, iii, iv, v, vi, ix, xi, 26, 28, 29, 30, 35, 38, $42,45,46,128$

Information Retrieval, 1, 2, iii, 2, 4, 9, 11, 288, 289, 290

in-memory, 14

Interweaved Posting List, 28

inverted document frequency, 19

inverted index structure, 2, 11, 109

irrelevant, 40 


\section{J}

java, 37,43

Jelinek-Mercer, 2, 24, 25, 35

JM. See Jelinek Mercer

joins, 8, 26, 27

\section{K}

keyword query, 7

\section{$\mathbf{L}$}

language model, $2,17,22,23,25,35,43,60,62,63,70,87$, $89,110,112,113,119$

likelihood principle, 23

Lucene, 10, 288

\section{M}

machine learning algorithm, 130

maximum likelihood estimation, 23

merge operation, 14

minimal joining networks trees, 7

MJNT. See minimal joining networks trees, See minimal joining networks trees

Movielens, 43

multinomial unigram model, 23

multivariate Bernoulli Naïve Bayes model, 20

MySQL, 37, 42, 43

\section{$\mathbf{N}$}

naïve Bayes model, 23

\section{$\mathbf{P}$}

performance, 42,43

PFD, 16

phrase search, 11

plan generator, 7

porter stemmer, 39

posting list, 11, 12, 14, 15, 16, 28, 29, 30, 31, 32, 33, 39

precision, $16,21,40,41,50,68,72,94,129,130$

primary key, 27, 30, 33

probabilistic model, 17, 20, 21, 23, 33, 67

pruning method, 16

punctuation, 39, 42

\section{Q}

query time, 129 query vector, 18

\section{$\mathbf{R}$}

RAM, vi, vii, viii, 29, 42, 45, 46, 47, 50, 55, 56, 60, 65, 67,

$68,70,72,73,74,76,81,85,87,90,91,93,95,97,98$,

$99,101,106,110,115,117,119,121,122,123,129$

ranked, 1, 2, 8, 9, 18, 20, 39, 40, 41, 50

ranked retrieval algorithms, 2

ranking principle, 20

rebuild, 15, 29

recall, $39,40,50,68,72,129,130$

references. See foreign keys

regular expressions, 11

relational database management system, 26

relevant, 2, 1, 16, 18, 20, 22, 23, 24, 31, 40, 41, 51

remerge, 15

retrieval algorithms, 2, 1, 21, 26, 30, 40

Retrieval Process, ix, 40

retrieval status value, 21

retrieved, 14, 26, 39, 40, 51

S

Sakila database, 42

schema, 1, 4, 6, 7, 8, 9, 26

schema-based, 4, 7, 8, 9

signature files, 11

S-KWS, 4, 9

SMART System, 18

smoothing, 2, 22, 24, 25, 35

sort-based, 14

SQL, 1, 8, 9, 42, 131, 135, 169

Steiner Tree problem, 10

Stemming, 39

stop words, 16, 43

suffix arrays, 11

suffix trees, 11

symbol table, 8

\section{T}

term frequency weight, 19

test queries, 42

TF. See term frequency weight

time complexity, 31

tokens, 11, 16

transfer rate, 29

trials, 40, 42, 45, 46, 49, 65, 95, 101, 119

two stage Bigram model, 25 


\section{U}

Ubuntu, 37

unigram language model, 23

unique terms, 19

unranked, 40, 41, 50

user's search query, 2, 5
V

vector space model, 10, 17, 18, 19, 21, 31, 33, 43, 49, 51, $53,66,115$

W 